Fall 1970

\title{
1970 Miracle Yearbook
}

\section{Cedarville College}

Follow this and additional works at: https://digitalcommons.cedarville.edu/yearbooks

Part of the Higher Education Commons, Organizational Communication Commons, and the Public Relations and Advertising Commons

\section{Recommended Citation}

Cedarville College, "1970 Miracle Yearbook" (1970). Yearbooks. 50.

https://digitalcommons.cedarville.edu/yearbooks/50

This Book is brought to you for free and open access by DigitalCommons@Cedarville, a service of the Centennial Library. It has been accepted for inclusion in Yearbooks by an authorized administrator of DigitalCommons@Cedarville. For more information, please contact digitalcommons@cedarville.edu. 


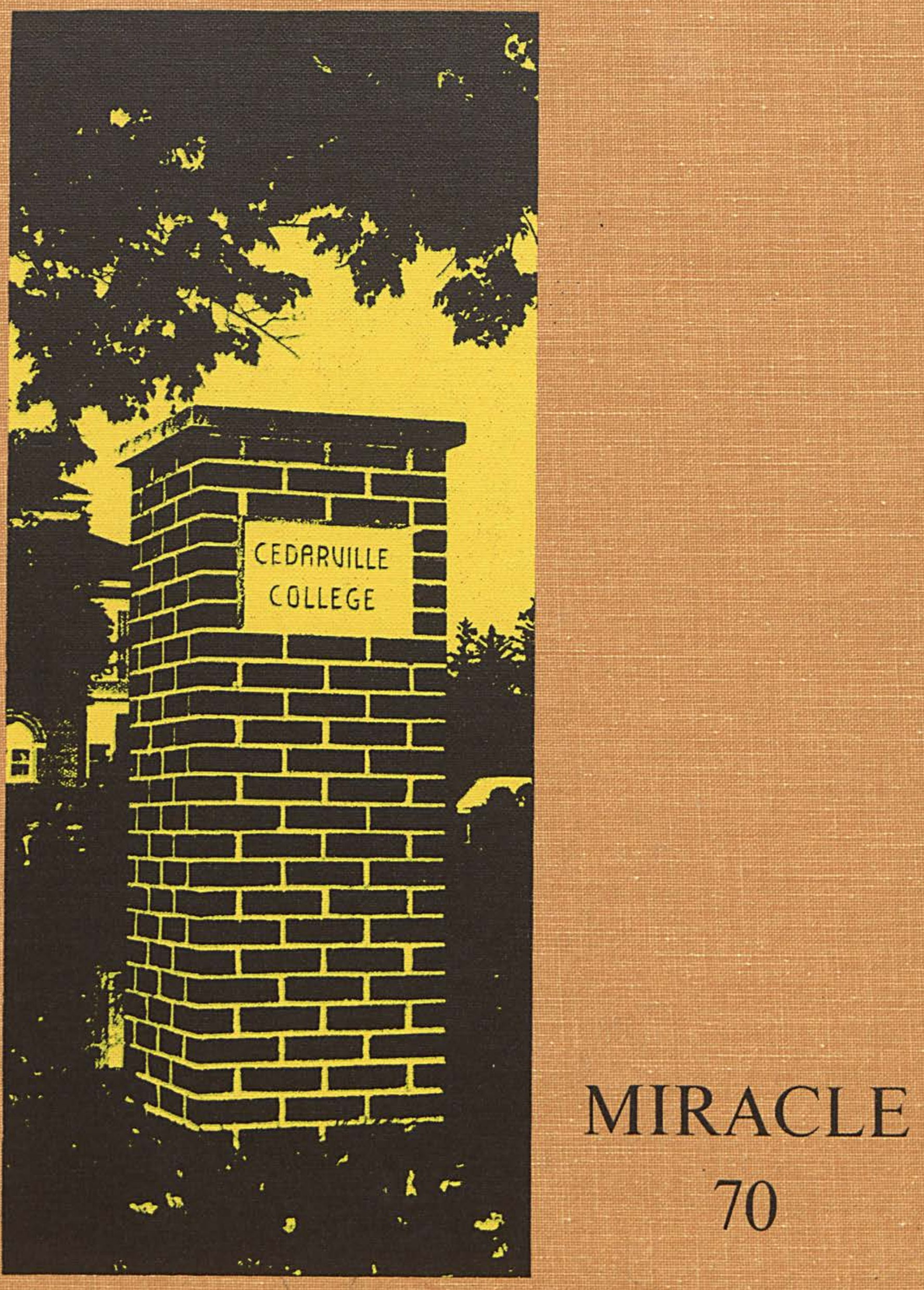




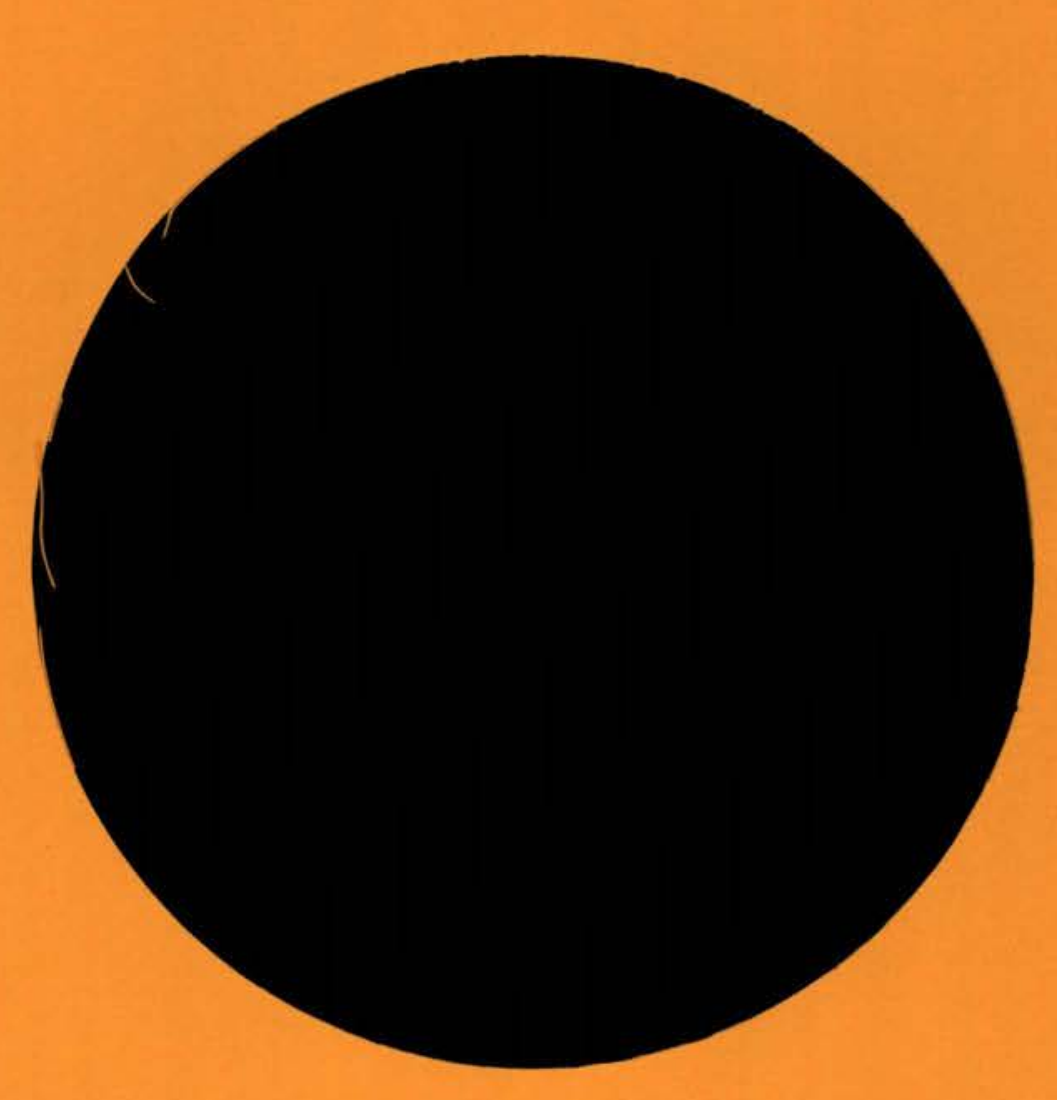




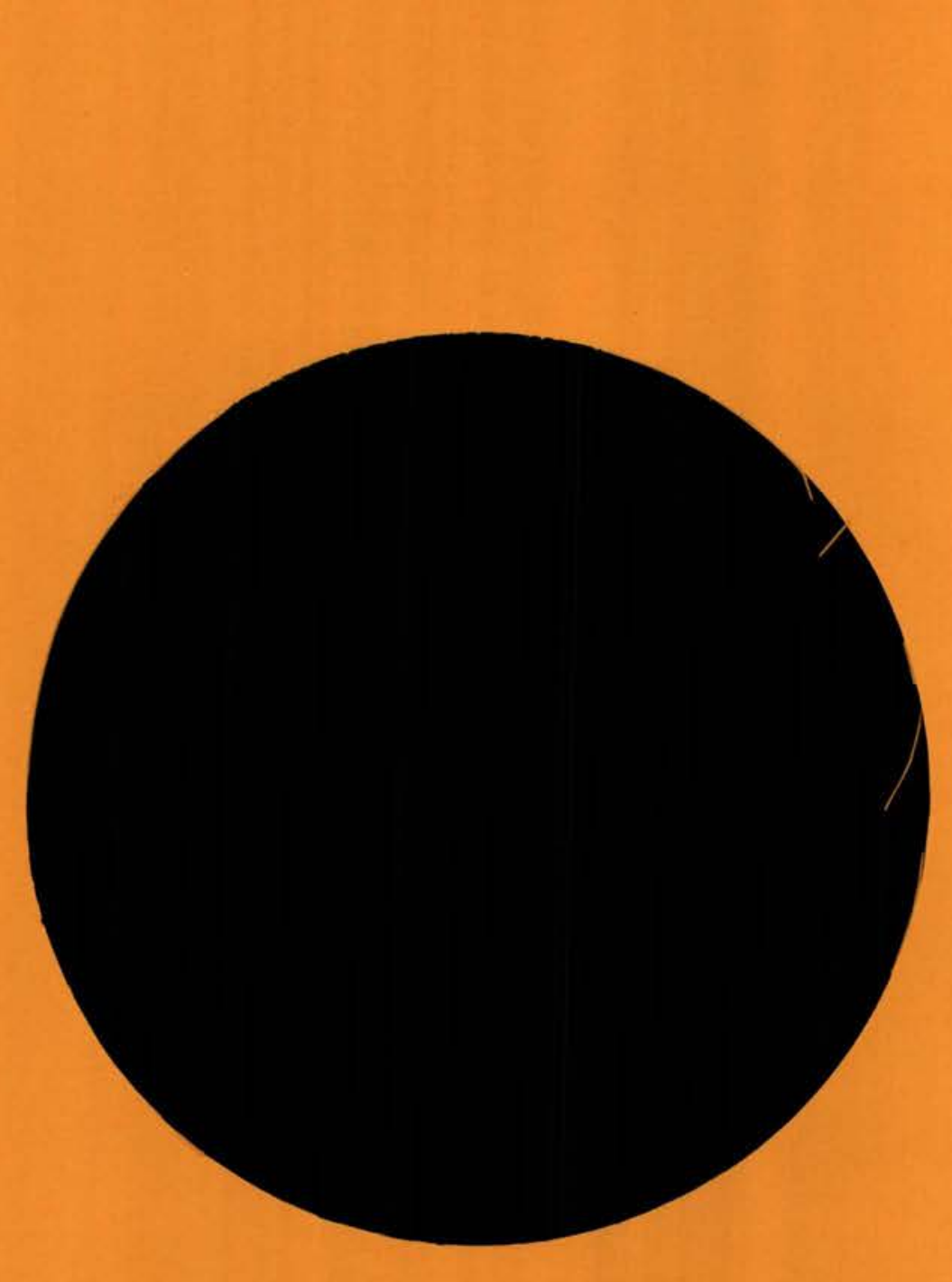


Q⿻

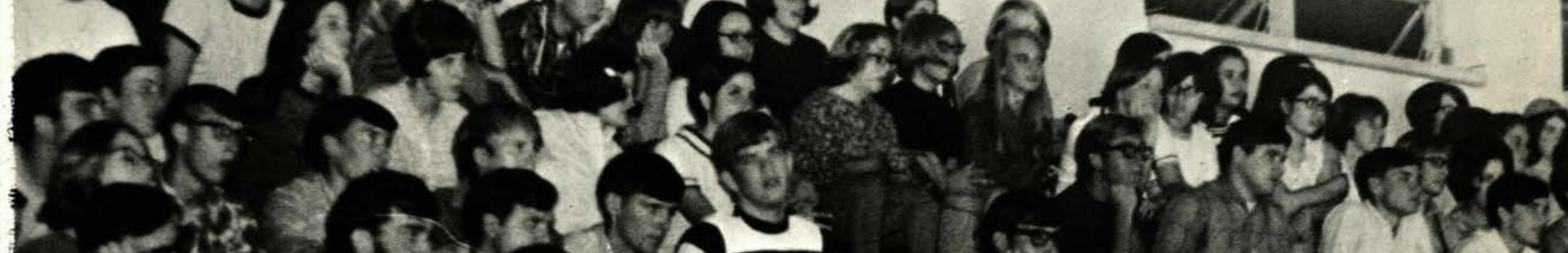

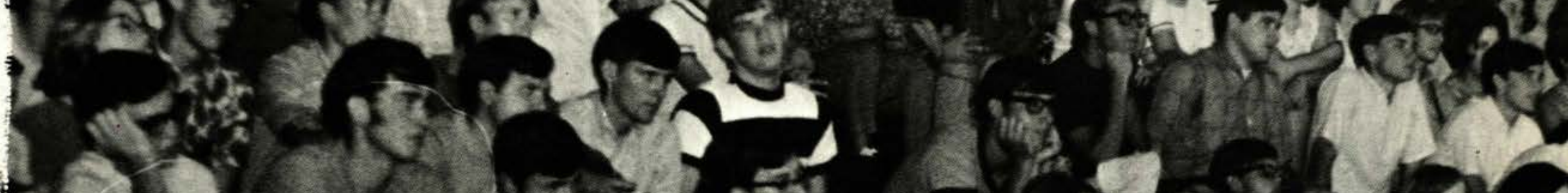

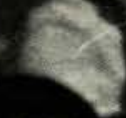

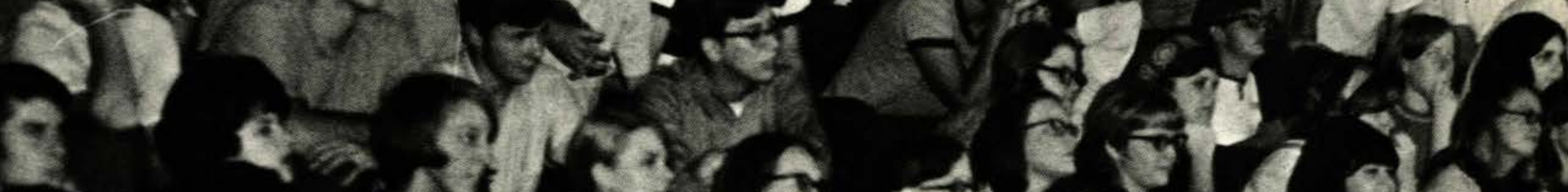

1. $=2$

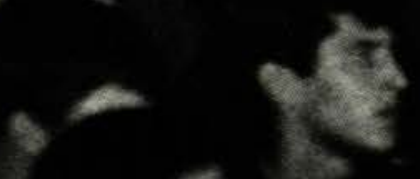
A. 19 a

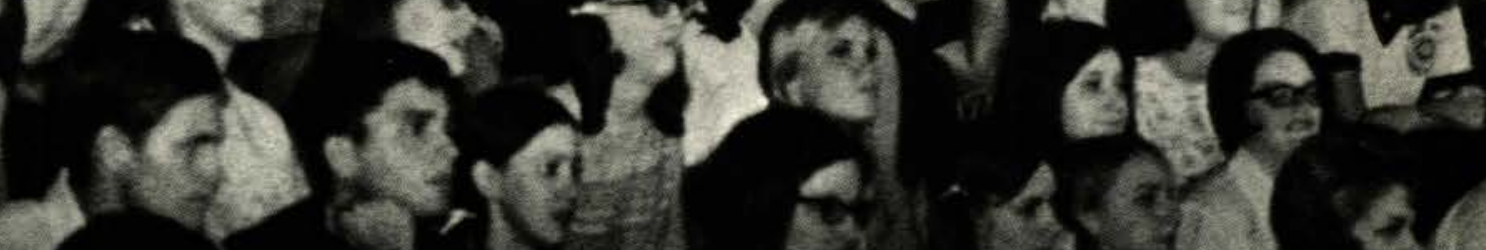

eीl acis (4) (3)

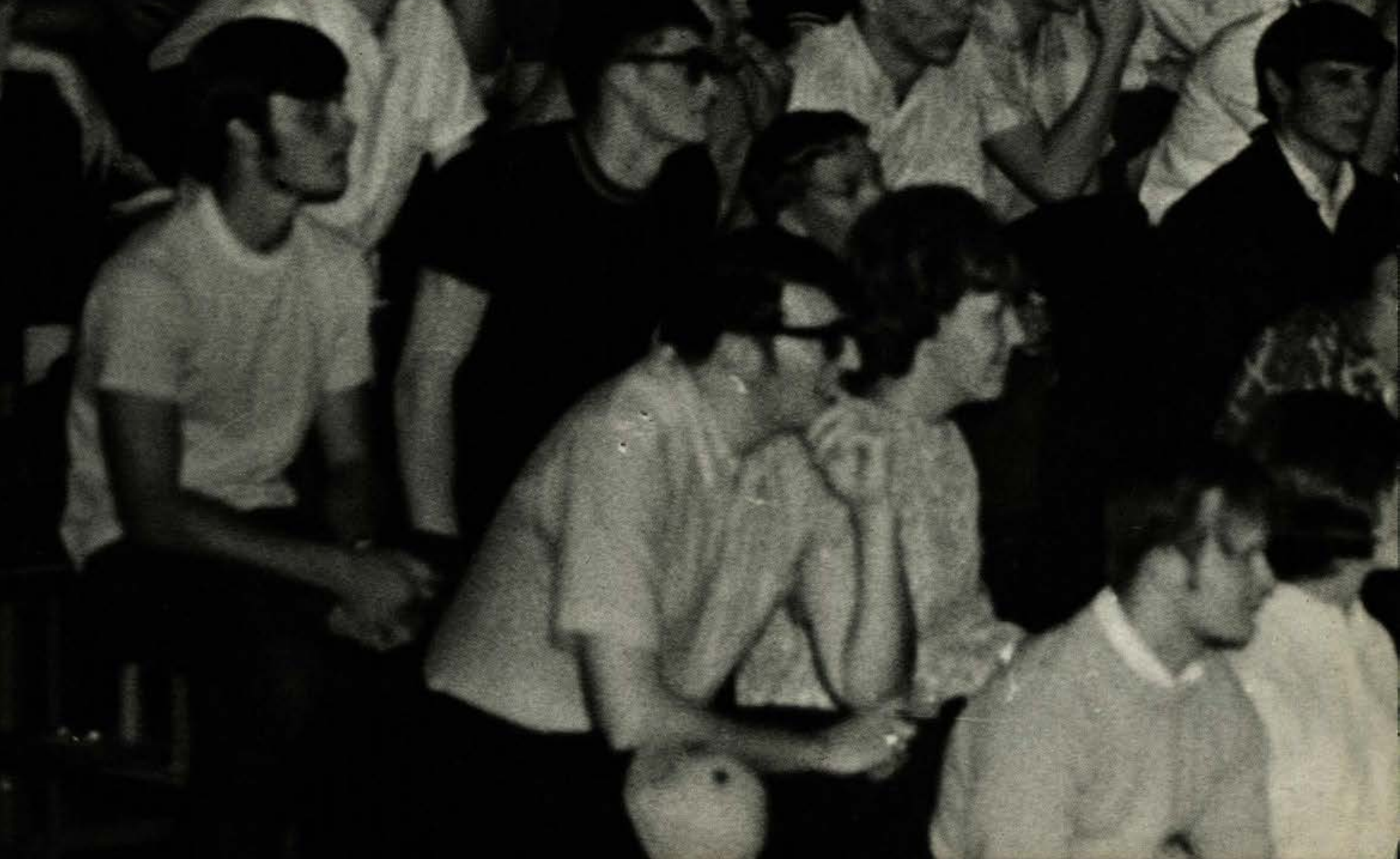




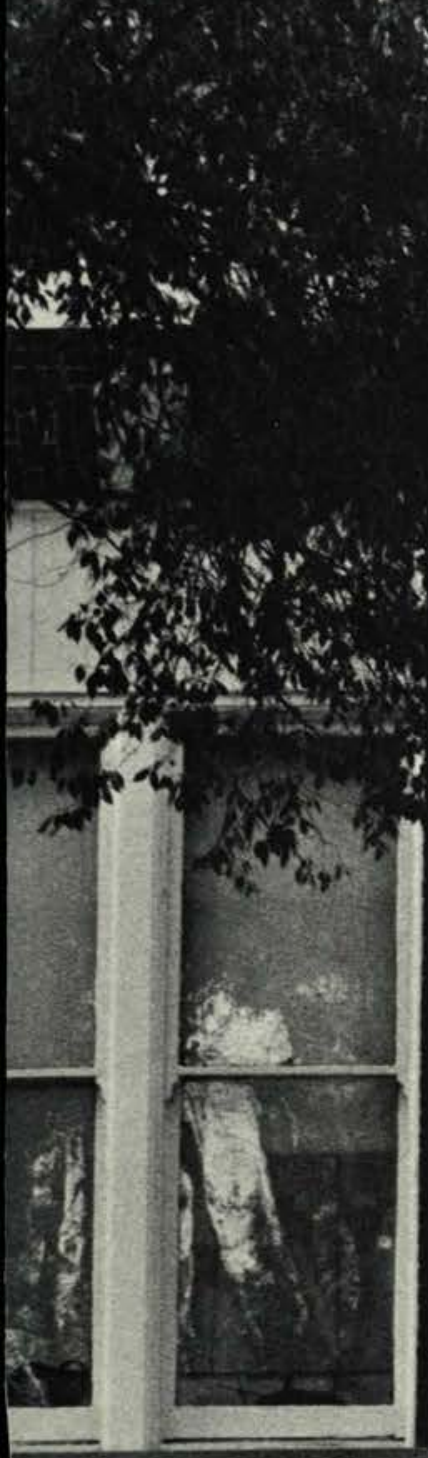

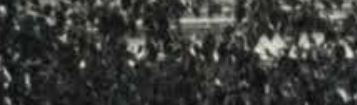




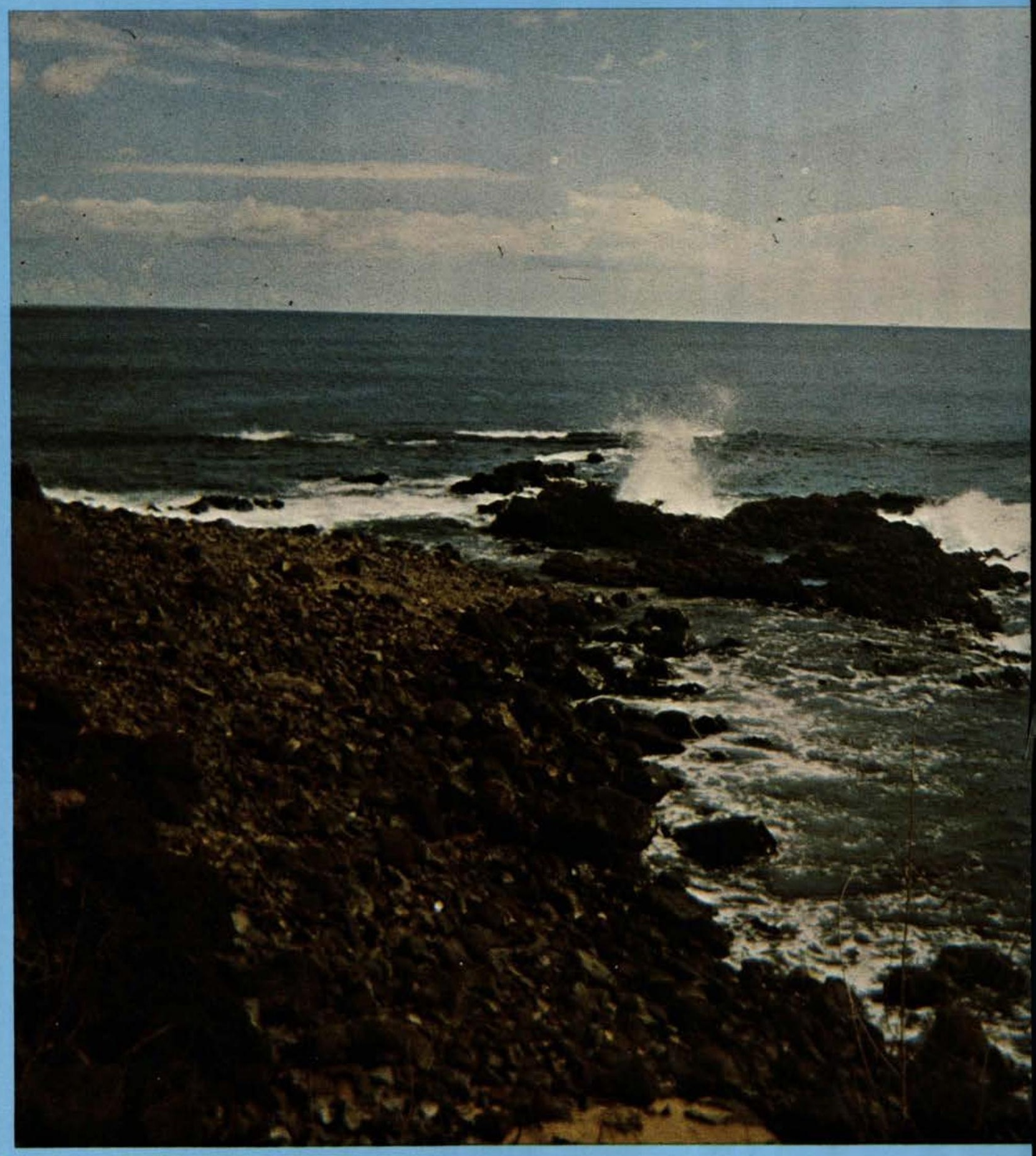




\section{独rolonue}

A Miracle. ...

That's how it all started,

The framing of a stellar galaxy

The calling out of oceans

The caressing of beasts,

Prelude to the nativity of humanity

Vouchsafed to walk the new earth

In the comeliness of repose. . .

Anon to hear the sage's echo

Of a seed to be planted in a virgin

And the birth of an only begotten Son

In something called the fullness of time

To walk an old earth

And make dark places light,

Until His darkest hour

Signaled the overture of miracles

Capstoned in a new birth,

Liable for the lives and steps,

Perceptible in the philosophy and dreams

Of Cedarville humanity

In this year of our Lord. . .

1970

Patti France 

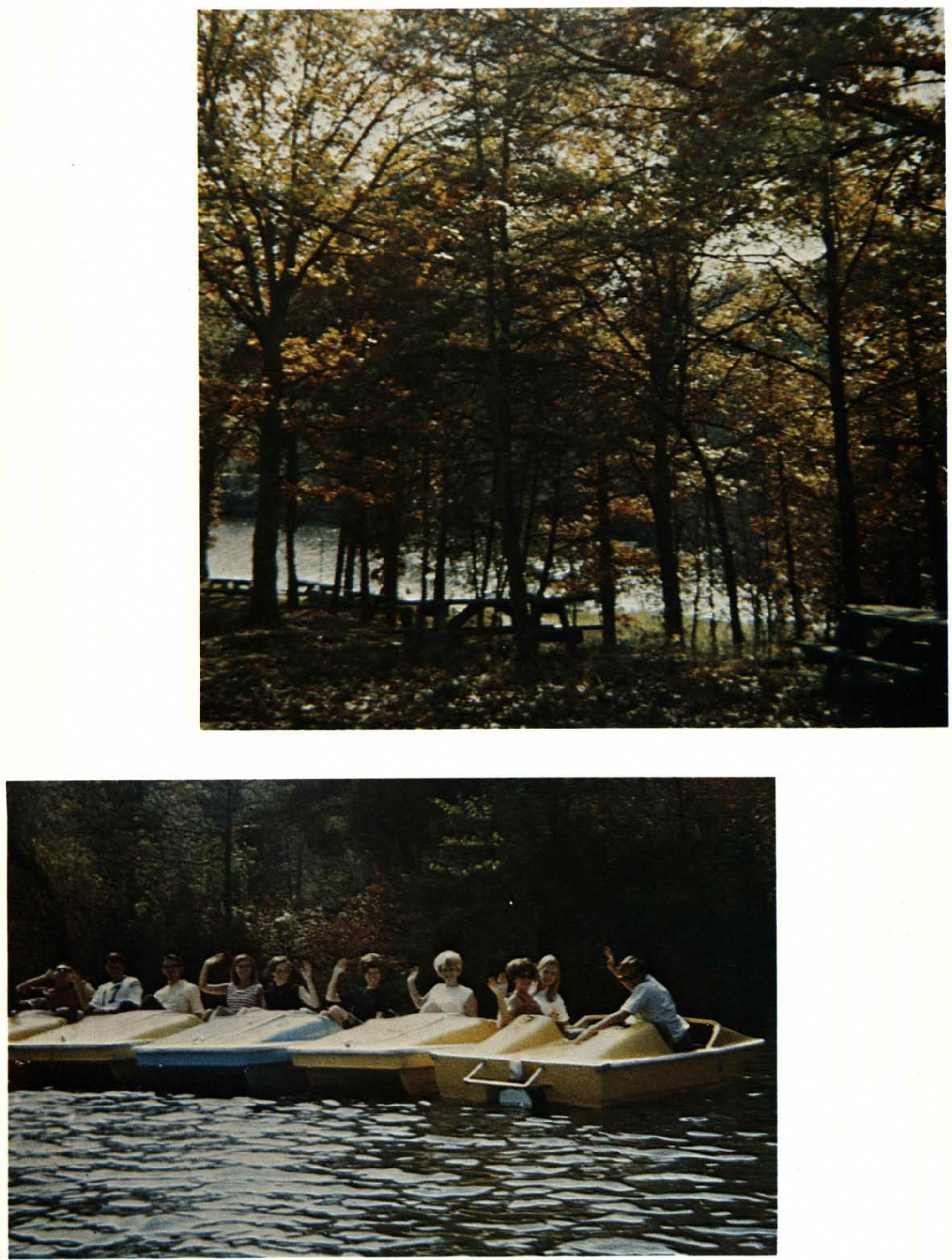

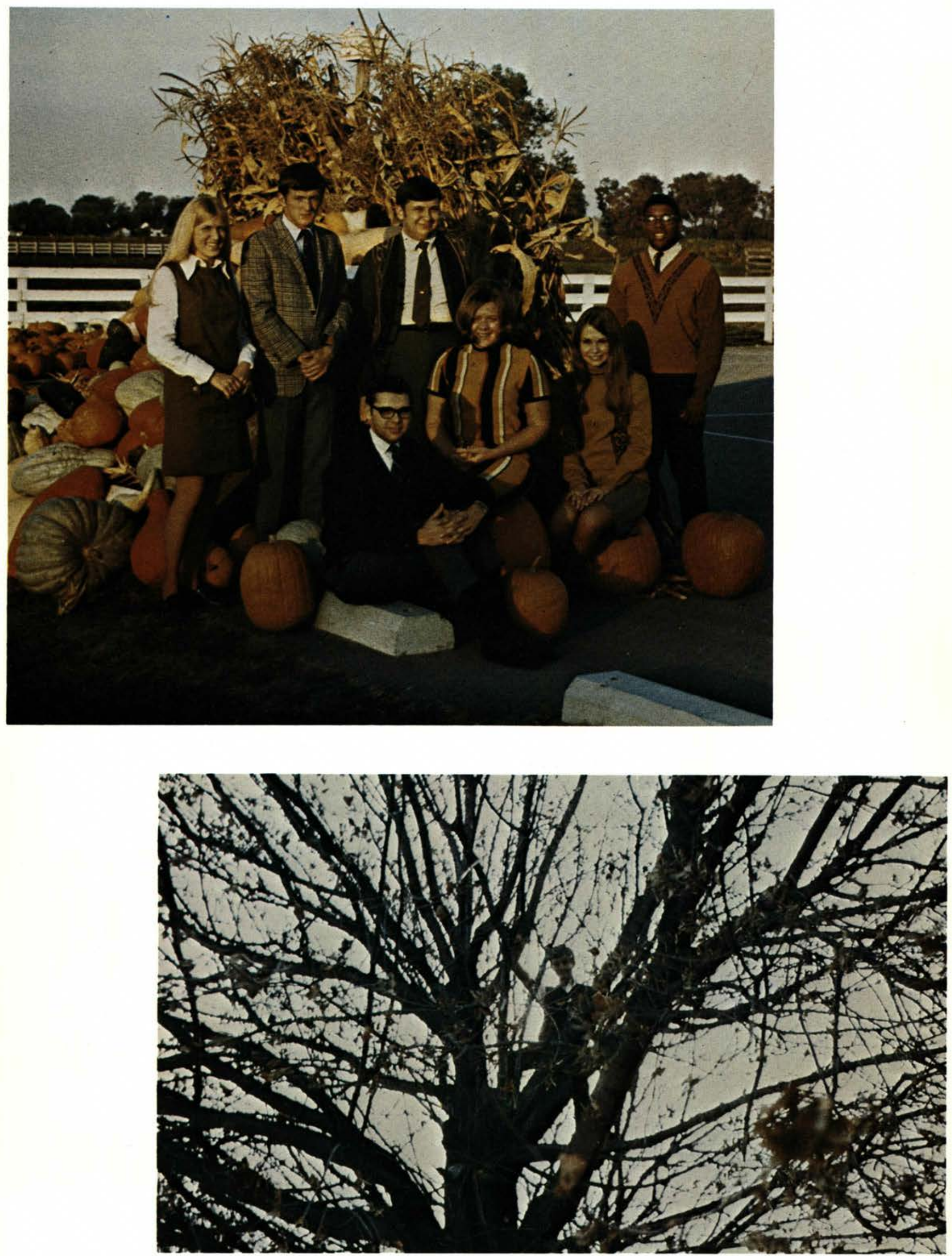


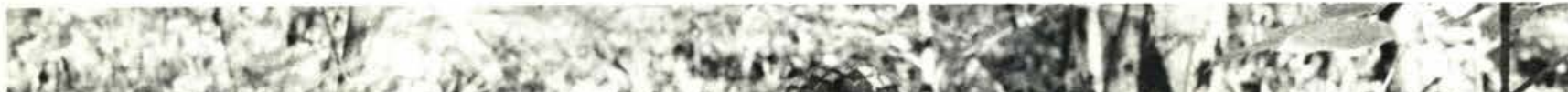
Q

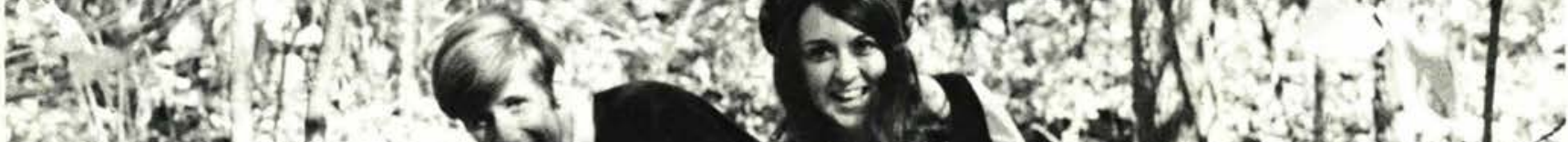

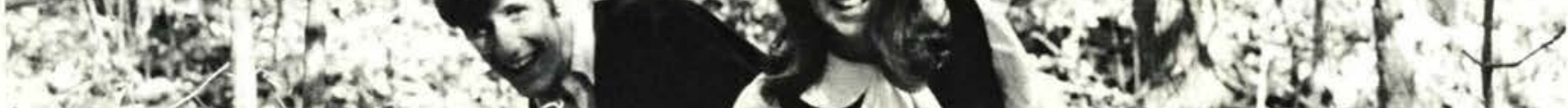

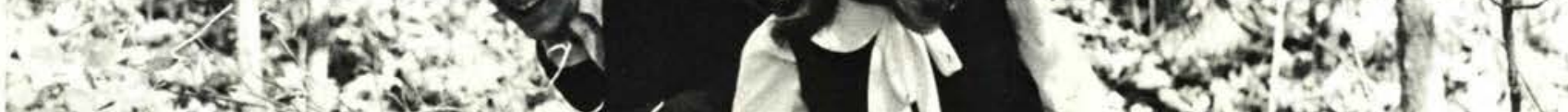

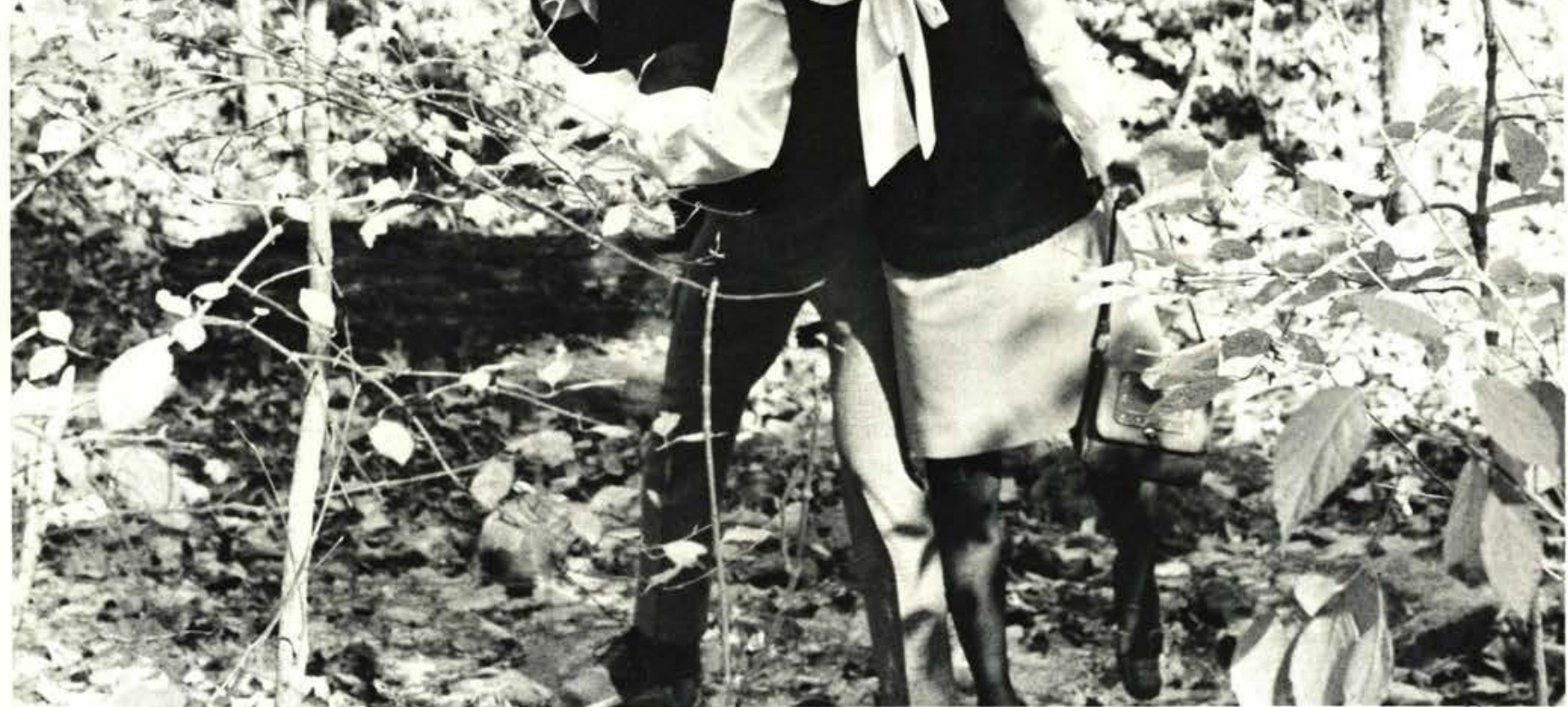
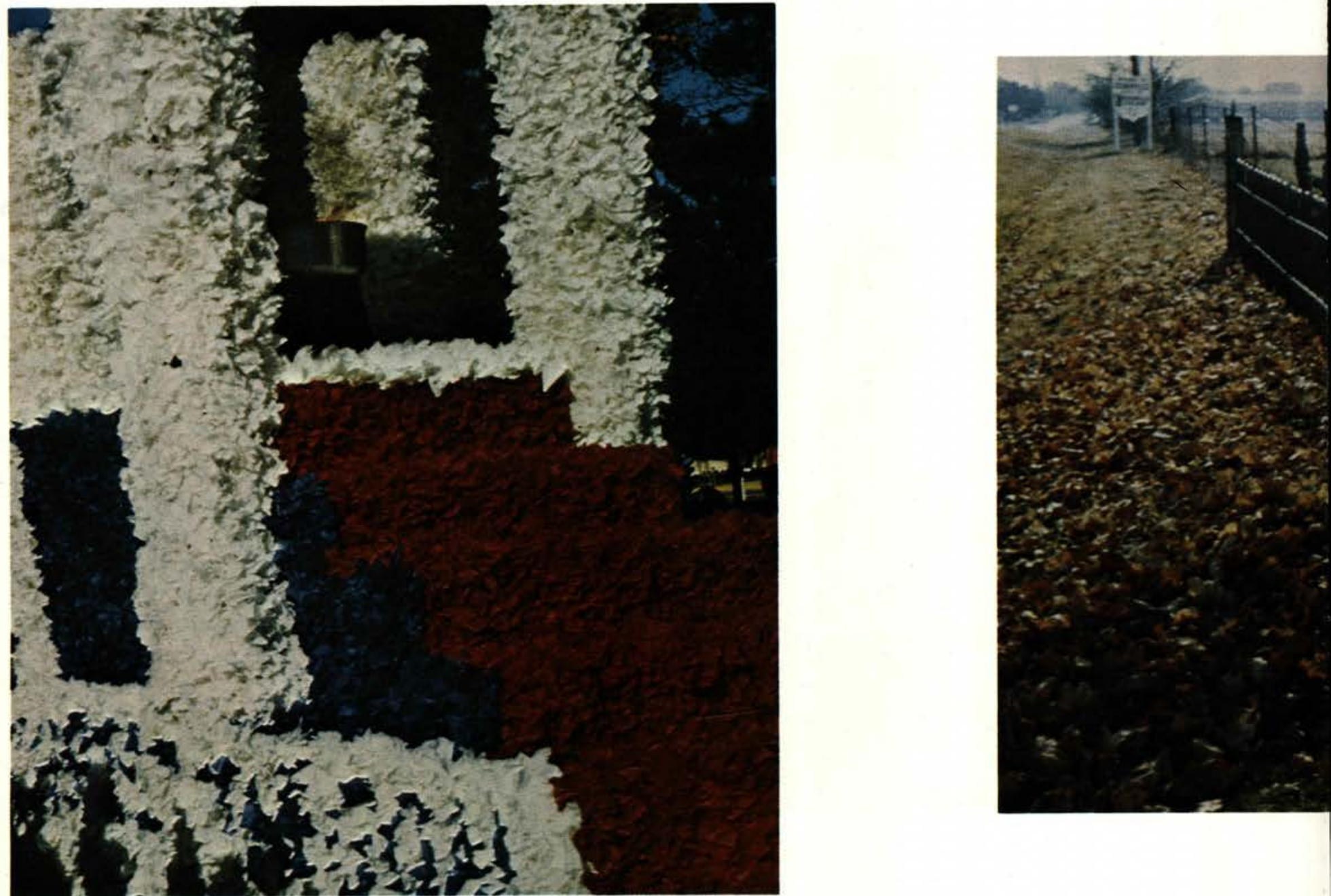

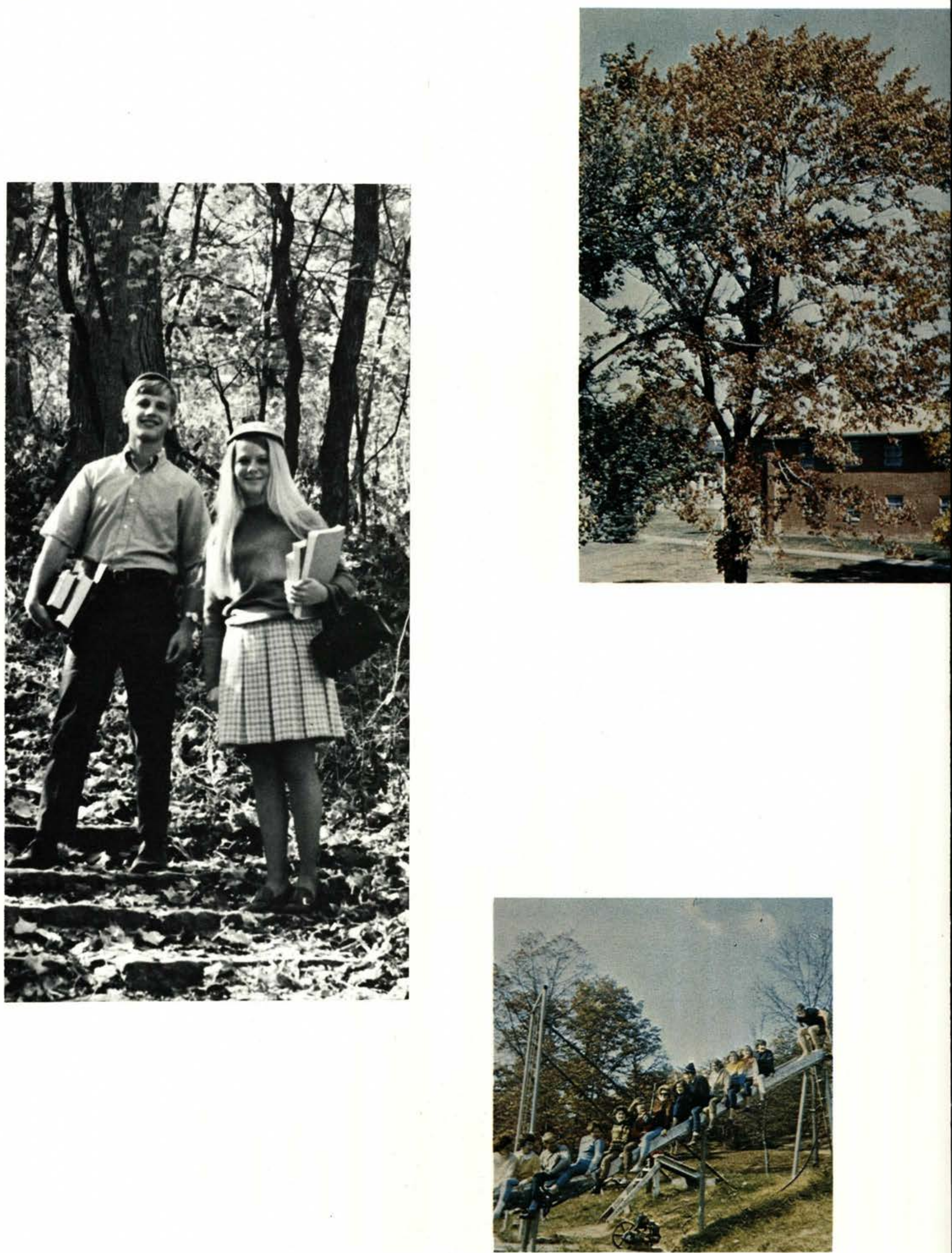

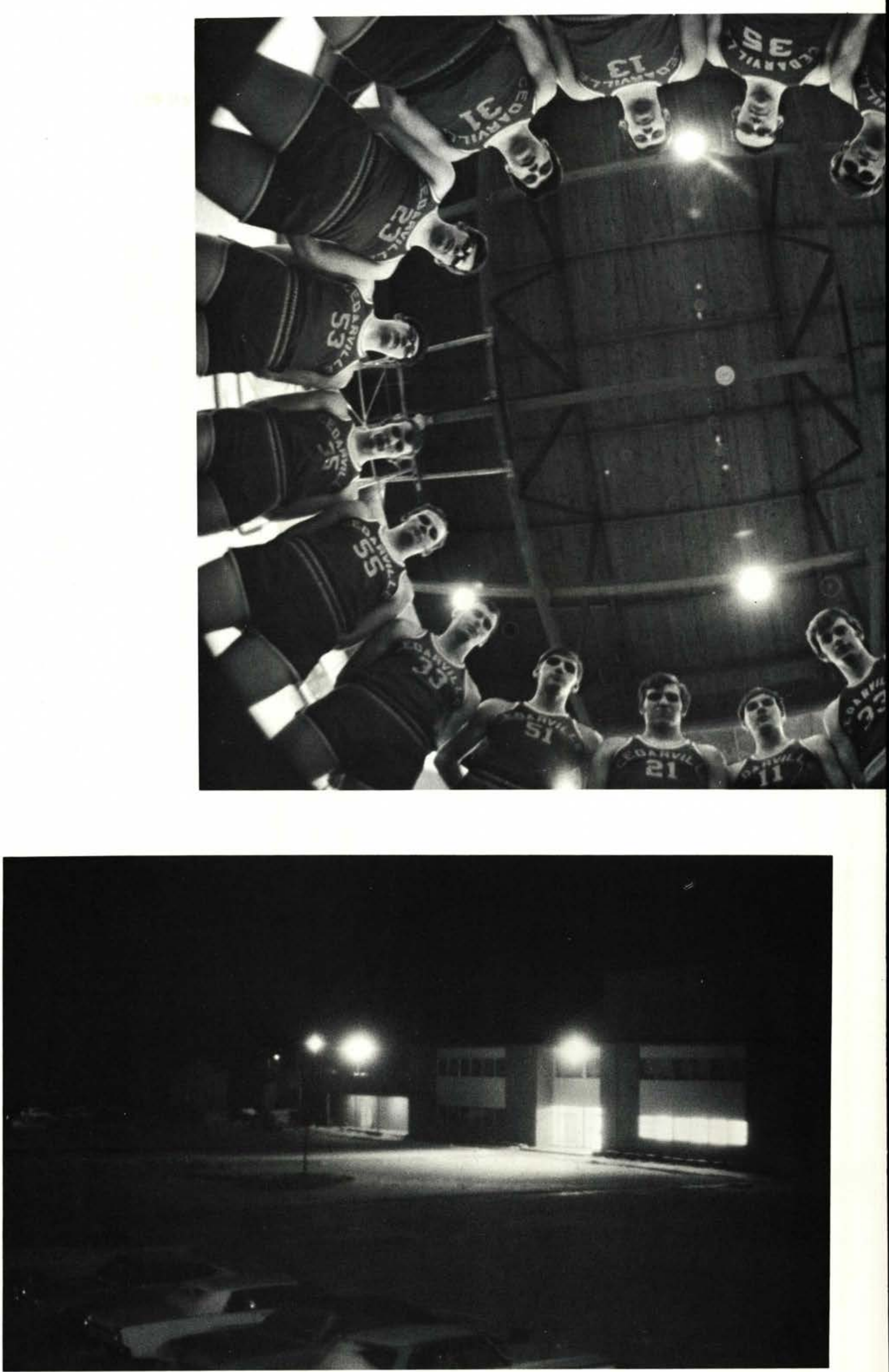

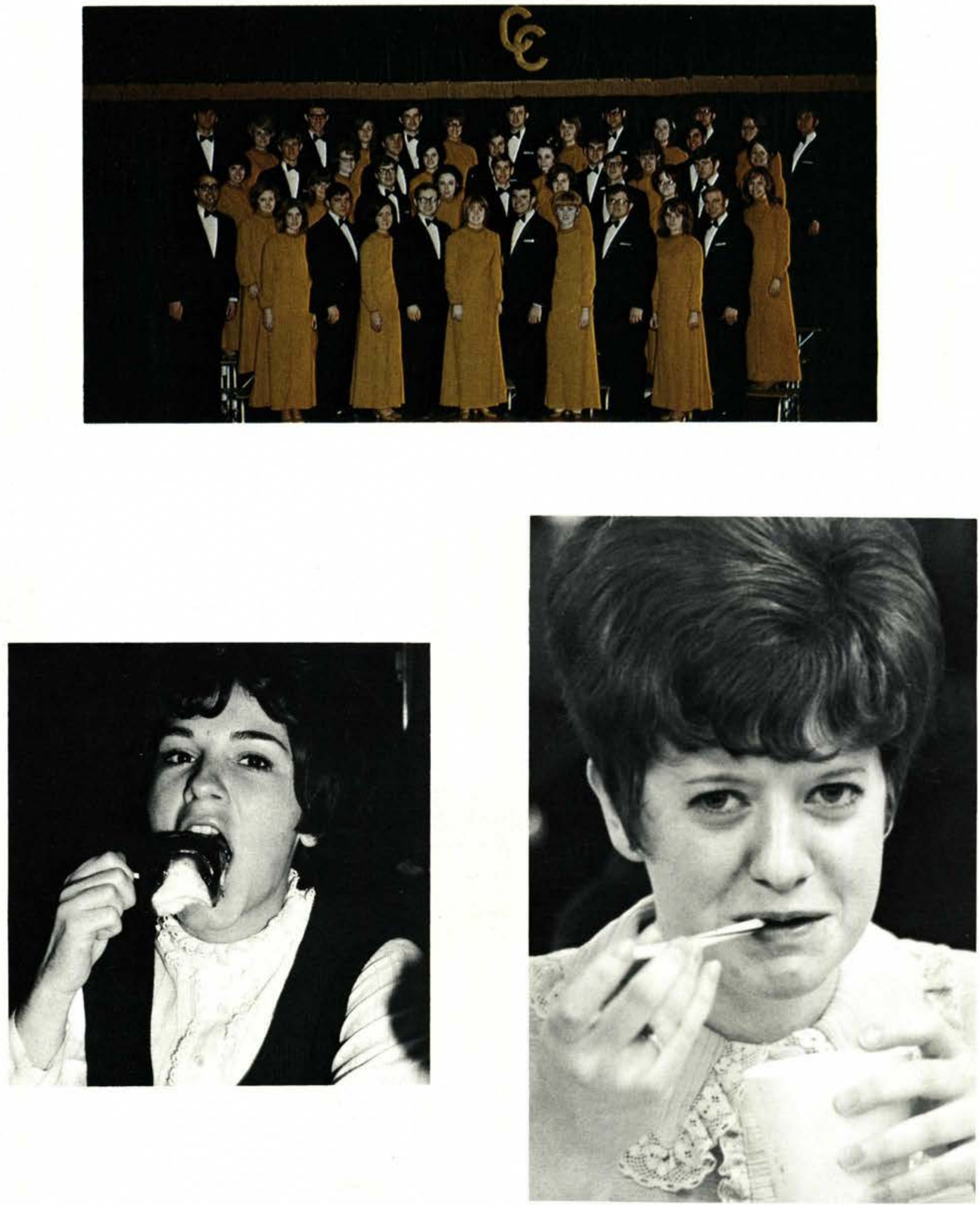

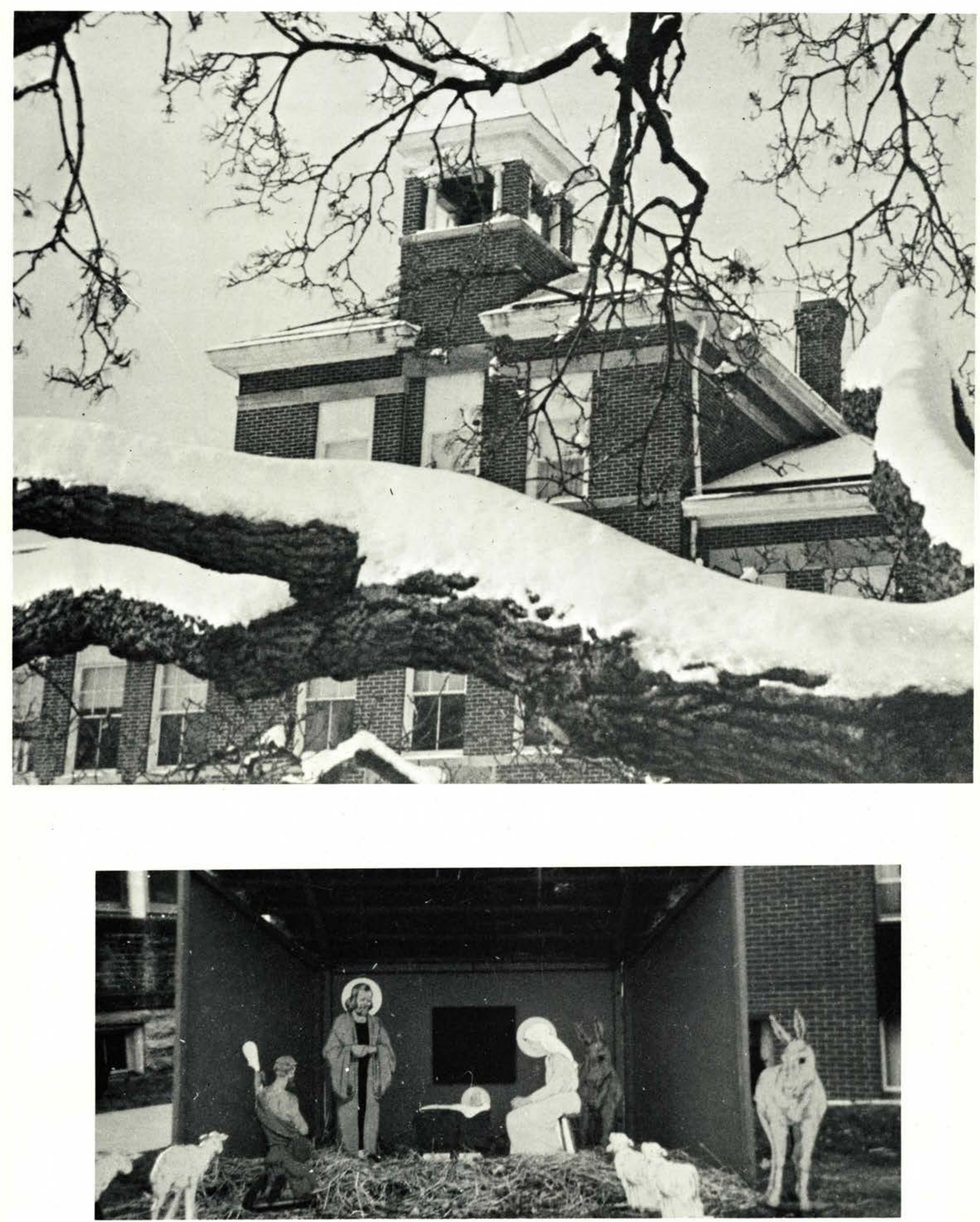
He: What'syourname?

She: Idon'tknow.

He: Whereyafrom?

She: Idon'tknow.

He: What'sthematter?

She: I'mafreshman.

$\mathrm{He}: \mathrm{Oh} \ldots$

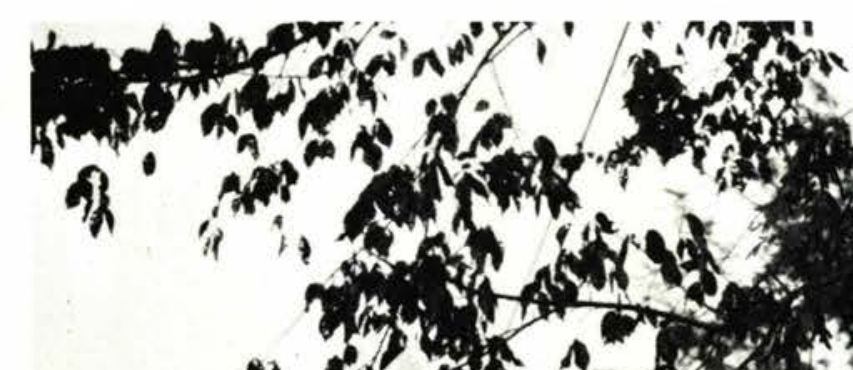

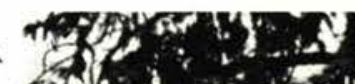

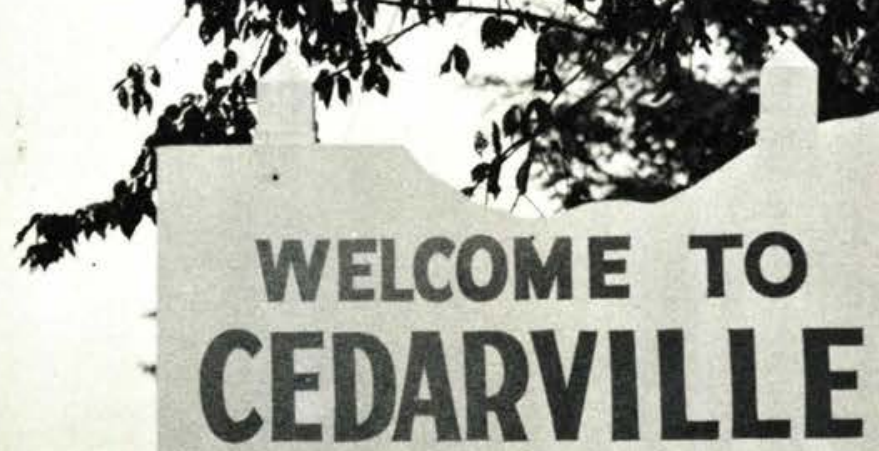

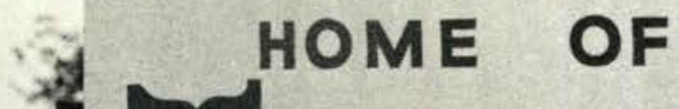

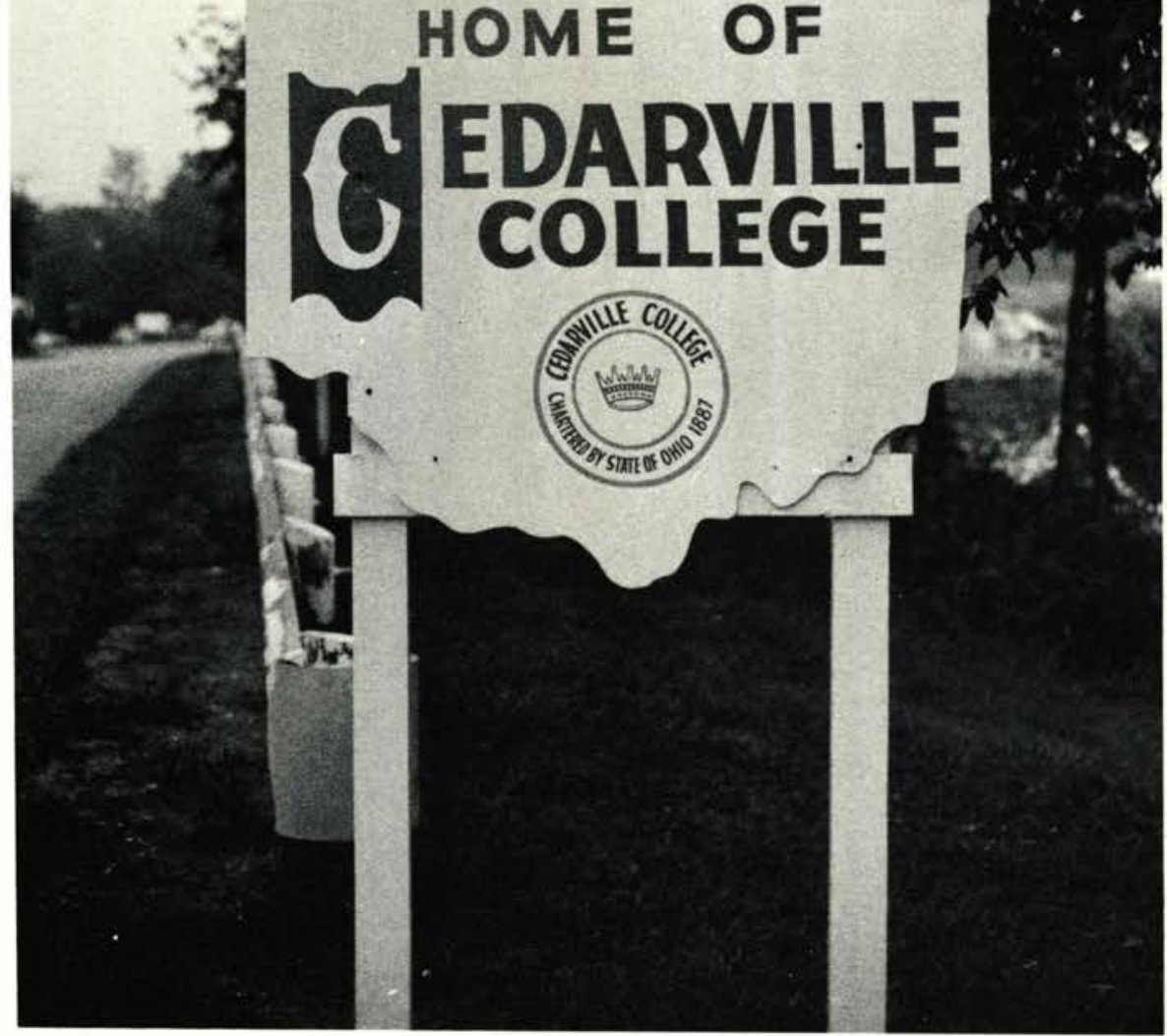

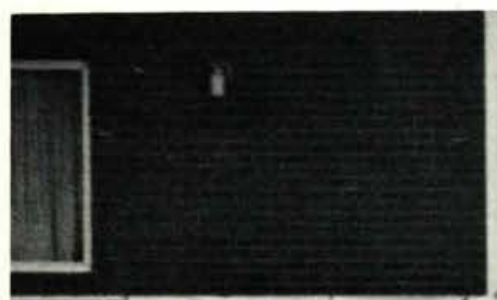
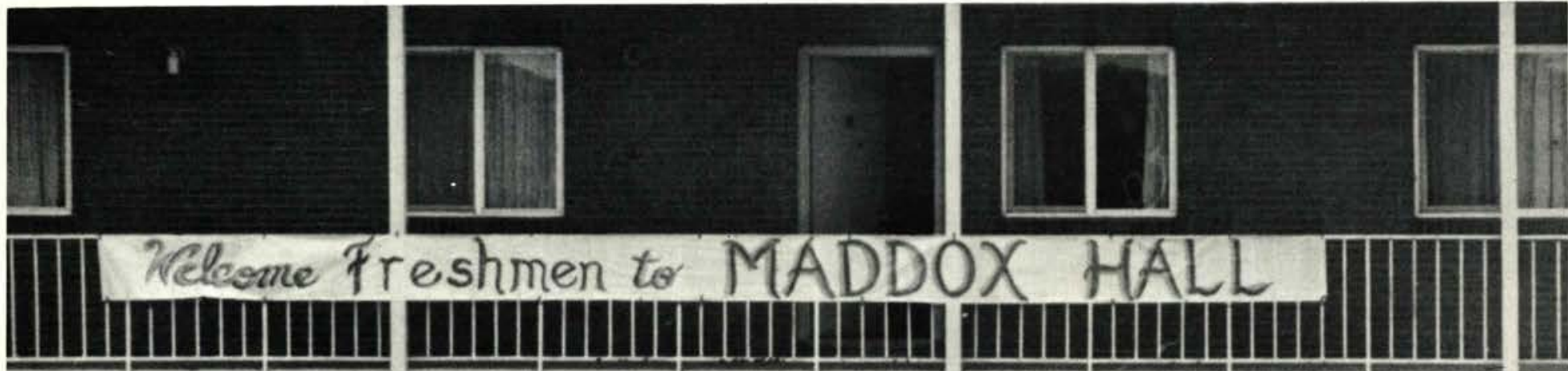

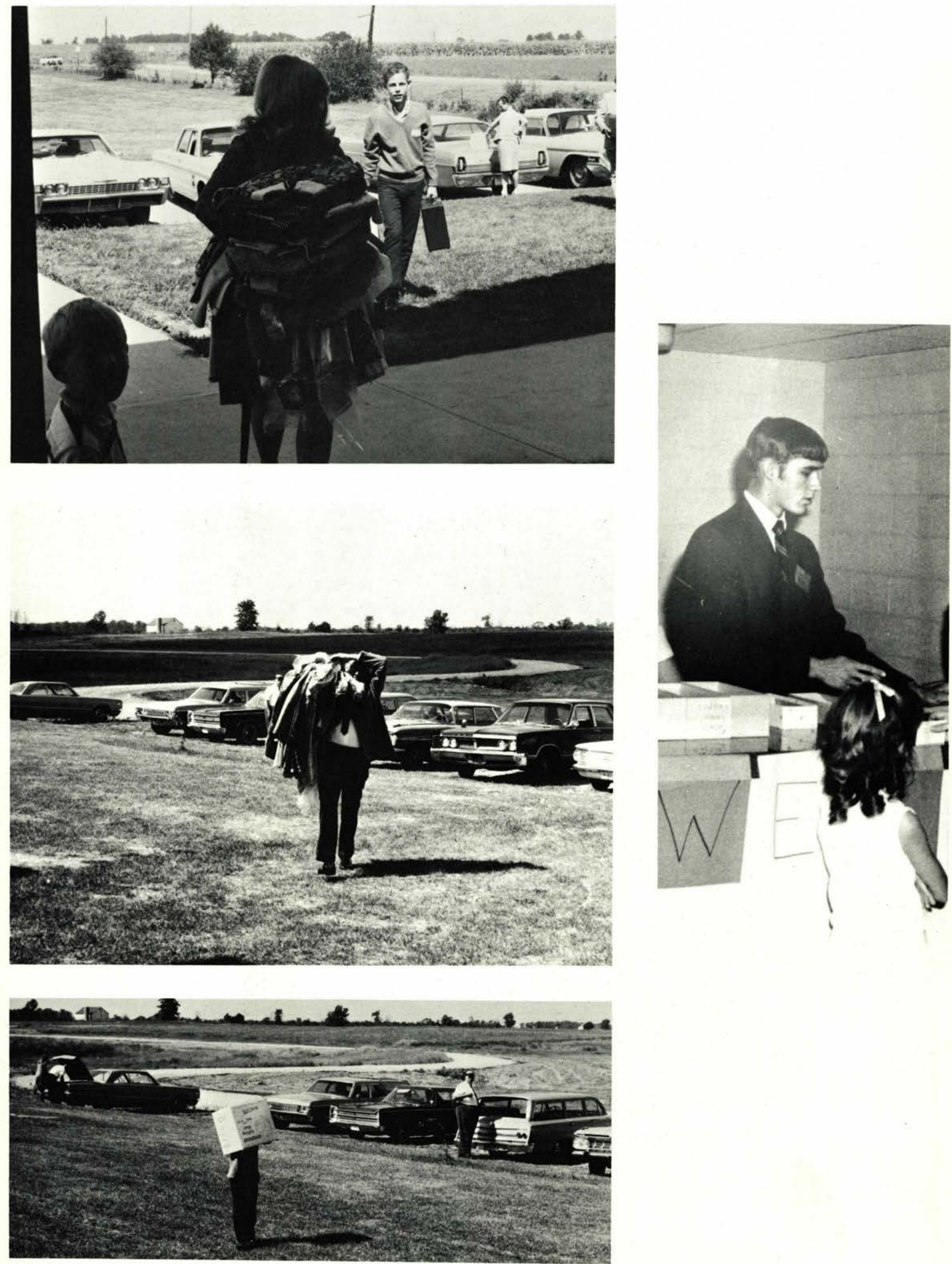


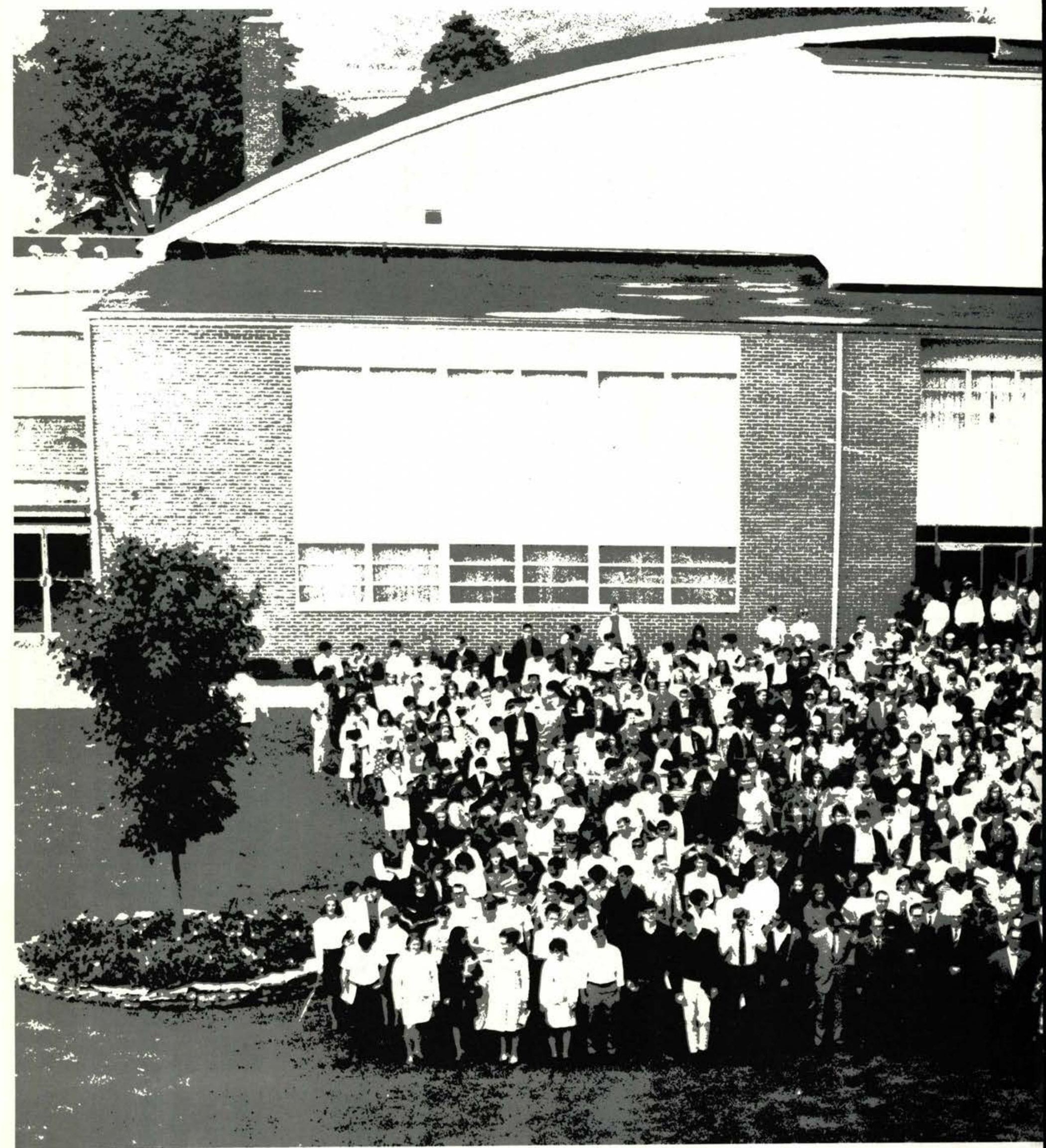

\section{WHAT HAVE YOU DONE THAT MADE}




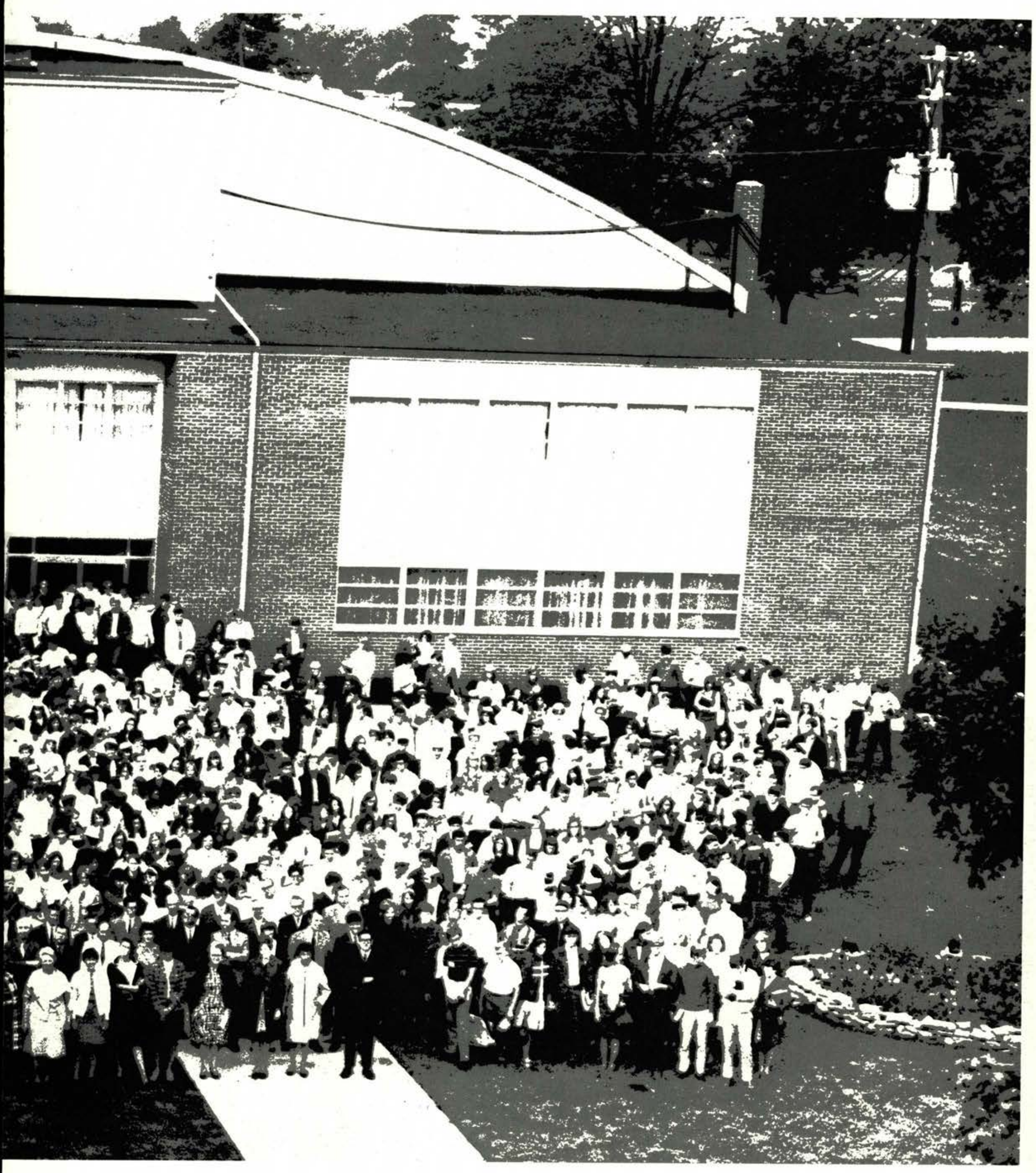

YOU ST AND OUT FROM THE CROWD? 


\section{WHO'S WHO AT CEDARVILLE}

"Those who are called to be leaders may profit from intelligence but can only justify their position by integrity."

Dag Hammarskjold

\section{Lyle Anderson}

Choir President and Student Director

Delta Sigma Alpha President Student Court Justice

Student Council Representative

Alpha Chi Talent Award Guardsmen Quartet Dorm Counselor

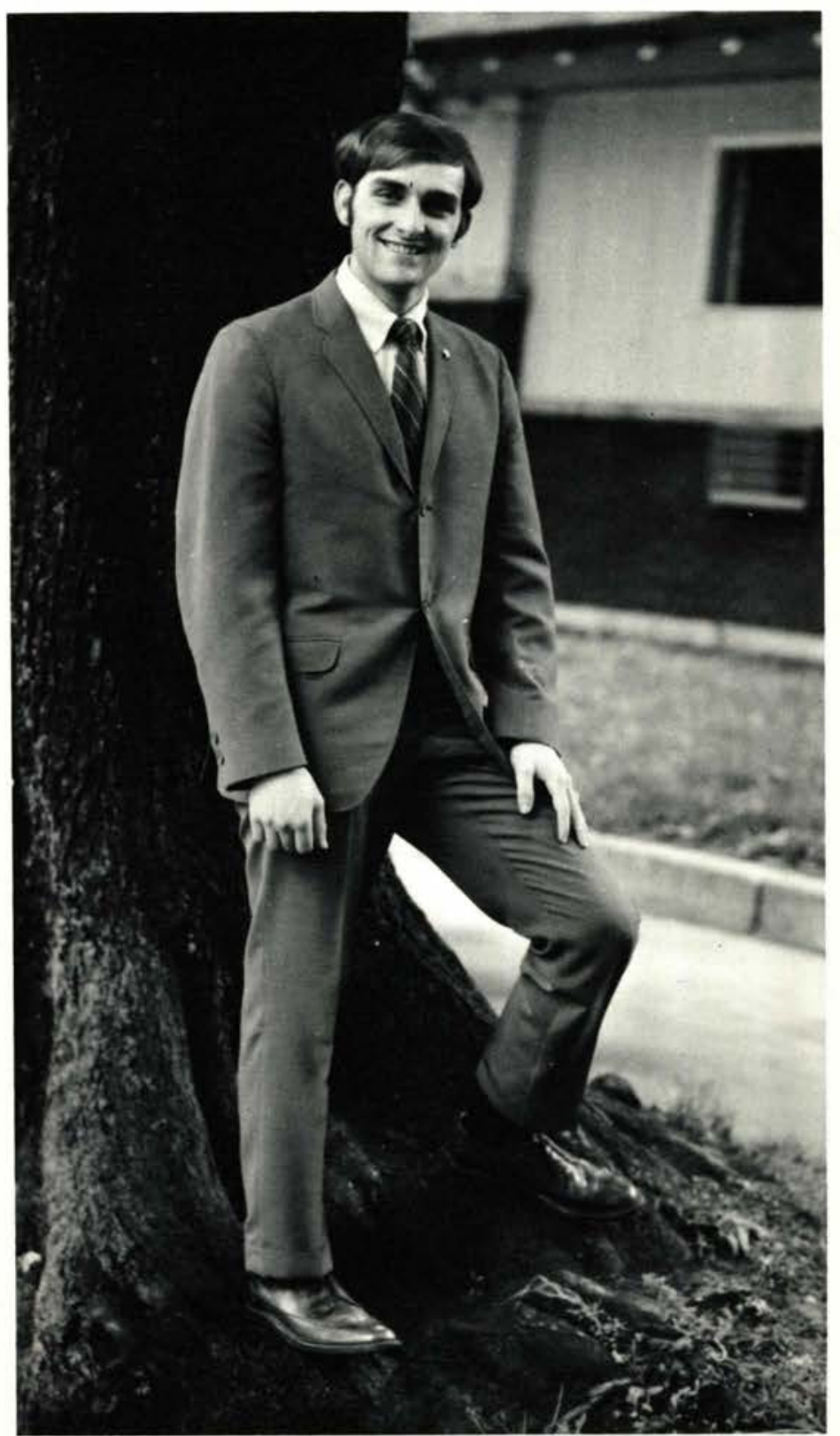




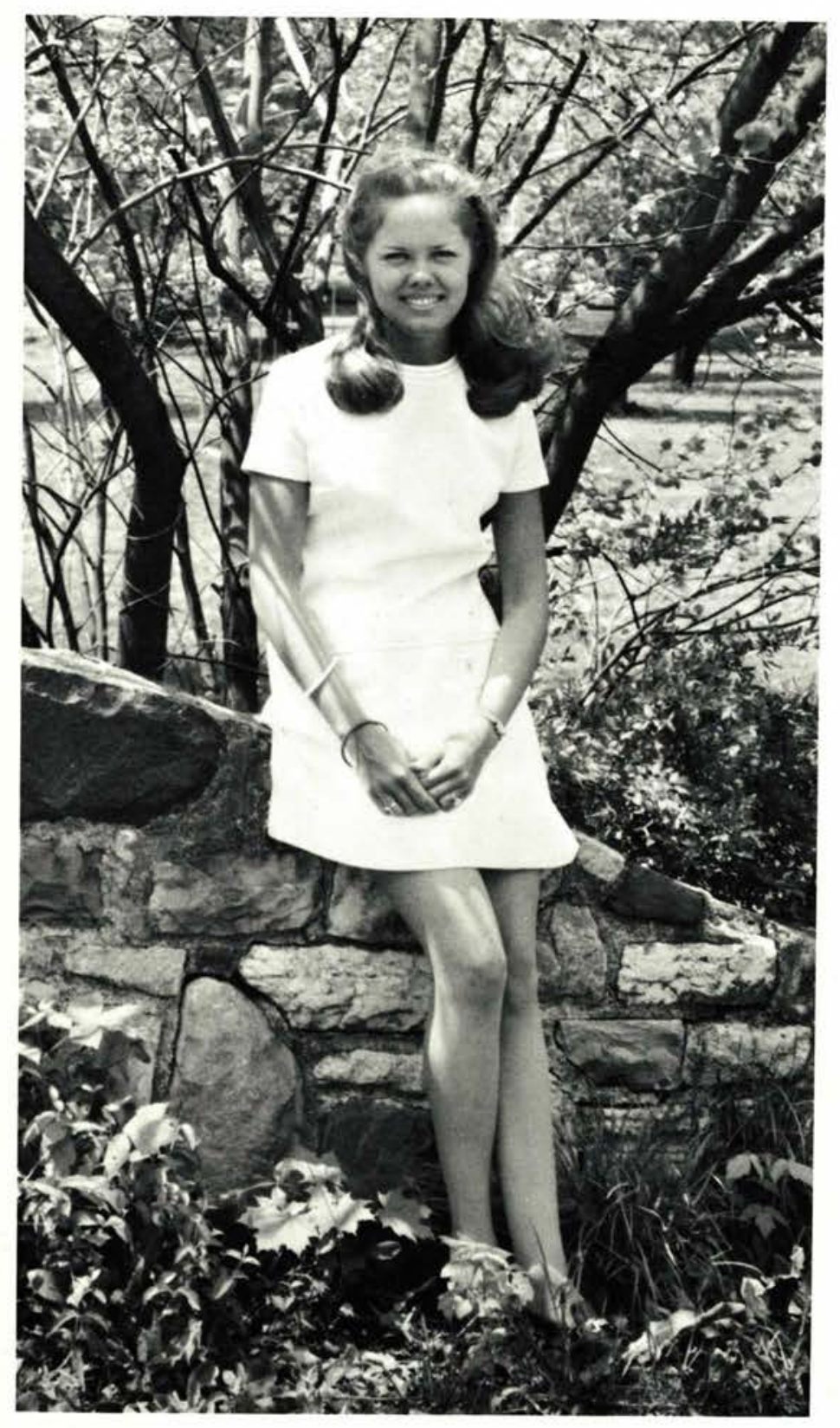

\section{Jan Beesley}

College Choir Student

Council Rep.

Delta Sigma Alpha Secretary

Homecoming Attendant

Dorm Counselor

Ambassadors Trio

\section{Ken Cole}

Student Council Vice

President

Outstanding Debater Award Class Treasurer

Dorm Counselor

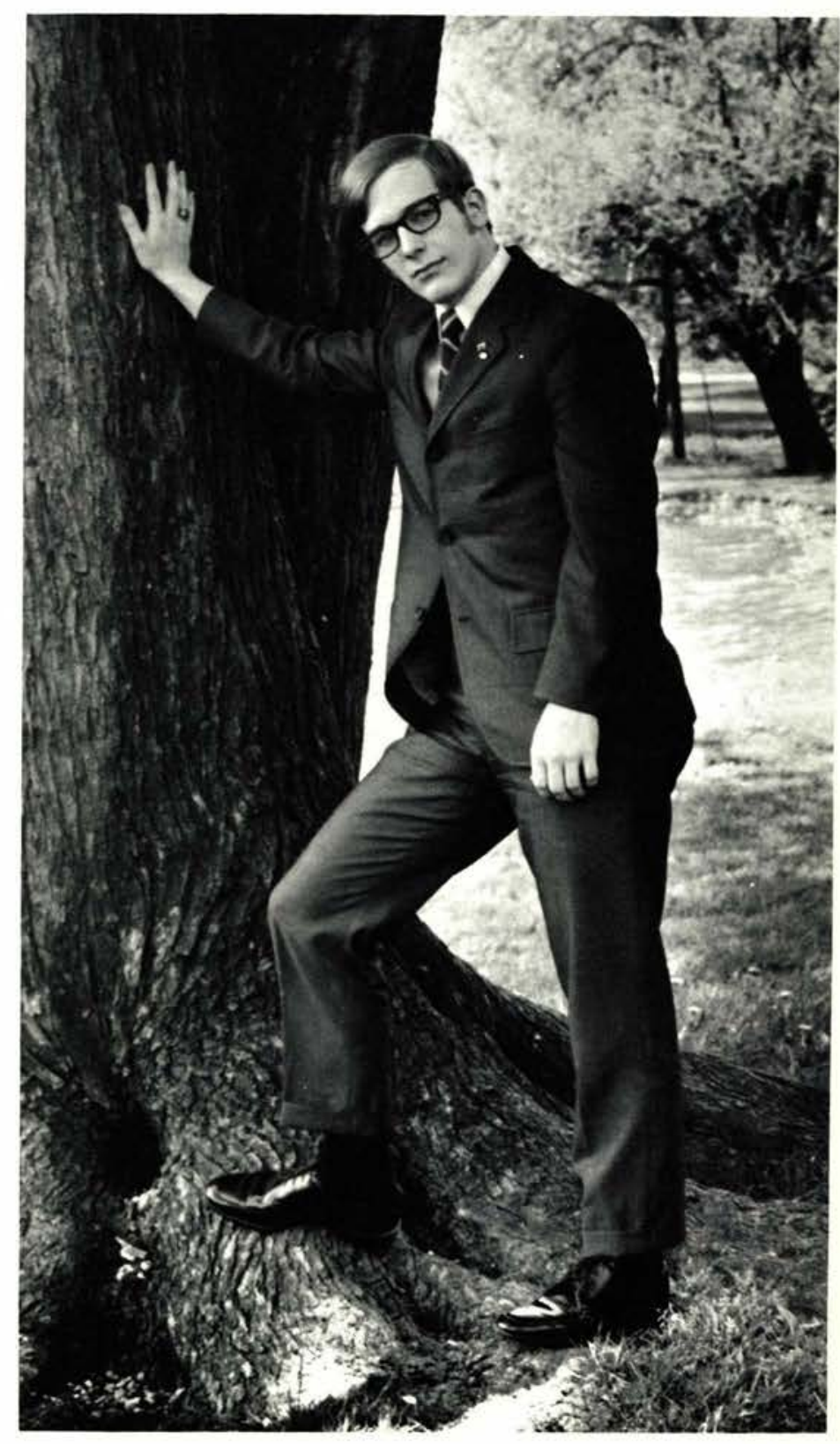




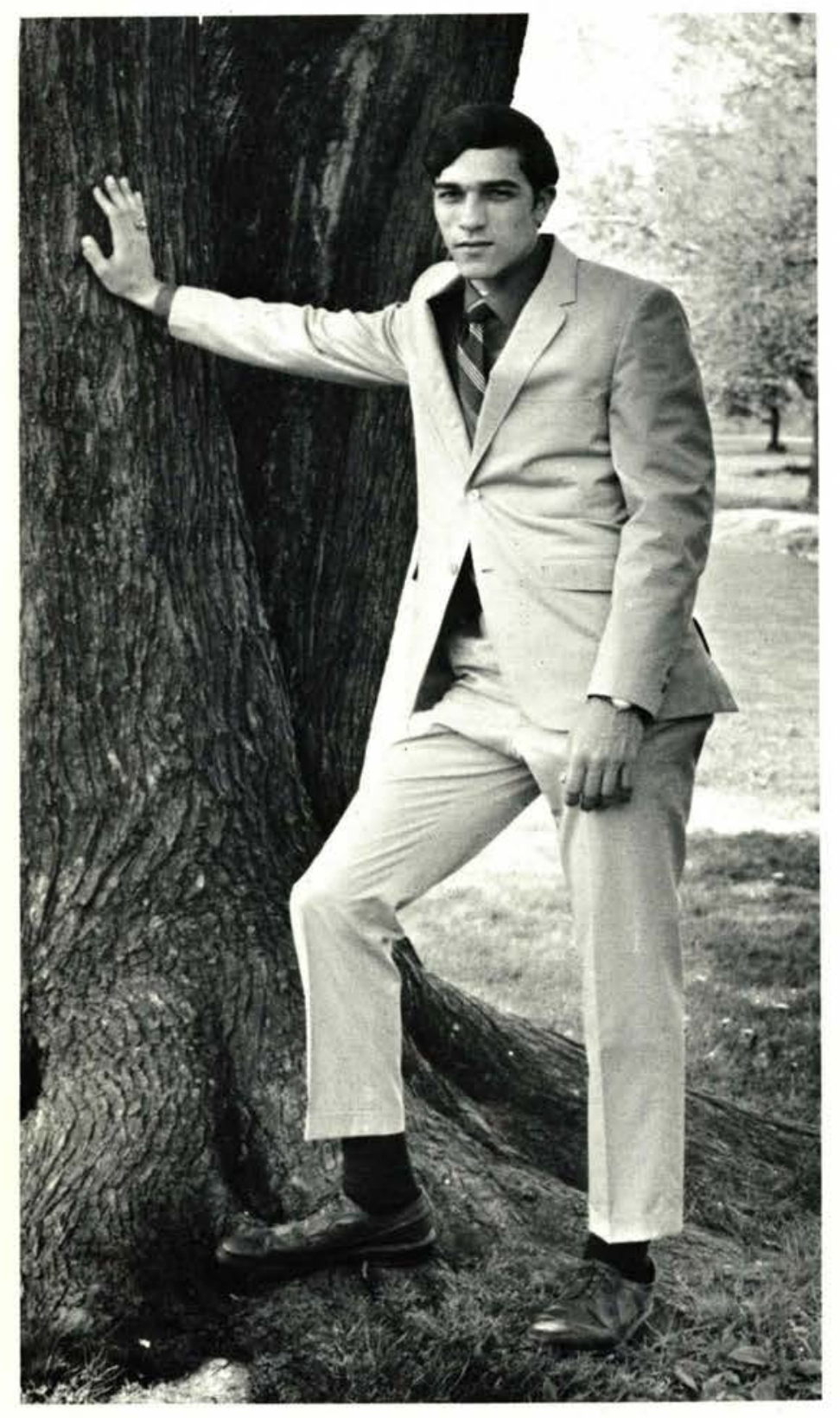

\section{Ken Curcio}

Varsity C President

Pi Sigma Nu Treasurer

Student Court Justice

\section{Ron Edwards}

FWM President

Sigma Delta Kappa Vice President

Student Court Justice

Student Council

Representative

Dorm Counselor

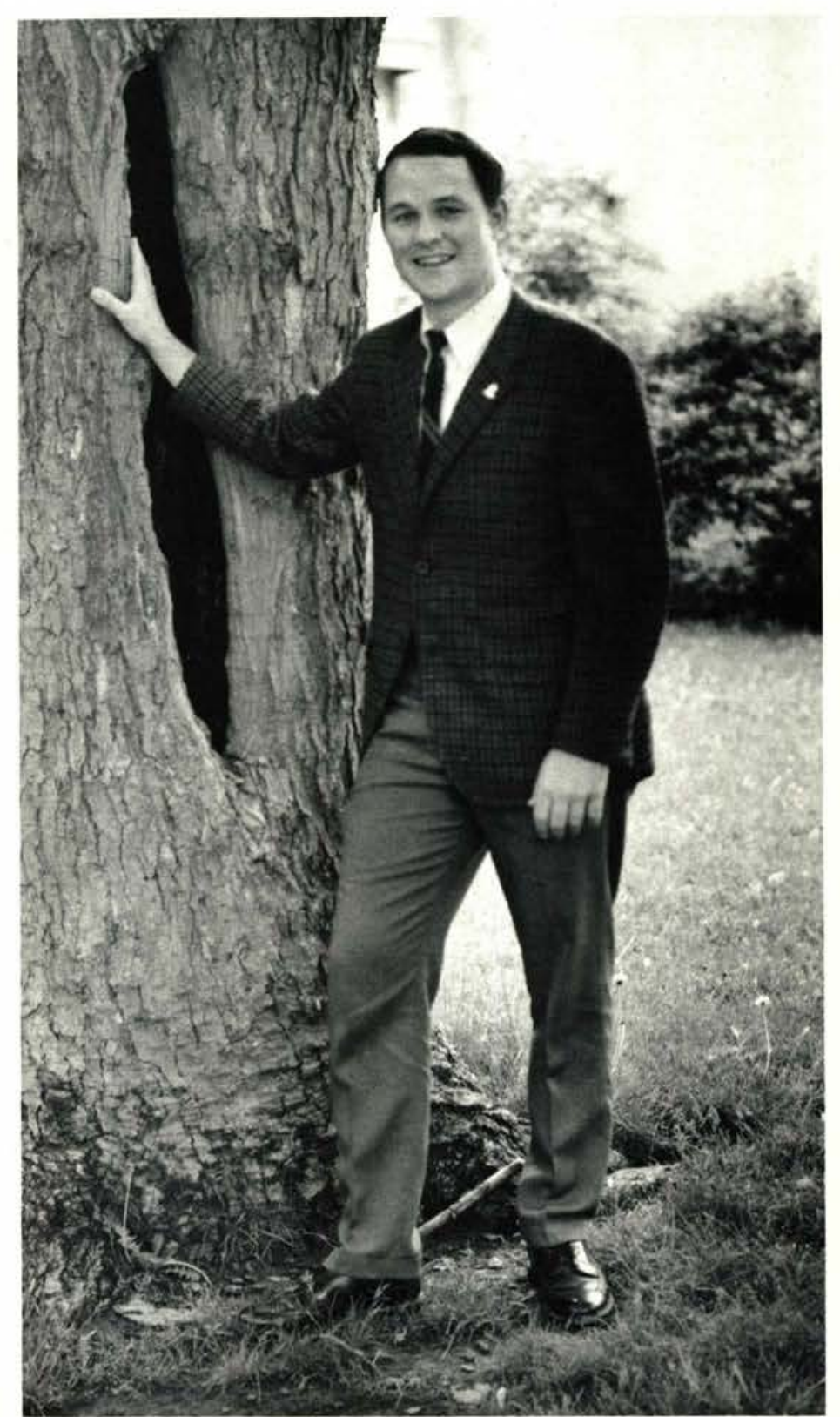




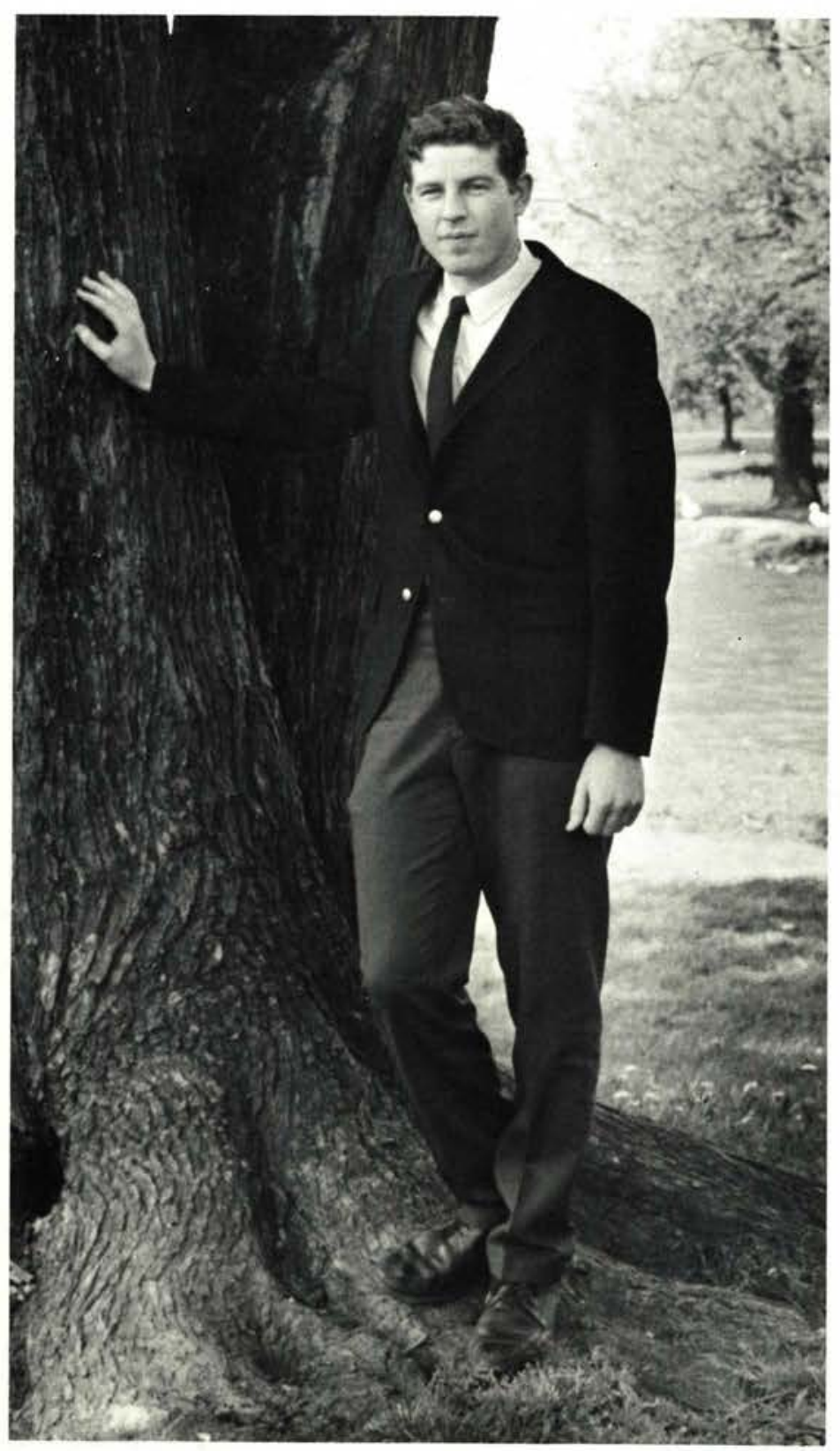

\section{Lee Eichelberger}

WCDR Production Director Outstanding Broadcaster Award Dorm Counselor

\section{Patti France}

\section{MIRACLE Editor}

Student Council Secretary

Gamma Chi Treasurer

Student Court Justice

Clara Monzell Milner Award Dorm Counselor

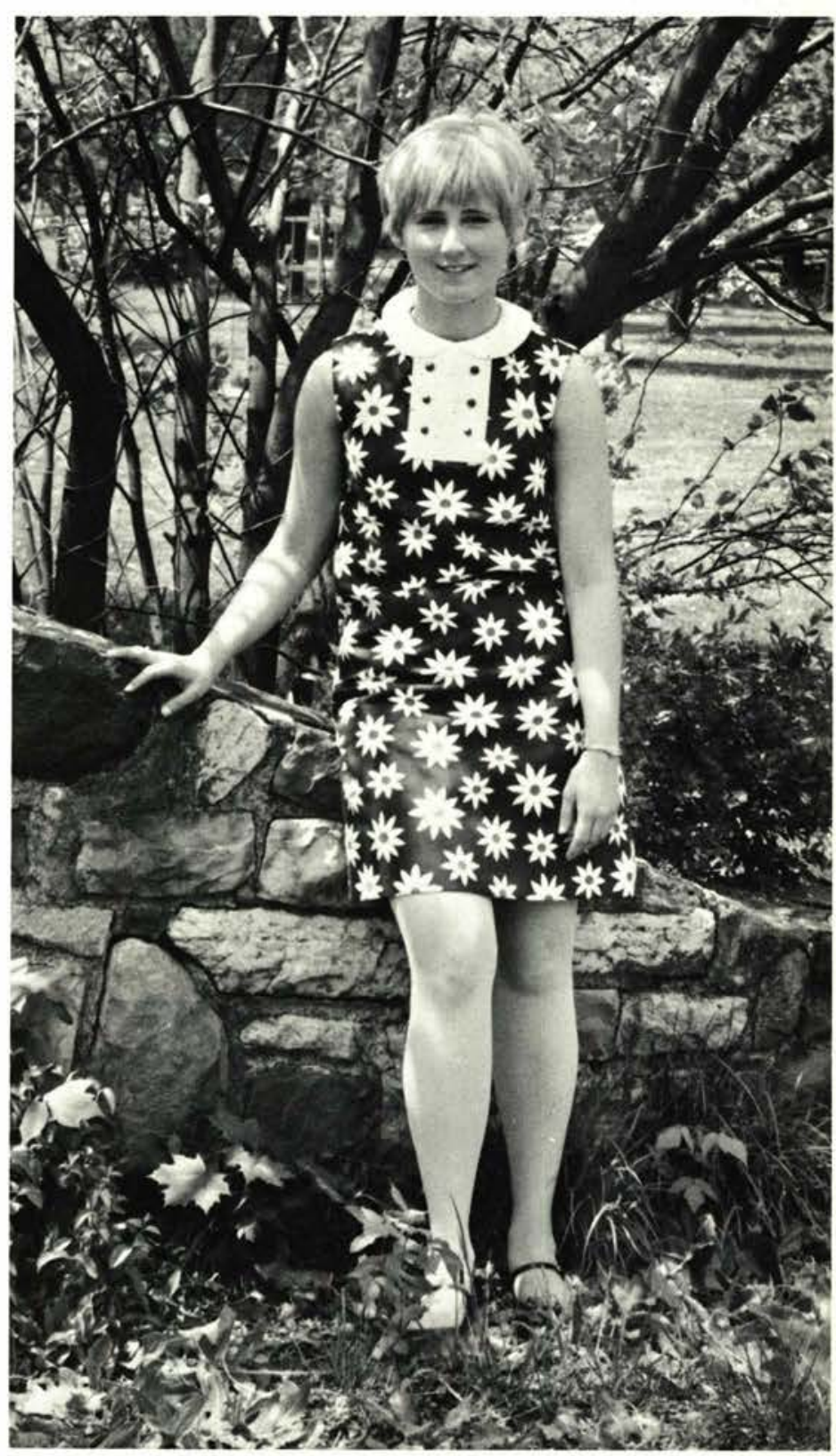




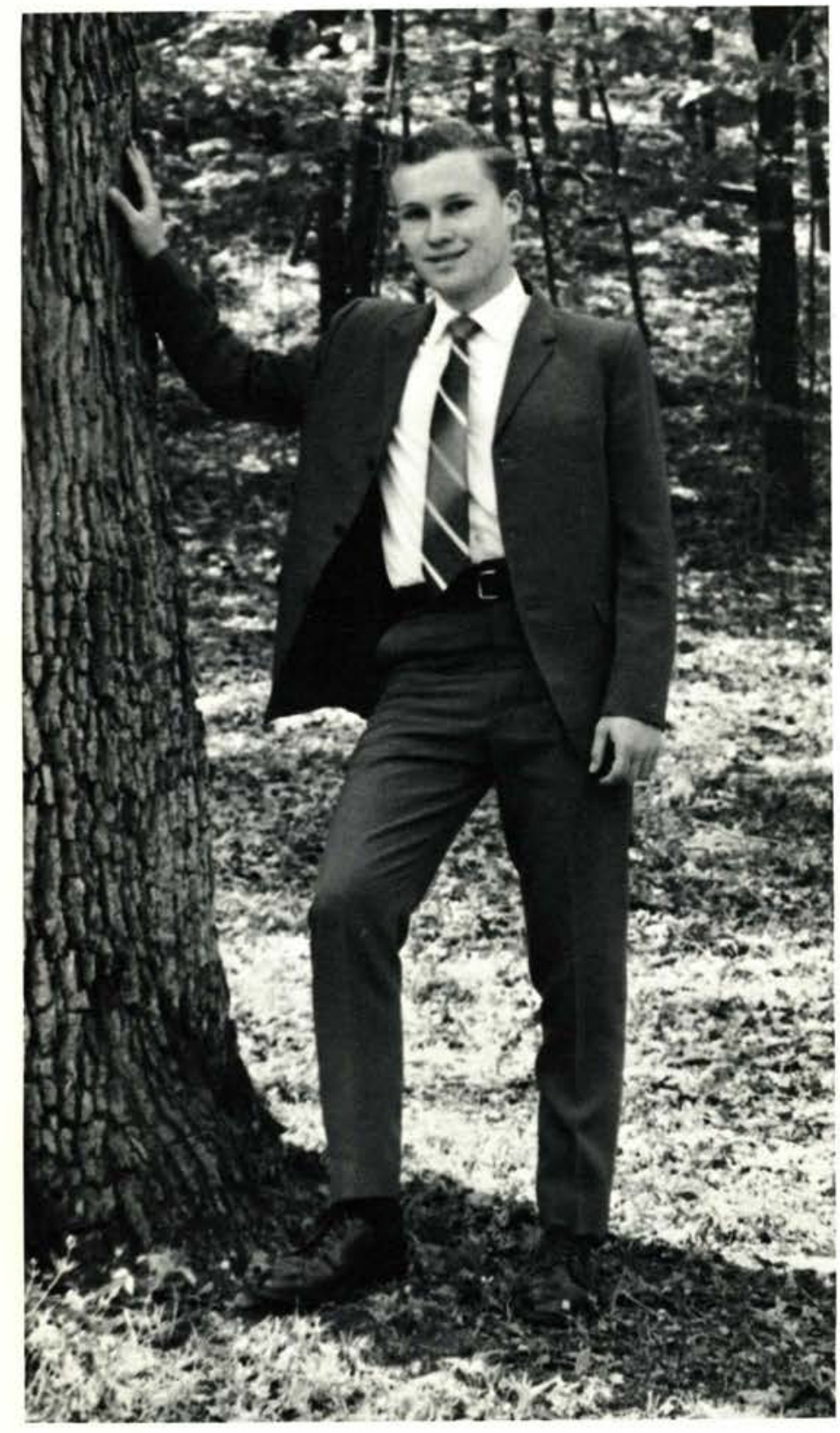

\section{Dave Kearney}

Student Council President College Choir Vice President Sigma Delta Kappa Secretary Dorm Counselor

\section{Joyce Motts}

MENC Student Council

Representative

Band Secretary

Music Department Secretary

Delta Sigma Alpha

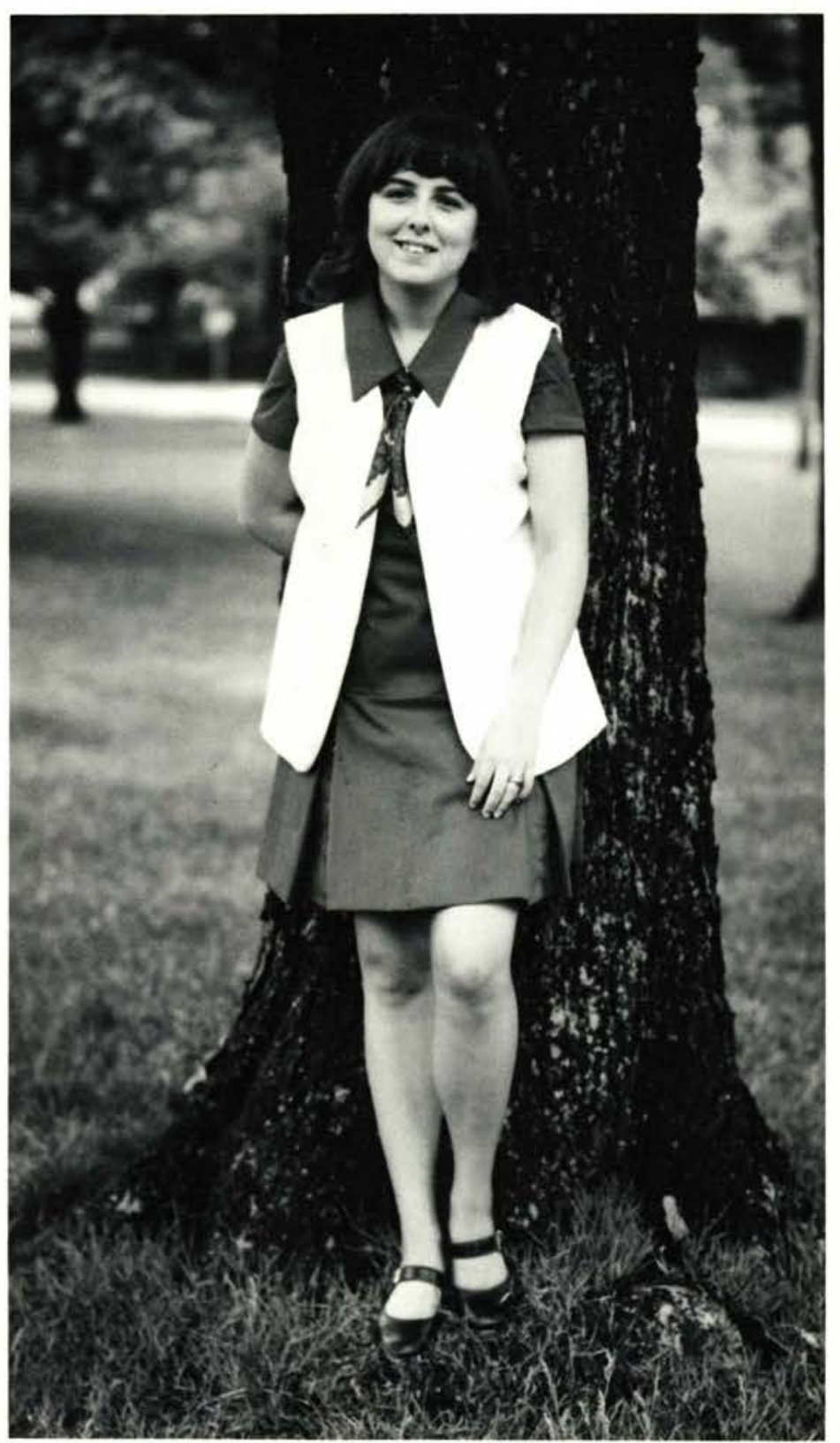




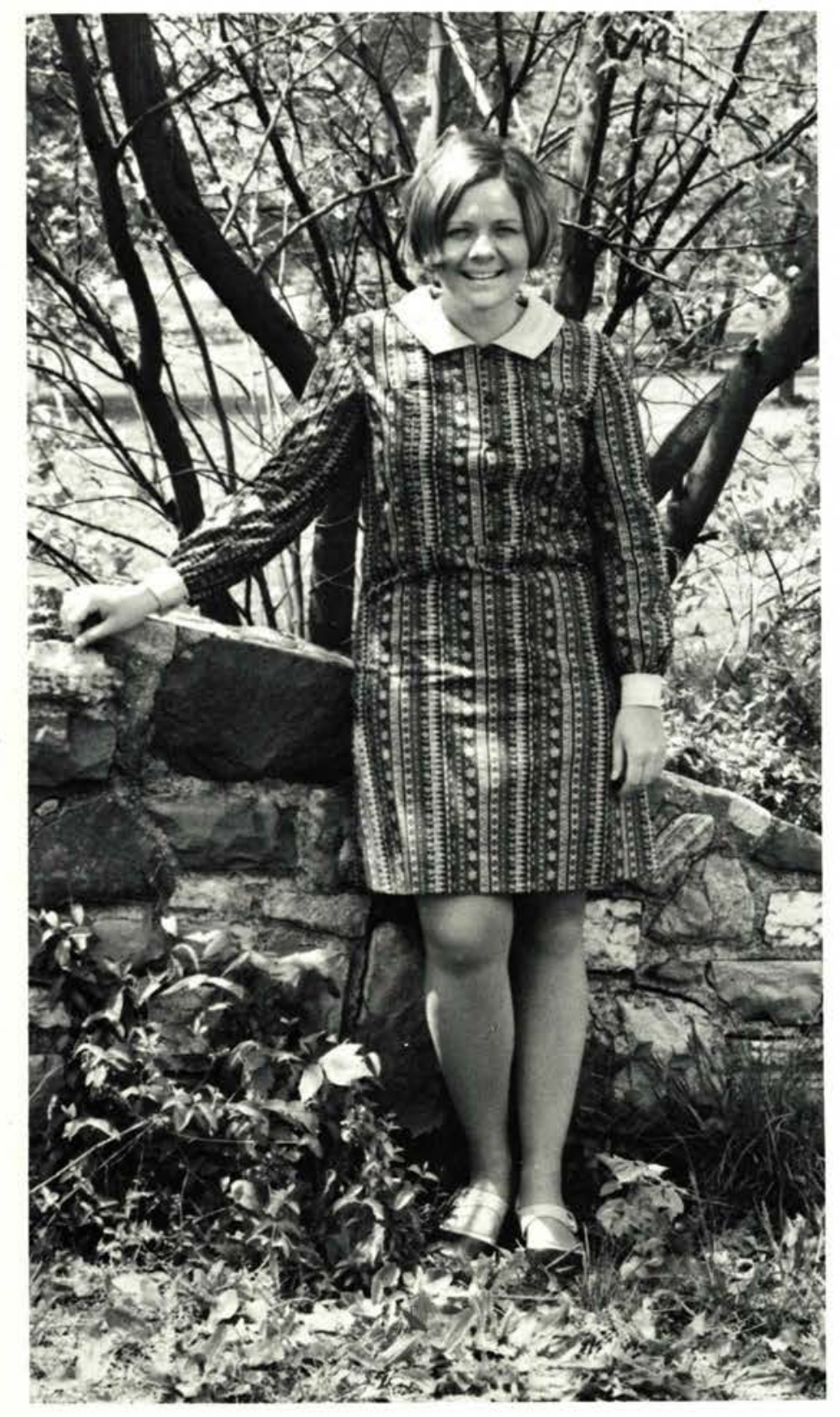

\section{Janet Phenix}

Gamma Chi President Senior Class Student Class Rep. Whispering Cedars Staff Dorm Counselor

\section{Gail Sears}

Alpha Chi Sweetheart Homecoming Attendant Senior Class Student Council Rep.

Gamma Chi Secretary WRA Treasurer Dorm Counselor

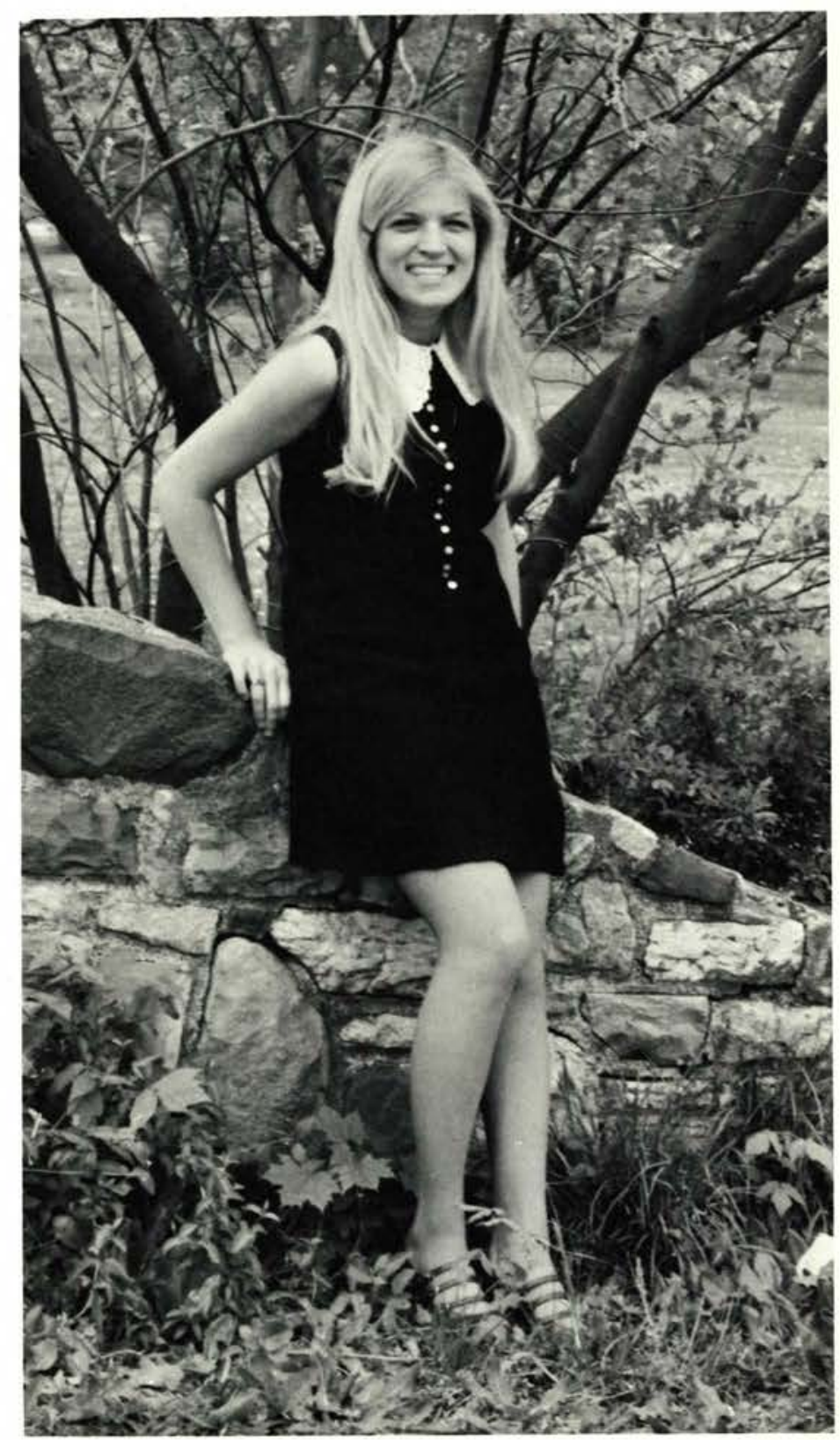




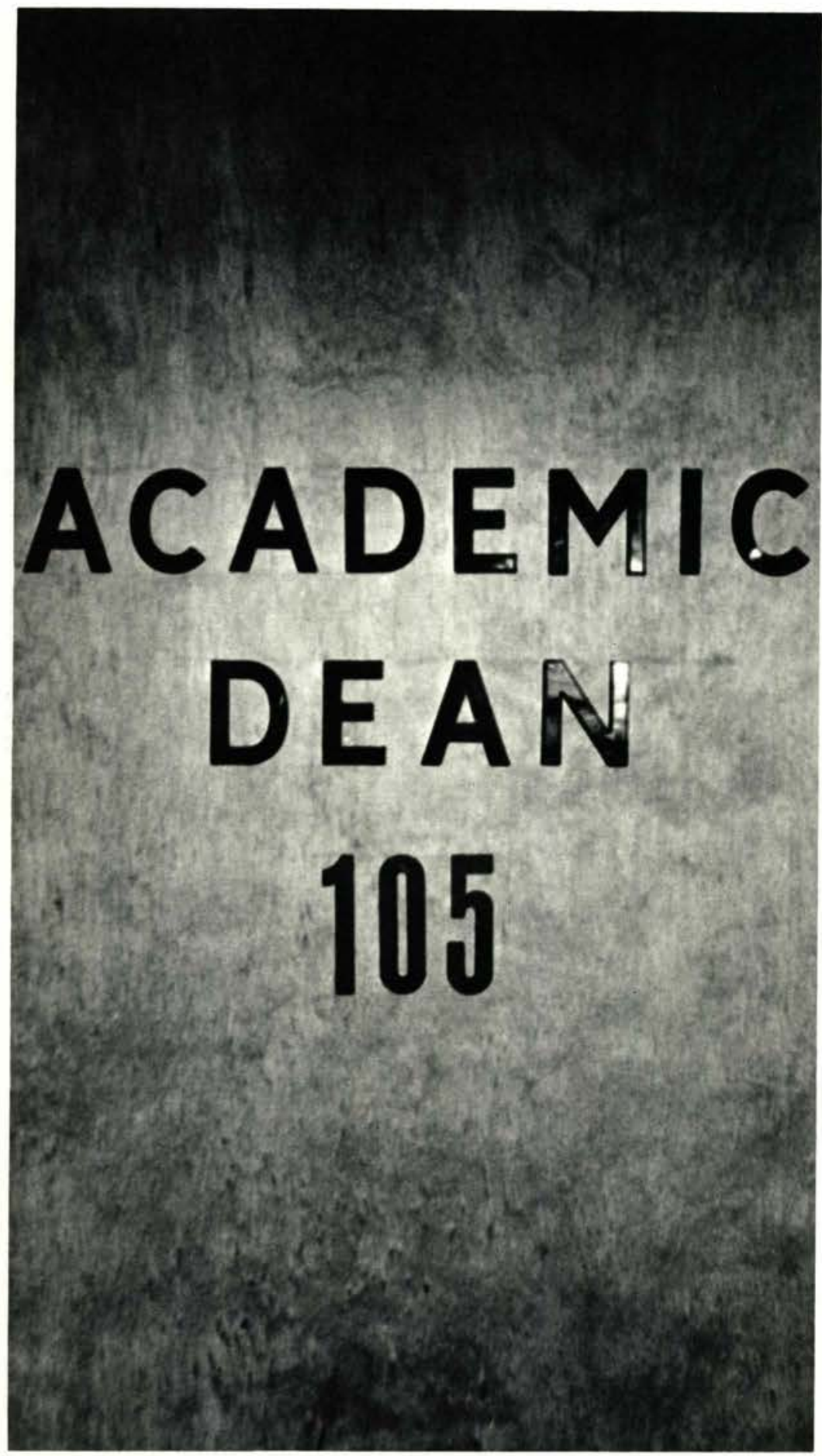

Academic Dean . . .

An ominous title,

A grave responsibility,

A sincere challenge.

A position requiring

Dedication to God,

Education,

And Cedarville College.

Dr. Clifford Johnson . .

A man

Equal to this position;

Spiritually consecrated, Intellectually stimulating,

Educationally motivating.

Dr. Johnson

Leads the academic life

Of the college

With genuine concern

For challenging the minds

Of Cedarville students.

In appreciation

of his services

As our academic

Standard-bearer

And friend,

We dedicate

The Miracle ' 70

To Dr. Clifford Johnson. 


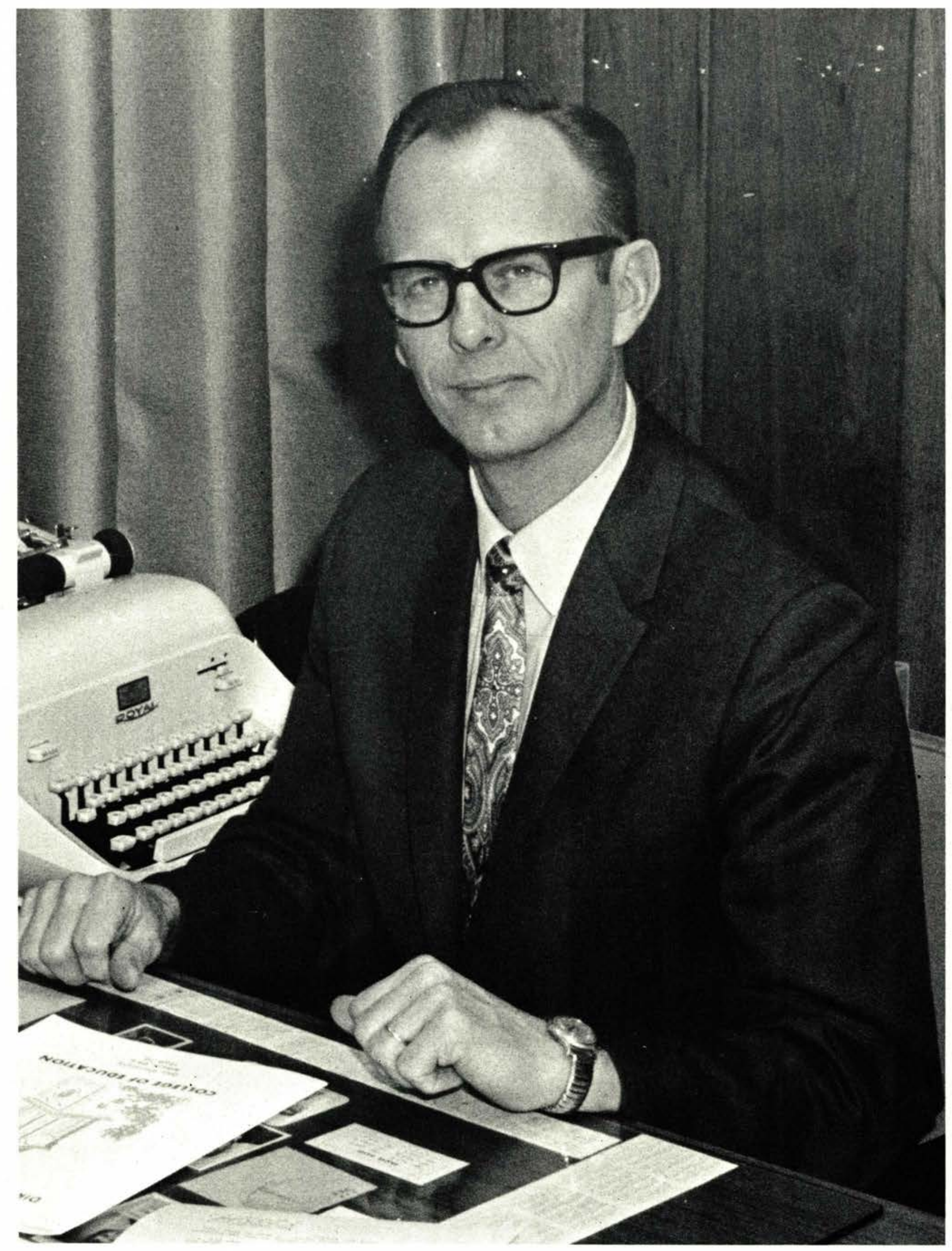




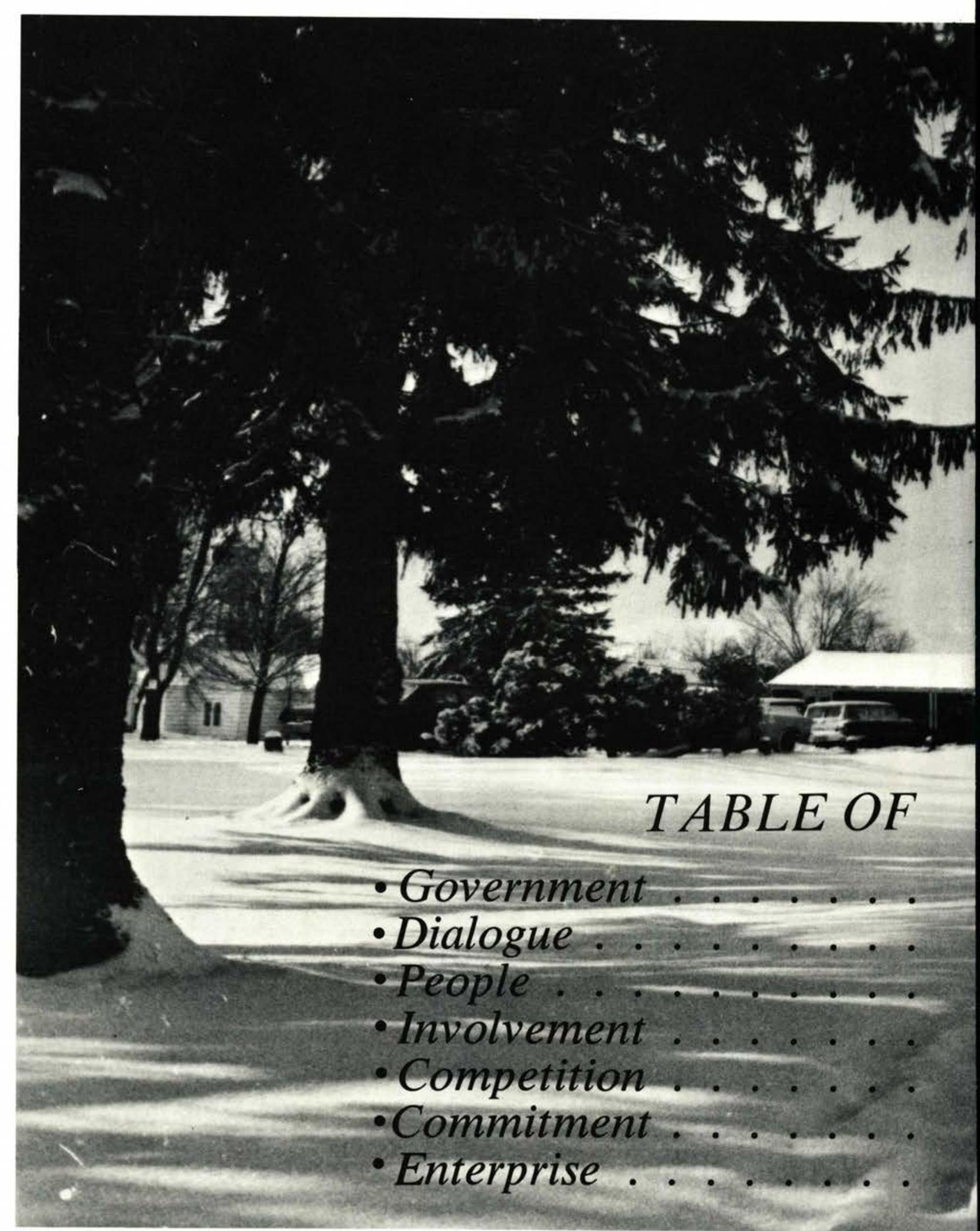




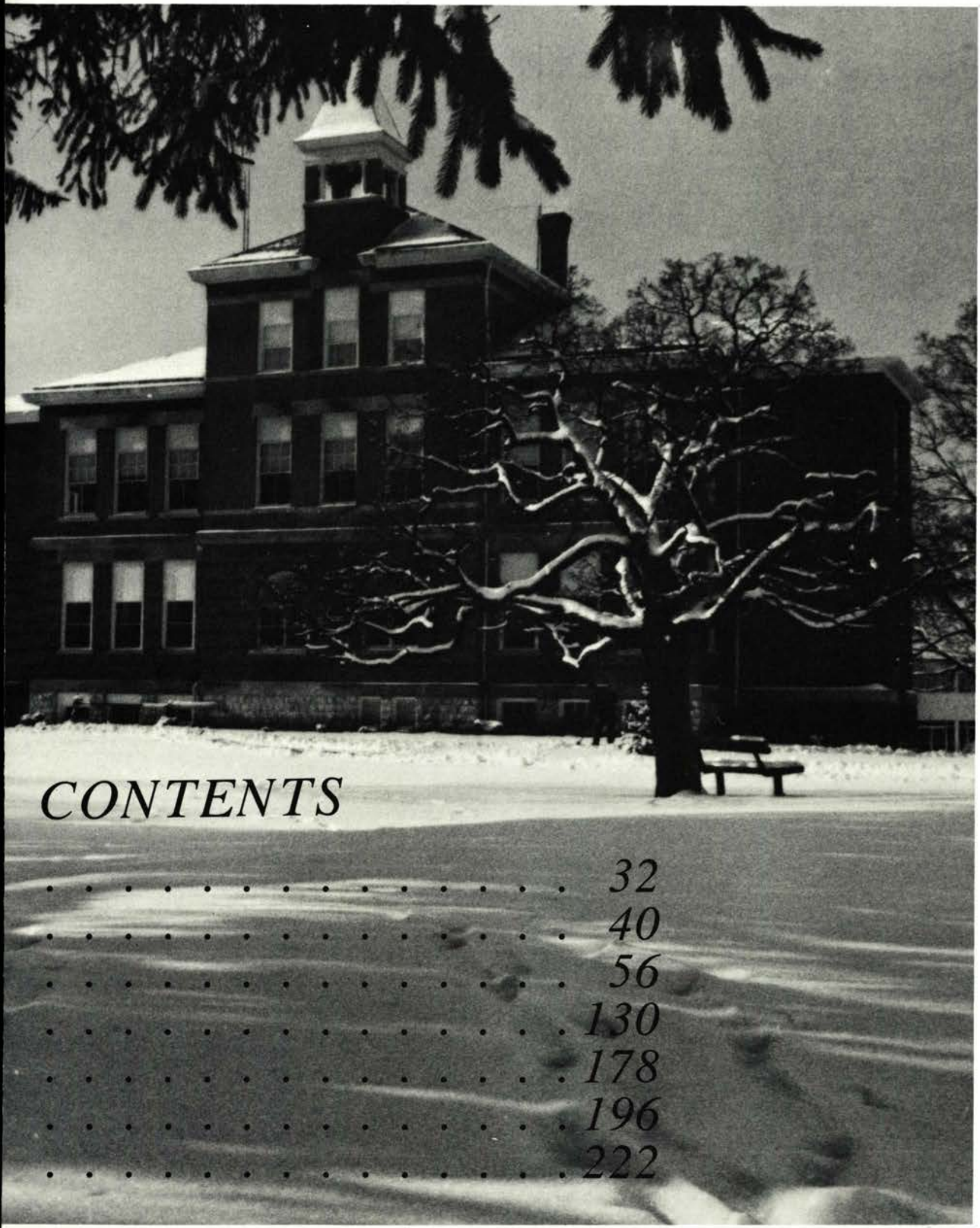




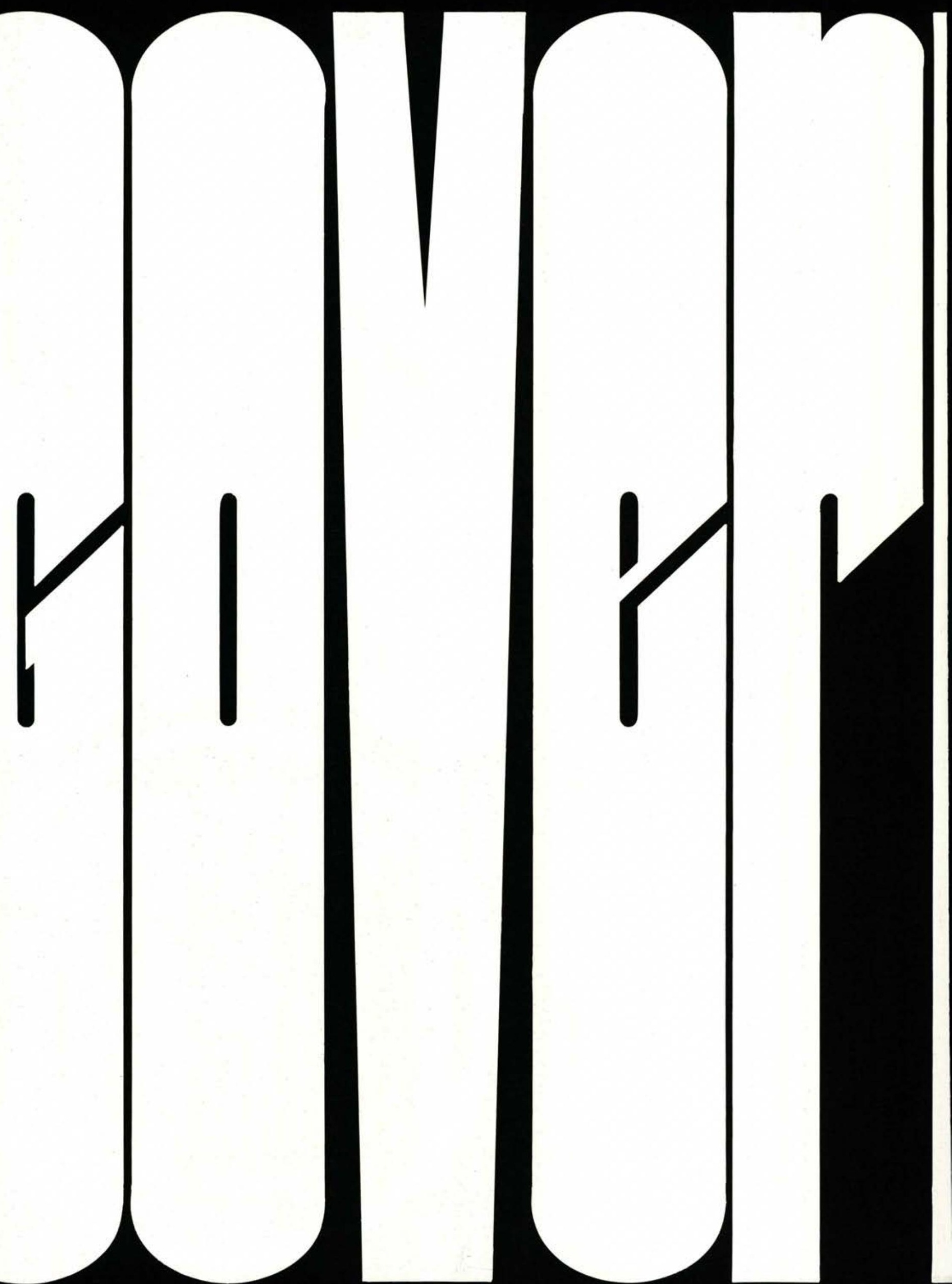




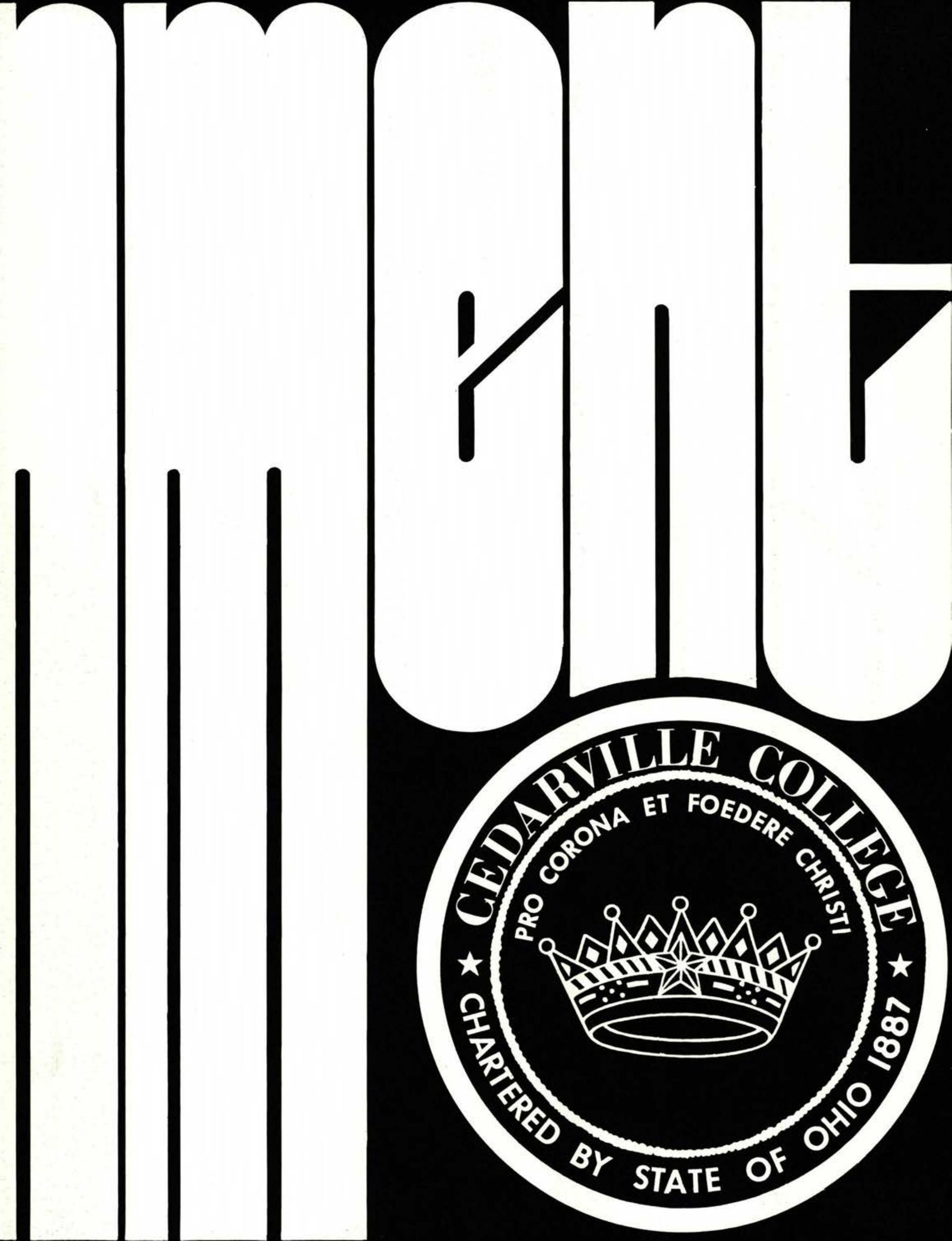




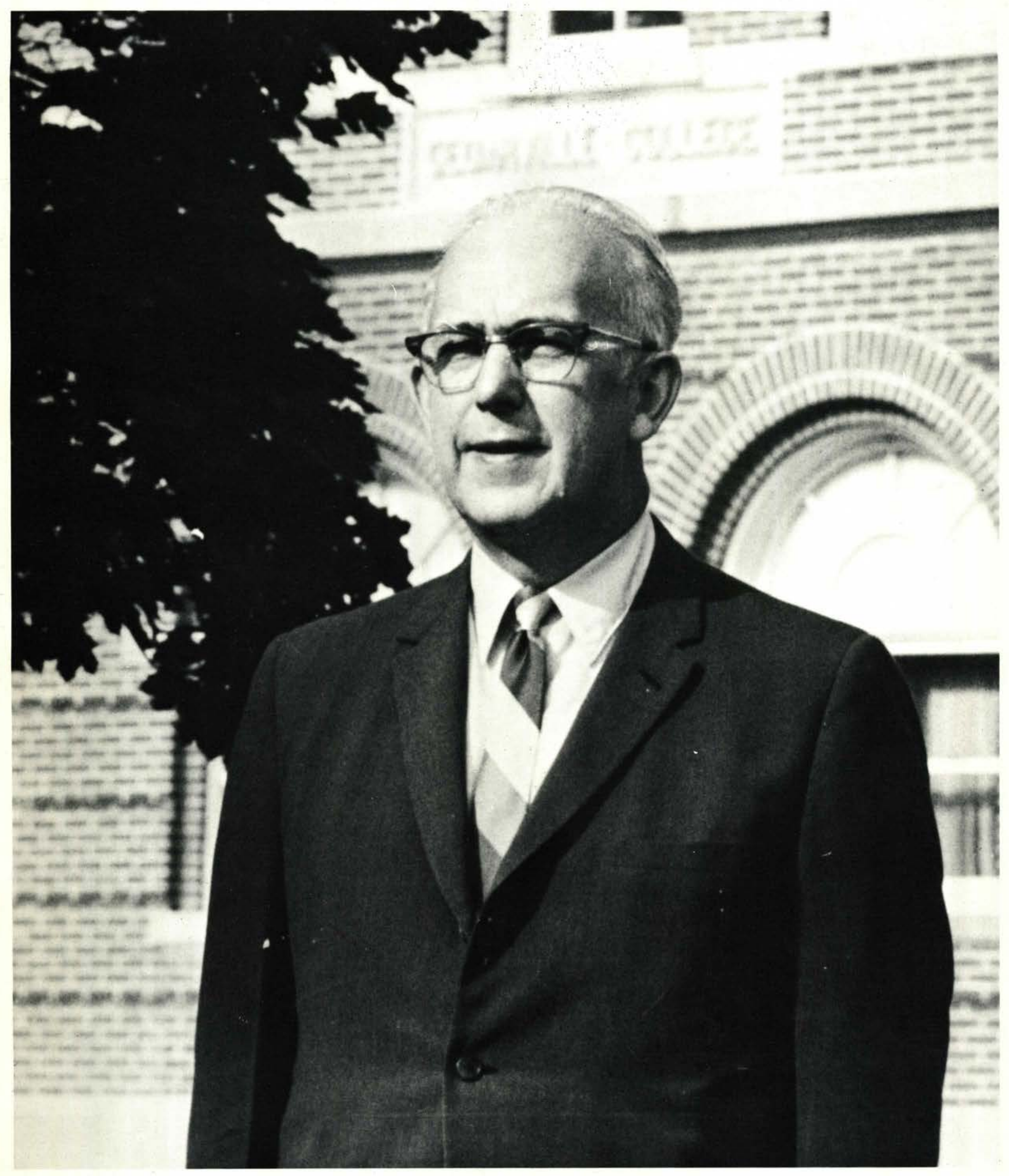

JAMES T. JEREMIAH CEDARVILLE COLLEGE PRESIDENT 


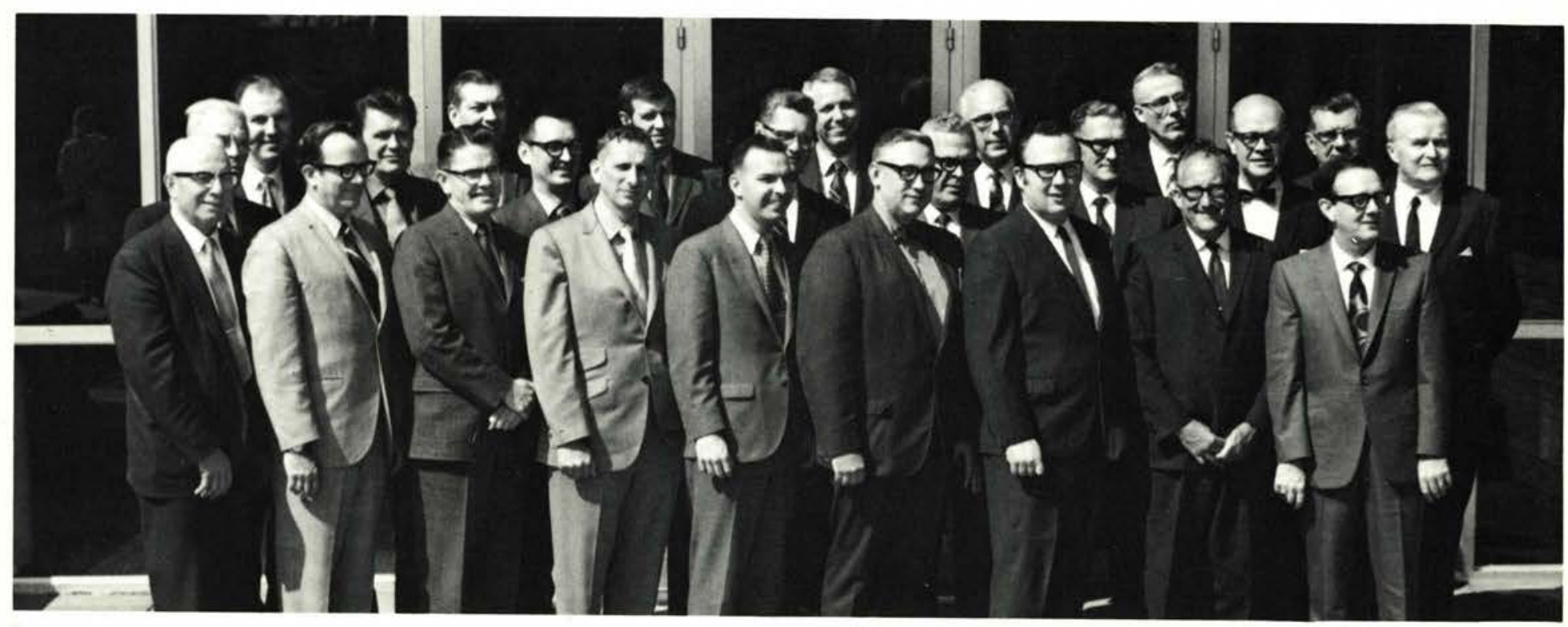

CEDARVILLE COLLEGE TRUSTEES

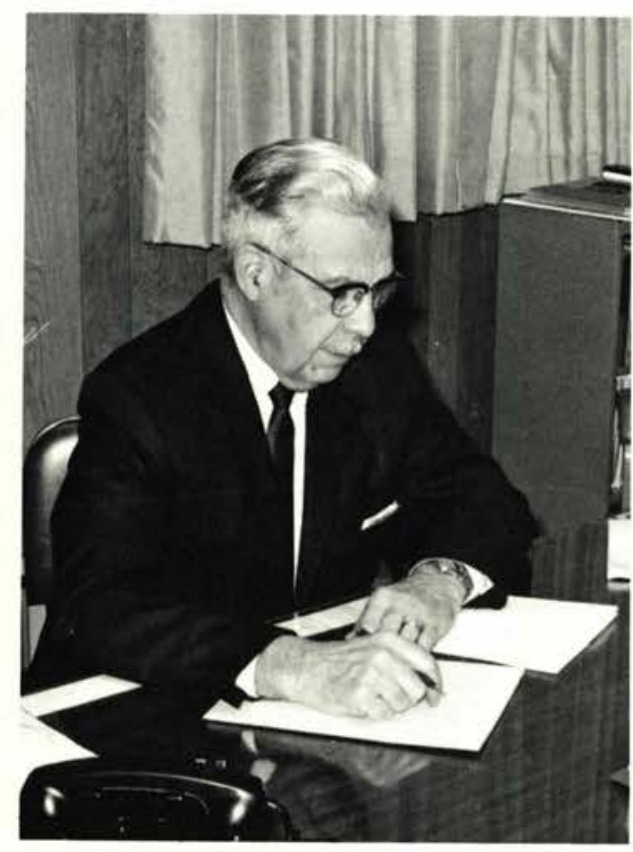

C. B. Hurst

Registrar

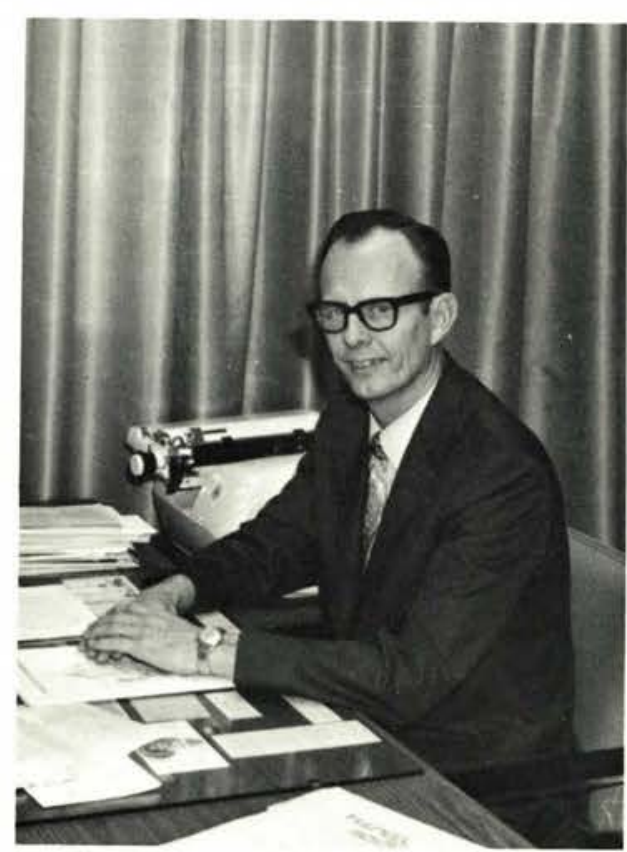

Clifford W. Johnson

Academic Dean

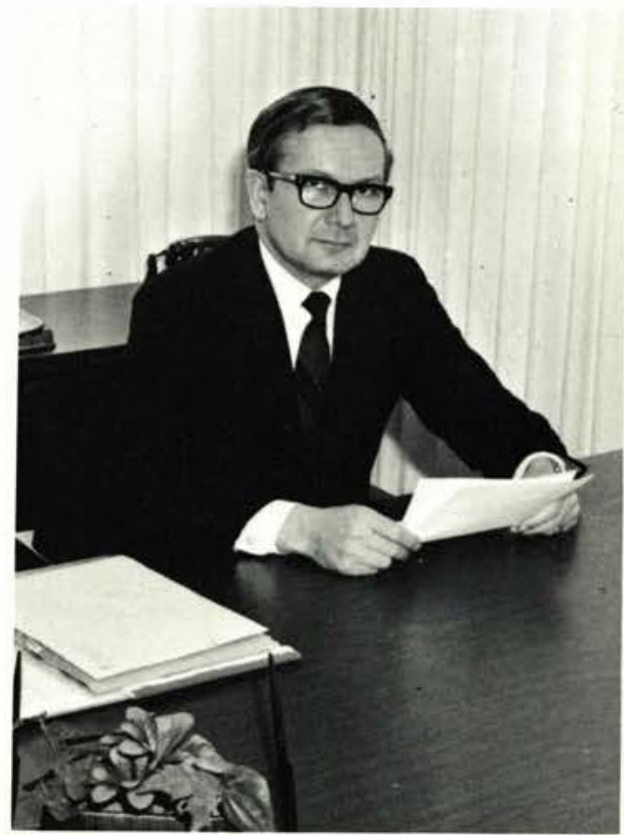

Kenneth H. St. Clair Business Manager 


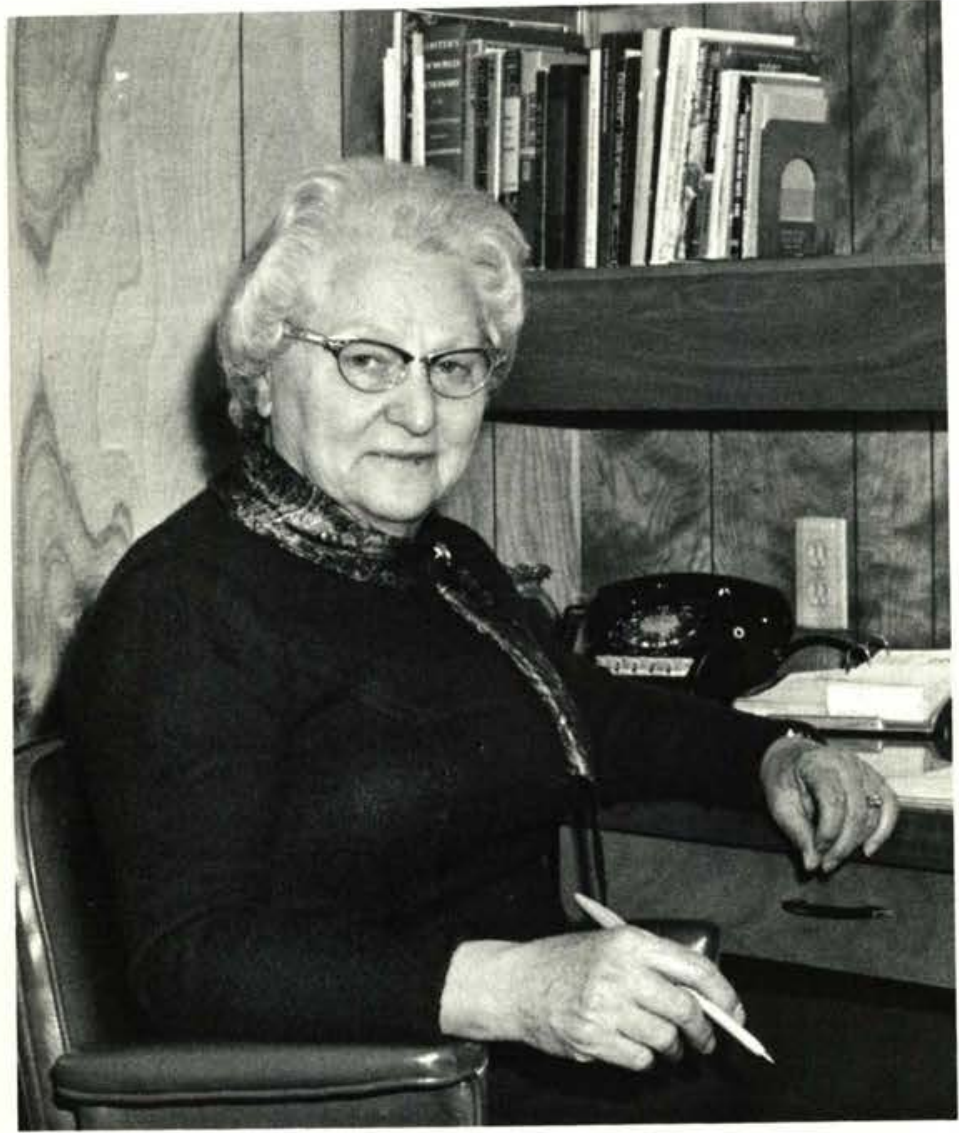

\section{OFFICE $O F$ \\ STUDENT \\ PERSONNEL}

Mrs. Mary Armstrong

Dean of Women

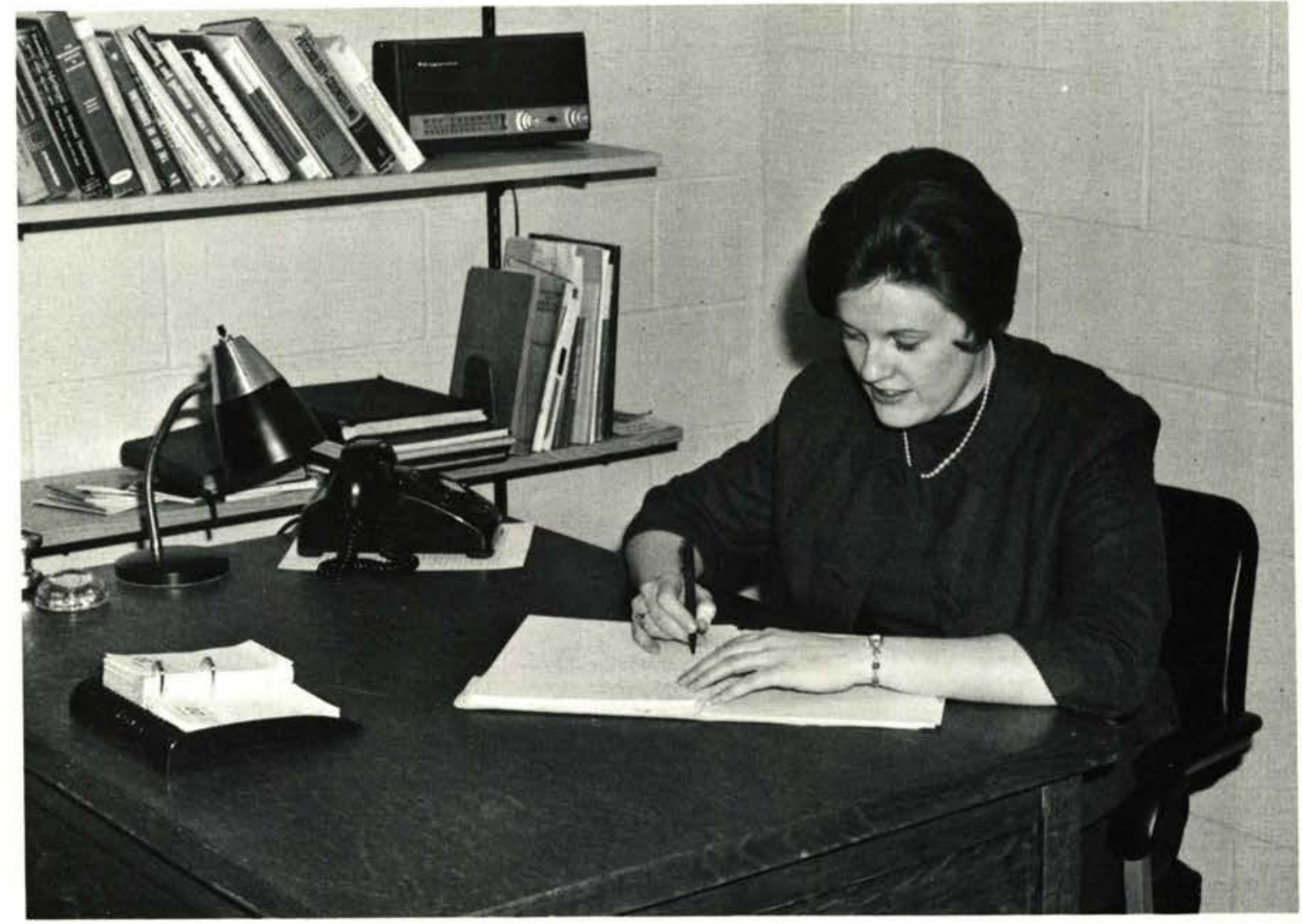

Miss Diane Lewis

Assistant Dean of Women 


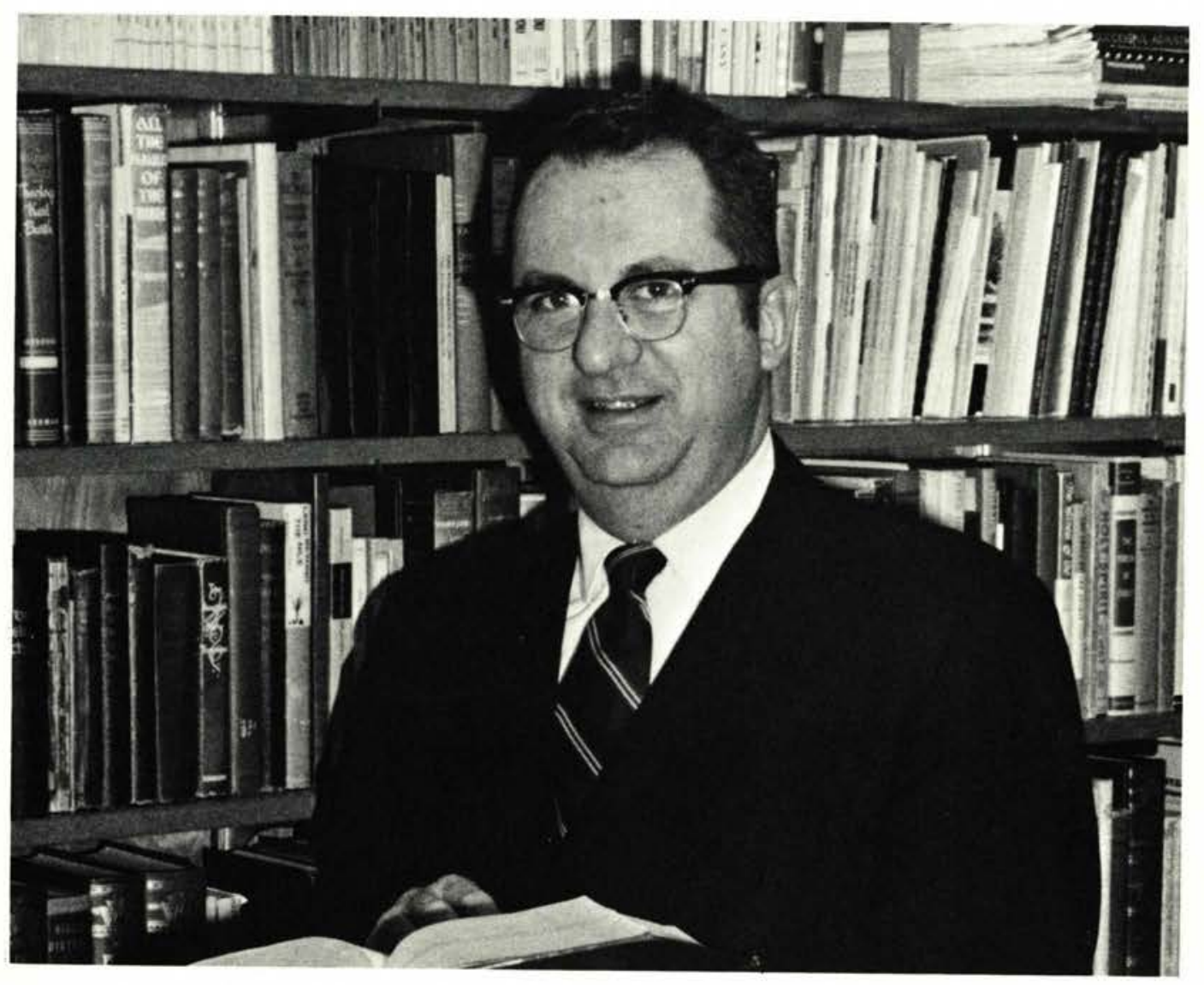

Mr. Richard McIntosh

Dean of Students

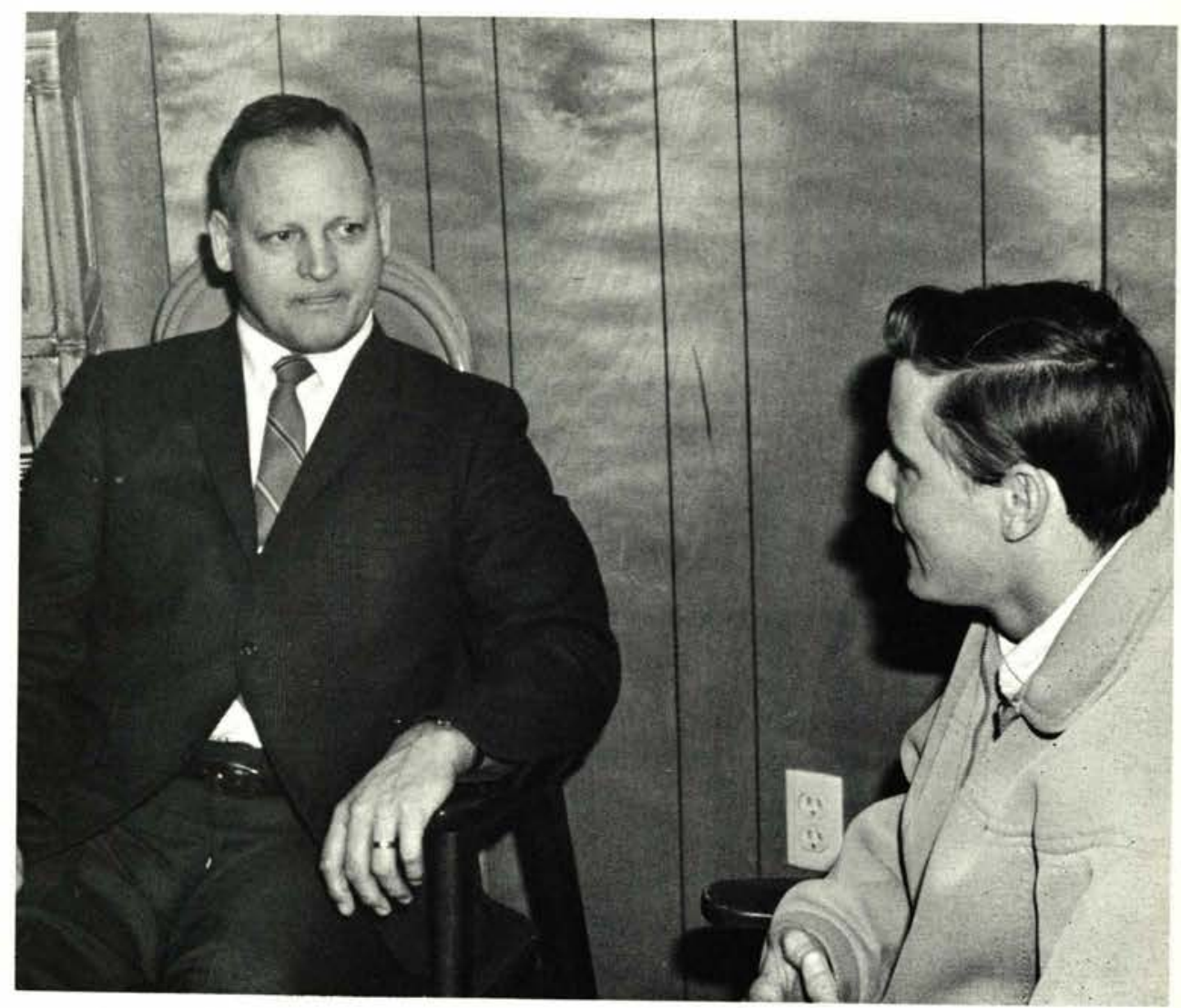

Mr. Thomas Loper

Dean of Men 


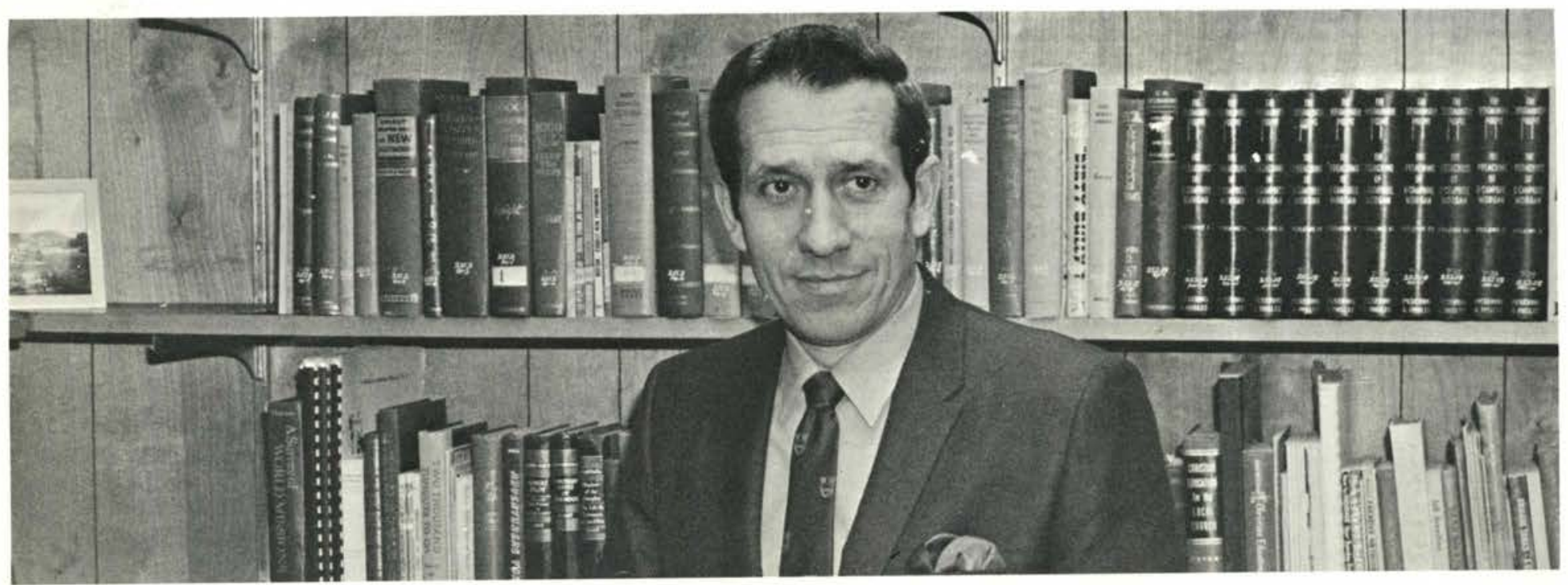

Mr. Lee Turner, Director of Development

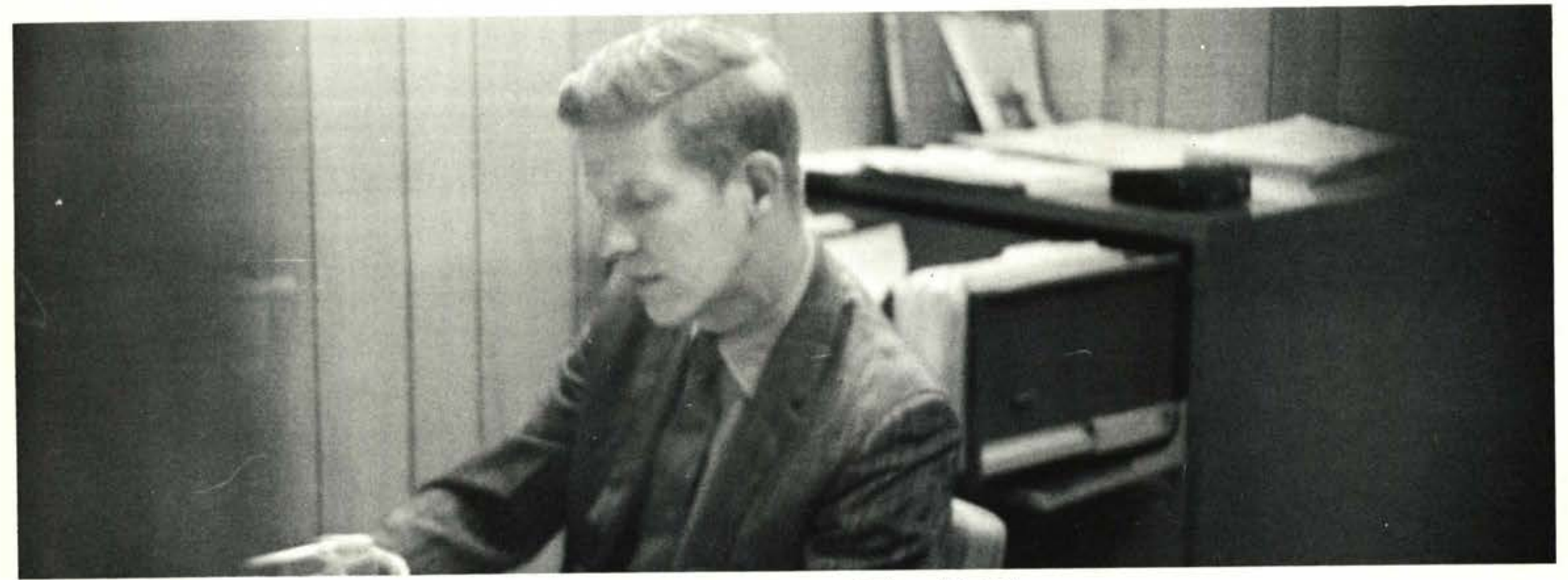

Mr. David Gidley, Director of Financial Aid

Mr. James Phipps, Director of Student Activities

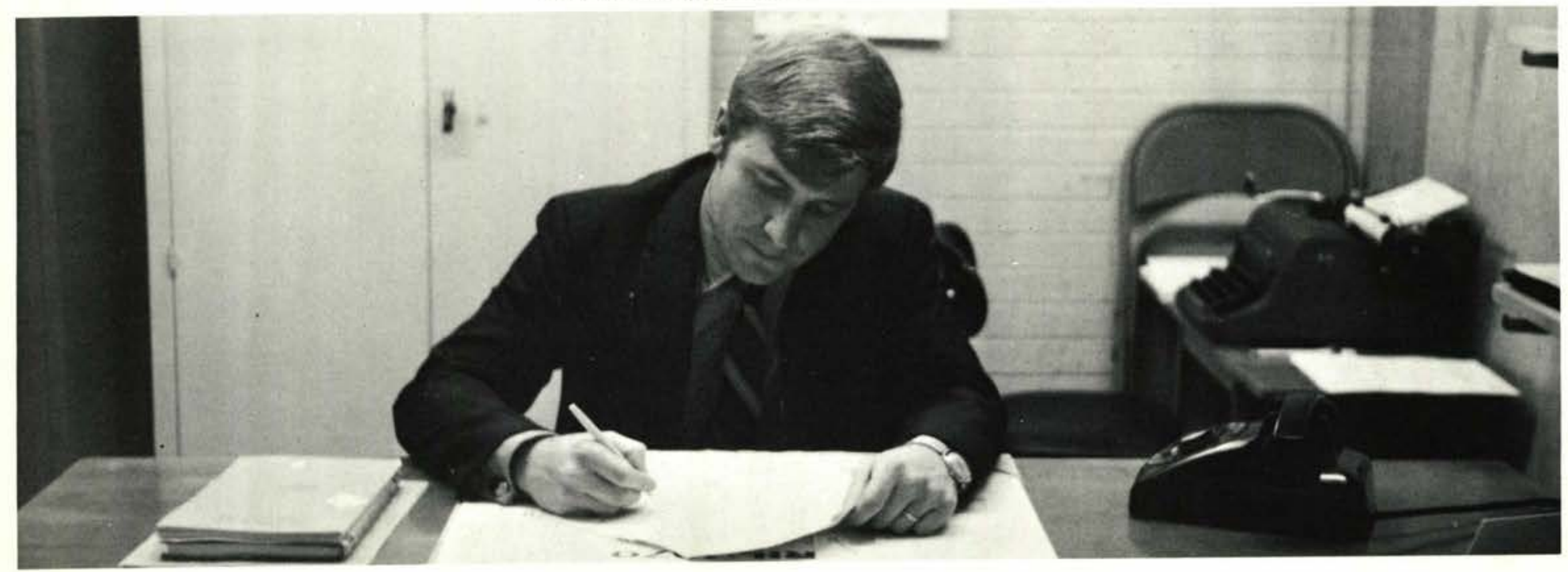




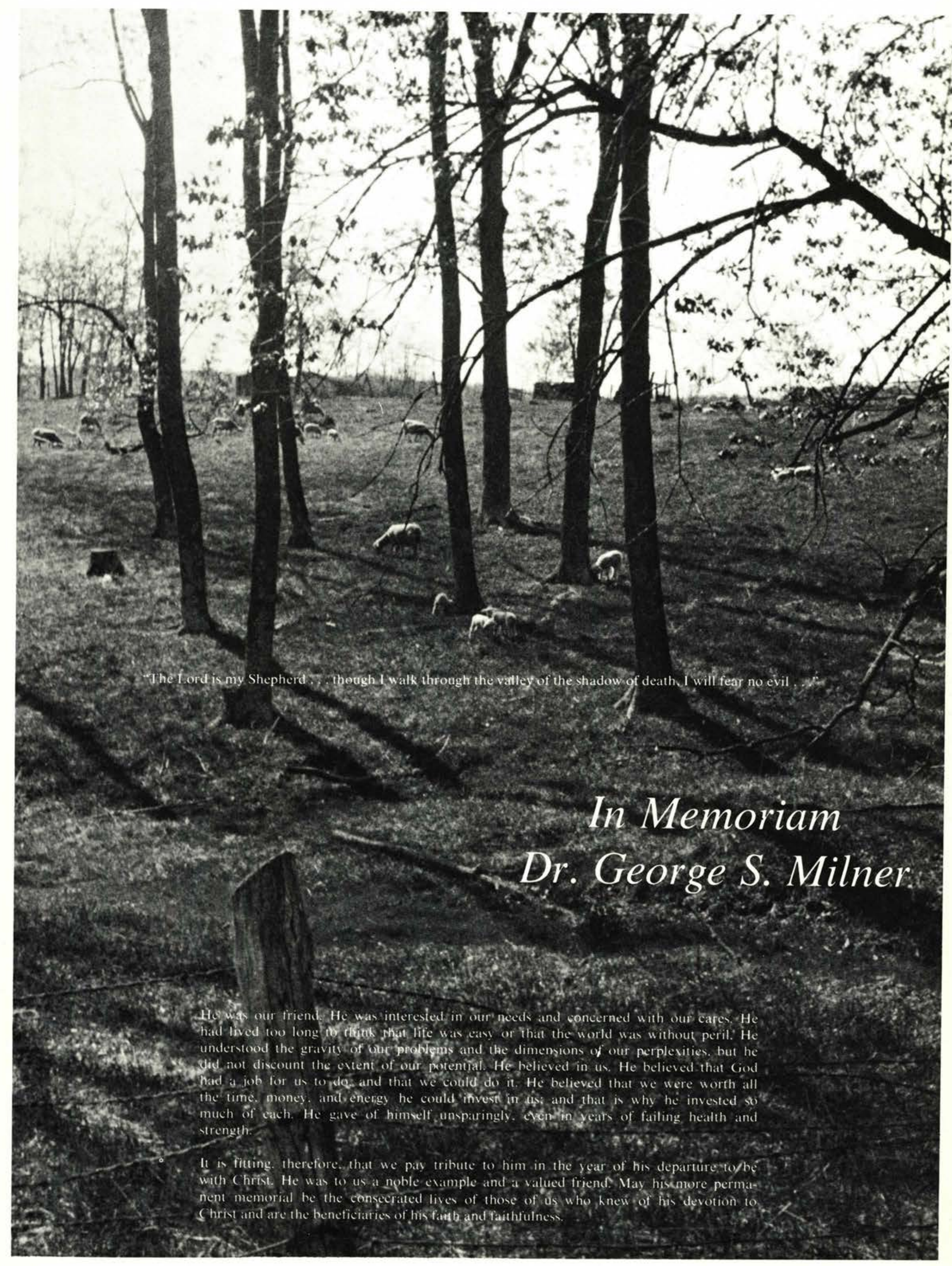




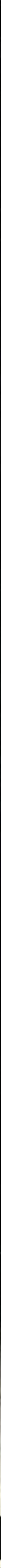




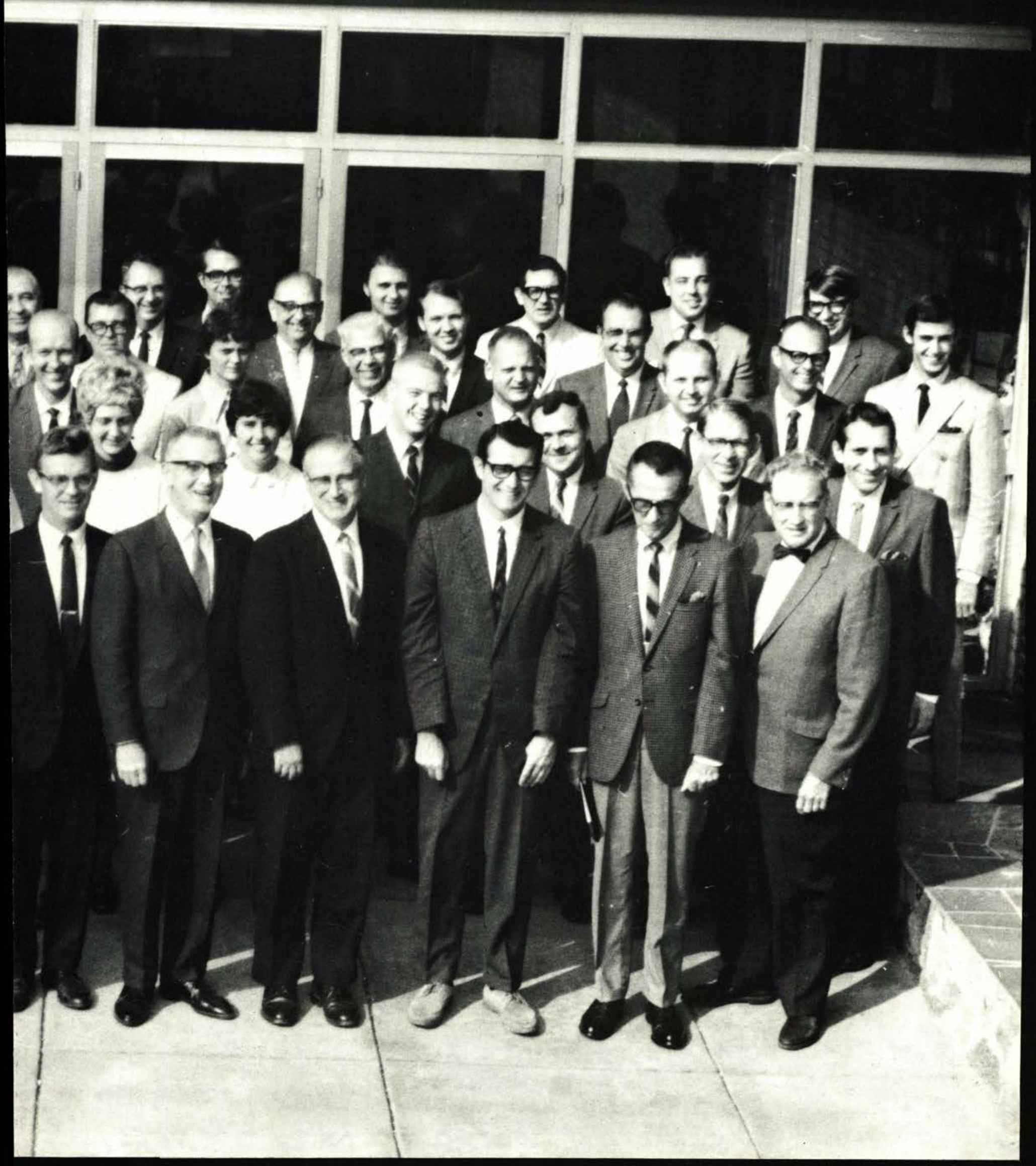




\section{$B I B L I C A L$ EDUCATION}

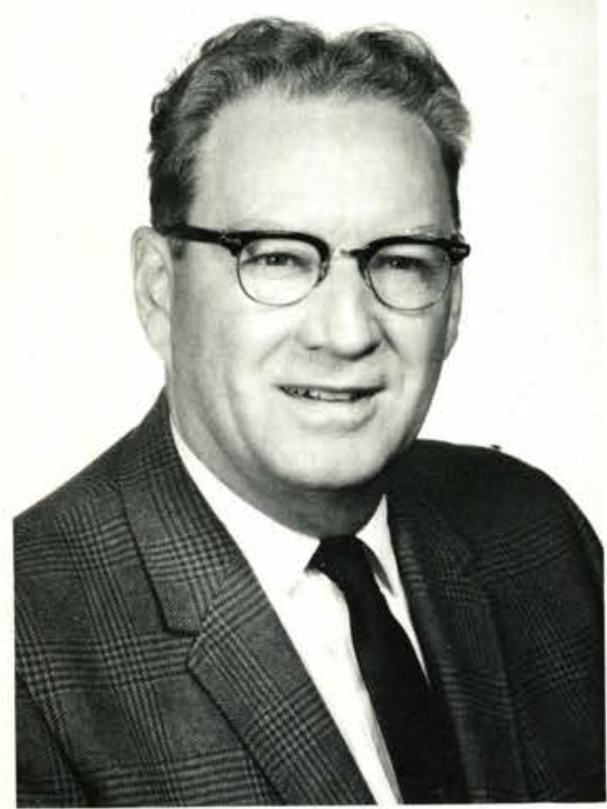

Mead C. Armstrong

U. of Pittsburgh, M. Litt.

Director of Christian Service

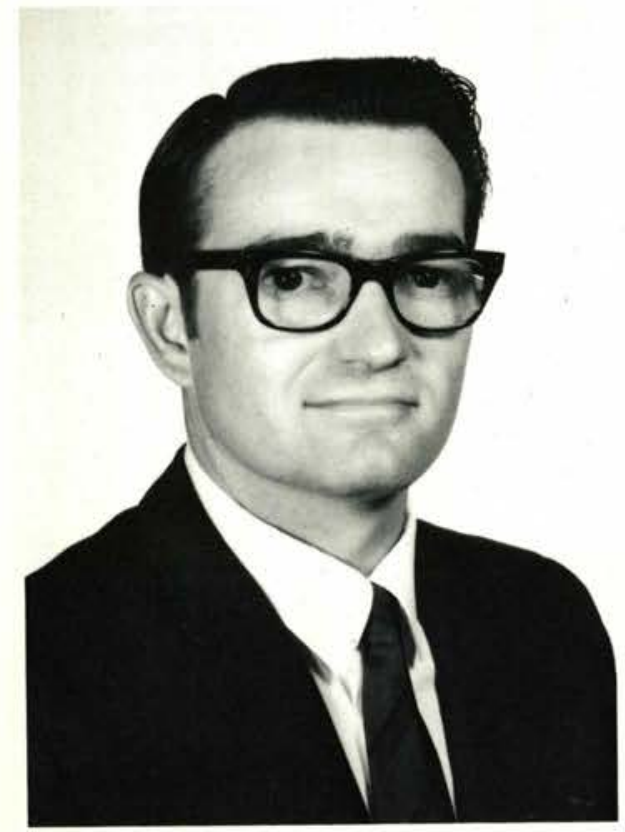

Robert Gromacki

Grace Theological Sem., Th.D. Golf Coach

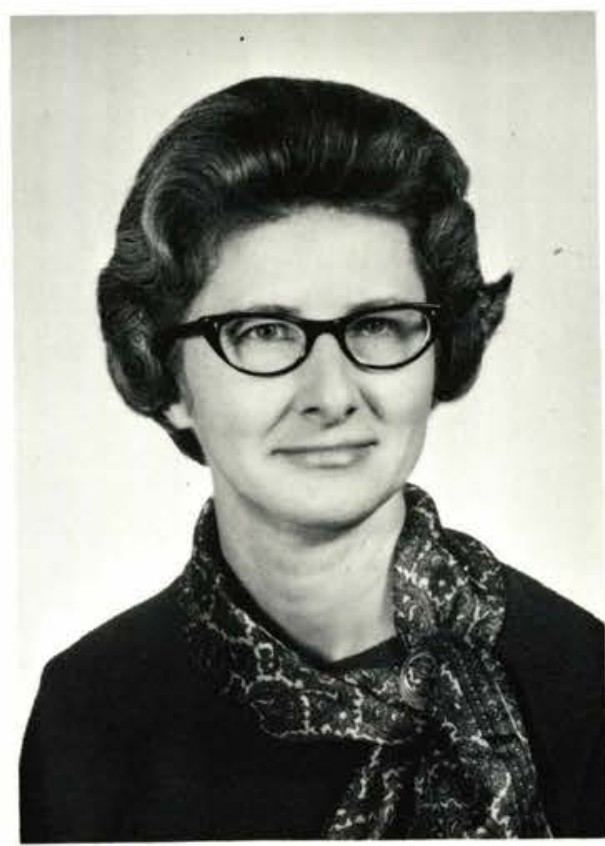

Jean Fisher

Wheaton College, M.A

Admissions Committee

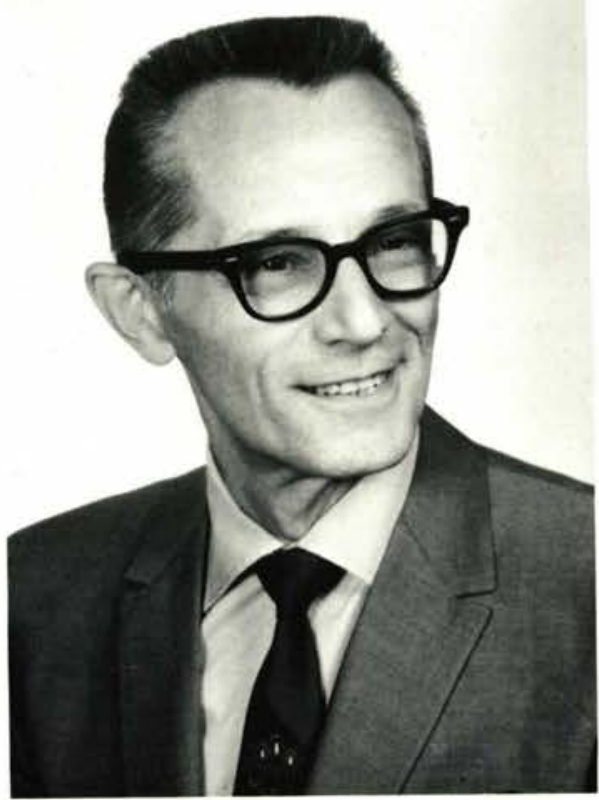

George L. Lawlor

Grace Theological Sem., Th.D.

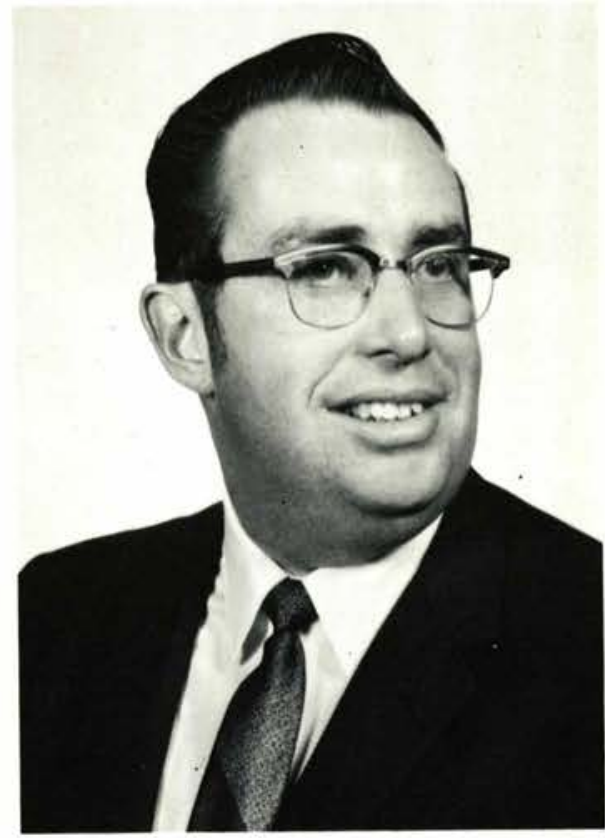

James M. Grier

Grace Theological Sem., M. Div.

Timalathian Advisor

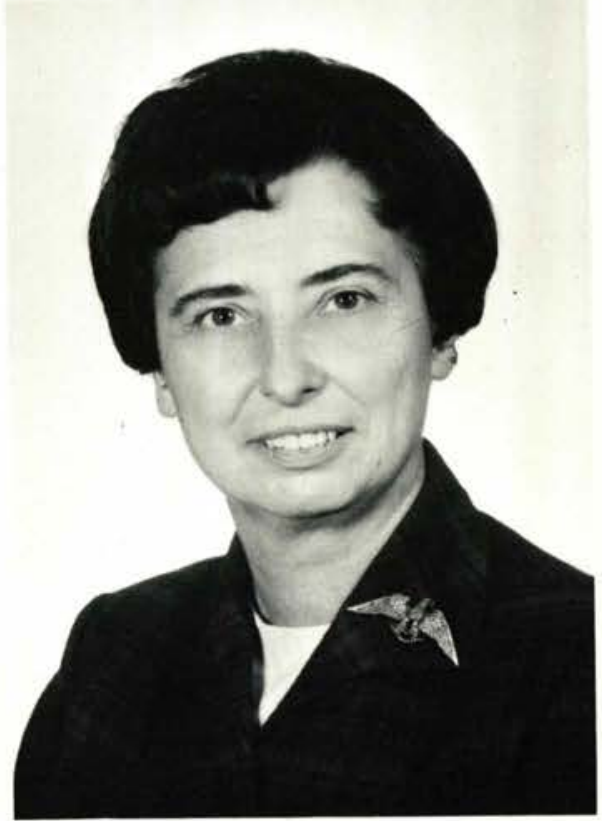

Joy Mackay

Wheaton College, M.A.

Summer Institute, Word of Life Island 


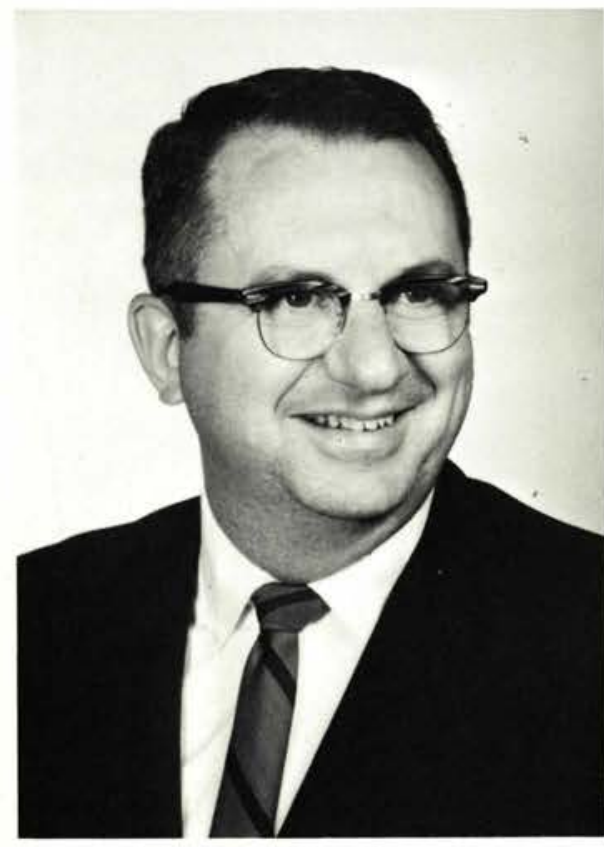

Richard T. McIntosh

Grace Theological Sem., Th.M.

Student Council Advisor
The Biblical Education Department is charged with the twofold responsibility of establishing the student in the Christian Faith and stimulating the student to evaluate all knowledge in the revelatory light of Scripture. The expansion of the department into the field of Christian Philosophy is intended to increase the student's ability to evaluate all knowledge.

The development of a Christian World and Life Philosophy is an absolute essential for each Christian to function properly in a God created and ordered world. The integration of all truth, as the consistent expression of the being and will of the Sovereign Creator, is the goal we must reach for the Glory of our God and our own spiritual well-being.

—James Grier

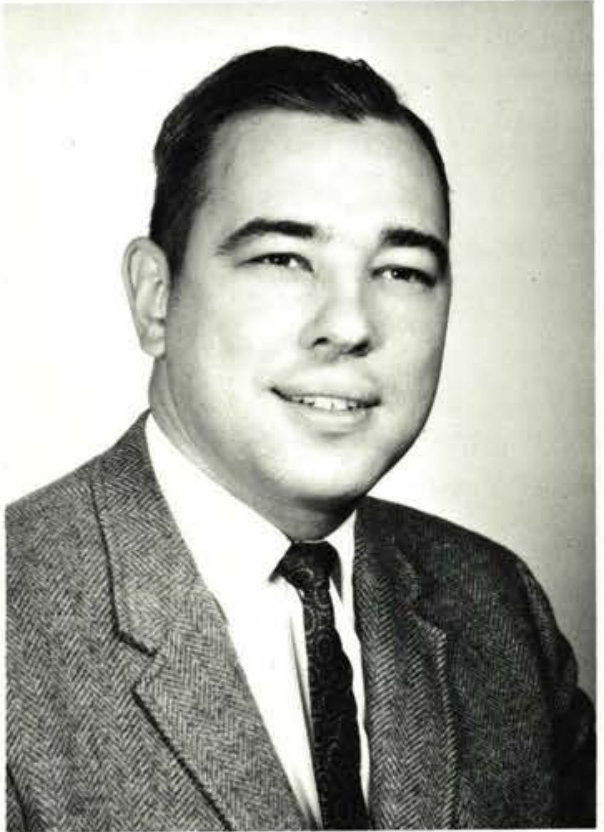

Jack R. Riggs

Dallas Theological Sem., Th.D.

F.W.M. Advisor

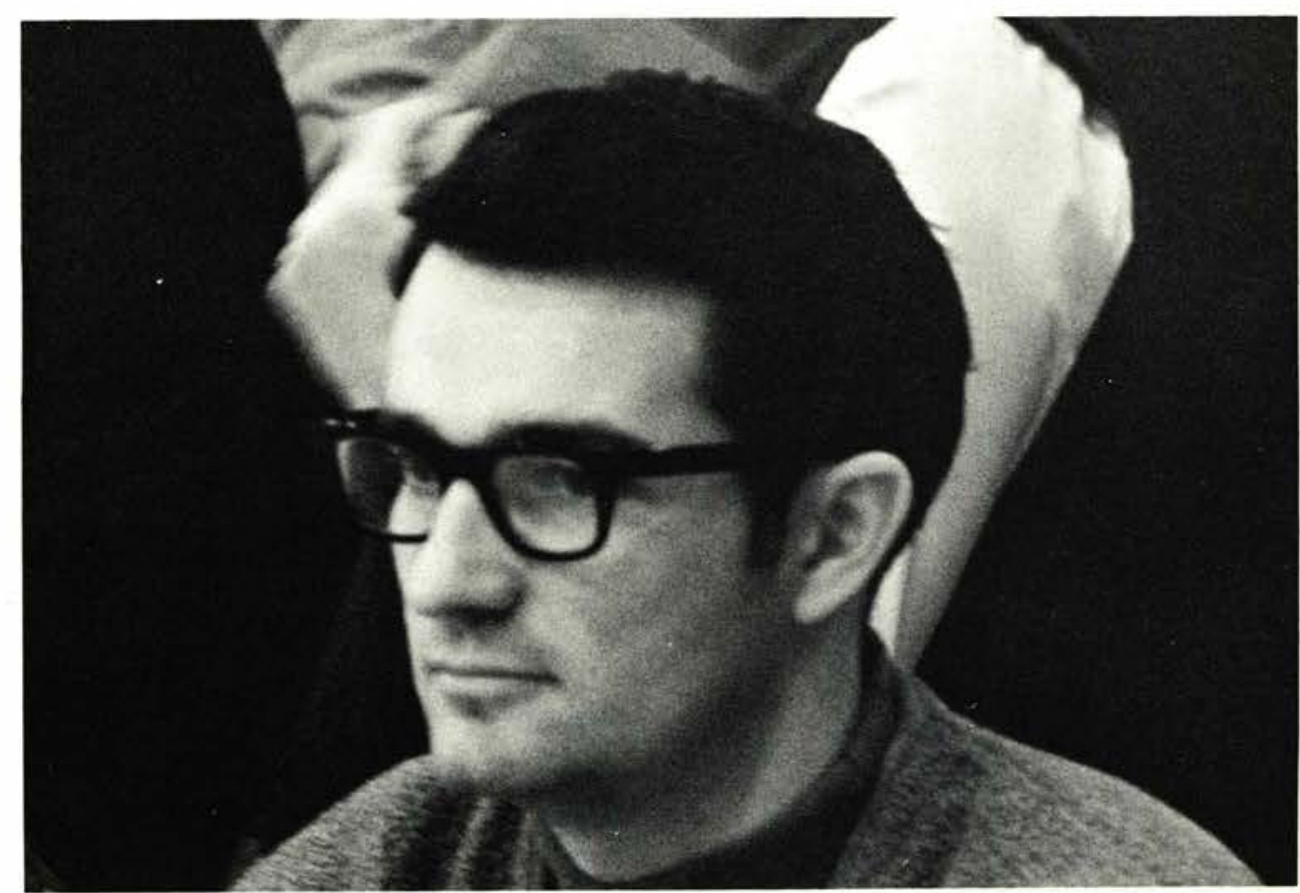




\section{EDUCATION}

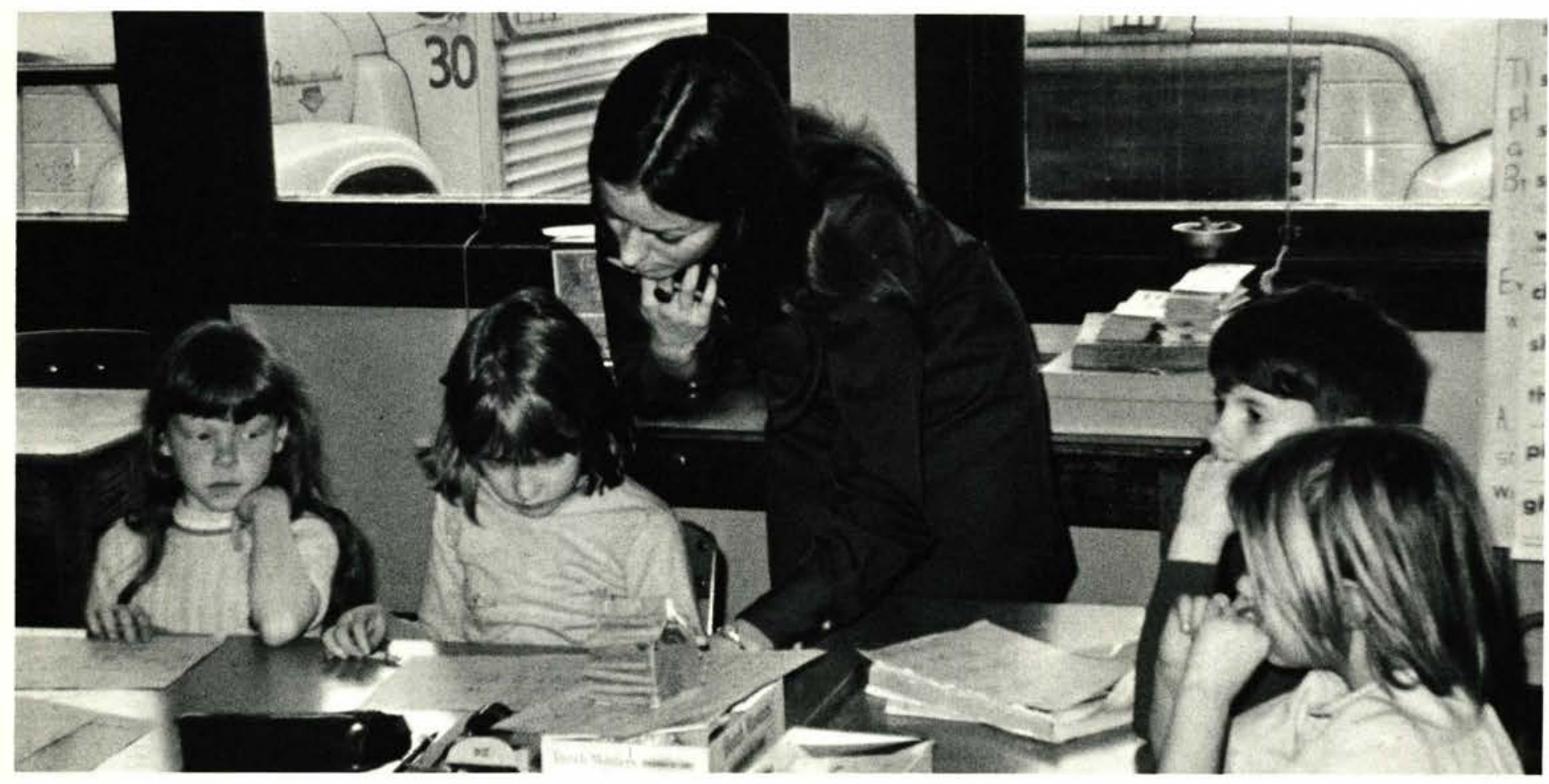

Modern higher education is coming under increasingly critical attacks for its "soul-less", technology-oriented, de-personalized methodology. As to specifics, students appear to be asking for more direct and personal involvement in classwork, more relatedness between what happens in class and life experience, and deeper, personal, more meaningful relationships with the more knowing member of the class experience, the professor. The encounter approach of contemporary psychology offers some hope to the too prevalent dehumanizing pressures of education. The encounter is a creative experience wherein one enters into the reality of a situation in terms of the conditions and requirements intrinsic to that situation. Openness, receptiveness, and relatedness are significant aspects of the encounter. The encounter is an immediate, imminent reality between persons engaged in a living communion, where there is a sense of mutuality. The application of these concepts to educational concerns is at once difficult, but not impossible.

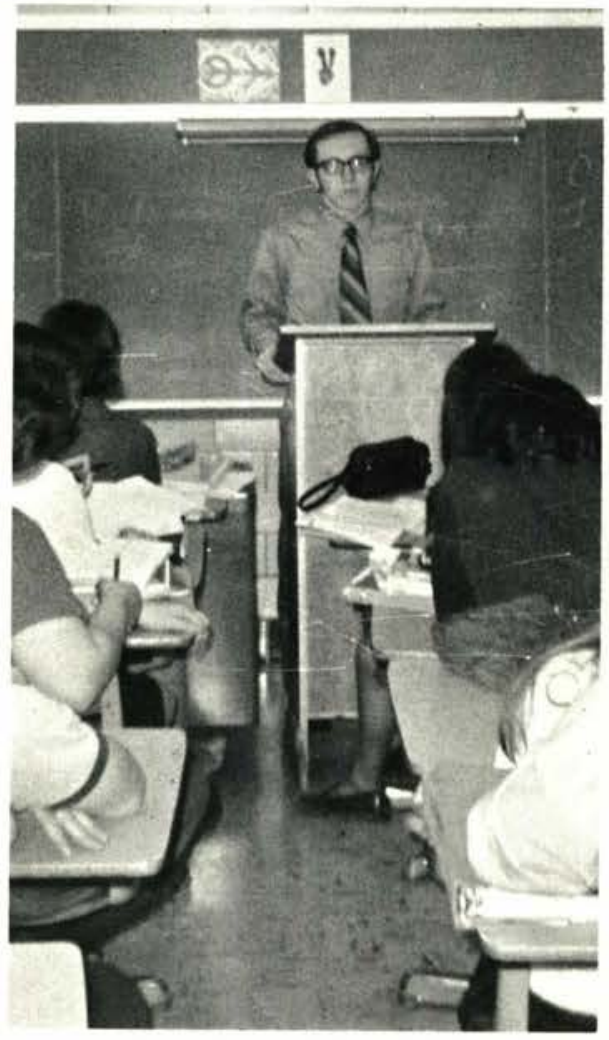




\section{PSYCHOLOGY}

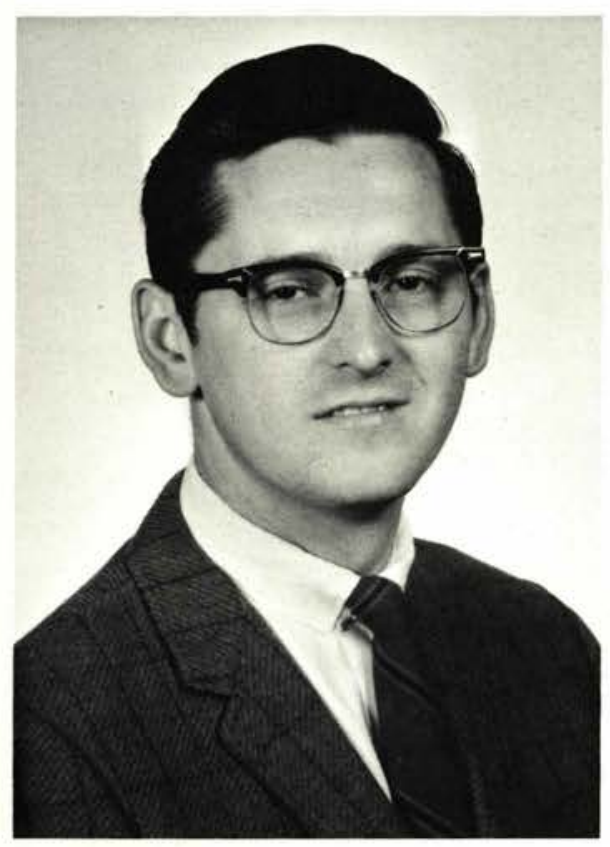

Merlin F. Ager

Ohio State University, Ph.D.

Advisor Class of 1971

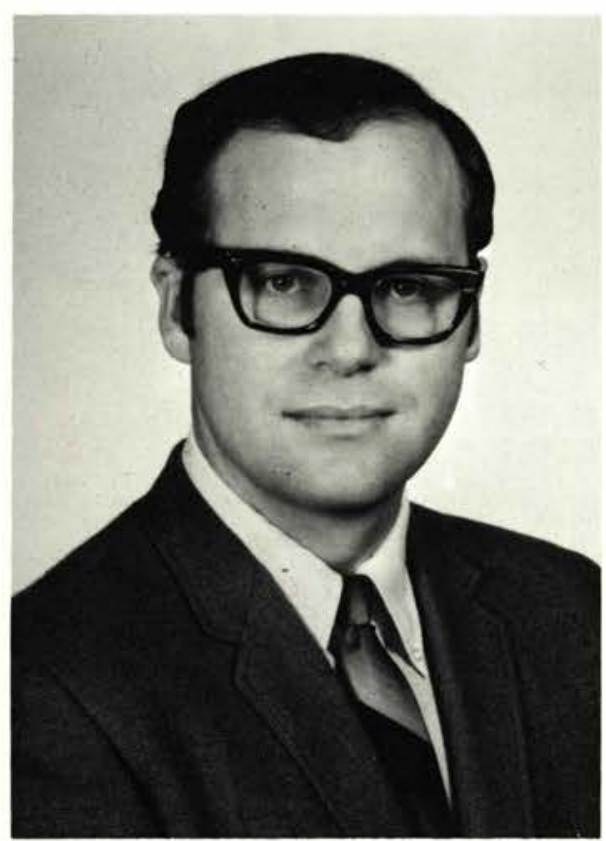

Dwayne I. Frank

College of Idaho, M.S.

College Residence Advisor

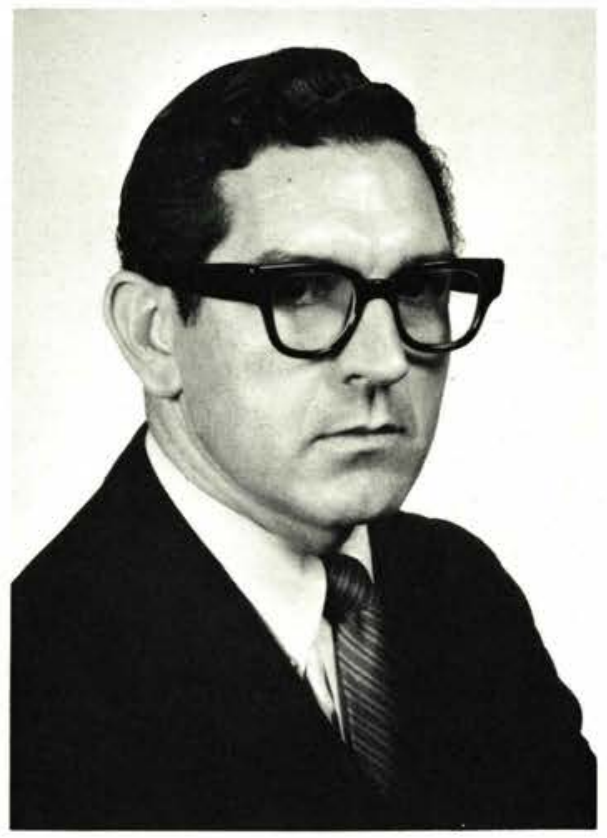

Stanley N. Ballard

N. Texas State University, M.S.

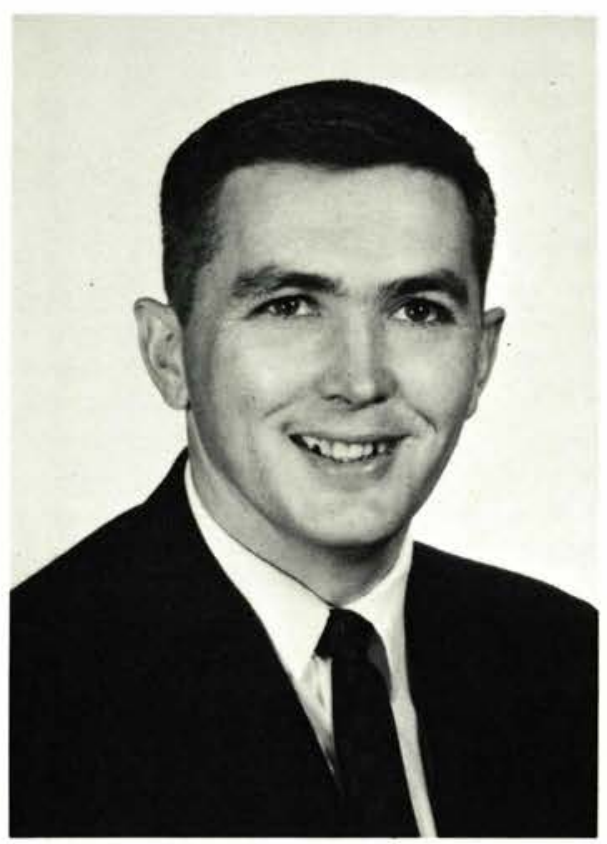

Brad E. Moore

Ball State University, M.A.

Pi Sigma Nu, S.E.A.C. Advisor

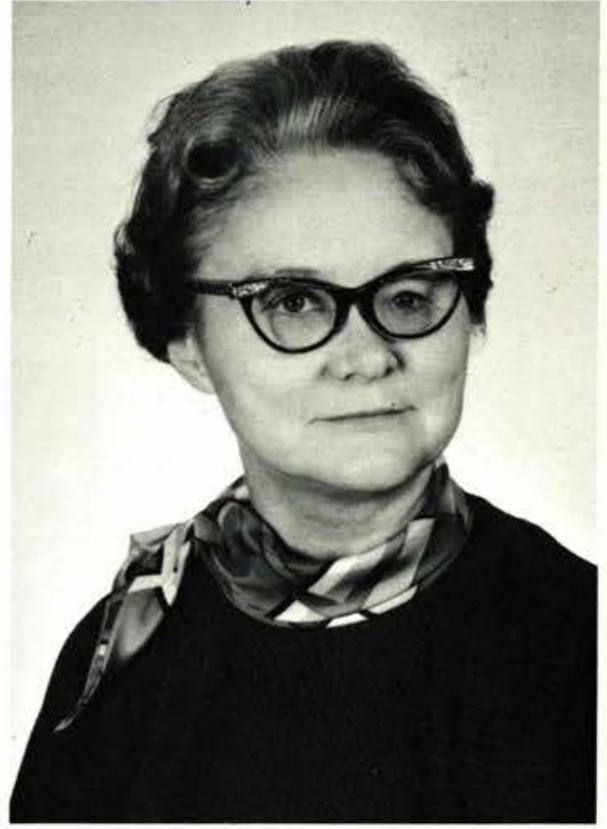

Irma M. Dodson

Indiana State Uni., M.A.

Educational Policies Committee

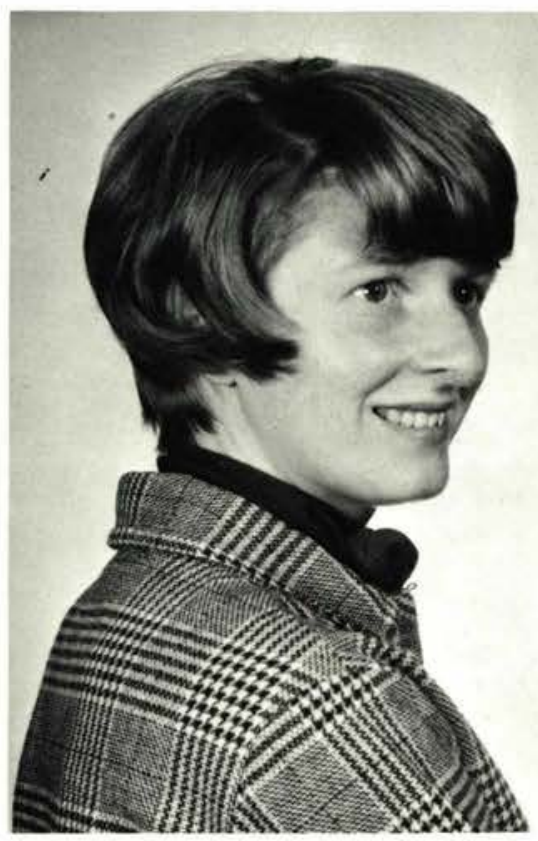

Lois Stockwell

Bowling Green State Uni., M.A. 


\section{LANGUAGE $A N D$ \\ LITERATURE}

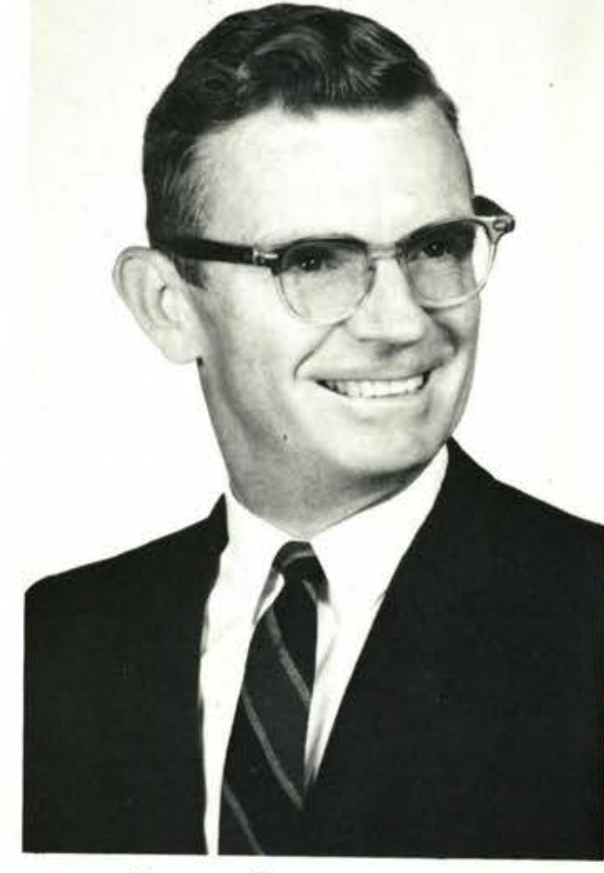

Harmon Bergen

Indiana University, M.A.

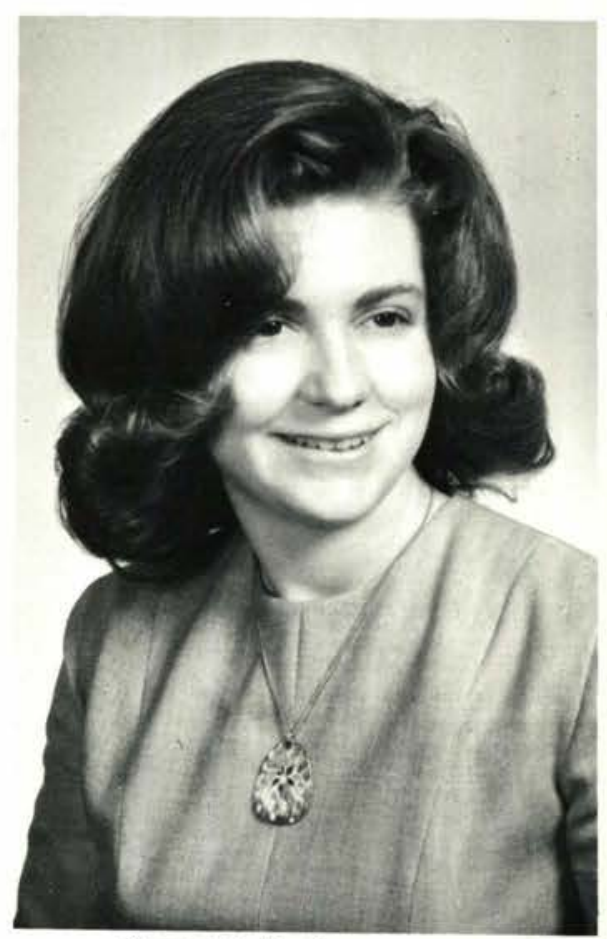

Sharon R. Boylan

Iowa State University, B.S. 


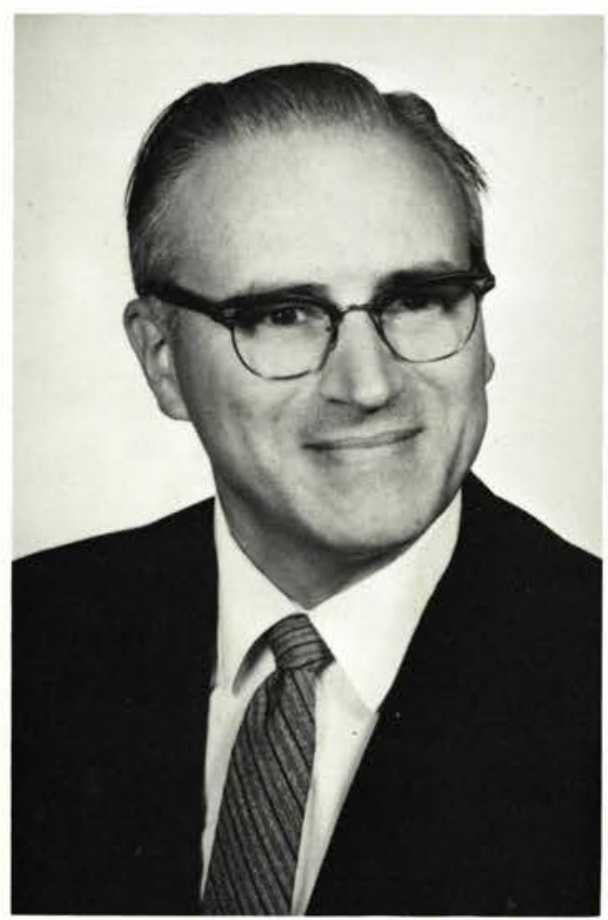

Edward L. Greenwood

University of Dayton, M.A.

Advisor, Class of 1972

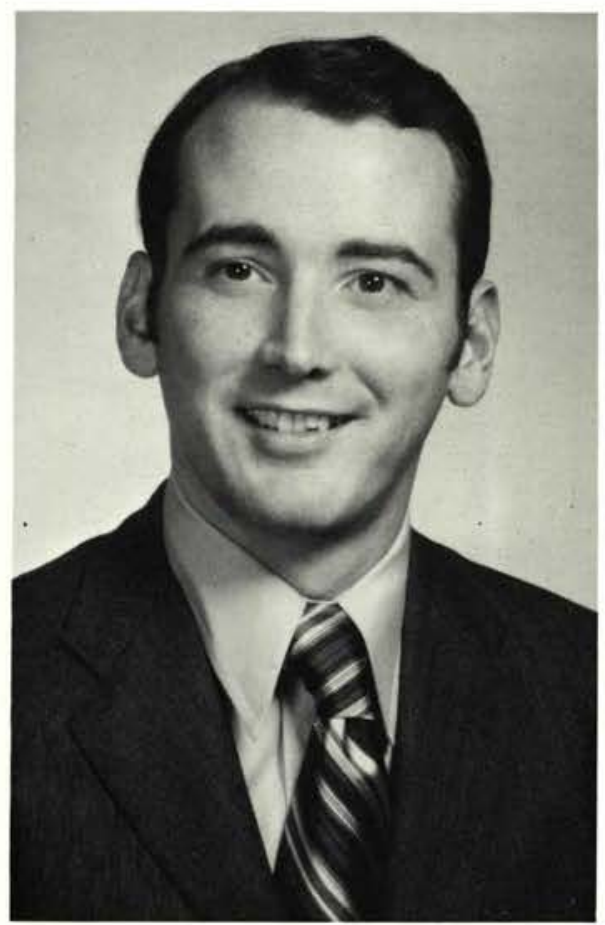

Ronald M. Grosh

Kent State University, M.A.T.

Student Affairs Committee

Literature: Aesthetically, verbally articulated mirror of life, reproducing and recreating what man has enregistered about the whole story of mankind. Literature does not set forth formulas for existence itself . . . it gives awareness, it awakens, it gives depth and understanding of the total human condition Christ came to redeem. Maturity sees wholeness and relatedness of all man's experiences. Enjoyment that becomes insight is perhaps the greatest gift literature has to offer.

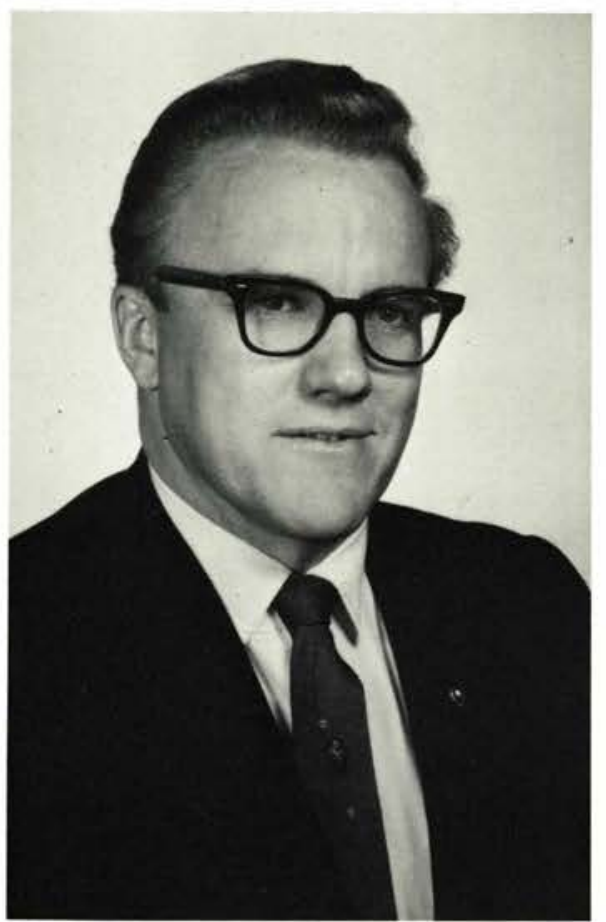

Helmuth C. Poggemiller

Kansas State Teacher's College, M.A.

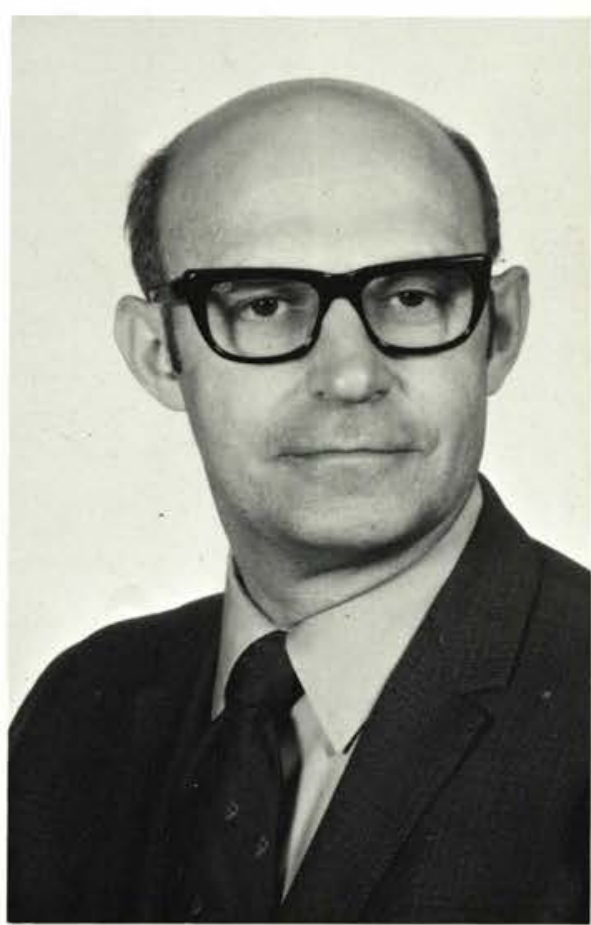

Edward E. Spencer

University of Dayton, M.A. Advisor, Class of 1970

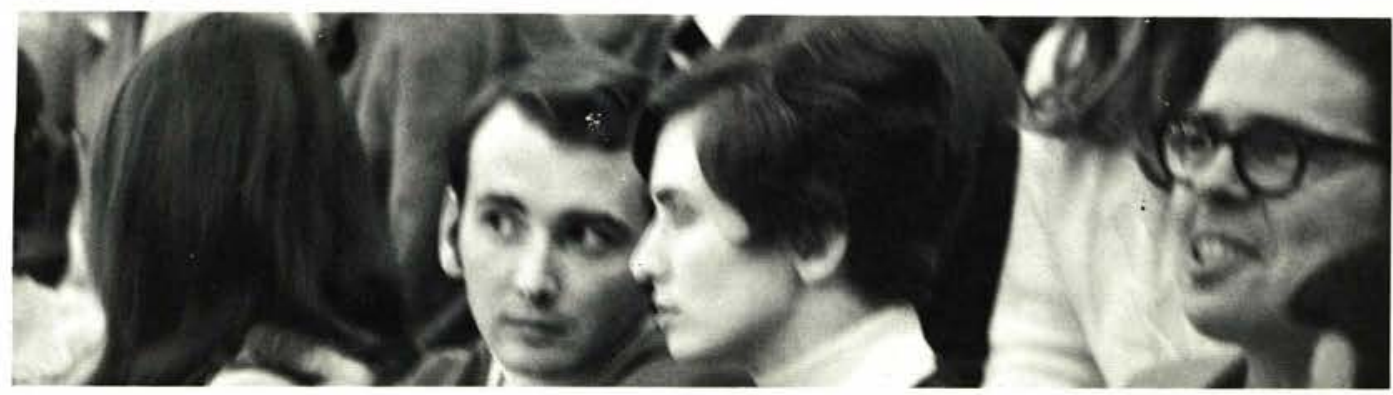




\section{SPEECH}

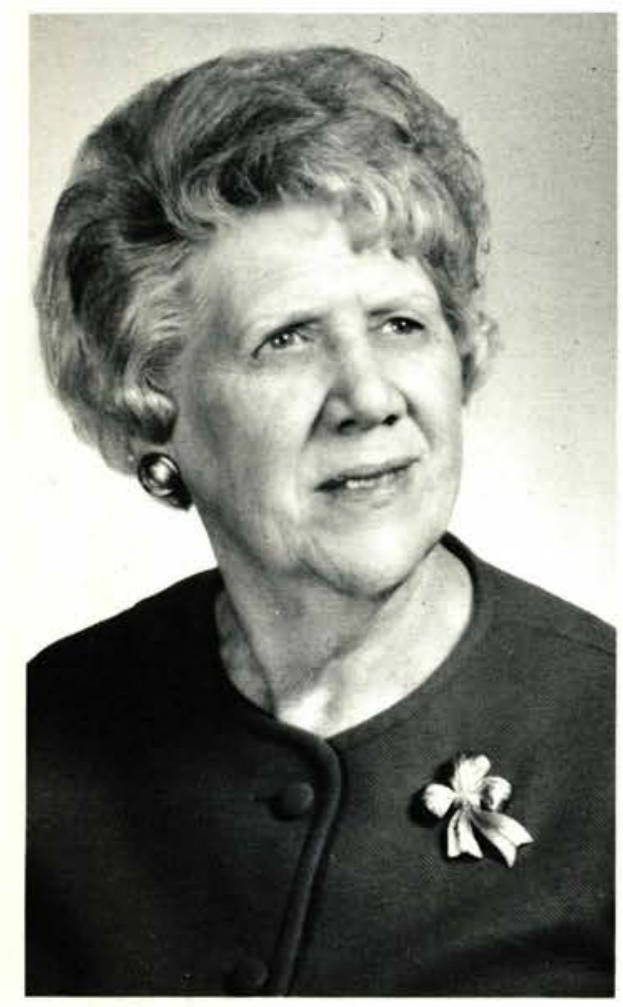

Miriam B. Maddox

Moody Bible Institute, B.A.

Gamma Chi Advisor

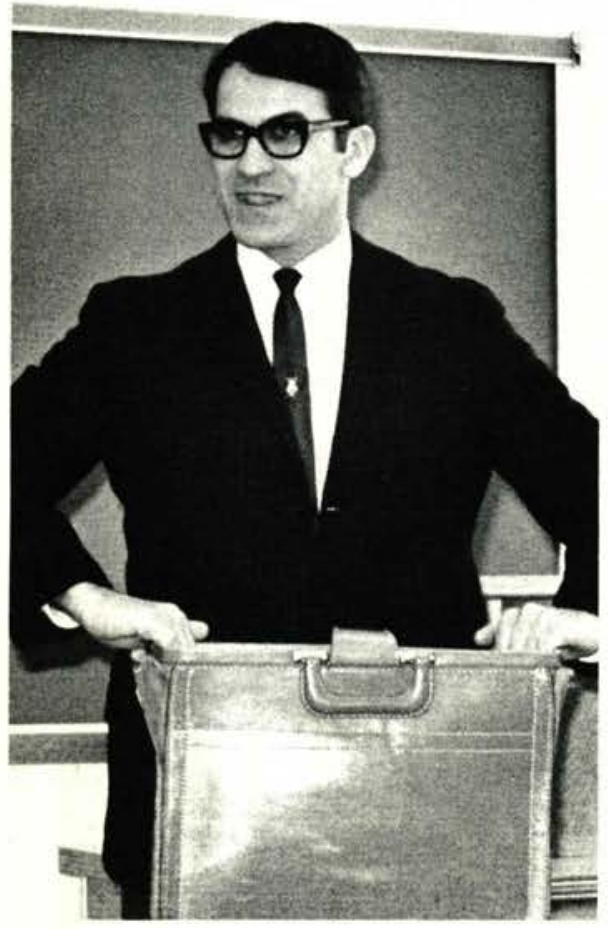

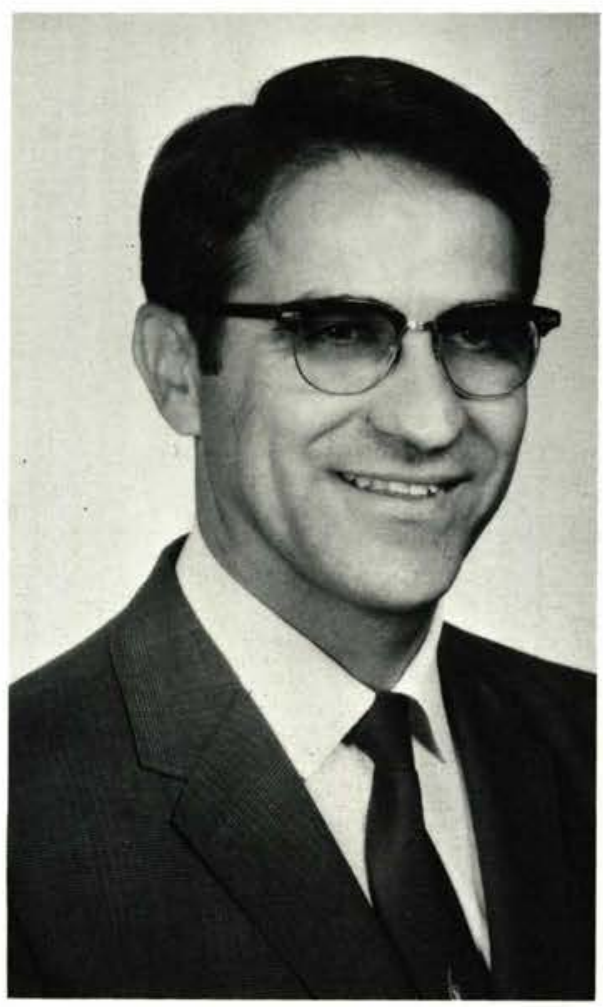

John W. Reed

Ohio State University, Ph.D.

Advisor, Class of 1970

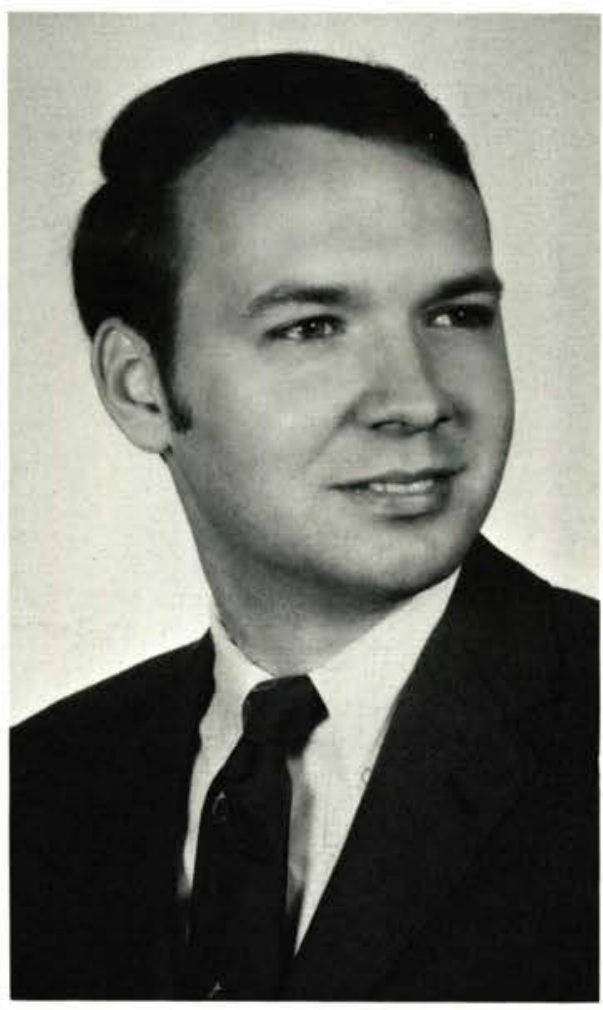

John C. Stockwell

Bowling Green State Uni., M.A.

Village Players Advisor

Long, long ago, so an old theatrical producer mused, a caveman foraging for his sustenance was approached by a savage stegosaurus who offered to eat him. A fearful fight ensued, but the stegosaurus was finally felled by a staggering wallop. Shaken, but triumphant, the hunter gathered his friends to relate the incident. But instead of just telling it, he acted it out. The spectators yowled and banged sticks and stones to show their appreciation. The drama was born.

Soon, however, the audience began to reflect. Some thought the stegosaurus hadn't been represented well at all. Others doubted that the hero was as brave as he pretended. So it began. So it continues. 


\section{SOCIAL SCIENCE}

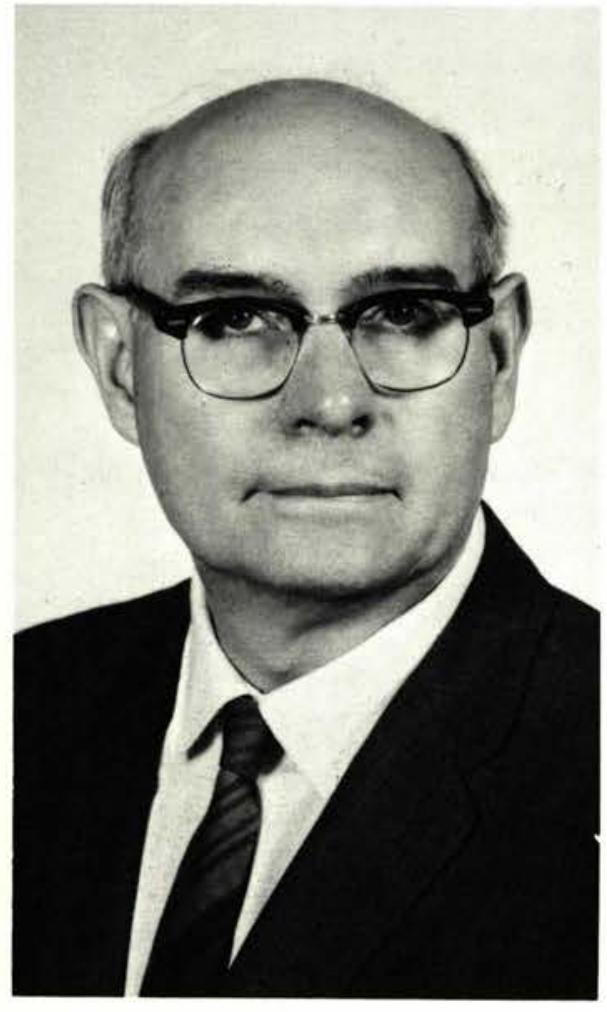

Cleveland McDonald

Ohio State University, Ph.D.

Faculty Committee to President

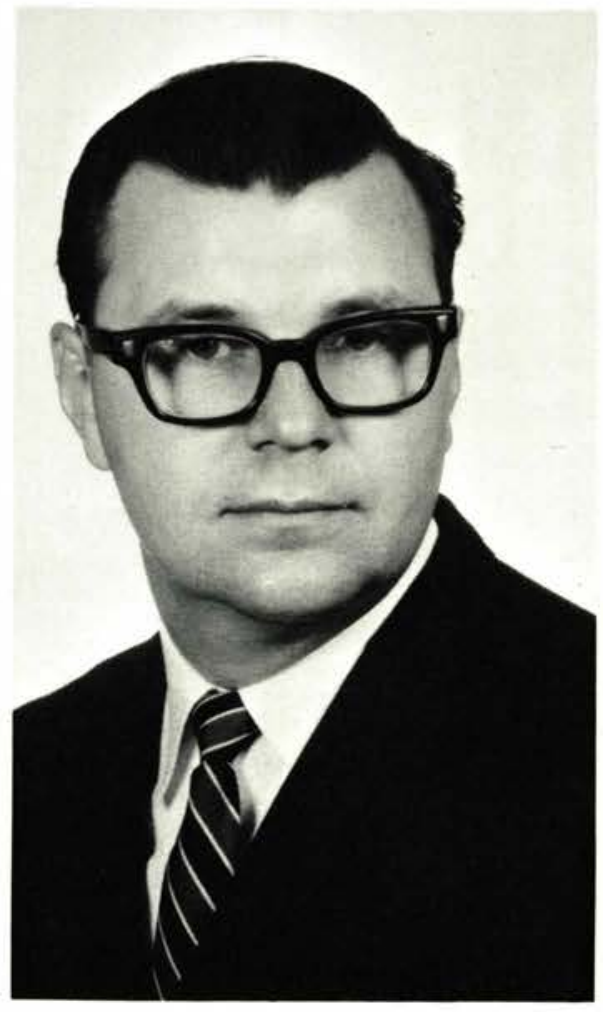

Allen L. Monroe

Montclair State College, M.A.

\section{FRECKLES}

One of the great crises facing mankind is freckles. All the people who have no freckles agree that the people with freckles are "different". The people who have freckles think that all the people without freckles are bigotted. The people without freckles say, "but some of those freckled people want to take over our country." But not all people with freckles agree. Furthermore, if the people without freckles can disagree among themselves - why can't the people with freckles? Perhaps someday they even could learn to agree and disagree with each other.

-J. Murray Murdoch

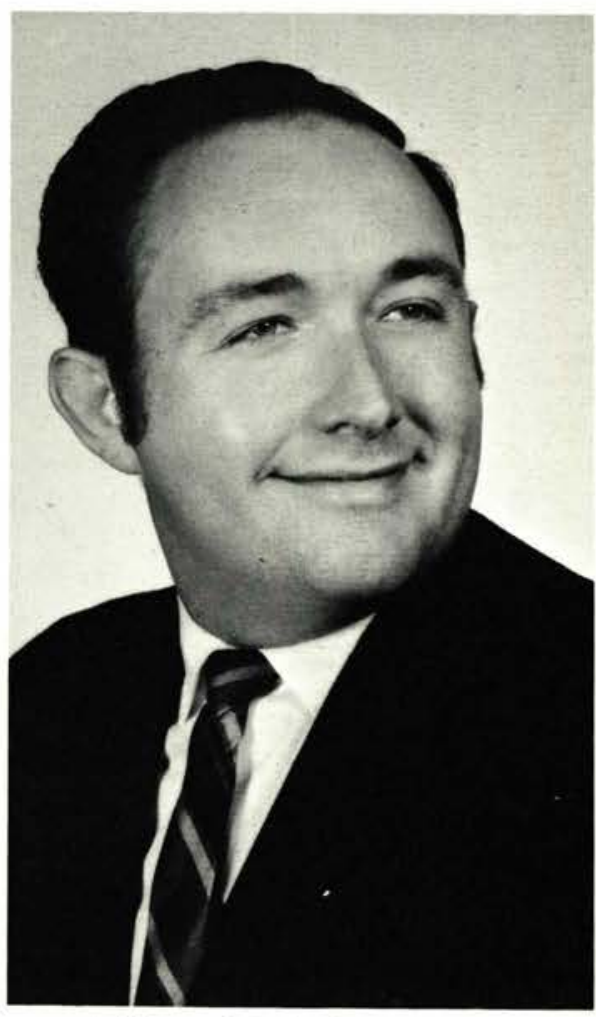

J. Murray Murdock

Northwestern University, M.A.

Advisor, Alpha Chi/Tennis Coach

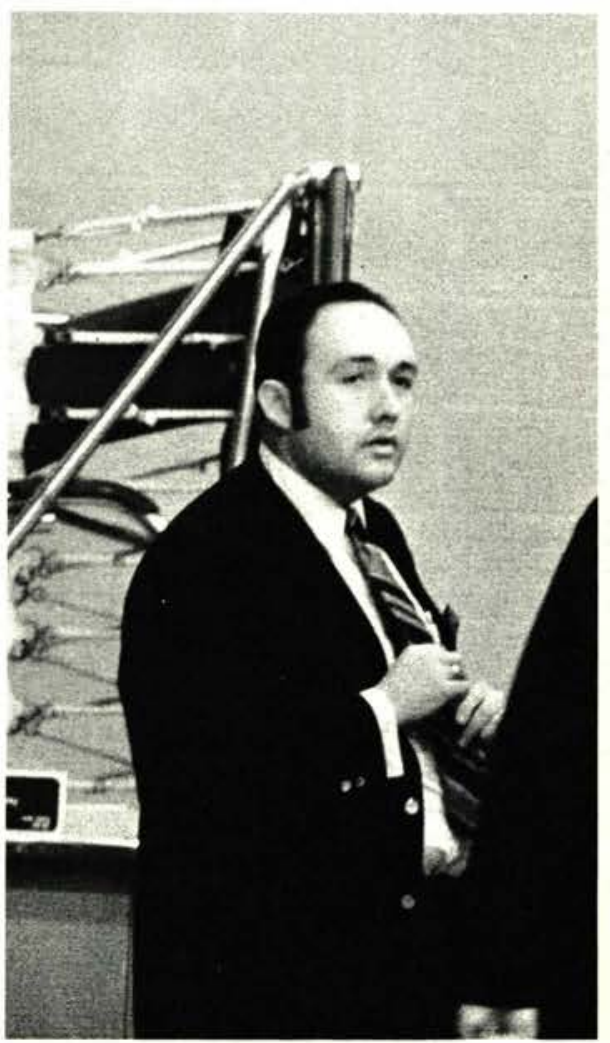




\section{SCIENCE $A N D$ $M A T H$}

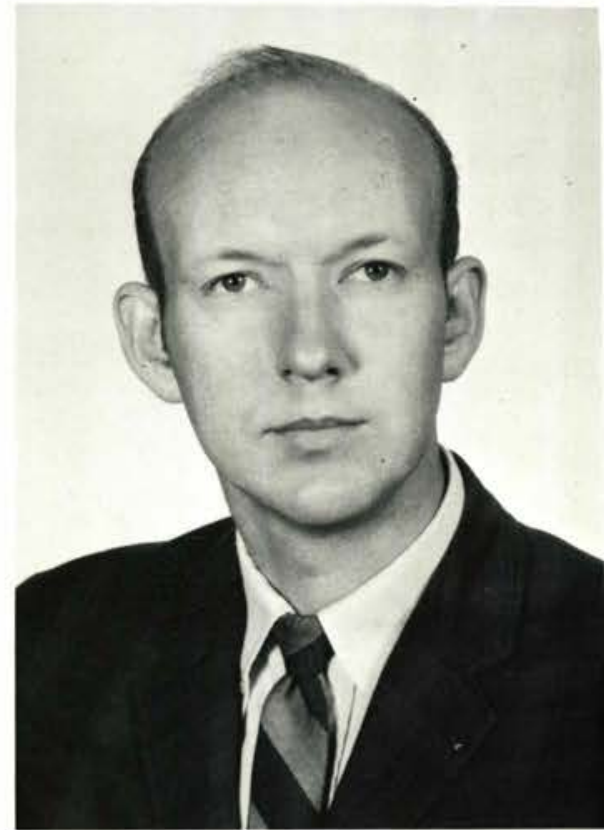

Donald P. Baumann

Iowa State University, Ph.D.

Yearbook Advisor

Current important topics in today's world include "ecology" and "pollution". Man is waging a futile battle to restore his environment which, because of a sinful nature, he is destroying. Any attempt by man alone will result in failure; he must have his "spiritual pollution" removed and a good relationship established with the Creator resulting in "spiritual ecology".

—Donald P. Baumann

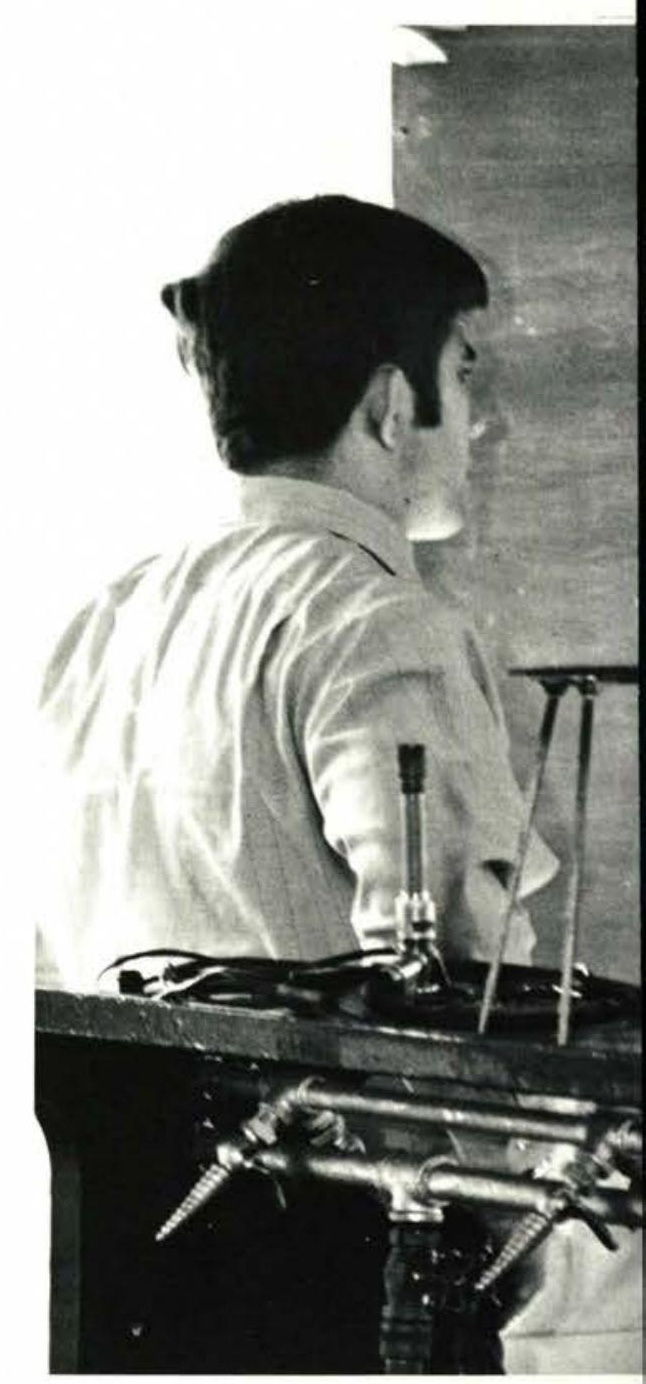




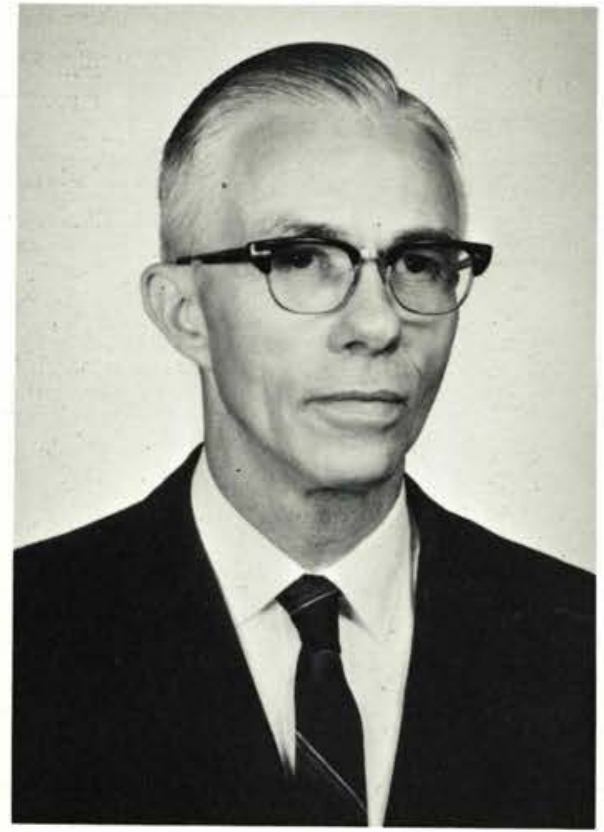

Austin D. Elmore

Indiana University, M.A.T.

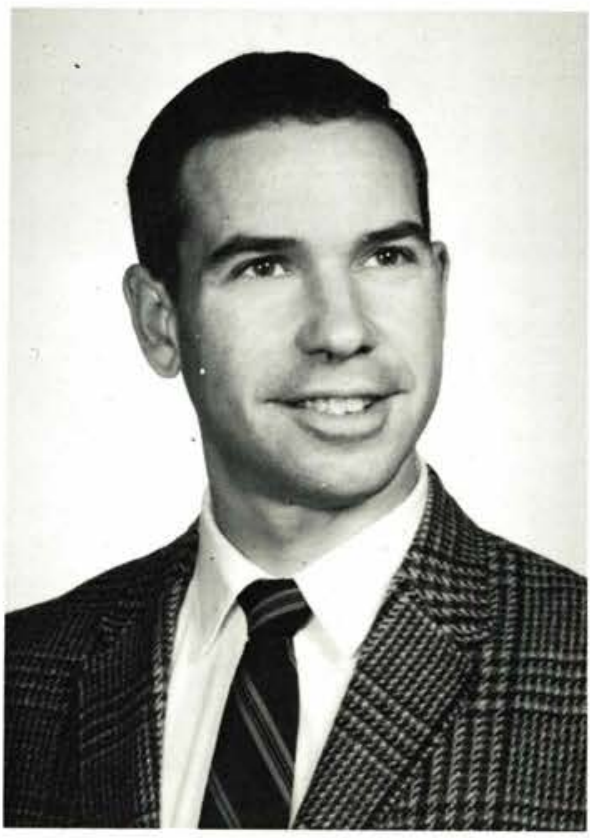

Larry S. Helmick

Ohio University, Ph.D.

Youth Group Sponsor

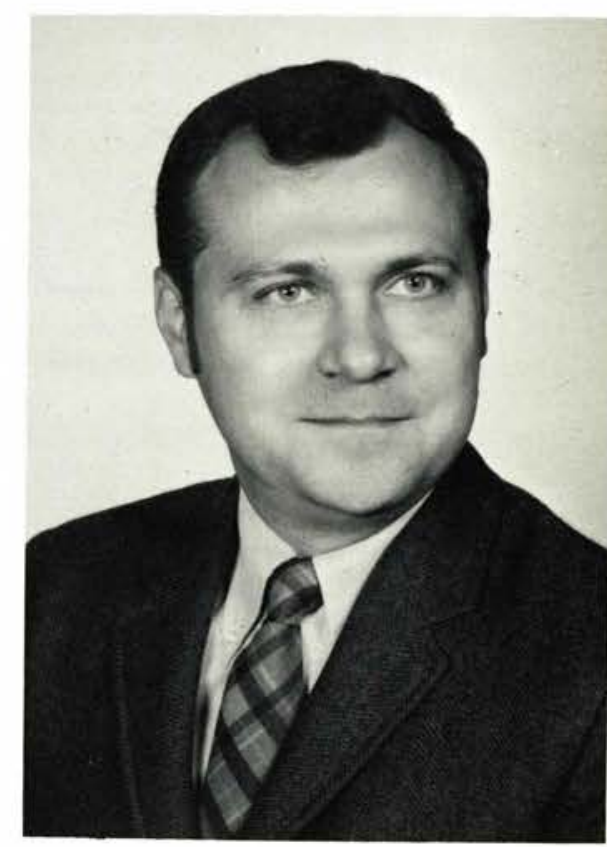

Lawrence N. Killian

Syracuse University, M.S.

Teacher Education Committee
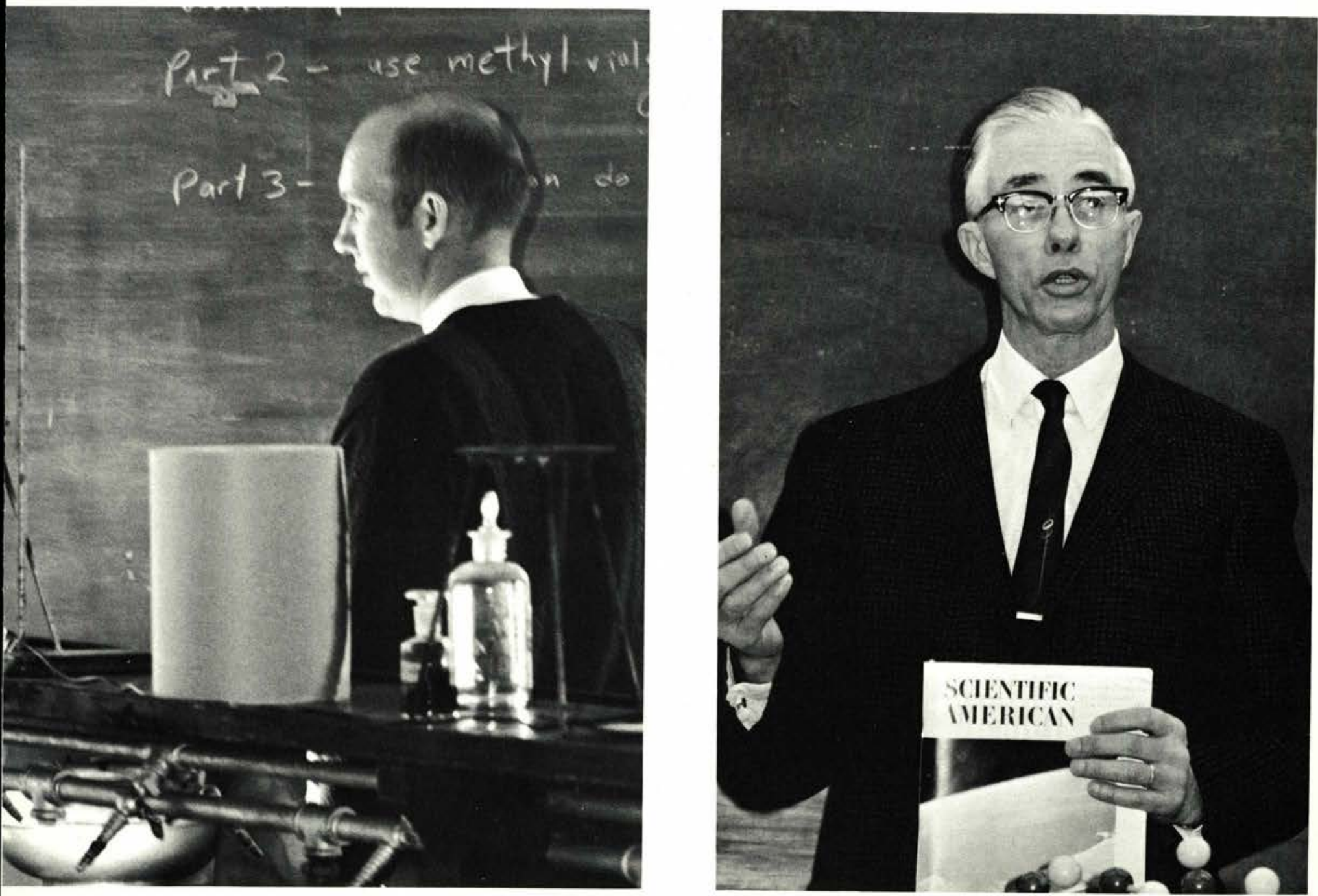


\section{BUSINESS}

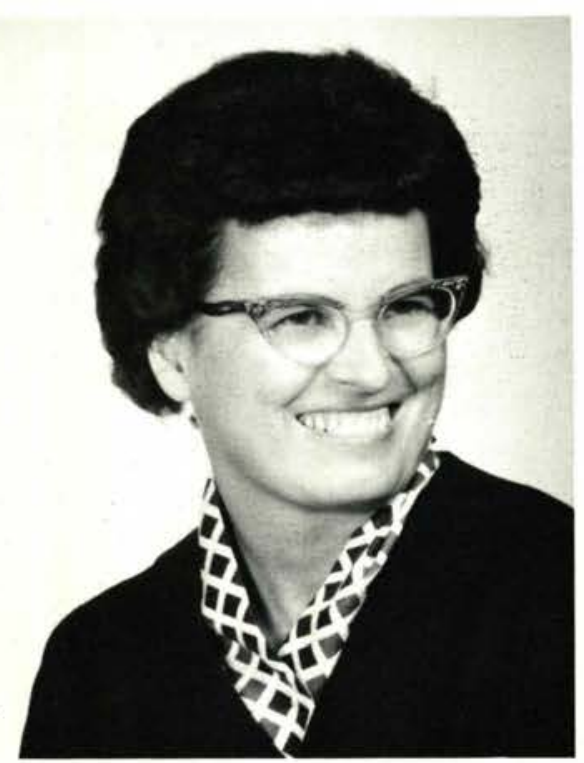

Ardeth Webber

Ohio State University, M.A.

Teacher Education Committee

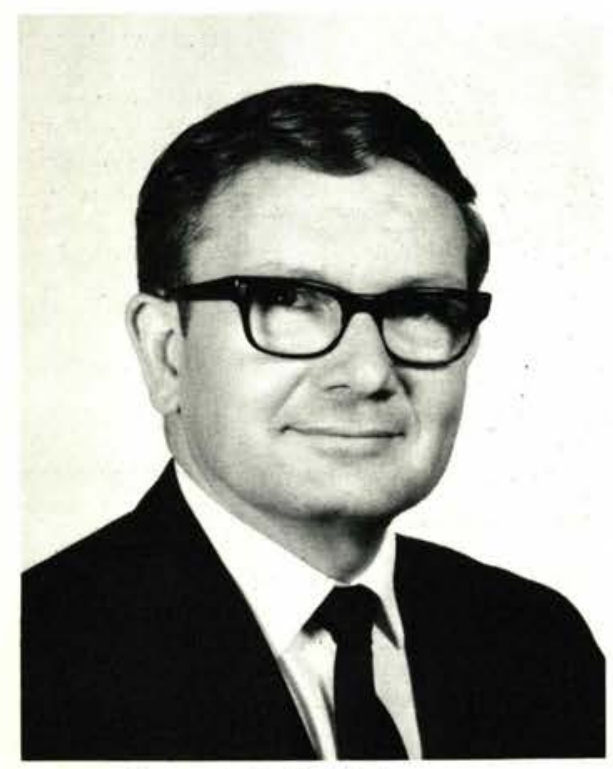

Kenneth H. St. Clair

University of Illinois, M.S.

Business Manager

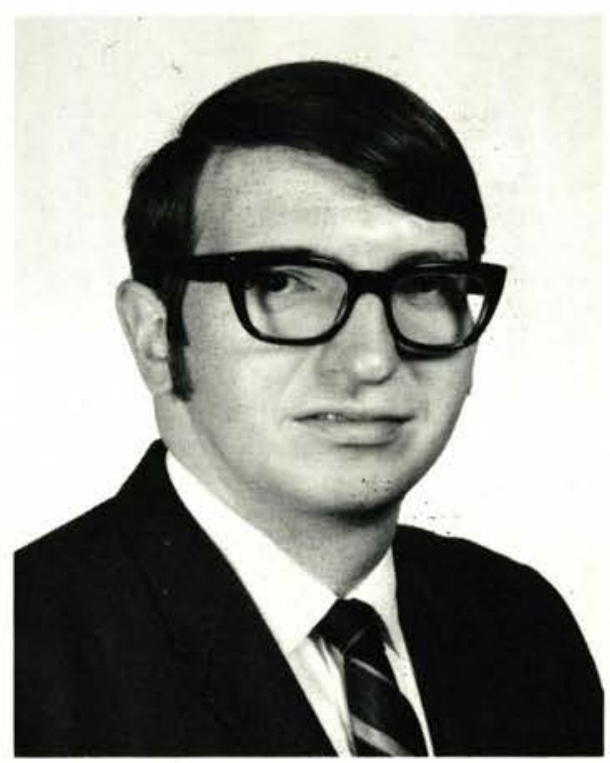

Donald T. Wilcoxon

University of Dayton, M.B.A. Advisor, Class of 1973

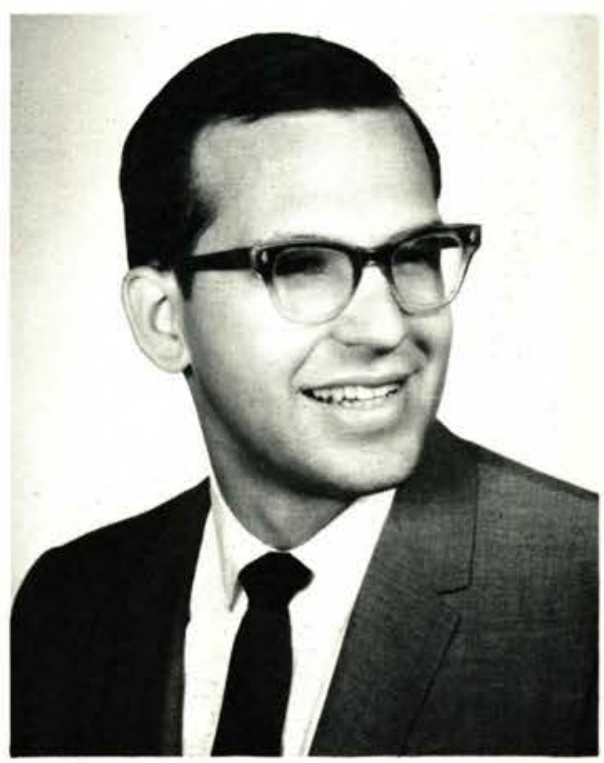

William R. Riter

Cedarville College, B.S.

State of Illinois, C.P.A

Ethics in business is a subject of much concern to many. Can Christ and business mix? To answer "NO" would be to relegate God to a place of no importance in that area of a man's life where he spends most of his waking hours.

"Whatsoever you do in business, do only that which will please God. If you know that it will please Him, then do it heartily, enthusiastically." (Paraphrase, Col. 3:23, by John Mitchell). 


\section{PHYSICAL EDUCATION}

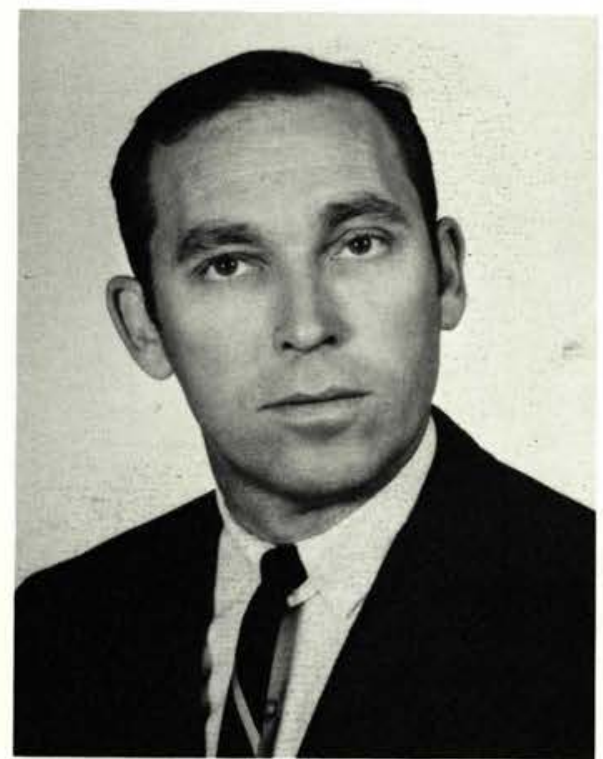

Donald Callan

Ohio State University, Ph.D.

Athletic Director

Basketball, Baseball Coach

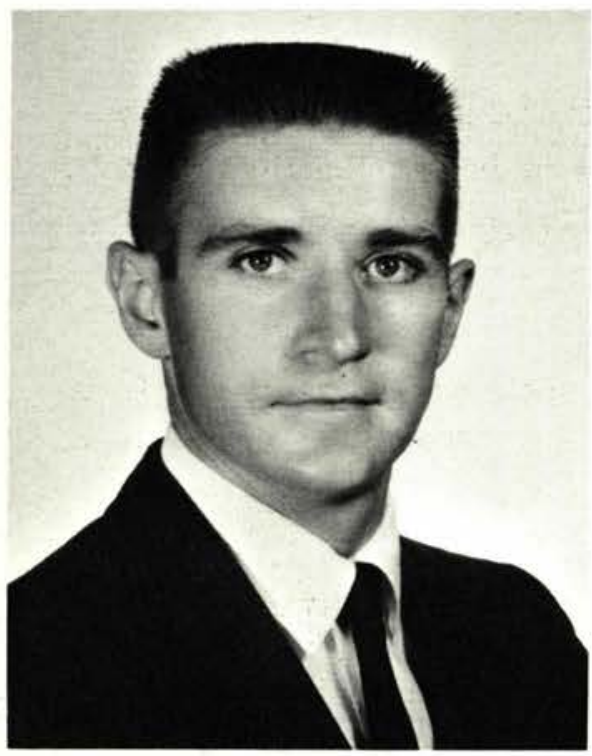

Elvin King

Bowling Green State University, M.Ed.

Track and Cross Country Coach

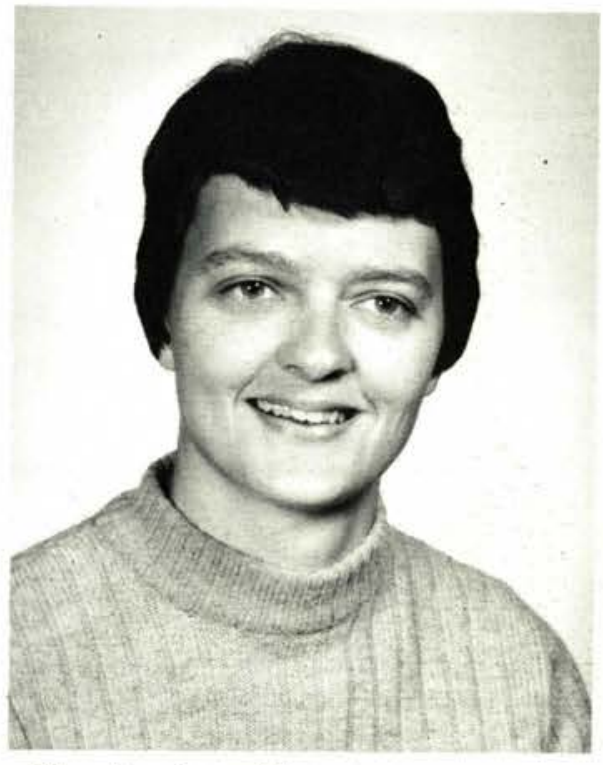

Maryalyce Jeremiah

Ohio State University, M.A.

Women's Intercollegiate Basketball Coach

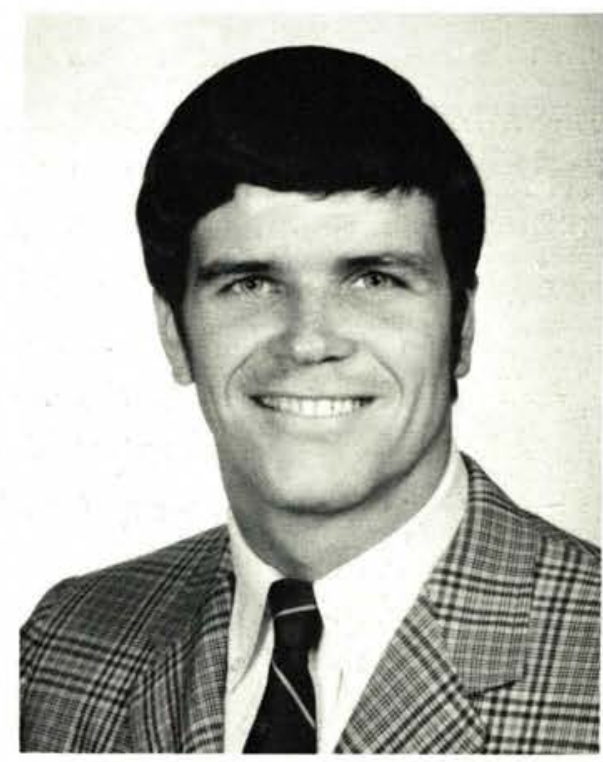

Randall Ross

Cedarville College, B.S.

Soccer Coach/Wrestling Coach

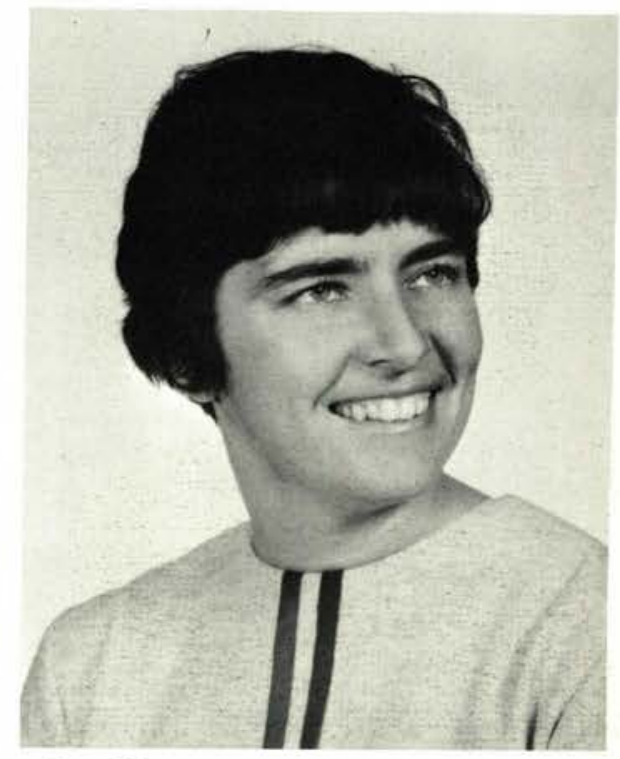

\section{June Kearney}

Wittenburg University, M.Ed.

Women's Field Hockey/Volleyball Coach

The great need for progressive sequential physical education prog r a m s in the elementary schools has prompted many colleges and universities to move toward the comprehensive program for physical education majors. The State of Ohio has granted certification for this type of program at Cedarville, and students desiring to teach physical education in the esementary schools as well as junior and senior high now have this opportunity for service.

—June Kearney 


\section{FINE ARTS}

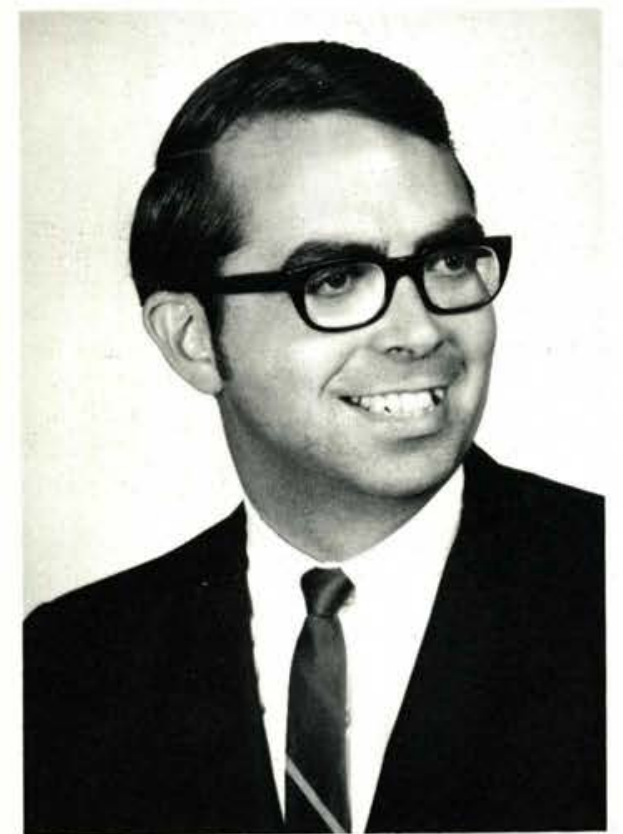

David L. Matson

Ohio State University, M.A.

Delta Sigma Alpha Advisor

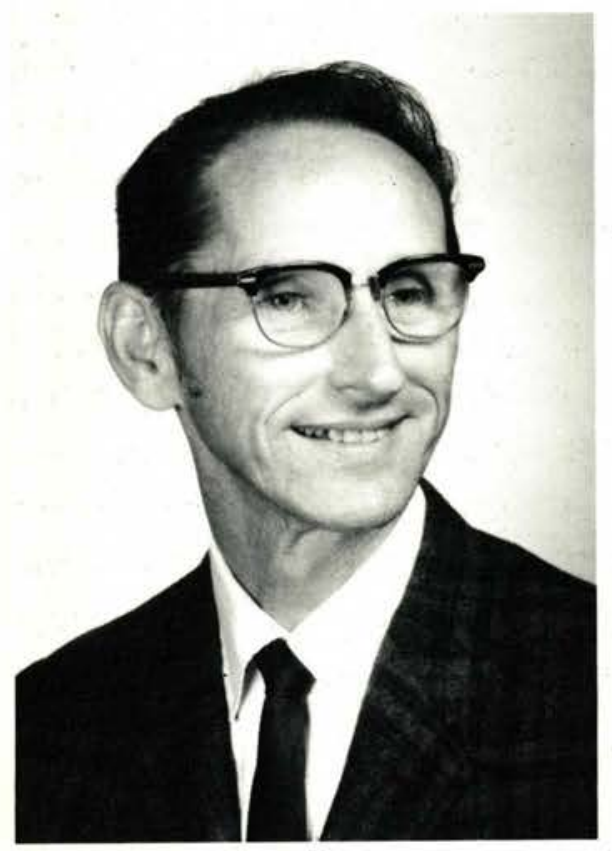

William D. Thorton

Colorado State College, Ed. S. Advisor, M.E.N.C.

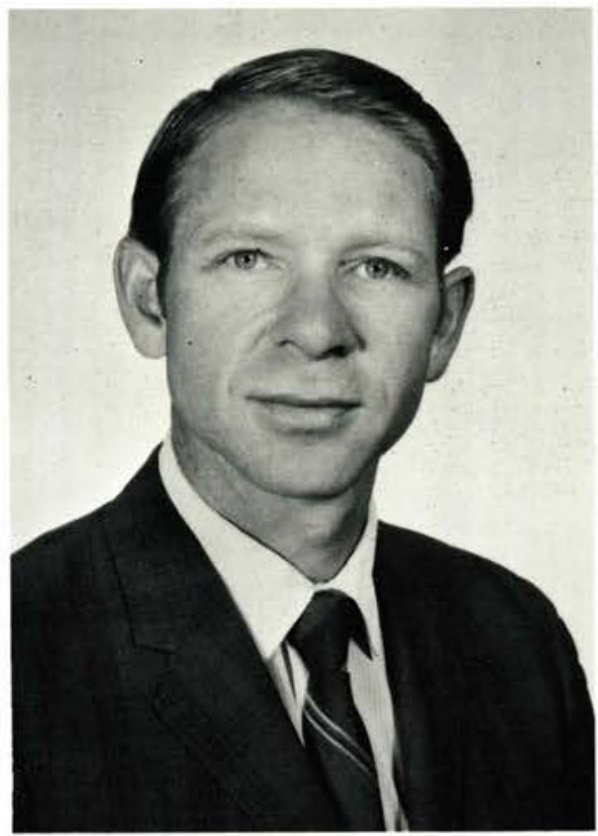

Jack R. Payne

Drake University, M.M.

Music Director Troy Baptist Temple

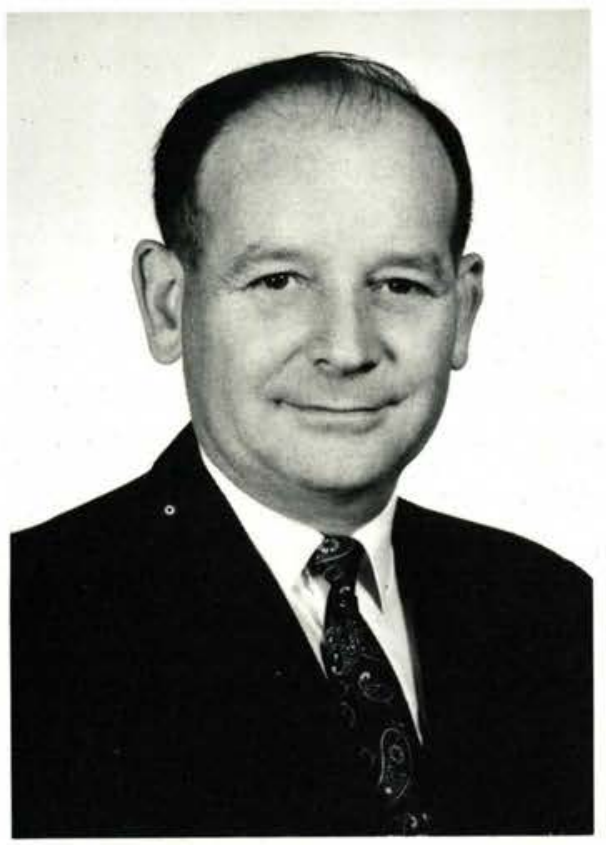

Warren L. Webber

Ohio State University, Ph.D.

College Band Director

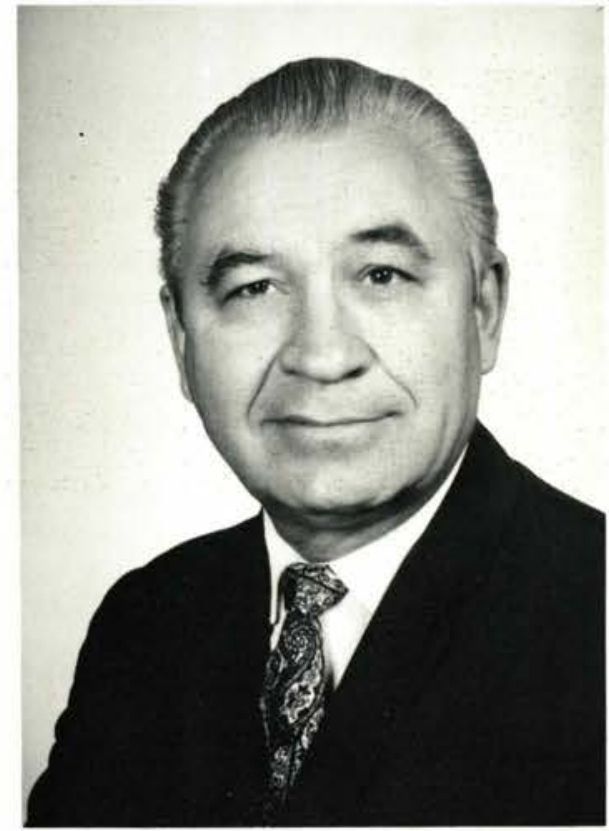

J. Emerson Russell

Ohio State University, M.A.

TeleBible Television Program

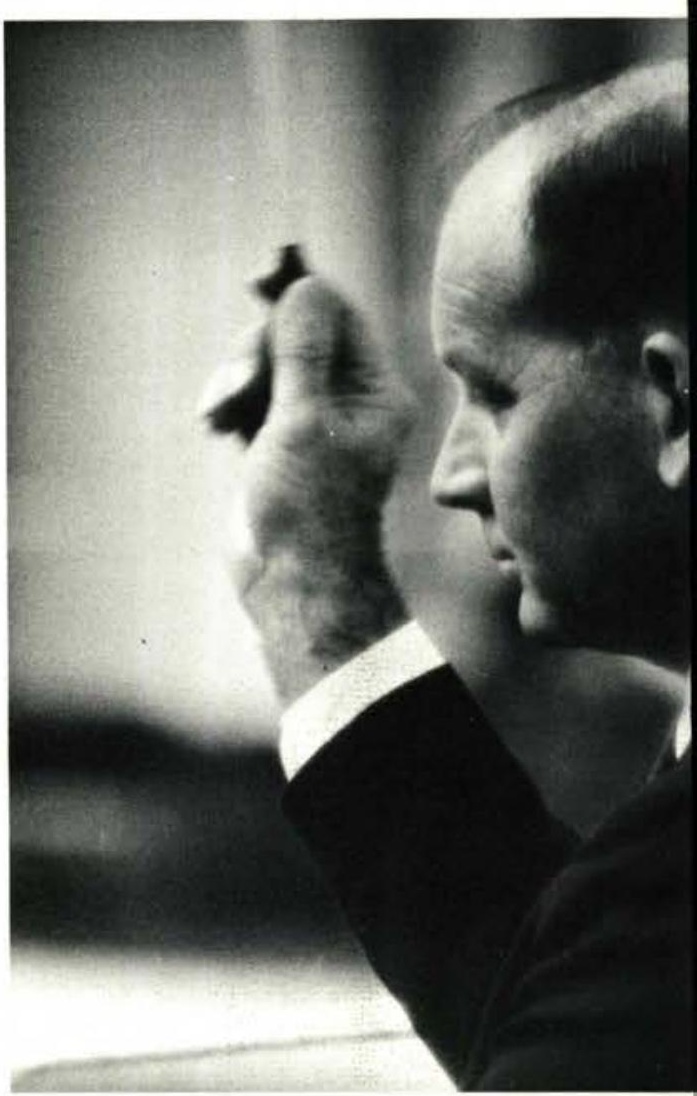




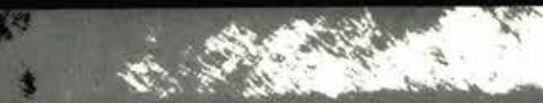

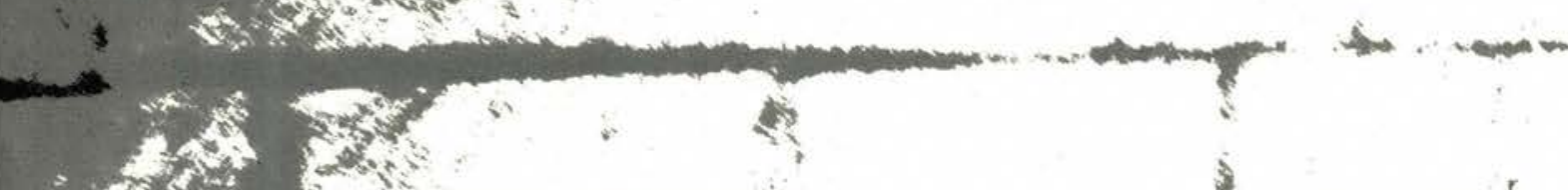

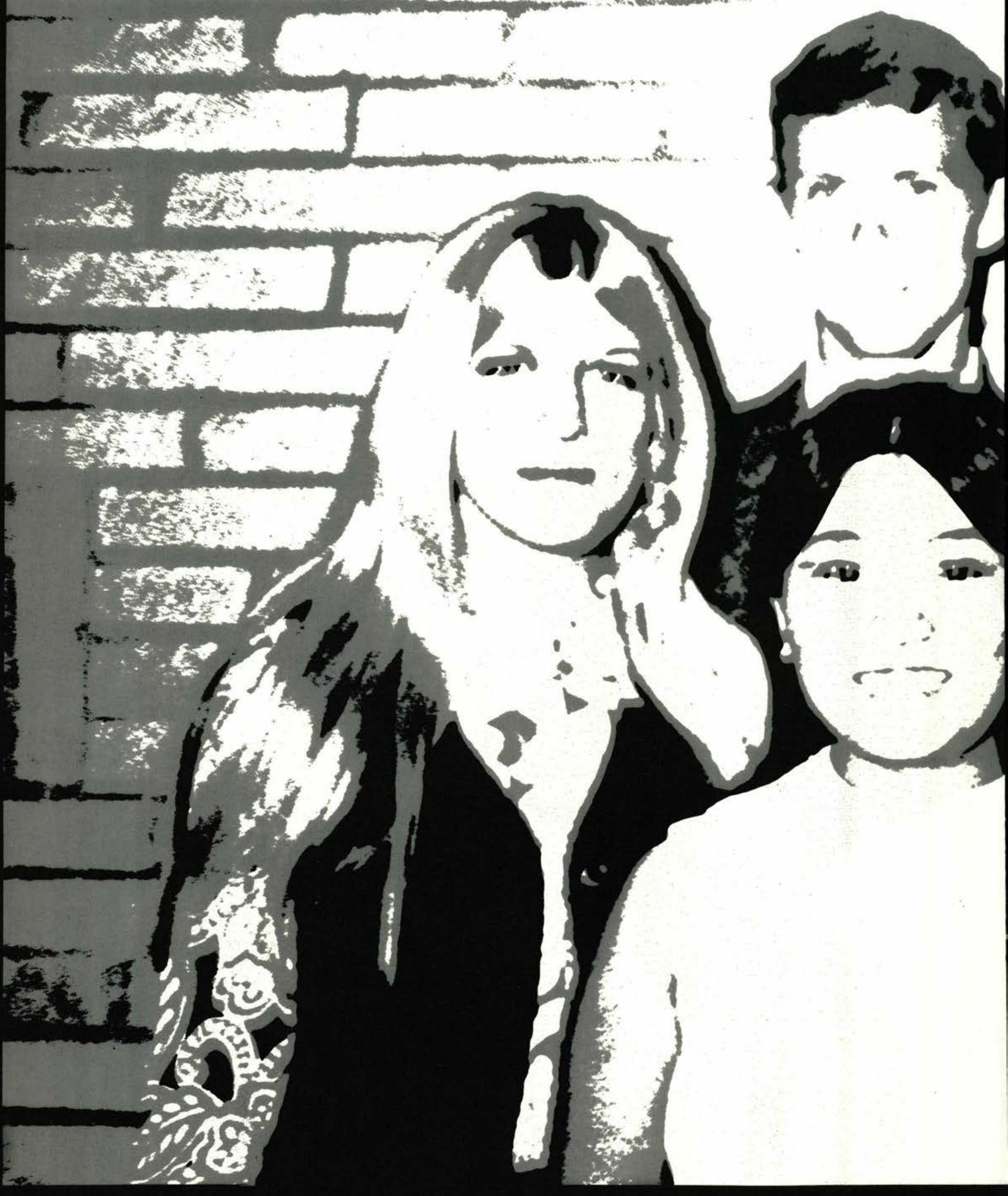




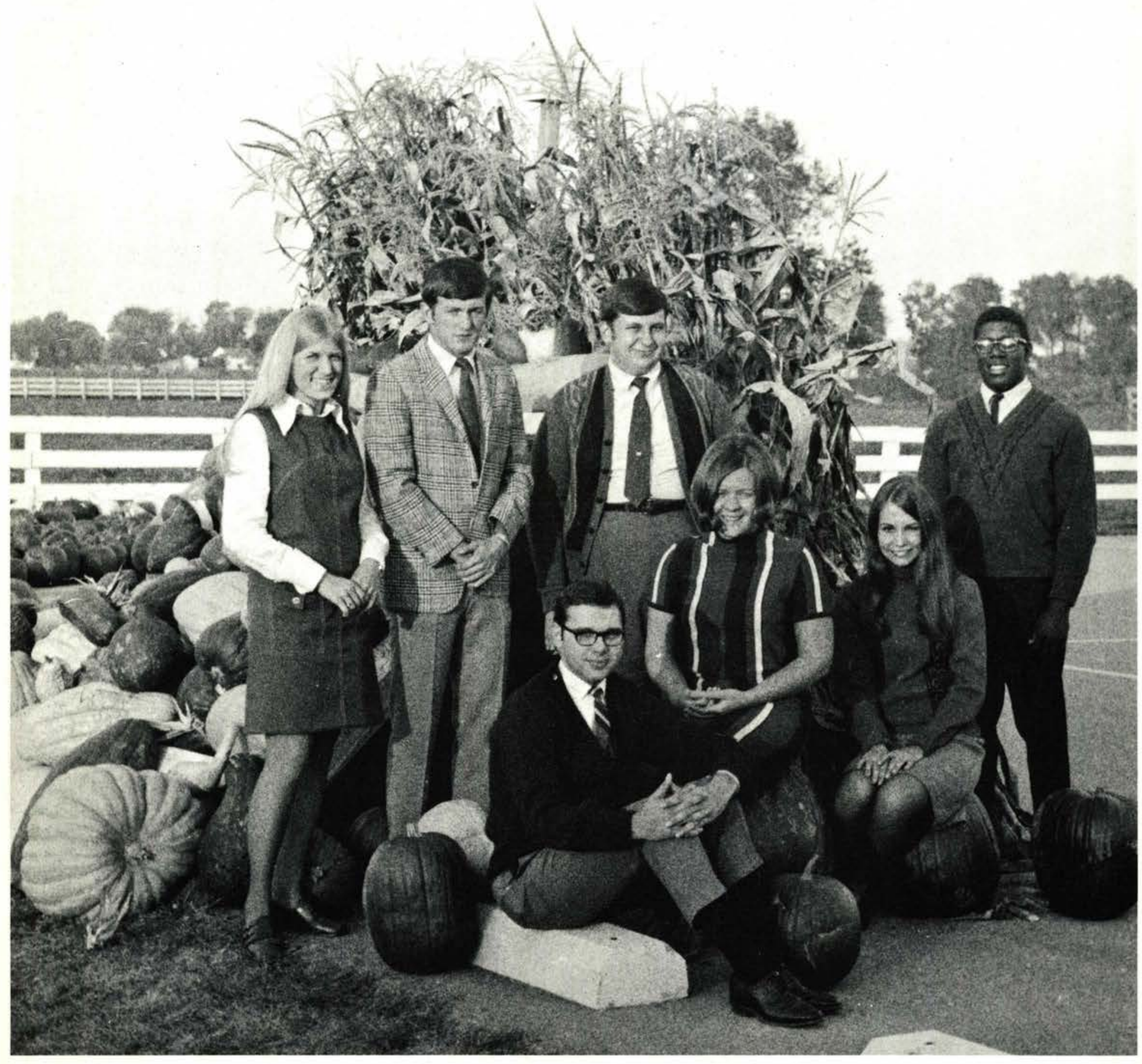

\section{SENIORS \\ CLASS OFFICERS}

\section{Standing:}

Gail Sears, St. Council Rep.

Jim Jeremiah, Vice President

Dan Liechty, President

Ken Hammons, Chaplain
Seated:

Paul Jones, Treasurer

Jan Phenix, St. Council Rep.

Joyce Saemenes, Secretary 
Dorothy L. Abrams

Skaneateles, New York English

Betty Allen

Newfield, N.J.

English

Lyle Anderson

Des Moines, Iowa

Music Comprehensive

Olivia Anderson

Berea, Ohio

Elementary Education

Thomas Andrews

Cedarville, Ohio

Bible/Education

\section{Ron Artrip}

Creston, Ohio

Elementary Education

Jane Austin

Bridgeton, N.J.

Elementary Education

Frank Bailey

Cambridge, Ohio

Social Studies

Nancy Bailey

Traverse City, Mich.

Physical Education

Donita Baker

Cardington, Ohio

English

Allen Beltle

Manasquan, N.J.

Business Administration

Beth Bennett

Chagrin Falls, Ohio

Elementary Education
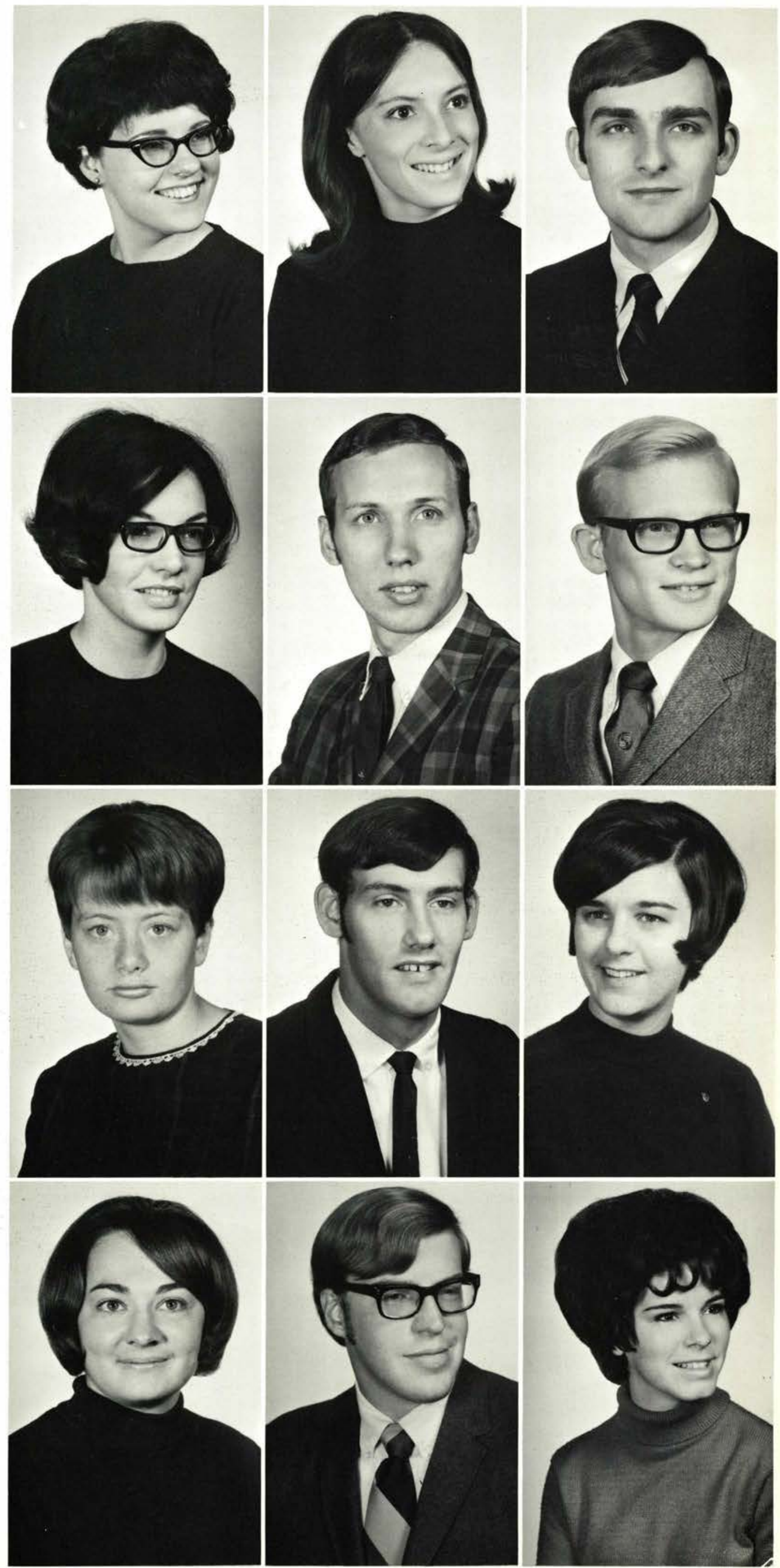

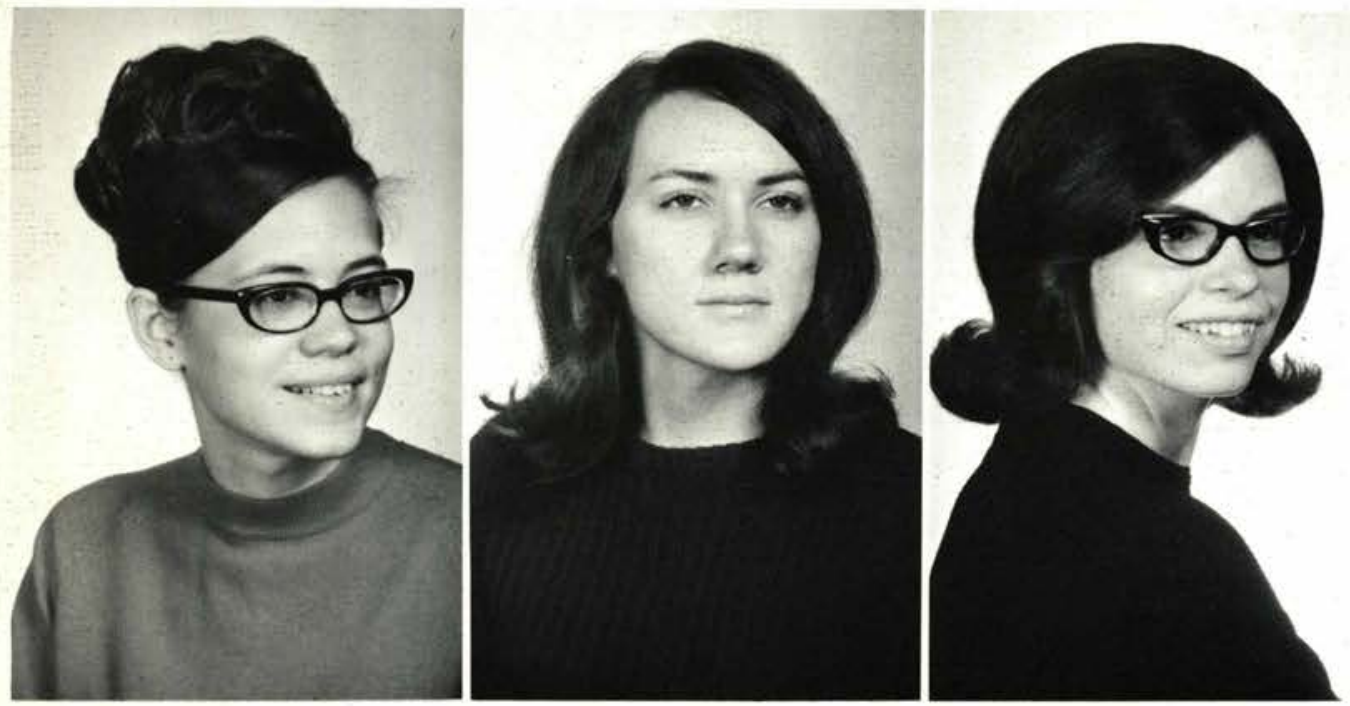

Barbara Biddison

Grand Blanc, Mich.

Elementary Education

Regina Bigley

Dunbar, $\mathrm{Pa}$.

Elementary Education

Pat Black

Coal Port, Pa.

Elementary Education
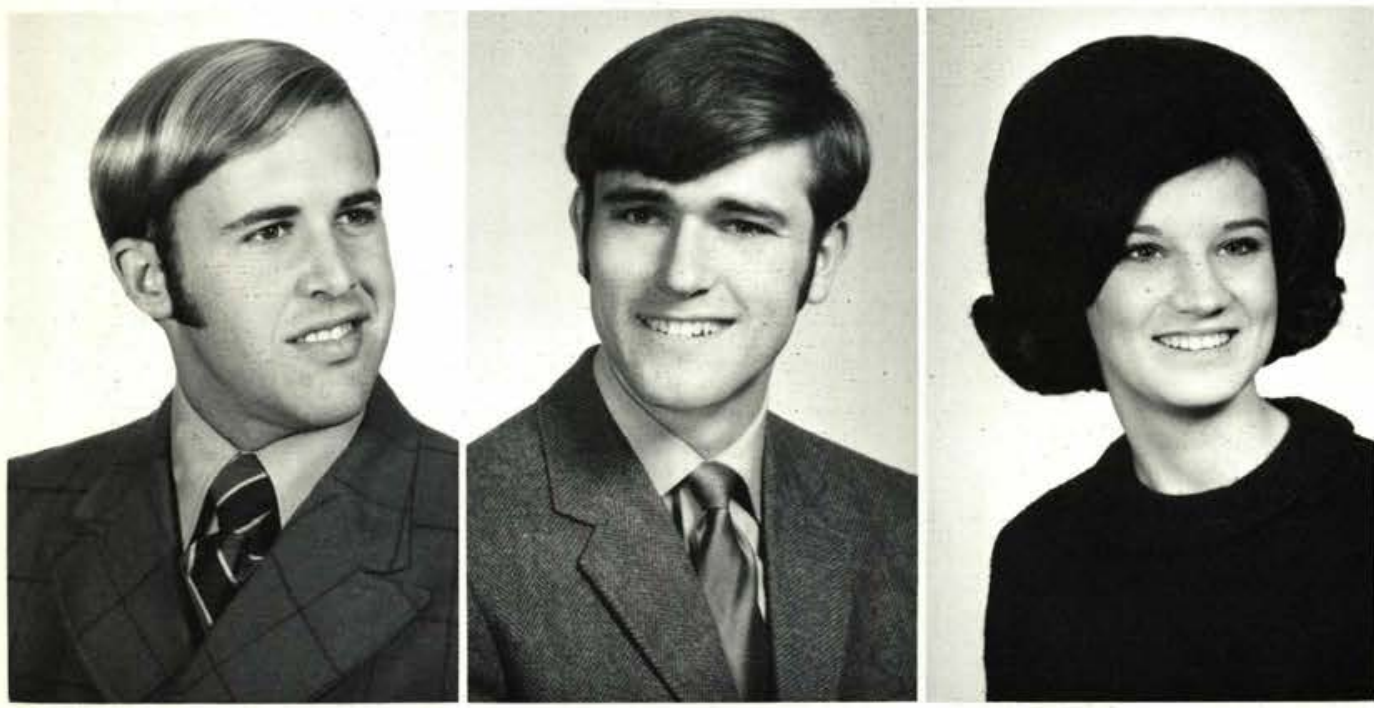

David Bodden

Oley, Pa.

Biology/Speech

Darwin Boertje

Lima, Ohio

Business Administration

Karen Borger

New Castle, Pa.

Elementary Education

Bernice W. Bowersox

Xenia, Ohio

Sociology/Psychology

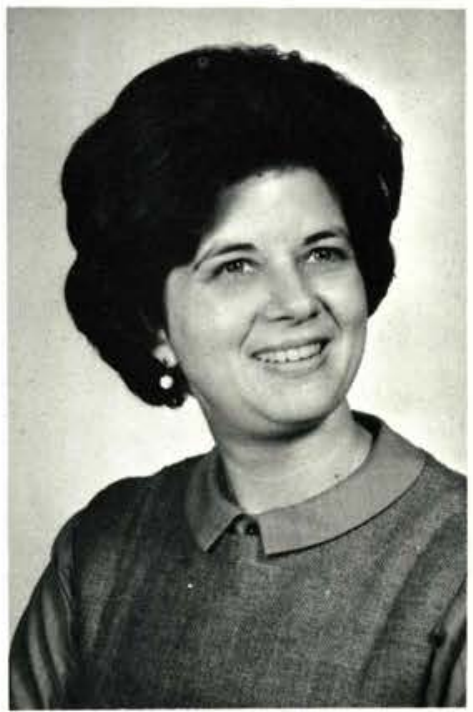




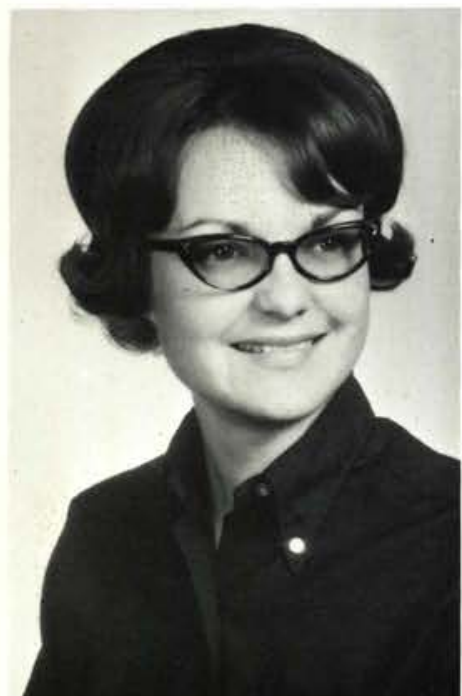

Linda Campbell

Ankeny, Iowa

Biology

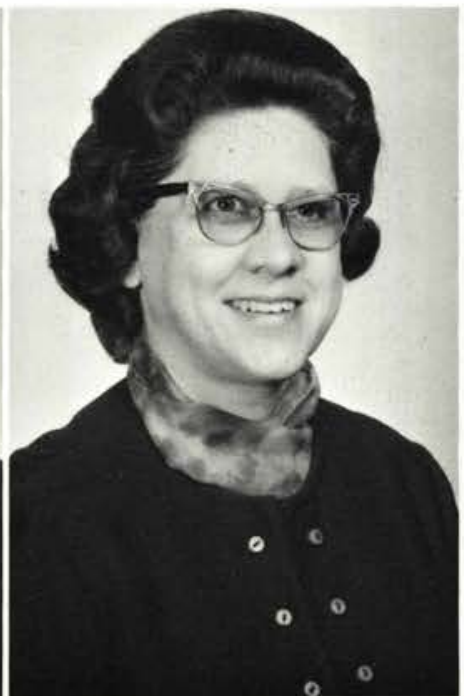

Annie L. Carlson

Bessemer, Alabama

Elementary Education

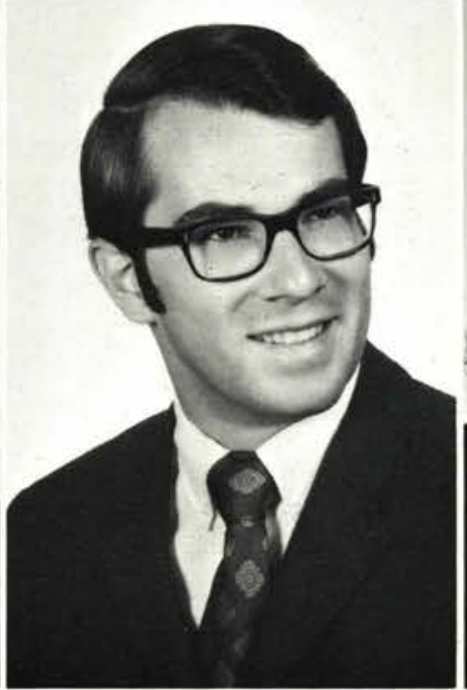

Robert Carmean

Dayton, Ohio

Speech

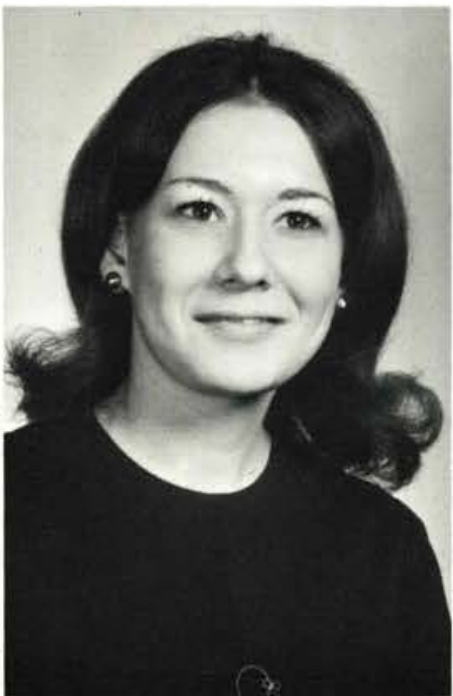

Cindy Cartner

San Francisco, Calif.

Elementary Education

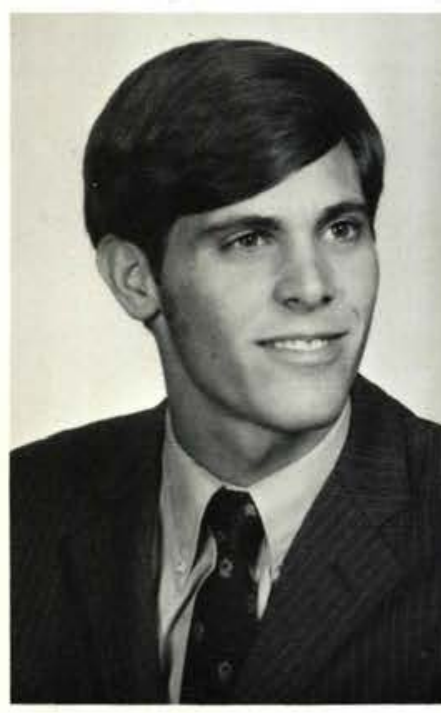

Mark Causey

Morton, Illinois

Psychology

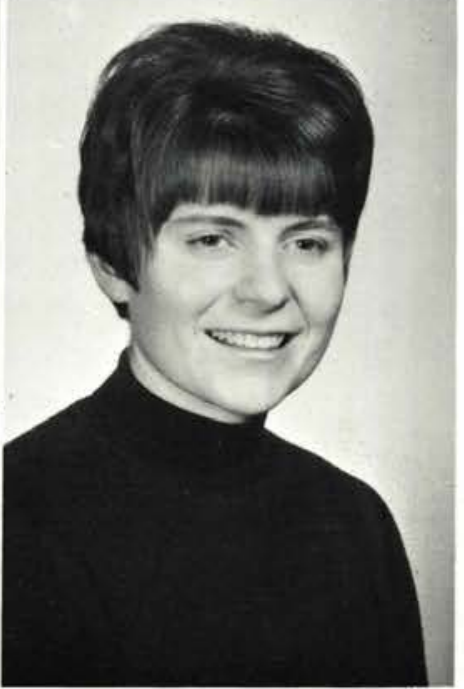

Carolyn Clark

Clarion, Iowa

Christian Education
Ken Cole

Gallipolis, Ohio

Chemistry

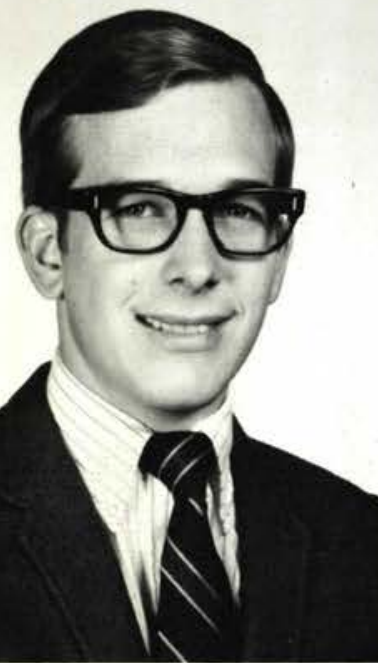

Martha L. Cox

Elkhart, Indiana

Elementary Education

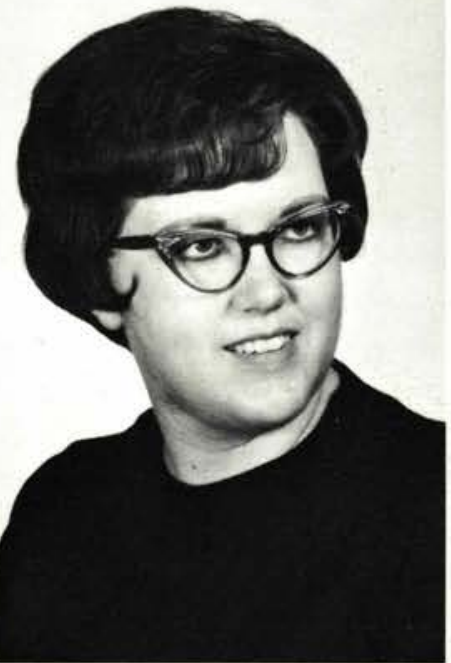

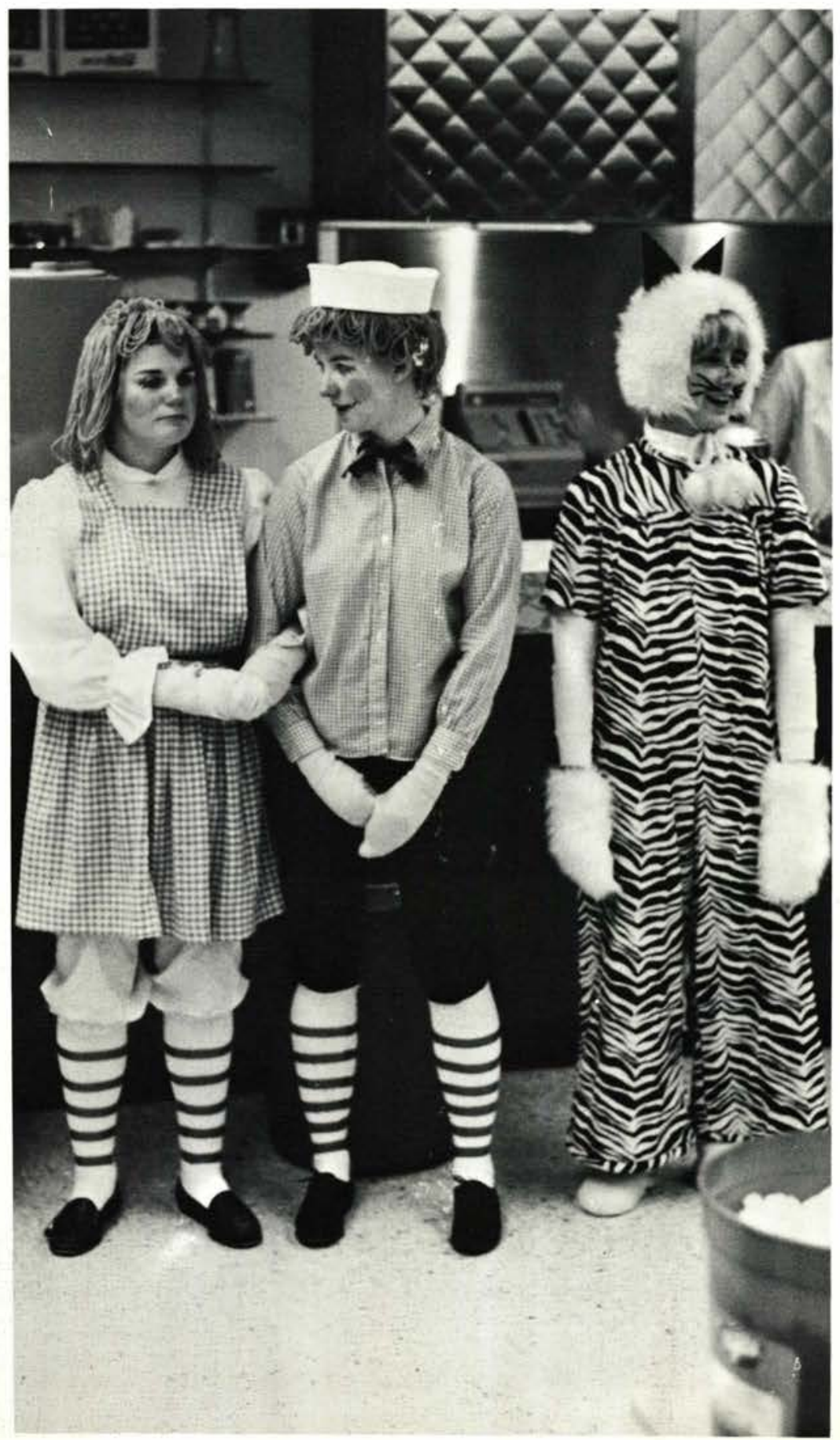




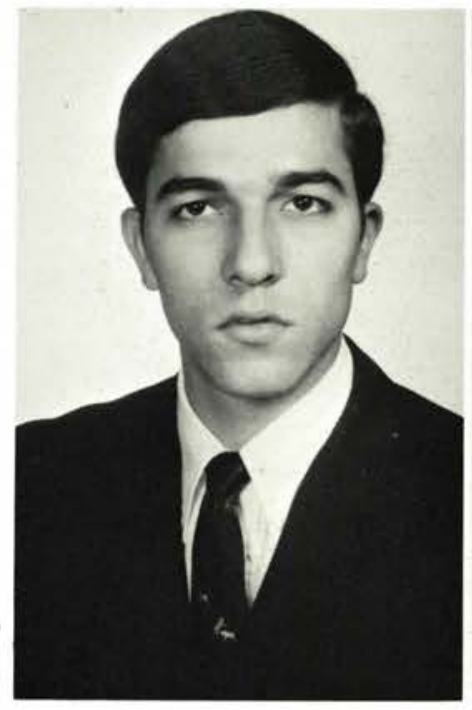

Ken Curcio

Sciotoville, Ohio

Accounting

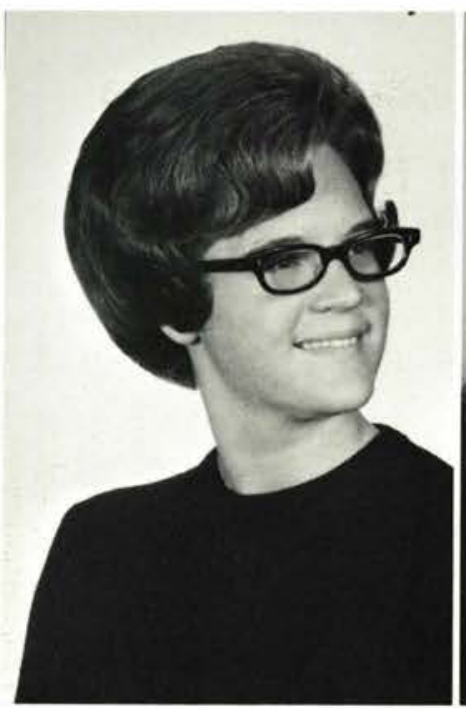

Joan Dautel

Harvey, Illinois

Physical Education

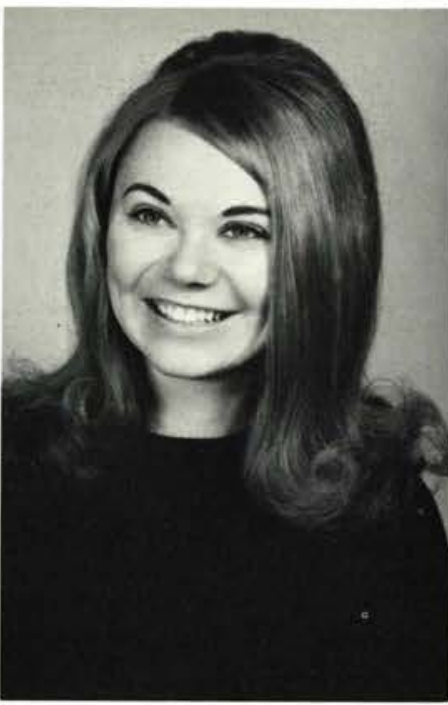

Nancy Davis

Grandview, Washington

Psychology

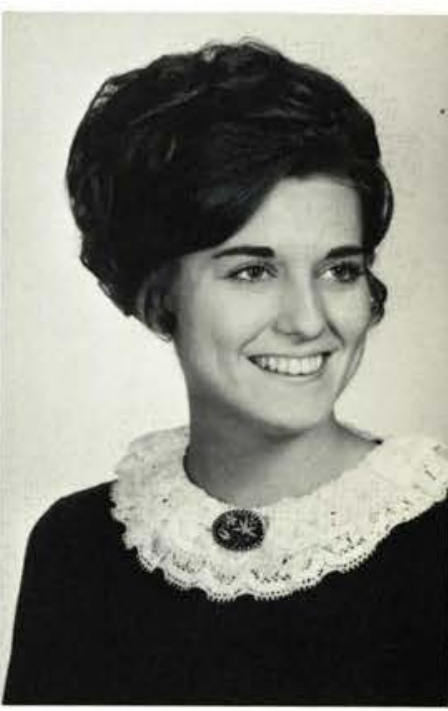

Becky Decker

Hinckley, Ohio

History/English

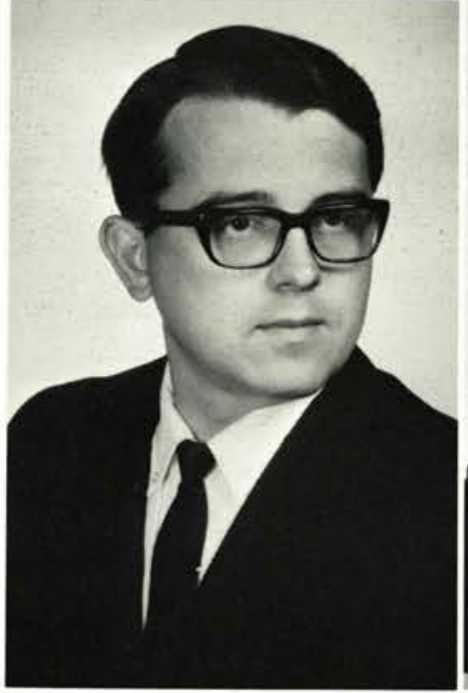

Richald De Walk

Hubbard, Ohio

Business

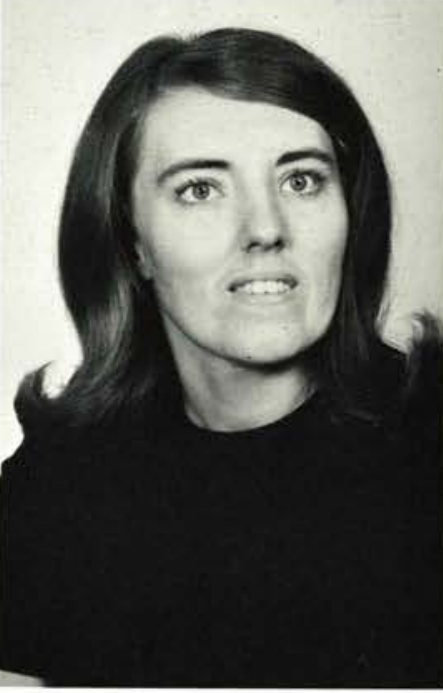

JoAnne Dillard

Springfield, Ohio

Social Studies Comp.

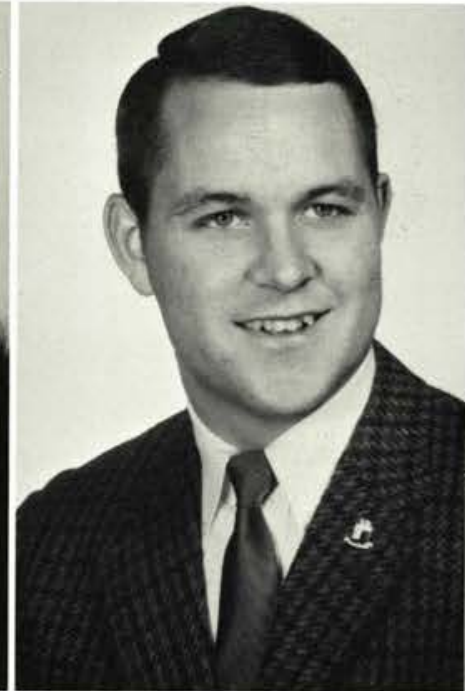

Ronald Edwards Kipton, Ohio

Pre-Seminary

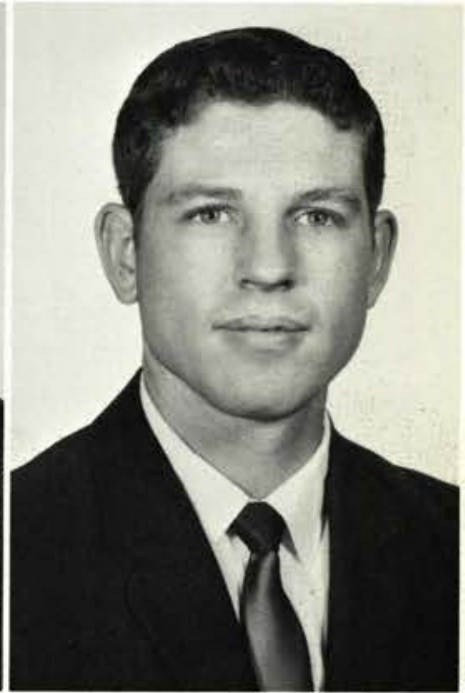

Leland Eichelberger Kana, Idaho

Speech
Elaine Eldridge

Francesville, Indiana

Elementary Education

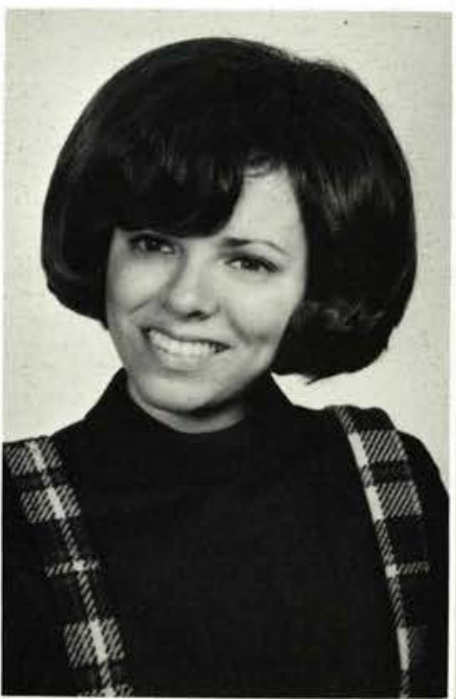

Patti France

Bedford, Ohio

Social Studies Comp.

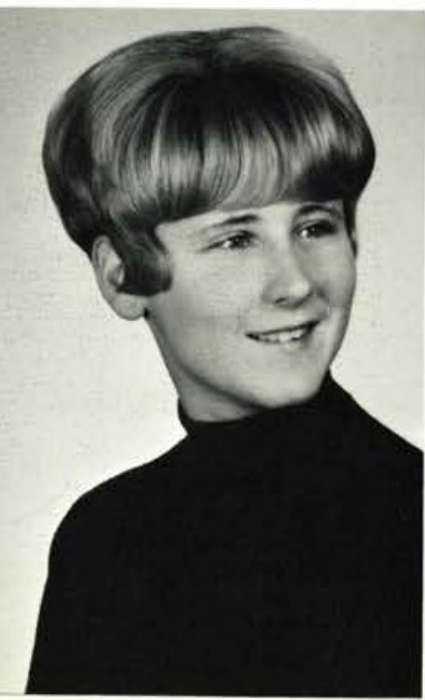

Paul Friedrich LaPorte, Indiana

Pre-Seminary

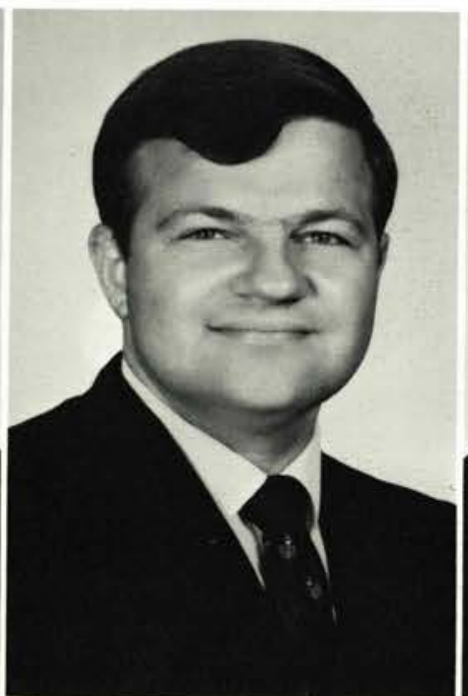

Walter Folkmann Brunswick, Ohio Biology

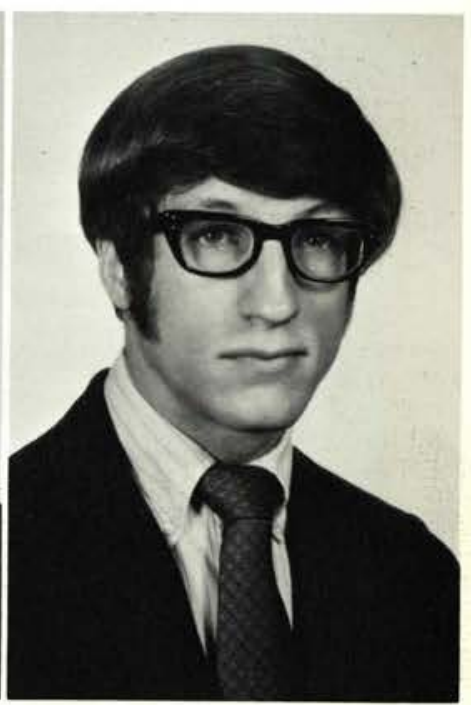




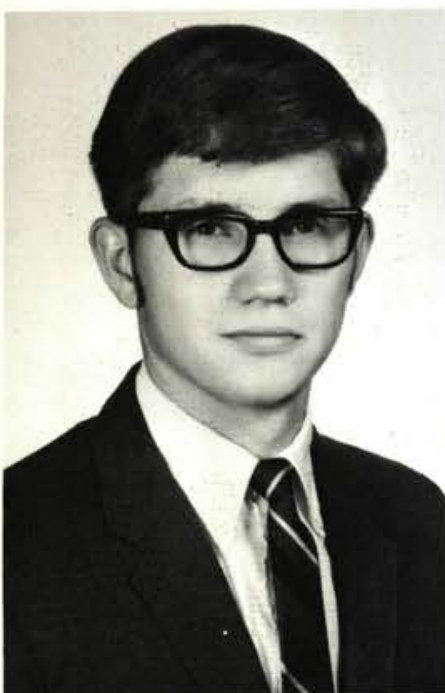

Andrew Gathany

Montague, Michigan

Bible

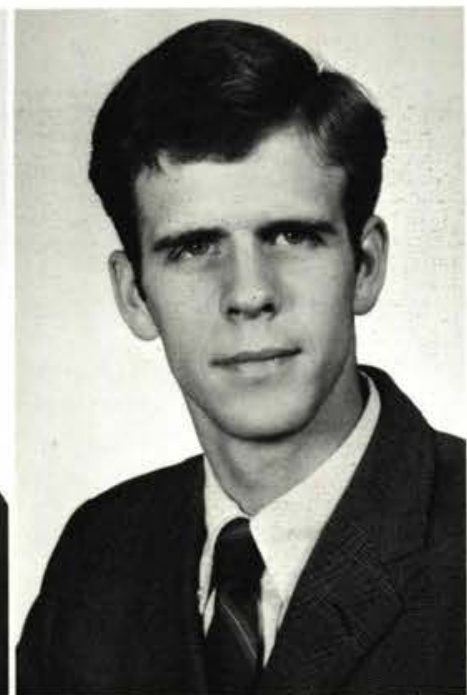

Tom Gelo

Cedarville, Ohio

Business Administration

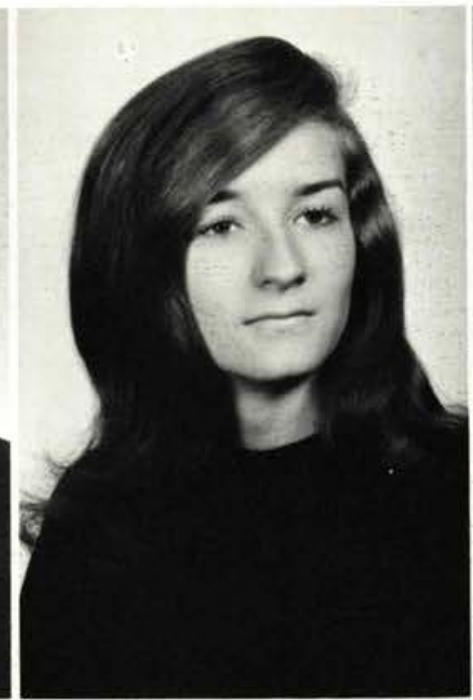

Susan Gift

Jackson, Michigan

Speech/English

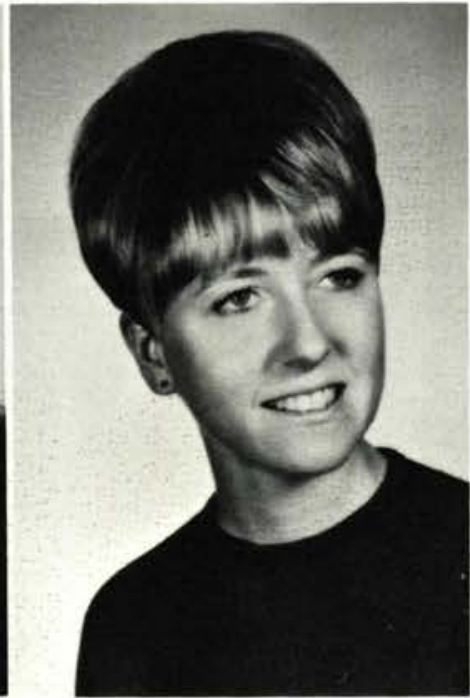

Glenda Gillenwater

Dayton, Ohio

Elementary Education

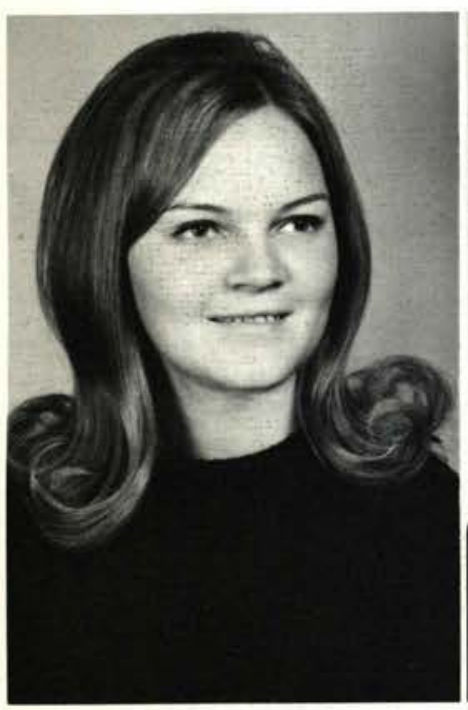

Sharon Graham

Aurora, Ohio

Elementary Education

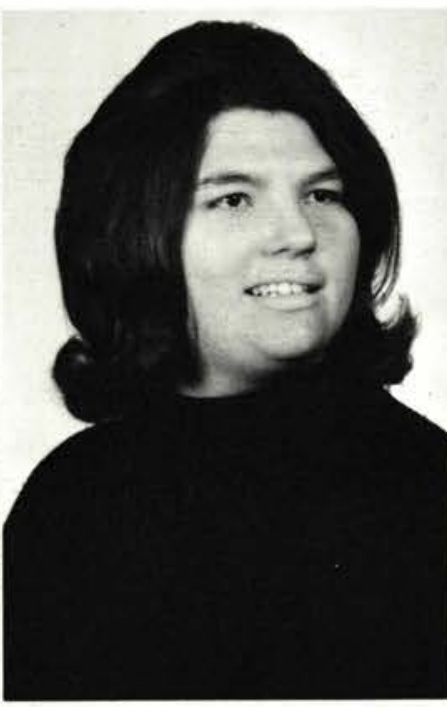

Lynn Grandy

Canajoharie, N.Y.

Elementary Education

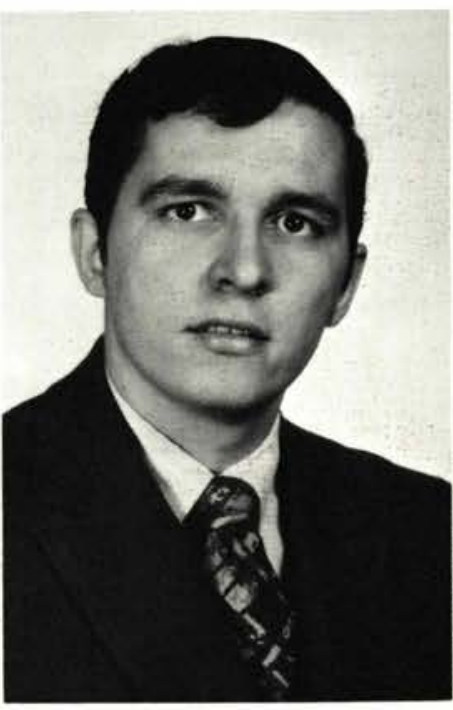

Randall Hamilton

Philadelphia, $\mathrm{Pa}$.

Speech

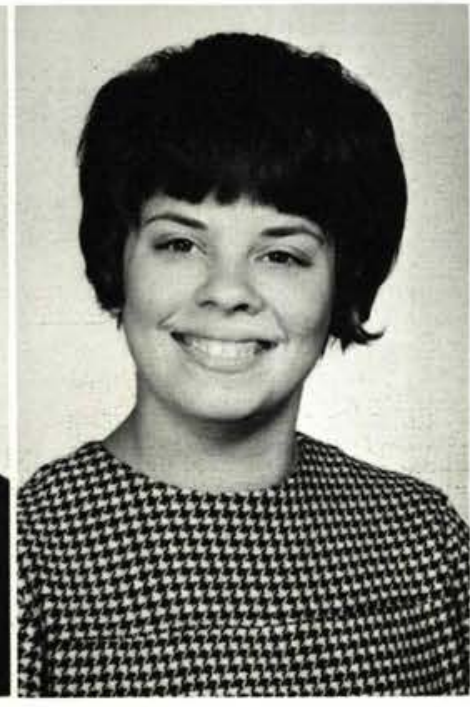

Faith Hammond

Laceyville, $\mathrm{Pa}$.

Elementary Education

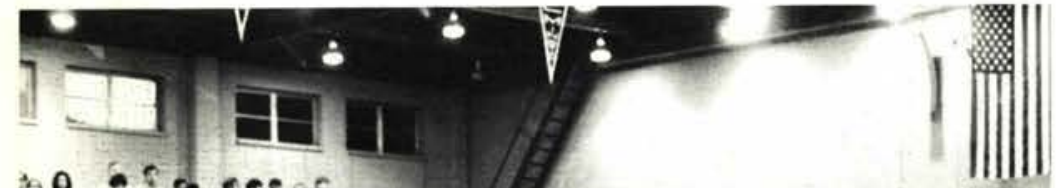

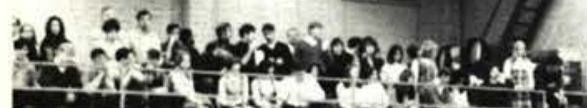

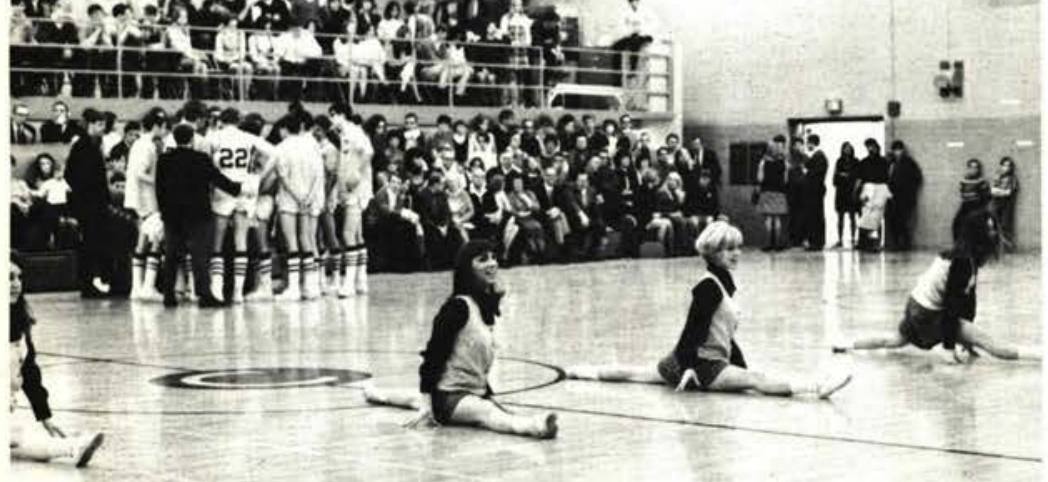

Ken Hammonds

Wilmington, Delaware. Pre-Seminary

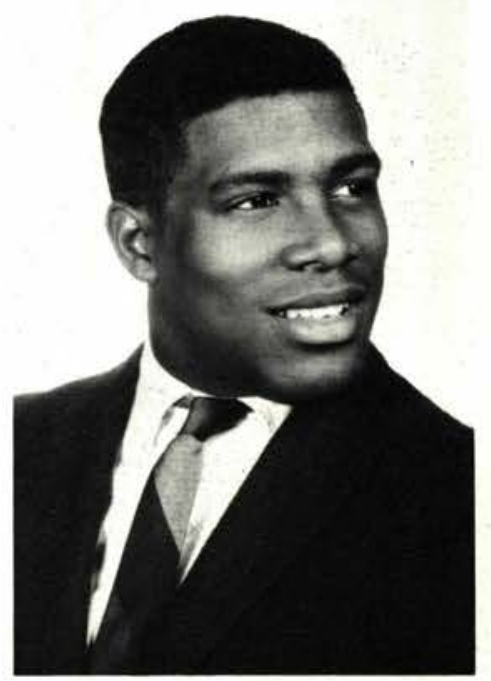




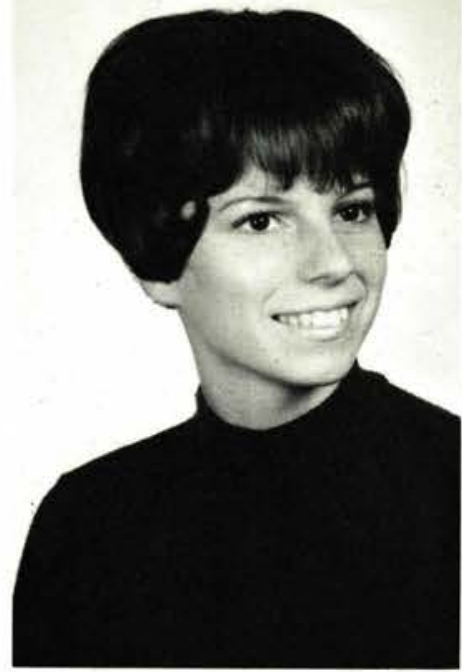

Evelyn Hare

Cedarville, Ohio

Elementary Education

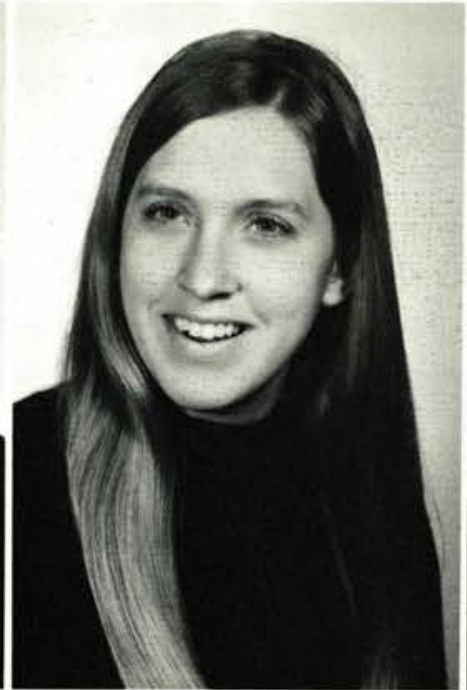

Becky Harman

Connellsville, $\mathrm{Pa}$

Mathematics

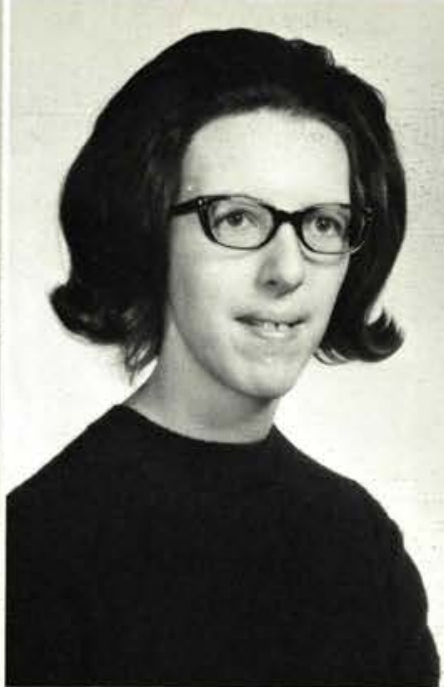

Linda Heater

Cedarville, Ohio

Business Administration

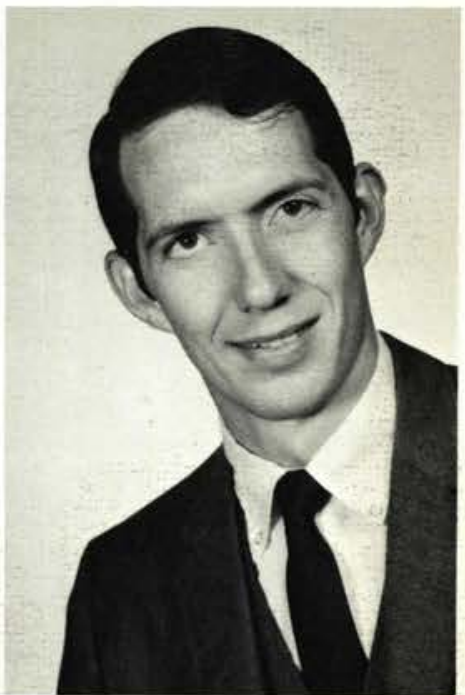

Kirk Heldreth

Brunswick, Ohio

Speech

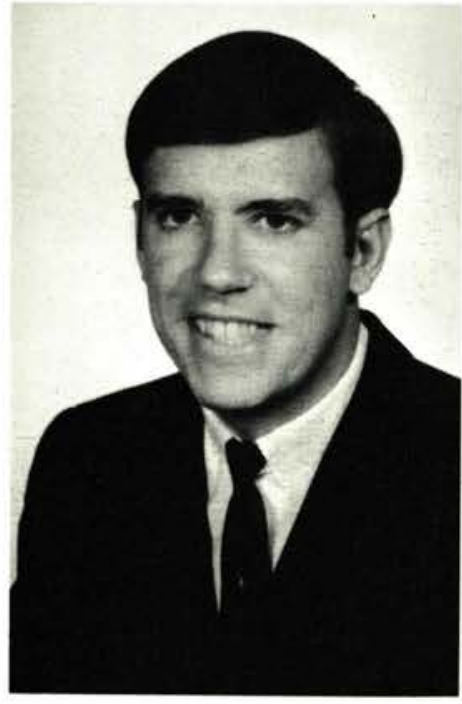

Ralph Helm

Villa Park, Ill.

History/Psychology

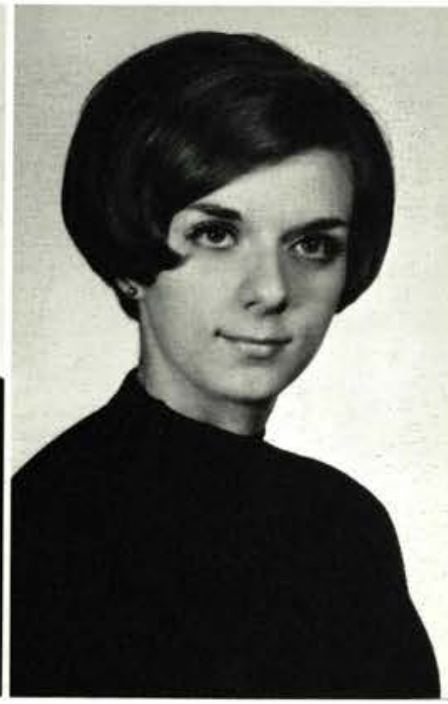

Sue Herrin

Cincinnati, Ohio

Elementary Education

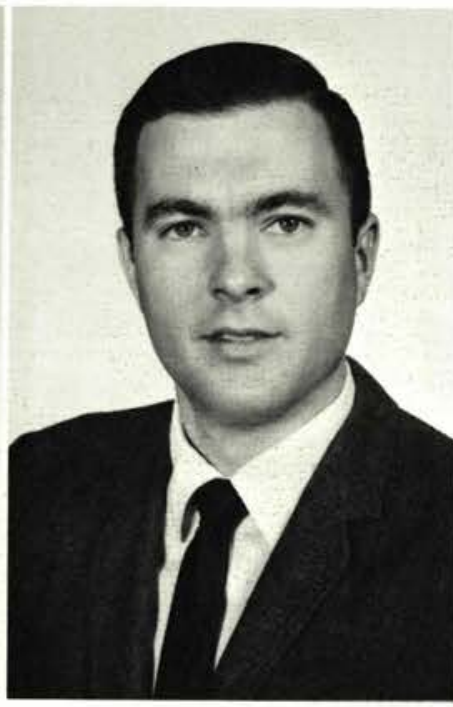

Sherrill Hesler

Cedarville, Ohio

Pre-Seminary

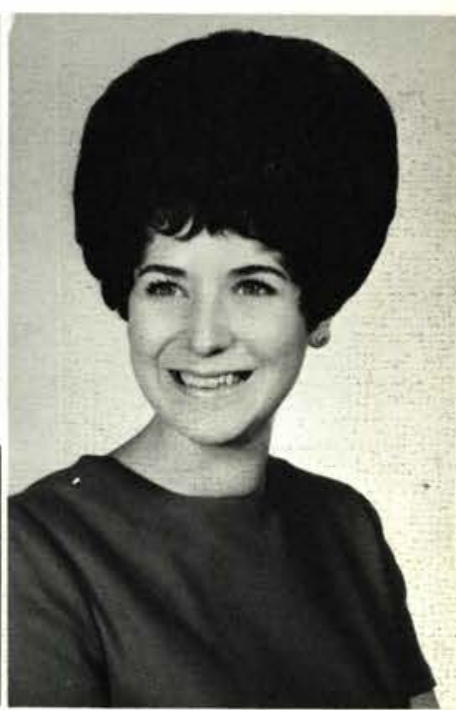

Sarah Hickam

Moline, Illinois Mathematics
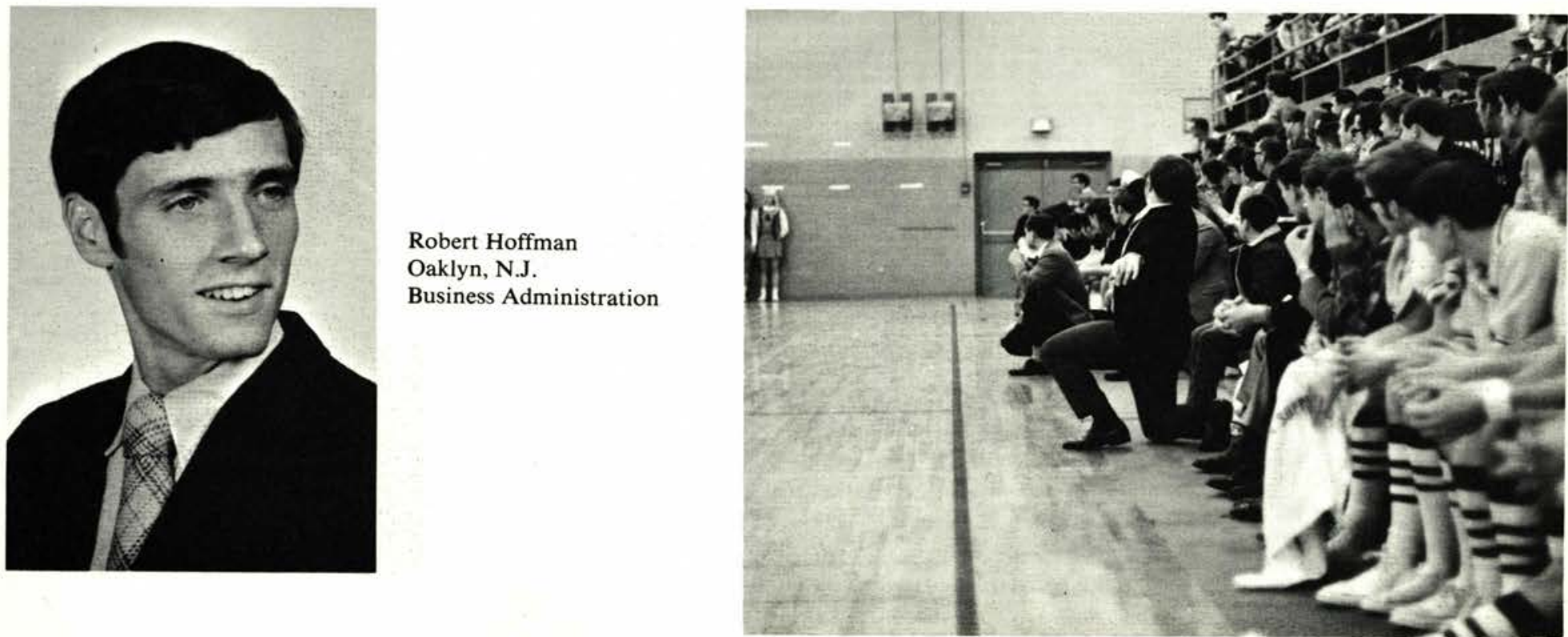

Robert Hoffman

Oaklyn, N.J.

Business Administration 


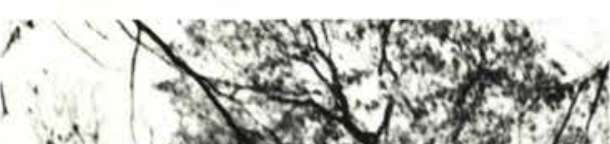
W

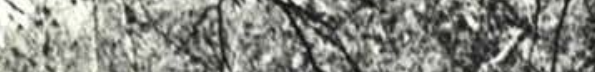

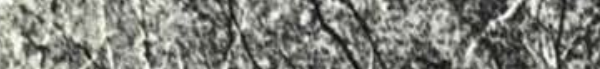
470 . $45450 \times 2$. 7 W

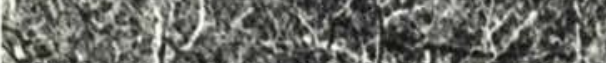

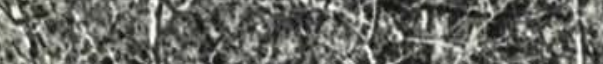

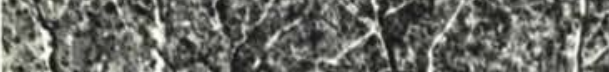

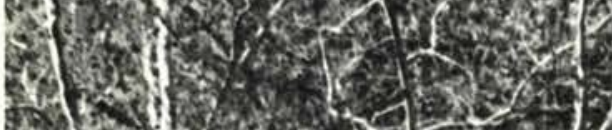

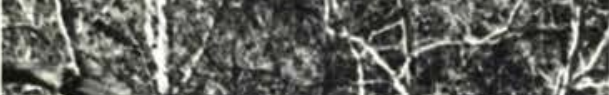

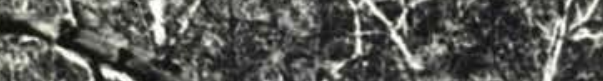

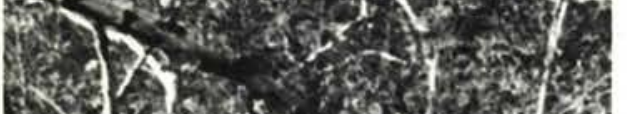
24 .

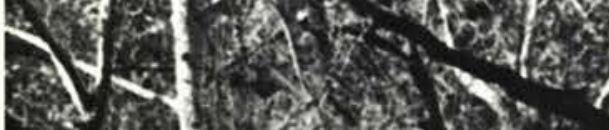
- $1212 x$

x a

s. (i)

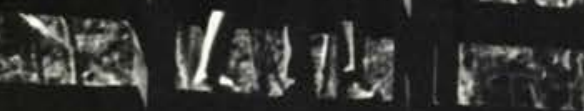
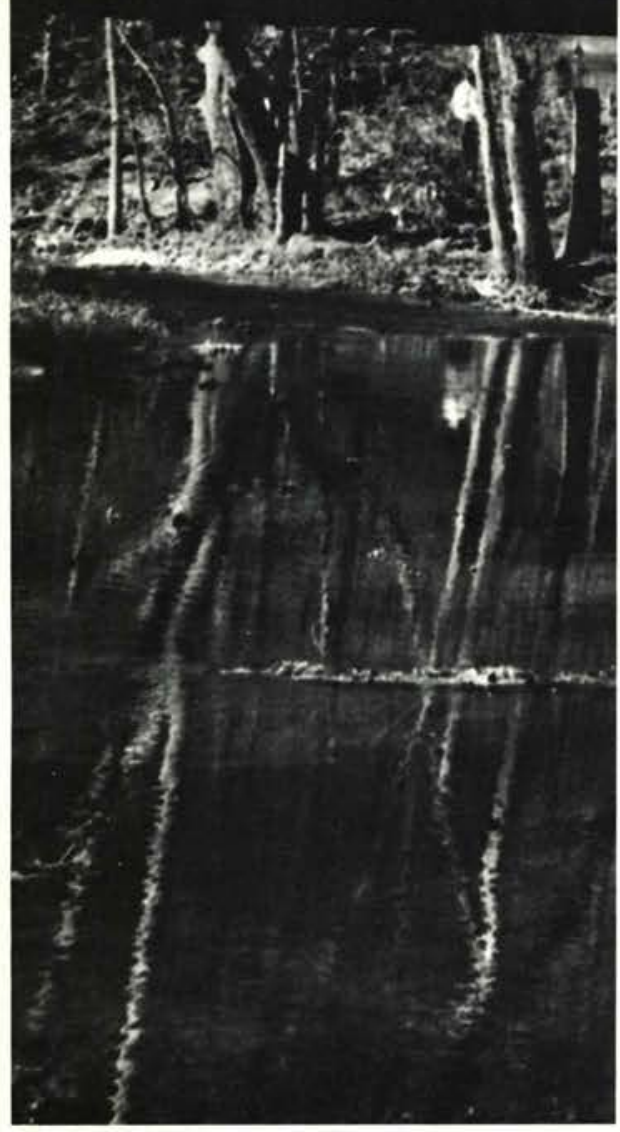

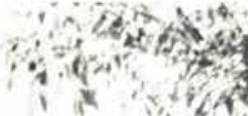

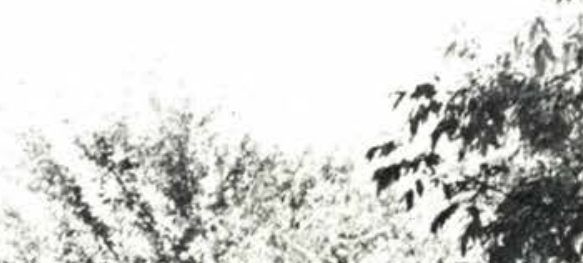

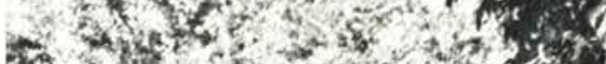

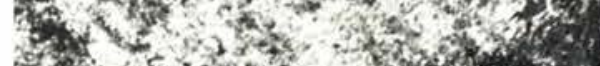

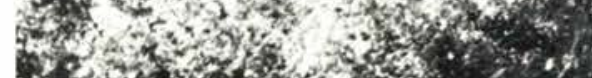

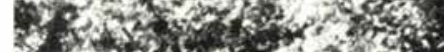

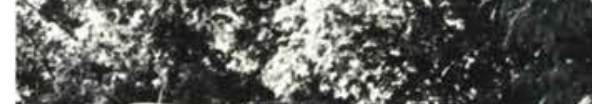
3.
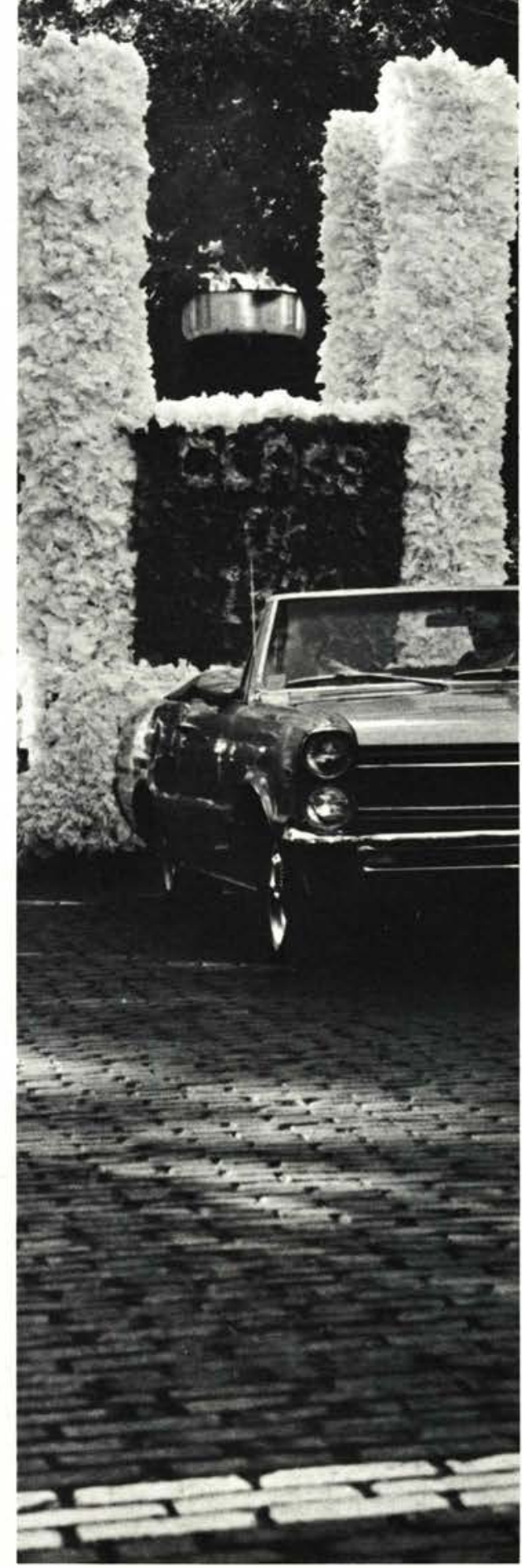

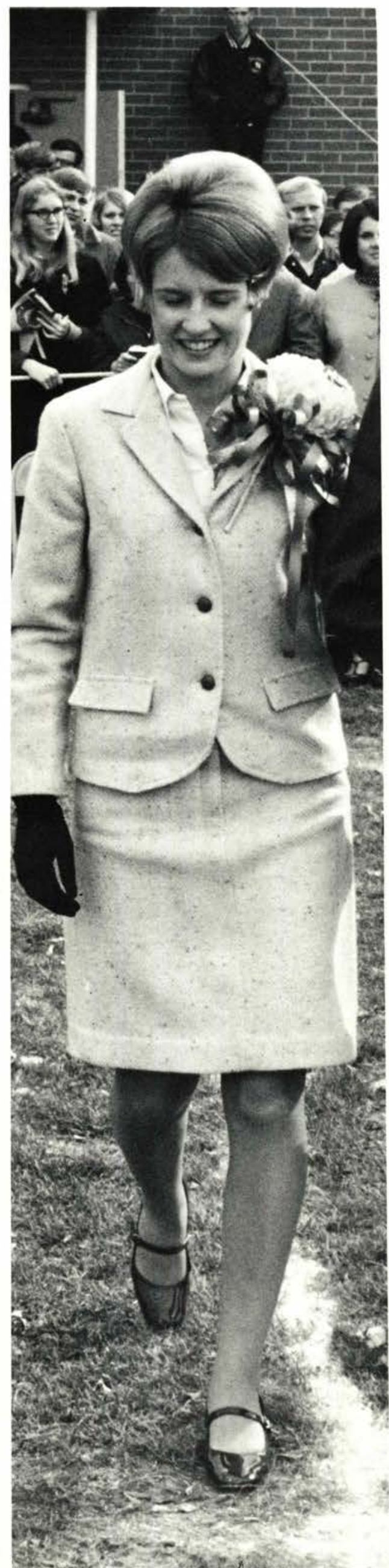




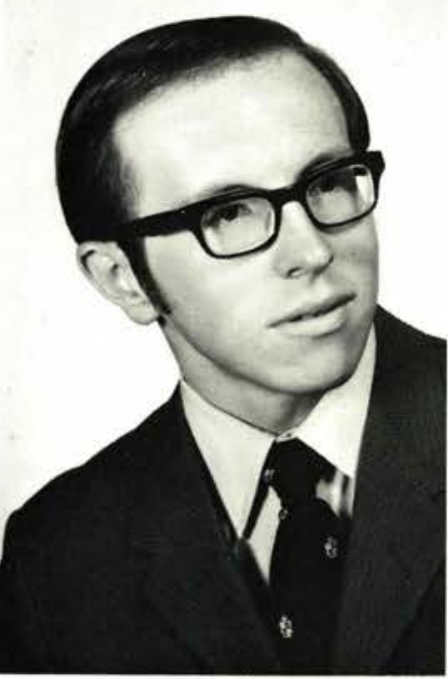

Jack Holliday

Richard, W.Va.

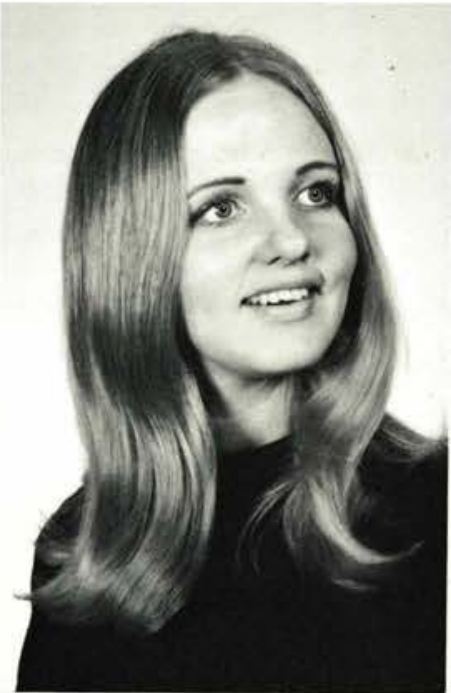

Barbara Hoyer

Cortland, N.Y

Elementary Education

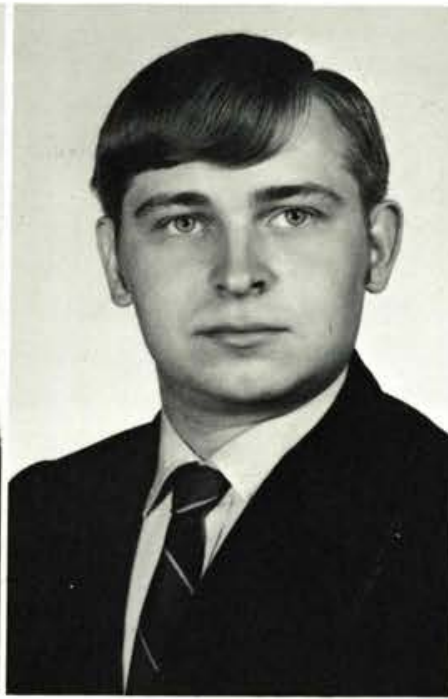

Dennis Huddleston

Dayton, Ohio

Business

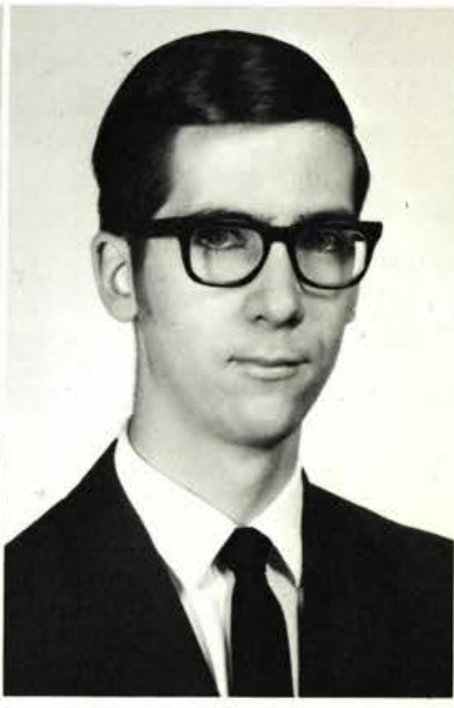

David Hull

Cortland, Ohio

Chemistry

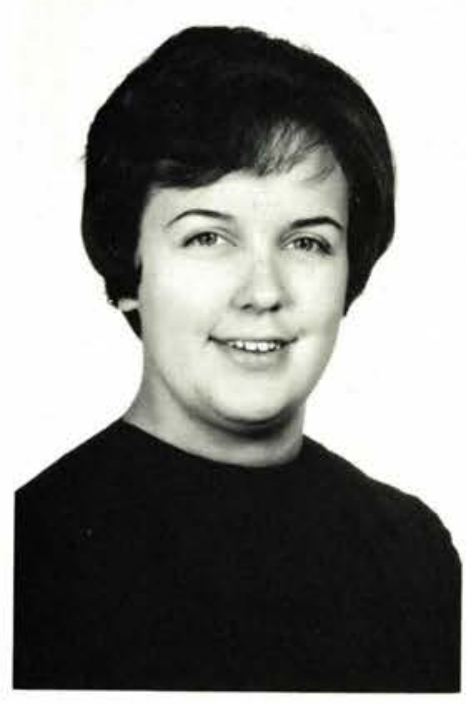

Kathy Hull

Washington, Iowa

Elementary Education

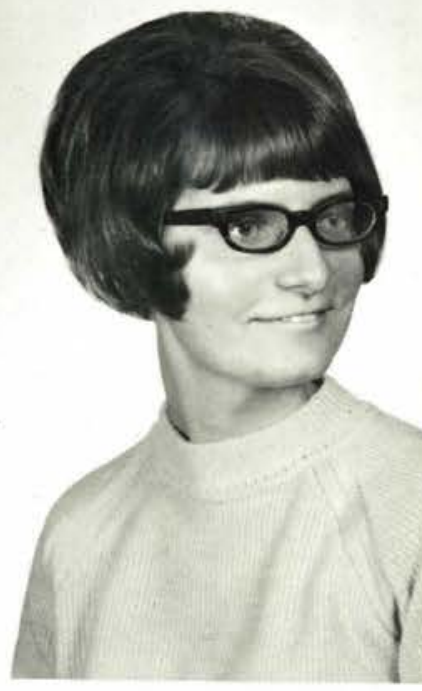

Dianna Hutchison

Columbus, Ohio

Elementary Education

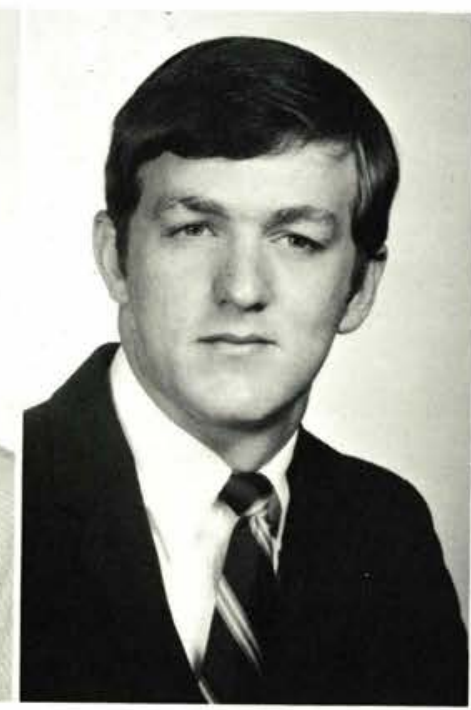

James Jeremiah Cedarville, Ohio Accounting

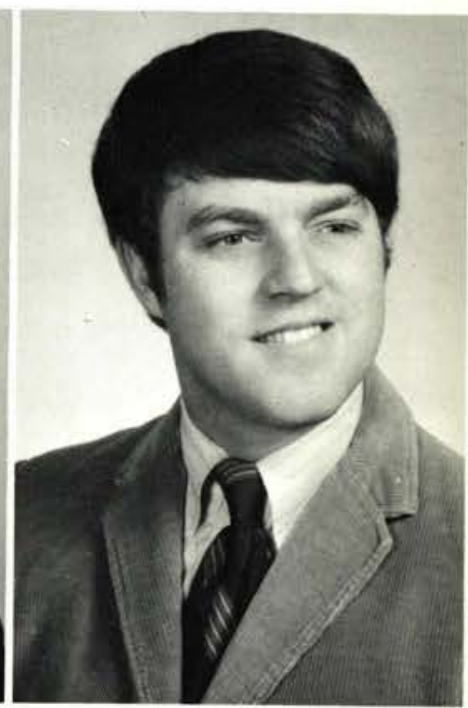

Philip Johnson

Decatur, Illinois Business/History
Ken Jones

Columbus, Ohio

Elementary Education

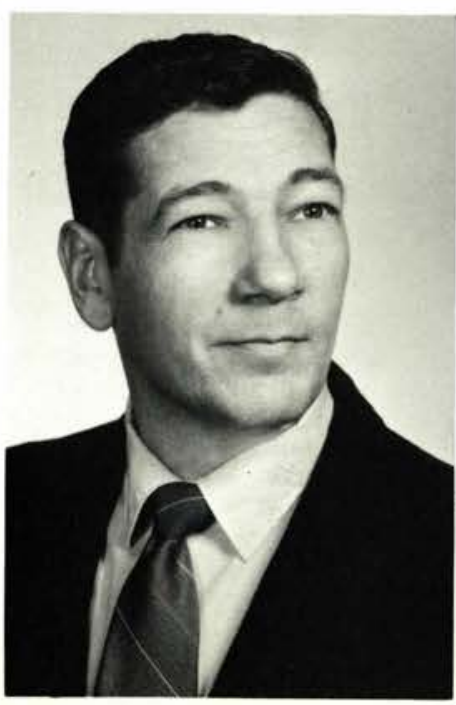

Paul Jones

Piqua, Ohio Speech

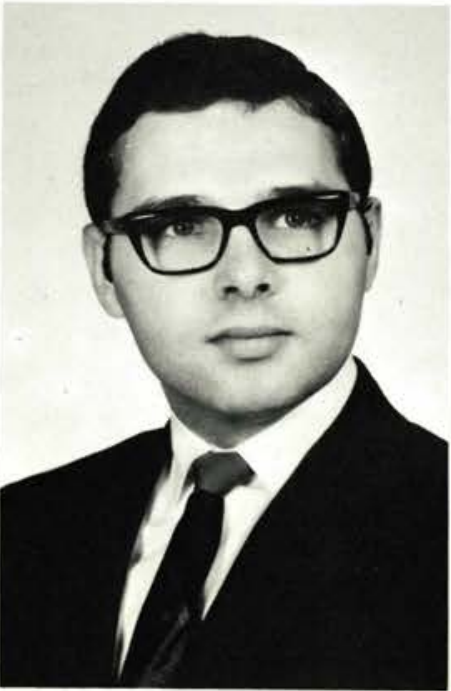

Rebecca Kern

Waterloo, Iowa

Elementary Education

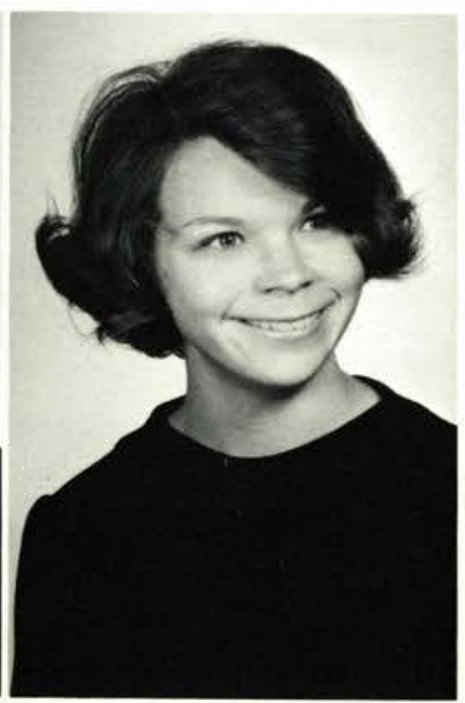

David Kimball

Chicago, Illinois 


$$
\begin{aligned}
& 0909 \\
& 8008 \\
& 8089
\end{aligned}
$$




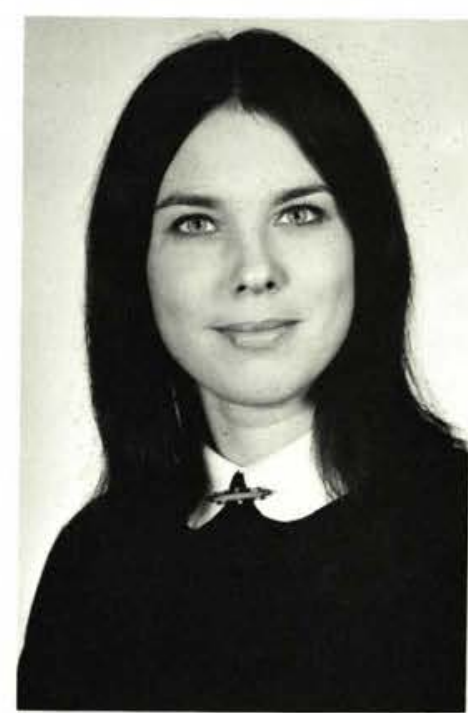

Beverlee Mason

Toledo, Ohio

Speech

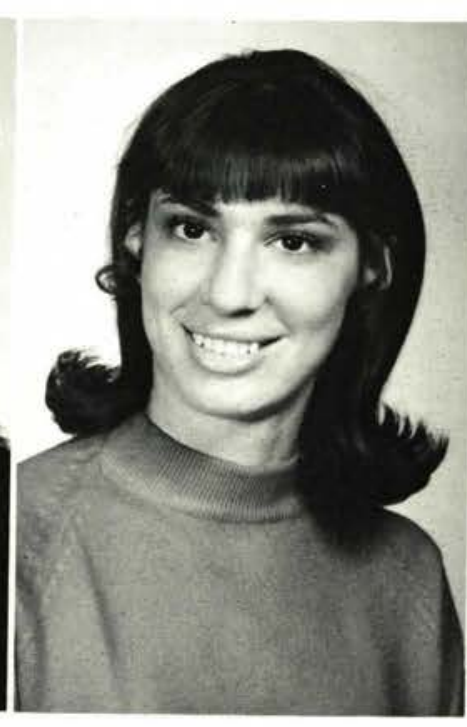

Judy McCann

Quincy, Michigan

Physical Education Comp.

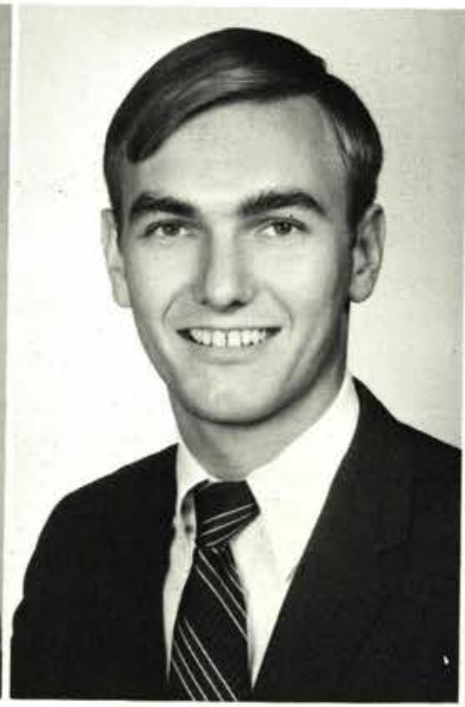

Max McCullough

Coshocton, Ohio

Pre-Seminary

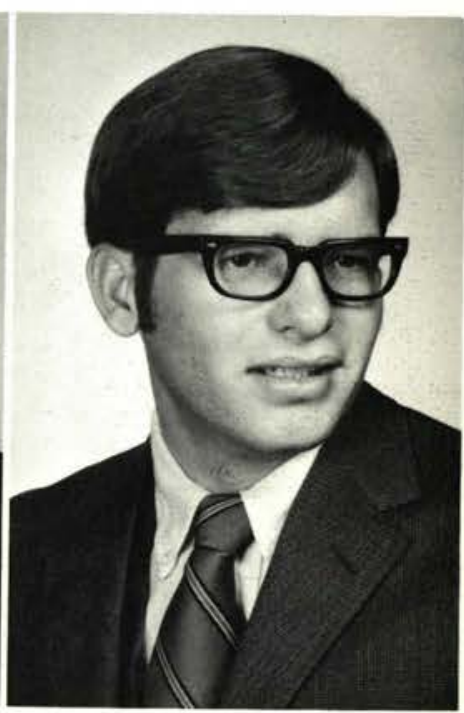

Gary McDowell

Hobart, Indiana

Social Studies Comp.

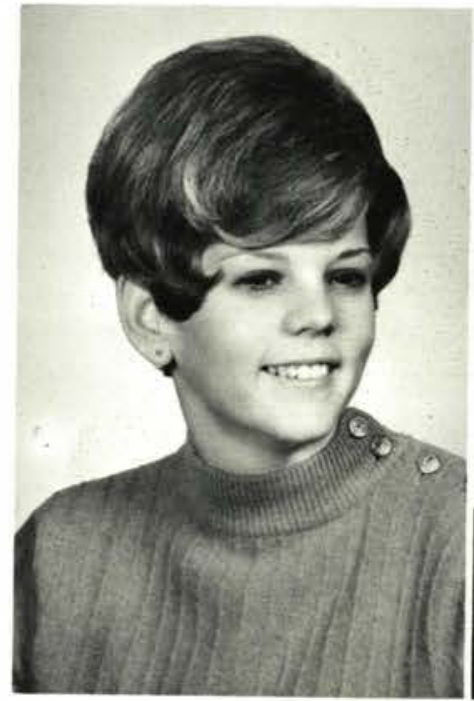

Winifred McFarlane

Portage, Indiana

Music

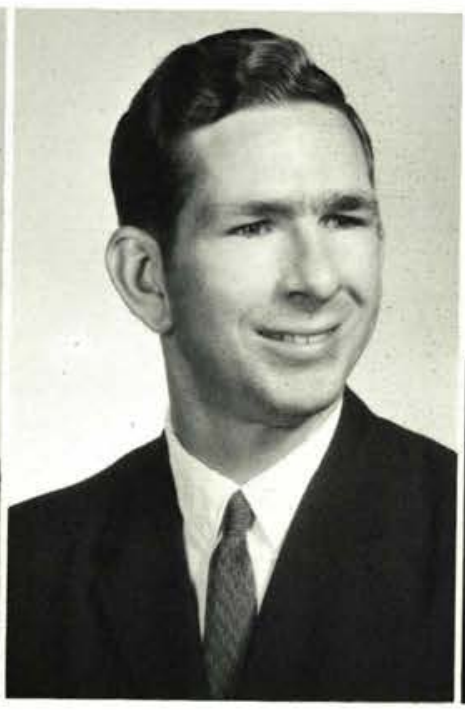

Roger McNamara

Harpursville, N.Y.

Bible

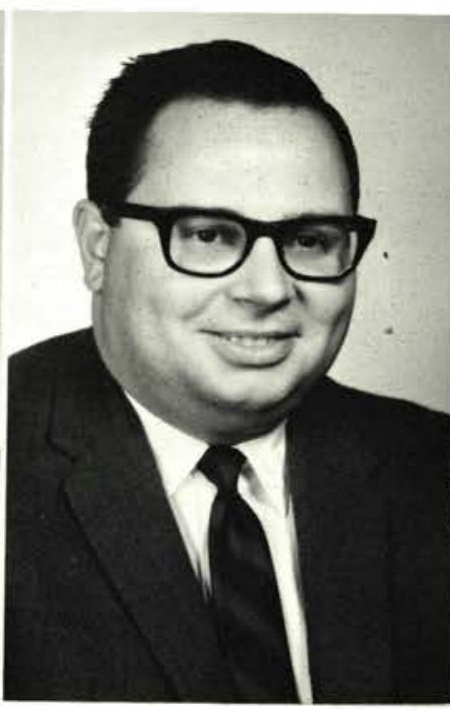

Don Meadows

Cedarville, Ohio

Elementary Education

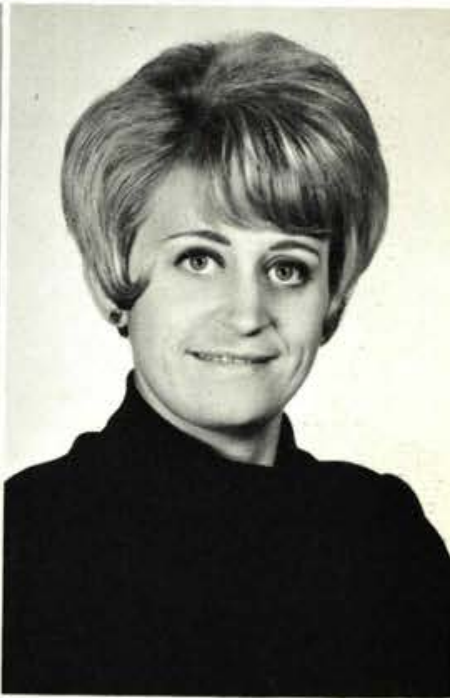

Viann Meyer

Waterloo, Iowa

English/Sociology
Ronald Meyers

East Moline, III.

Chemistry/Mathematics

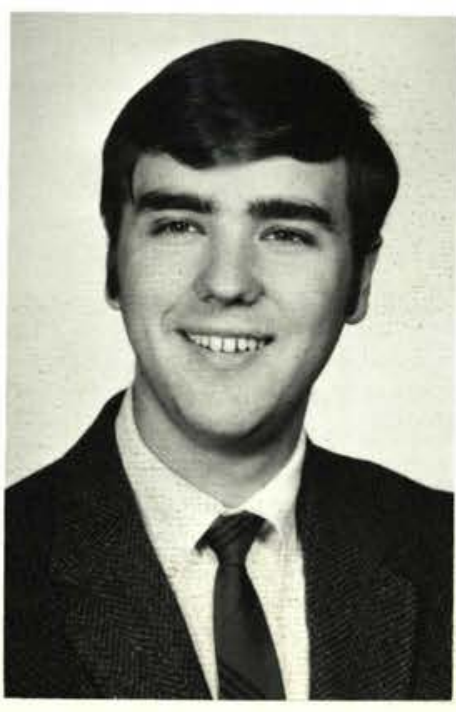

Joyce Miller

Williamston, Mich.

Sociology
Richard Mitchell

Malaga, New Jersey History
Alice Moorman

Xenia, Ohio

Elementary Education
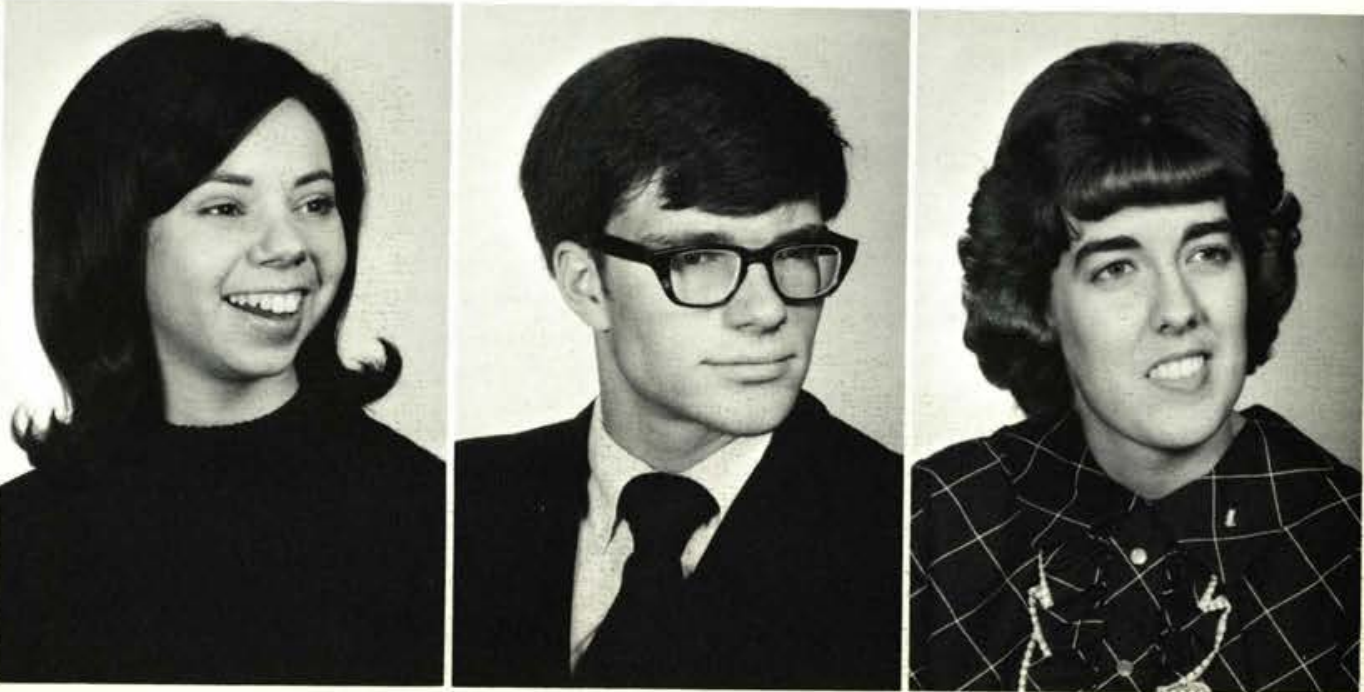


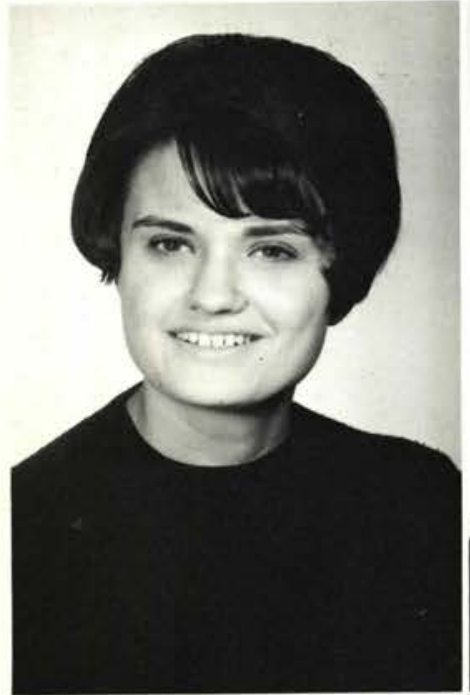

Carol Morrell

Indianapolis, Ind.

Elementary Education

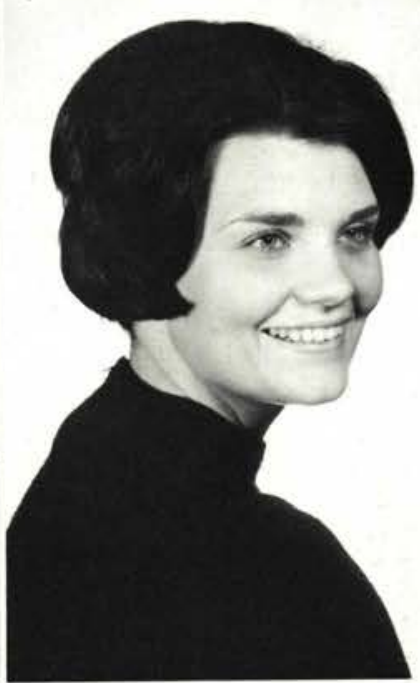

Gwendolyn Mosby Indianapolis, Ind. Elementary Education

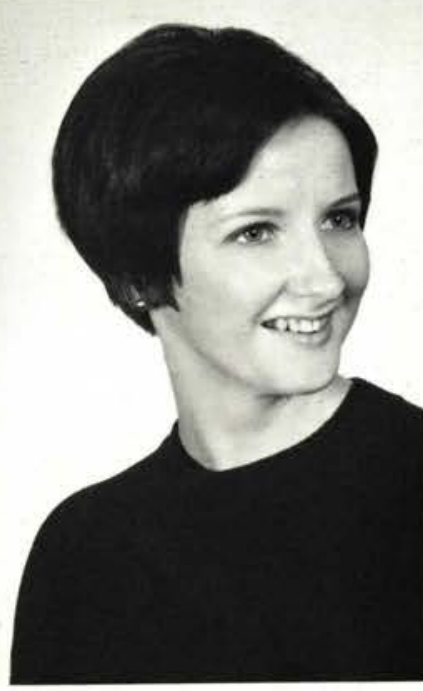

Shirley Mott

Angelica, N.Y. Spanish

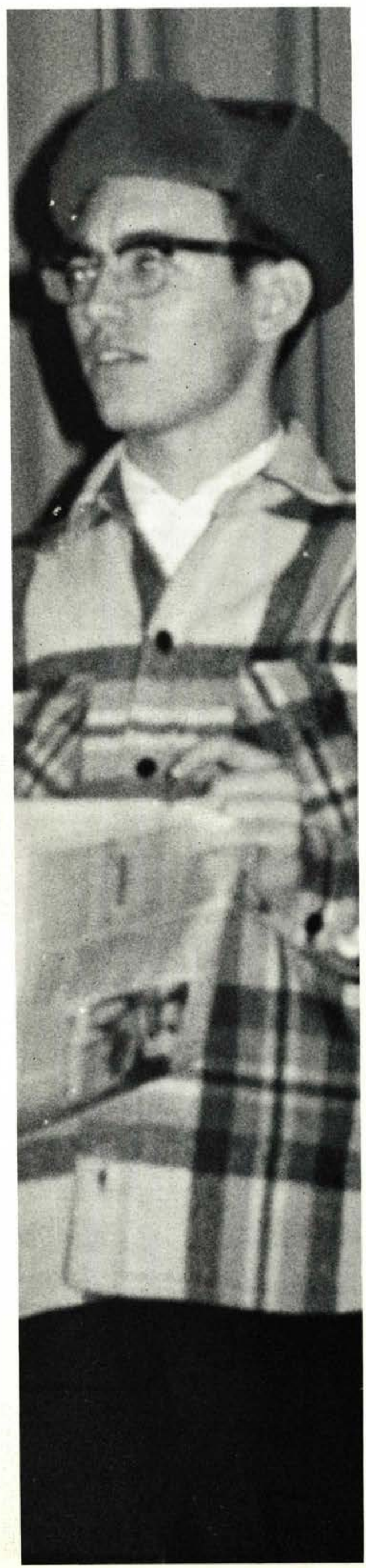

Joel Nitz

Greensburg, Pa.

Business Administration

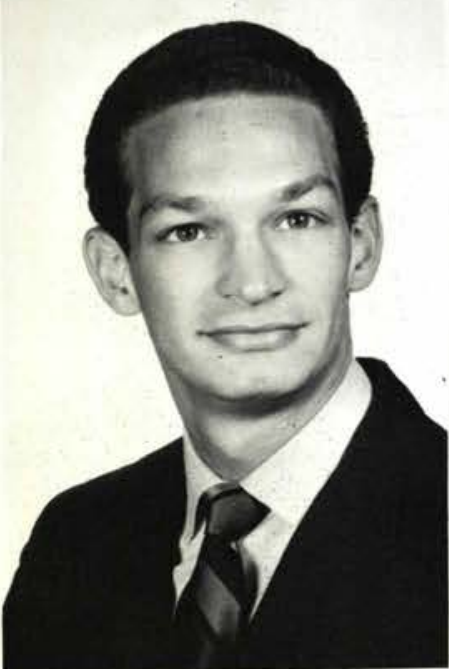

Loretta Nutter

Delaware, Ohio

Elementary Education

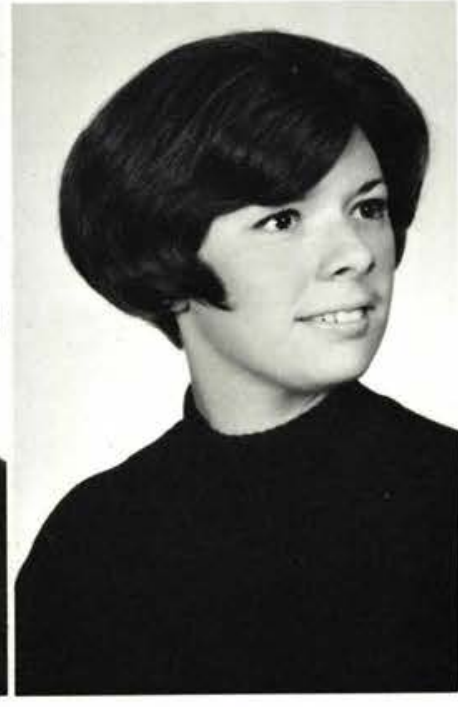

Penny Nichols

Crawfordsville, Ind.

Elementary Education
Peter Oehmcke

Wauwatosa, Wis.

Social Science Comp.

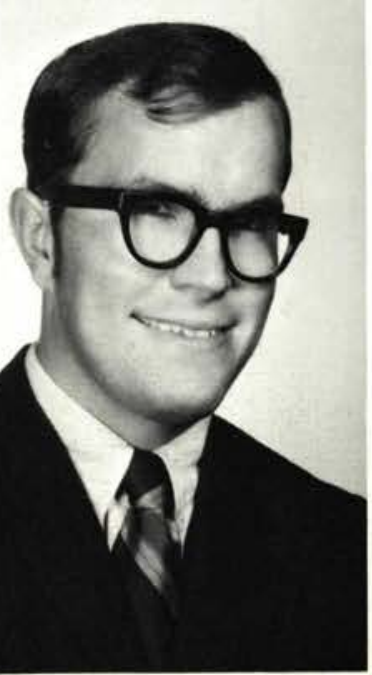




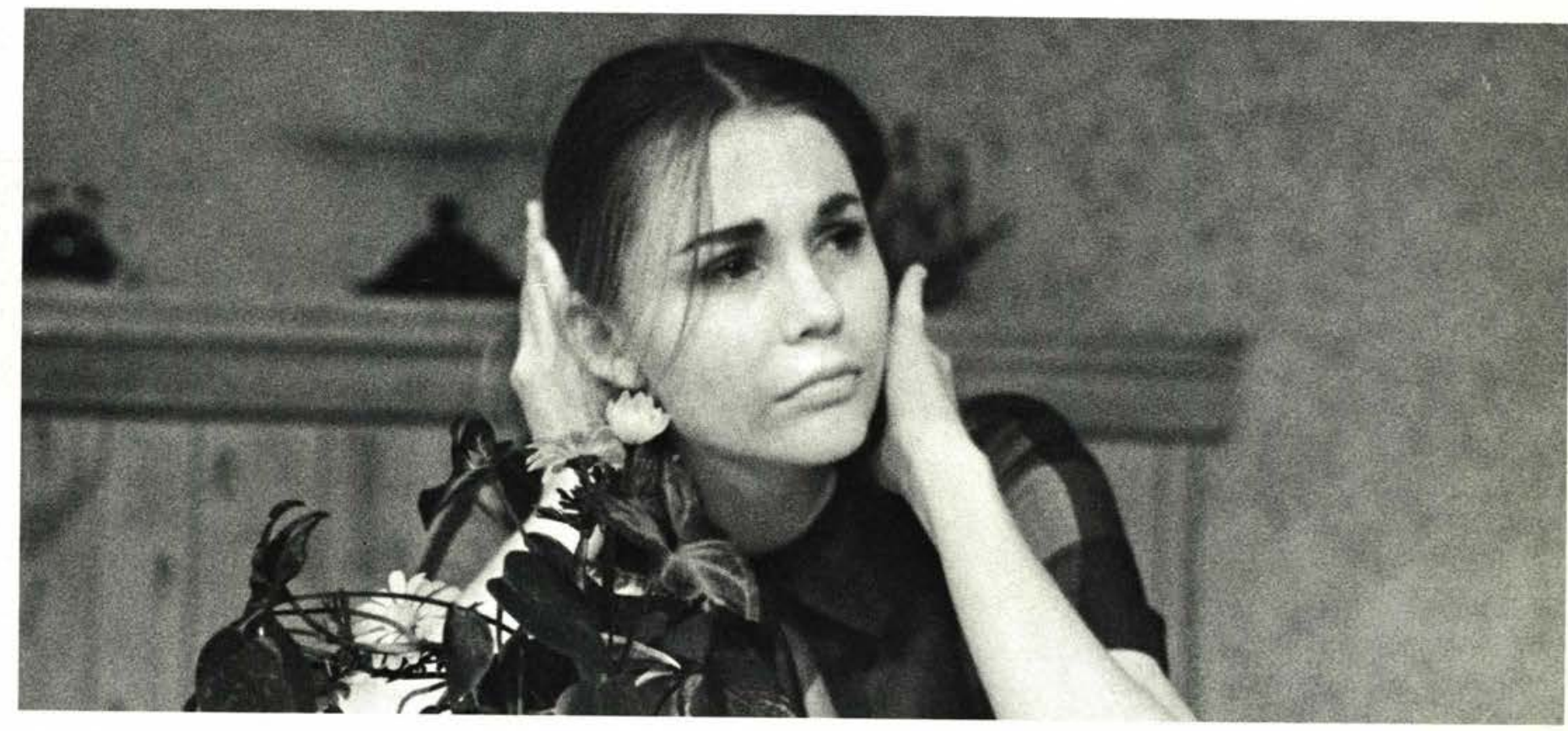

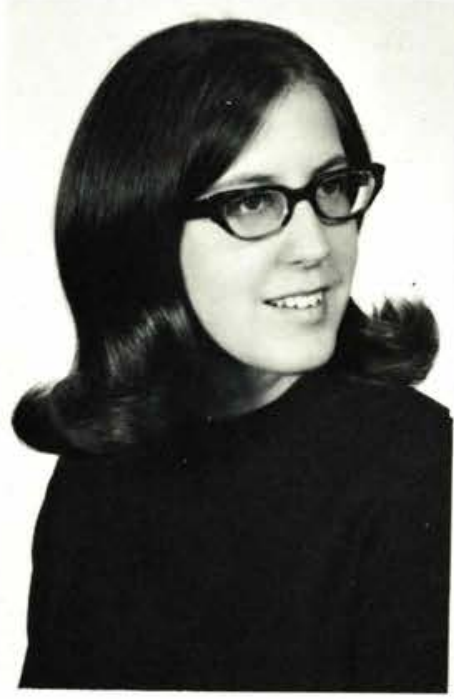

Beth O'Keefe

Parma, Ohio

English

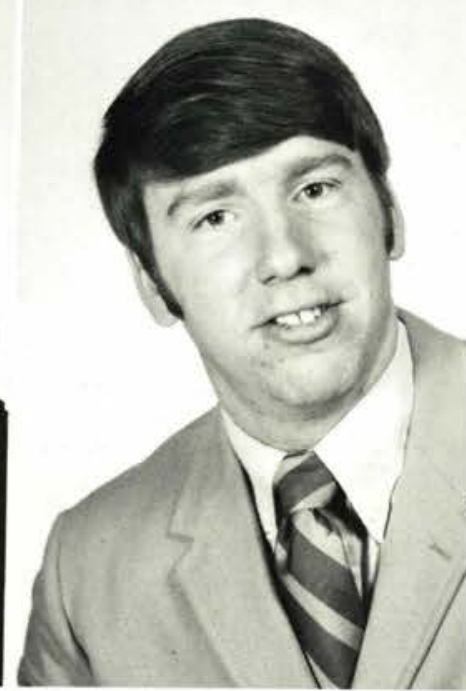

James O'Keefe

Wesleyville, $\mathrm{Pa}$

Psychology/Sociology

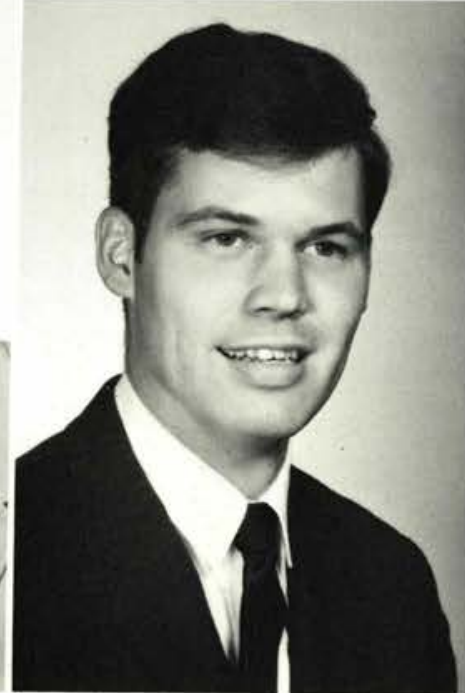

Samuel Olsen

Lima, Peru

English

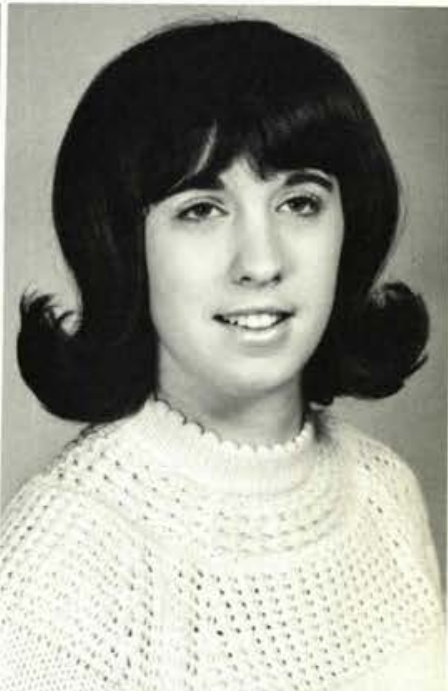

Susan Pauling

Allenwood, $\mathrm{Pa}$.

Elementary Education

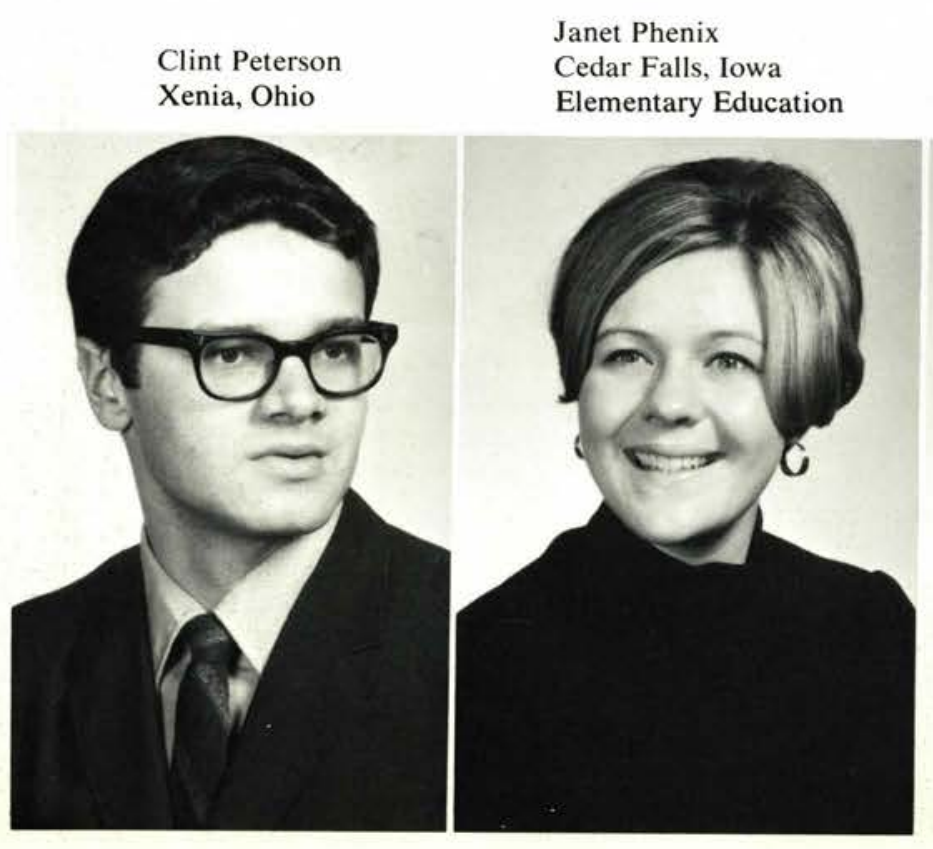

Edith Phillips

Plainfield, Ind

Mathematics

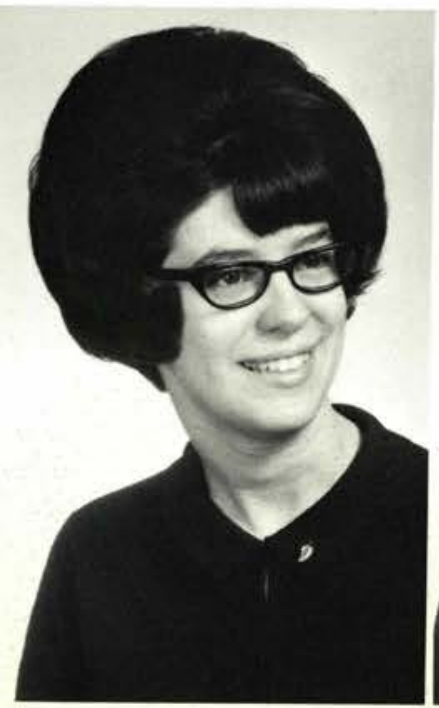

Pat Phipps

Portsmouth, Ohio

Elementary Education

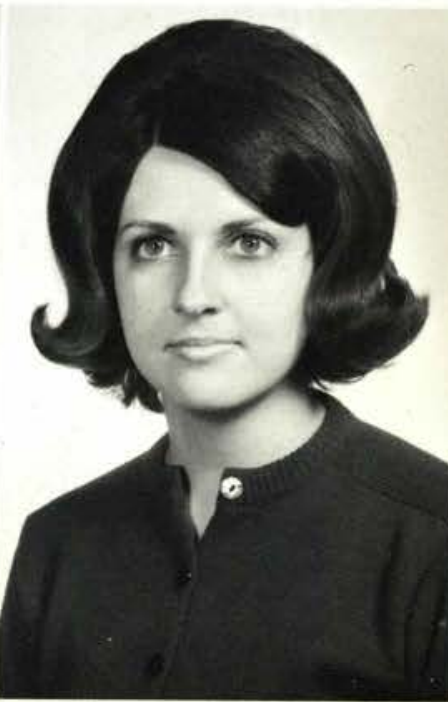




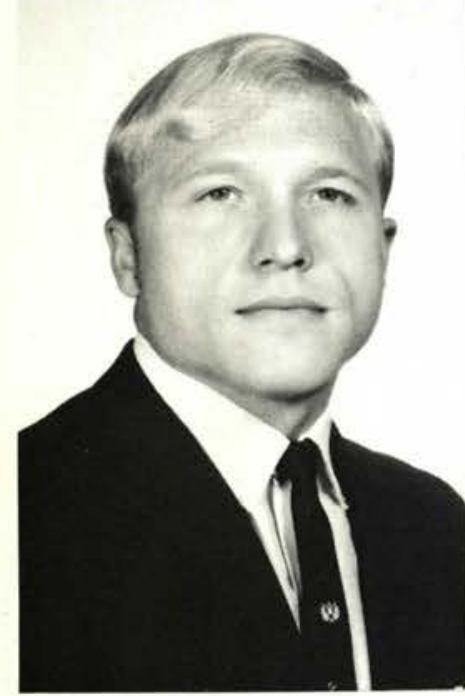

Terry Phipps

Concord, Calif.

Biology

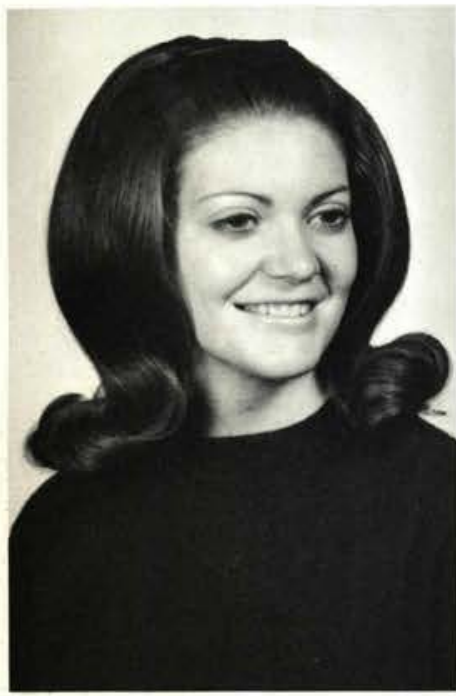

Betty Jean Reese

Lock Haven, $\mathrm{Pa}$.

Sociology/English

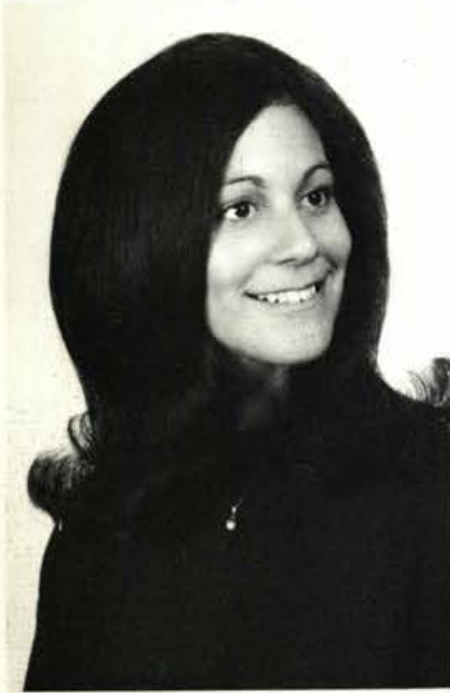

Ruth Rodrigues

Greenlawn, N.Y.

Social Science

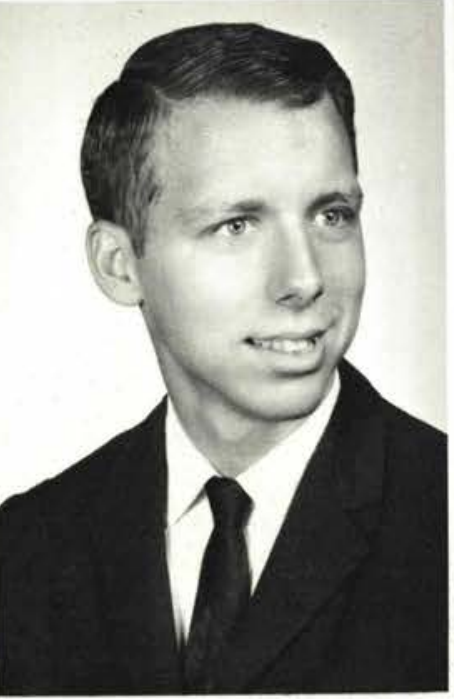

Larry Radcliff

Caldwell, Ohio

Mathematics

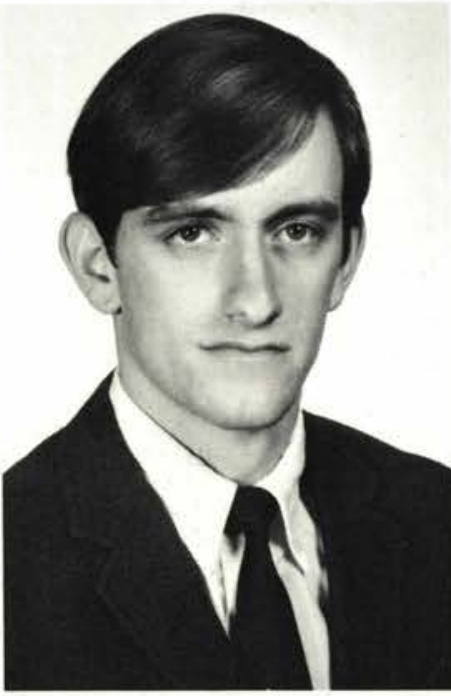

Robert Rehkopf Petoskey, Mich.

Accounting

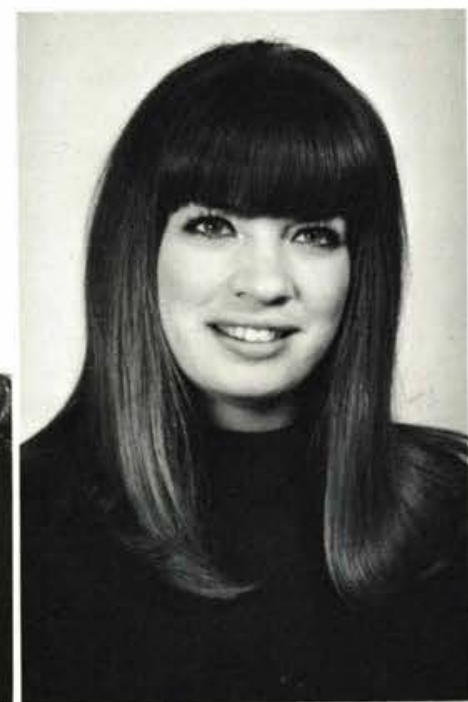

Ruth Rogers

Madison, Ohio

Music/Elementary Education

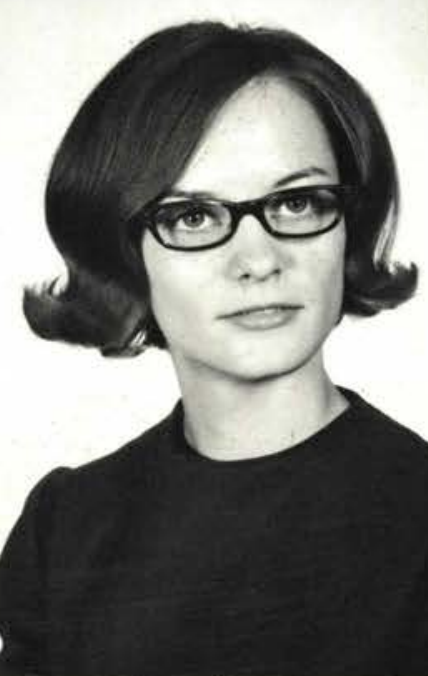

Diana Radcliffe

Wakeman, Ohio

Elementary Education

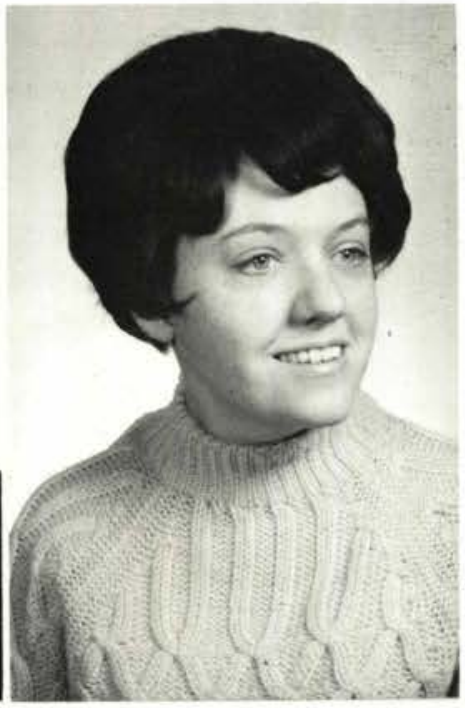

Sue Ray

Decatur, Illinois

Elementary Education

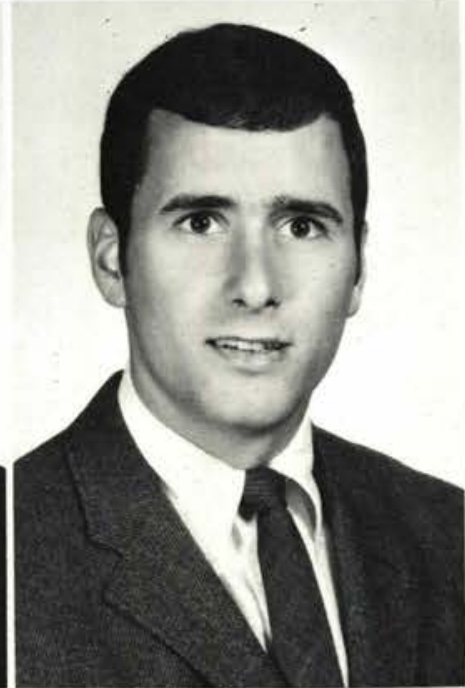

Loren Reno

Wheaton, Illinois

Social Studies Comp.

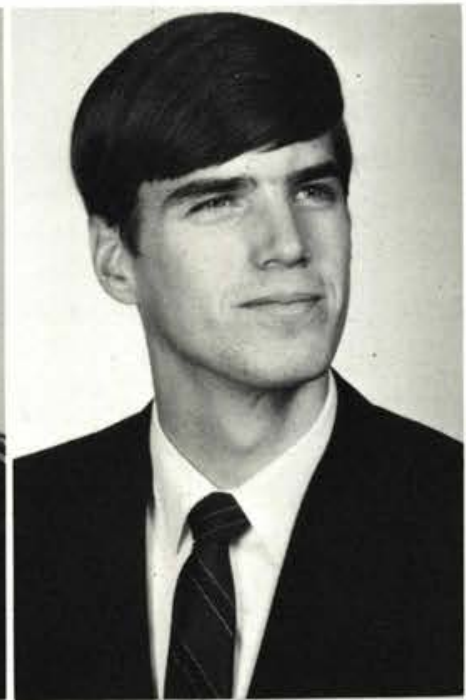

Philip Rohler

Twinsburg, Ohio

Speech

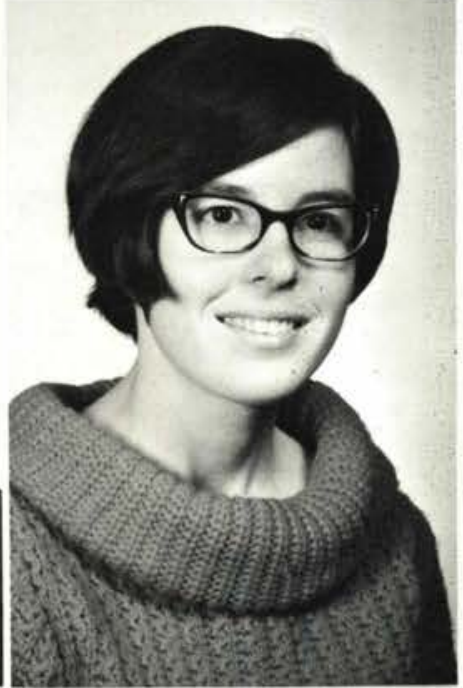

Rebekah Rochelle

Atlanta, Georgia

Elementary Education

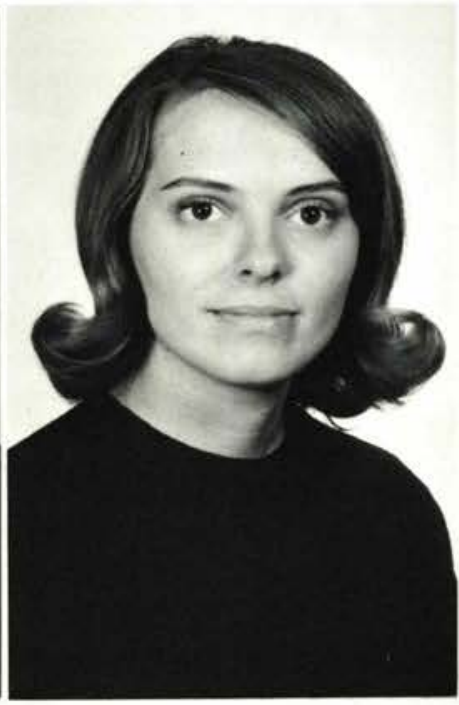

Cecilia Romine

Huntington, W. Va.

Elementary Education 


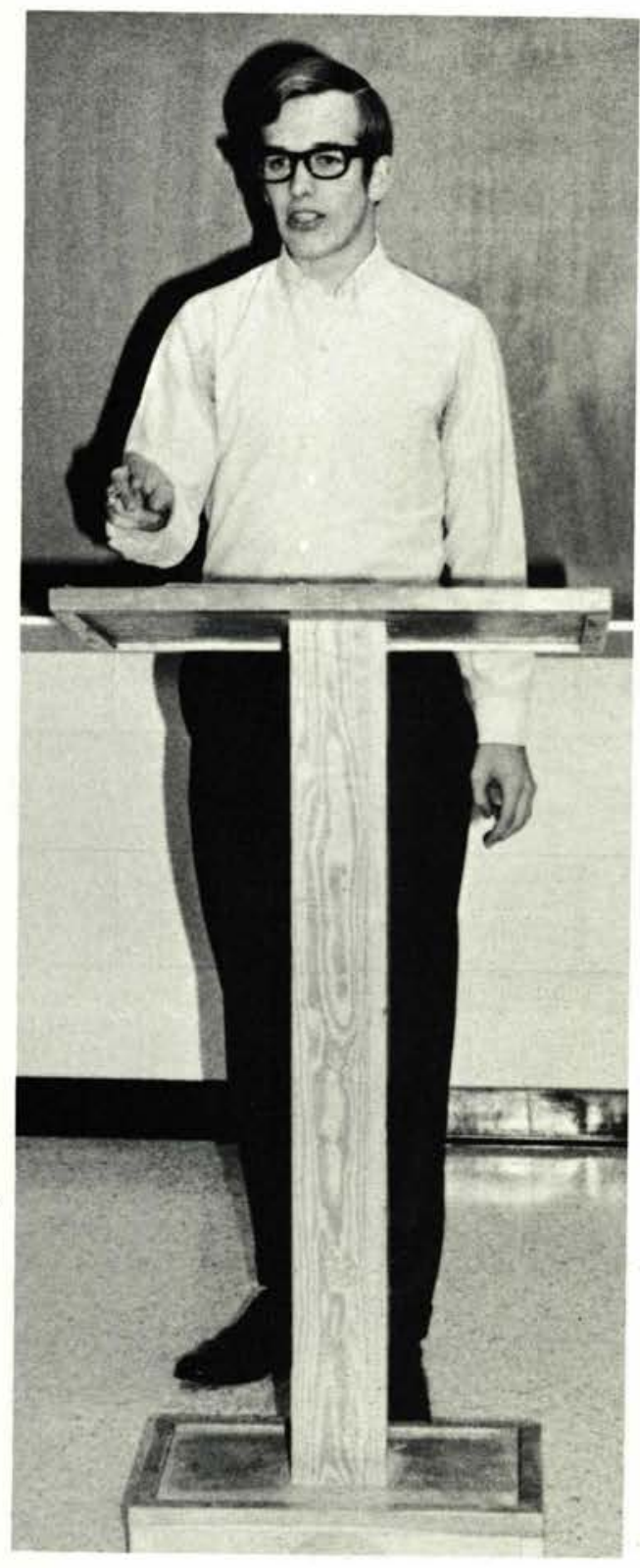

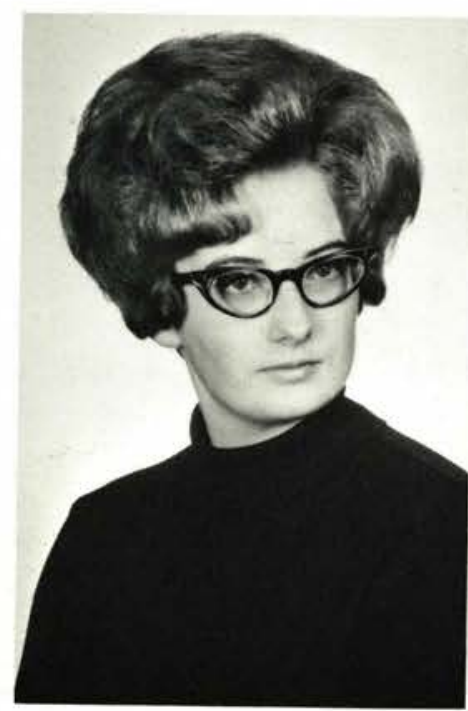

Lois Rumage

Hallstead, $\mathrm{Pa}$.

Business Administration

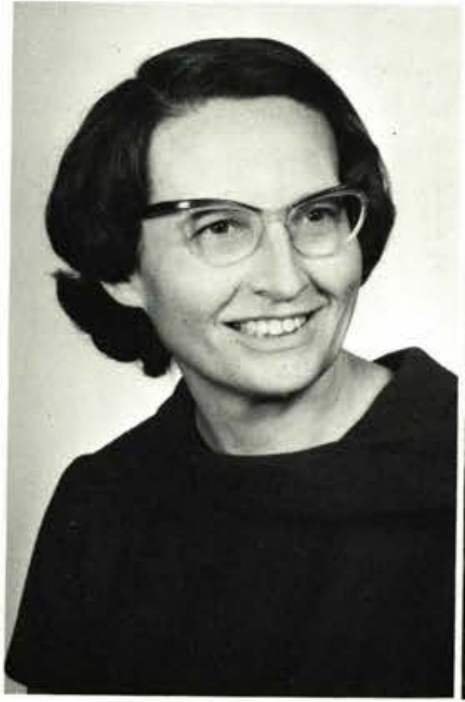

Marian Schwenk

La Porte, Ind.

English

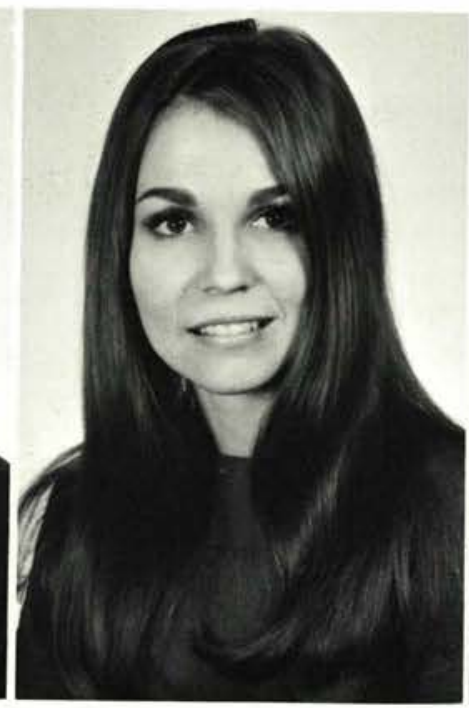

Joyce Saemens

Phoenix, Arizona

English

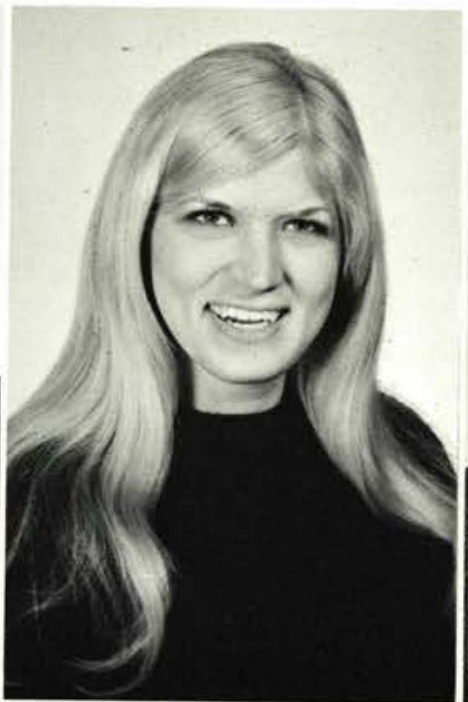

Gail Sears

Grinnell, Iowa

Elementary Education

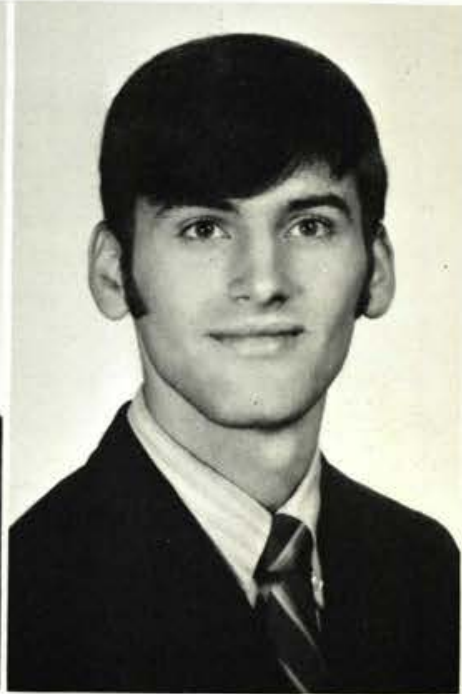

John Schneider

Hammond, Indiana

History

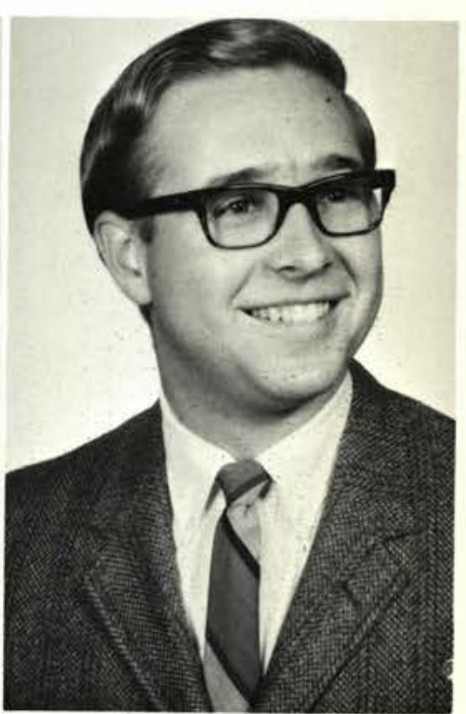

Phillip Senseney

Linwood, N.J.

Music

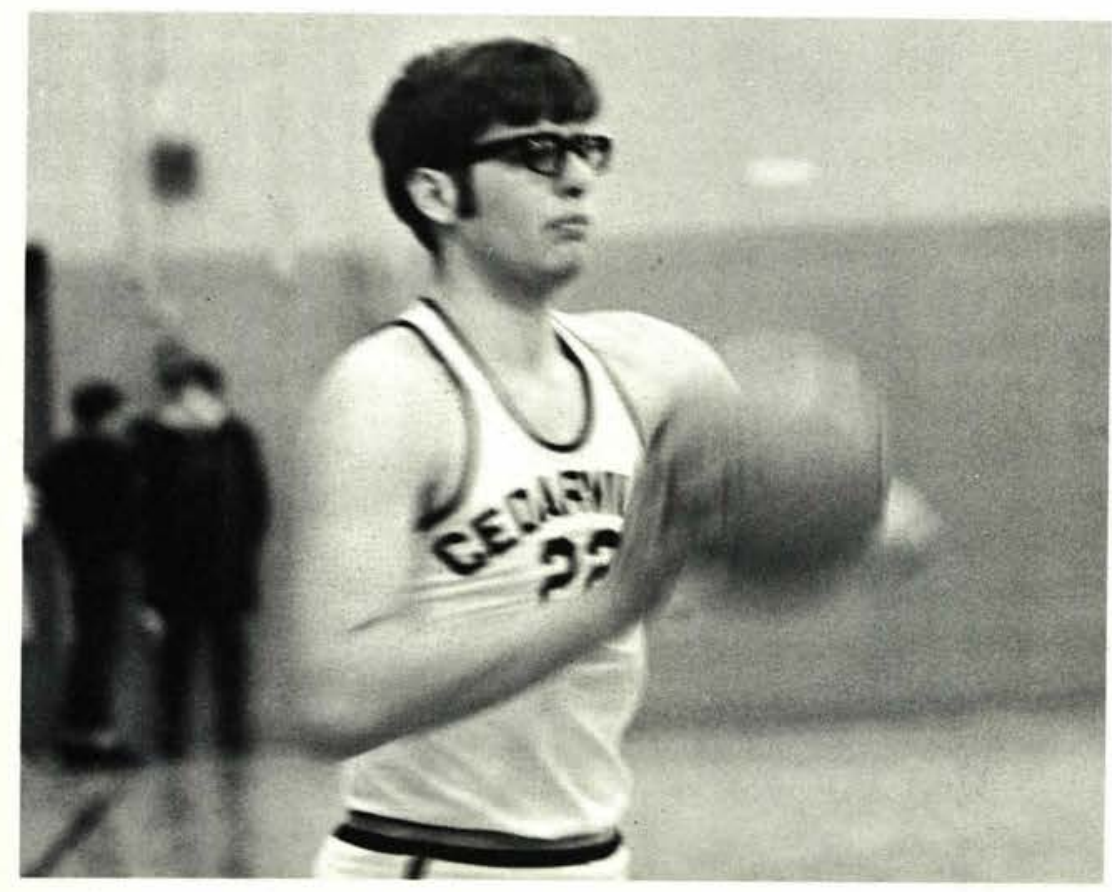

Becky Sharp

Columbus, Ohio

Elementary Education

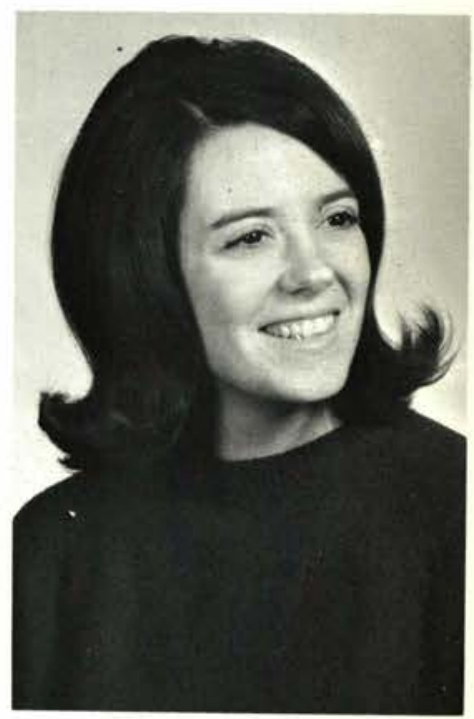




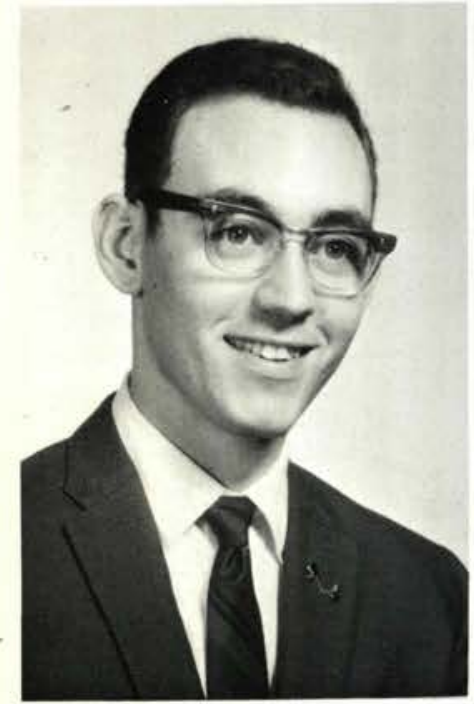

Art Shuter

Carroll, Ohio

Music

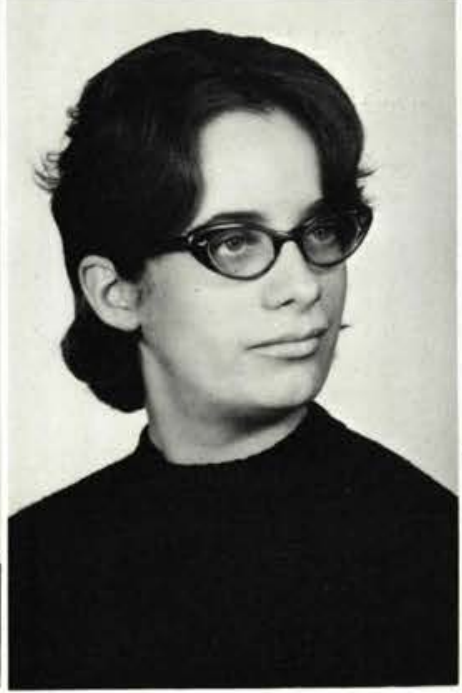

Carol Smith

Ashland, Kentucky

Elementary Education

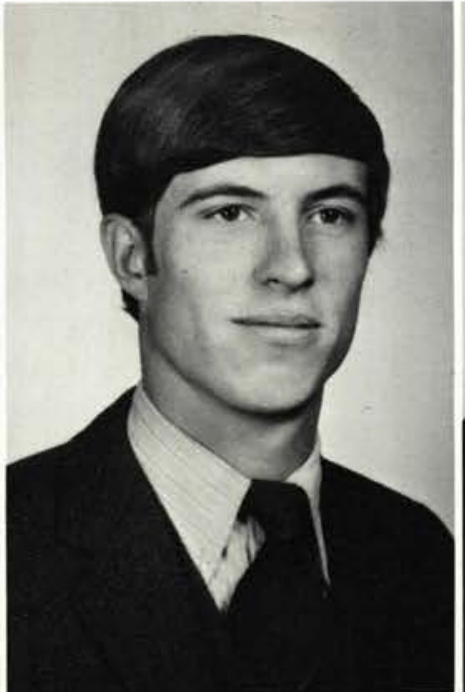

Dan Smith

Boise, Idaho

Music

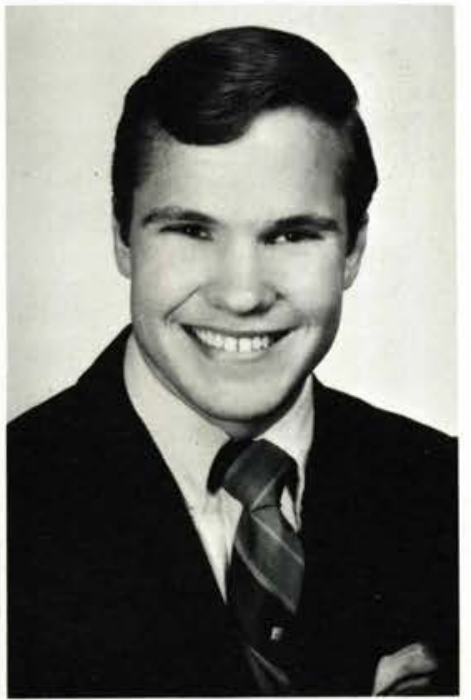

William Smith

Milford, Illinois

Mathematics/Bible

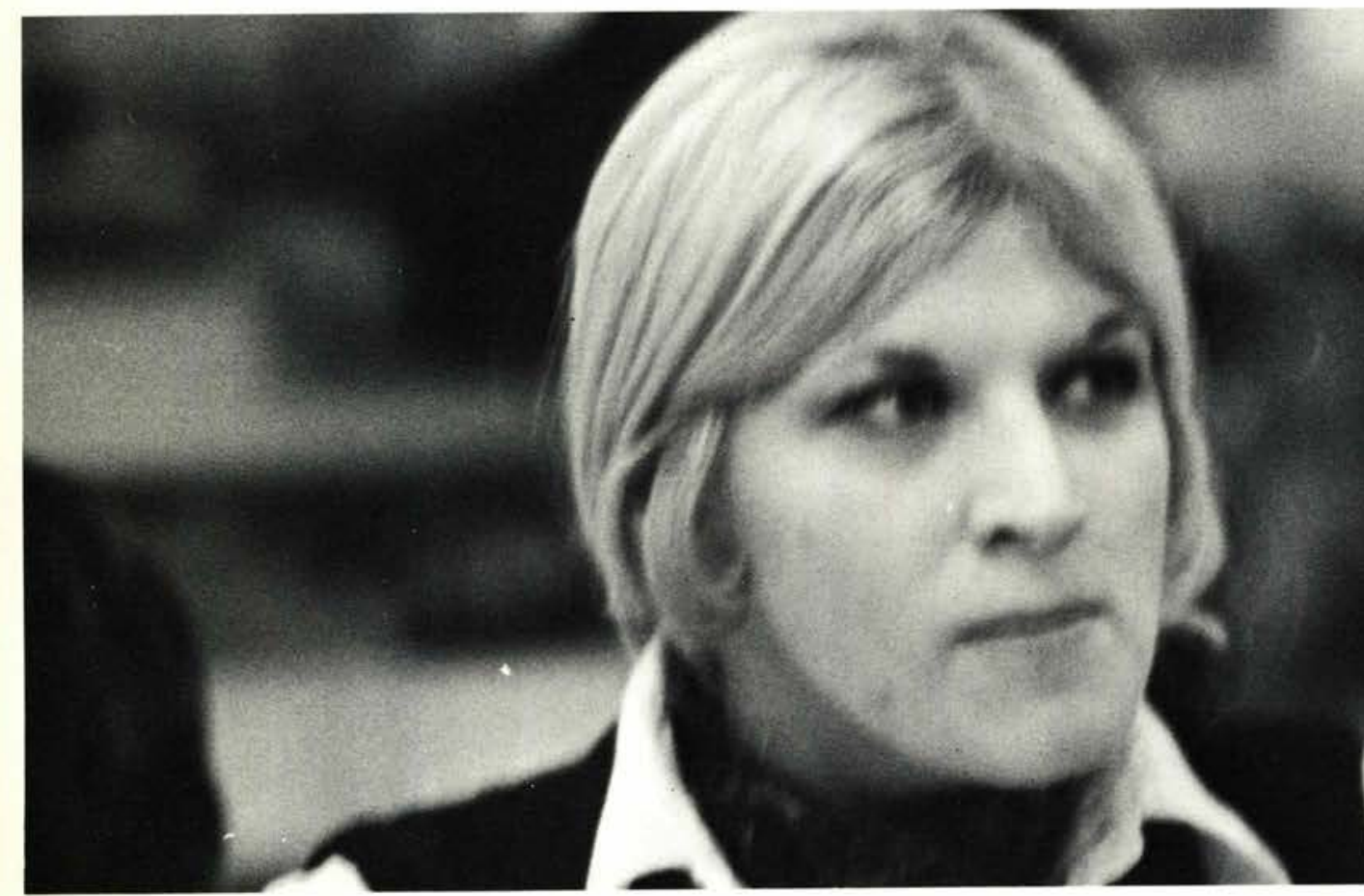

Philip Sommerfeld

San Diego, Calif. Speech

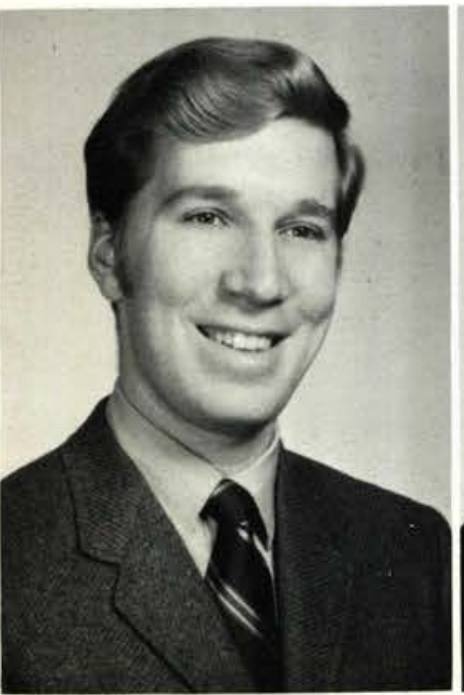

David Southwell

Mancelona, Mich.

Mathematics

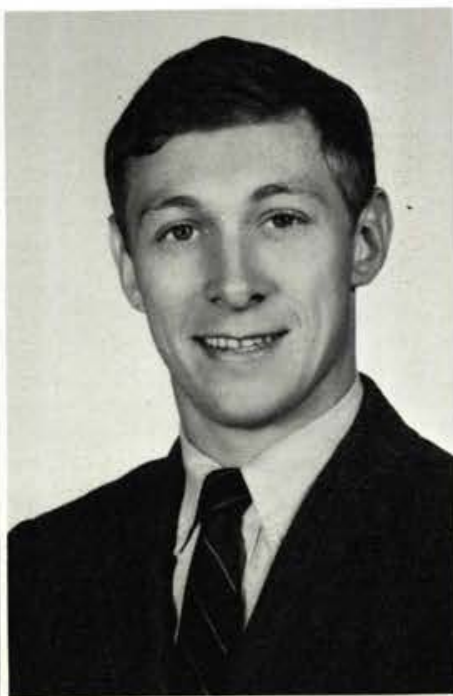

Judy Stamatis

Glen Cove, New York

Physical Education
Pat Sturdevant

Union City, $\mathrm{Pa}$

El. Ed/Christian Ed.

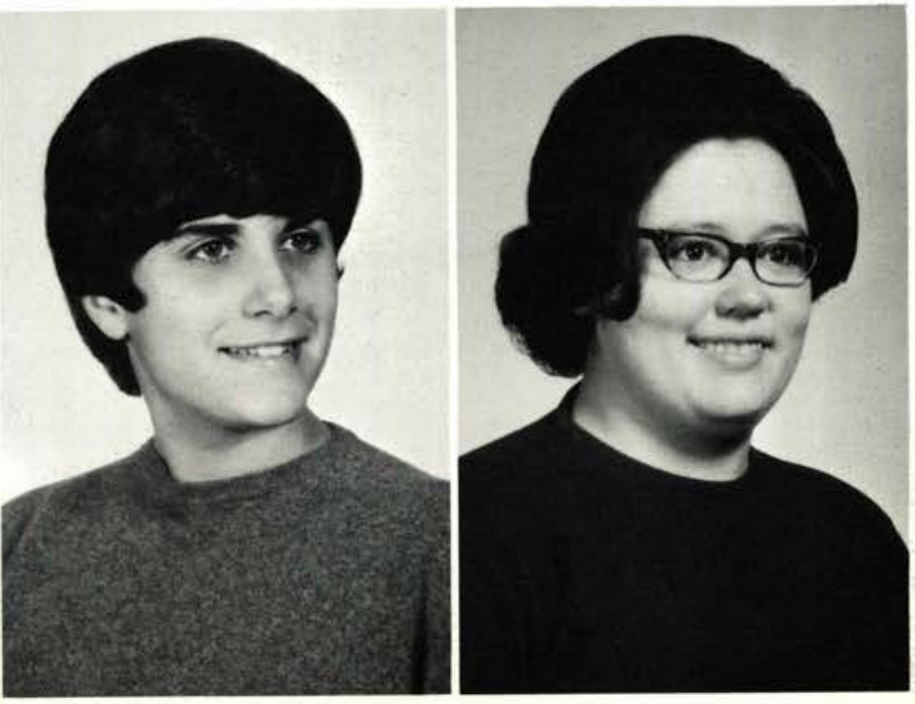




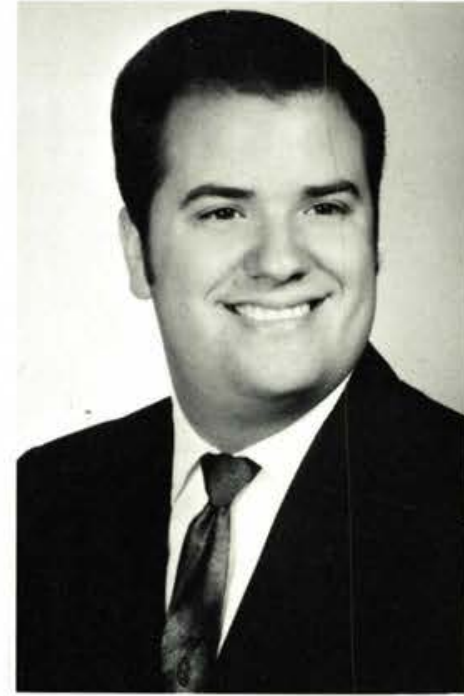

Richard Taylor

Cleveland, Ohio

Business Education

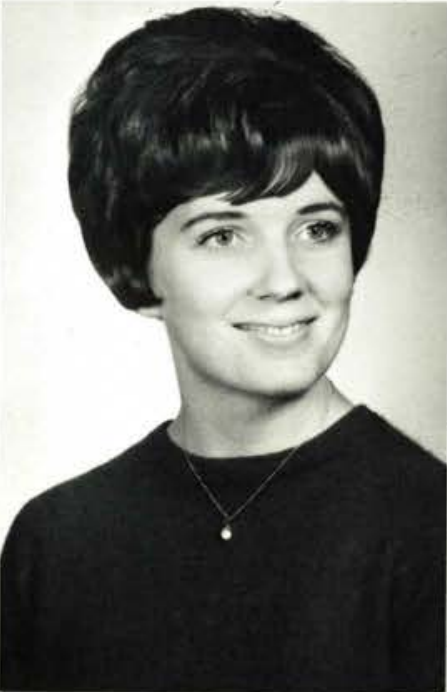

Sandy Taylor

Middleburg Heights, O.

Accounting

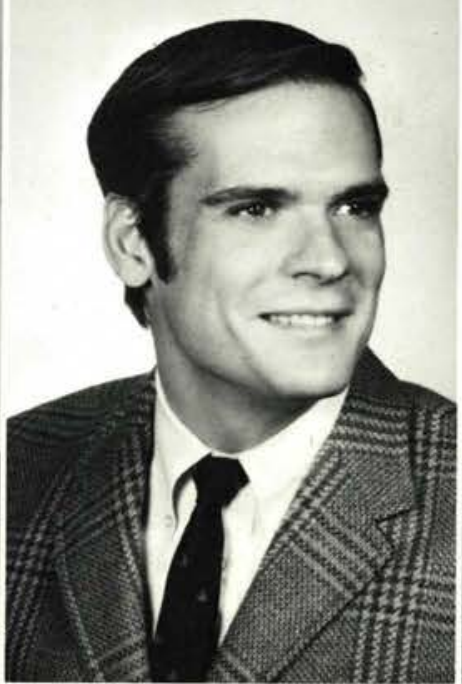

Steve Taylor

Philadelphia, $\mathrm{Pa}$.

Psychology

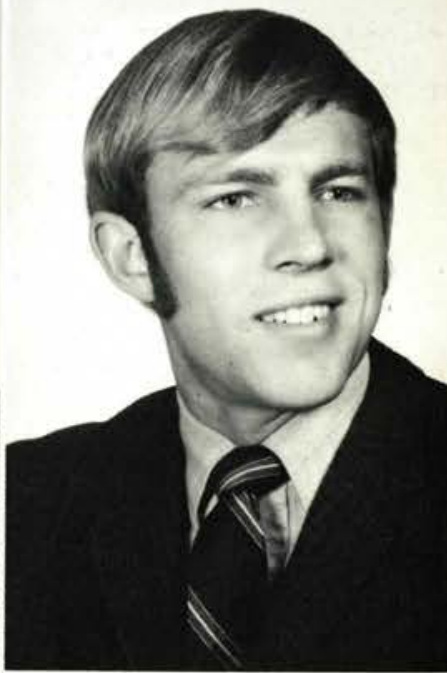

Kenneth Treat

Galetown, Pa.

Physical Education

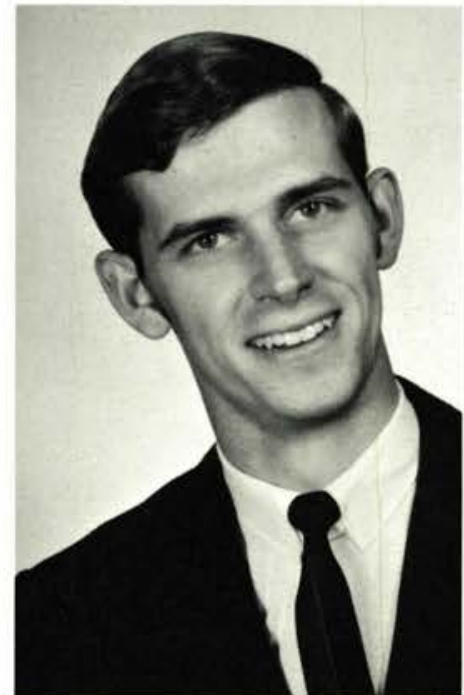

Bill Tripp

New London, $\mathrm{O}$.

Biology

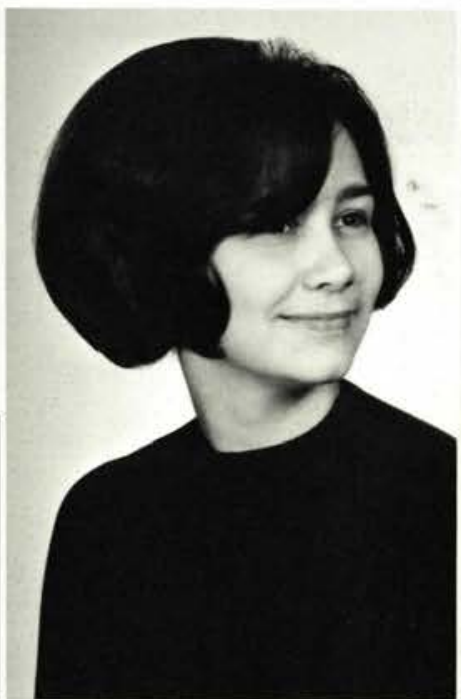

Mary Trumphour

Warren, Ohio

Biology

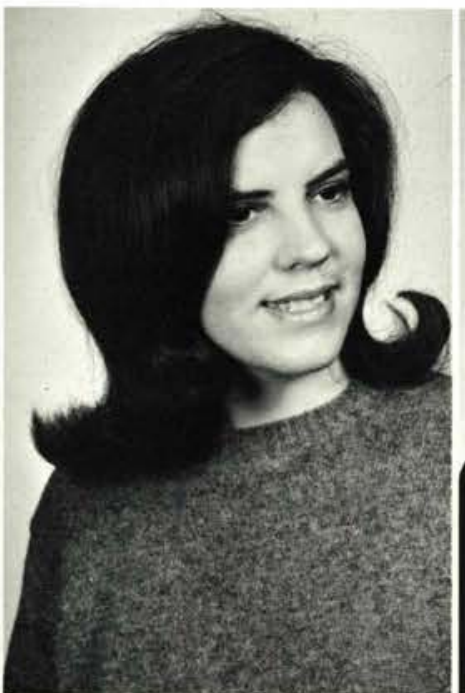

Nola Watson

Wakeman, Ohio

Music

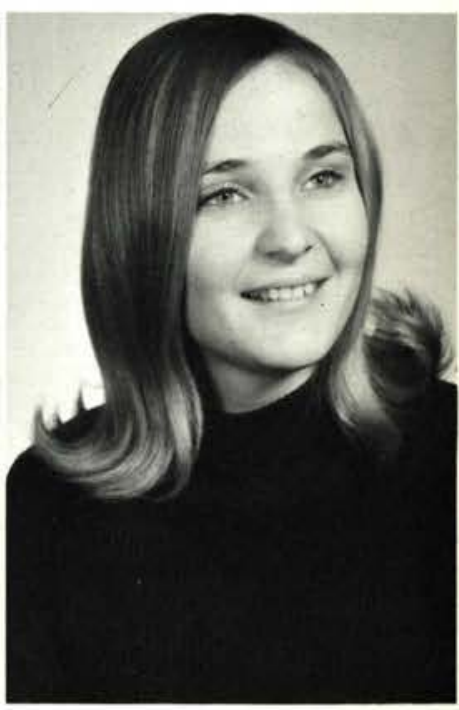

Lynda Wessel

Newton, Iowa

Social Studies Comp.
Shirley Witt

Waterloo, Iowa

Elementary Education

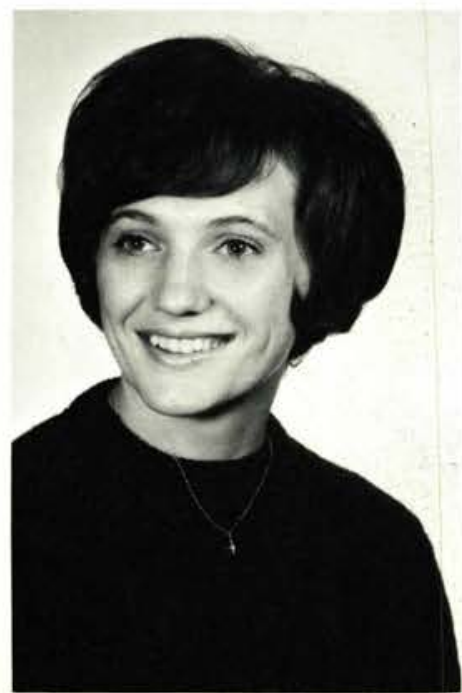

Pat Wissinger

Warren, Ohio

Physical Education

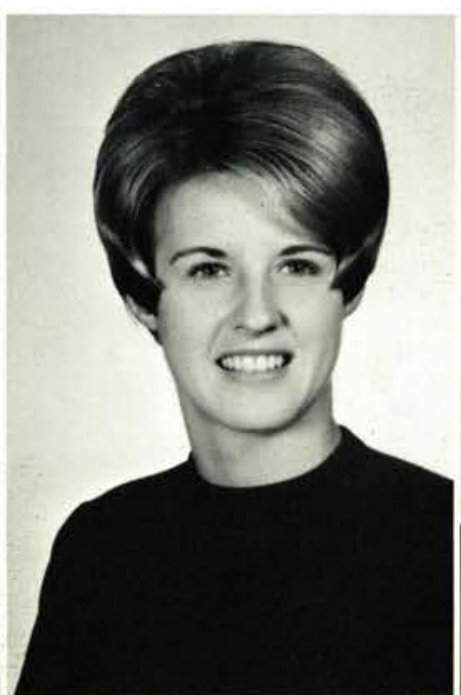

Gary Yates

Dayton, Ohio

English

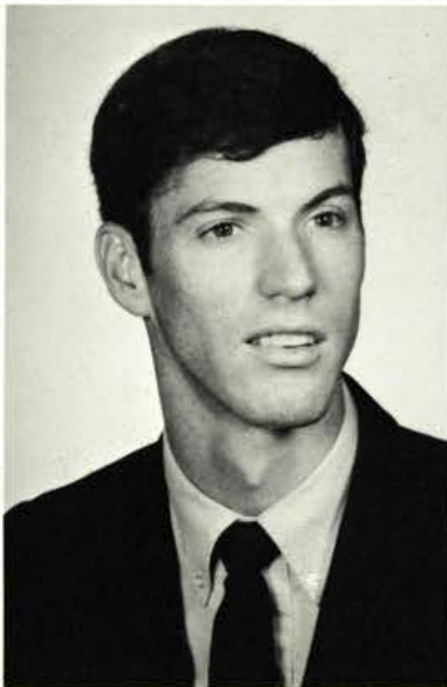

Joyce Young

Endwell, N.Y.

Elementary Education

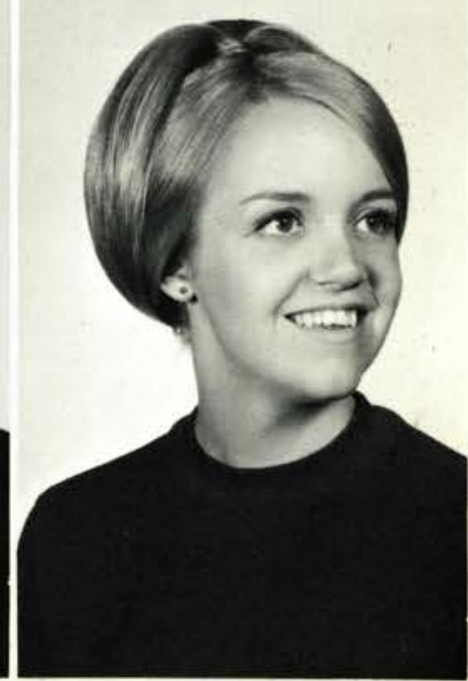




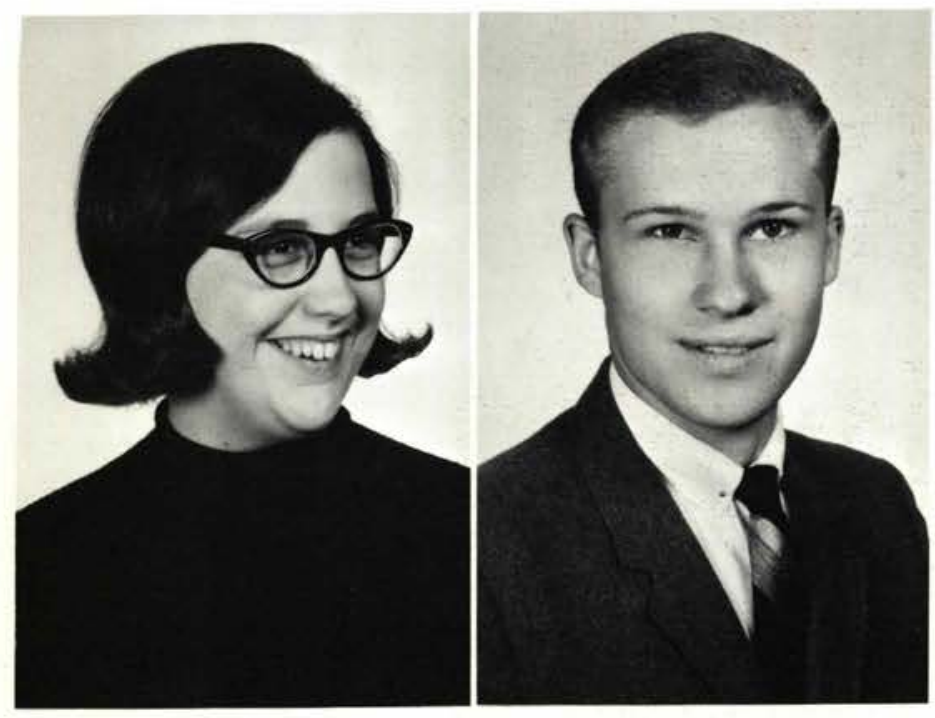

Cynthia Zickefoose

Warren, Ohio

Elementary Education

David Kearney

Elyria, Ohio

Speech
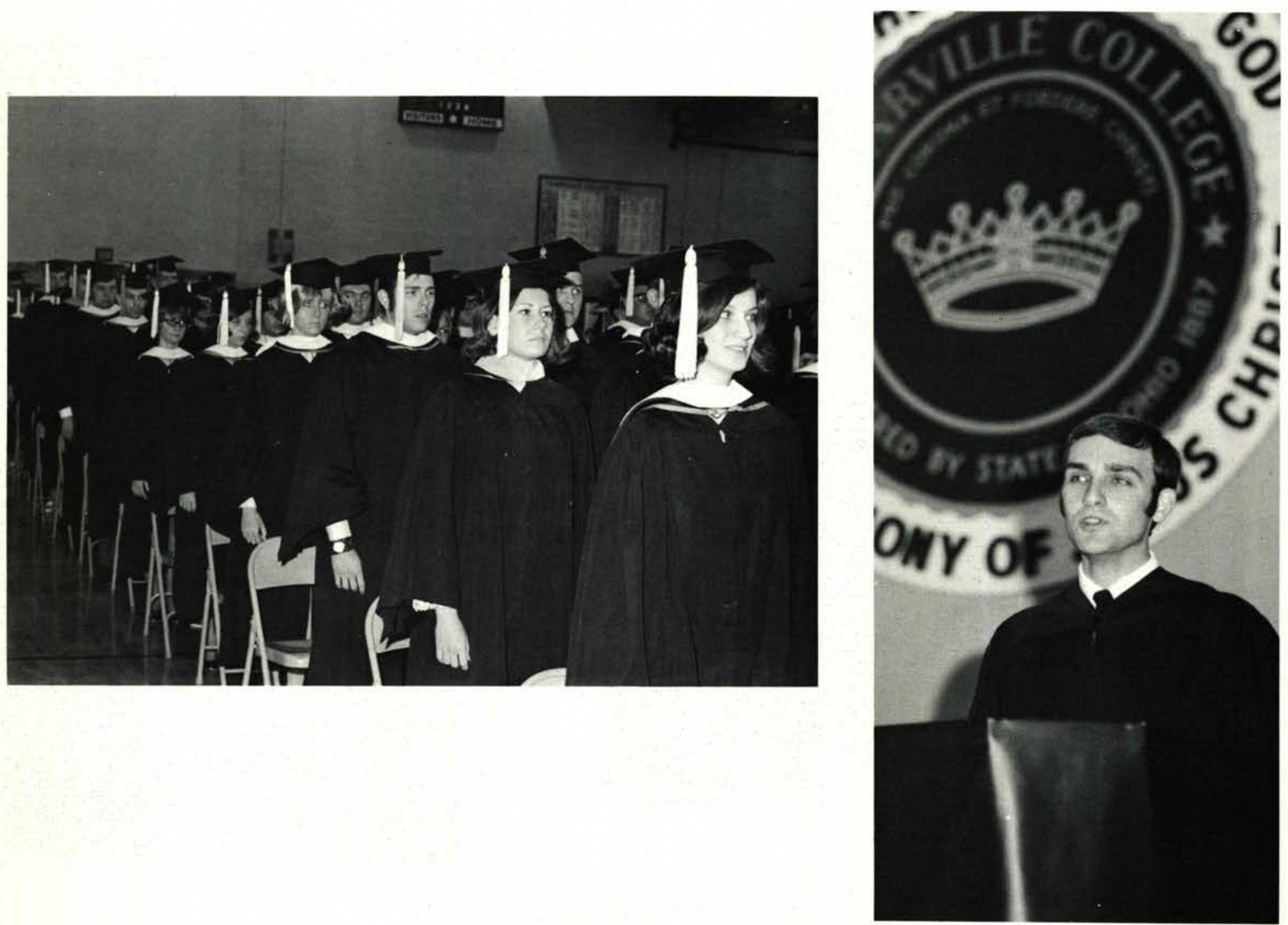

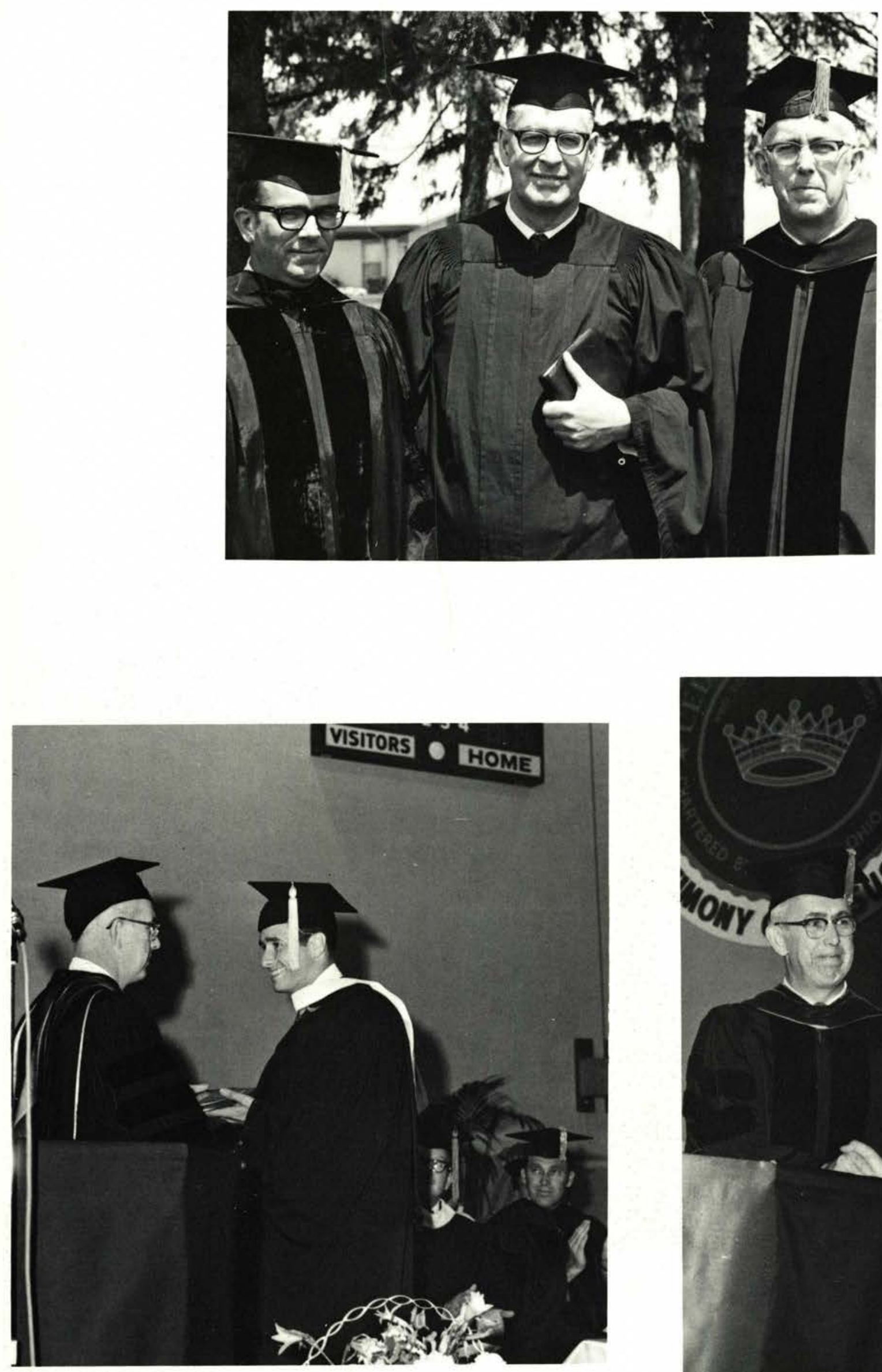

"Try not to become a man of success but rather to become a man of value"

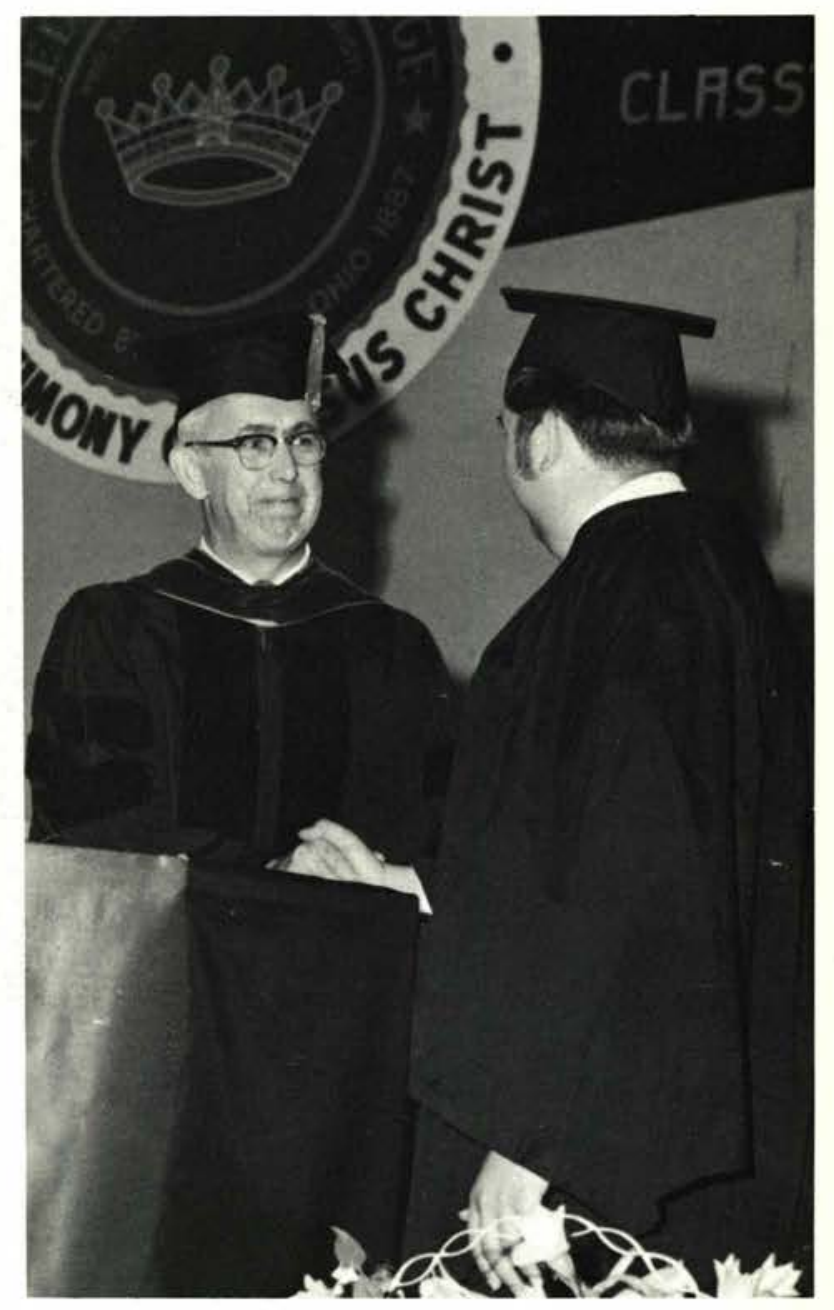
Dr. Albert Einstein 


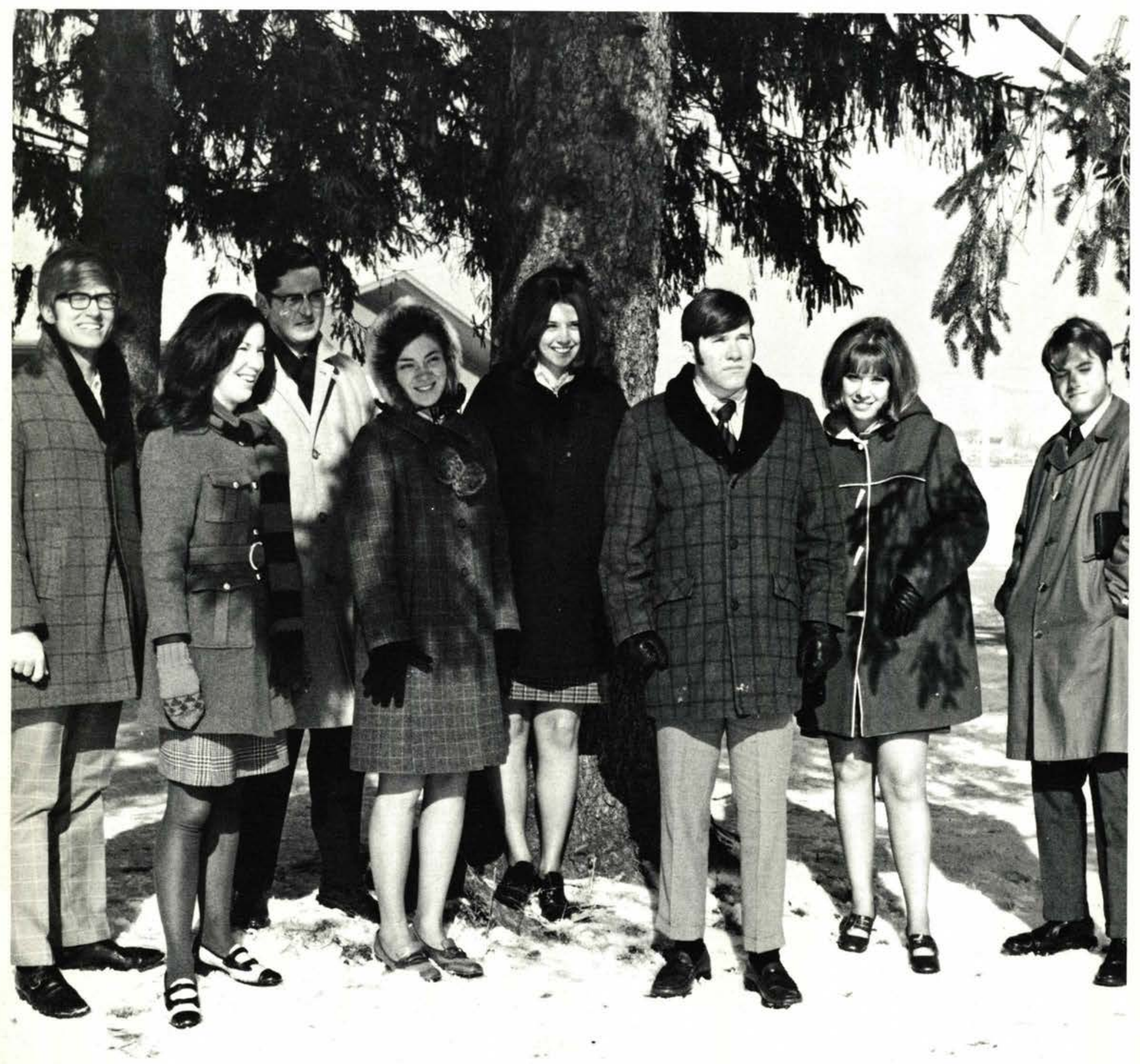

\section{JUNIORS}

\section{CLASS OFFICERS}

Jeff Seeley, St. Council Rep.

Judy Leach, Secretary

Dr. Ager, Advisor

Rita Deatley, St. Council Alt.
Not pictured:

Bob Brown, Vice President
Melinda McNiece, Treasurer

Dan Manross, President

Connie Busho, St. Council Rep.

Craig Roberts, Chaplain 
William Amstutz

Cherry Hill, N. J.

Connie Averitt

Richmond, Ind.

Frank Bale

Ontario, Canada

Jacqueline Barlow

Villa Park, Ill.
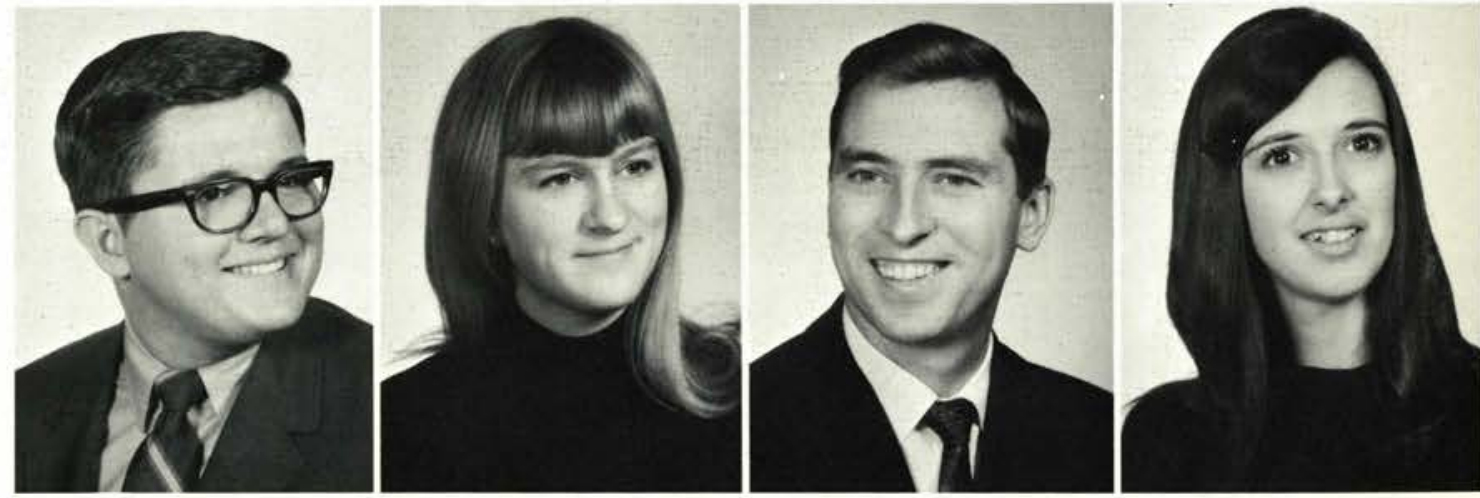

Mary Bartlett

Schiller Park, III.

Dave Beck

Harvey, Ill.

Janet Beesley

Monroe, Iowa

Mareanne Beitler

Medina, Ohio
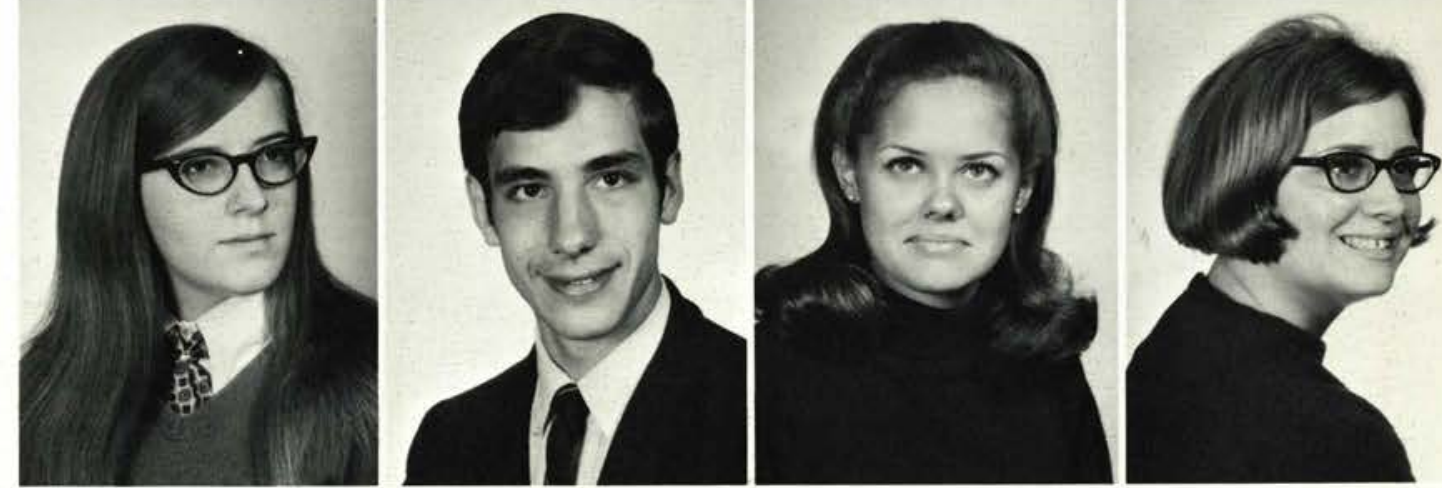

William Berkheiser Cedarville, Ohio

Timothy Bosworth Columbus, Ohio

Joan Boyd

Millersburg, Ohio

Joyce Brookhart Lima, Ohio
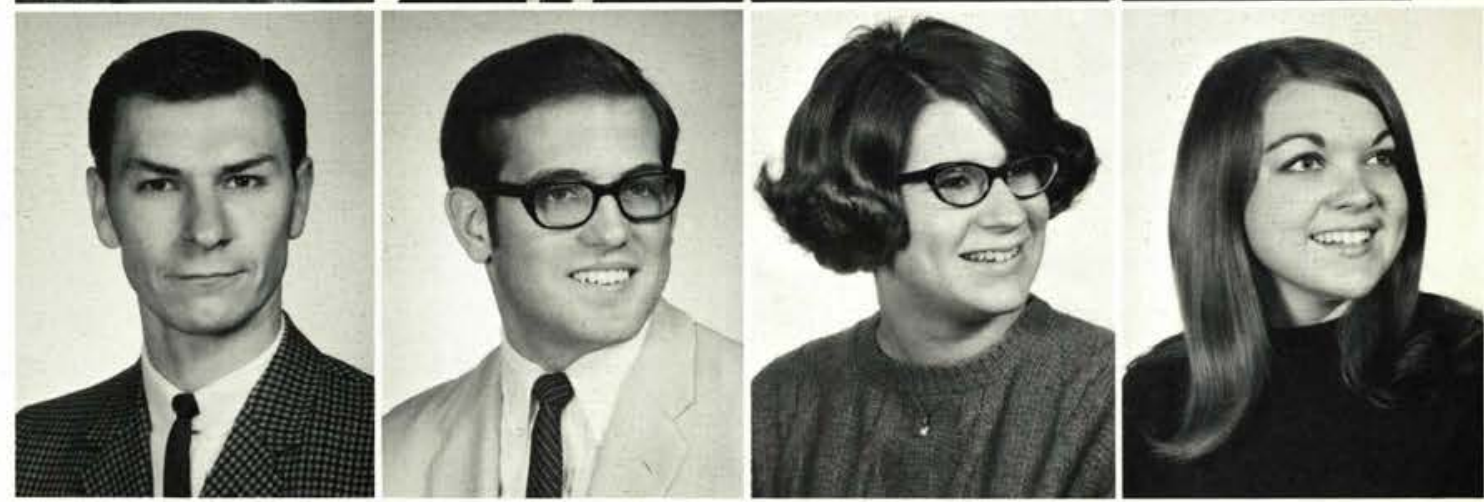

Bob Brown

Brunswick, Ohio

Nancy Brown

Taylors, S. Car.

Linda Browning

Alton, IIl.

Connie Busho

Austin, Minn.
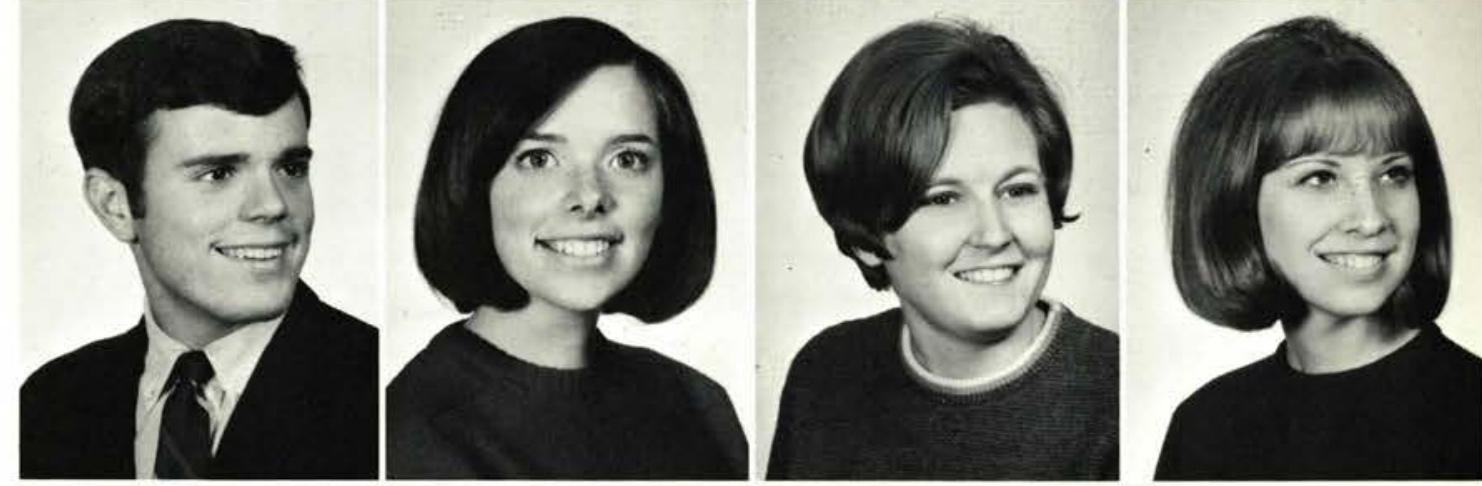

Jim Buzzard

Distant, Pa.

Raymond Cagwin Eden, New York

Marcia Carity Houston, Ohio

Cathy Clark

Hobart, Ind.
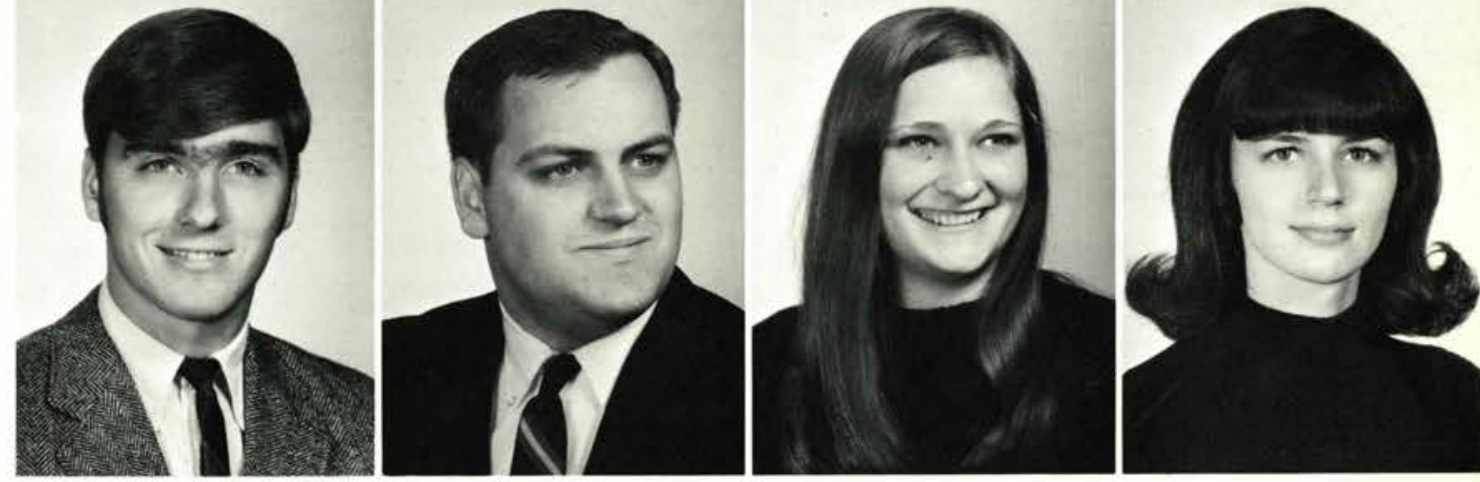

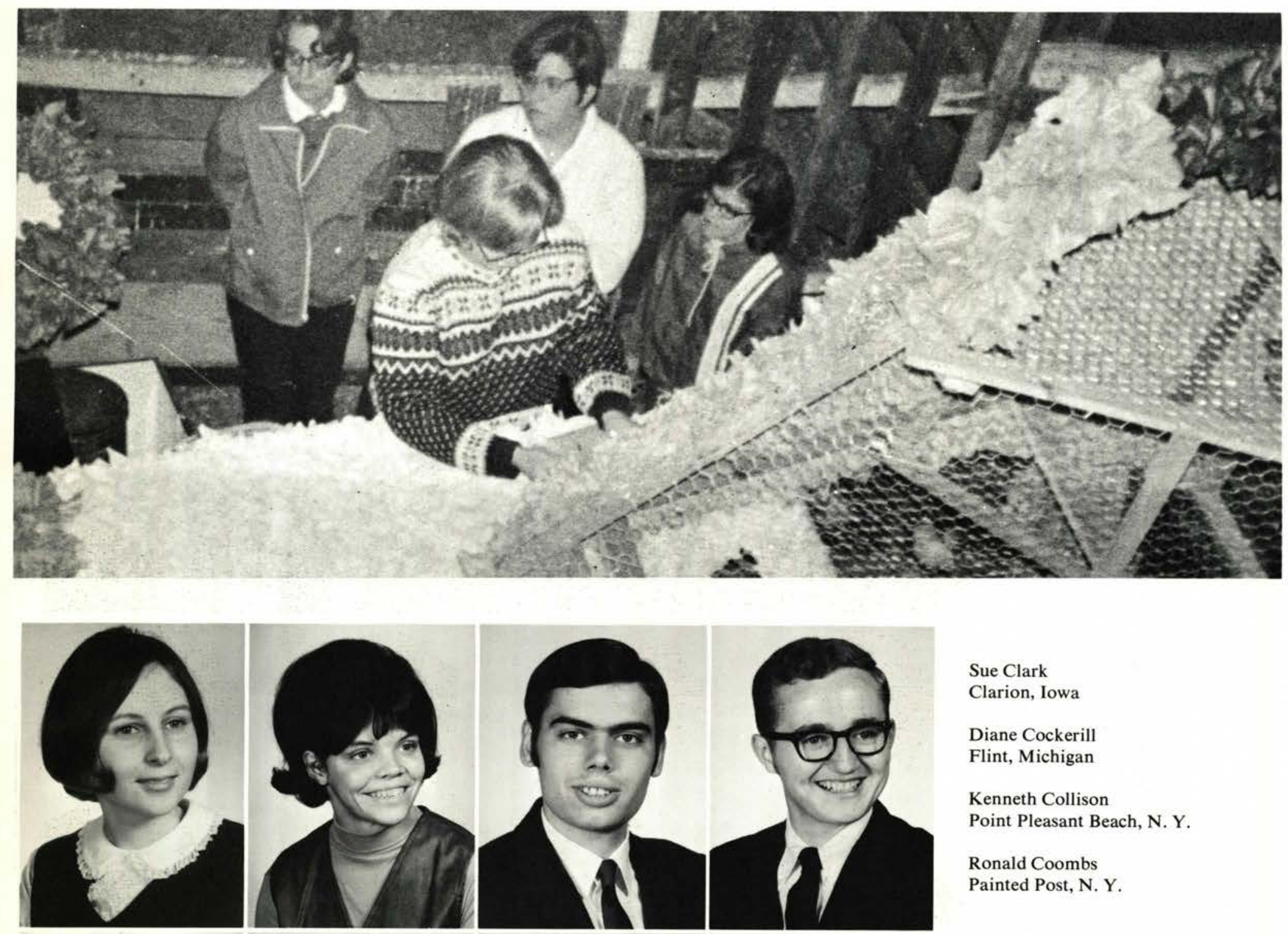

Sue Clark

Clarion, Iowa

Diane Cockerill

Flint, Michigan

Kenneth Collison

Point Pleasant Beach, N. Y.

Ronald Coombs

Painted Post, N. Y.
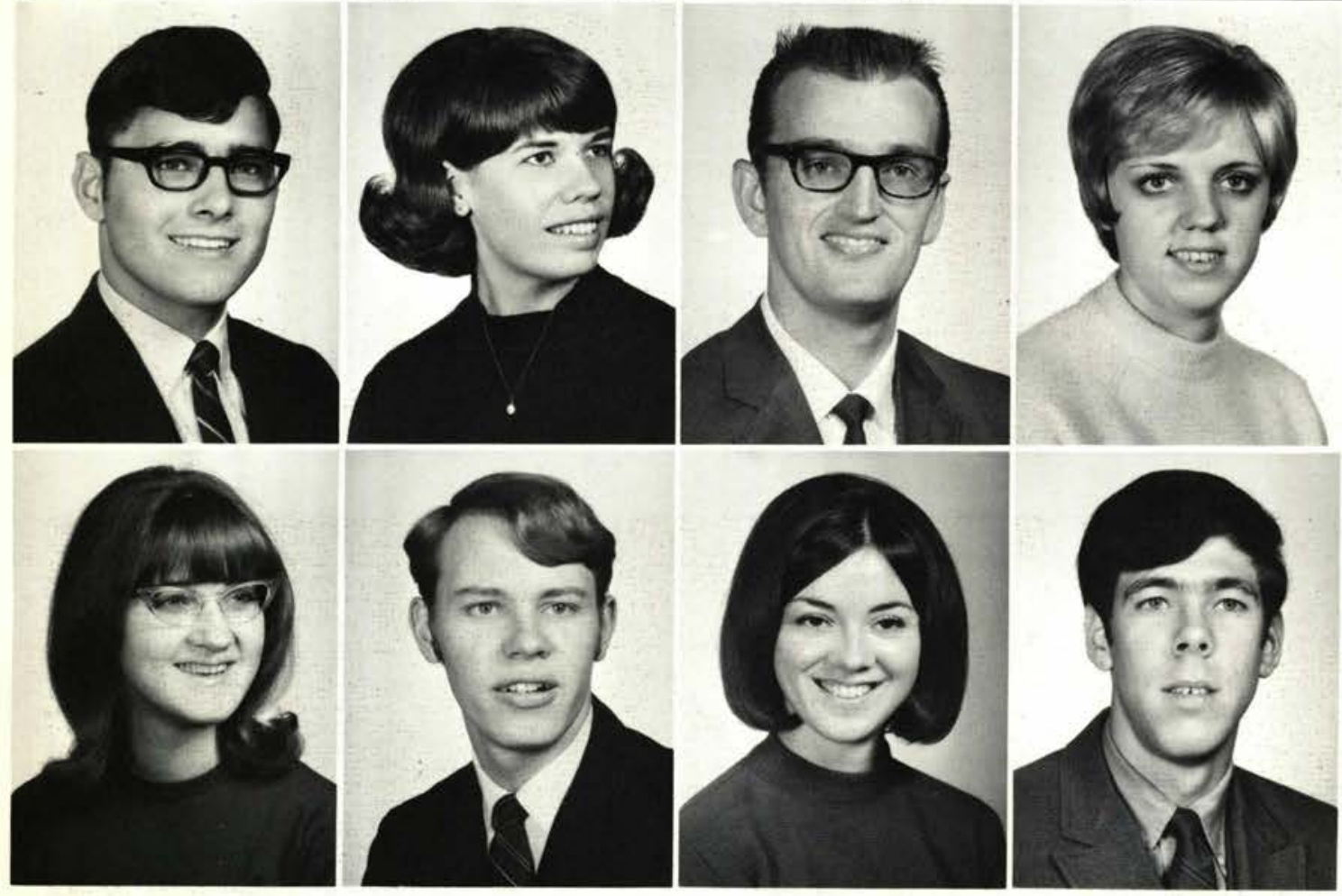

Gary Creviston

Fort Wayne, Ind.

Cathy Getz

Lyle Culberson

Pana, Illinois

Jane Cunningham

Aracanum, Ohio

Lynette Davis

Akran, N. Y.

Gary Dawson

East Moline, Ill.

Rita DeAtley

Minford, Ohio

Ray Decker

Vienna, W. Vir. 
Dennis Dieringer

St. Marys, Ohio

Howard Dilks

Giard, Ohio

Regina Duel

Galeton, $\mathrm{Pa}$.

David Durham

Cleveland, $\mathrm{O}$.
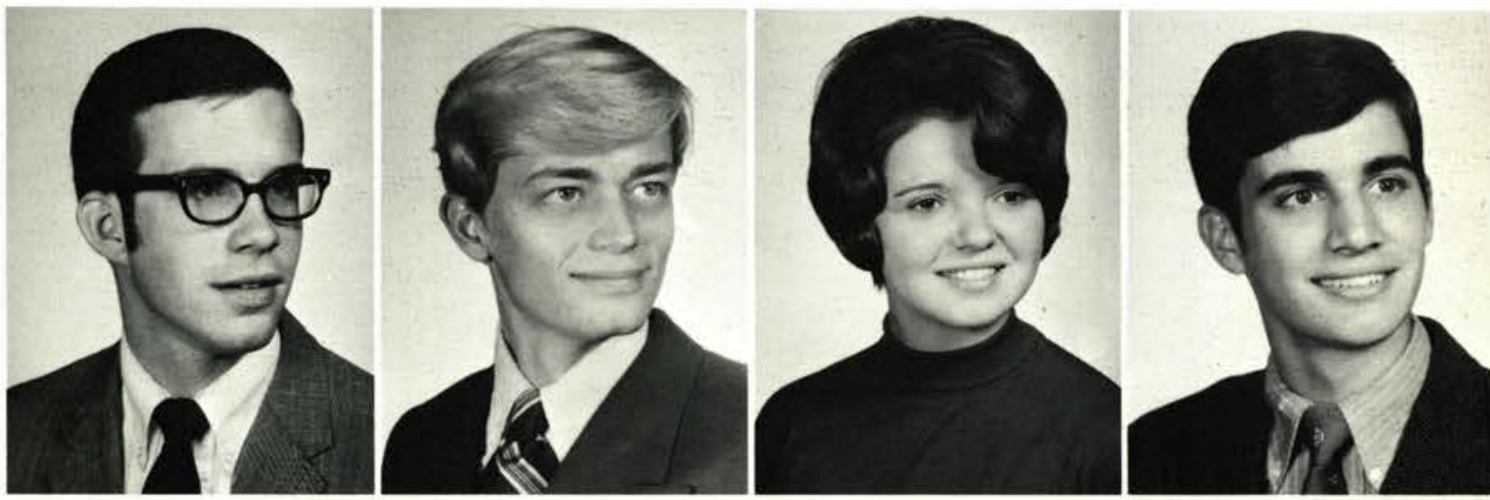

Charles Dutton

Lapeer, Mich.

Carol Engle

Hamburg, N. Y

Janet Epps

Davenport, Iowa

Kenneth Evans

Plymouth, Mich.

Jean Eveland

Tunkhannock, $\mathrm{Pa}$.

Robert Farrar

Portsmouth, $\mathrm{O}$.

Mark Flint

Warren, Ohio

Mark Frields

Huntingsburg, Ind.
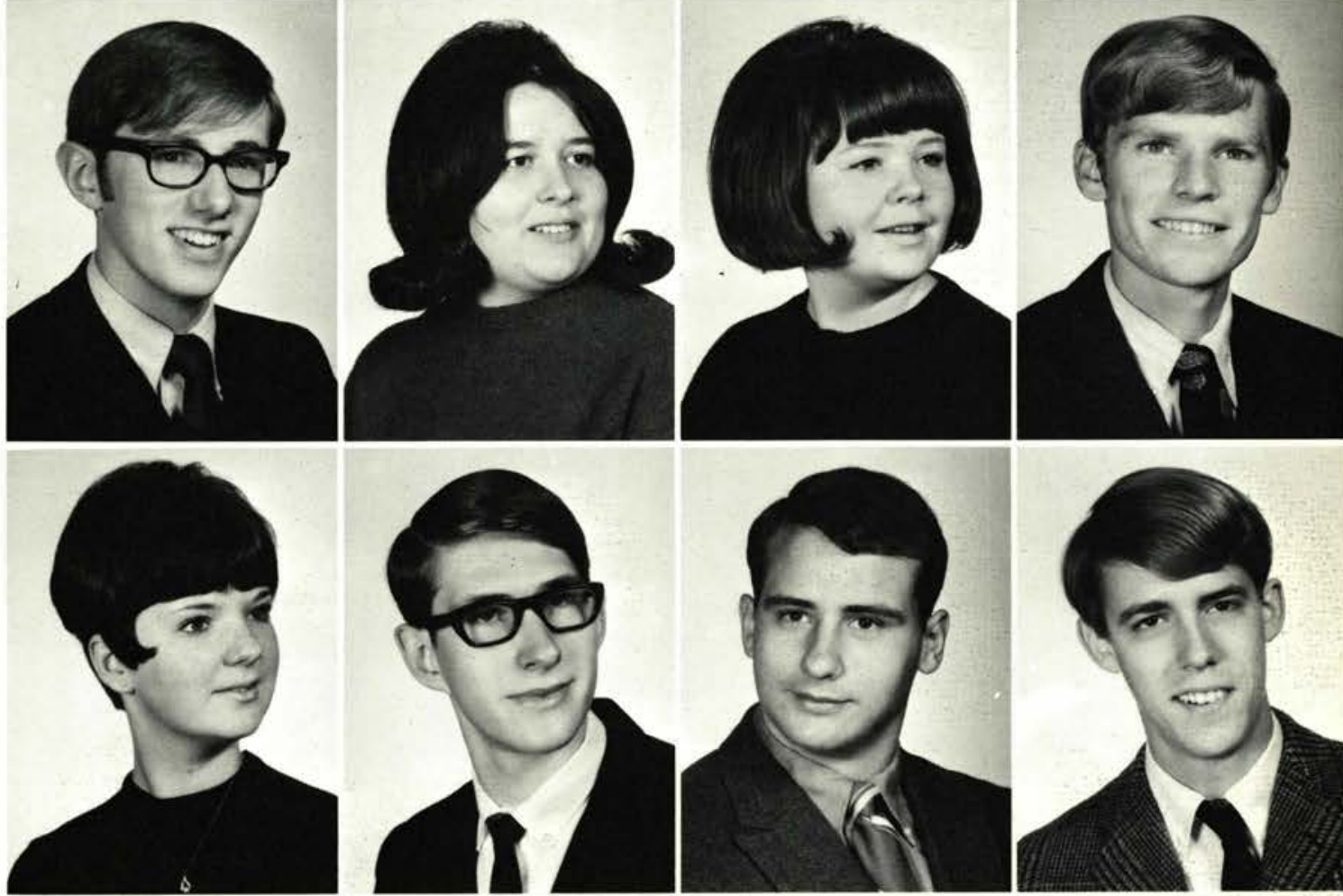

Jon Frye

Xenia, O.

Janet Gabriel

Chicago, Ill

Lonnie Gerber

Berlin, $\mathrm{O}$.

Richard Goldschmitt Pittsburgh, Pa.
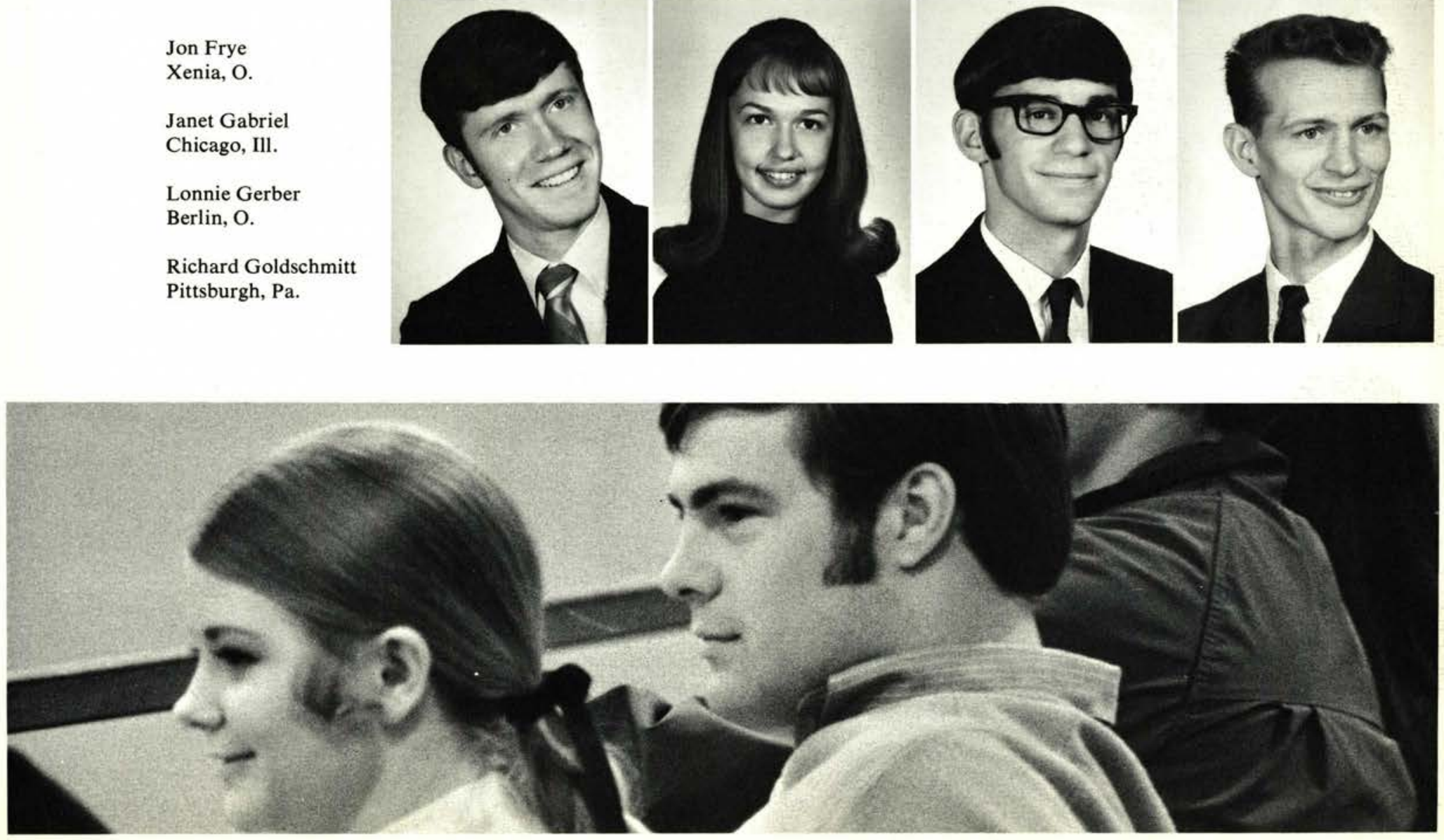


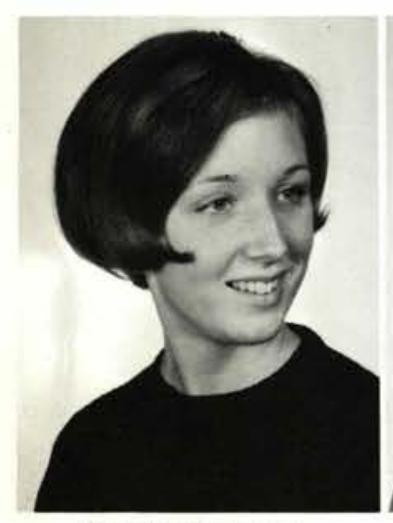

Sandra Gowman Detroit, Mich.

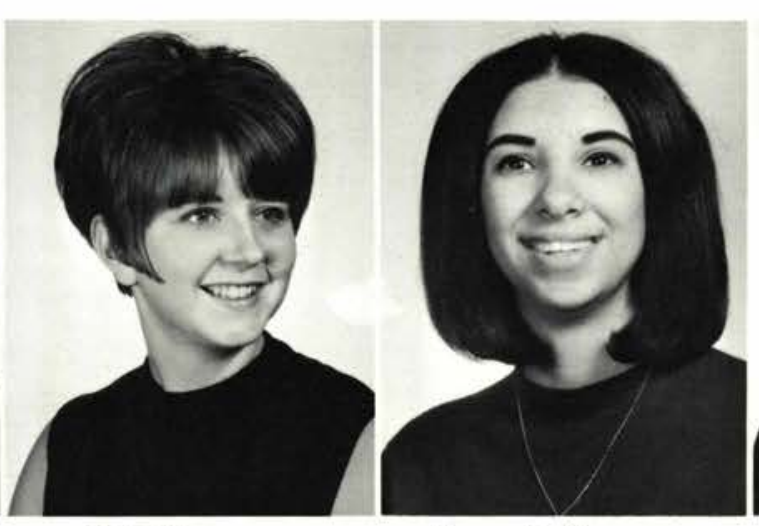

Judy Grover Whitehall, Wis.

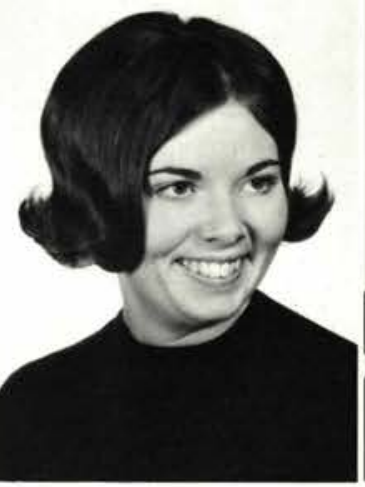

Joan Hamilton Wilmington, Del.

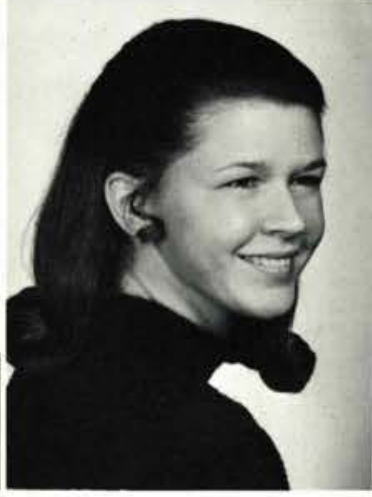

Barbara Healey Brunswick, Ohio

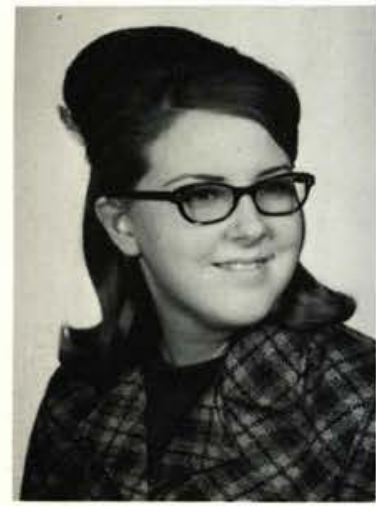

Linda Hirschy

Koumra, Rep. of Chad

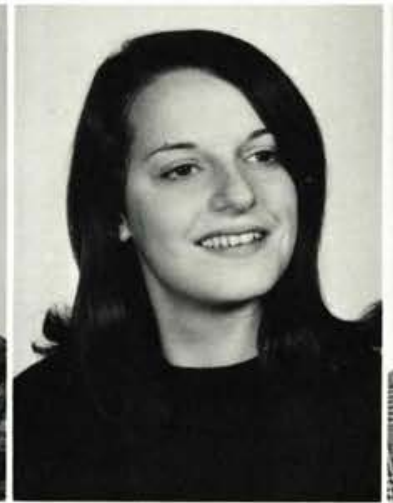

Julie Hoerath

Westerville, $\mathrm{O}$.

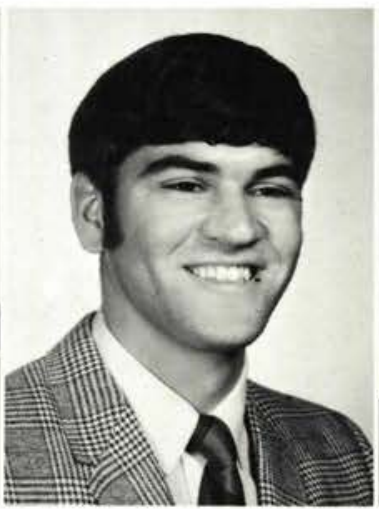

Richard Inghram

Morning Sun, Iowa

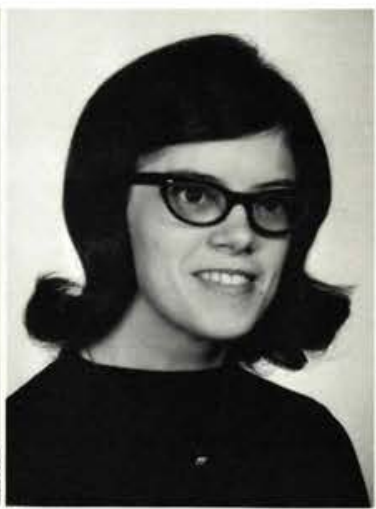

Norma Irwin

Tidioute, $\mathrm{Pa}$.

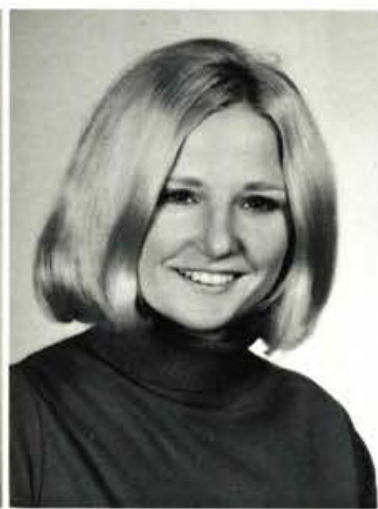

Patti Jo Irwin

Swartz Creek, Mich.

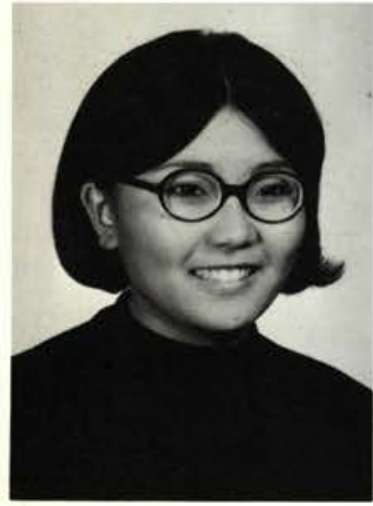

Geraldine Ishwin Seabrook, N. Y.

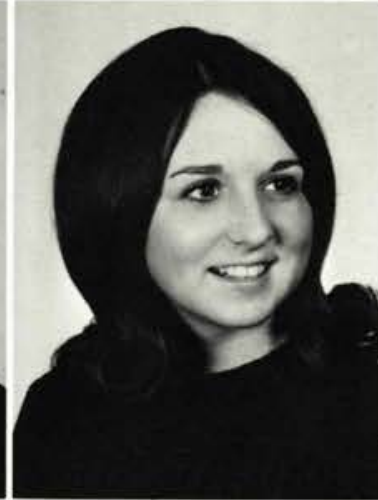

Patti Jenkin Cleveland, $\mathrm{O}$.

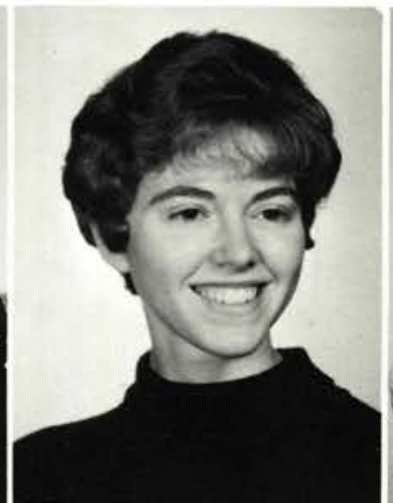

Faye Johnson Eastlake, O.

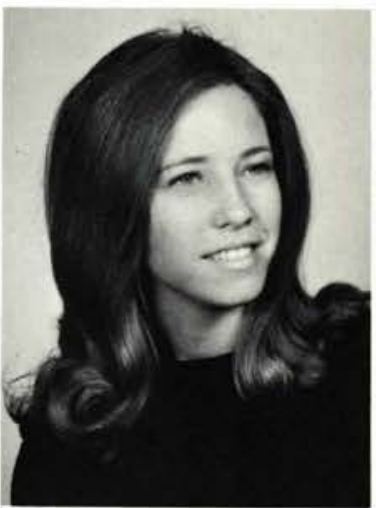

Sheila Jones

Concord, Calif.

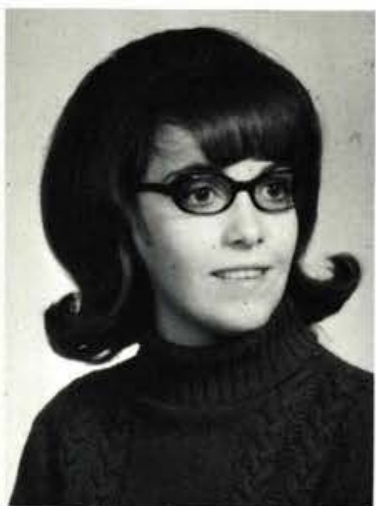

Verna Jones

Kingsley, $\mathrm{Pa}$.
Jean King

Olmstead Falls, $\mathrm{O}$.

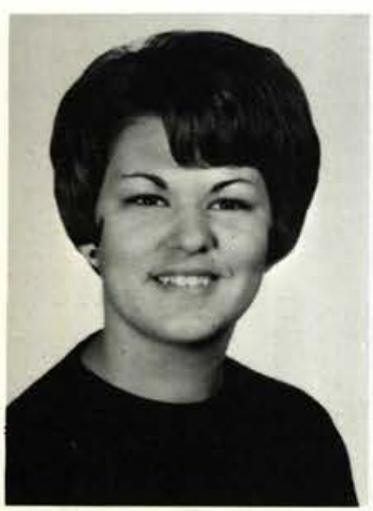

Florence Krikke

Greenwich, O.

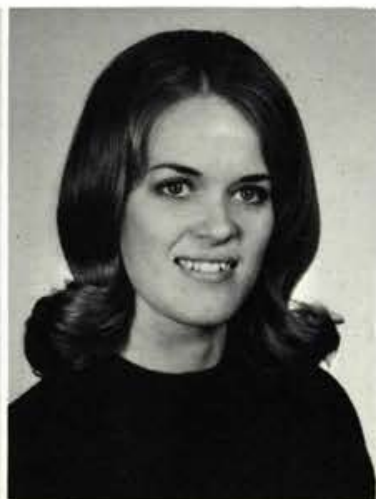

Annie Kusumoto

Seabrook, N. Y

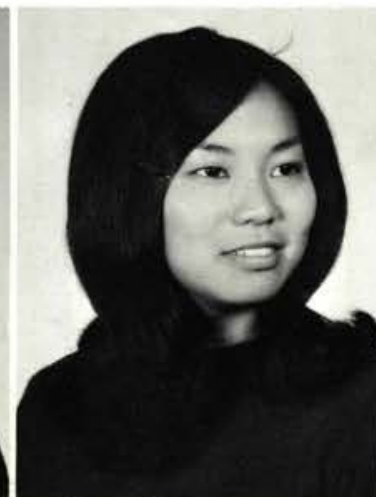

Paul Large

Iquitos, Peru

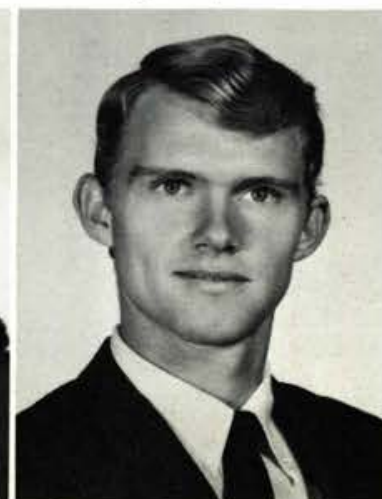

Judy Leach

East North Port, N. Y.

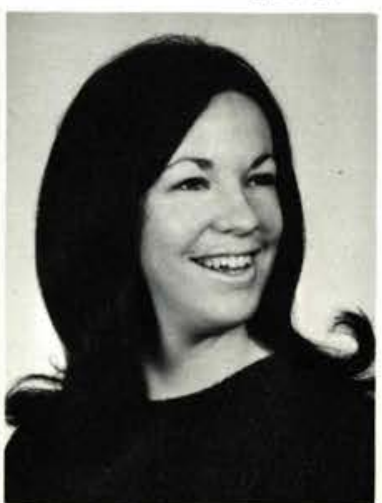




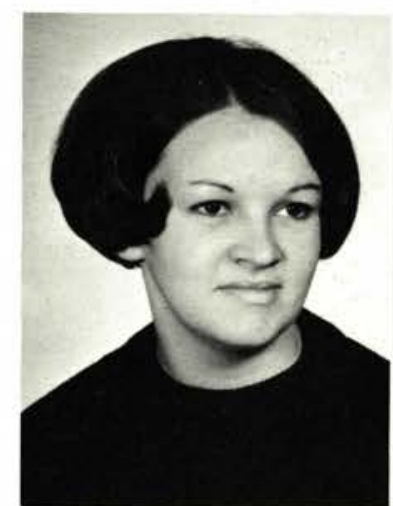

Patricia Lear

Derry, Pa.

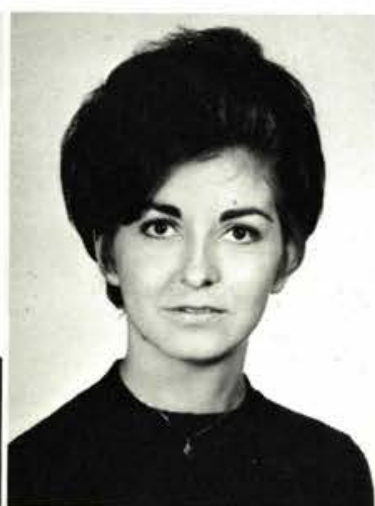

Kathy Leeser

West Palm Beach, Fla.

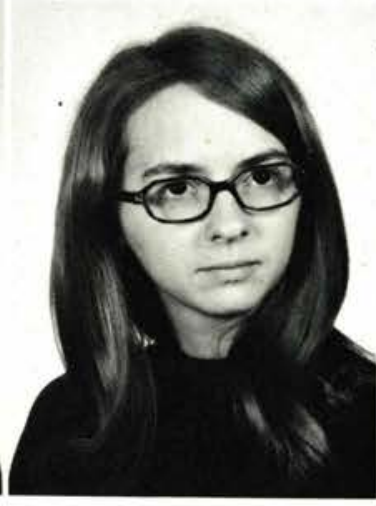

Darlene Lewis Maine, N. Y.

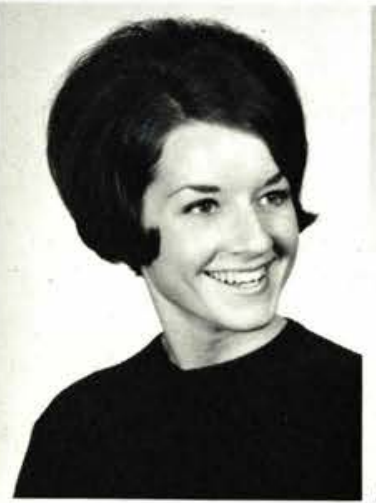

Judy Lewis

Kokomo, Ind

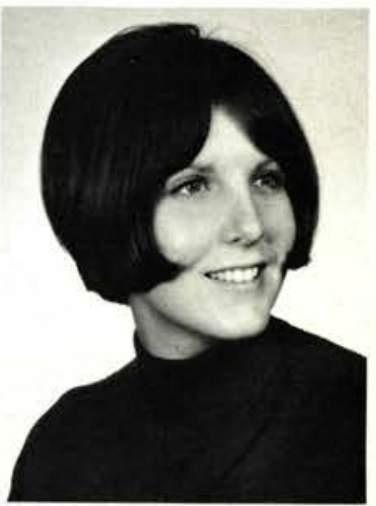

Barbara Lewright Eagle Grove, Iowa

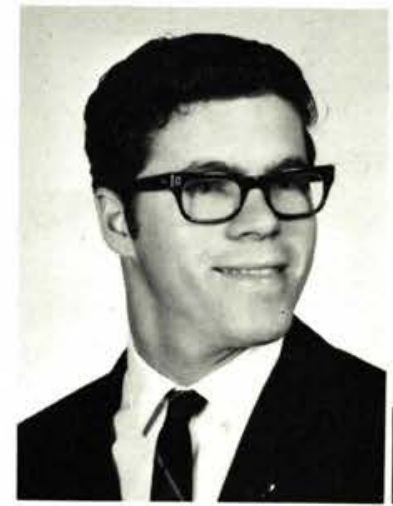

Donald Long

Worthington, $\mathrm{Pa}$.

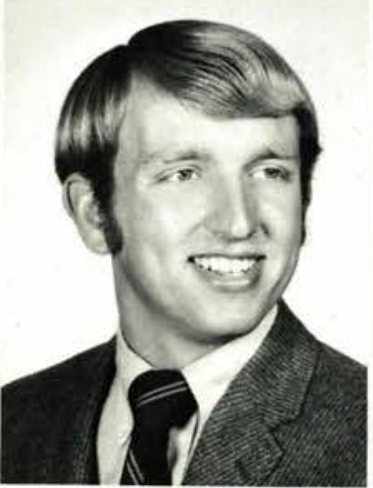

Robert Lunney

Port Huron, Mich.

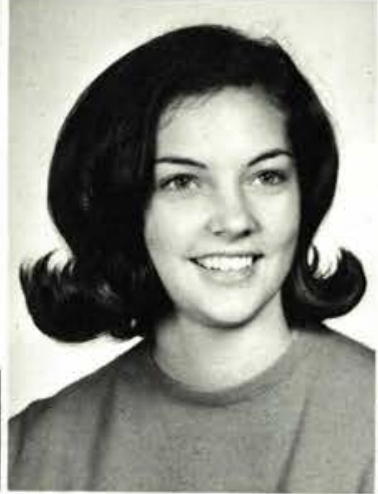

Linda Madsen Hamburg, N. Y.

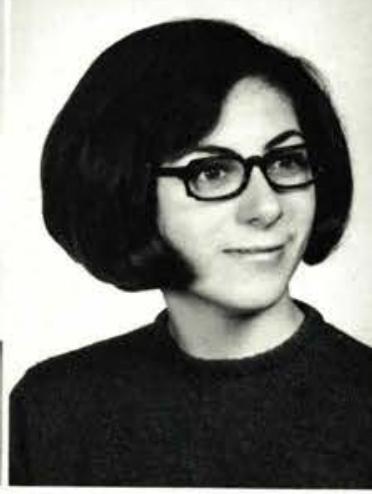

Janet Malmstrom Silvis, Illinois

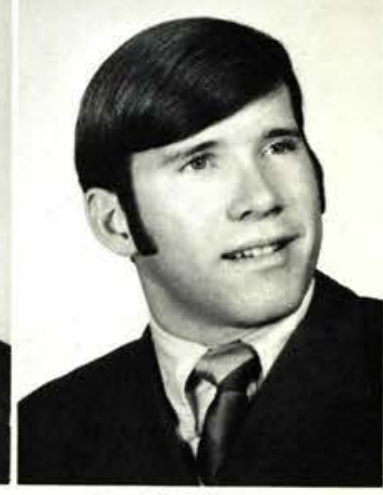

Daniel Manross Akron, $\mathrm{O}$.

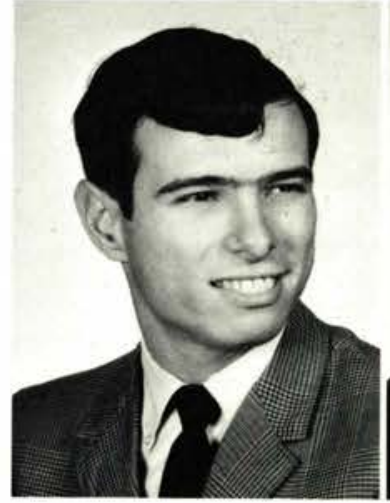

Dee Mays

Chillicothe, $\mathrm{O}$.

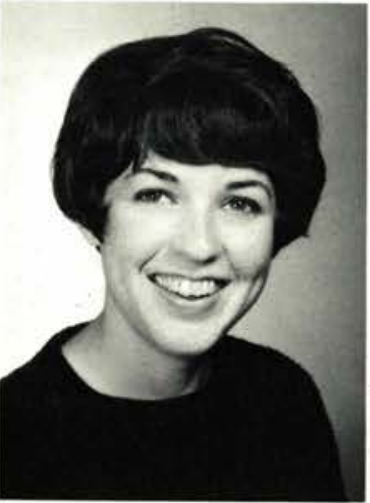

Tanis McDaniel

Mancelona, Mich.

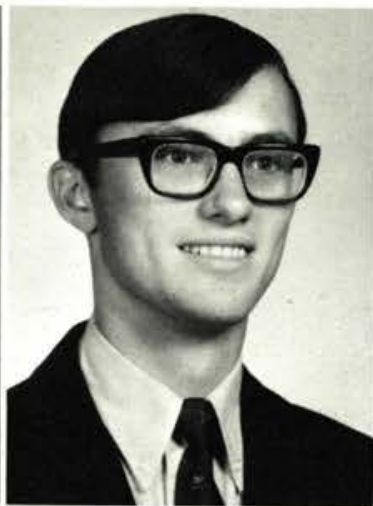

Samuel McMillen

Xenia, Ohio

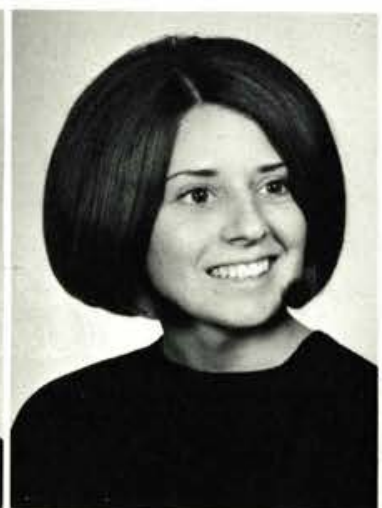

Melinda McNiece

Valparaiso, Ind.

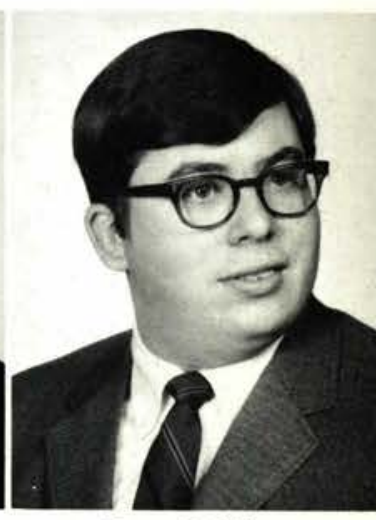

Darryl Miller Xenia, Ohio
Joan Miller Allegan, Mich.

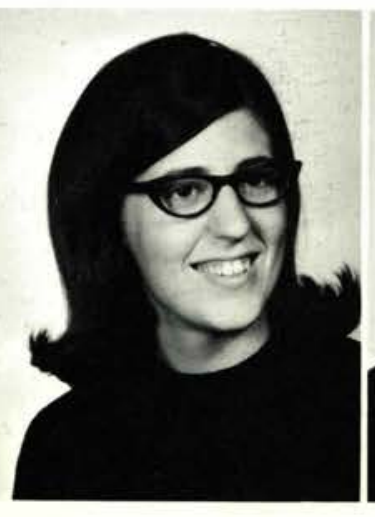

Beverly Mix Meadville, $\mathrm{Pa}$.

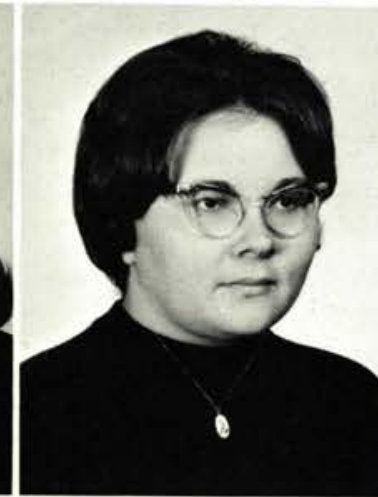

Beverly Moore Twinsburg, $\mathrm{O}$.

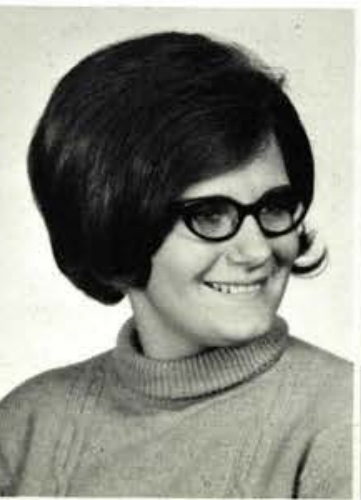

Coletta Morrow

Kenton, O.

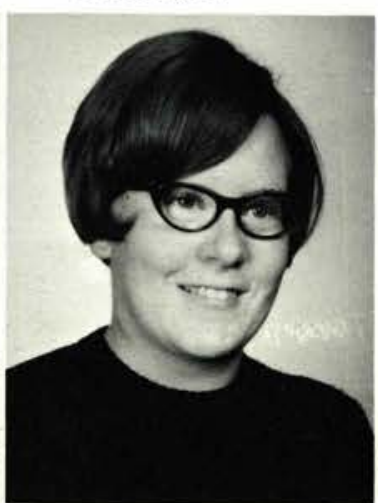

Shirleen Morrow Amherst, Ohio 


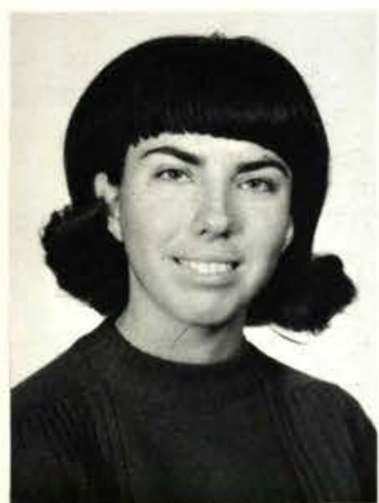

Carole Morse

Flint, Mich.

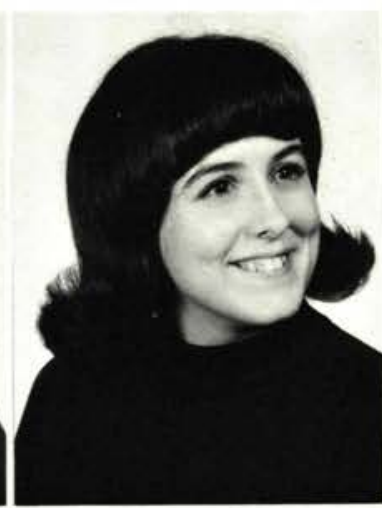

Joyce Motts

Northfield, O.

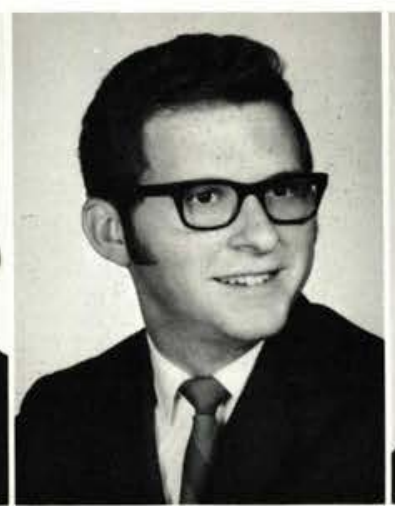

Steve Moulder

Sharpsville, Ind.

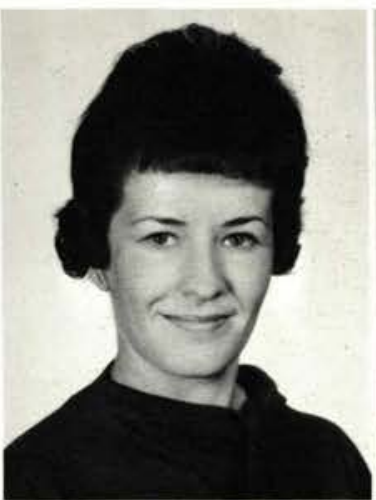

Phyllis Murphy Cedarville, Ohio

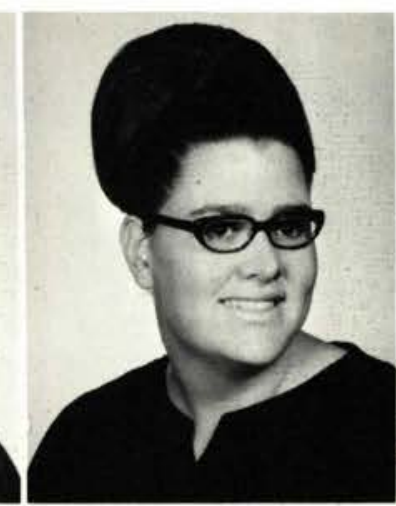

Edith Musser

Marshallville, Ohio
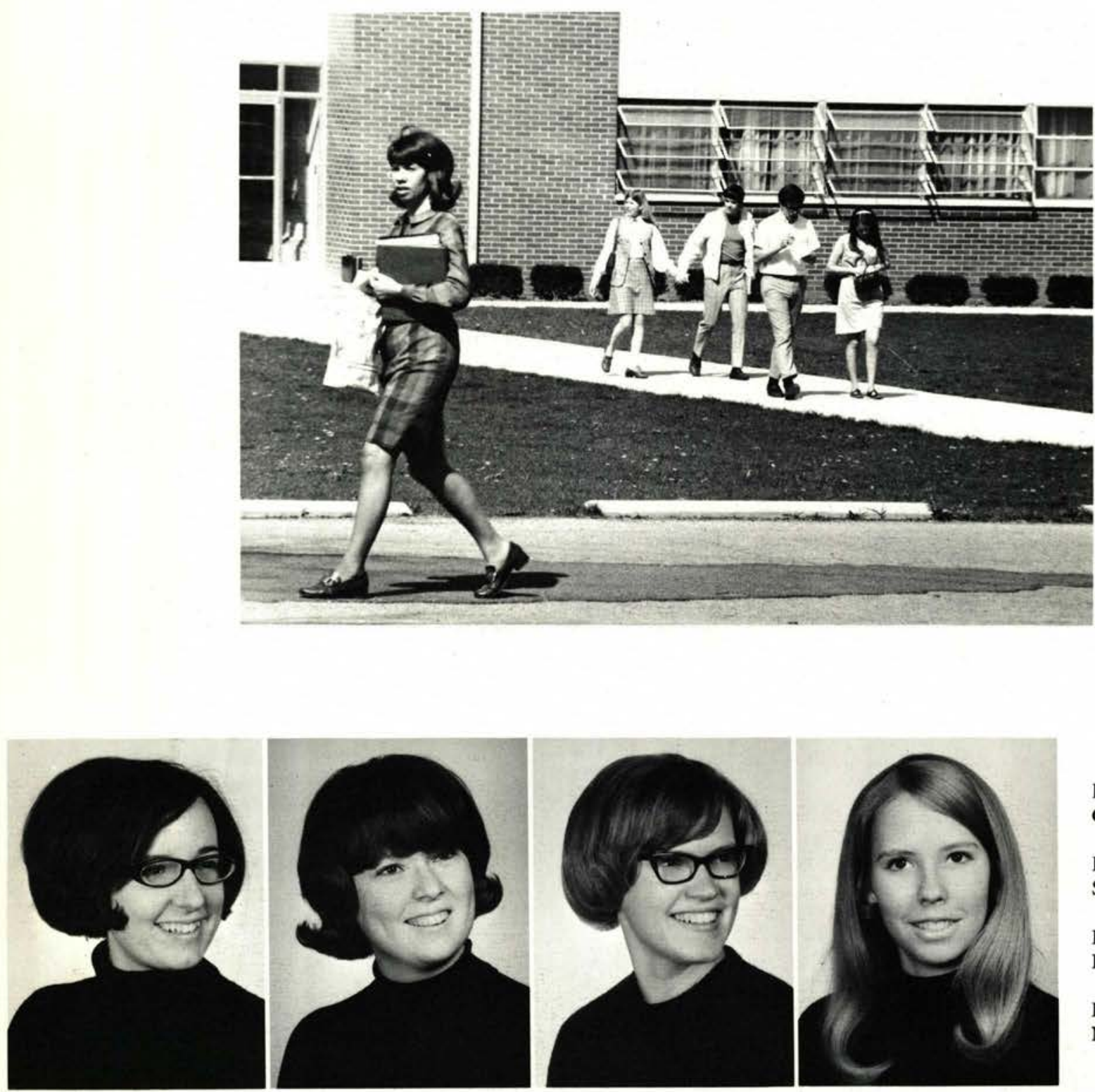

Dawn Myers

Chicago, Ill.

Brenda Nelson

Shawnee, Kan.

Linda A. Nelson

Frazee, Minn.

Linda D. Nelson

Nalaybalay, Bukidnon, P.I.
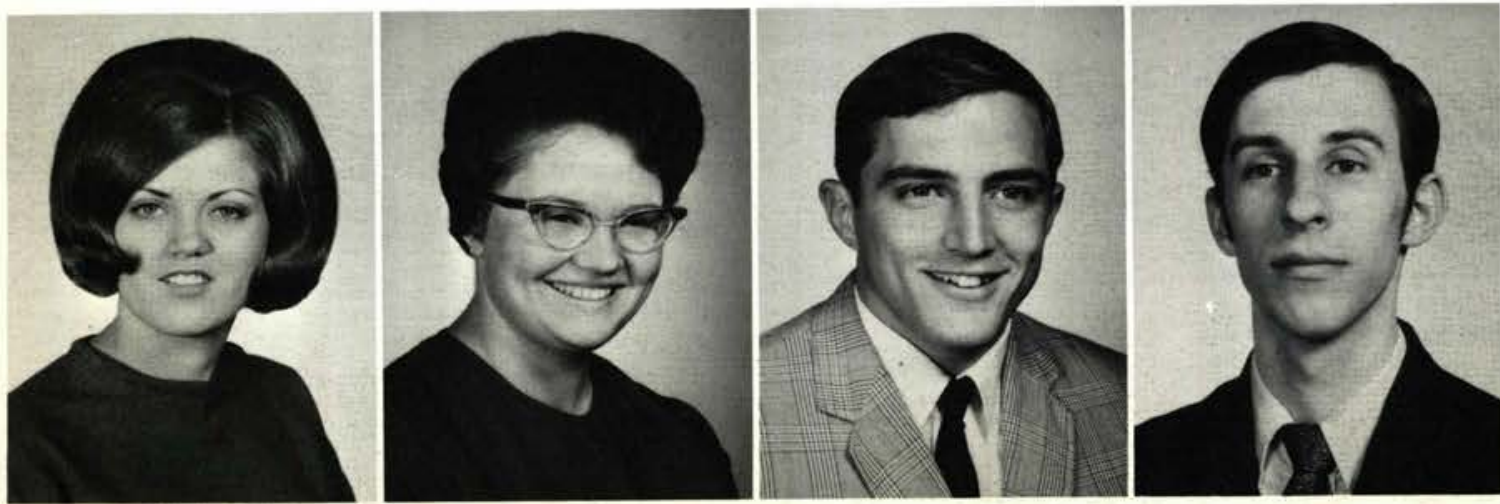

Karen Nelson

St. Louis, Mo.

Kathy Newton

Columbus, Ohio

Tim Northey

Medina, Ohio

Dan Norton

Columbus, Ohio 


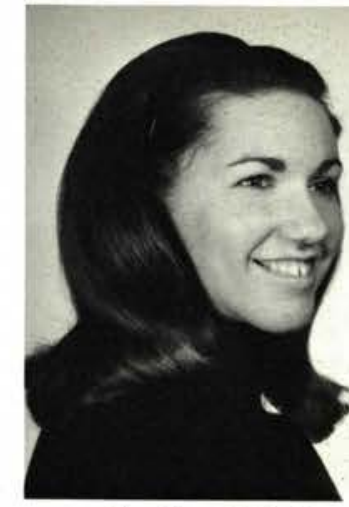

Carolyn O'Keefe Wesleyville, $\mathrm{Pa}$.

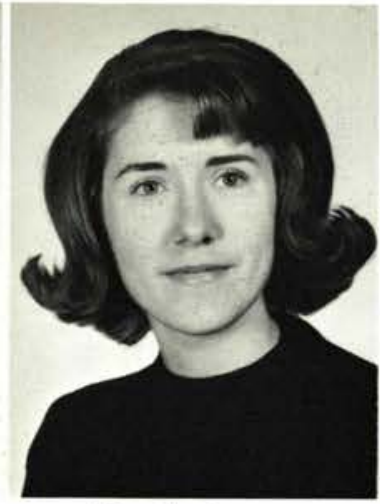

Marsha Osman Jamestown, Ohio

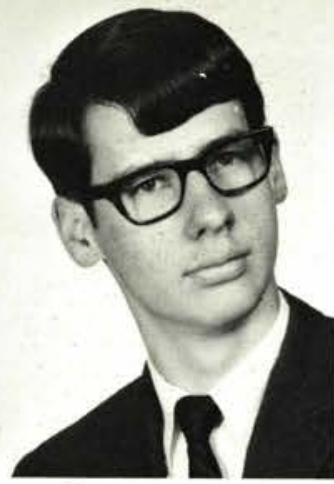

George Parcher Pittsburgh, $\mathrm{Pa}$.

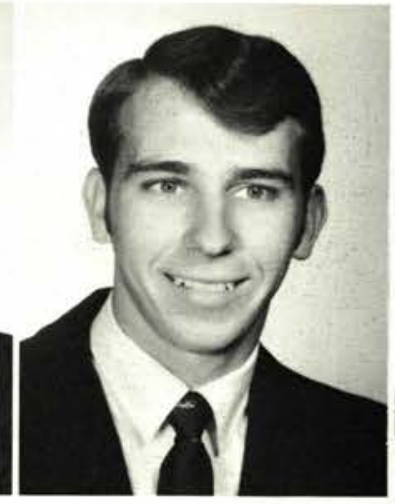

Randy Patten Caldwell, Ohio

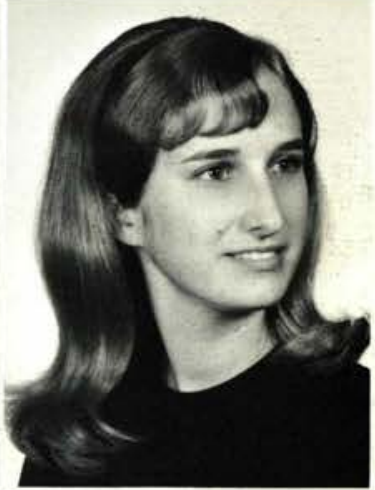

Nancy Paugh

Oakland, Maryland

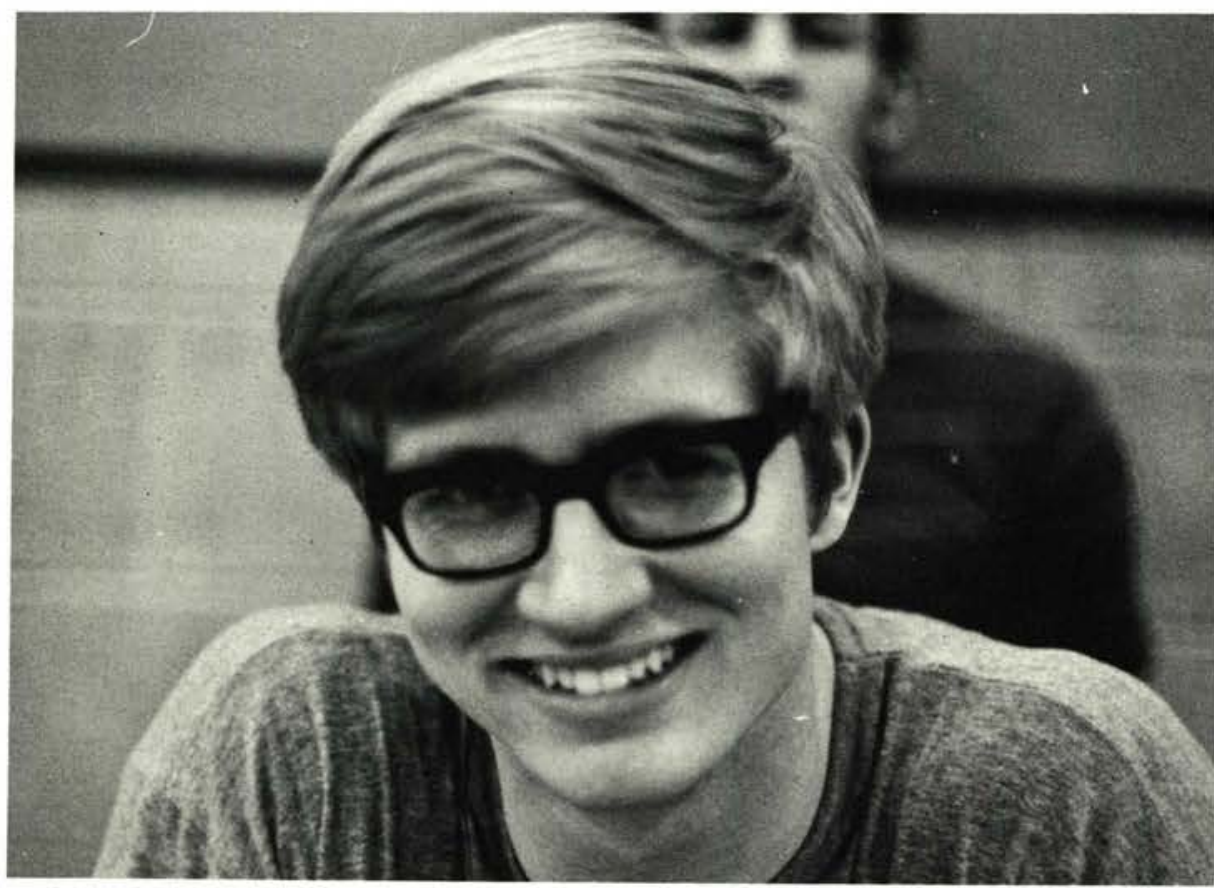

James Pollard

Washington Court House, $\mathrm{O}$.

Peggy Porterfield

Austin, Minnesota

Robert Powley

Lake Huron, Michigan

Sandra Prather

Kettering, Ohio
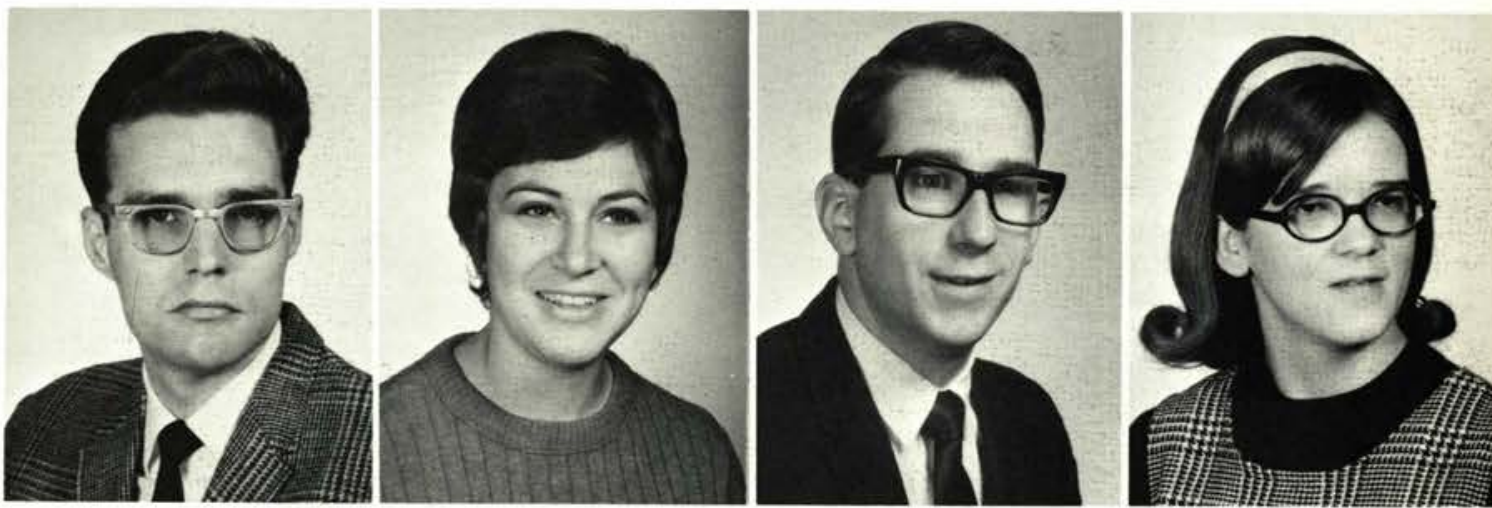

Judy Price

Haddon Heights, N. J.

James Rickard

Cedarville, Ohio

Craig Roberts

Des Moines, Iowa

Dane Rohm

Audubon, N. J.
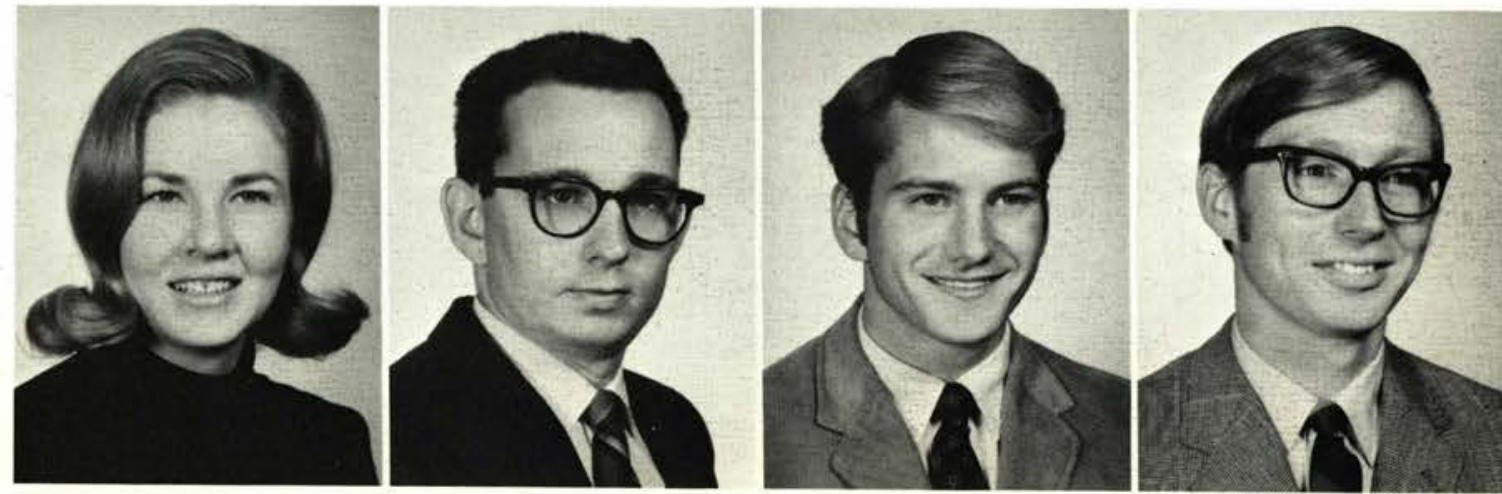


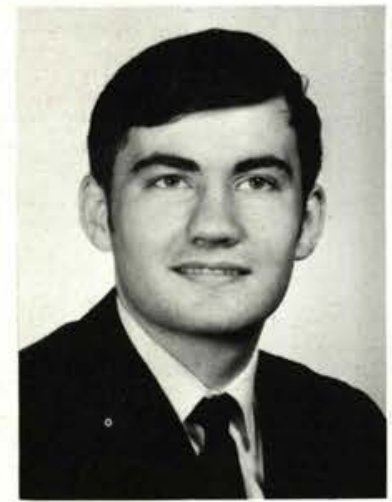

Charles Rowe

De Ruyter, N. Y.

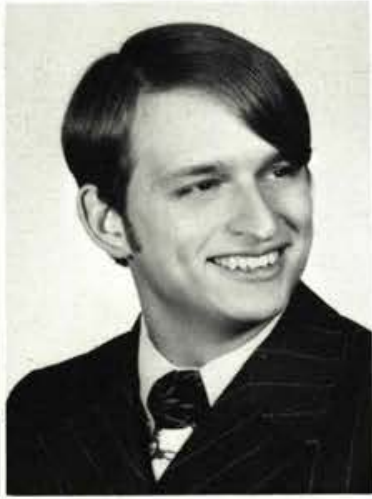

Dale Schilling

Novelty, Ohio

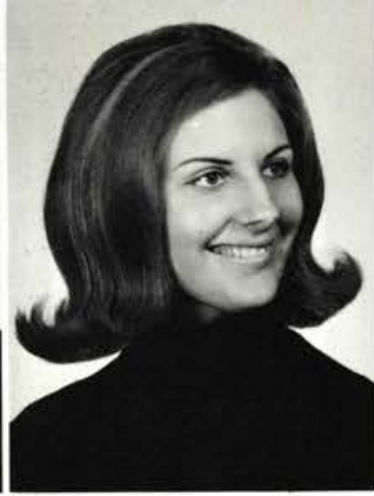

Marikay Schneider Hammond, Indiana

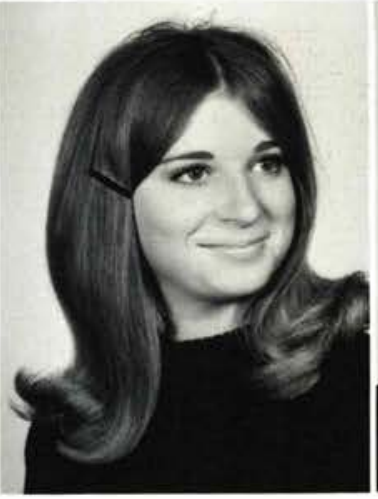

Norma Schwarm Barberton, Ohio

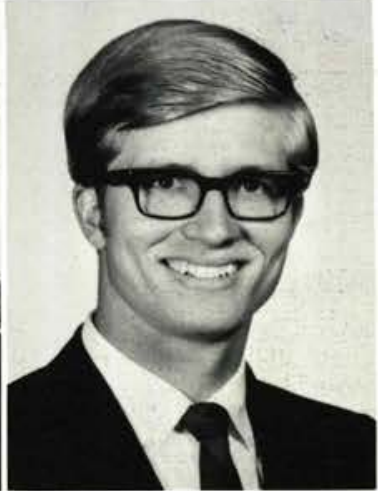

Jeffrey Seeley Oberlin, Ohio

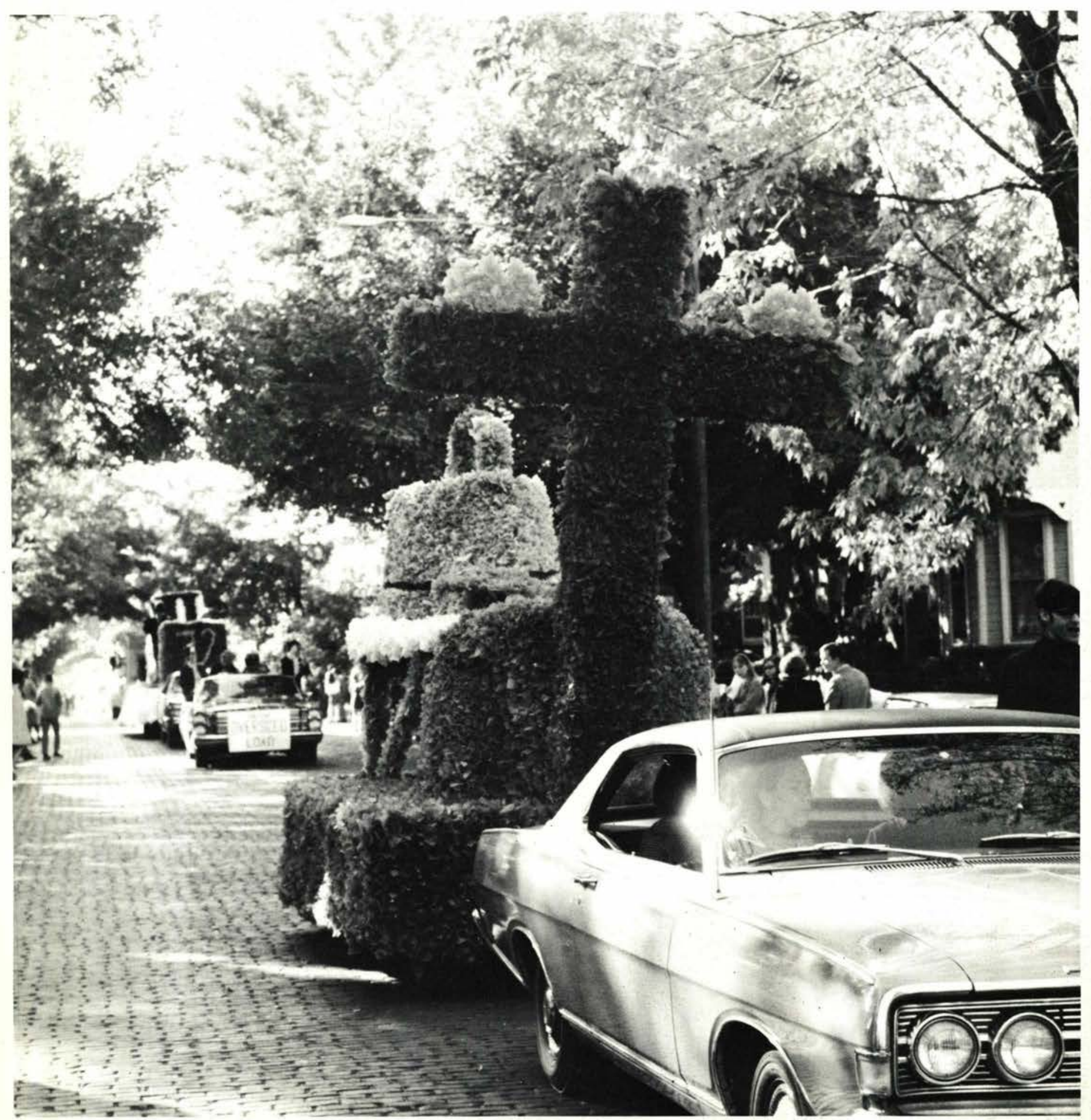




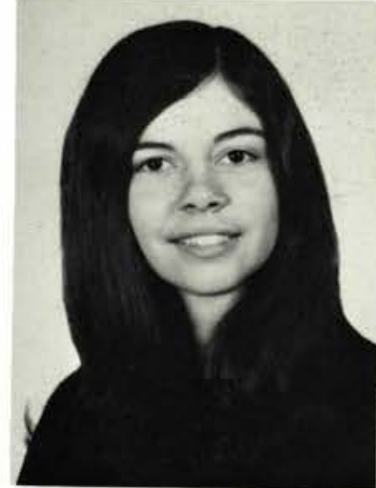

Cheryl Shafer

Hagerstown, Indiana

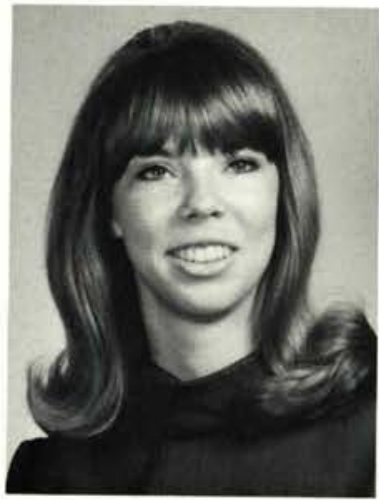

Renee Silverthorn

Centerline, Mich.

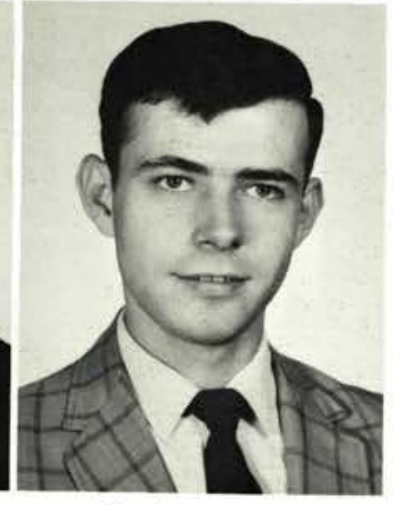

David Shreve

Meadville, $\mathrm{Pa}$.

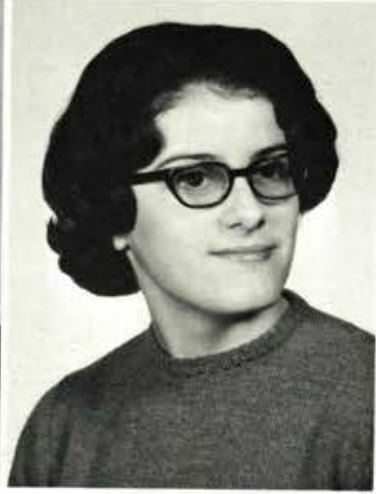

Linda Slates

St. Louis, Mo.

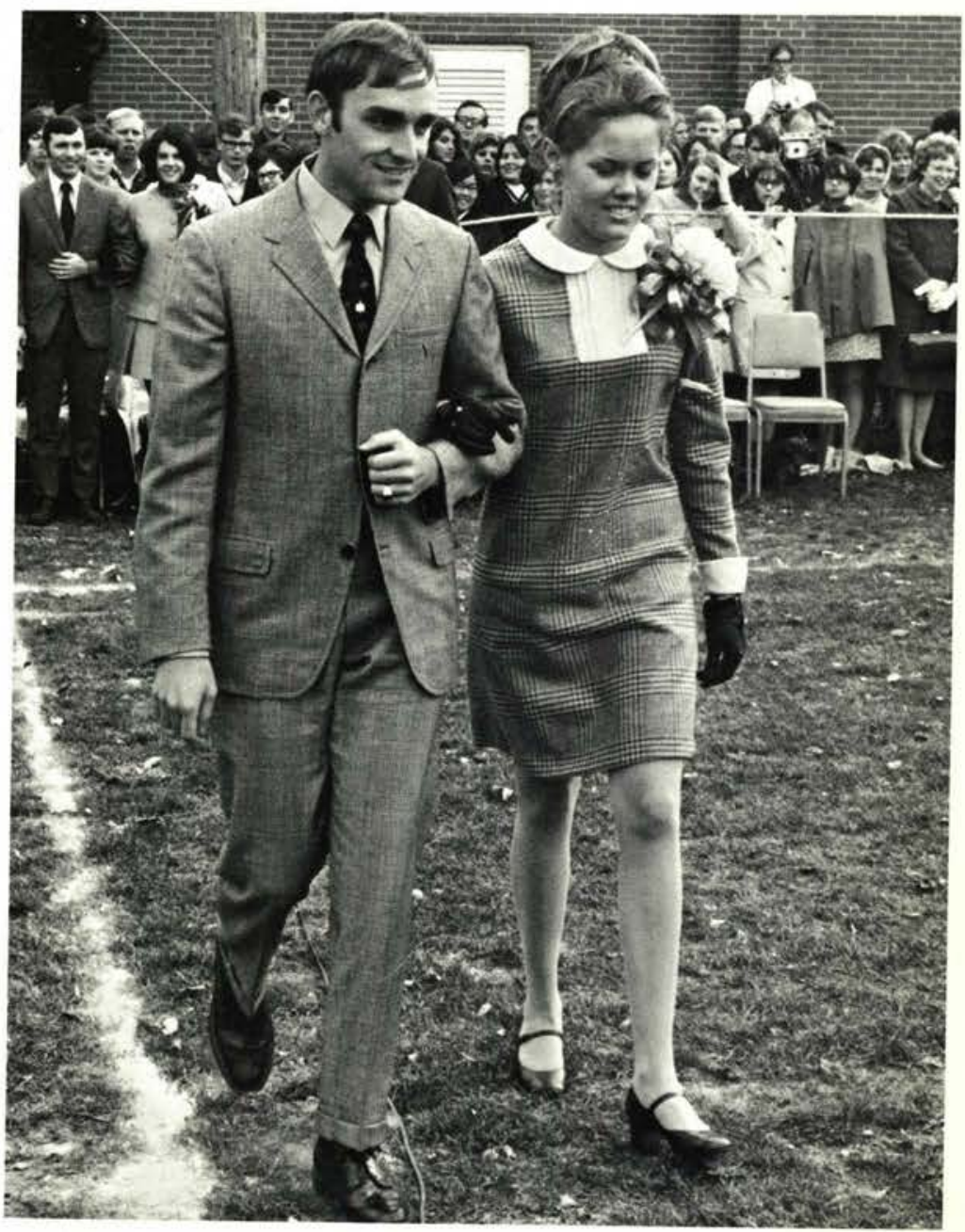

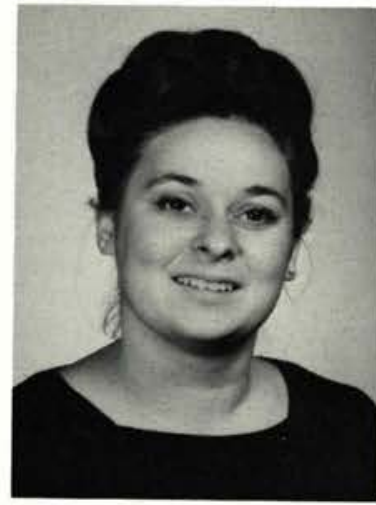

Bonnie Smith

Indianapolis, Ind.

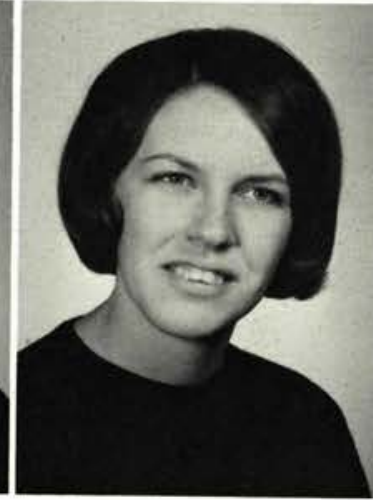

Nancy Snider

Barberton, $\mathrm{O}$.

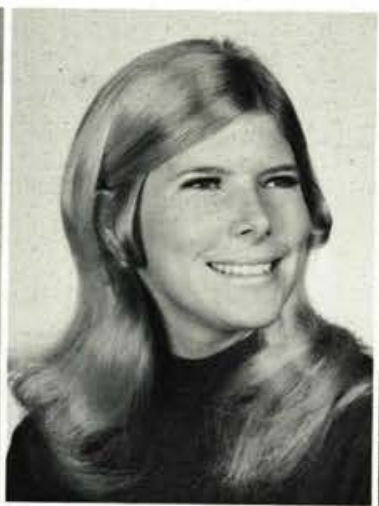

Joyce Sommerfeld San Diego, Calif.

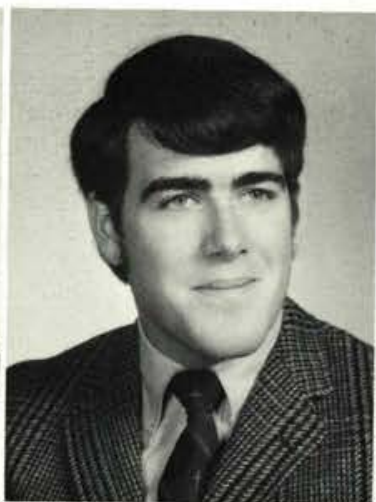

Jim Spaulding

Indianapolis, Ind.

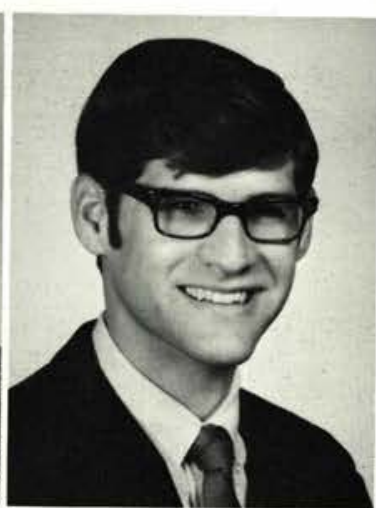

Ronald Spieth

East Gary, Ind.
Hannah Stowell

Hackensack, N. J.

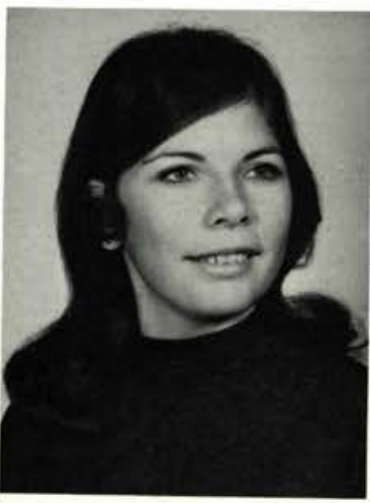

Gary Stutzman

Berlin, Ohio

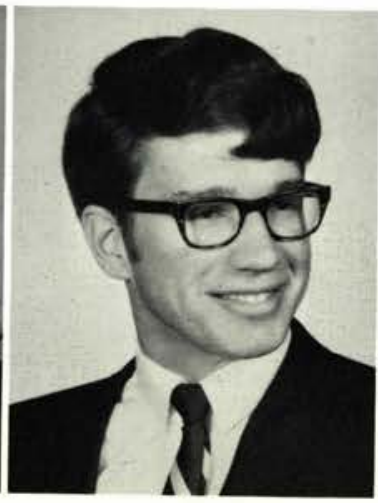
Creston, Iowa
Verna Tallmon

Mercy Tennant Bay City, Michigan

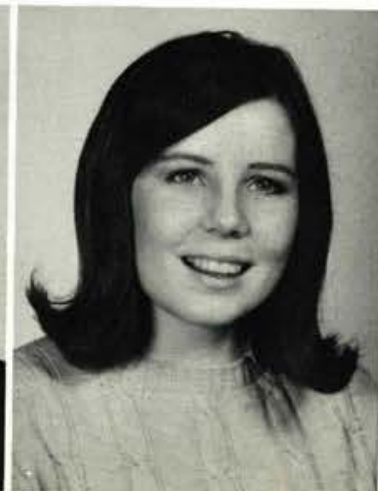

Billie Thomas

Columbus, Ohio

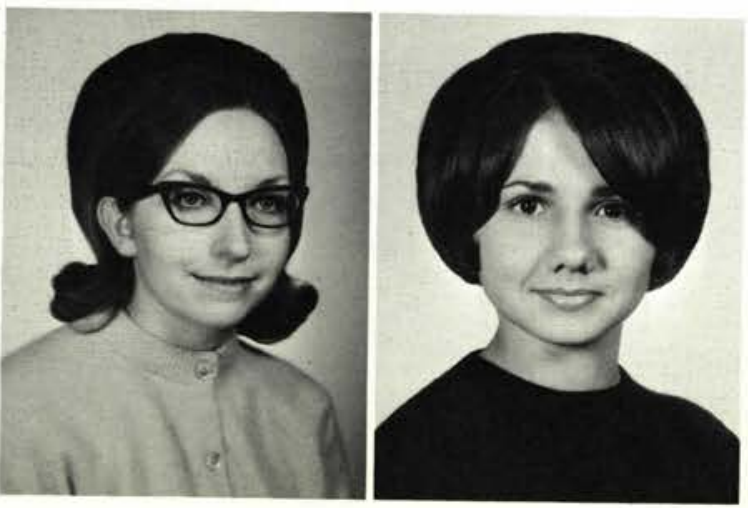




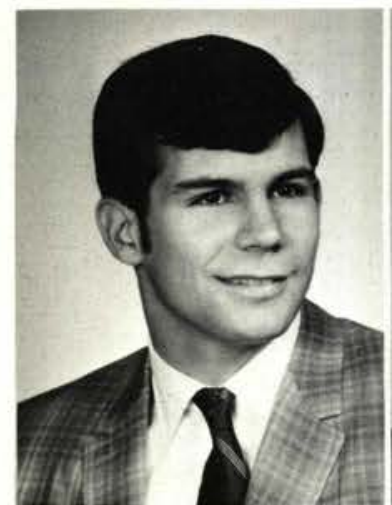

William Trefzger

Cincinnati, Ohio

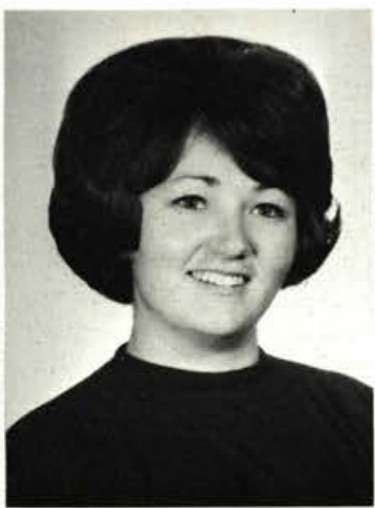

Sharon Trotter

Liverpool, Ohio

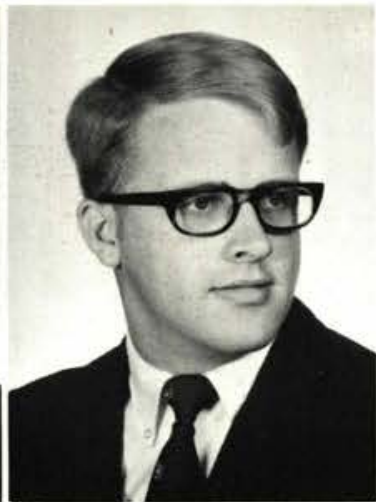

Dave Turner

Chillicothe, $\mathrm{O}$.

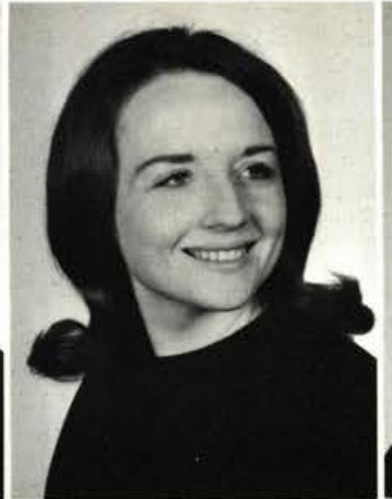

Pat Waggoner

Coatesville, Ind

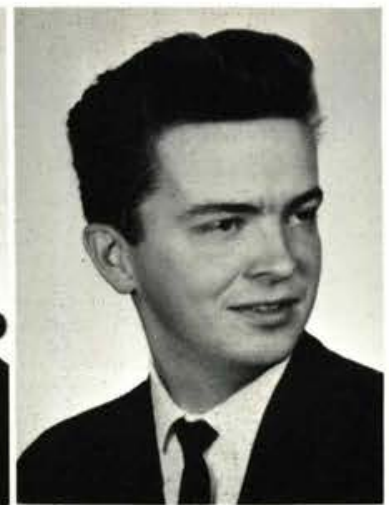

James Ward

London, Ohio

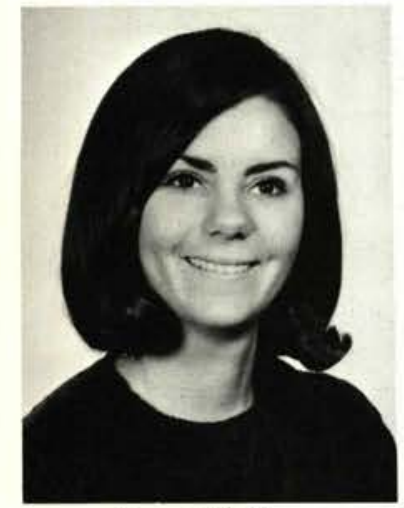

Susan Webb

Kokomo, Ind.

Pat Whitaker

Columbus, $\mathrm{O}$.

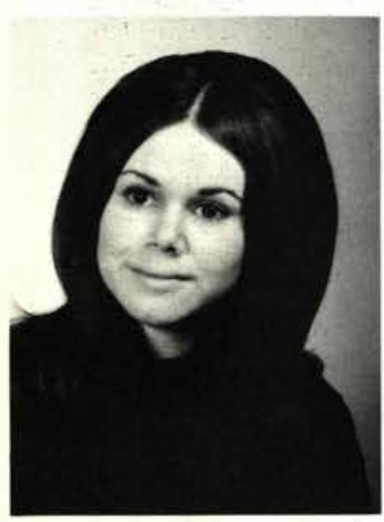

Herb Wilson

Dennsville, N. J.

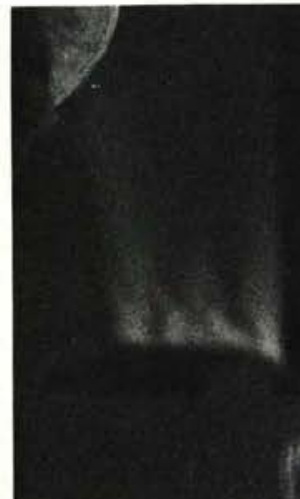

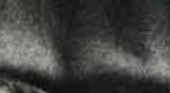

N. $5 x \rightarrow$
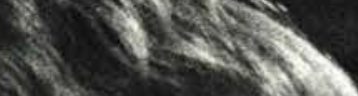

Ain.

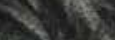

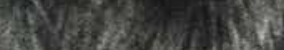

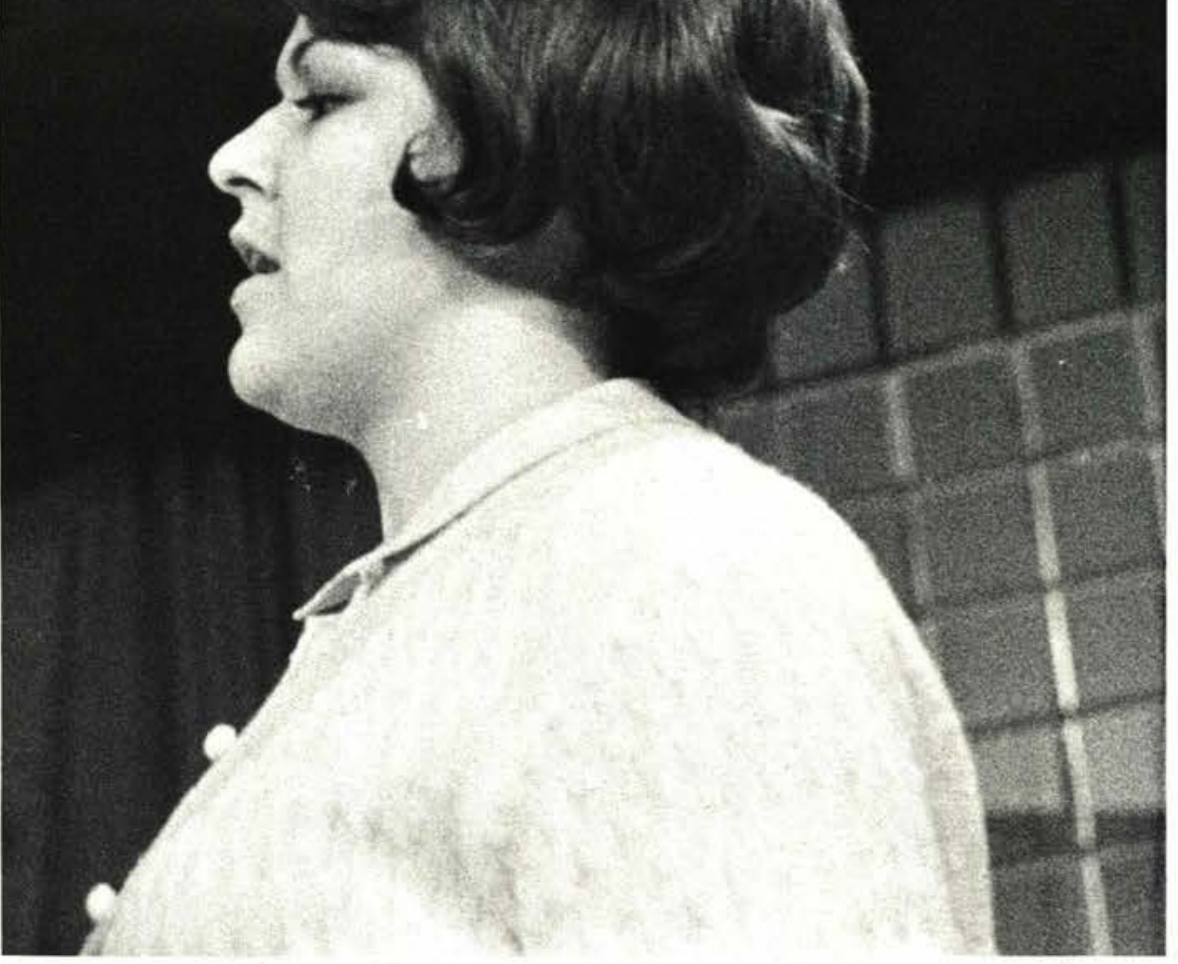

anet Yardlay

Troy, Ohio
Wendy Work Gettysburg, $\mathrm{Pa}$.
Darlyne Wuest Billings, Mont.

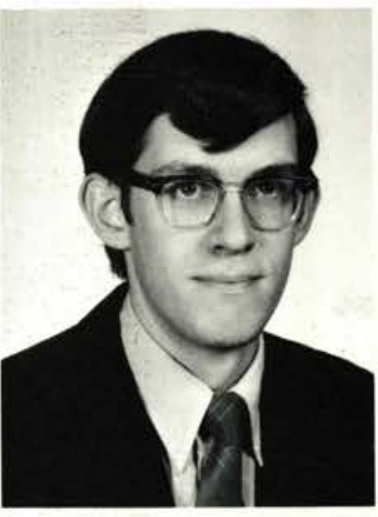

Kenneth Weddle Prosperity, $\mathrm{Pa}$.
Mark Wilhite

Corwith, Iowa

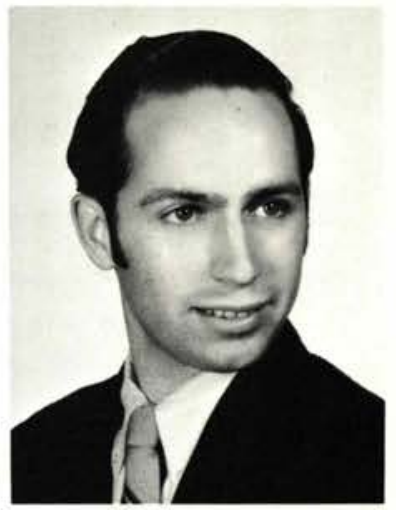

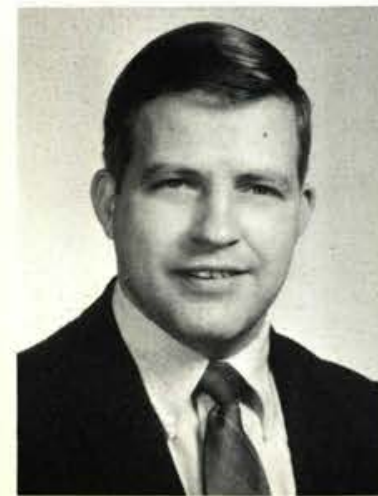

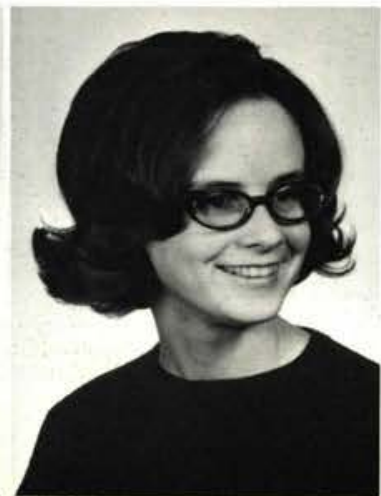

Cathy Zwiesler

West Alexandria, $O$.
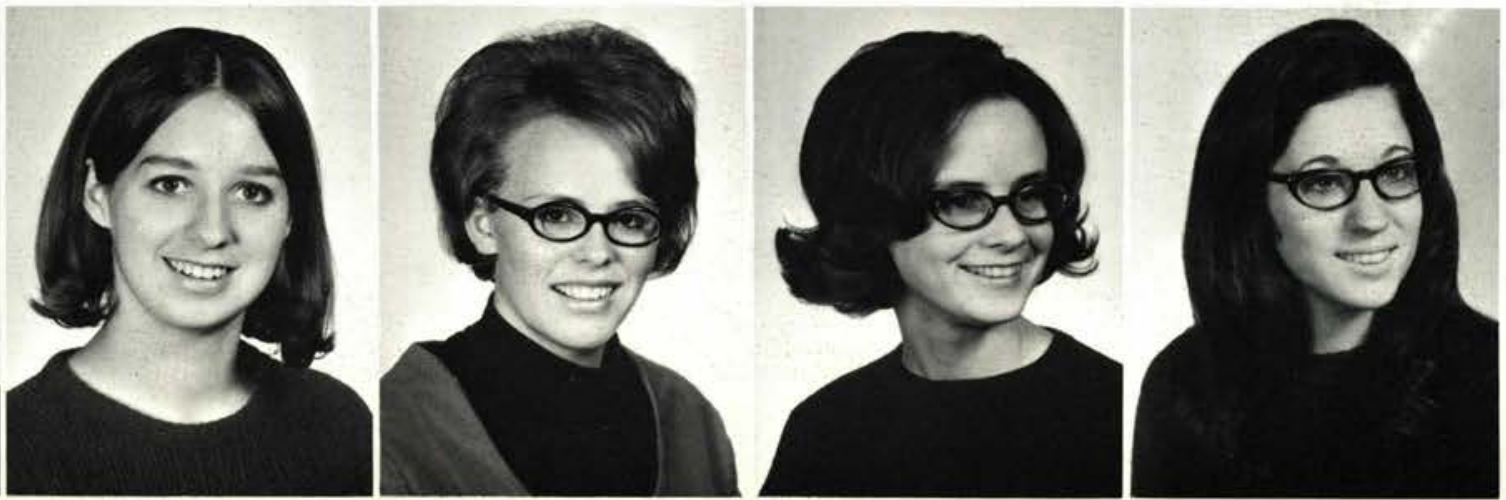

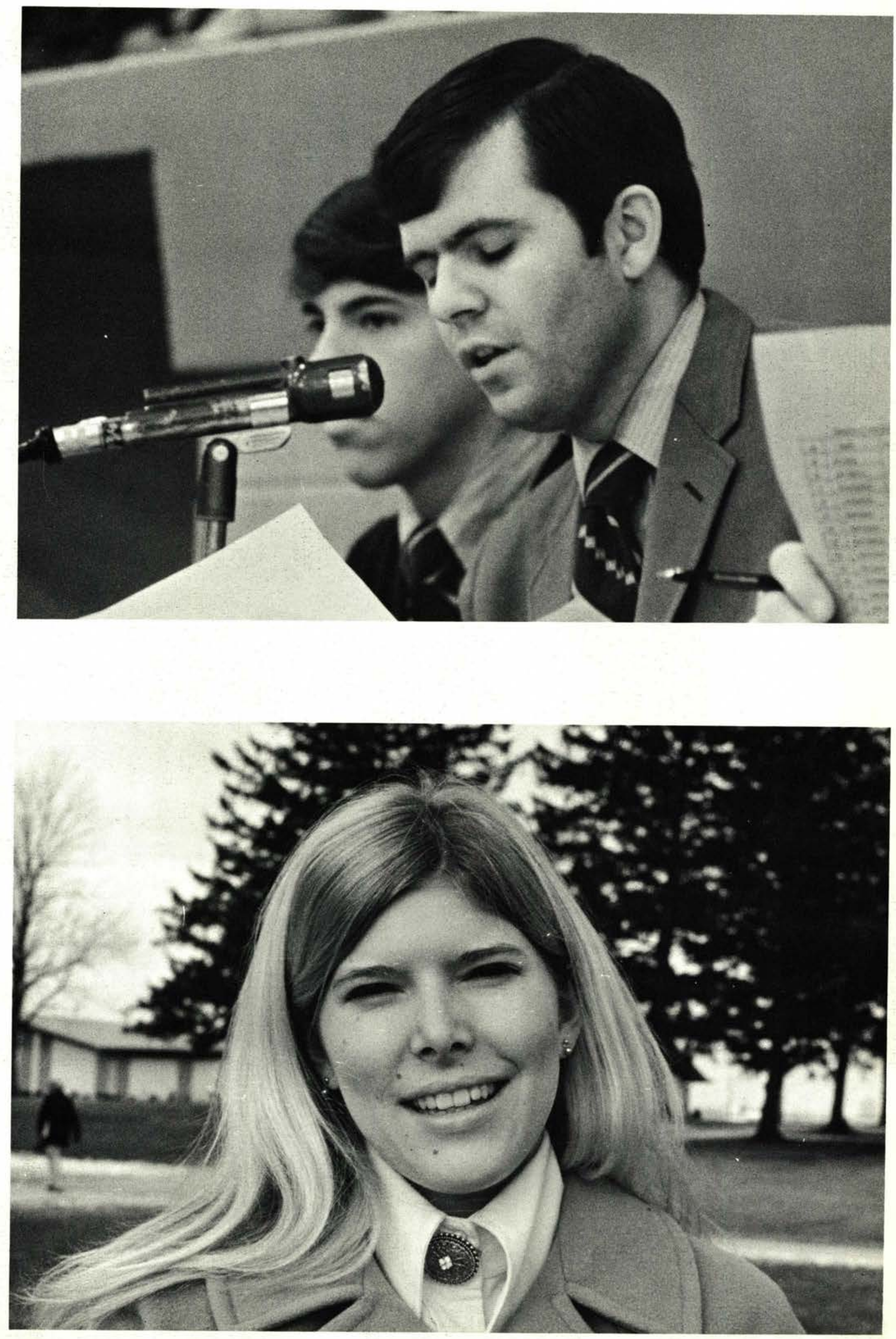


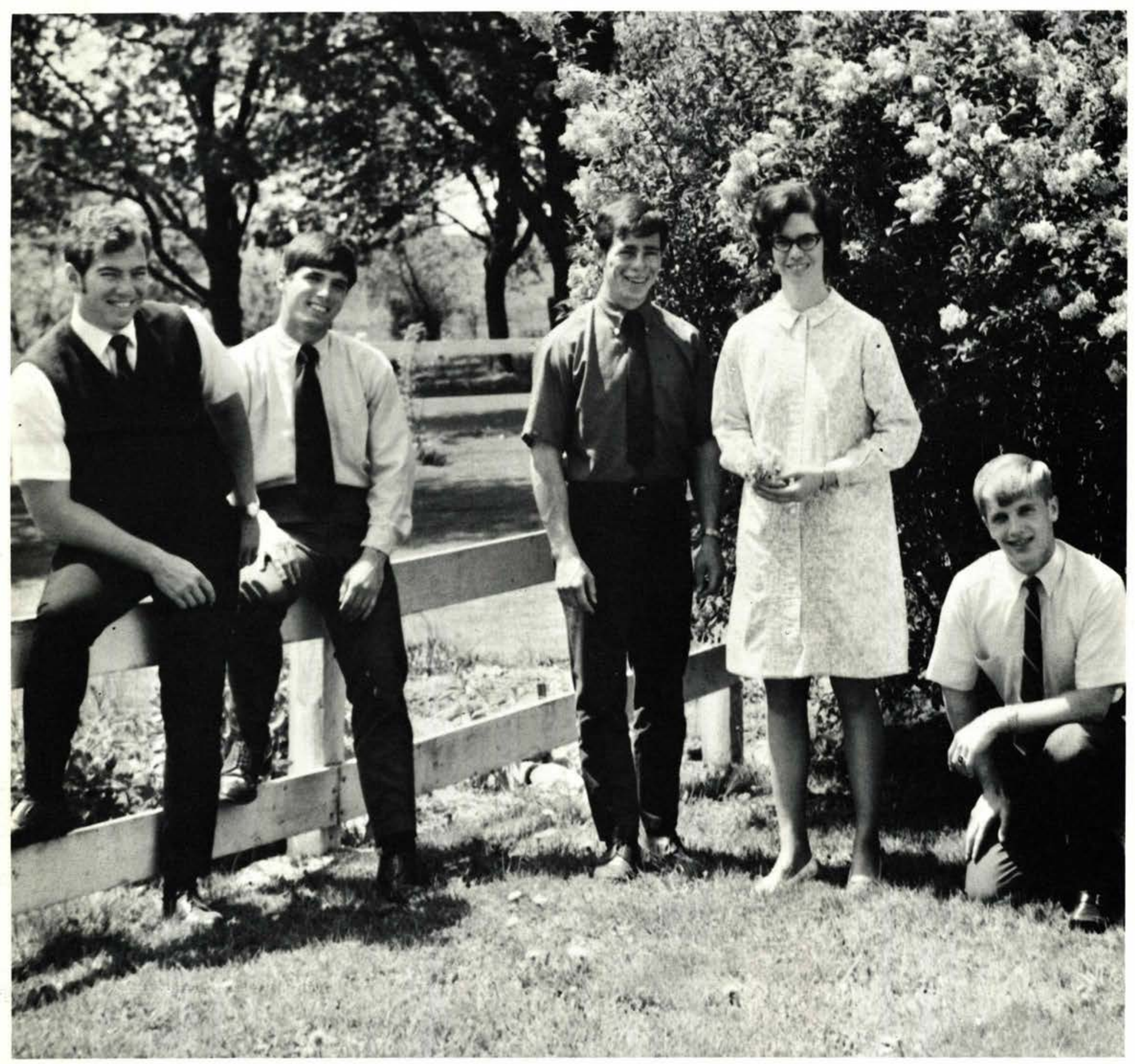

\section{SOPHOMORES CLASS OFFICERS}

Seated:

Mike Wilhite, Vice President

Ken Boothe, Chaplain
Standing:

Ralph Perkins, President

Dianne Kemp, Secretary 
JoAnne Adams

Dayton, Ohio

Sandi Adams

Cortland, $\mathrm{O}$.

Nancy Addleman Hillsdale, Mich.

Kathy Aldrich

Ithaca, New York
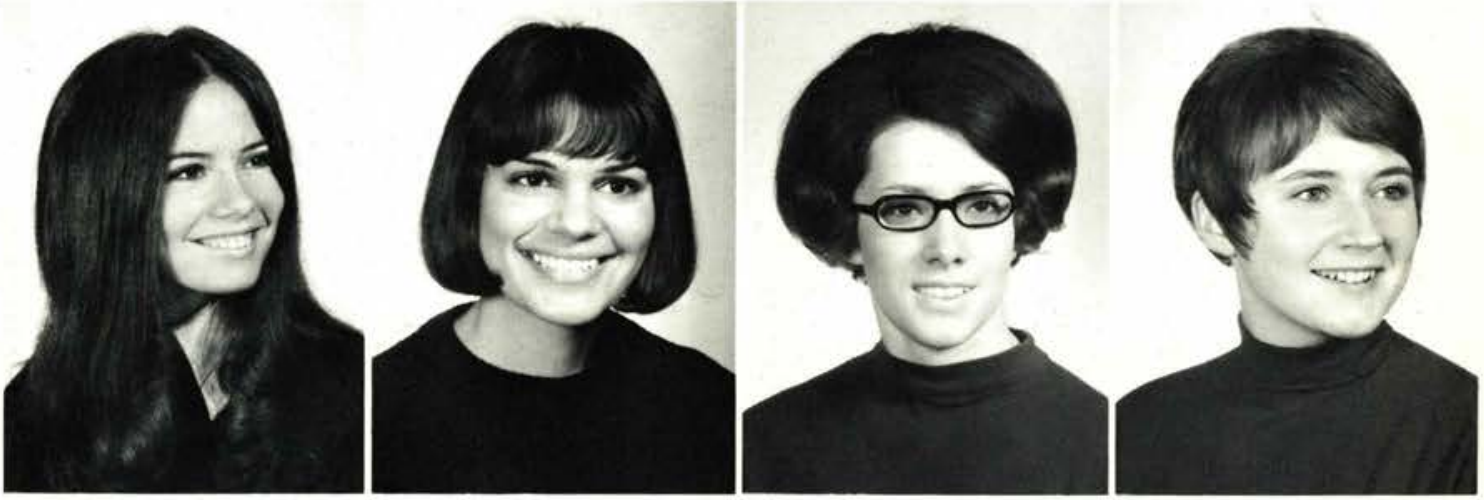

Steve Alexander

Stryker, Ohio

Robert Austin

New Milford, N. J.

Franklin Ayers

Eav Gallie, Florida

Judy Baker

Schenectady, N. Y.
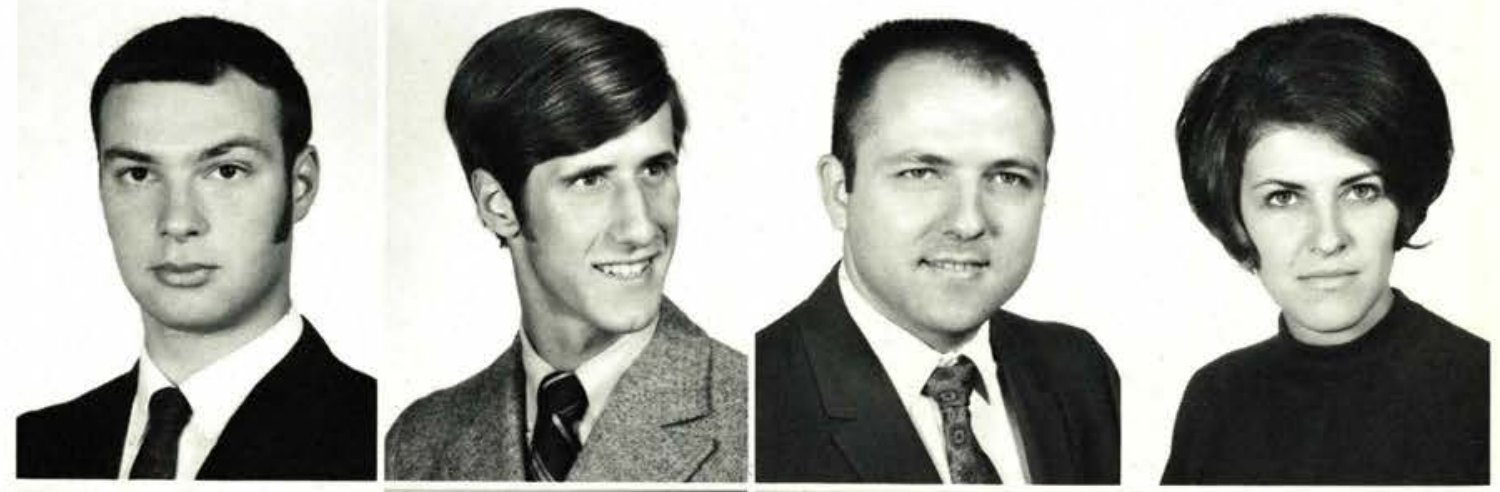

Rodney Baker

Cardington, Ohio

Brenda Bale

Cedarville, $\mathrm{O}$.

Candy Barr

Elyria, O.

Margaret Beck

Oswego, N. Y.
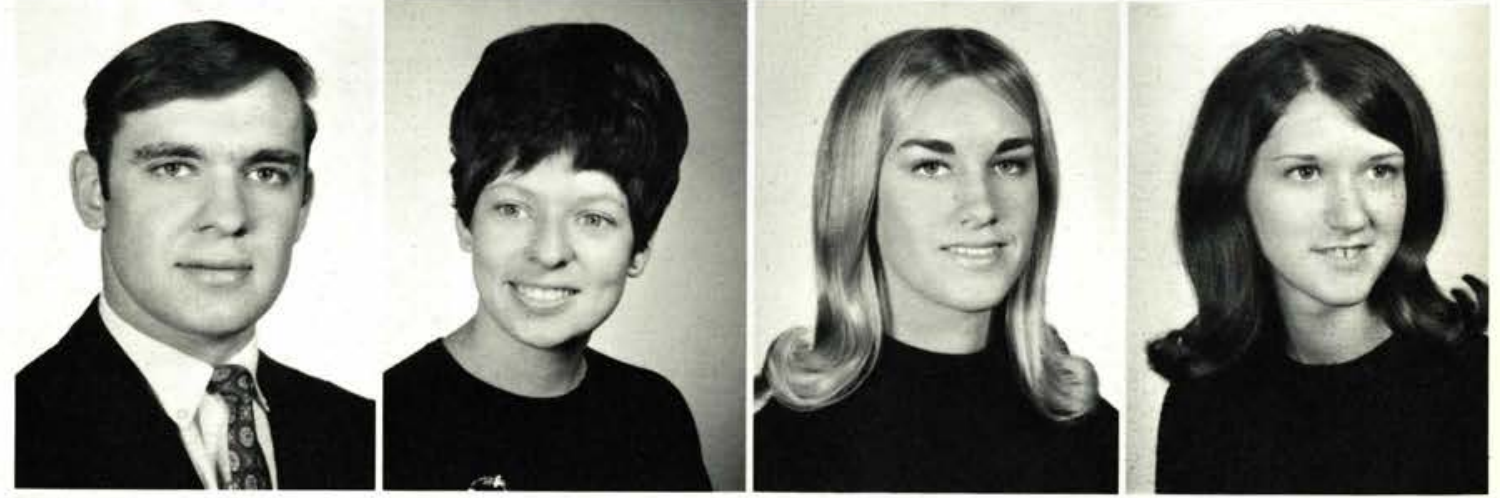

Mary Beitz

Cheektounga, New York

Karen Bernath

Stryker, Ohio

Linda Betts

Wellington, Ohio

Pamela Betts

Bunker Hill, Ill.
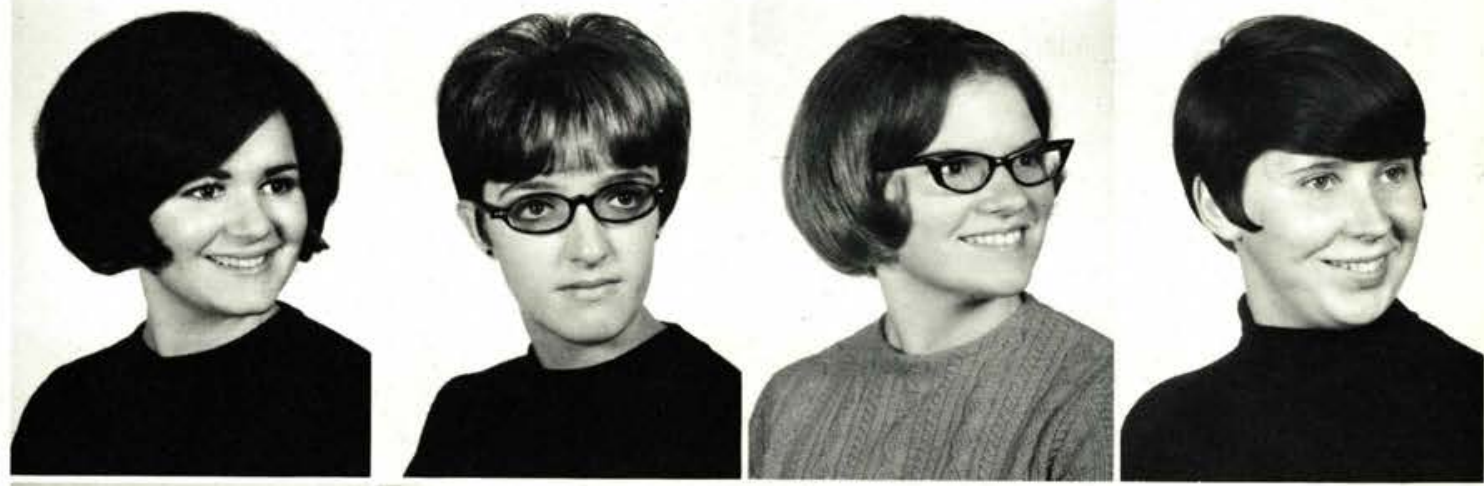

Rebecca Bittner

Tacoma, Washington

Thayne Bodenmiller Homerville, Ohio

Hilda Boothroyd Chicago, Illinois

Nancy Boysen Austin, Minnesota
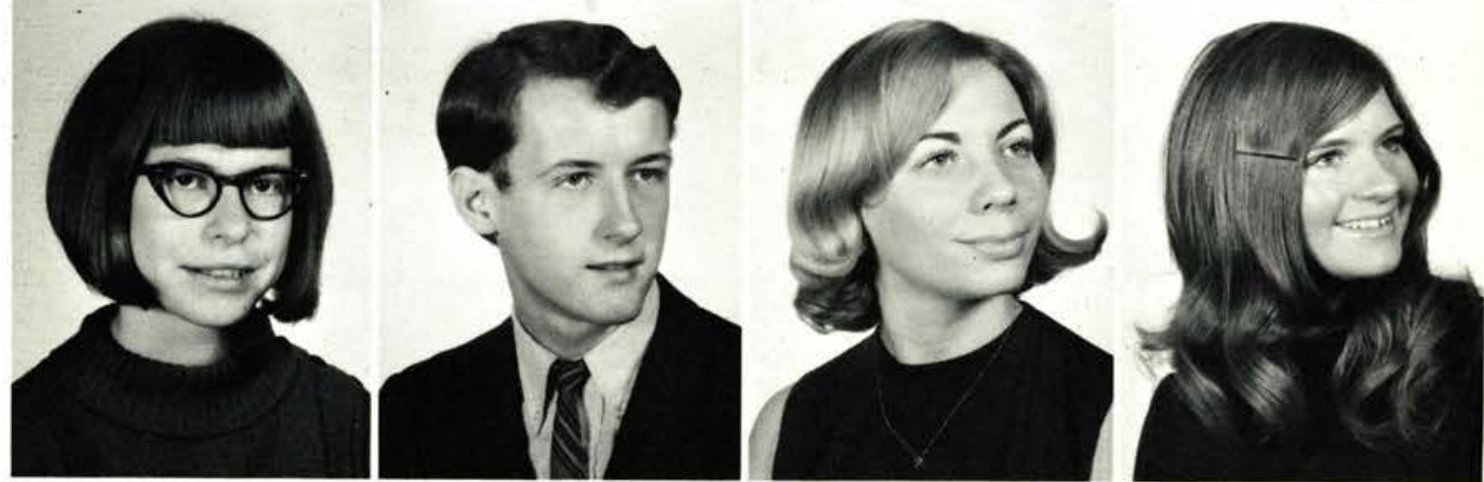


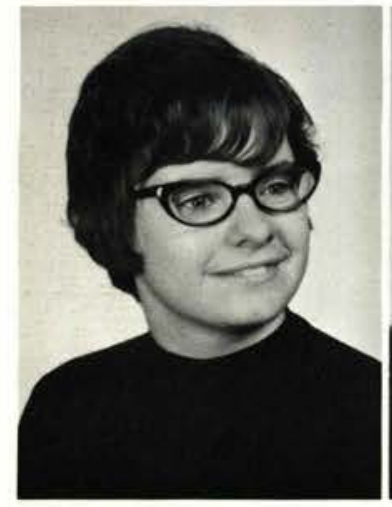

Pamela Branning

Binghamton, New York

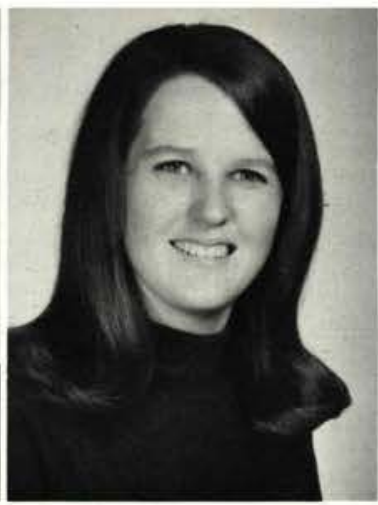

Marty Brooker Sebring, Florida

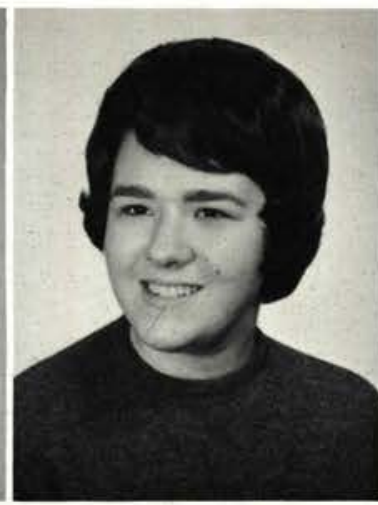

Linda Brown

Milton, Indiana

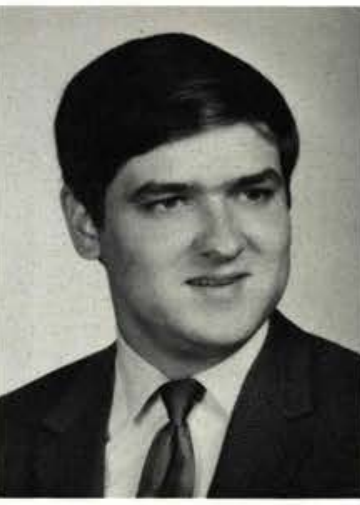

David Butler

Millburg, Ohio

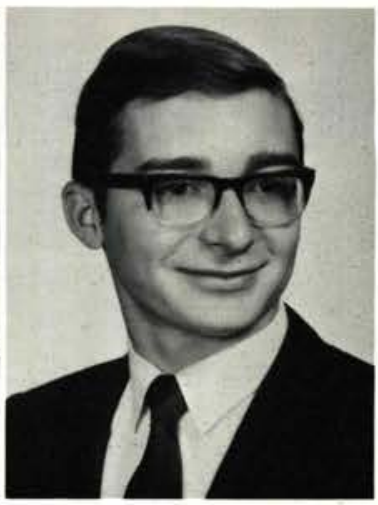

Roy Calvin

Salem, Ohio

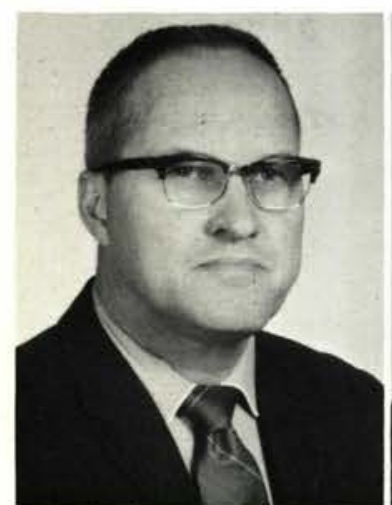

Eric Campfield

Xenia, Ohio

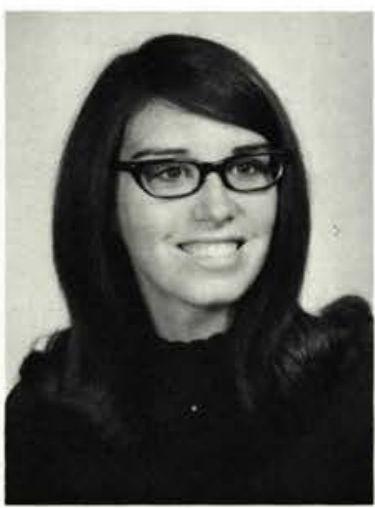

Helen Carter Royalton, Ohio

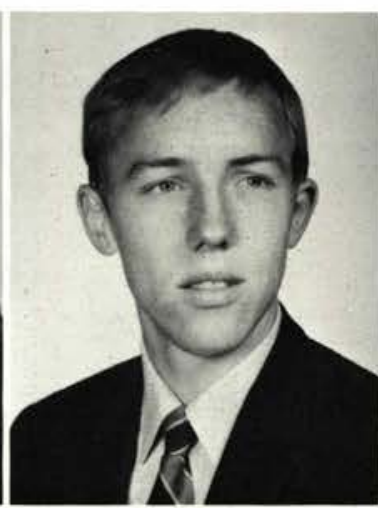

Jim Cavey

Sao Paulo, Brazil

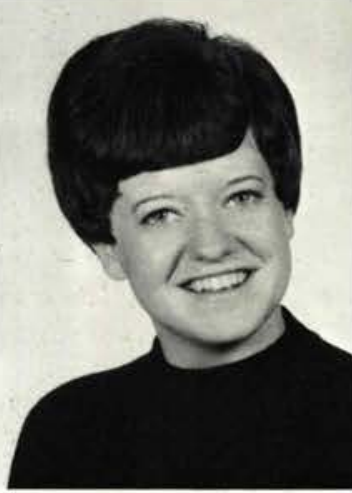

Fran Chambers

Grand Island, New York

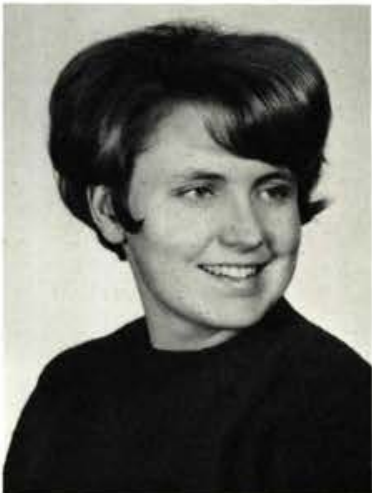

Linda Christiansen Des Moines, Iowa

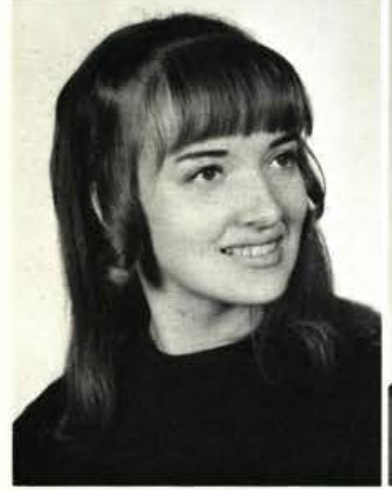

Annette Clark Galloway, Ohio

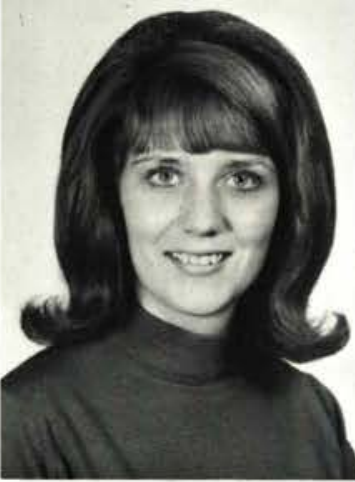

Connie Clark

Spokane, Washington

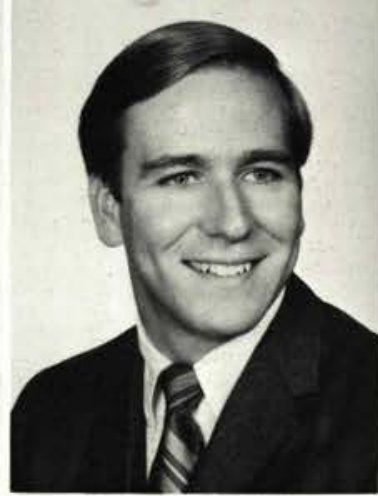

John Clauer

Springfield, Ohio

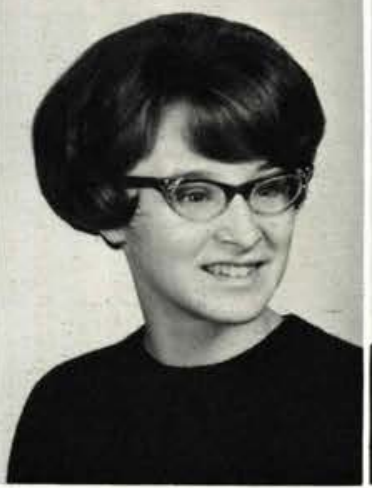

Pamela Closson

Berea, Ohio

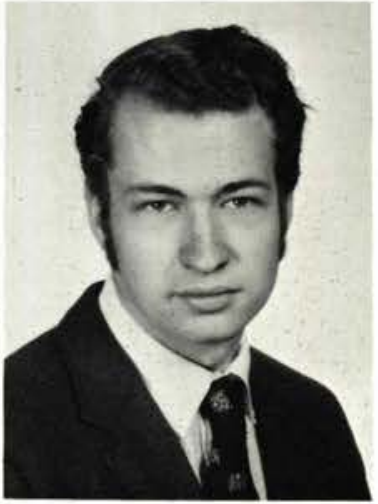

John Conant

Iloila City, Philippines
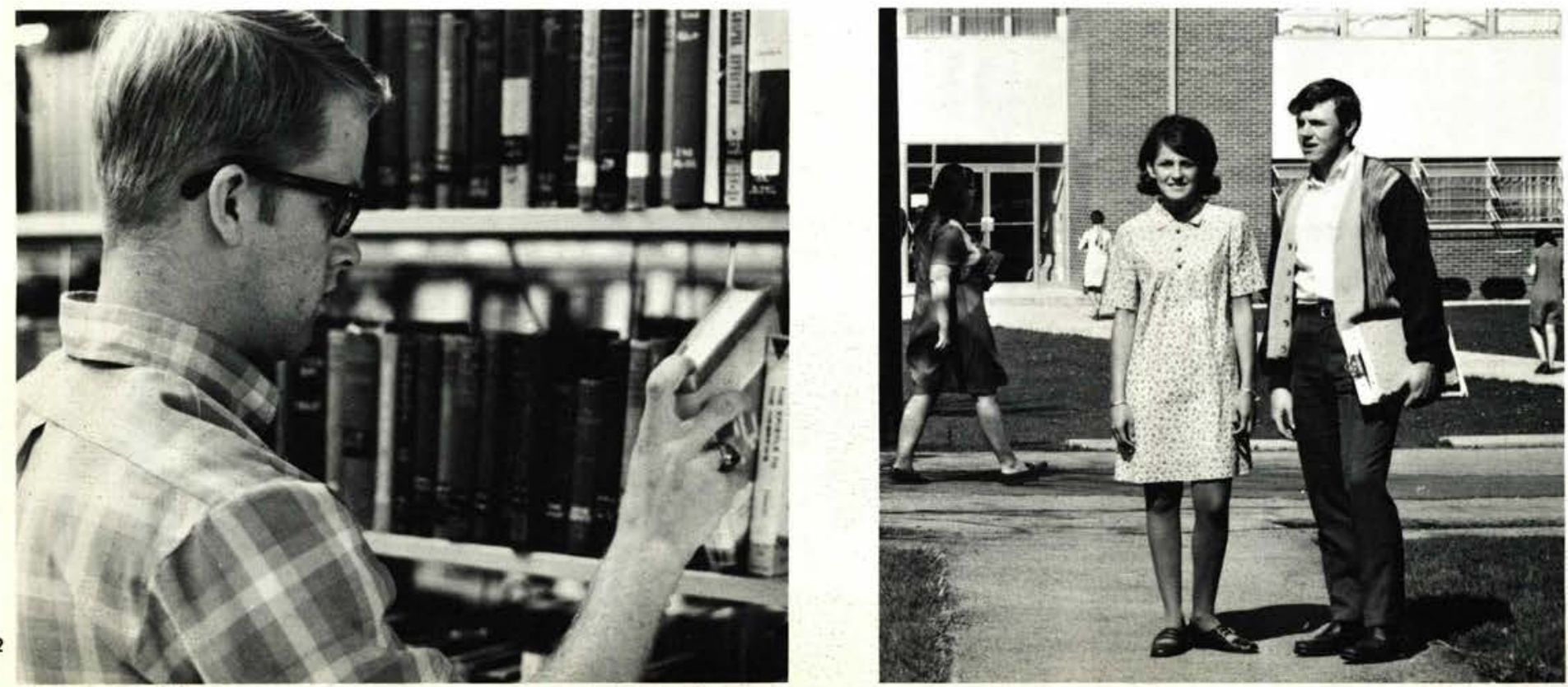


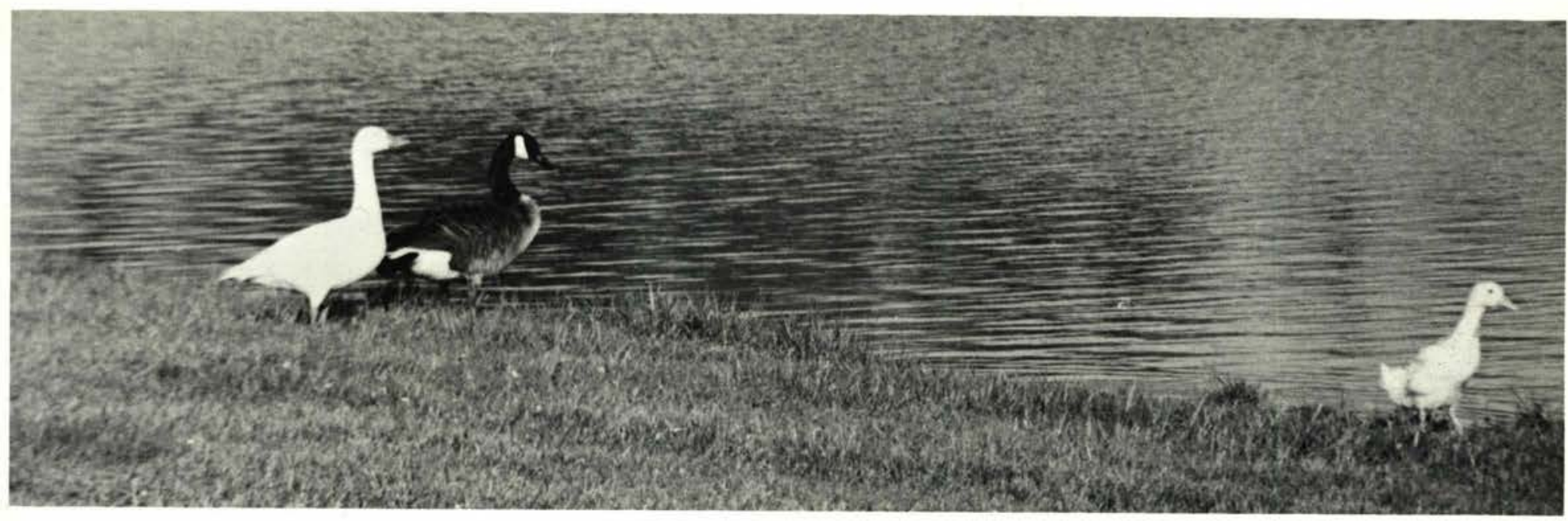

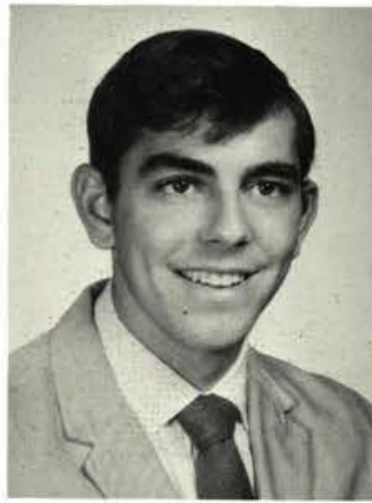

Roger Curtis

Newark, Ohio

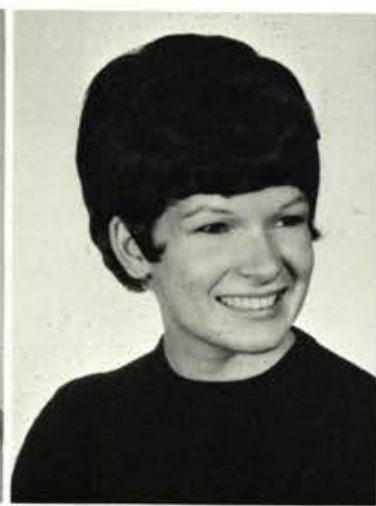

Glenda Dalton

Wakeman, Ohio

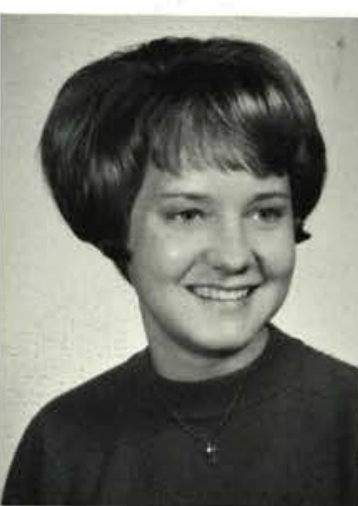

Cynthia Davis

Nampa, Idaho

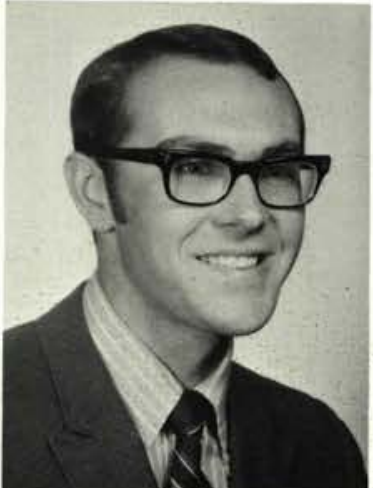

Bruce Dawley

Rochester, Michigan

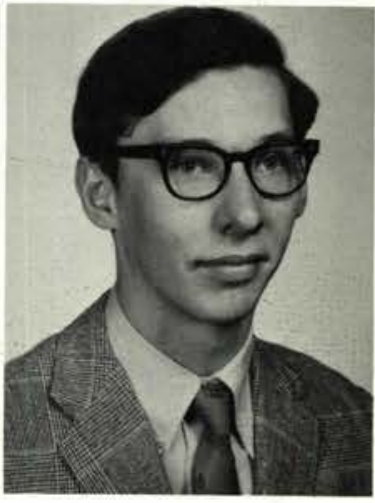

Michael DeBoer

Reinbeck, Iowa

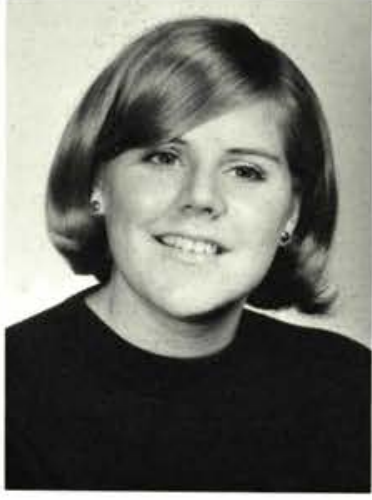

Carol DeBoer

Hollandale, Minnesota

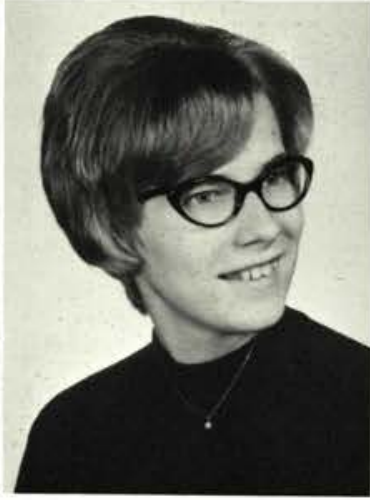

Elaine Dixon

Spokane, Washington

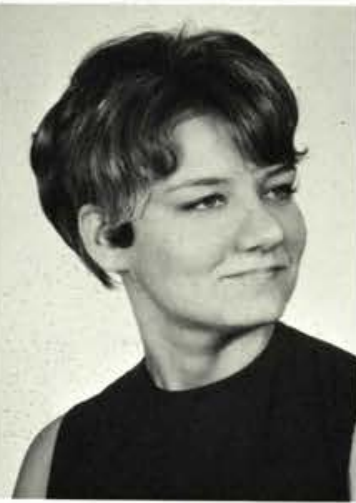

Sandra K. Douglas Ava, New York

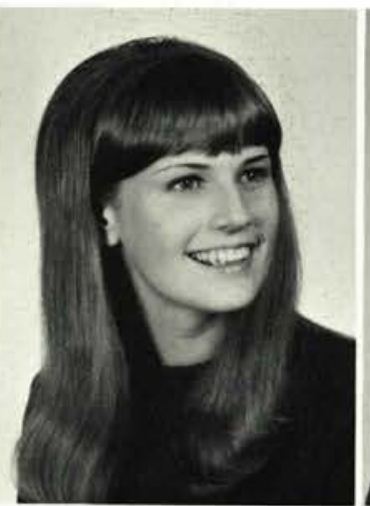

Meda Edelblute

Gallipolis, Ohio

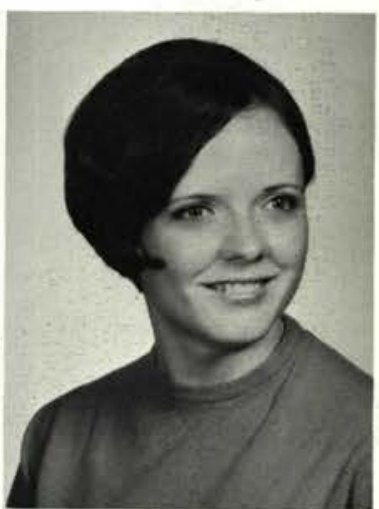

Brenda Edmunds Hillsdale, Illinois
Marsha Edwards Albion, Iowa

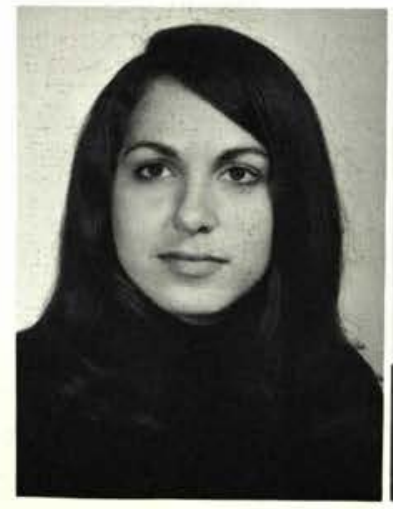

Clinton Eichelberger Kuna, Idaho

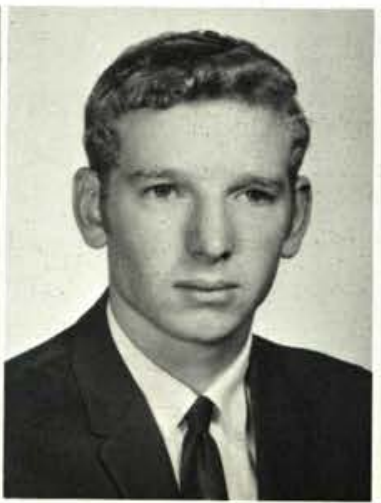

Connie Endicott Springfield, Ohio

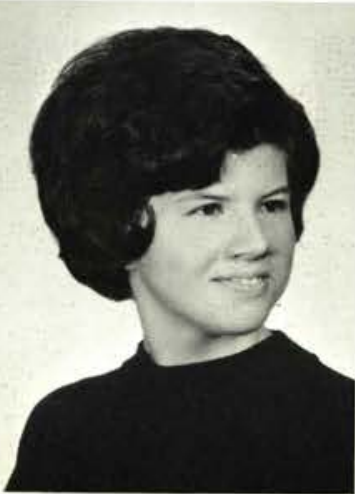

Todd England Geneva, Ohio

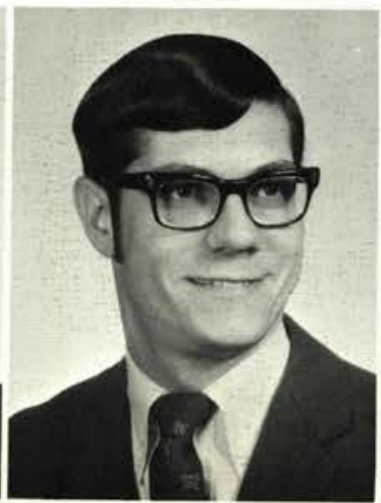

Laura Entenman Clearwater, Florida

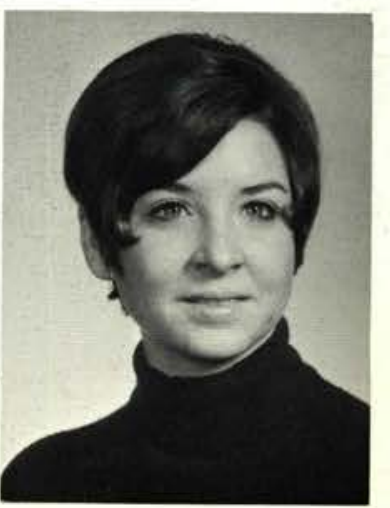



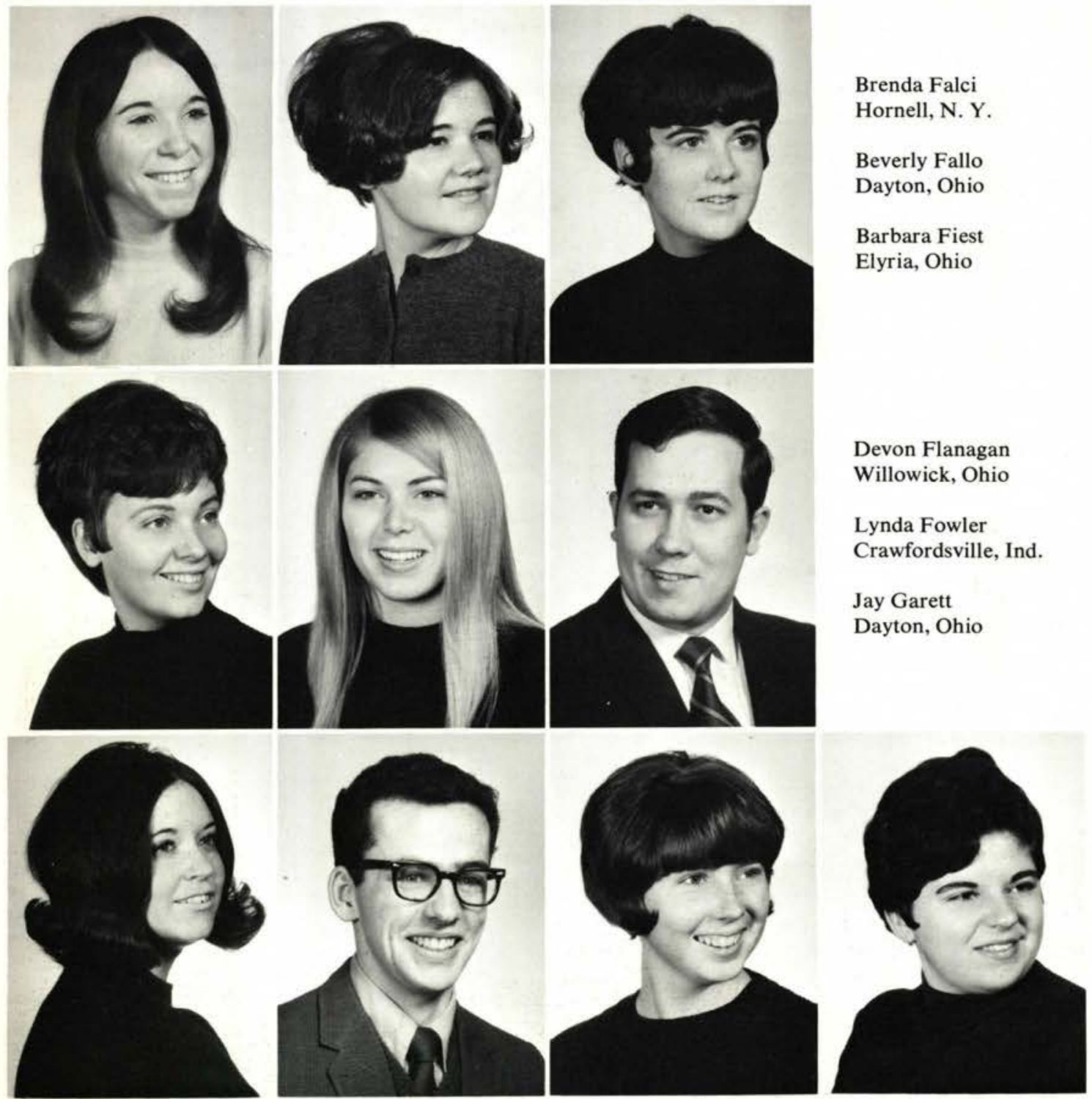

Devon Flanagan

Willowick, Ohio

Lynda Fowler

Crawfordsville, Ind.

Jay Garett

Dayton, Ohio

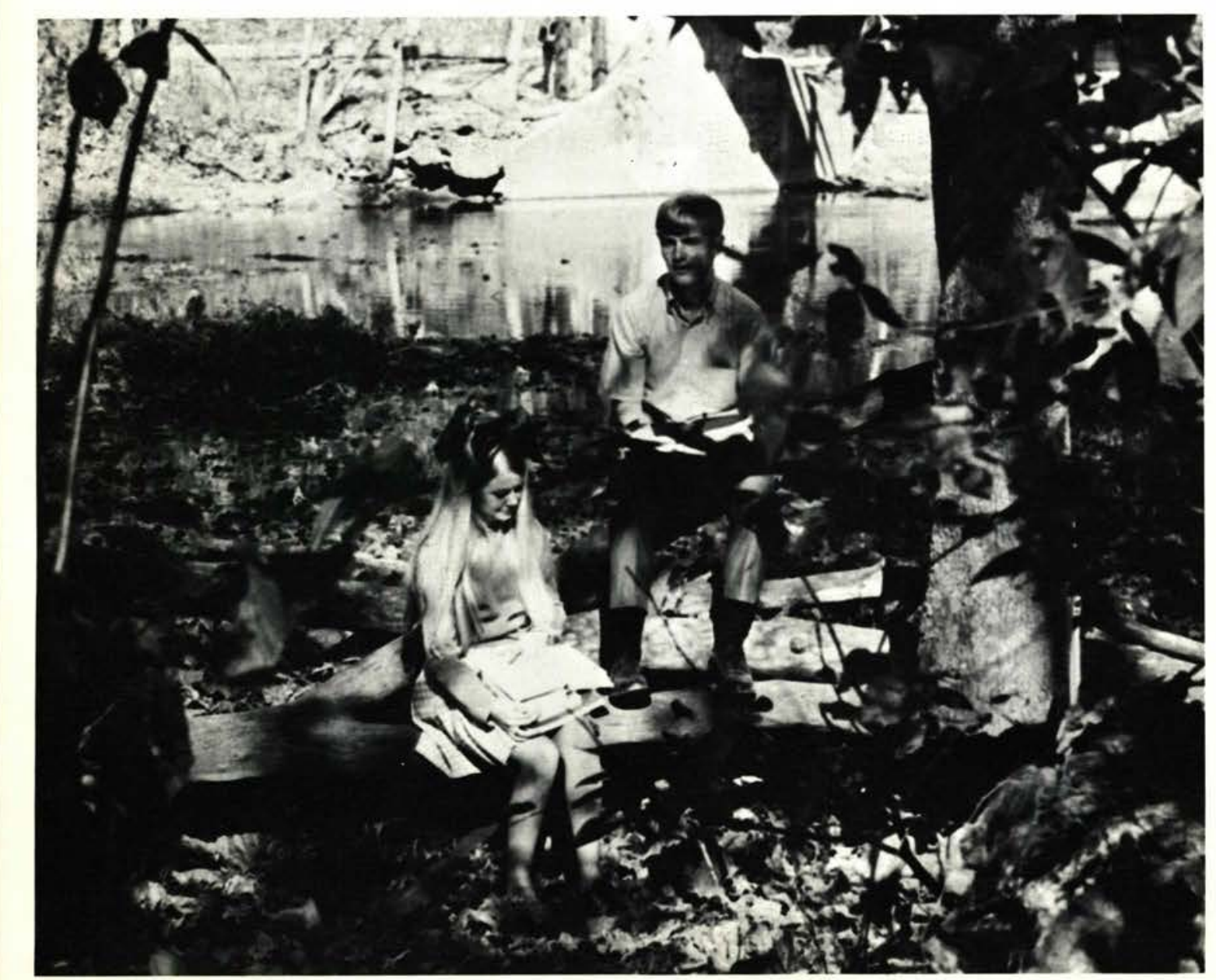

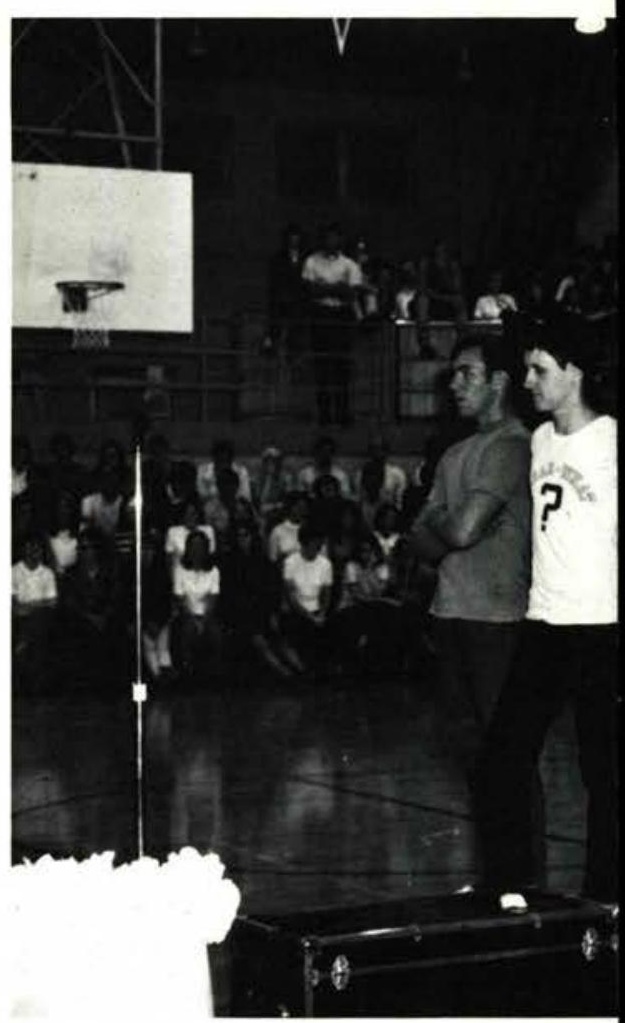

Kathy Gelo

Silvis, IIl.

Steve Glazier

Troy, Ohio

Carolyn Golliher

Richland, Mich.

Debbie Good

New Castle, Del.
Laura Lee Grable

Wheaton, Md.

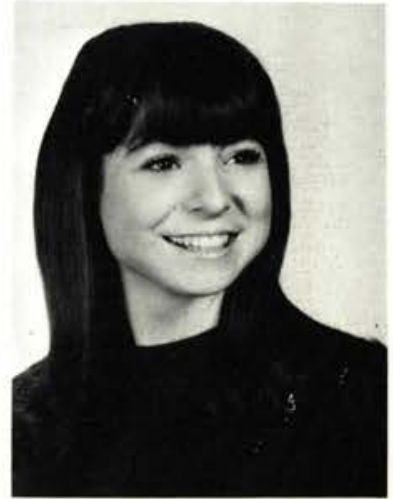




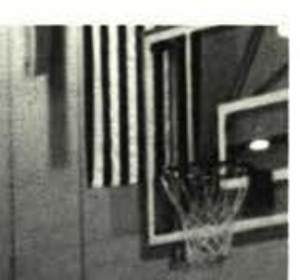

David Grant

New Hyde Park, N. Y.

Sarah Graydon

Cleveland, Ohio

Jerry Grayson

Prescott, Ariz.

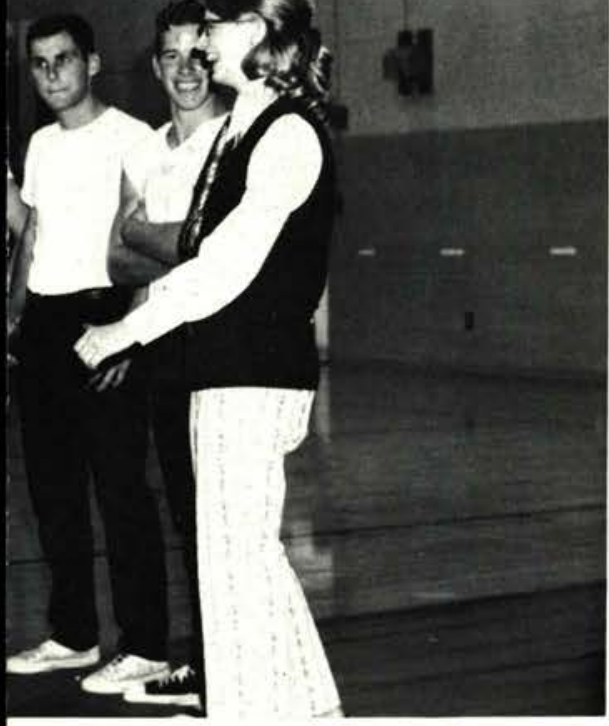

Beverlee Hare

Sao Paulo, Brazi

Susan Harold

Huntington, W. Va.

John Harrison

Richmond, Ohio

Barbara Hart

Des Moines, Iowa

Jim Greening

Johnson City, N. Y.

\section{Carol Hales}

Buffalo, N. Y.

Keith Hague

Fletcher, Ohio
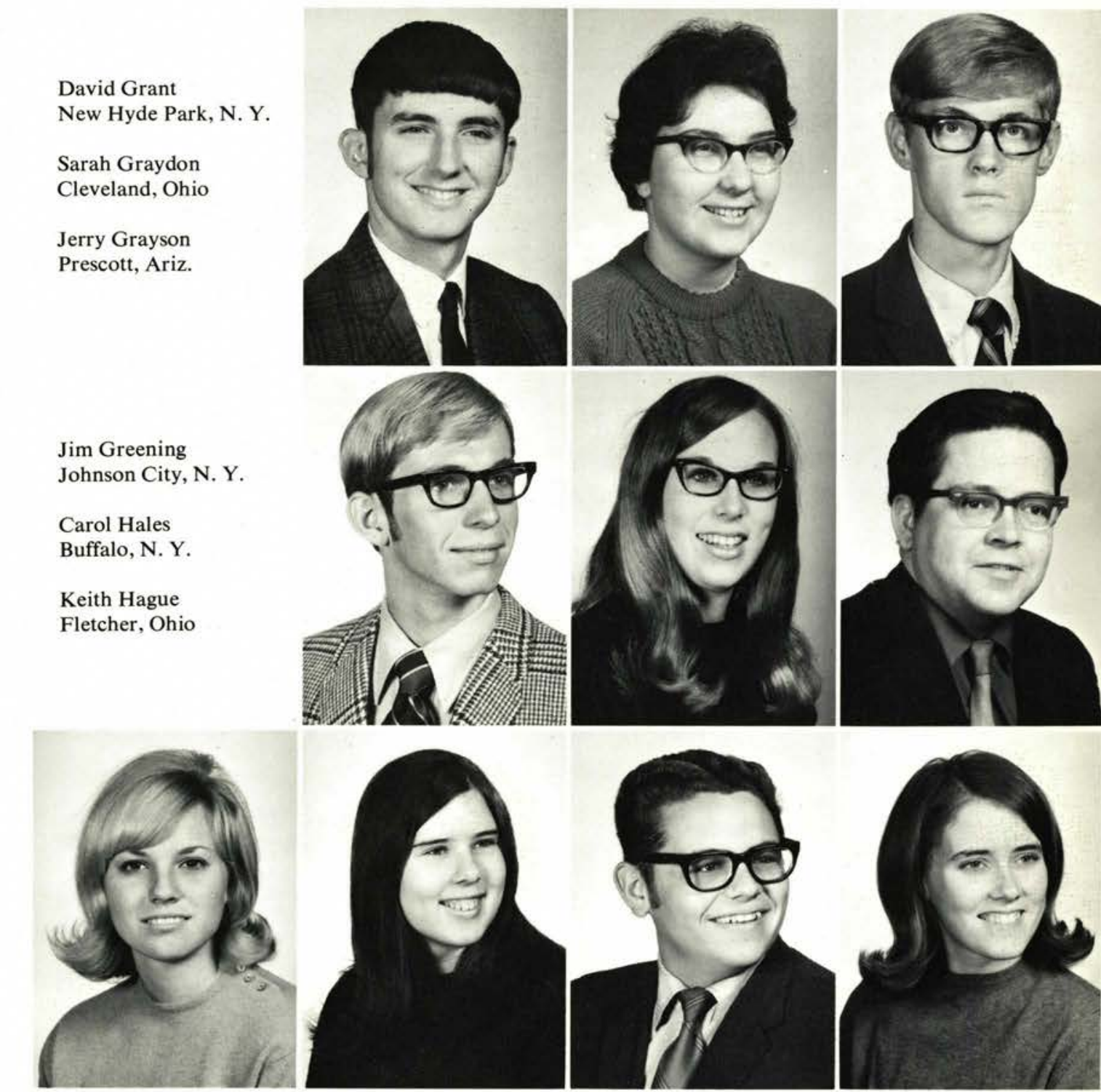

Marsha Hawley

Tonawanda, New York
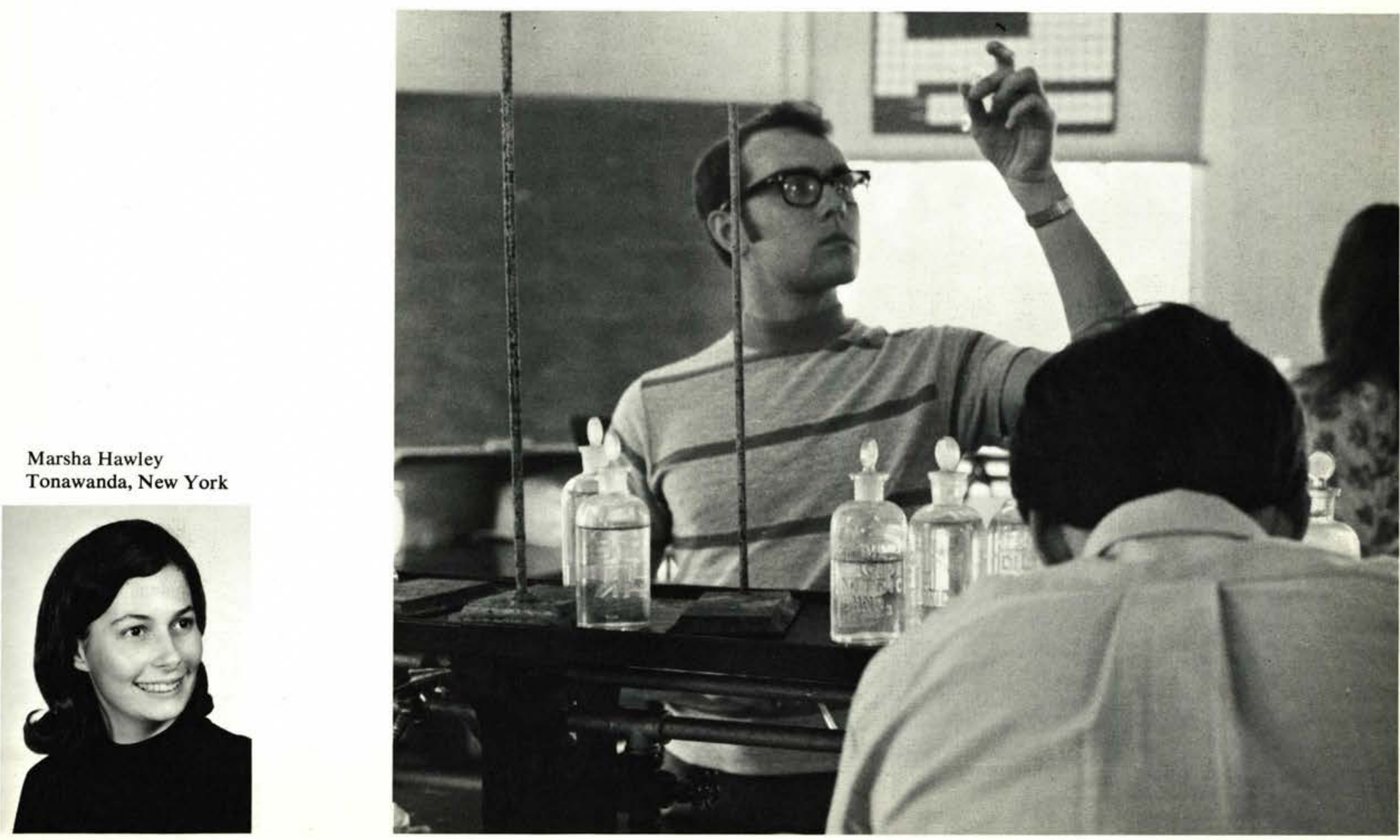

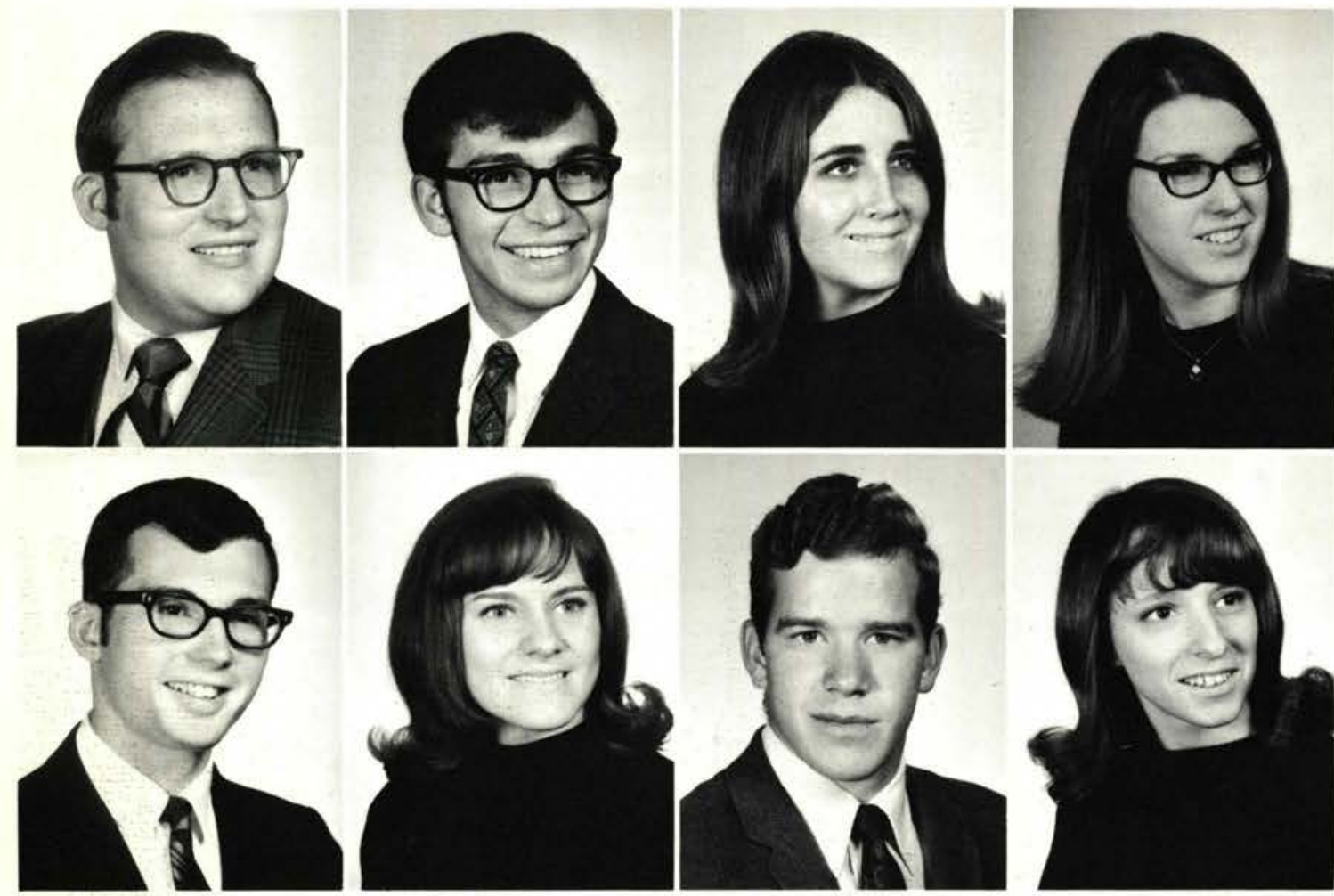

James Hostetler

Kokomo, Indiana

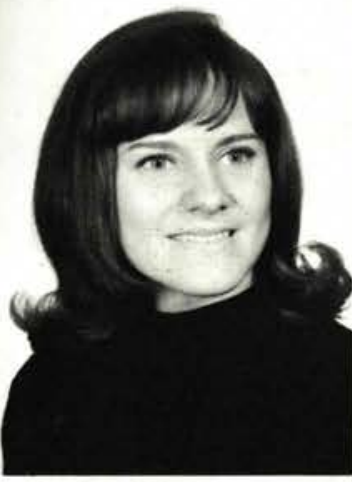

Andrea Houchen

Kansas City, Kansas

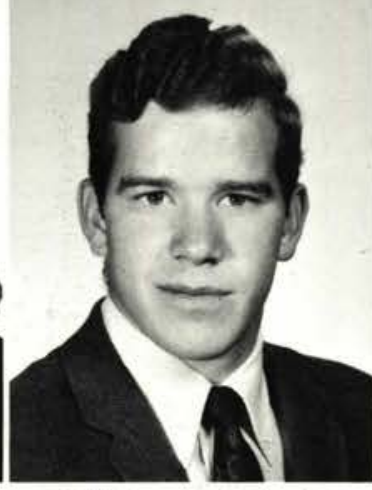

William Hodyshell

Pickerington, Ohio

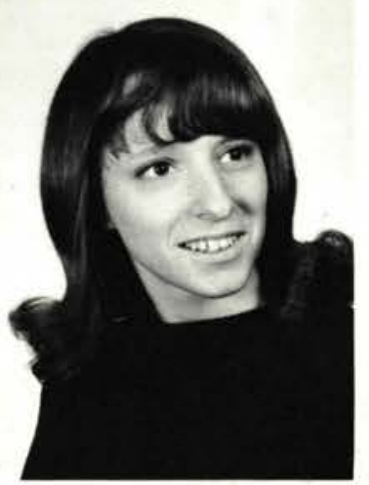

Judy Hubbard

Glastonbury, Conn.

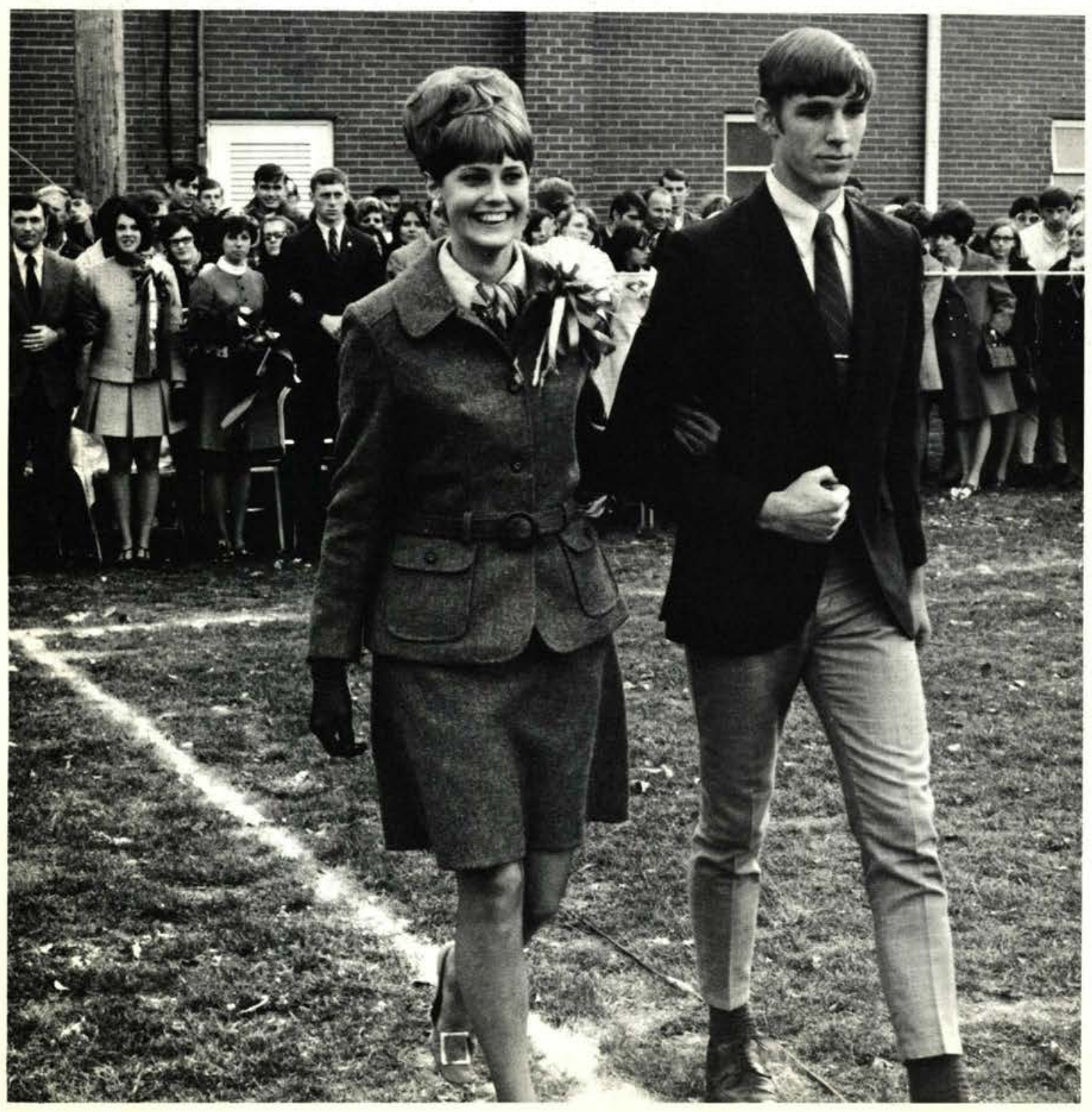

Bill Heater

Cedarville, Ohio

Bill Henry

Plainesville, Ohio

Wilma Hopkins

Carry, Pa.

Bonita Hostetler

Smithville, Ohio

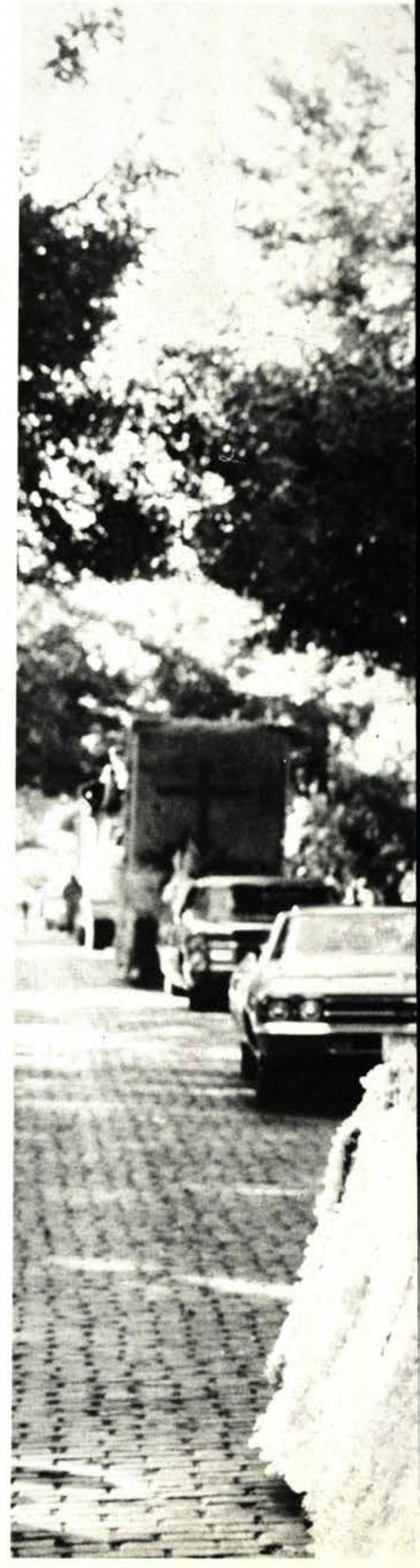


Rebecca Hull

Ithaca, New York

Rebecca Hunter

Warsaw, Indiana

Dan Inghram

Morning Sun, Iowa

Michael Jackson

Bloomfield, Iowa
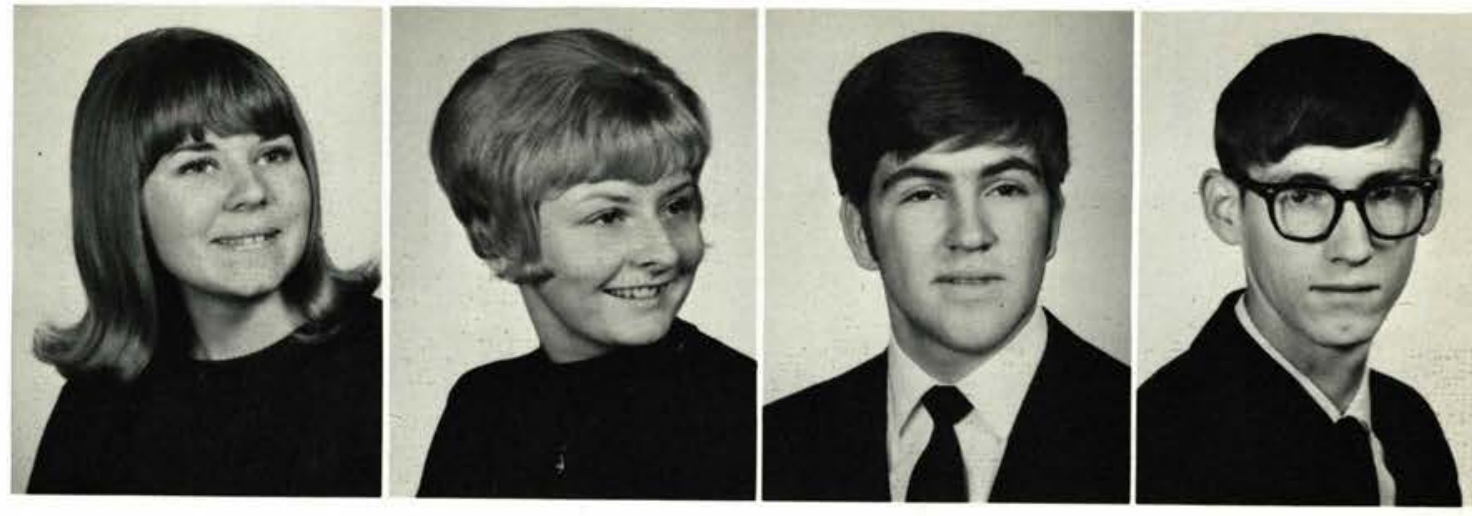

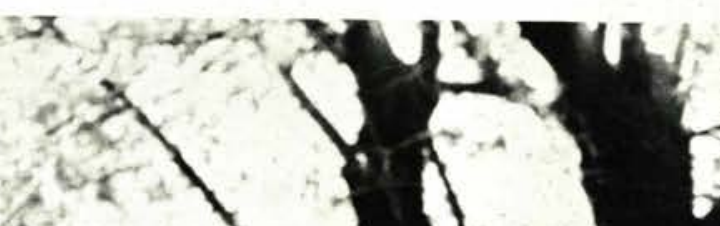

1.

6

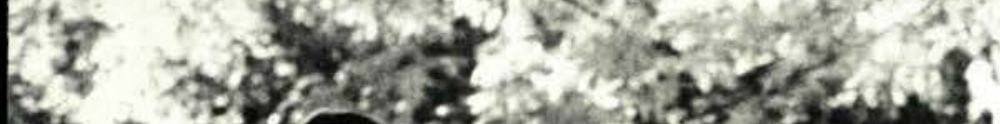

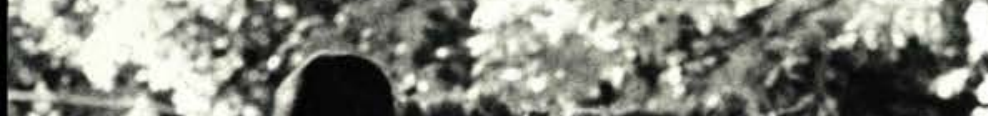

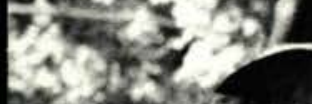
$\sum_{i \pi}^{2}$

4 a. 34 and tis.

\section{.}

$1+1$

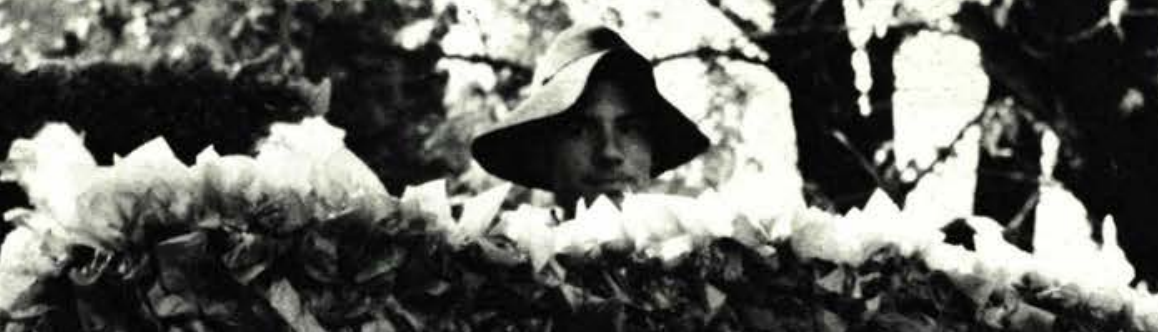
P,

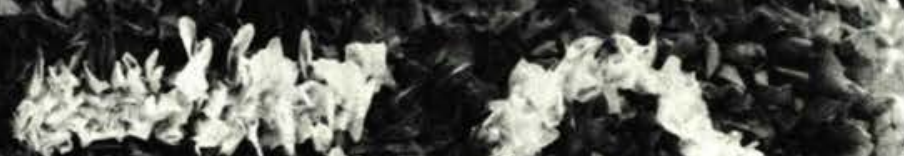

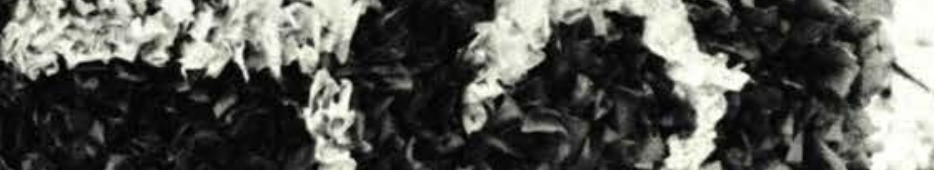
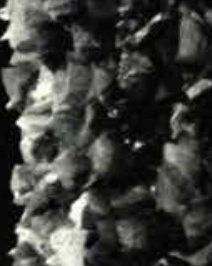

b.

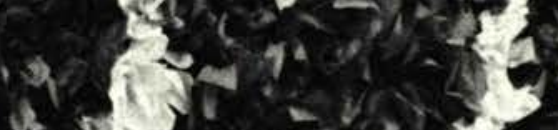

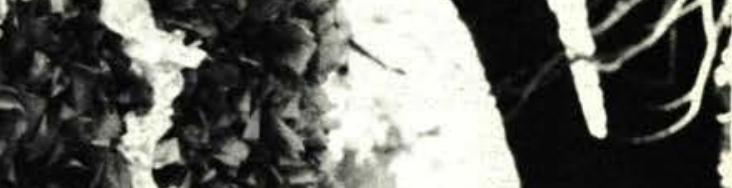

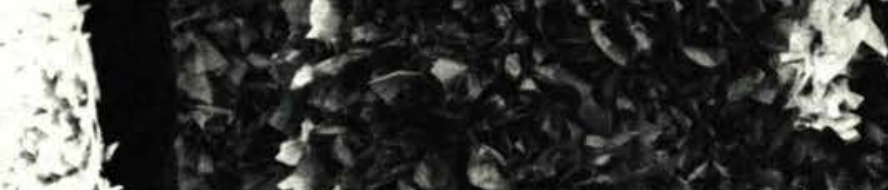
in $2 x+5$

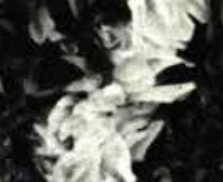

32

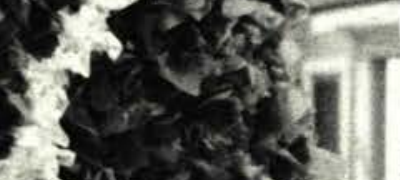
axis $x+2$

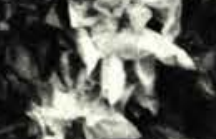

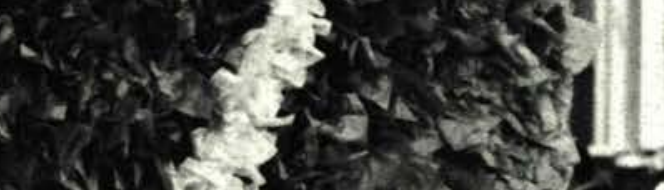

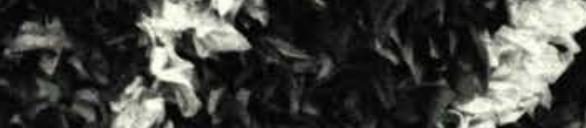

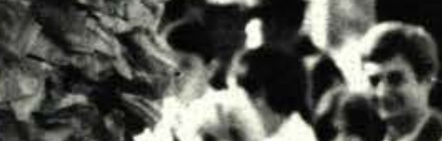

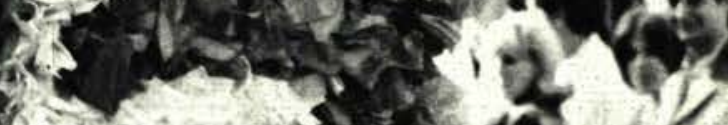

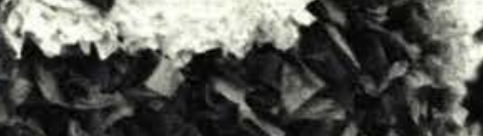

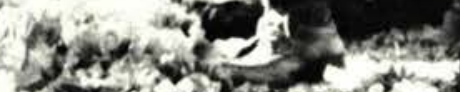
(4)

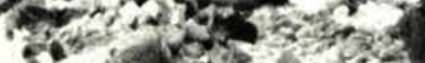
4 ans at 1

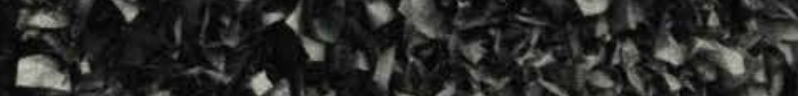

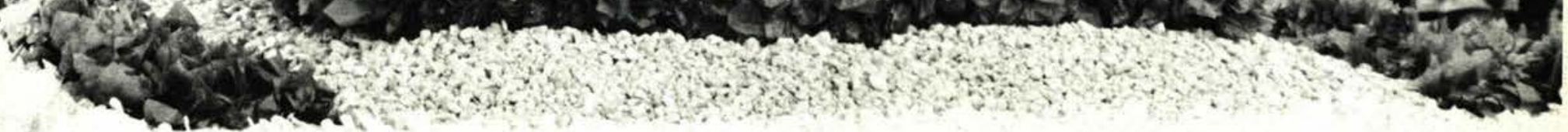




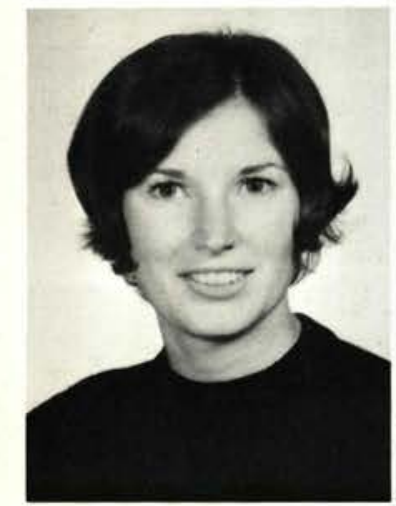

Mary Jacobs

Vestal, N. Y.

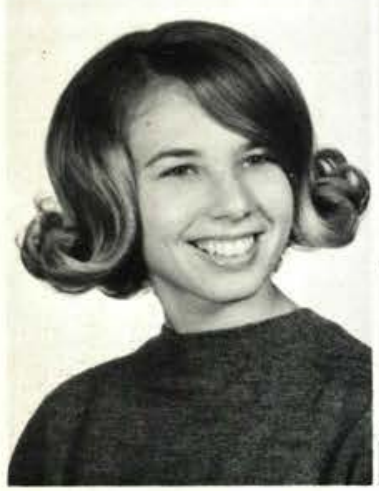

Laura Jones

Uniontown, Ohio

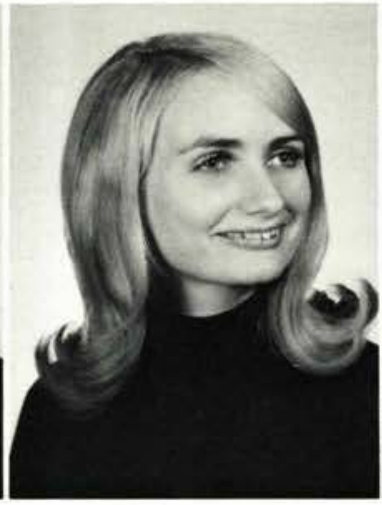

Elaine Jacoby

Lima, Ohio

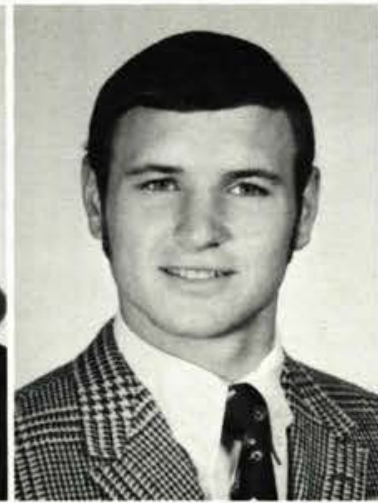

Warren Jenkins

McClaim, Va.

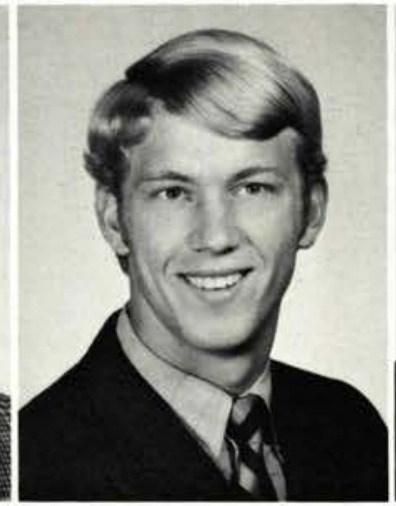

Clif Jensen

Caro, Mich.

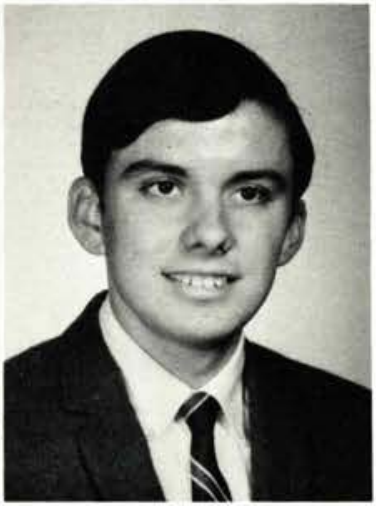

David Jewell Elyria, Ohio

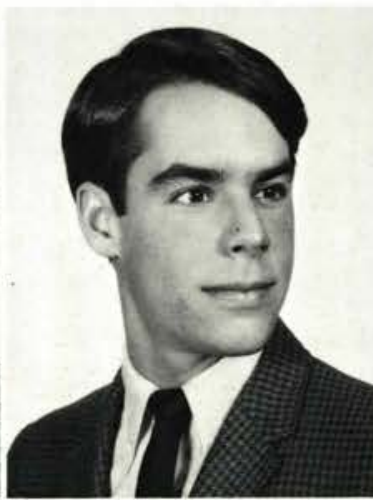

Steve Journell Kettering, Ohio

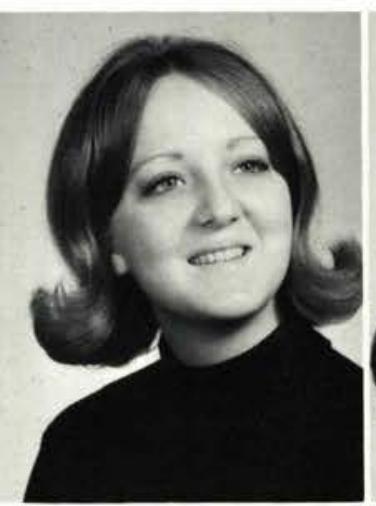

Donna Kahler Leanwood, Kan.

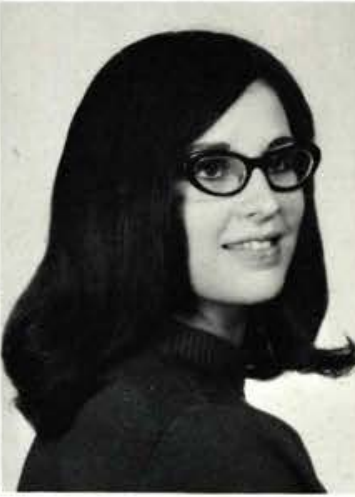

Faun Kauffman Webster City, Iowa

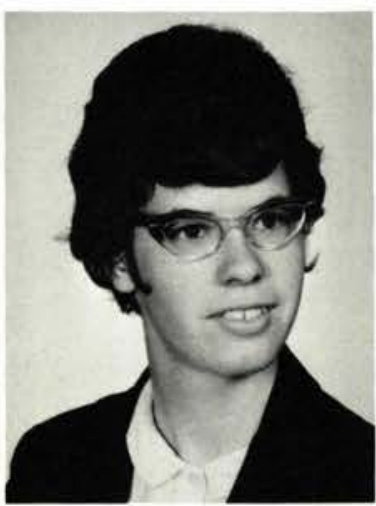

Diane Kemp Coal City, Ill.

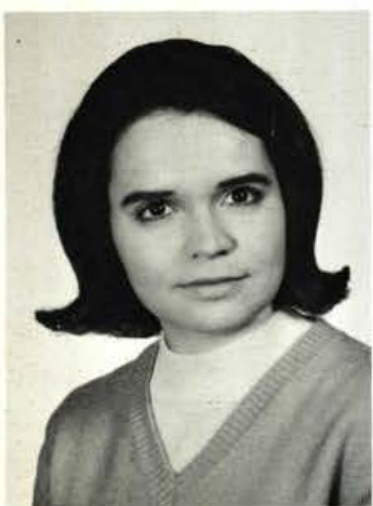

Pamela Kendrich

Bangui, Cen. Afr. Rep.

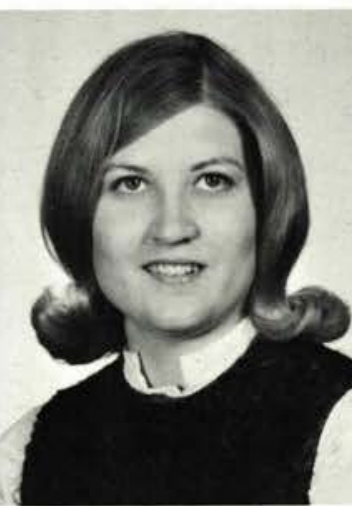

Sherian Kinniburgh Marion, Mont.

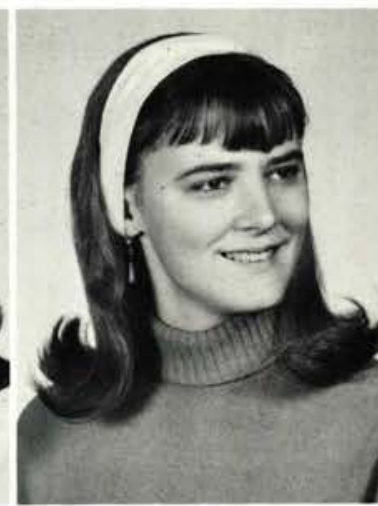

Ruth Kircher

Haddon Heights, N. J.

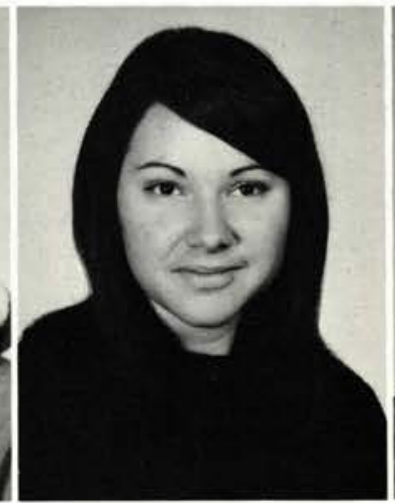

Raydina Kissel Mansfield, Ohio

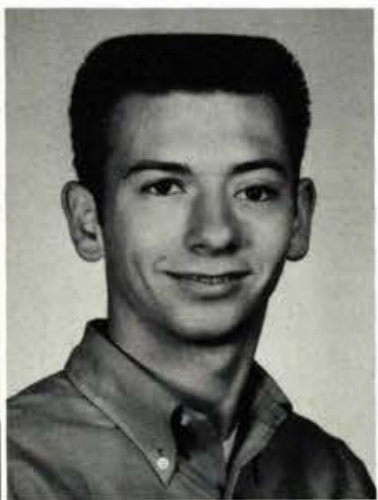

Harvey Klamm Union City, Pa.
Larry Klemm Clarkston, Mich.

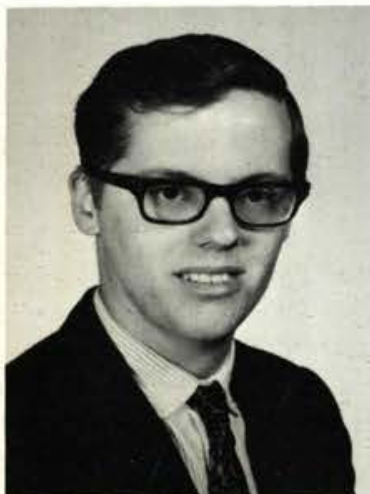

Julie Kleymeer

Wyoming, Mich

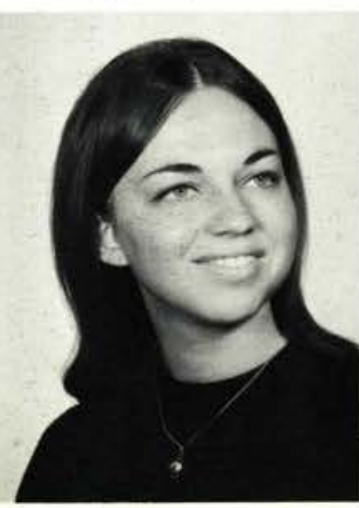

Tim Kline

Bristow, Iowa

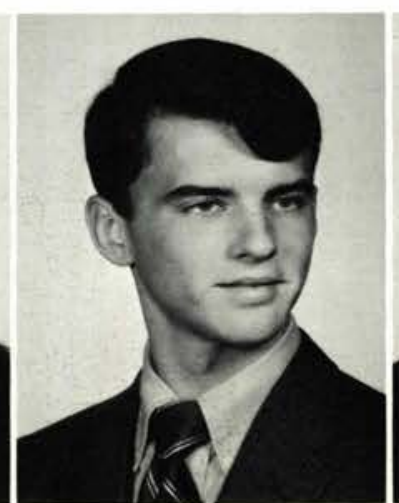

Allen Klinger

Grampian, $\mathrm{Pa}$.

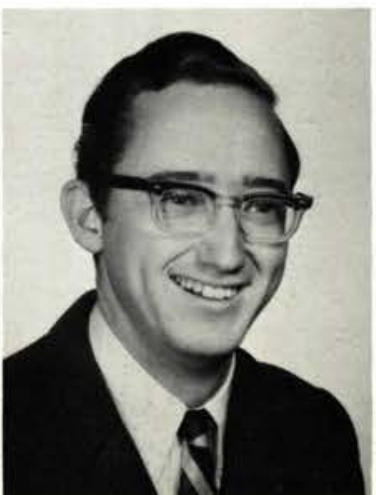

Victor Lakes

Dayton, Ohio

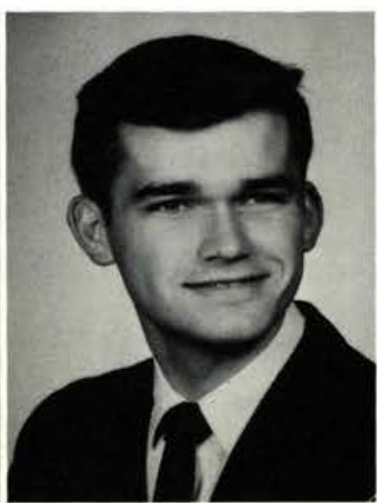




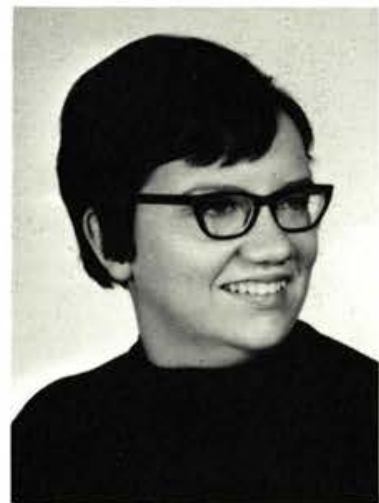

Carolyn Lambert

Rittman, Ohio

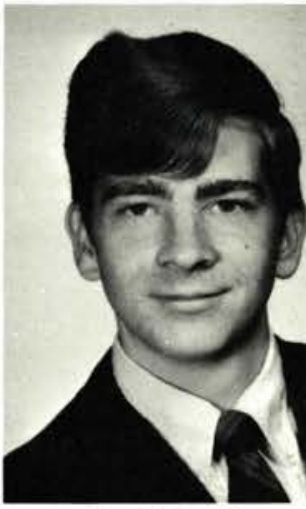

Frank Leslie

Meshoppen, $\mathrm{Pa}$.

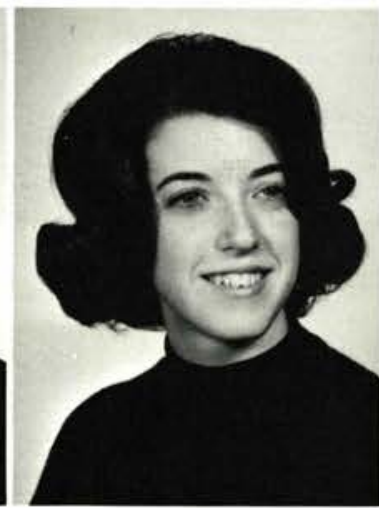

Dawn Lantz

Broomall, $\mathrm{Pa}$

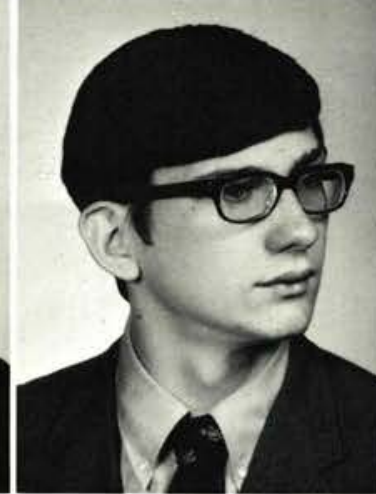

Curtis Larson

Allegan, Mich.

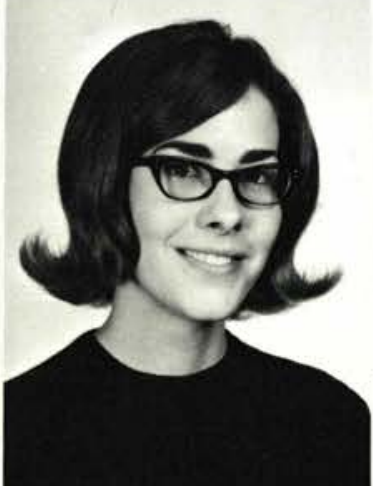

Sue Lathrop

Downers Grove, Ill.

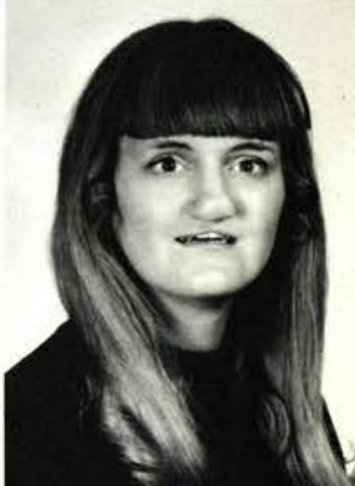

Sue Leonard

Des Moines, Iowa

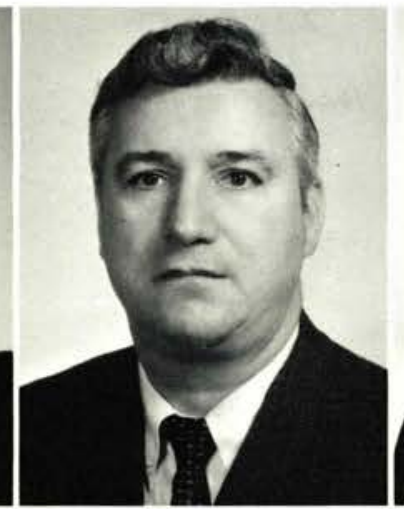

Joseph Lewis

Middletown, Ohio

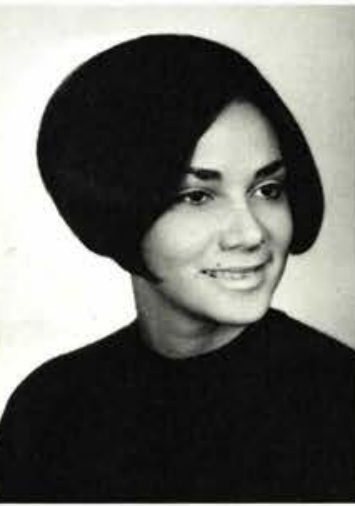

Iris Lima

Rio Piedras, Puerto Rico

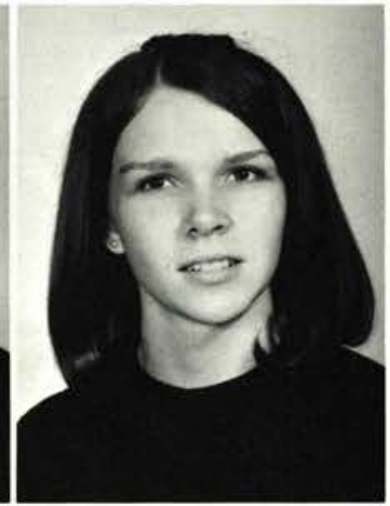

Linda Linger Northfield, $O$.

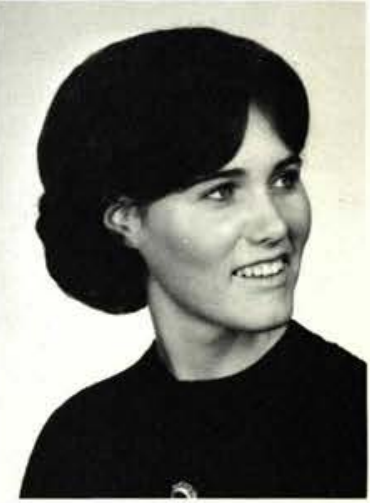

Sharon Lixey

Romulus, Mich.

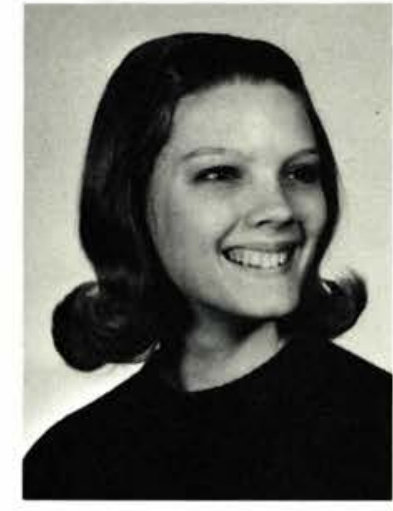

Joyce Love

Huntington, W. Va.

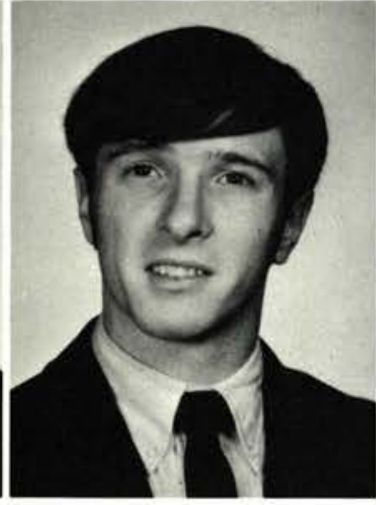

Michael Mays

Chillicothe, $\mathrm{O}$.

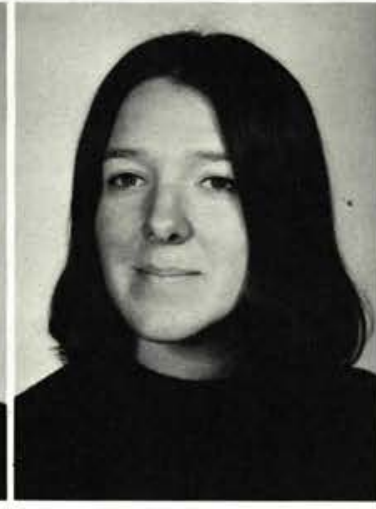

Catherine Marlowe

Oxford, Mich.

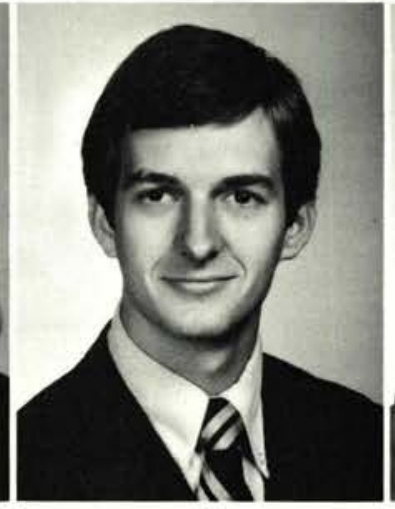

Dan Martin

Crawfordsville, Ind.

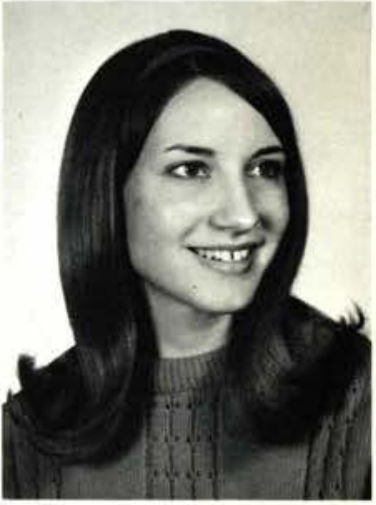

Lin Mason

Flint, Mich
Dan Mead

Fremont, Mich.

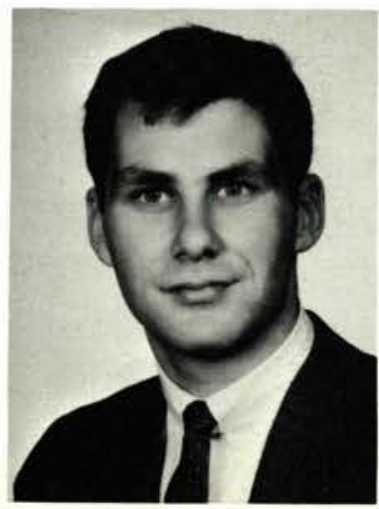

Judy Meyer

Willoughby, $\mathrm{O}$.

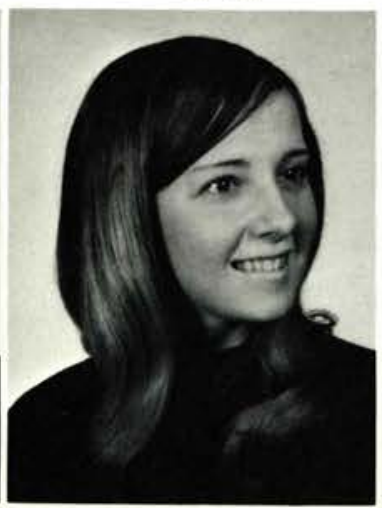

Barry McCoy Dayton, Ohio

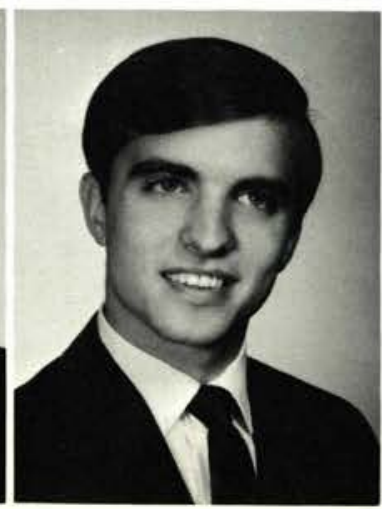

Chip McDaniel Mancelona, Mich.

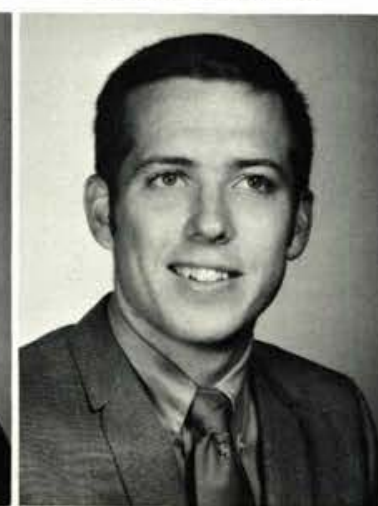

Rita McDowell Hobart, Ind.

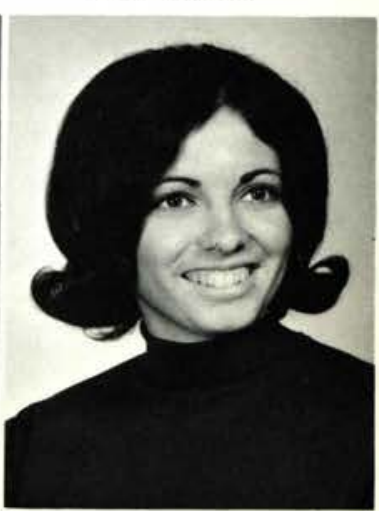



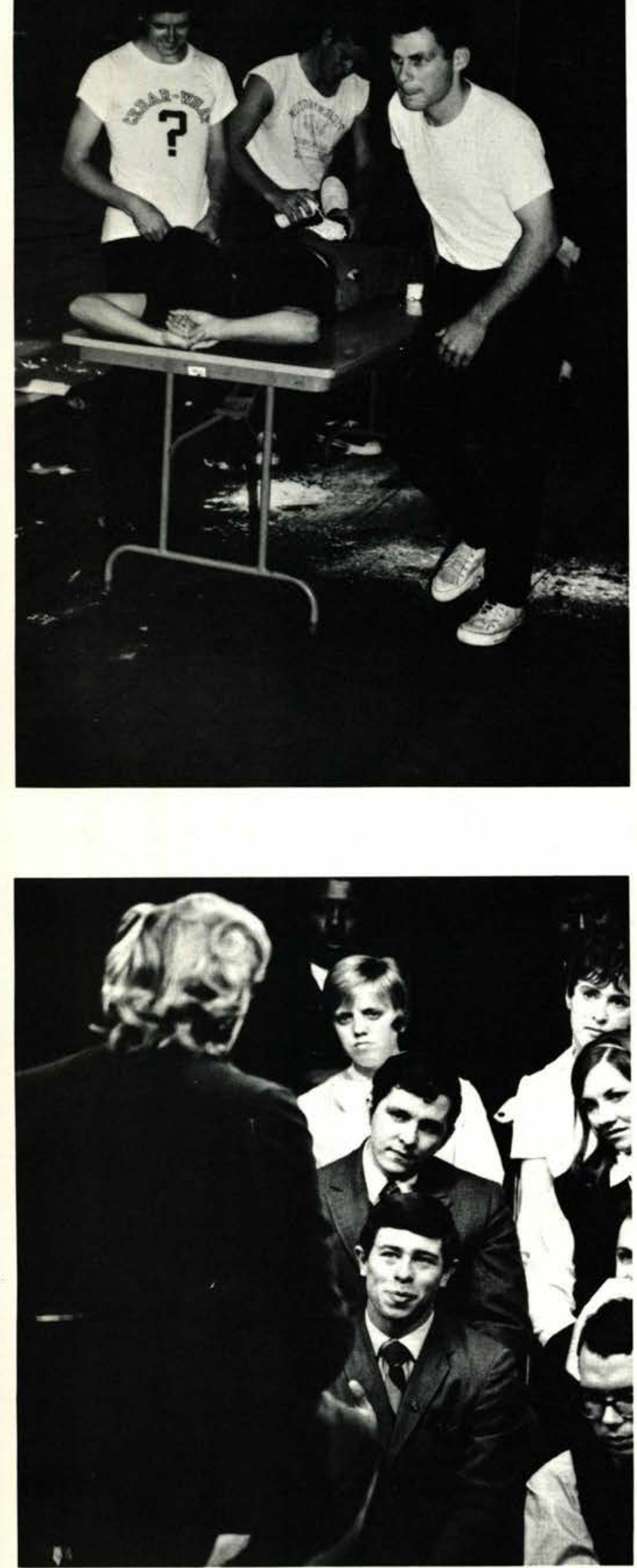

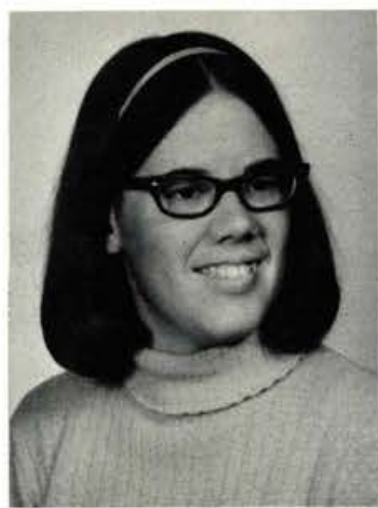

Mary McMurtry

Pittsburg, Pa.

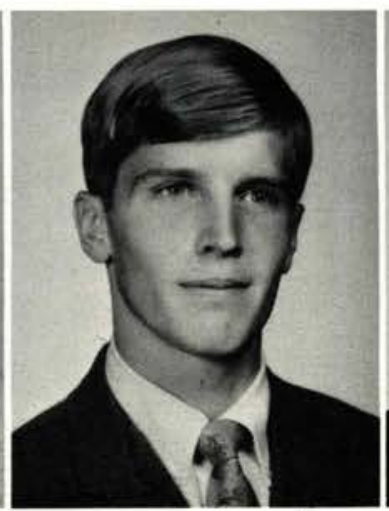

Lyle Miller

St. Charles, Iowa

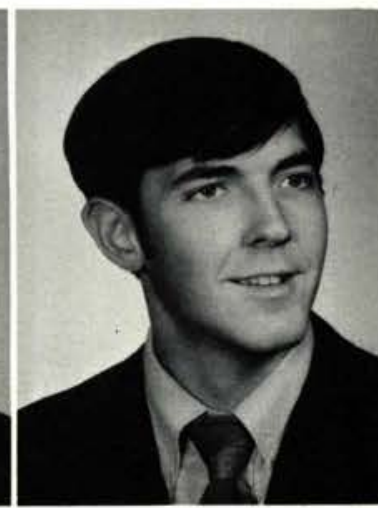

Roger Miller

Allegan, Mich.

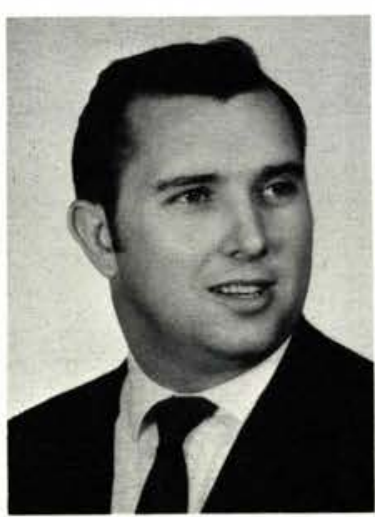

John Milligan

Jamestown, $\mathrm{O}$.

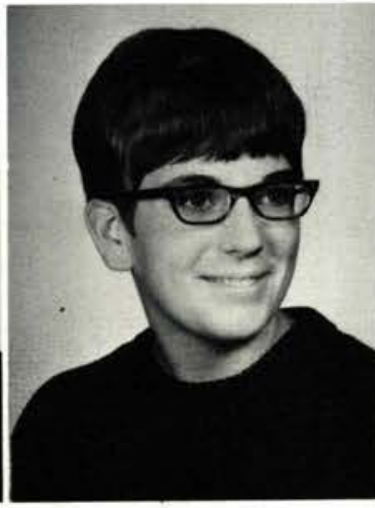

Virginia Mitchell Marovia, N. Y.

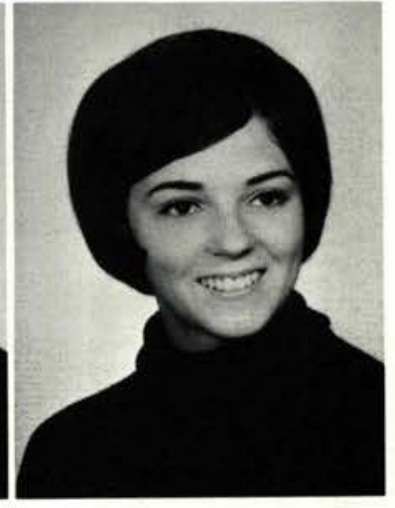

Ruth Moats

Webster City, Ia.

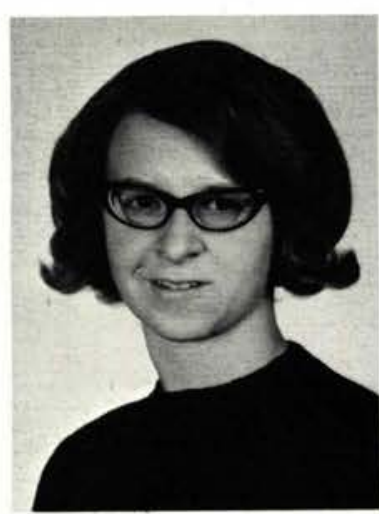

Rebecca Mohr

E. Syracuse, N. Y.

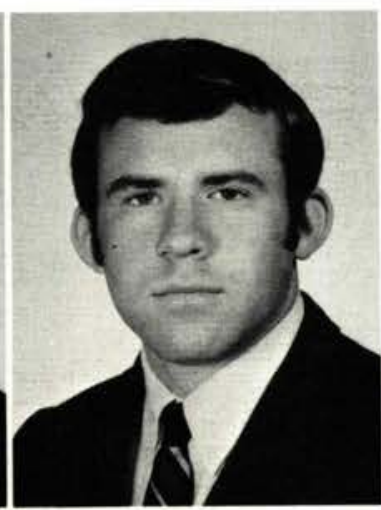

Philip Moon

Freeport, N. Y.

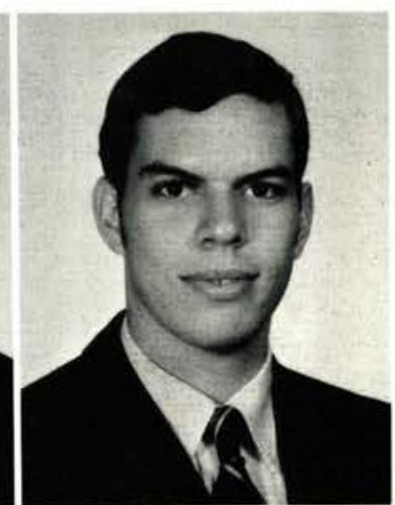

Gerald Mooney New Castle, Pa.
Shirleen Morrow Amhurst, Ohio

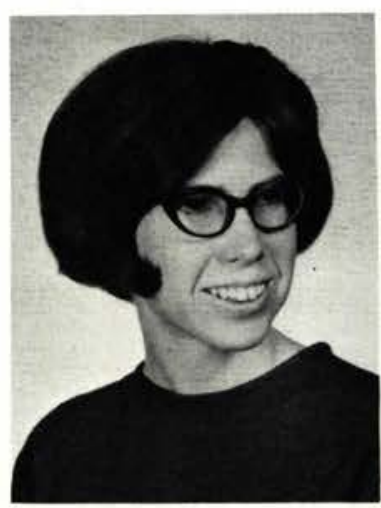

Sharon Mummery Hobart, Ind.

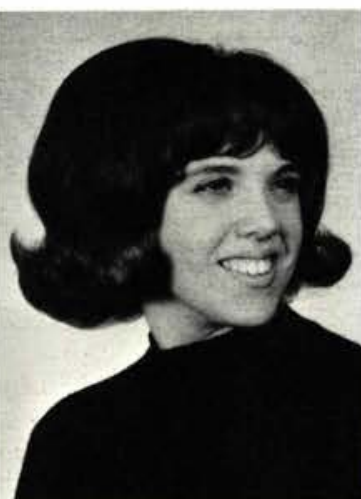

Cheryl Muzzy

Dayton, Ohio

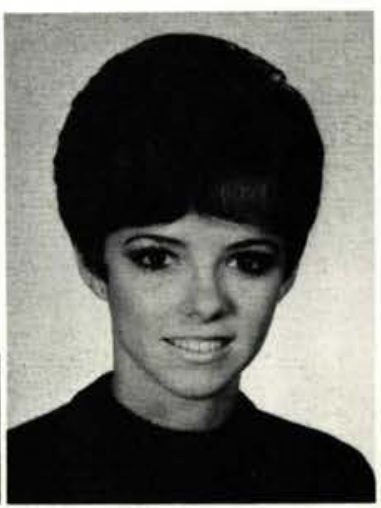




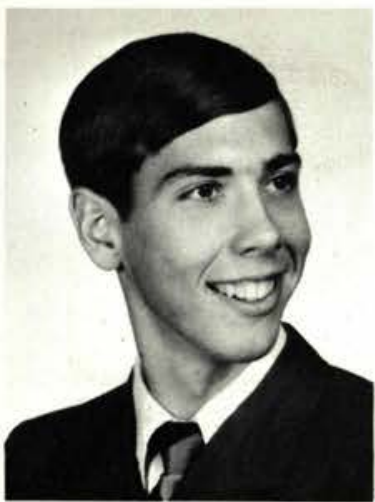

David Nelson

Santa Barbara, Calif.

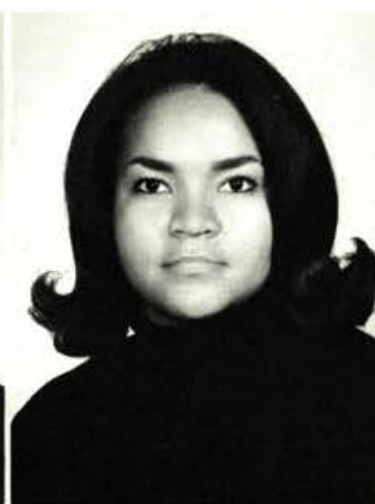

Sherie Nelthropp

St. Thomas, Virgin Islands

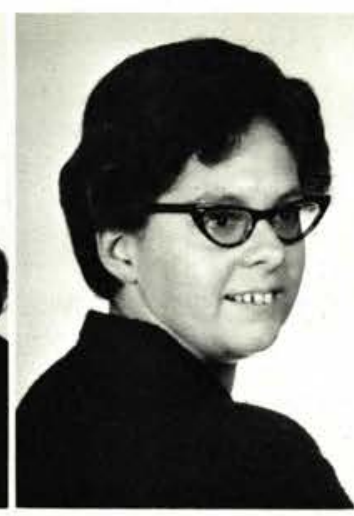

Bonnie Nolen

Wheelersburg, Ohio

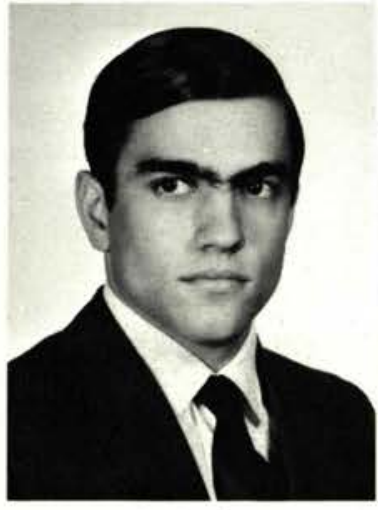

Ted Northey

Medina, Ohio

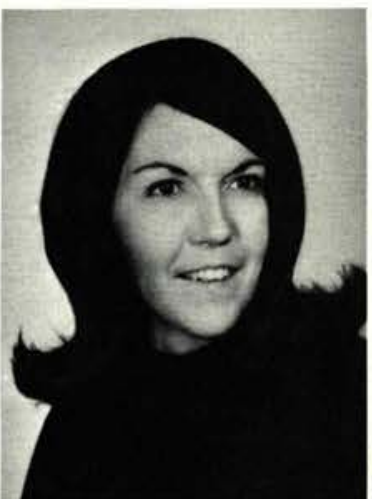

Jeanette Peek

Fort Wayne, Ind.

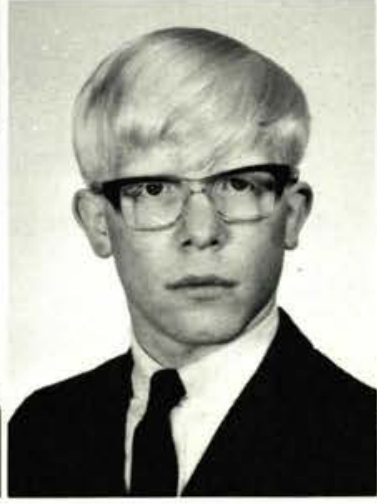

Mark Oklak

Mishawaka, Ind.

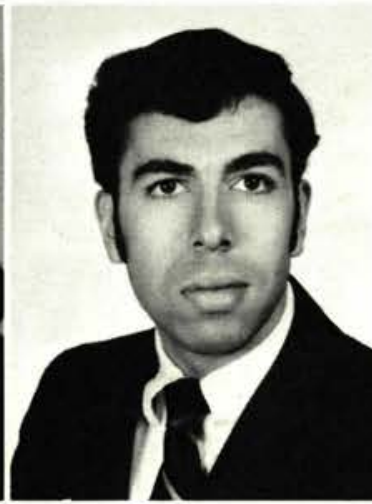

John Pereira

Kings Park, N. Y.

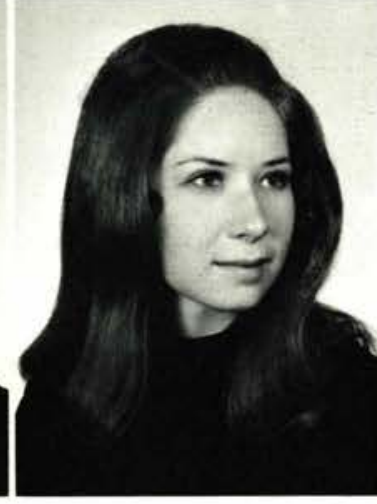

Laura Payne

Cedarville, $\mathrm{O}$.

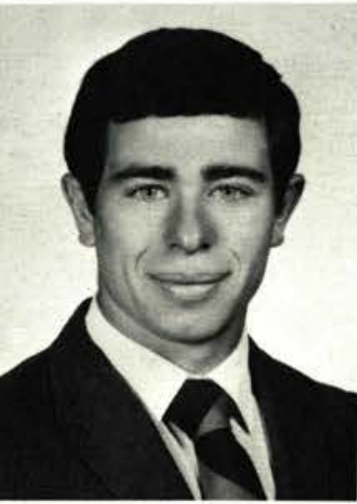

Ralph Perkins Denver, Colo.
Bruce Perry

Tiffin, $O$.

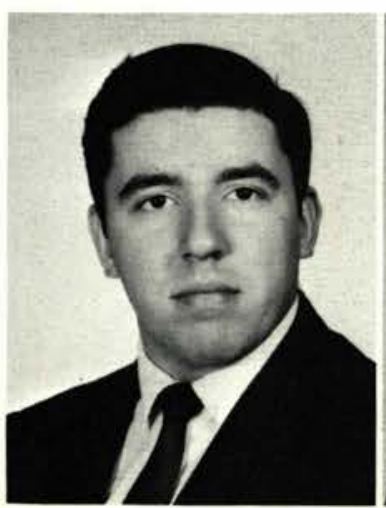

Cheryl Persing

Lodi, Ohio

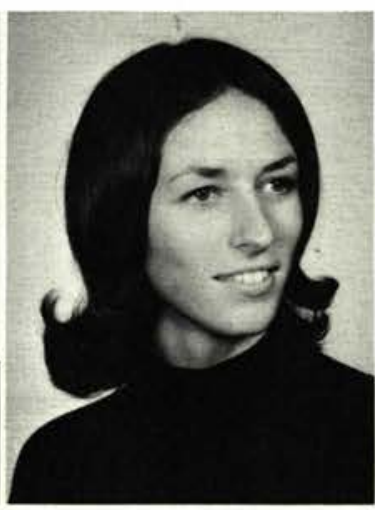

Bev Pestel

Ladysmith, Wis.

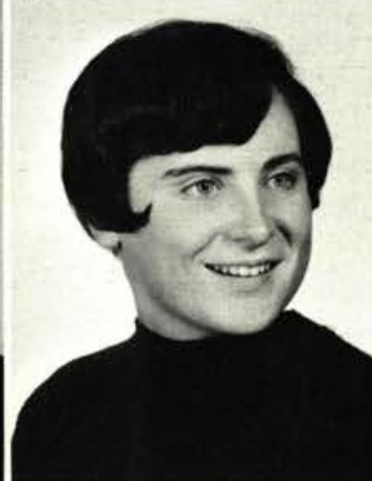

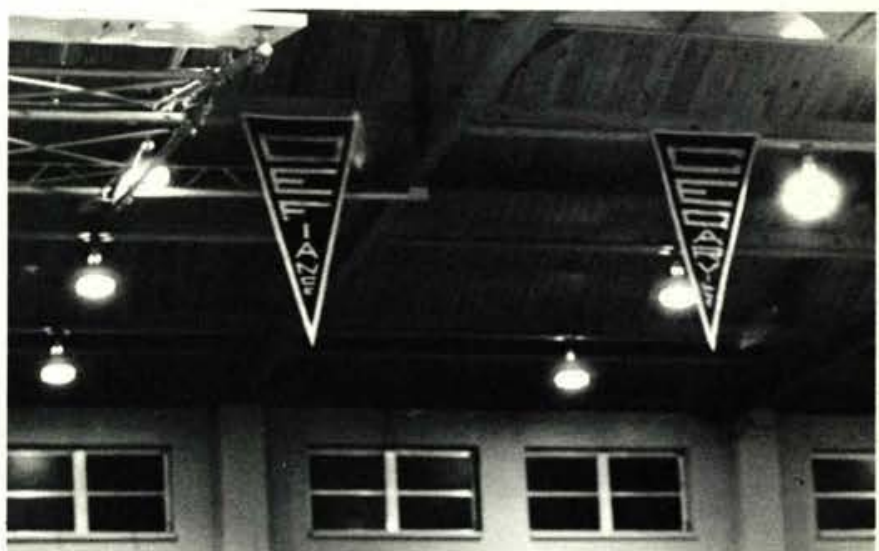

4.

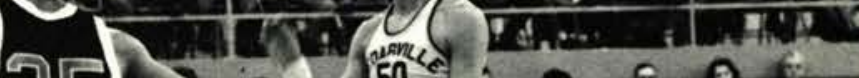

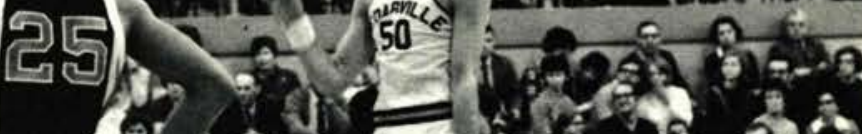

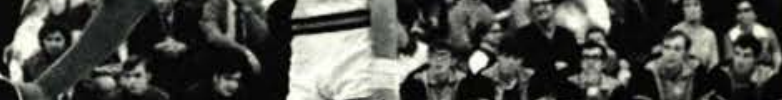

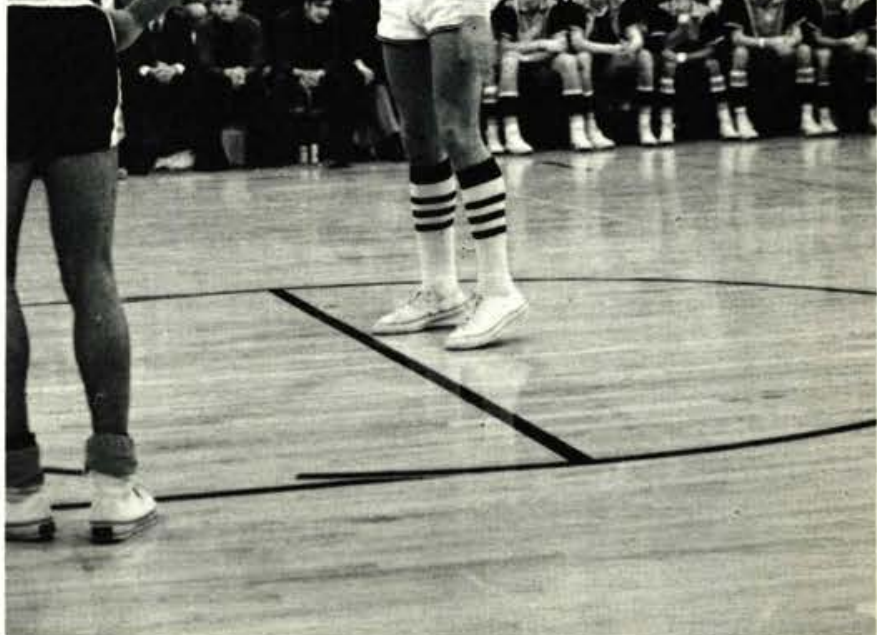




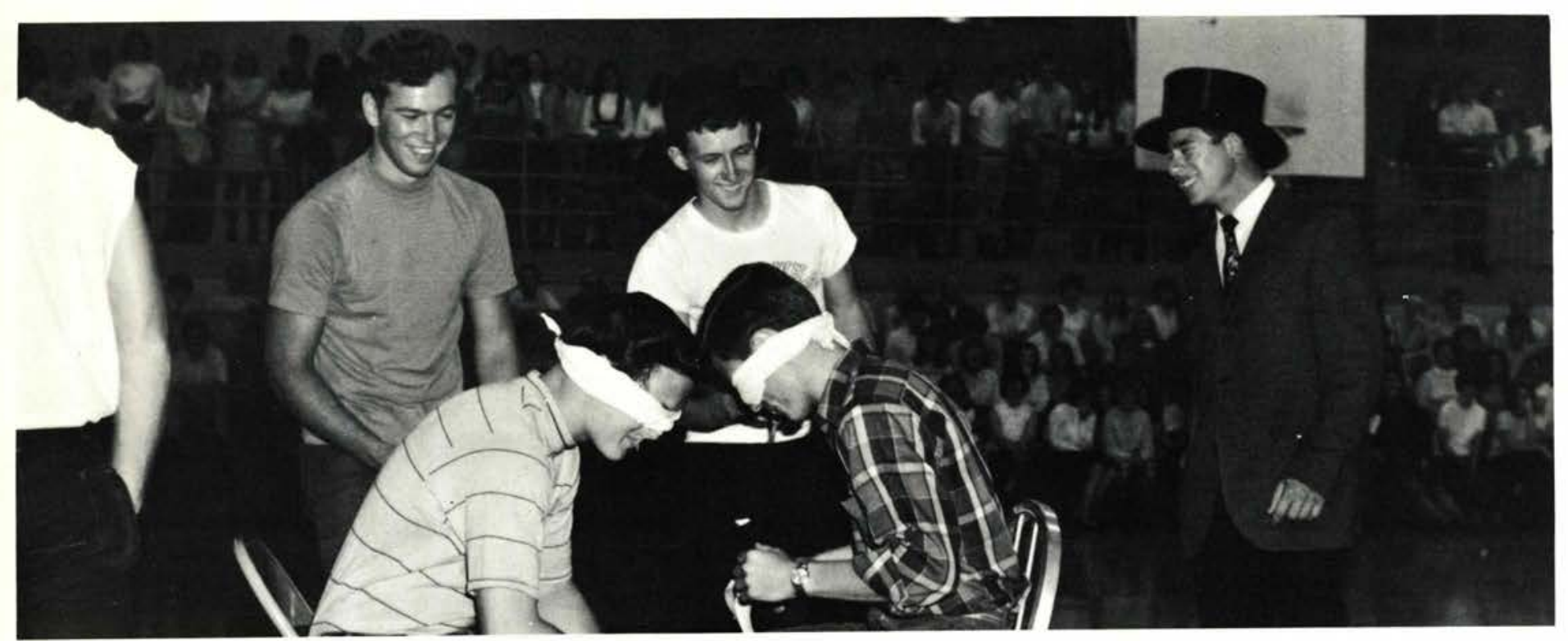

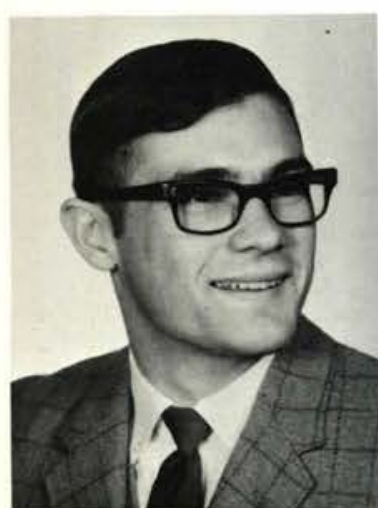

Dave Peters

Troy, Ohio

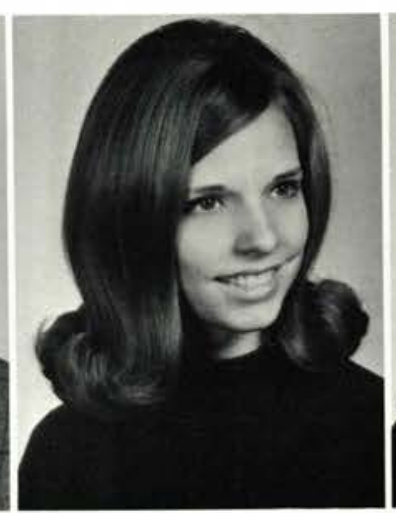

Karen Peterson

Colona, Illinois

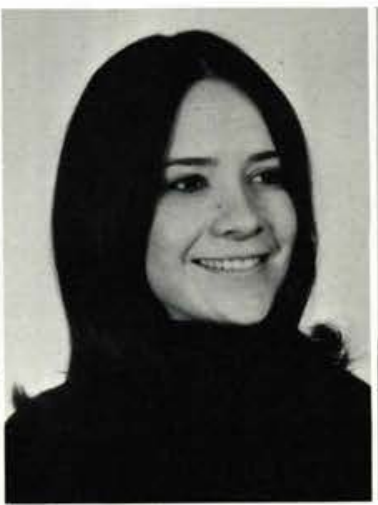

Kay Phenix

Cedar Falls, Iowa

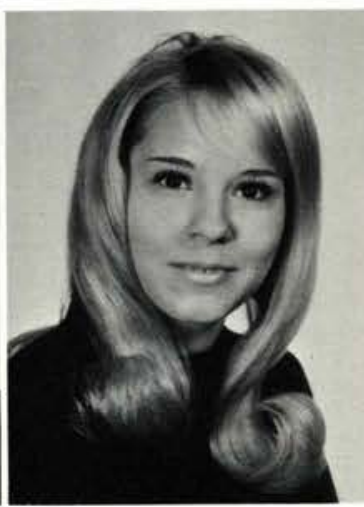

Rita Phipps

Indianapolis, Ind.

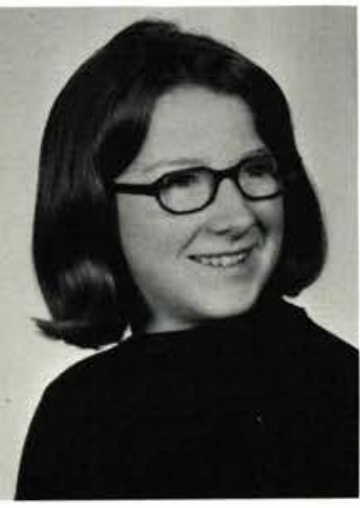

Tamera Porter Onaway, Mich.

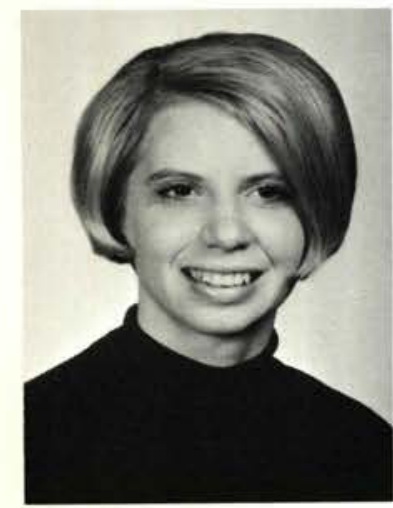

Sharon Pribble

Royal Oak, Mich.

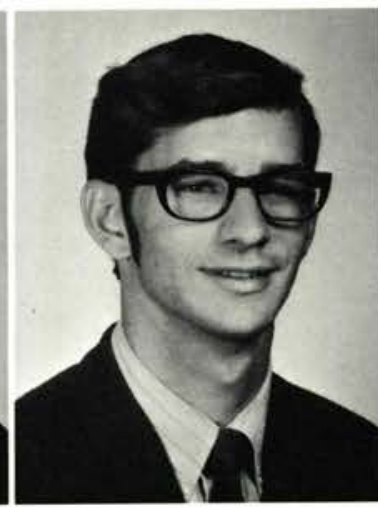

Daryl Prindle

Dowagine, Mich.

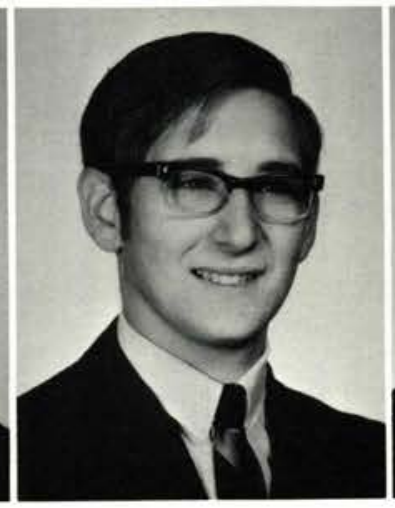

John Pruden

Prudenville, Mich.

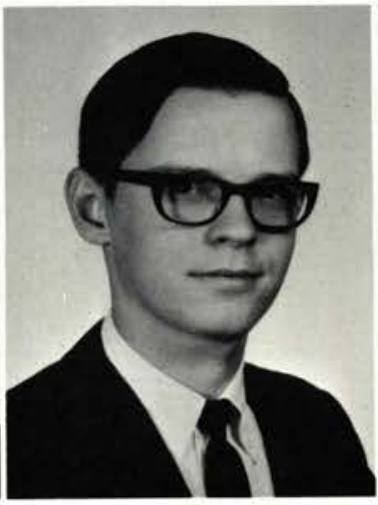

Paul Radcliff

Caldwell, Ohio

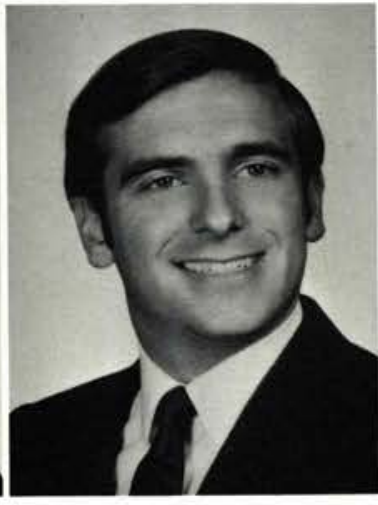

David Railsback

Olmstead Falls, O.
Deborah Reed Norwalk, Ohio

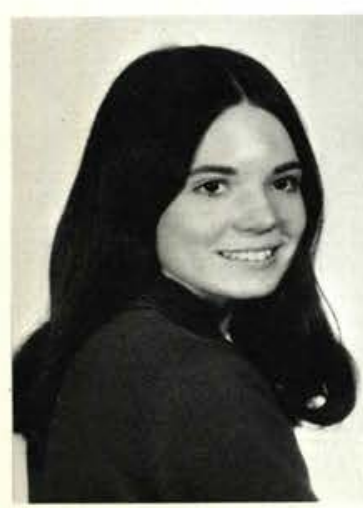

Tom Reeder

Youngstown, $\mathrm{O}$.

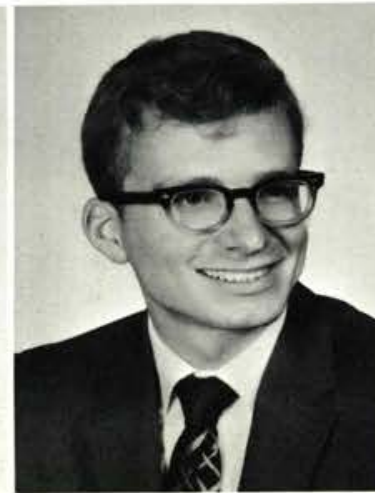

Curtis Rexroth

Silvis, Ill.

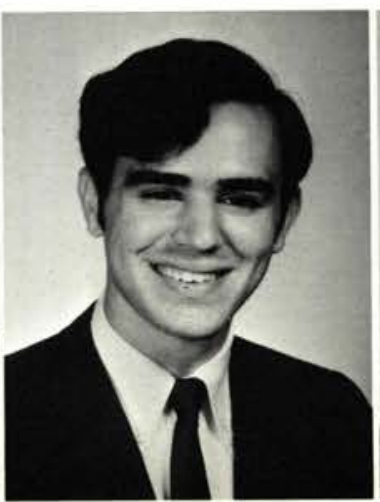

Patricia Riley

Newark Valley, N. Y.

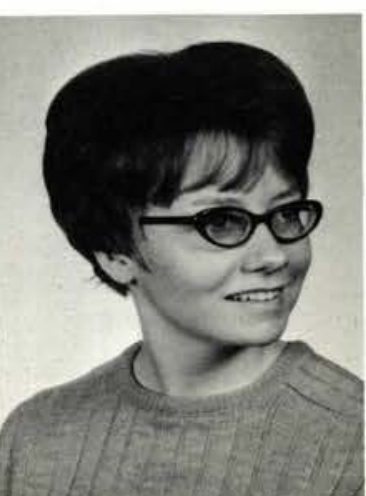

Lawrence Robinett

Todi, Ohio 


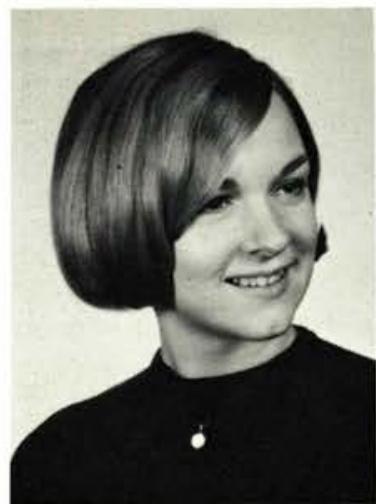

Shirley Rocher Amherst, Ohio

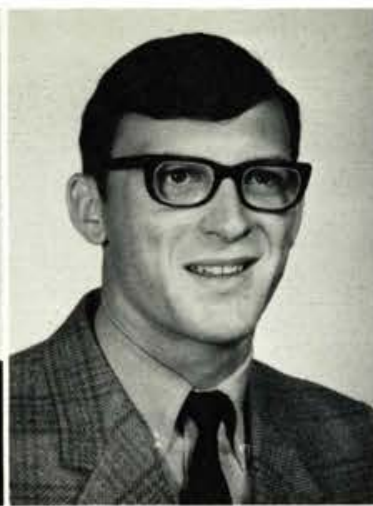

Lyle Rodman

Tower Hill, Ill.

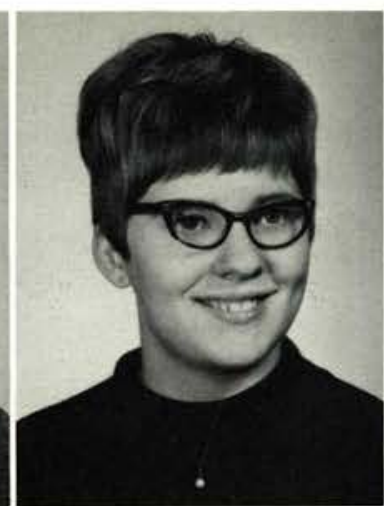

Sue Roloff

Charles City, Iowa

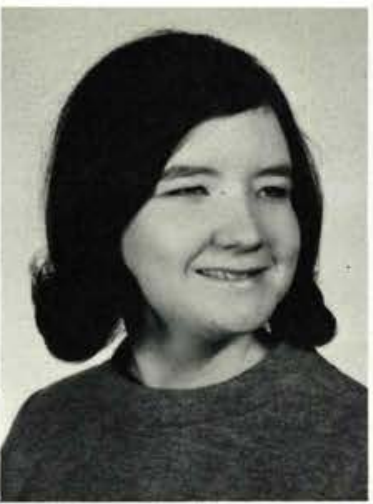

Ruth Ronsbottom

Pittsburgh, Pa.

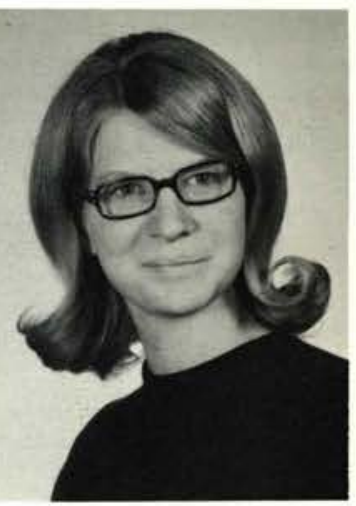

Darlene Rouch

Warren, $\mathrm{Pa}$.
Susan Scott

Key West, Fla.

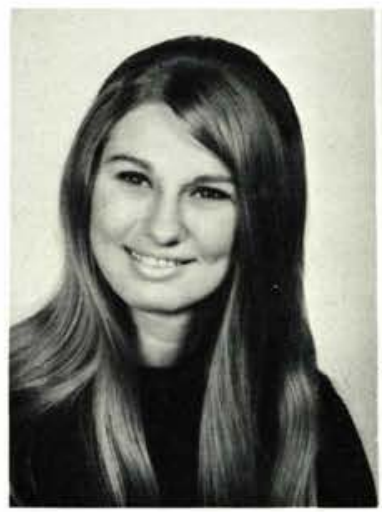

Suzanne Slater

Glen Aubrey, N. Y.

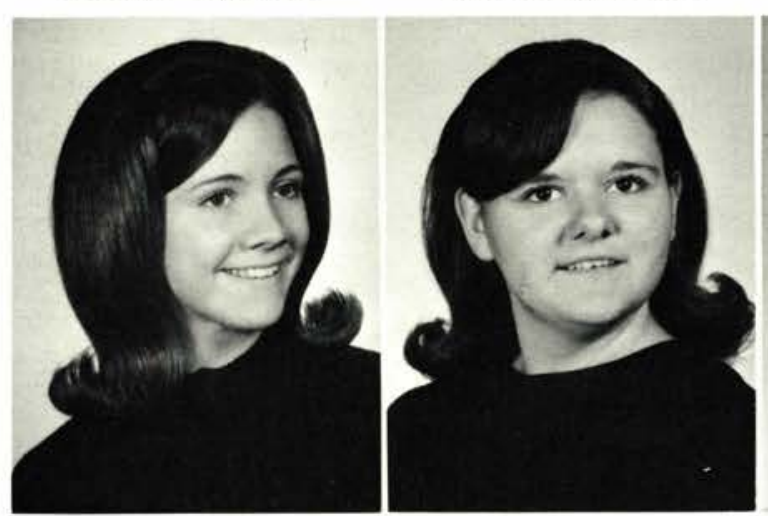

Tom Seidler

Cincinnati, $\mathrm{O}$.

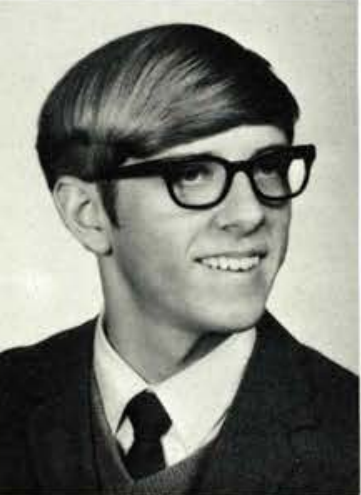

Deborah Slavers

Crawfordsville, Ind.
Bob Senseney

Linewood, N. Y.

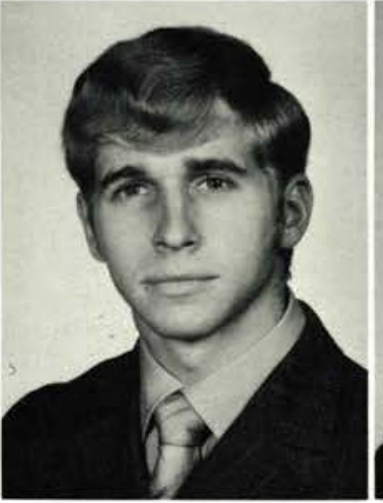

Donna Smith

Detroit, Mich.

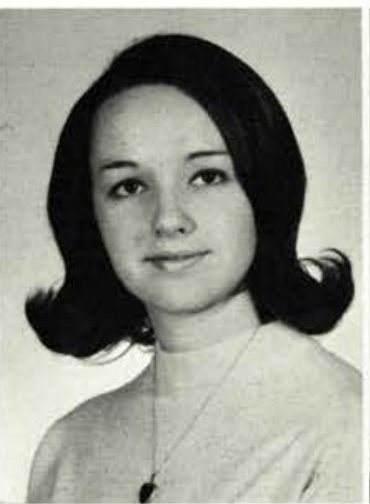

Cathy Shaw

Xenia, Ohio

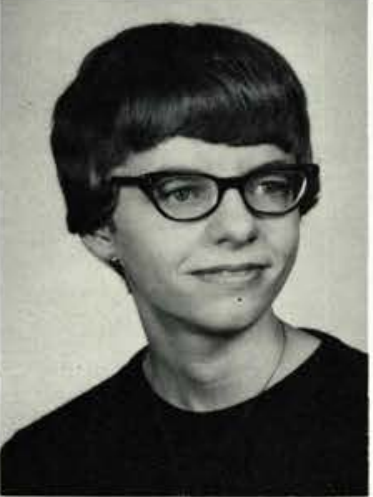

Nancy Smith

Sinclairville, N. Y.

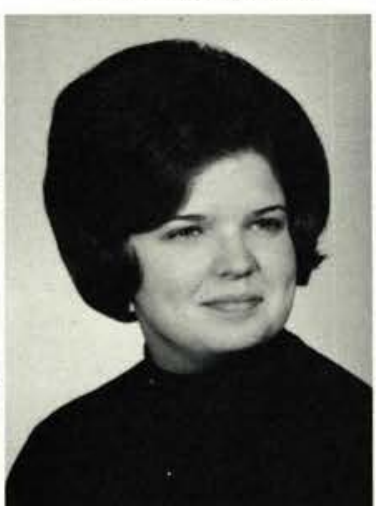

Gloria Shriver

Ursa, Illinois

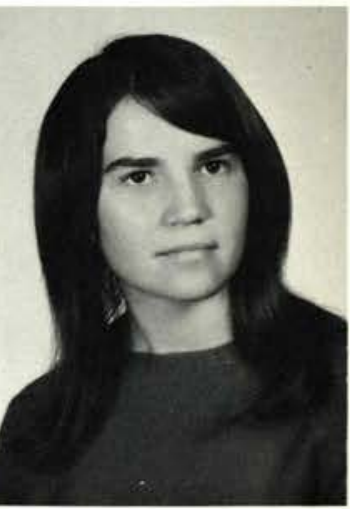

Bob Snyder

Ft. Wayne, Ind.

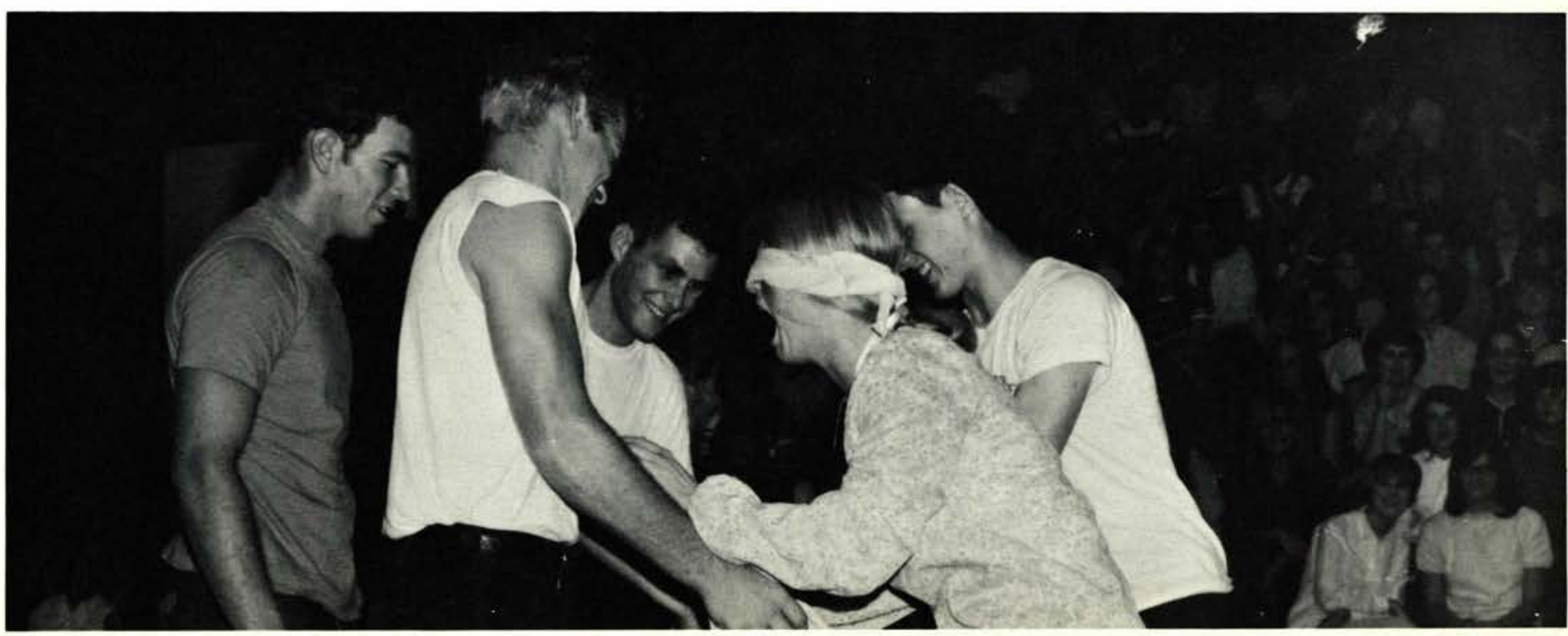




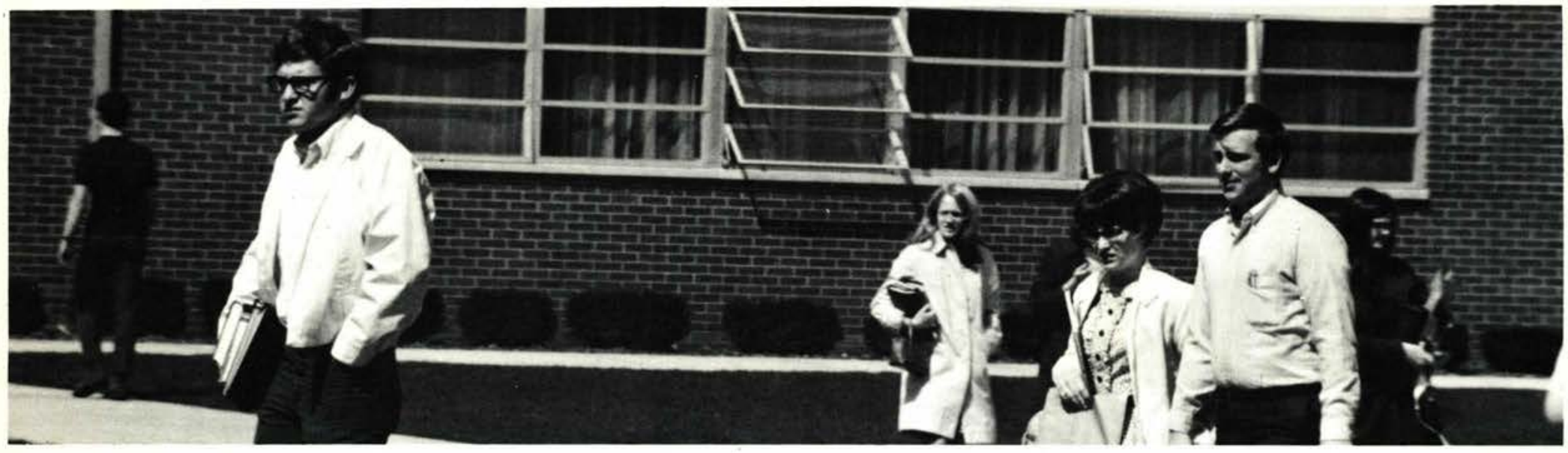

Linda St. Clair Cedarville, Ohio

Cherie Stock Lansing, Mich.

Mary Storey Erie, $\mathrm{Pa}$.
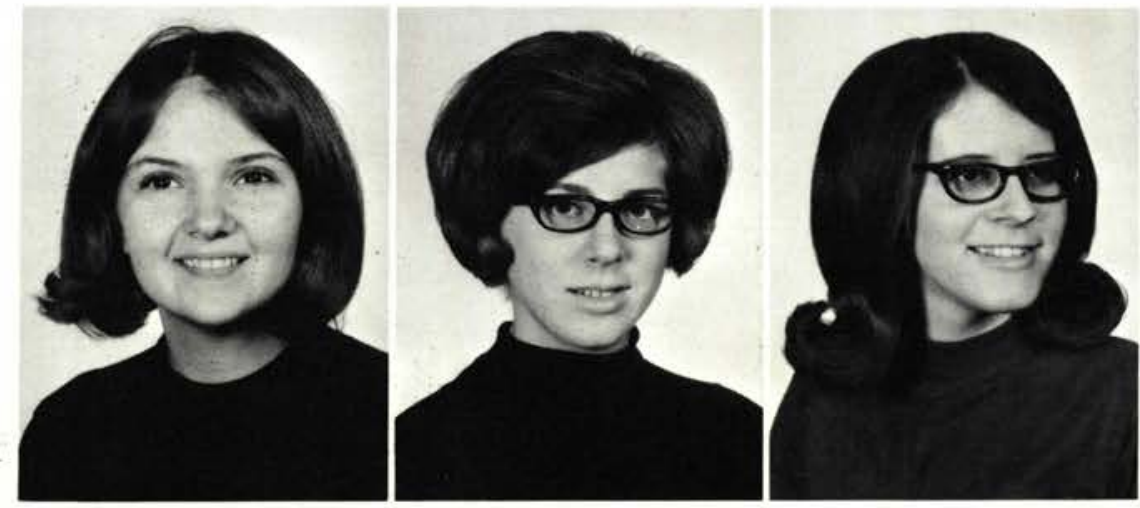

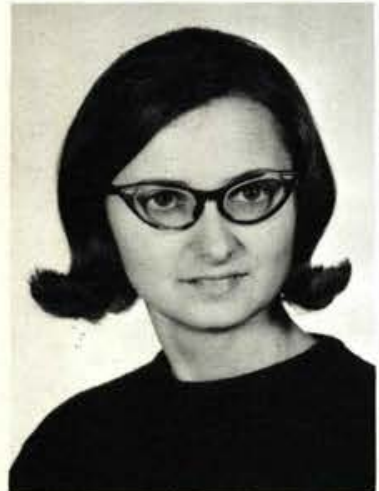

Carolyn Stubrich

Hubbard, O.

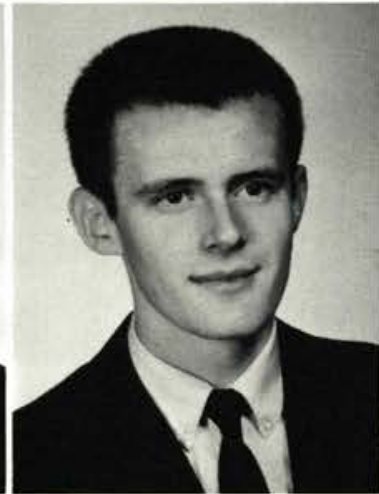

Roger Swanson

Rockford, III.

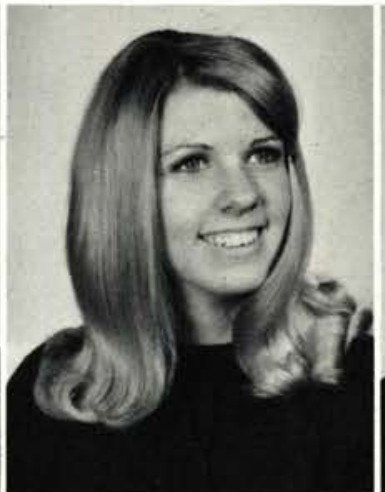

Karol Taylor

Steam Boat Rock, Ia.

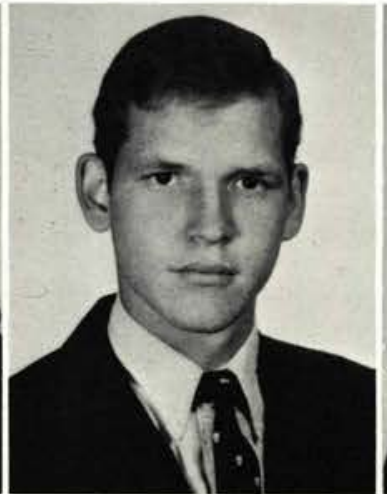

Louis Tewis Cedarville, $\mathrm{O}$.

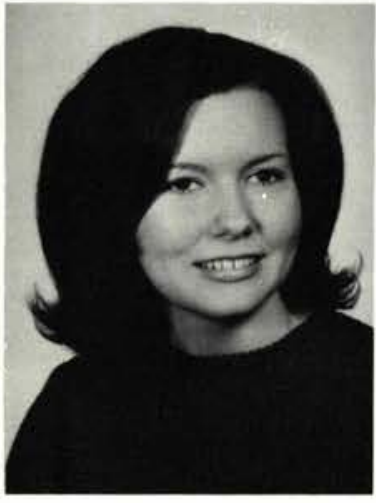

Janice Timpe

Clear Water, Fla.
Vicki Tobias

Port Allegany, $\mathrm{Pa}$.

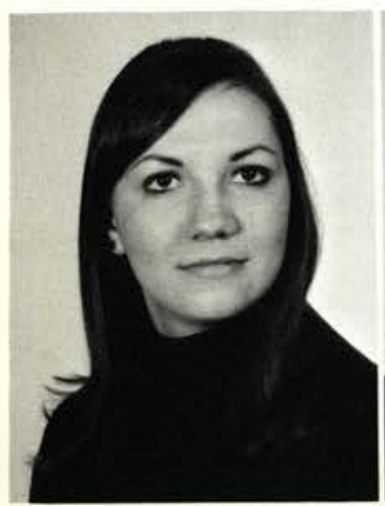

Connie Tompkins Sherman, N.Y.

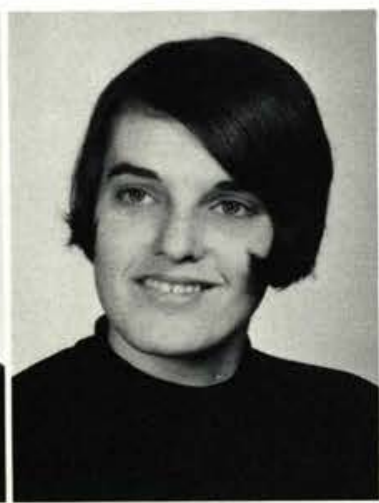

Mary Tress Apollo, $\mathrm{Pa}$.

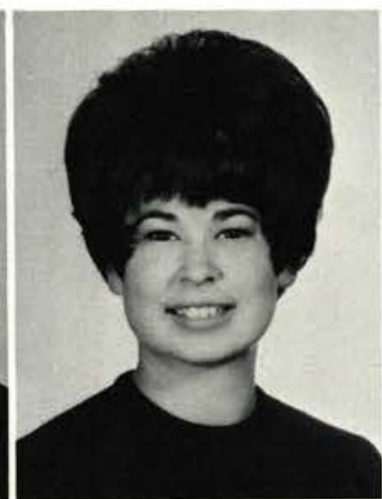

Mary Ubbink Bay City, Mich.

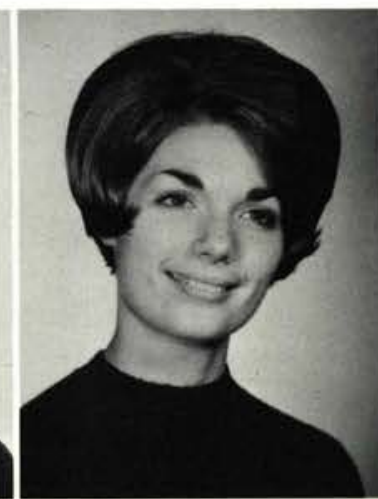

Carol Urban Denver, Colo.

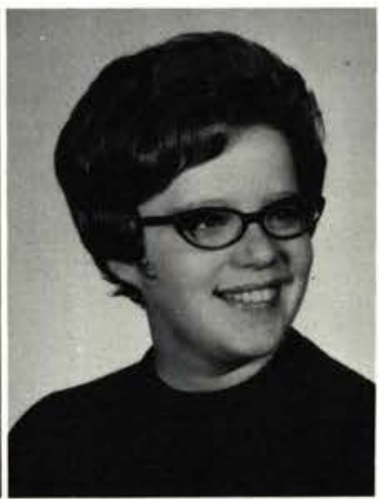




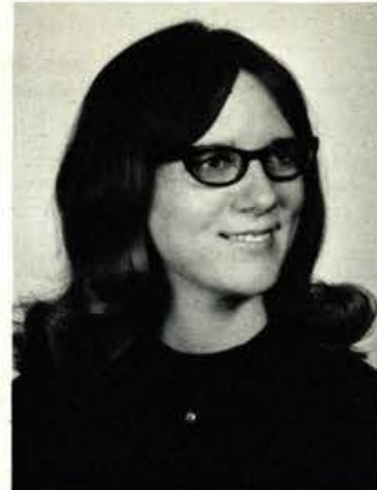

Mary Vanderpoo

Tekonsha, Mich.

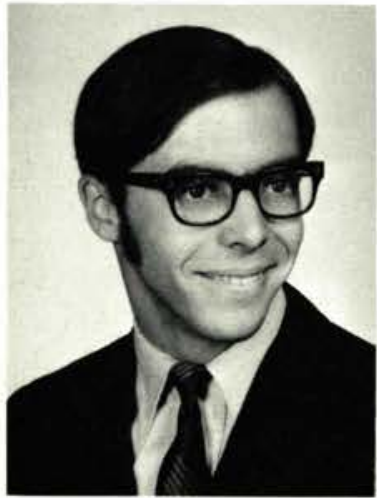

Tony Wall

Amherst, O.

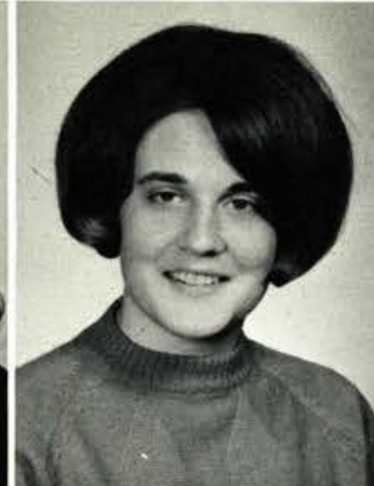

Elizabeth Viall

Austin, Minn

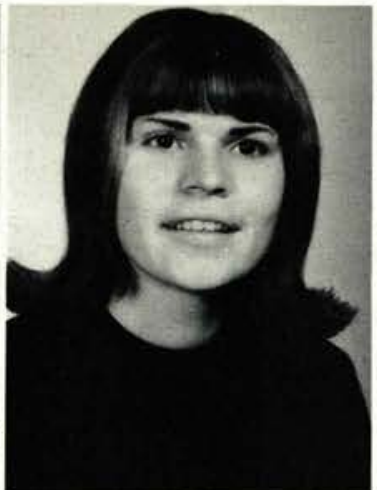

Janice Vlug

Wyoming, Mich.

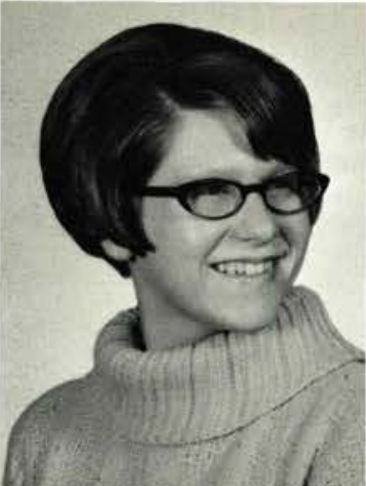

Corinne Waldron

Haviland, $\mathrm{O}$.

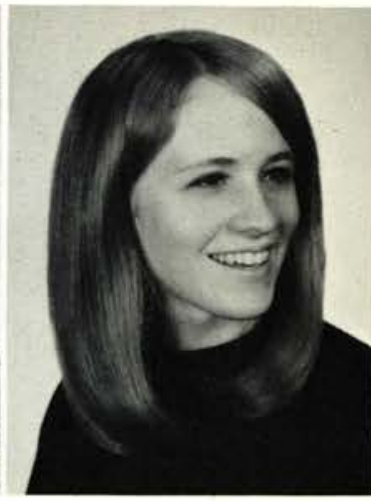

Connie Walker Kirtland, O.

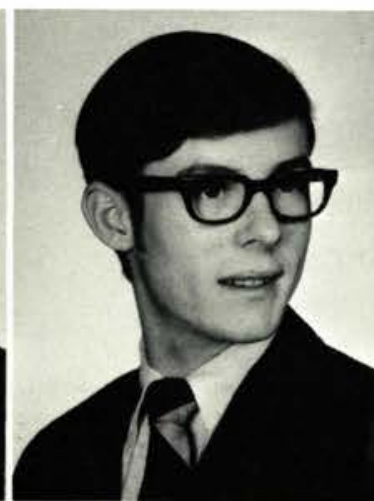

Carl Wallner

Jacksonville, Fla.

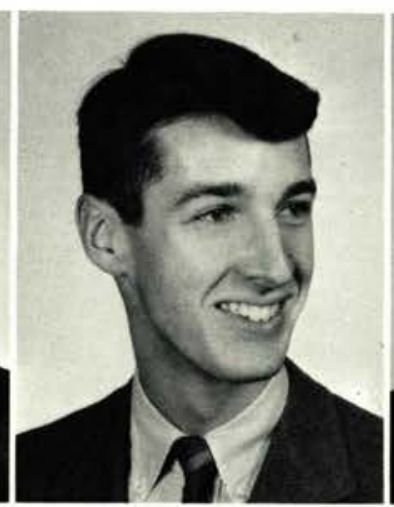

Paul Wheeler

Huntington, W. Va.

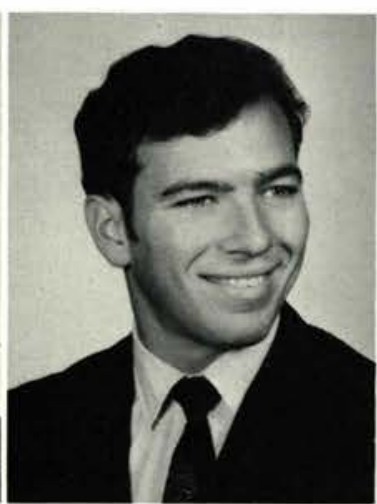

Mike Wilhite

Clayton, Ind.

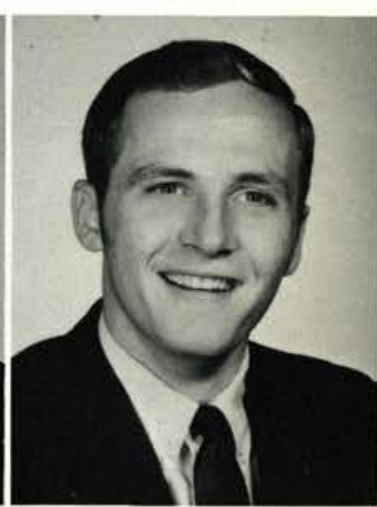

Charles Williams

Nebraska City, Nebr.

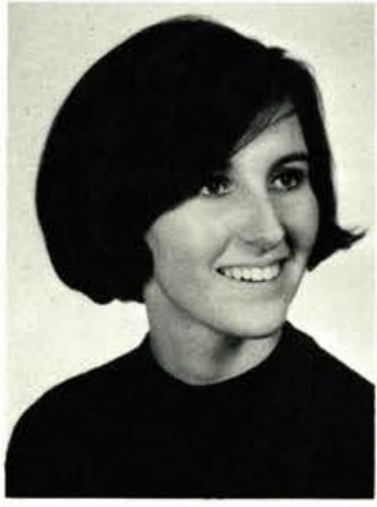

Diane Williamson Chicopee, Mass.

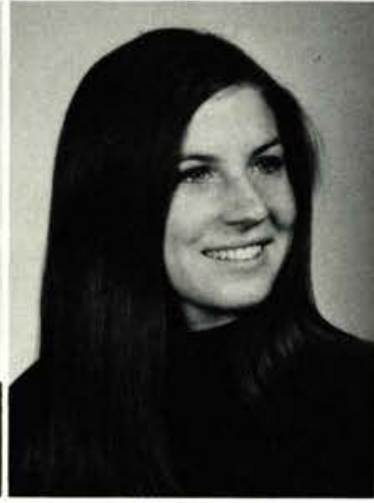

Charlotte Willis Rochester, Mich

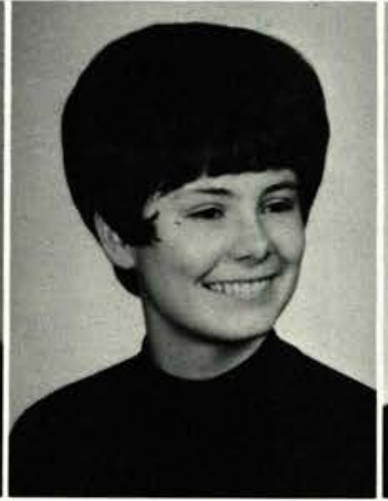

Alice Wilson

Crook, Colo.

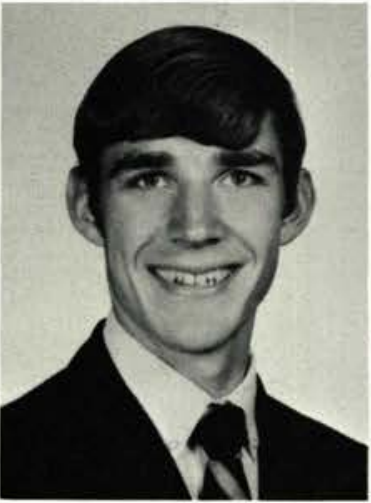

David Wood

Clarkston, Wash.

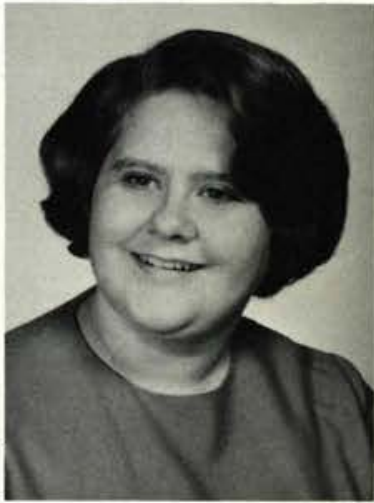

Rebecca Wood Kokomo, Ind.
Jeffrey Woodcock Jamestown, N. Y.

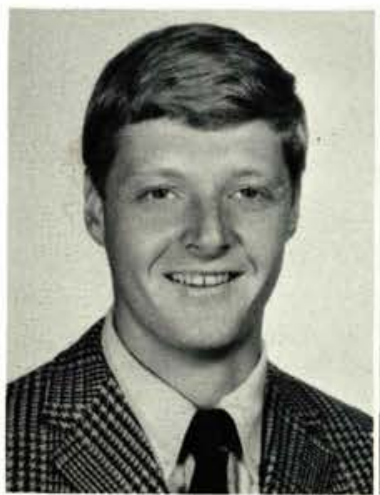

Rebecca Wright Grafton, $\mathrm{O}$.

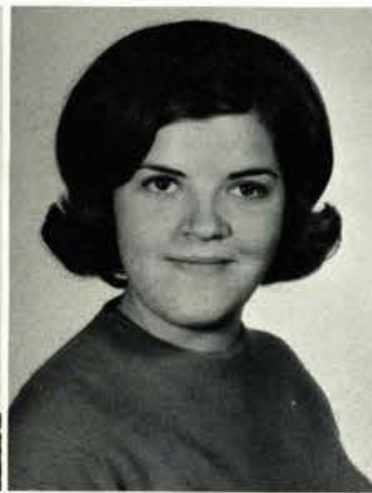

Ellen Young St. Paris, Ohio

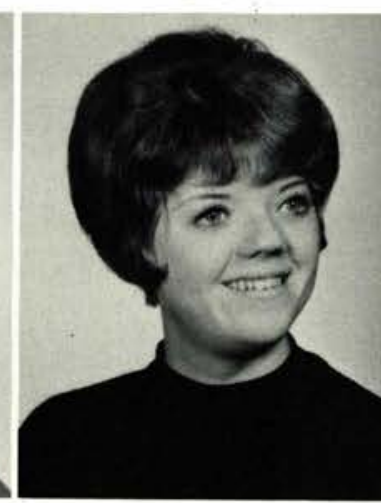

Mabel Young Mt. Pleasant, Mich.

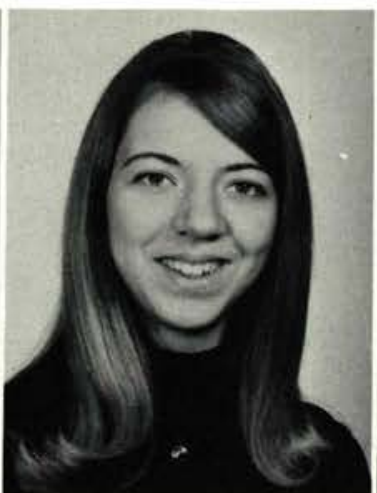

Monica Zimmerman Fuhushima, Ken, Japan 


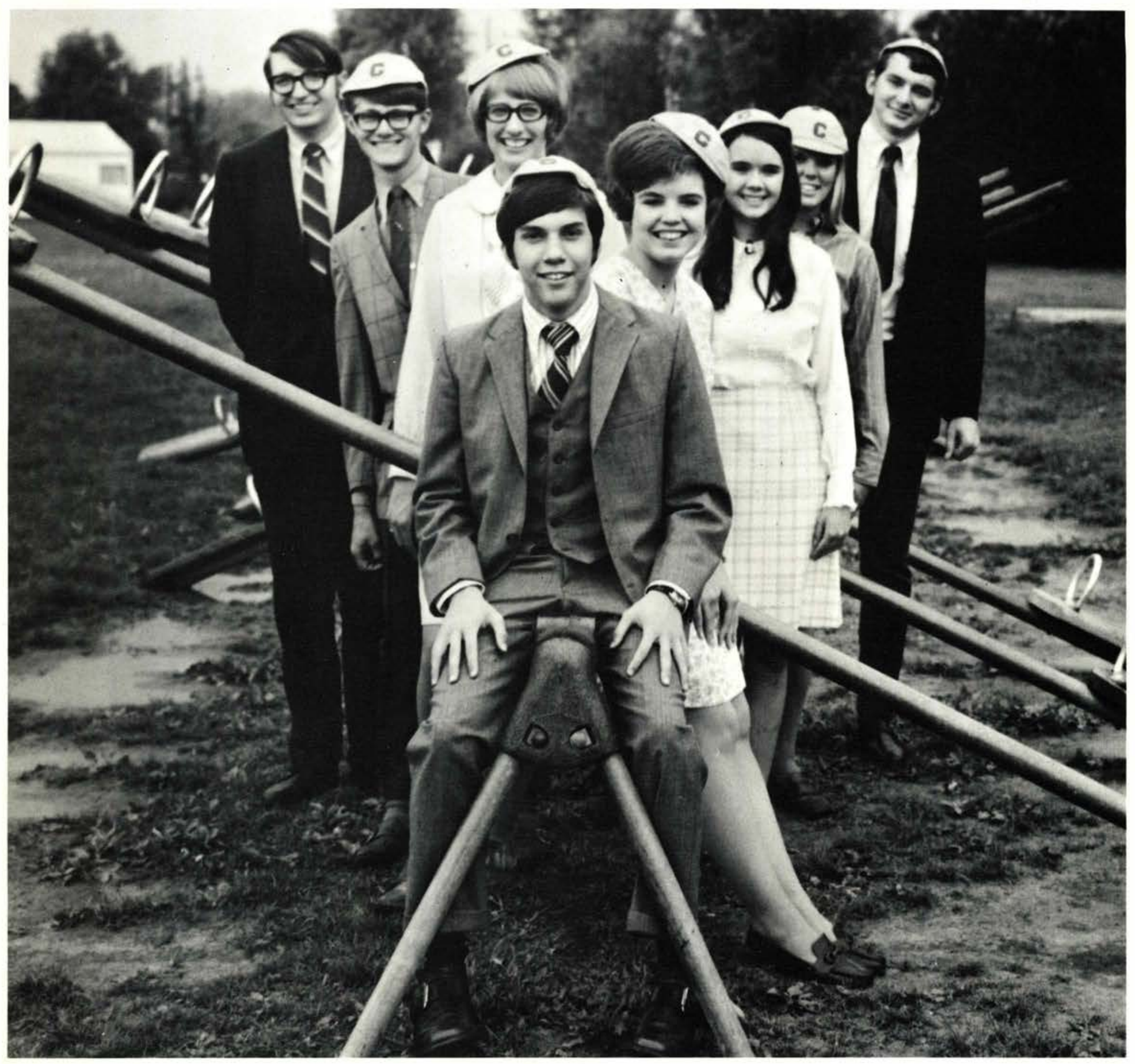

\section{FROSH CLASS OFFICERS}

Standing:

Seated:

Ron Sumner, President

Barb Cunnagin, Vice President
Rita Gast, Secretary

Linda Billington, Treasurer

Steve Hodgin, St. Council Rep.

Holly Wenner, St. Council Rep.

Mr. Wilcoxon, Advisor

Tim Andreanapolis, Chaplain 
Donald Acton

Mentone, Ind.

Timothy Adams

Silvis, Ill.

Donna Alberding

Wheatfield, Ind.

Mary Aldrich

Ithaca, N. Y.
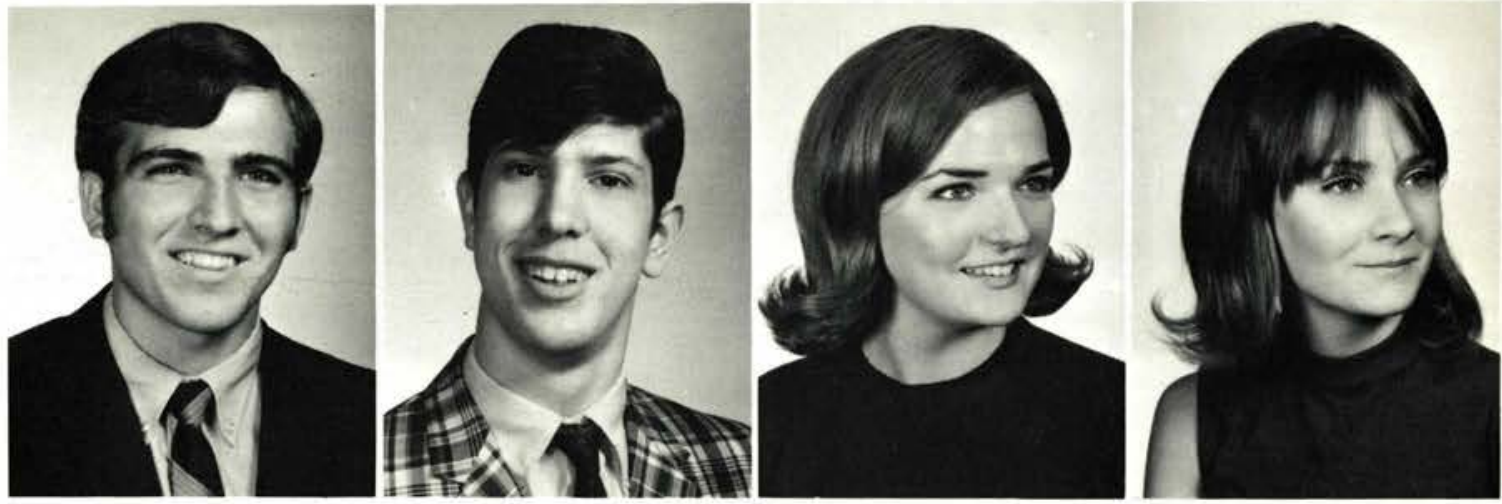

Mark Alexander Stryker, Ohio

Larry Alfrey

Adrian, Mich.

Kristen Anderson

Grand Rapids, Mich.

Tim Andreanopoulos Oakland City, Ind.
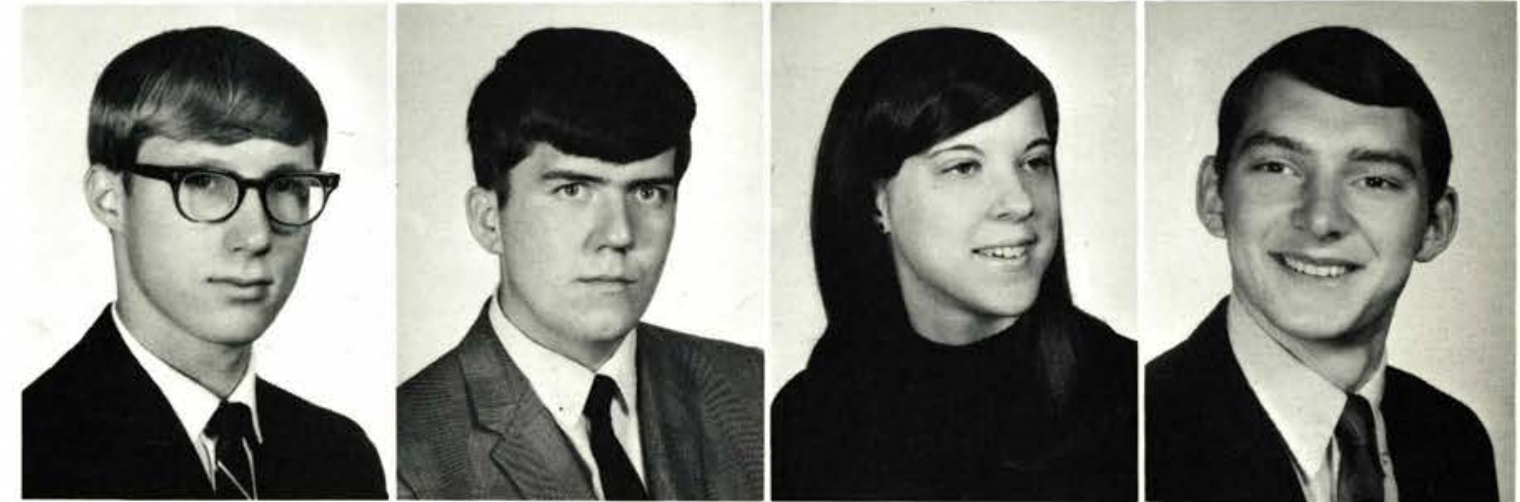

William Bacheller Cortland, New York

Anne Bailey

Endicott, New York

Keith Bailey

Charleston, West Va.

Marilyn Bailey

Bronson, Michigan
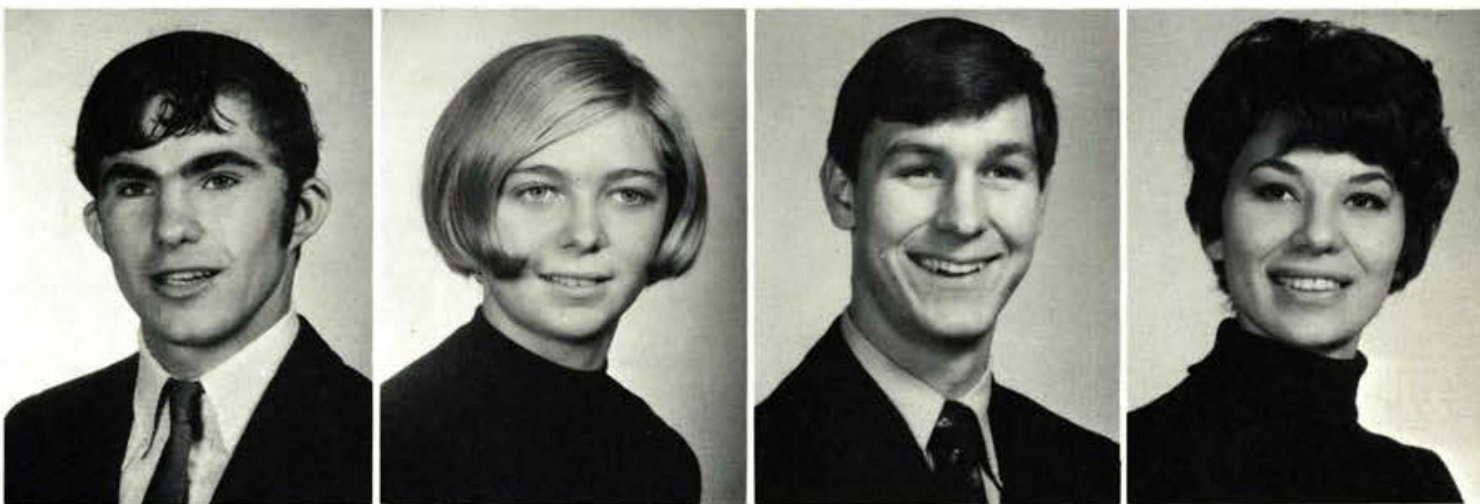

Randy Bailey

Warren, Ohio

Linda Baker

Randolph, New York

Deborah Bard

Linda Bernett

Tonawanda, New York
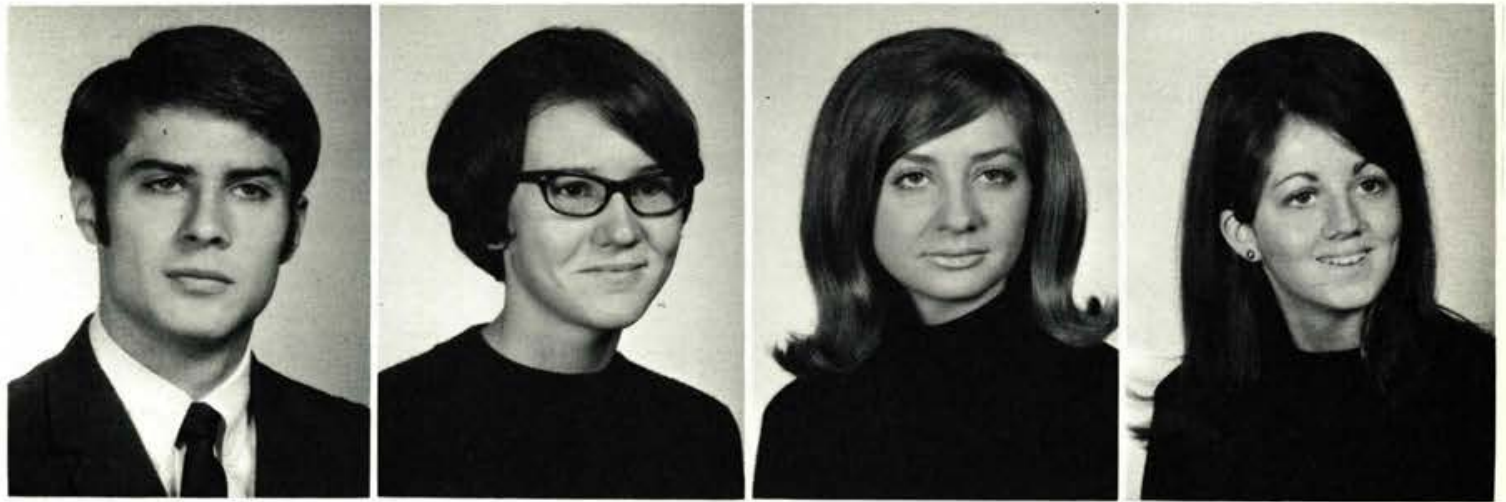

Monica Bayne

Little Hocking, Ohio

Gloria Beard

Nancelona, Michigan

Jim Bechtel

Des Moines, Iowa

Jon Bell

Colfax, Iowa
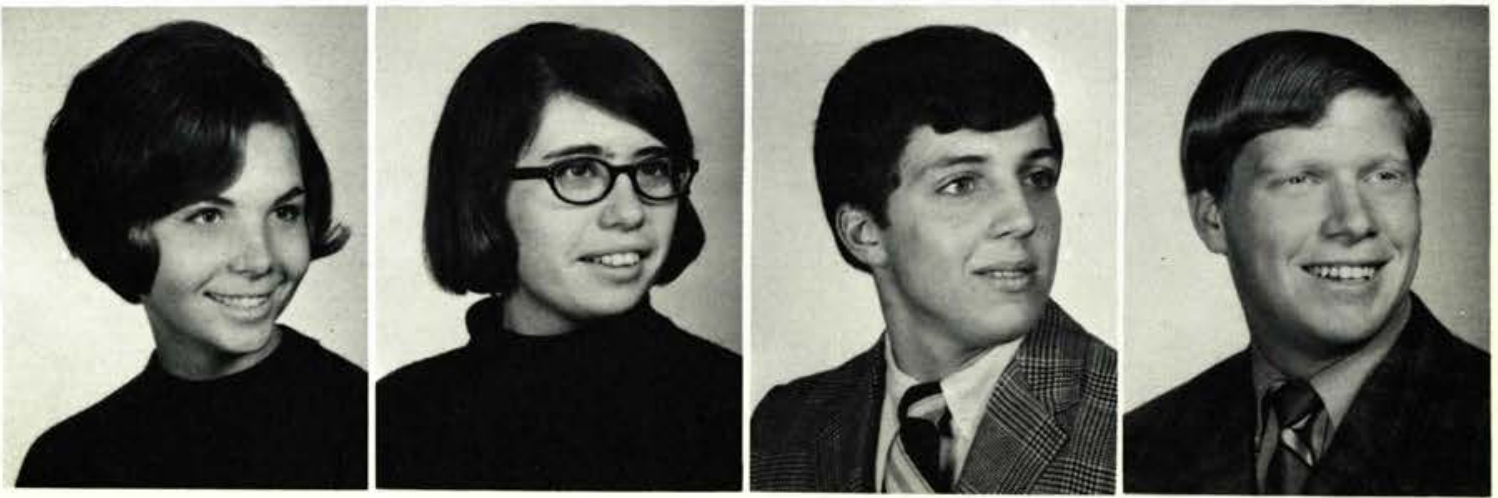


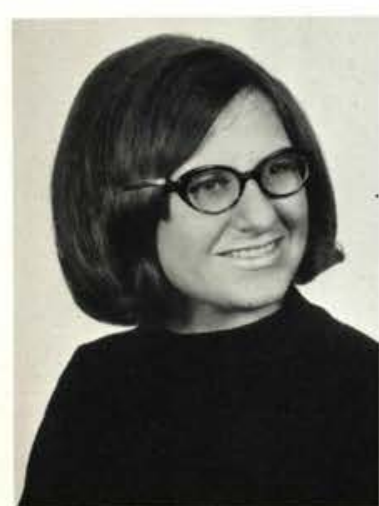

Debbie Berry

New Boston, Mich.

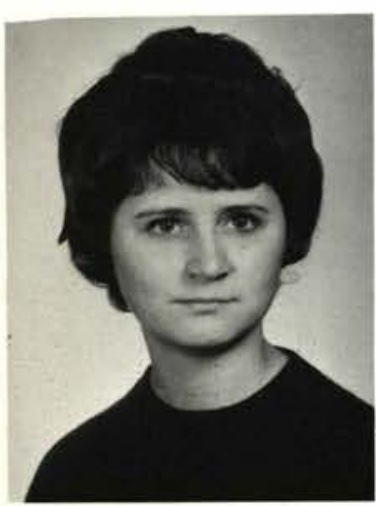

Bonny Bodenmiller Homerville, Ohio

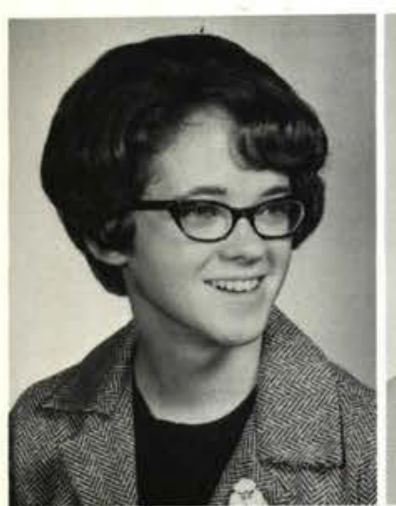

Marilyn Brown

Holly, Mich.

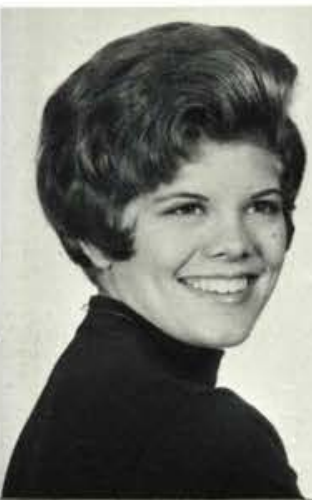

Carol Bierbaum

Bunker Hill, Ill.

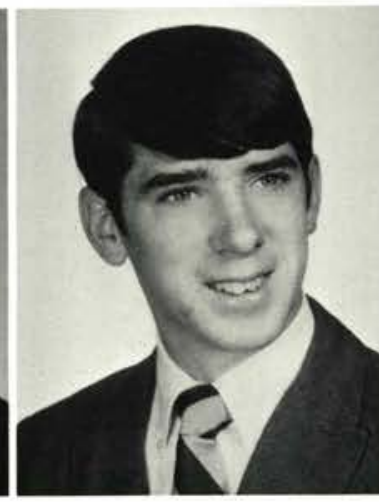

Daniel Bogert

Sherman, N. Y.

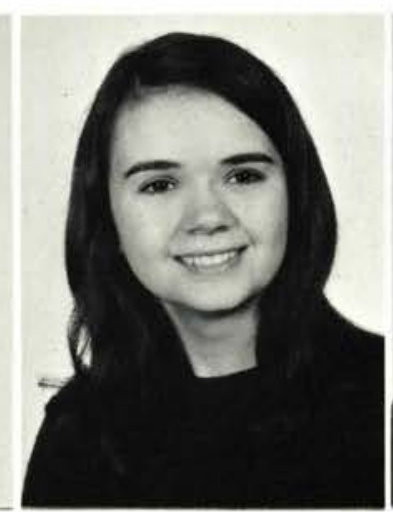

Linda Billington

Fostoria, Ohio

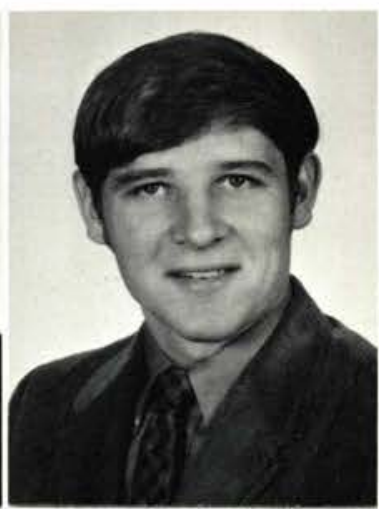

Tim Black

Algona, Iowa

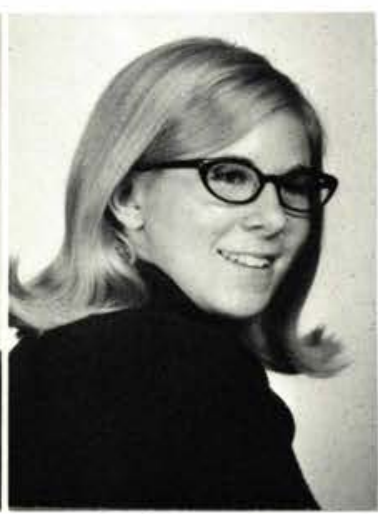

Carol Anne Bo

Rochester, N. Y.

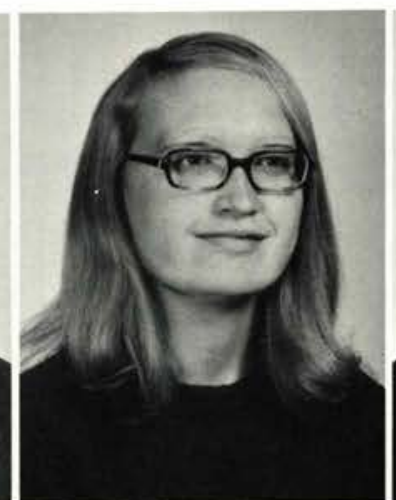

Pamela Borg

Flint, Mich.

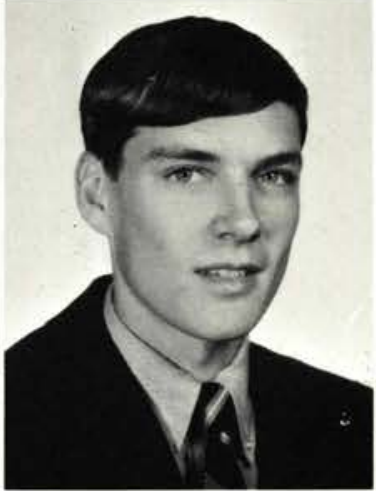

David Branon

Portsmouth, O.

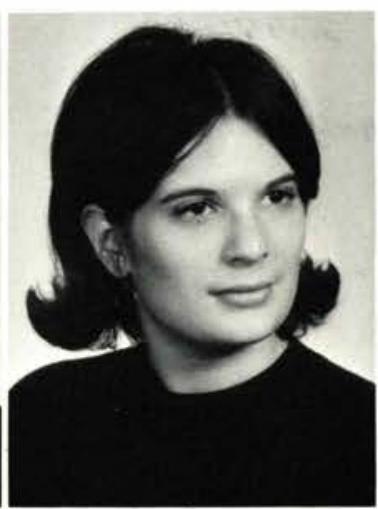

Pam Briggs

New Ipswich, N. H.

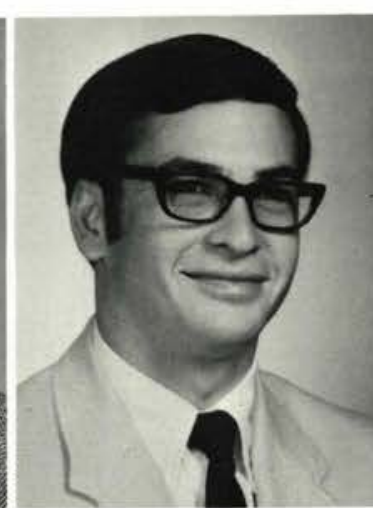

Randy Brown Arcanum, Ohio

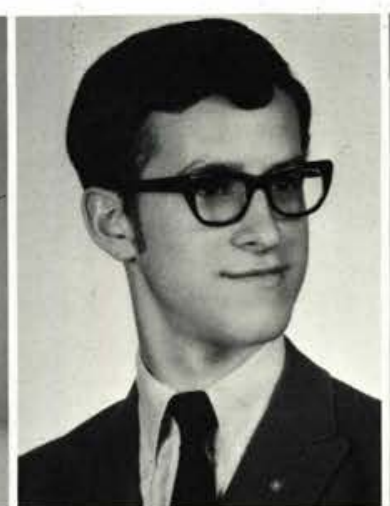

Jim Bucholtz Arcanum, Ohio

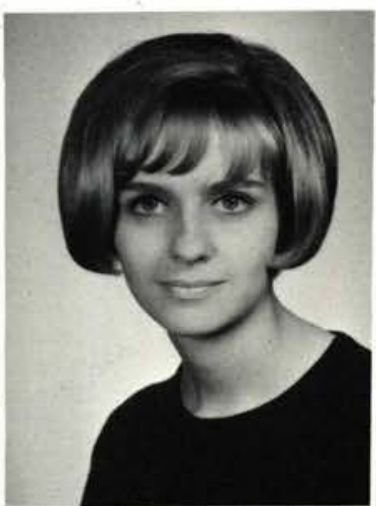

Cheryl Buck

Cleveland Heights, $O$.

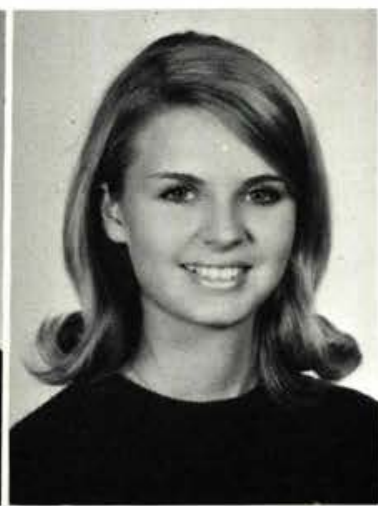

Ginger Burget Toledo, Ohio
Kathy Burkhard

Fort Lauderdale, Fla.

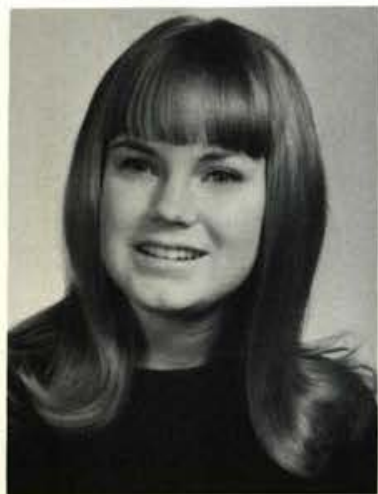

Lois Burlingame Almont, Michigan

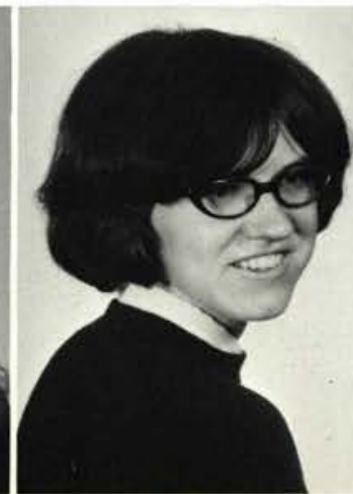

Sherry Burns

New Madison, $\mathrm{O}$.

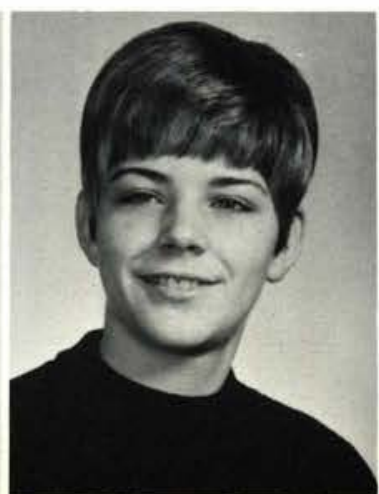

JoAnn Buschman Widefield, Colo.

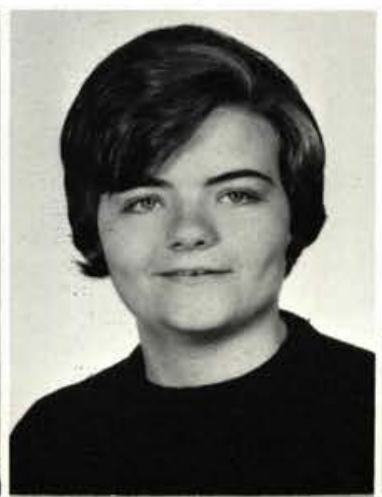

Mary Ann Butcher Alexandria, Vir.

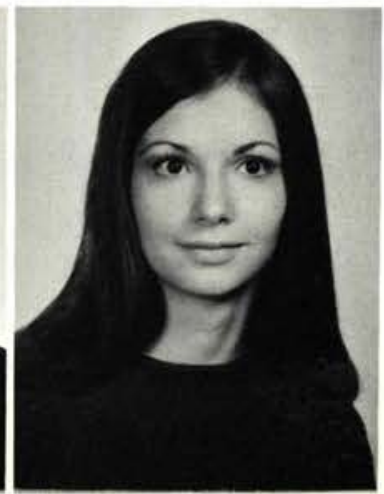




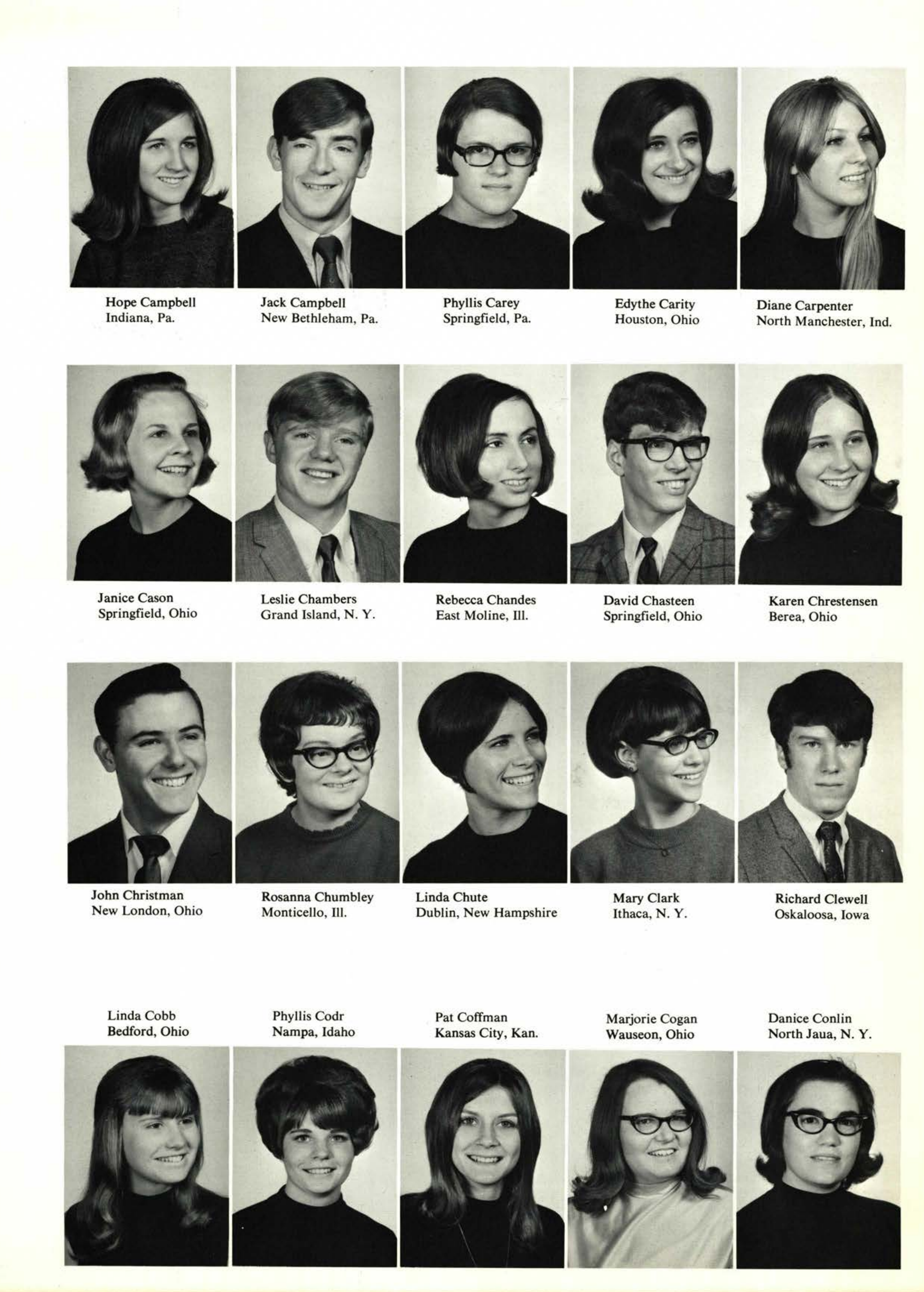




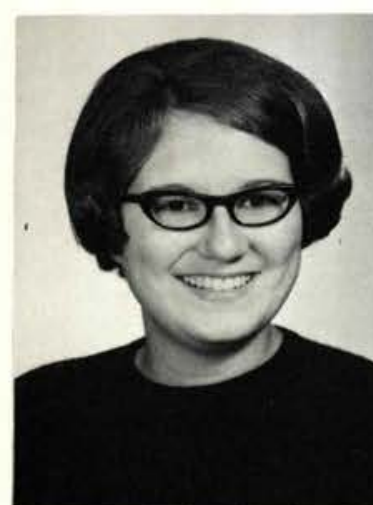

Ruth Coombs

Painted Post, N. Y.

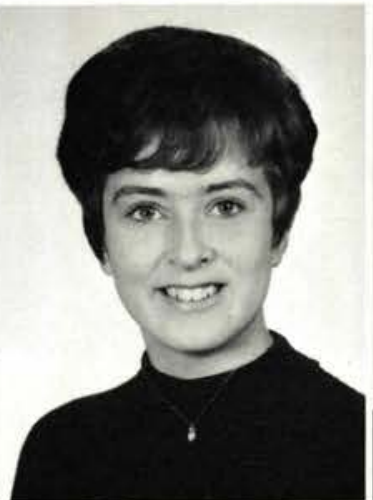

Barbara Cooper

N. Tonawanda, N. Y.

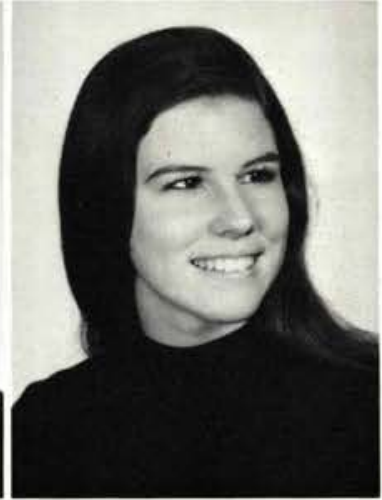

Yvonna Cope

Crawfordsville, Ind.

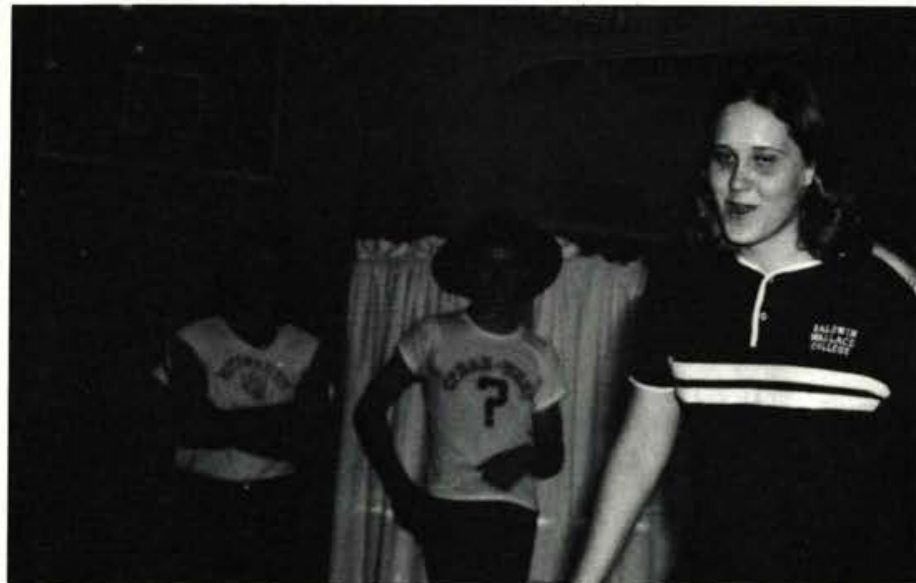

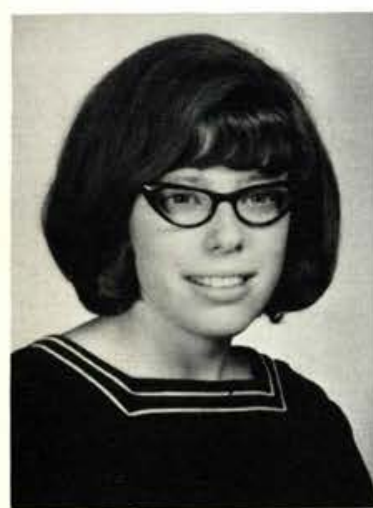

Bonnie Cowley

Pontiac, Mich.

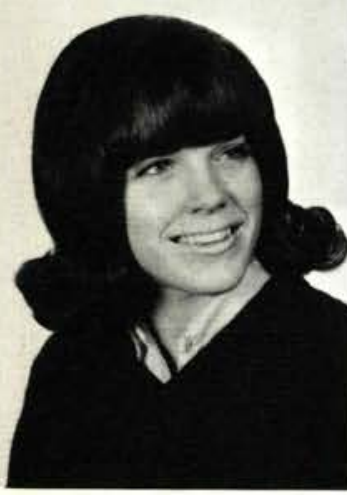

Pamela Davison Arcanum, Ohio

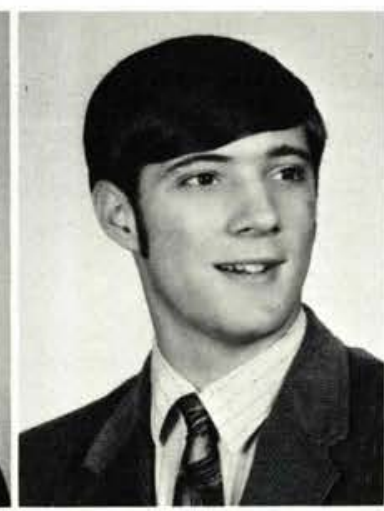

Ron Craig

Springfield, O.

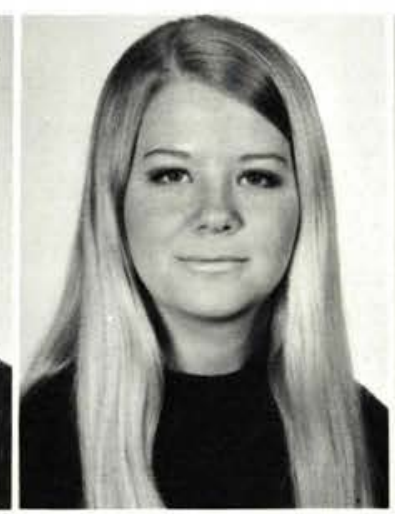

Geri Crotty

Detroit, Mich.

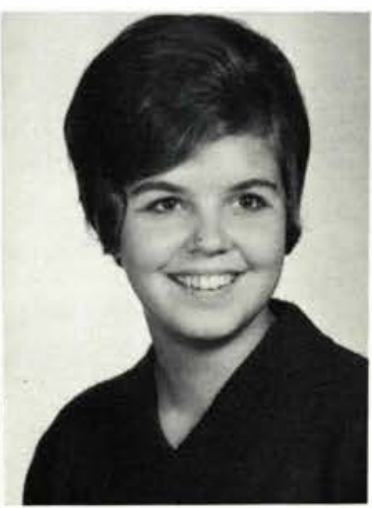

Barbara Cunnagin

Dayton, Ohio

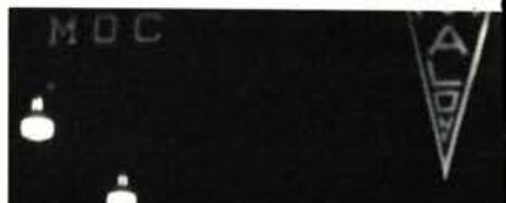

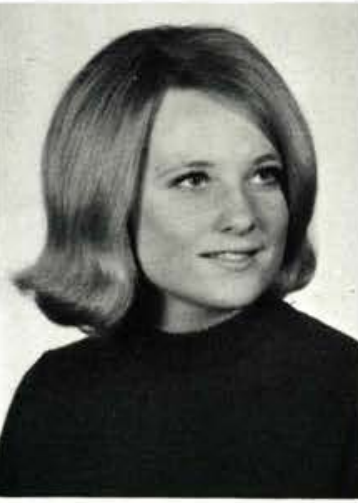

Roxane Davis

Chautauqua, N. Y.

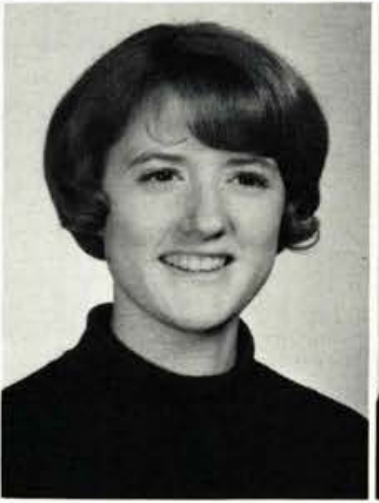

Judy Dawson Peru, Ill.

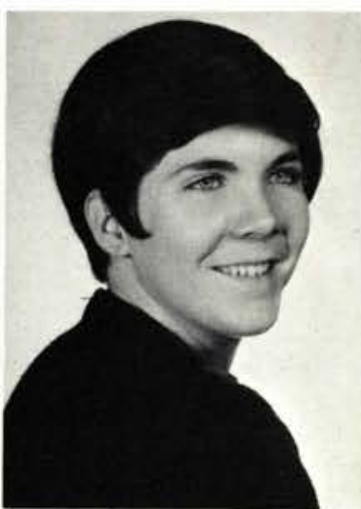

Rebecca Decker

Vienna, W. Va.

Sandy Delong

Troy, Ohio

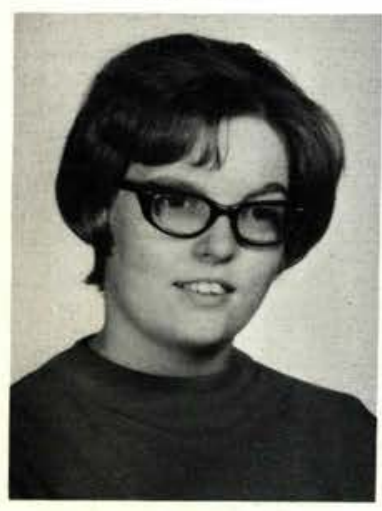

Karen DeMars Columbus, Ohio

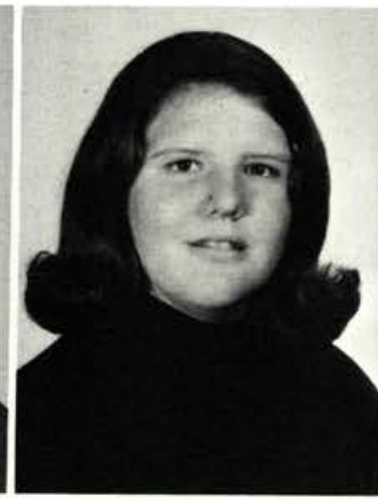

Carol Demos

Dublin, Ohio

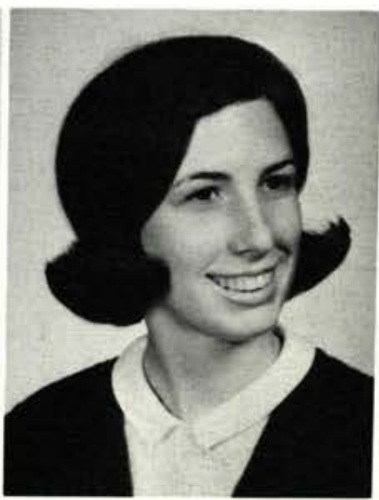

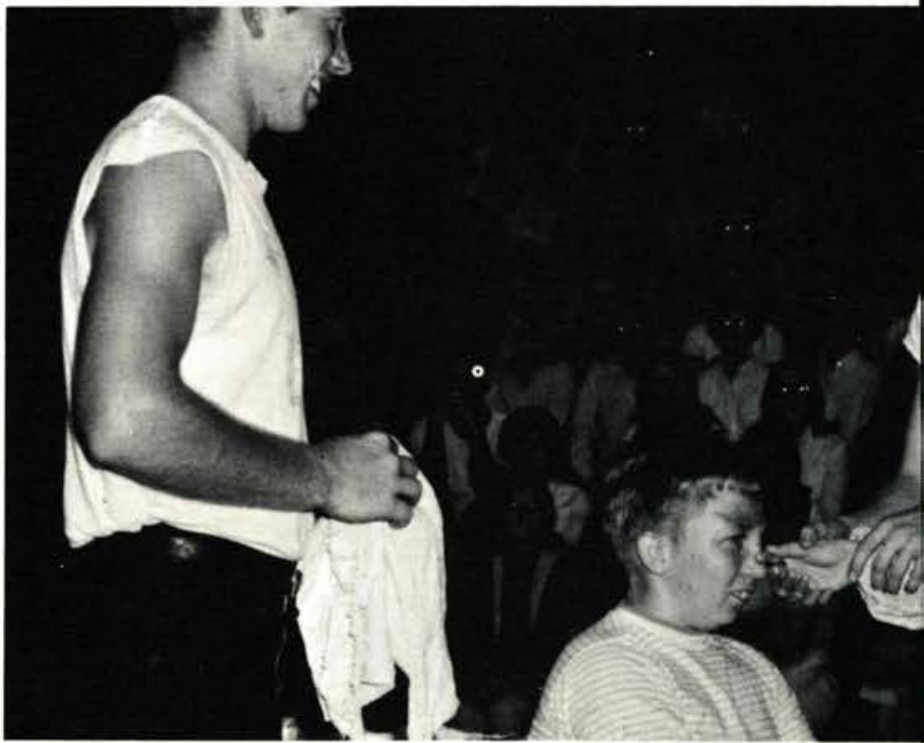



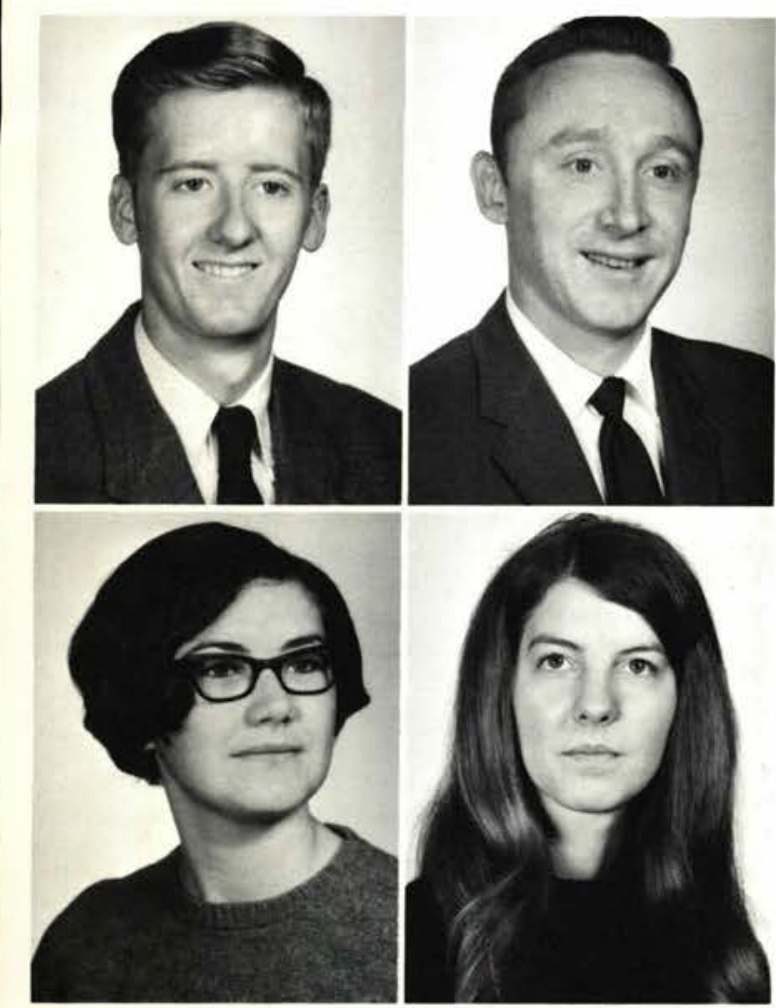

Rae Belle Fisher

New Milford, $\mathrm{Pa}$

Daria Fitzgerald

Tiffin, Ohio

Becky Fleming

Springfield, O.

Don Fleming

Lancaster, $\mathrm{O}$.

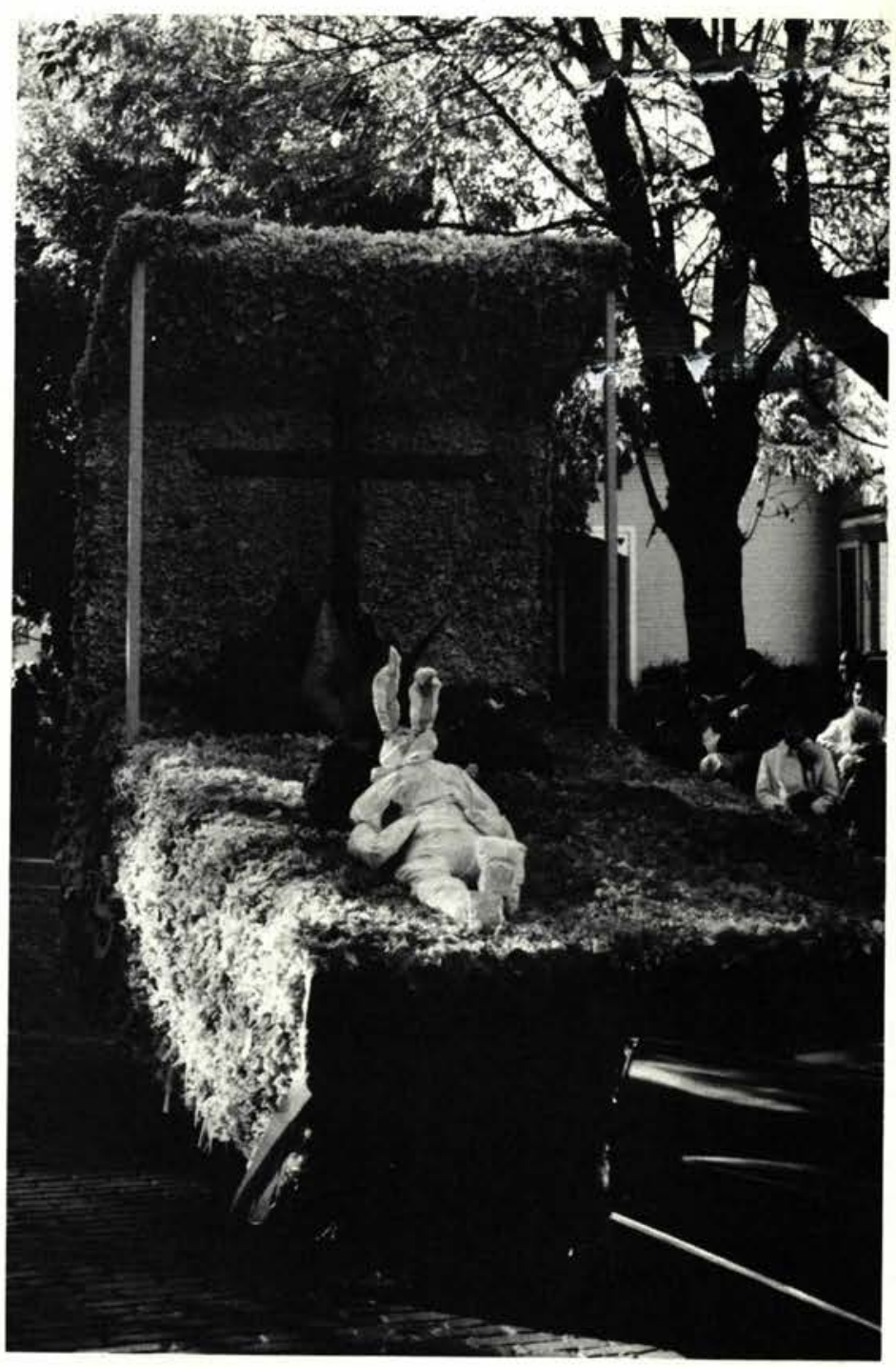

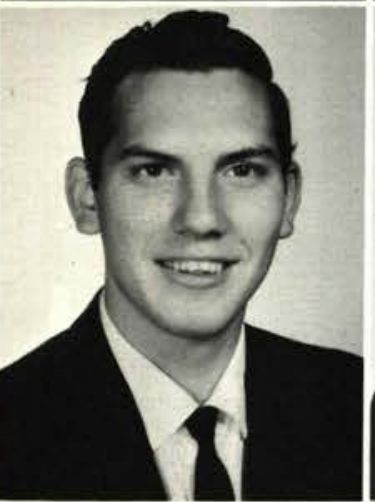

Harold Flood

Lake Orion, Mich.

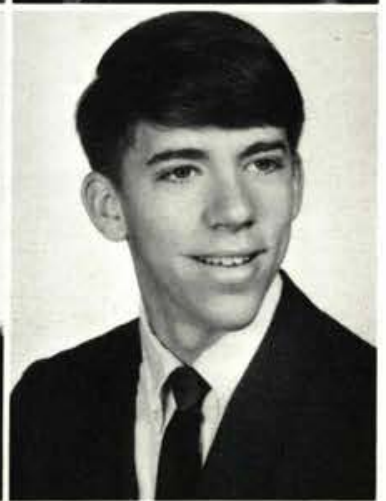

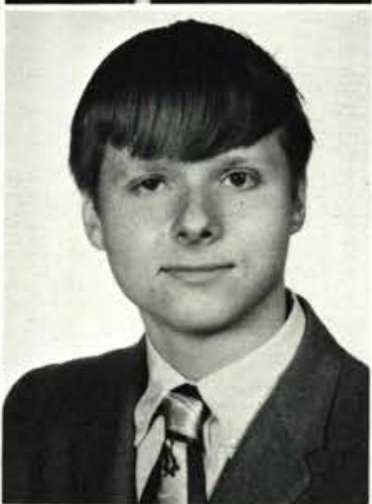

Dennis Forbis

Waterloo, Iowa

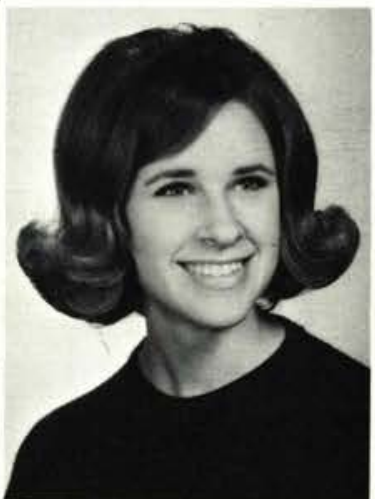

Janice Freed

Englewood, O.

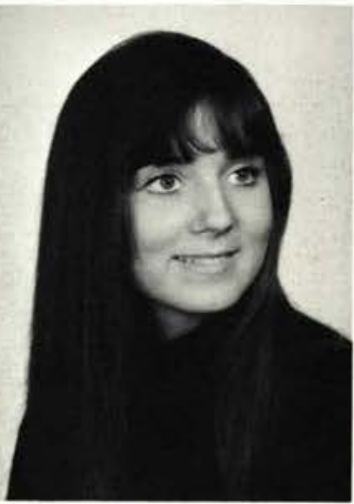

Lesley Freehauf

Warren, N. J.

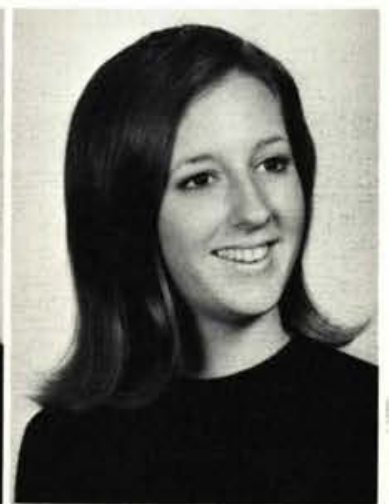

Kathie Frields

Huntingburg, Ind.
Vicky Fringer

Milwaukee, Wis.

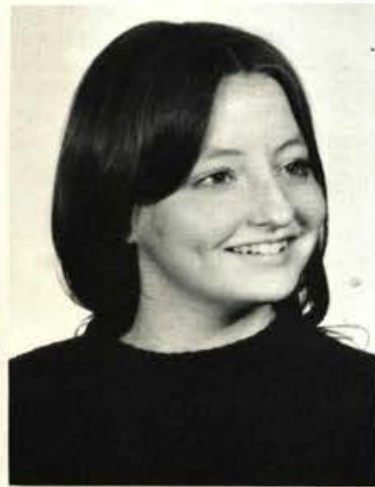

Michael Gallogly

Sunbury, Ohio

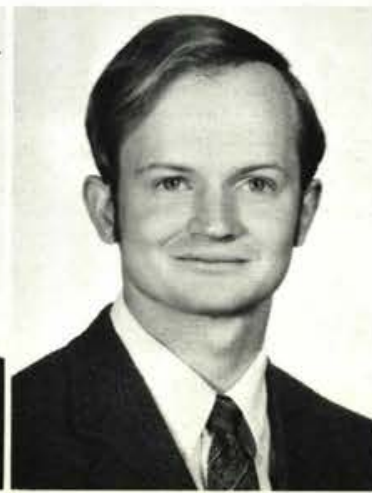

Jerold Garland

Romulos, Mich.

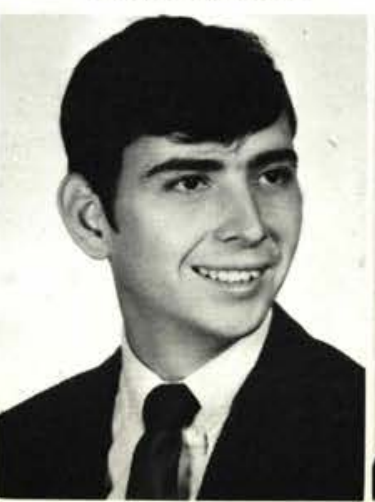

Rita Gast

Eldora, Iowa

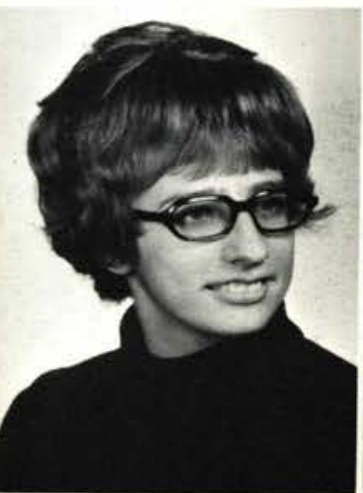

Kim Gerber

Berlin, Ohio

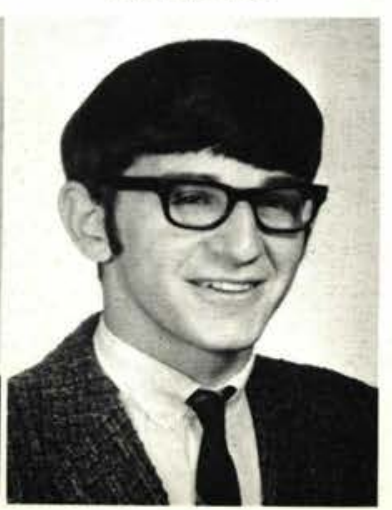




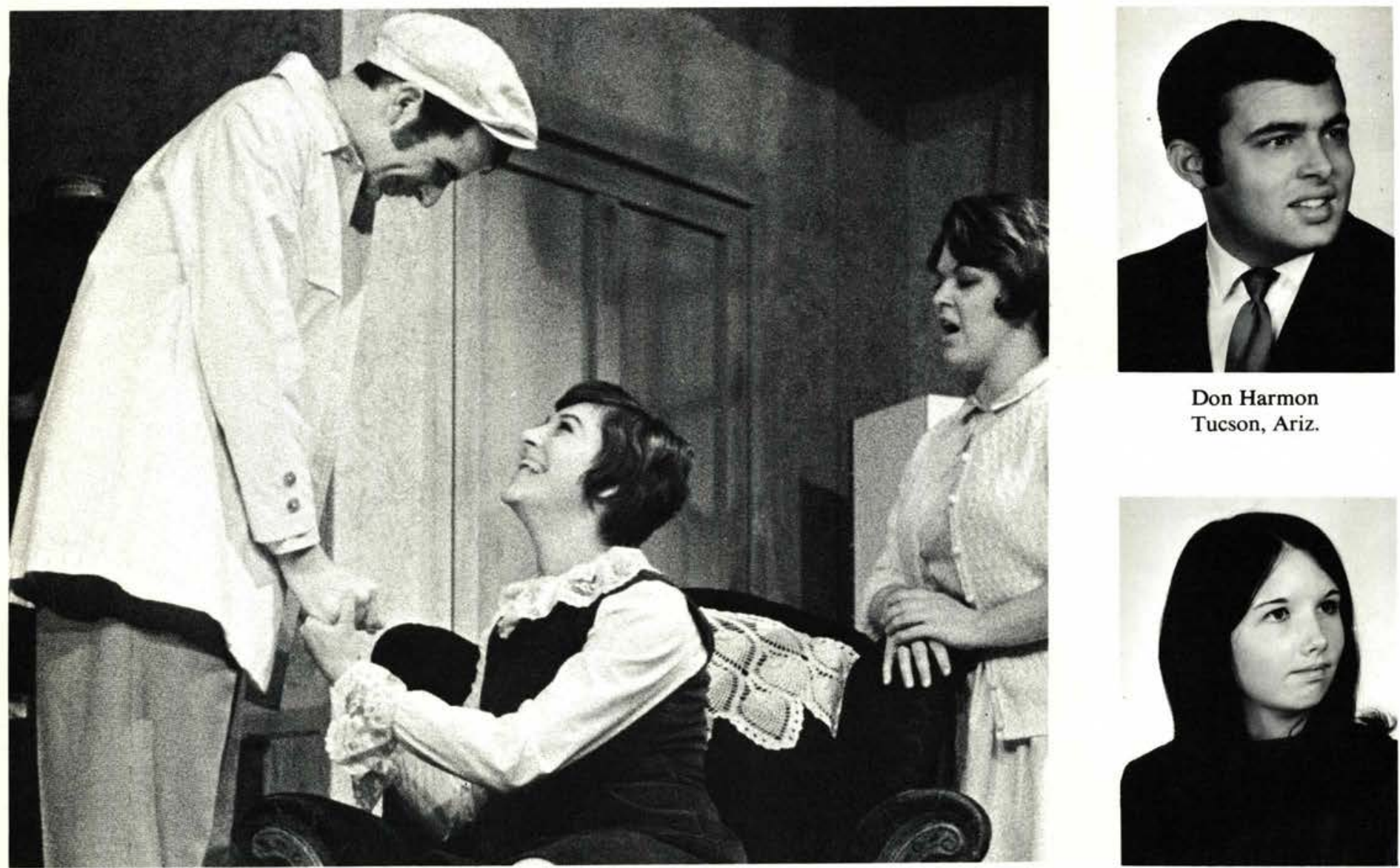

Don Harmon

Tucson, Ariz.

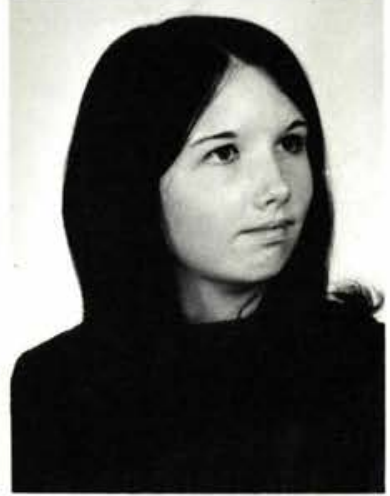

Jeanne Harmon

Delaware, $\mathrm{O}$.

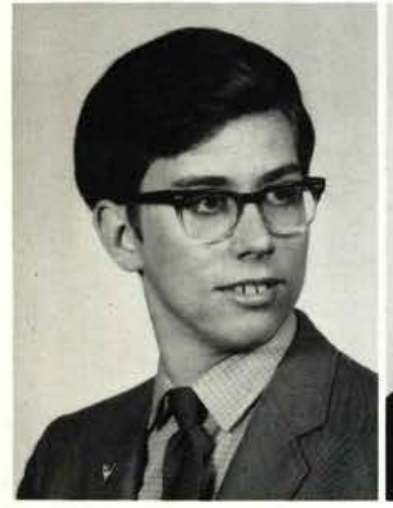

Stephen Harriman Indianapolis, Ind.

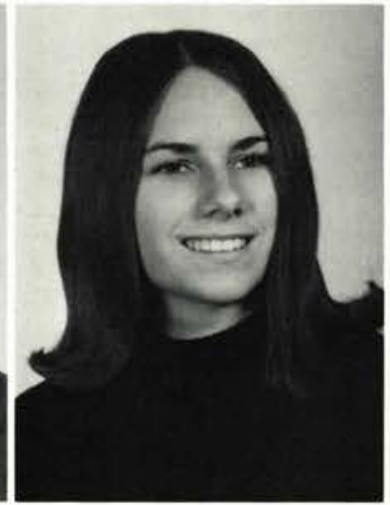

Janalee Harris

Cedar Falls, Iowa

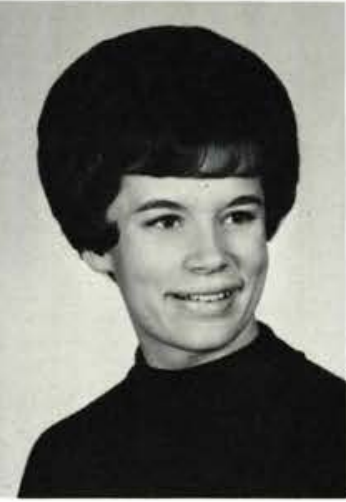

Virginia Heal

Bunker Hill, Ill.

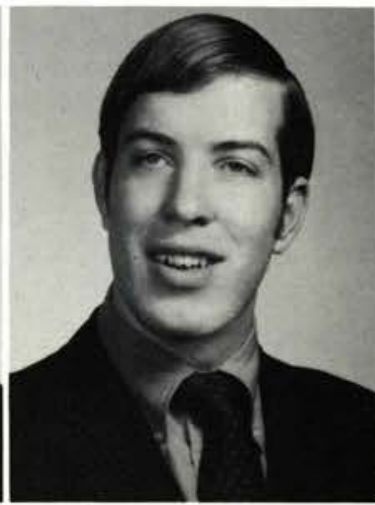

Tim Hegg

Spokane, Washington

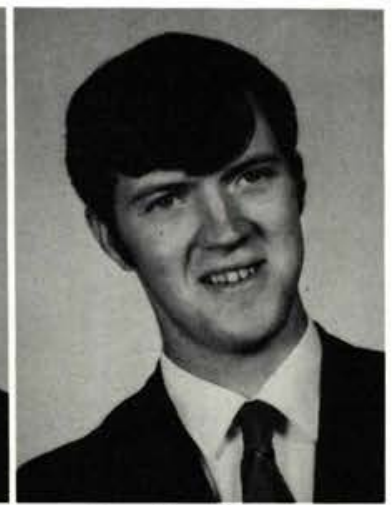

Don Hendrickson

Friendsville, $\mathrm{Pa}$.
Judy Hepworth Montague, Mich.

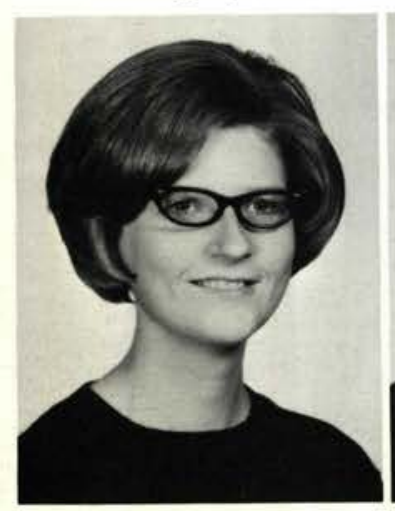

Stephen Herman Johnson City, N. Y.

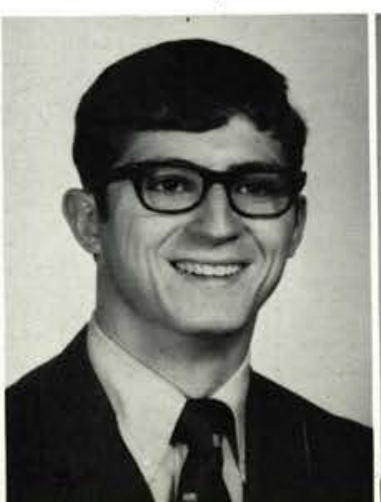

Doug Hess

Grundy Center, Iowa

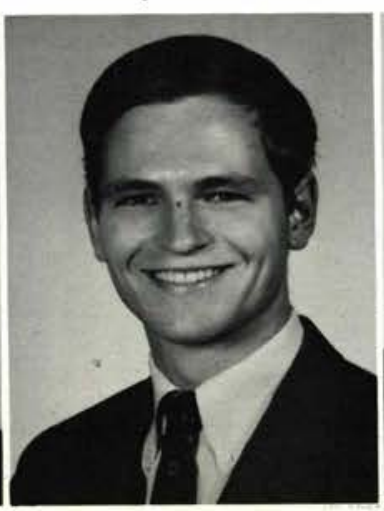

Terry Hignite Centerville, $O$.

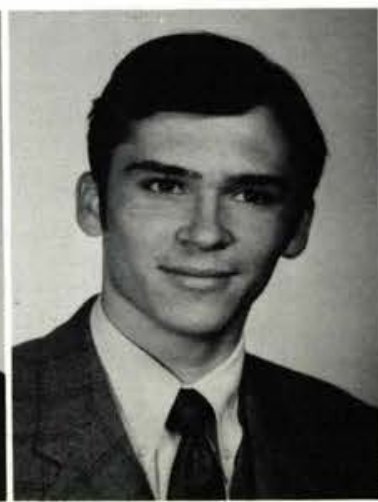

Dale Hilgar

Gibsonia, Pa

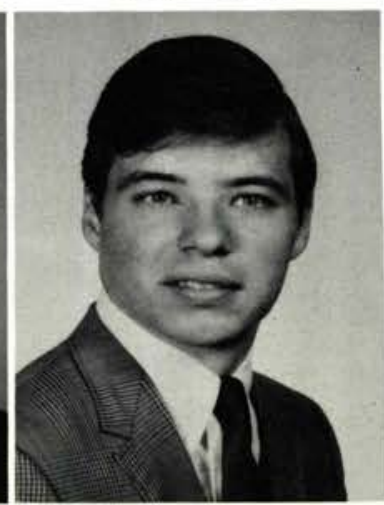




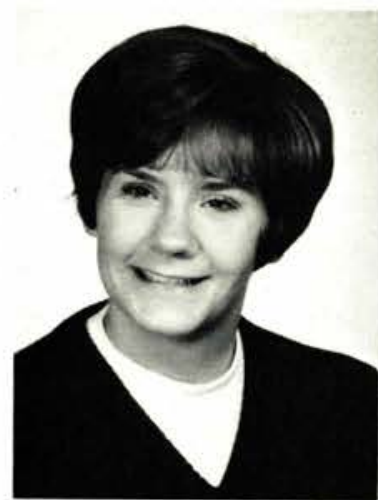

Janet Hillery

Alton, Ill.

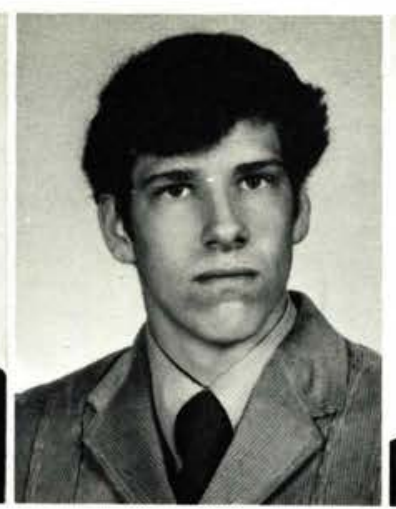

Craig Hilliker

Northfield, Mass.

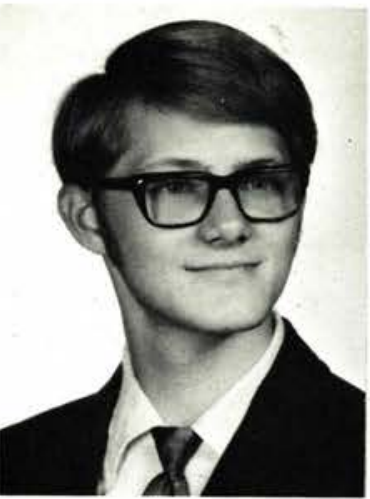

Steven Hodgin Plainfield, Ill.

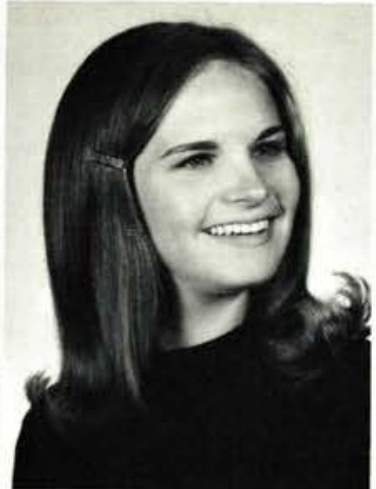

Cynthia Hofert Kenmore, N. Y.

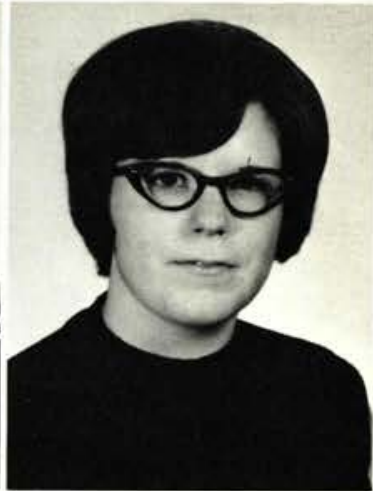

Rosemary Holingsworth Waynesville, Ohio

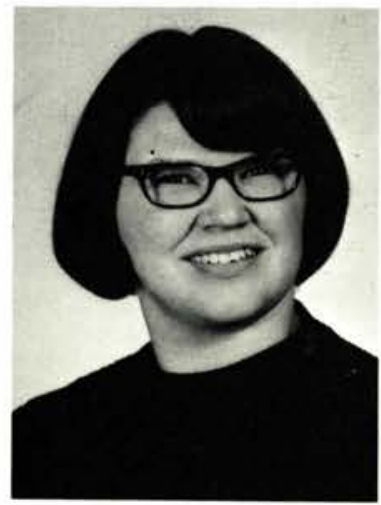

Cheryl Holtz

Muscatine, Iowa

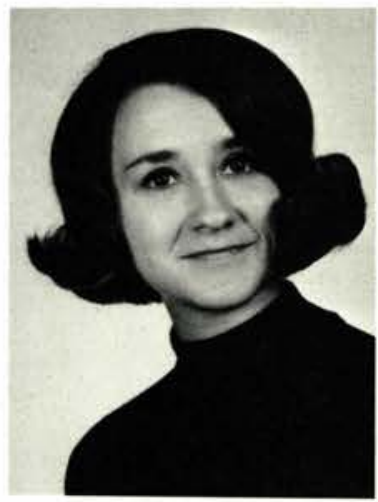

Patricia Howard Tipp City, Ohio

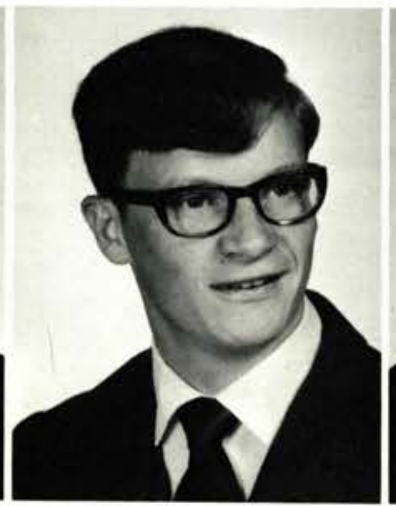

Bernard Horn

Lima, Ohio

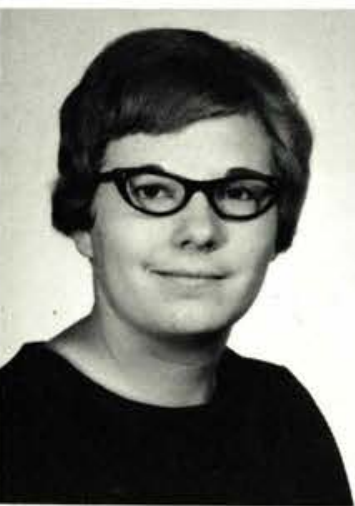

Sharon Hothem

Fresno, Ohio

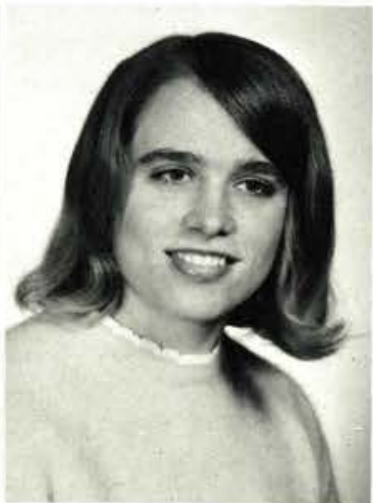

Joan Hovingh

Allendale, Mich.

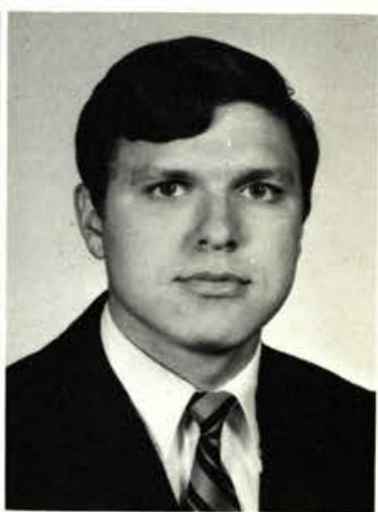

Lynn Howard Amherst, $\mathrm{O}$.

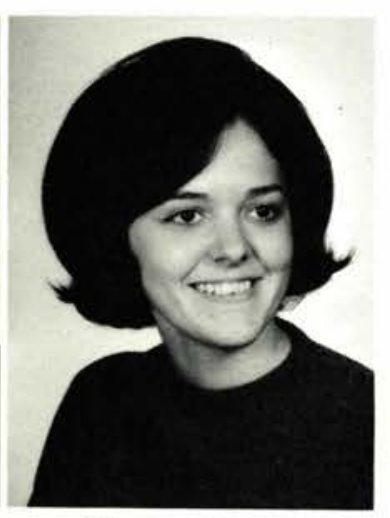

Rebecca Howard Findley, Ohio

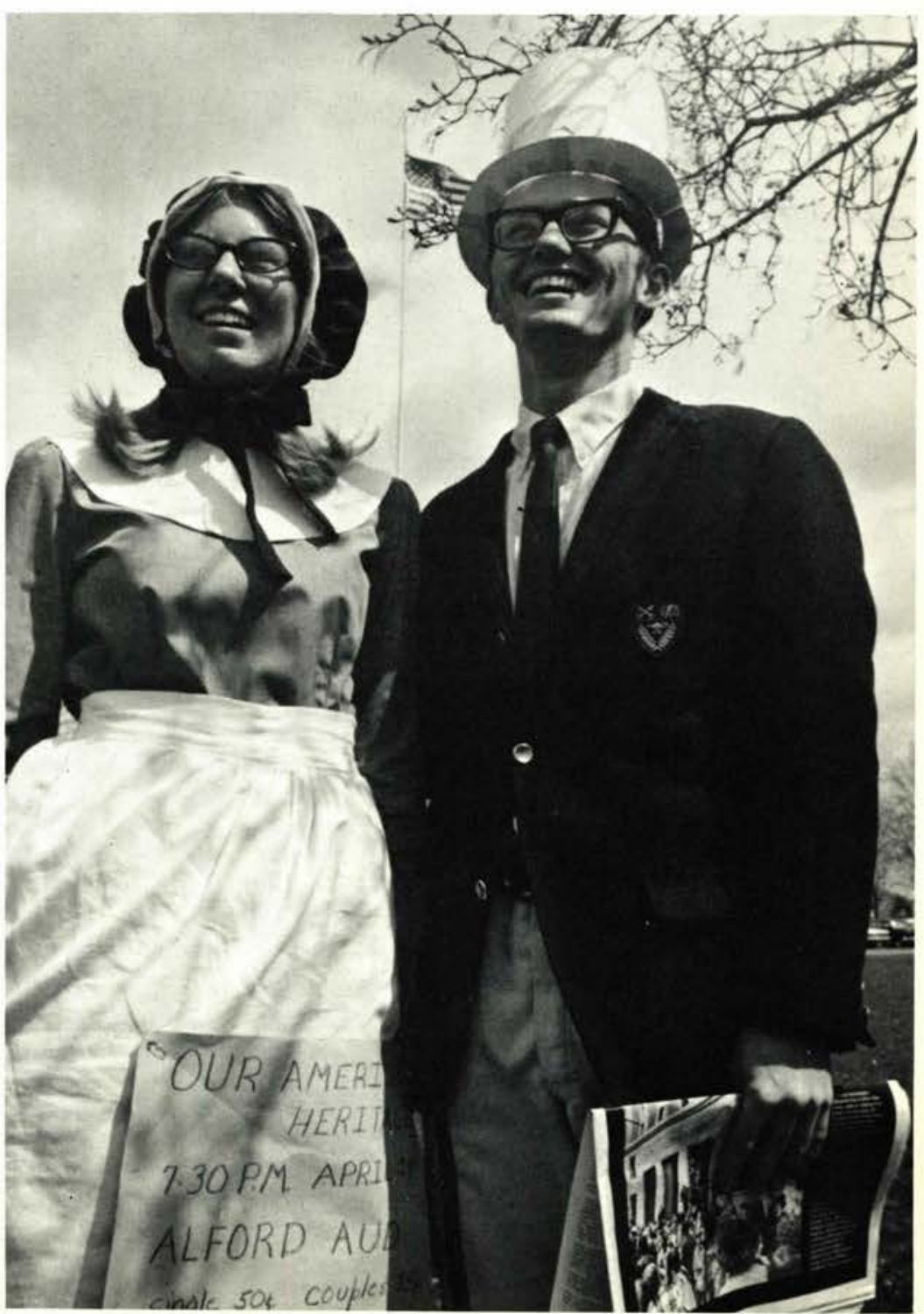

Bill Hyatt

Cedarville, $\mathrm{O}$.

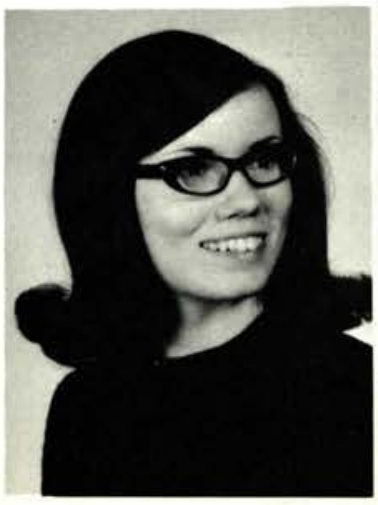

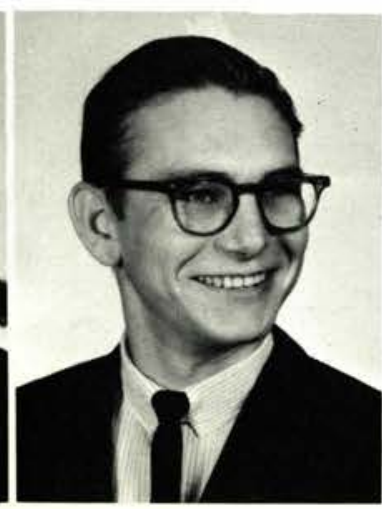




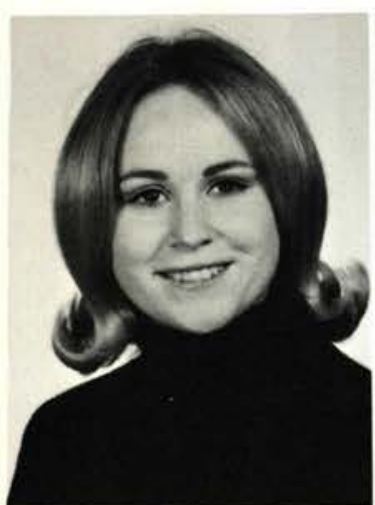

Rebecca Jackson

Carroll, Ohio

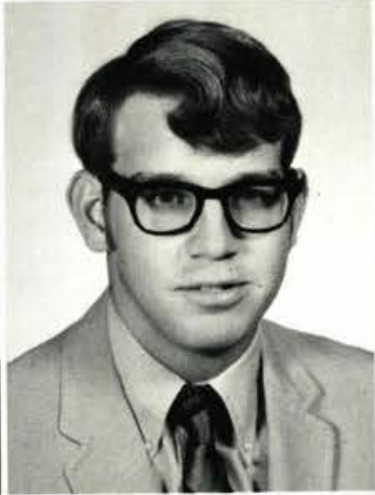

Timothy Jarvis Cedarville, $\mathrm{O}$.

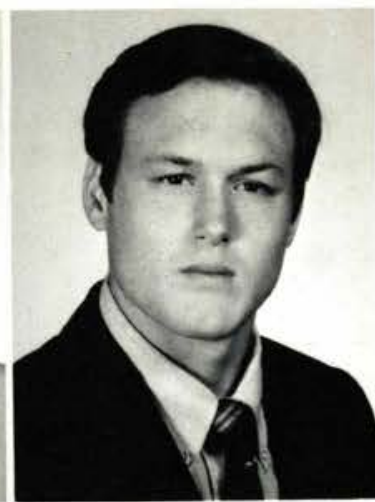

Russell Jenkins McLean, Va

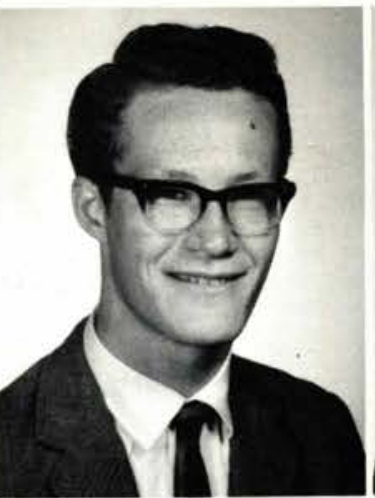

James Jenney

Cortland, N. Y.

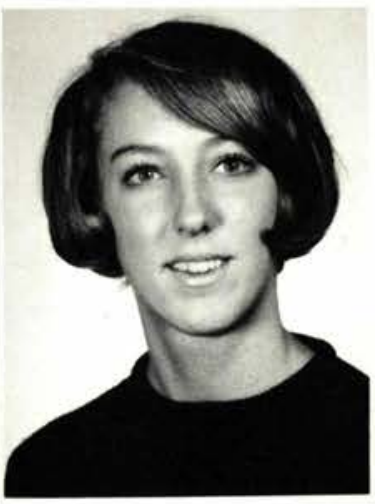

Julianne Jensen Caro, Michigan

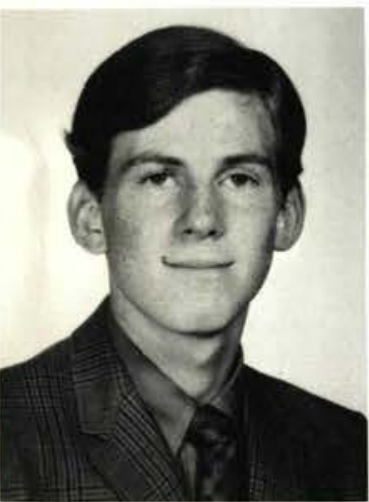

Douglas Jingst

Henry, Ill.

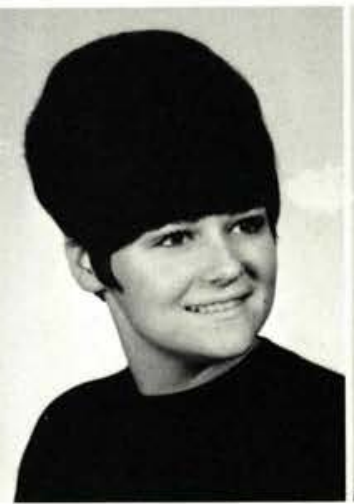

Barbara Johnson

Dayton, Ohio

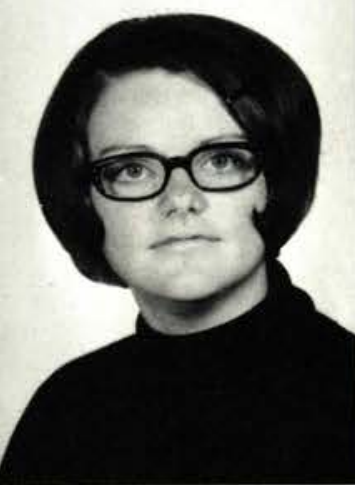

Judy Johnson

Akron, Ohio

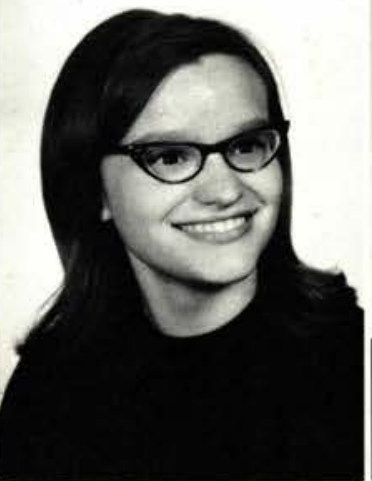

Rhea Johnson

Cedarville, Ohio

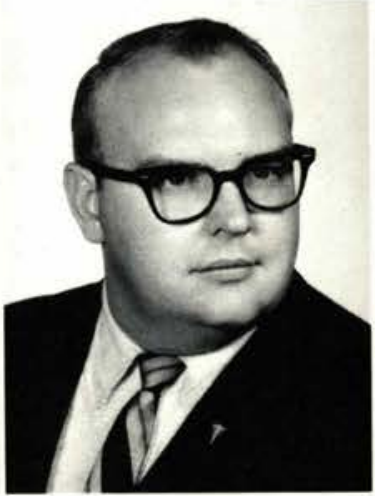

Roy Kern

Waterloo, Iowa

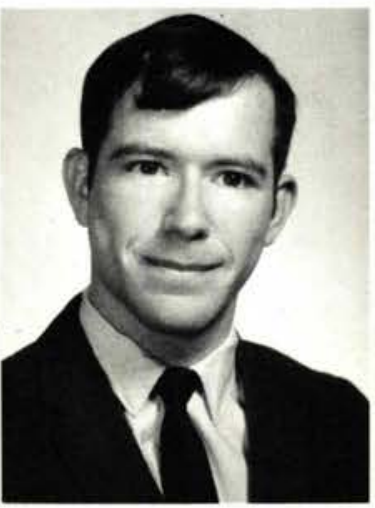

David Jones

Cedarville, $\mathrm{O}$.

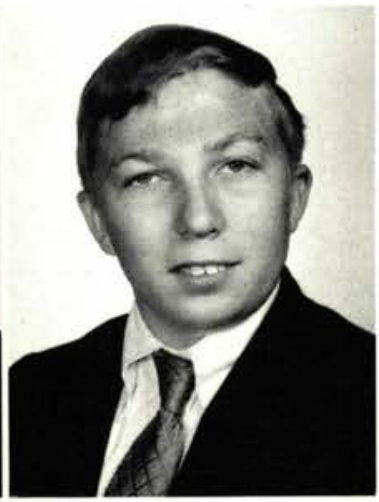

Hal King

De Graff, Ohio
Lyons, Ohio
Beverly Kemp
Des Moines, Ia.
Melanie King Dayton, Ohio

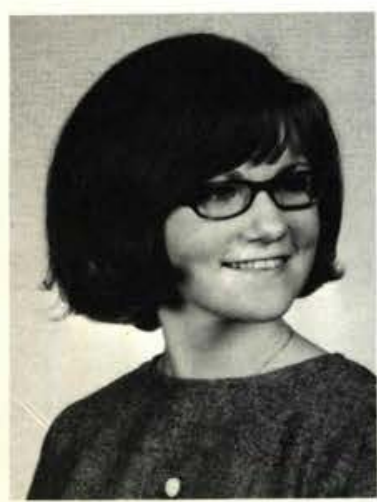

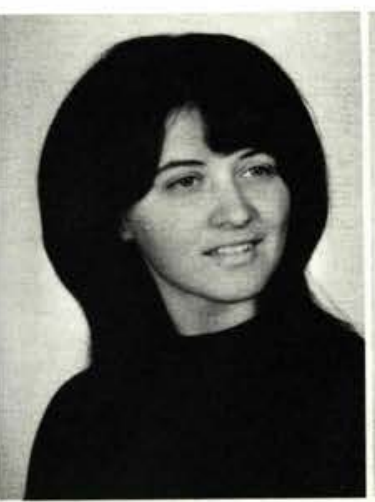

Susan Kinney

Des Moines, Ia.

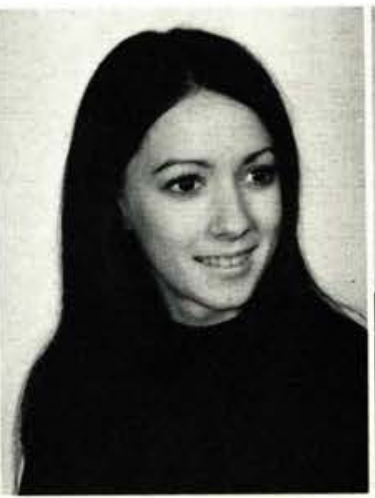

Deborah Kitting Tucker, Georgia

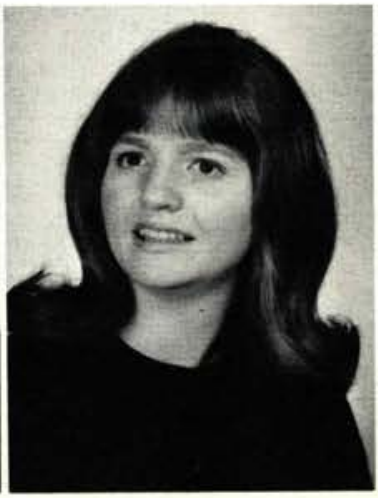

Cheryl Klomparens Grand Rapids, Mich.

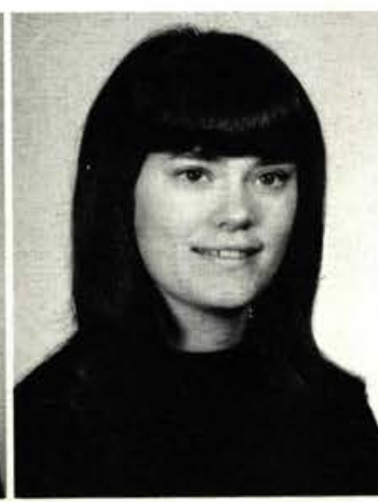




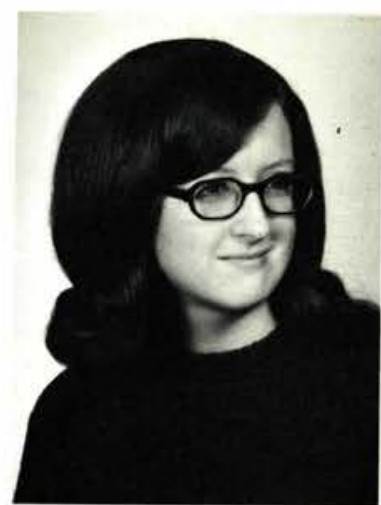

Arlene Knight Austin, Minn.

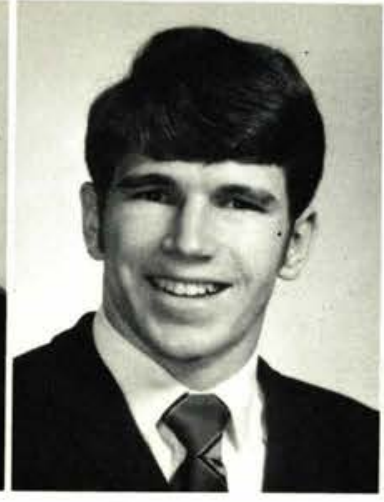

Denver Kohli Wauseon, O.

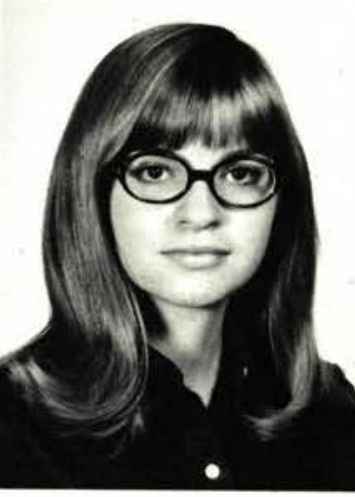

Sally Koncsol McDonald, Ohio

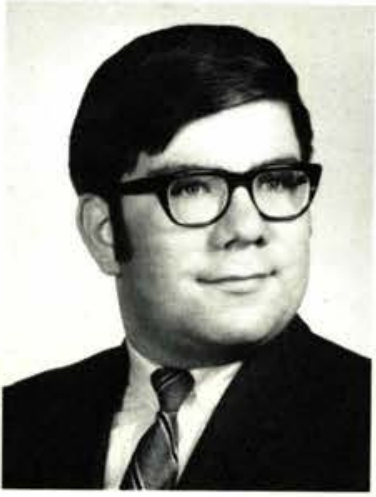

Gregory Kunz Dayton, Ohio

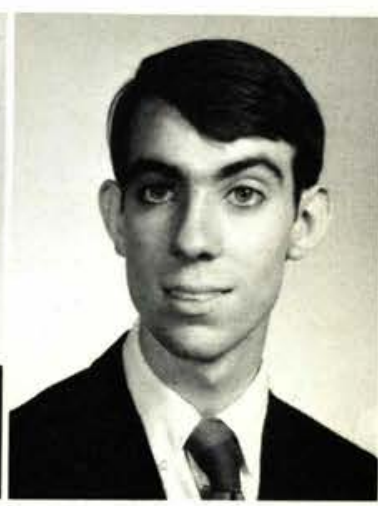

Dana Larrick

Wilmington, $\mathrm{O}$.

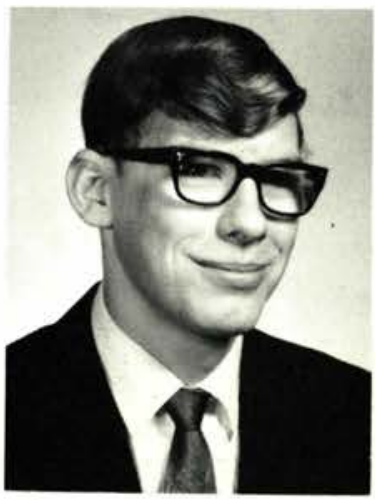

Charles Lemaster

Xenia, Ohio

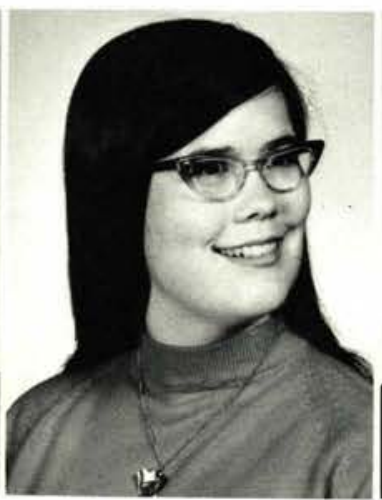

Diane Lenox

Columbus, $\mathrm{O}$.

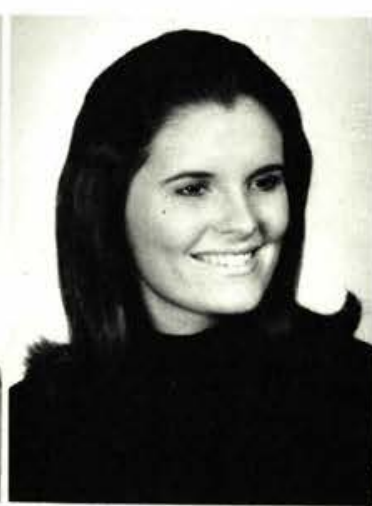

Sharon Lewis

Mentone, Ind.

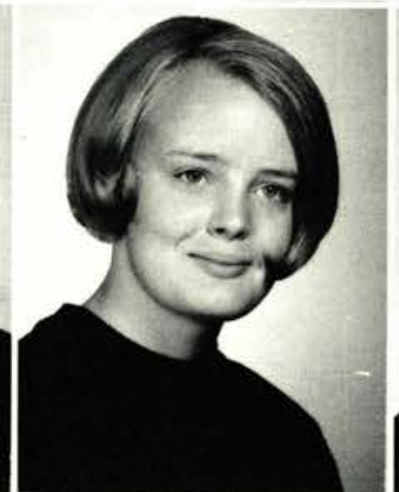

Lorraine Linderman Franklinville,N.Y.

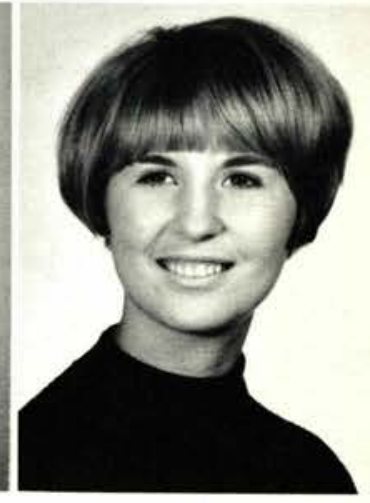

Barbara Lister St. Charles, Mo.

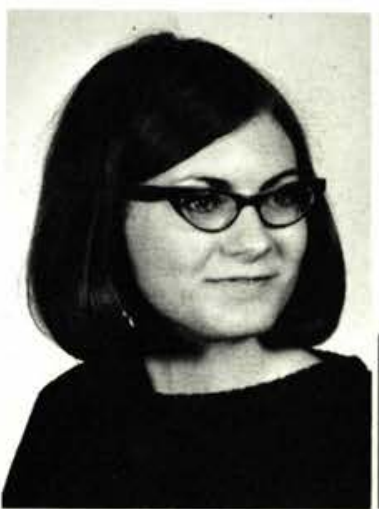

Diane Long Avon, Ohio

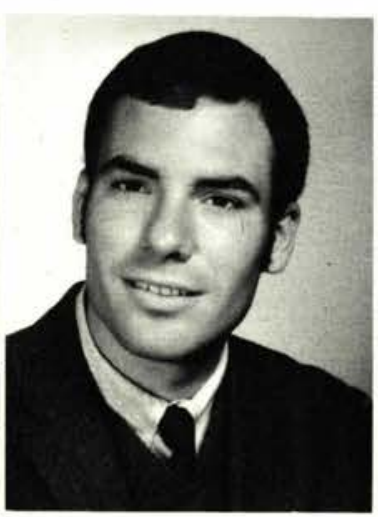

Jeff Lough Berea, Ohio

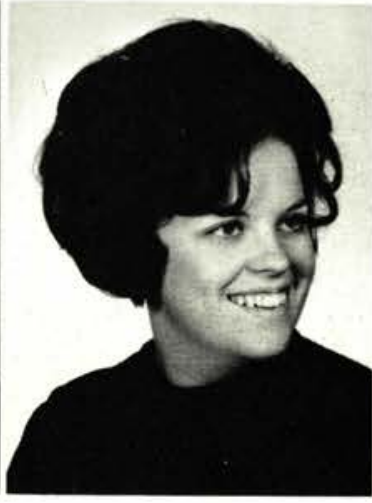

Diane Love

Fairborn, O.

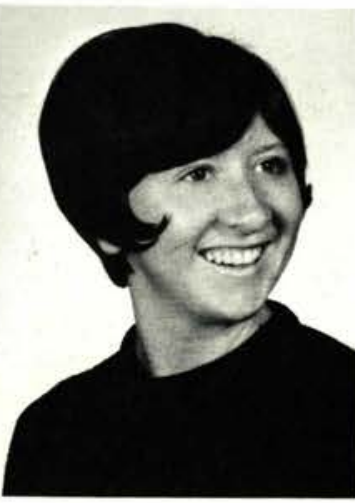

Landra Lowery

Warsaw, Ind.

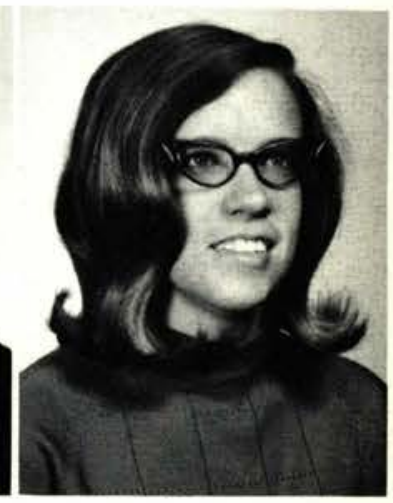

Roberta Lutton Delta, Ohio
Rebecca Lyons Kimbolton, O.

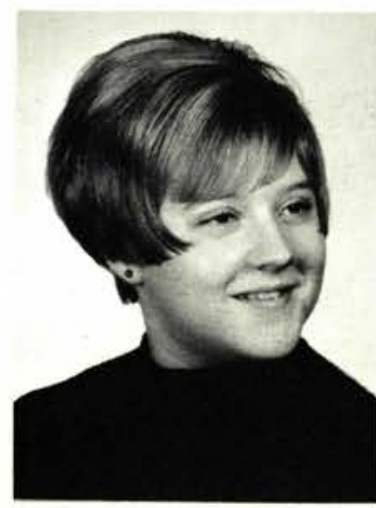

Jo Mackey

Plainfield, Ia.

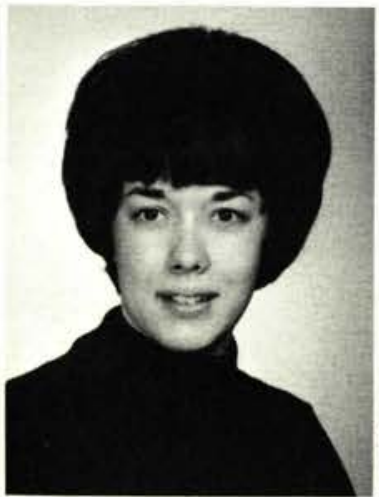

Cathy Mahoney Lowell, Ohio

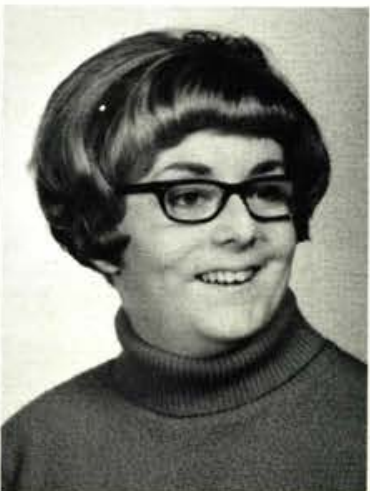

Marilyn Markley Troy, Ohio

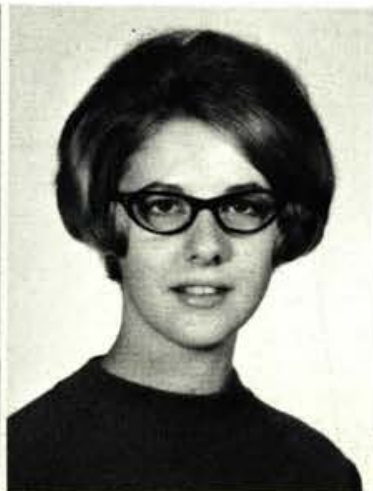

Janice Martin

Scott Depot, W. Va.

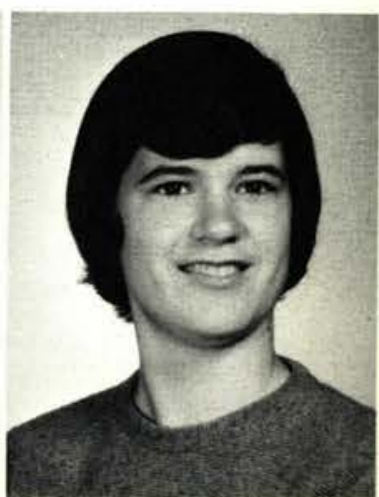



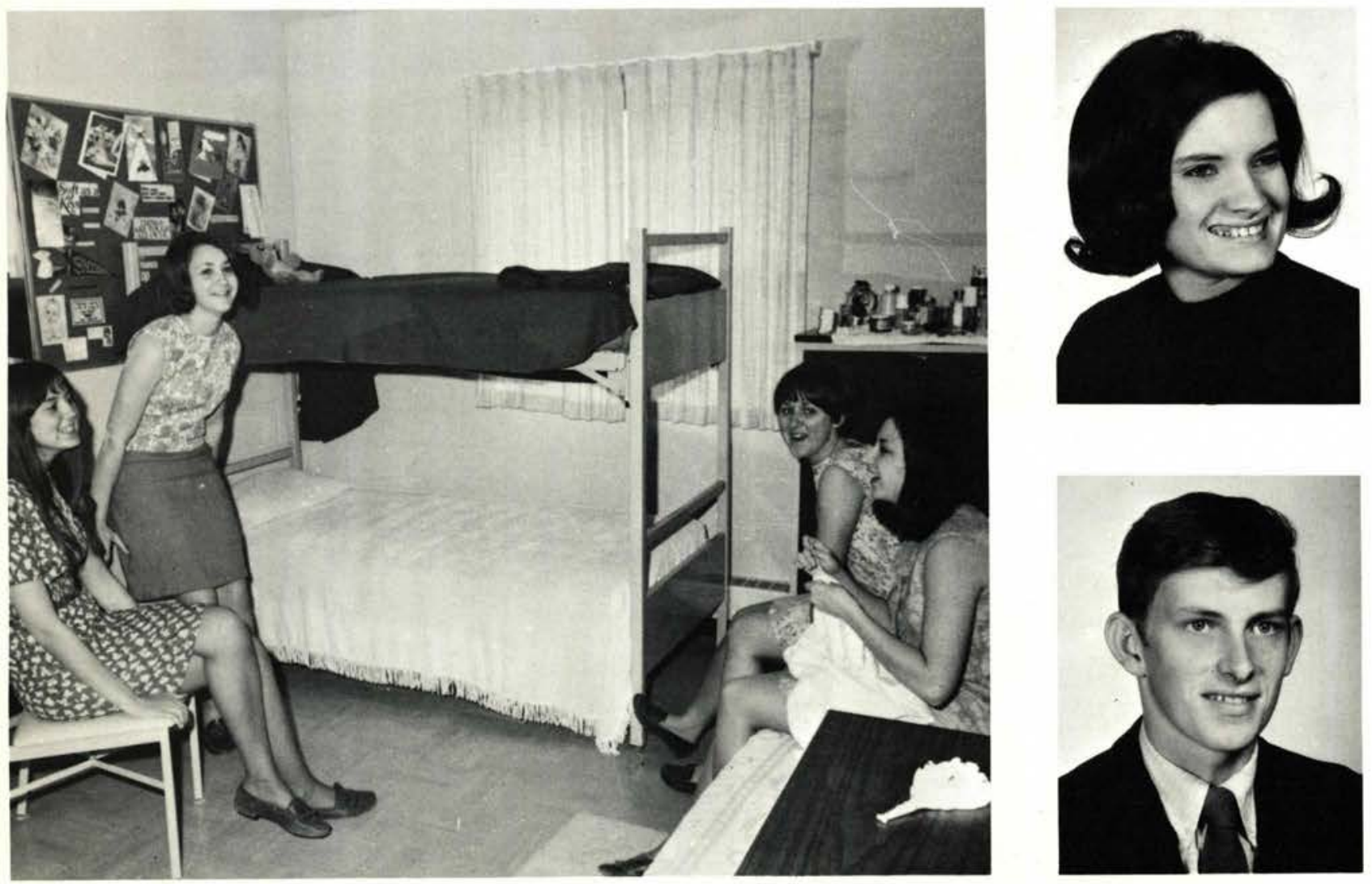

Shari Martin

Painesville, $\mathrm{O}$.

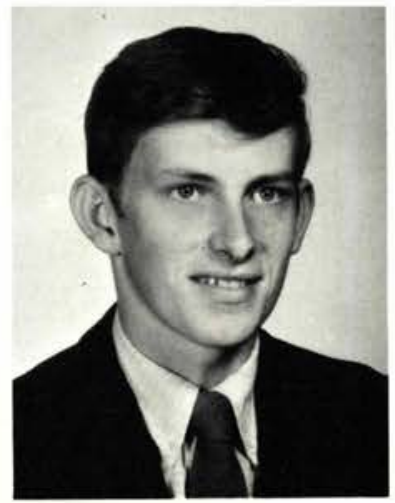

William McComb Gill, Mass.

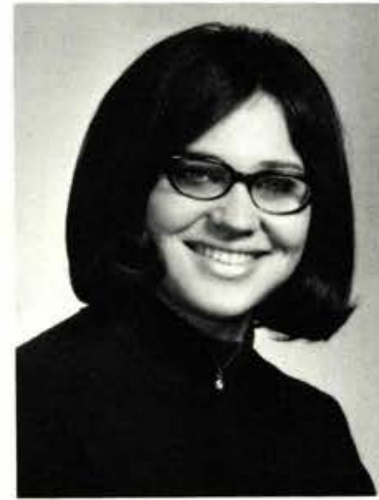

Thelma McDonald

Ypsilanti, Mich.

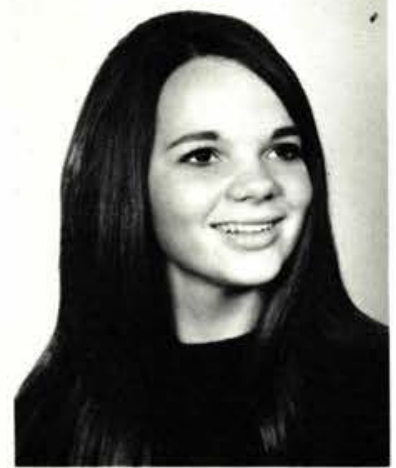

Bonita McNeill

Charleston, W. Va.

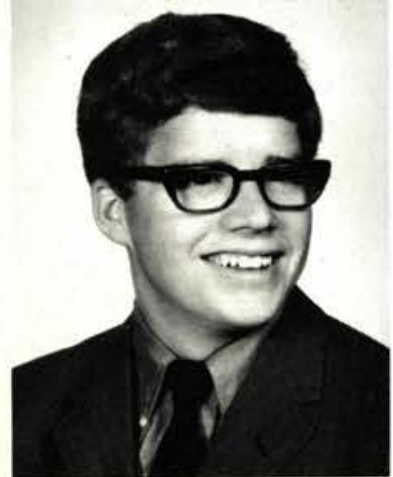

William McNiece

Valparaiso, Ind. 


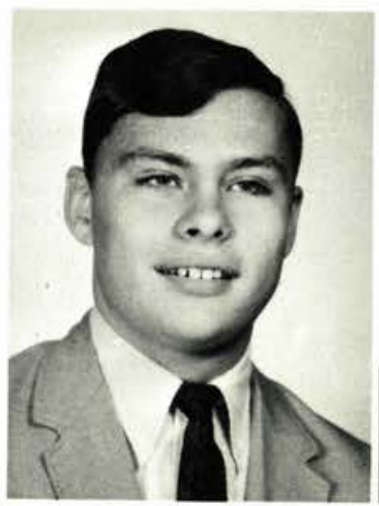

Janes Meadows

Blanchester, O.

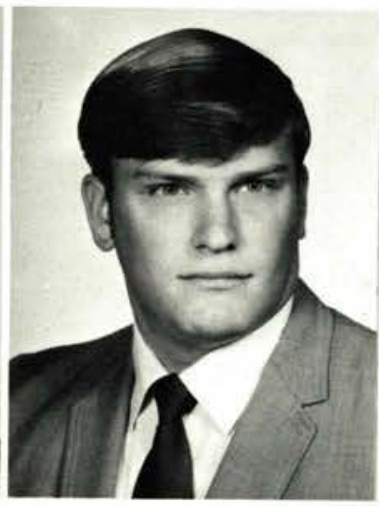

Paul Metcalf

Phoenixville, $\mathrm{Pa}$.

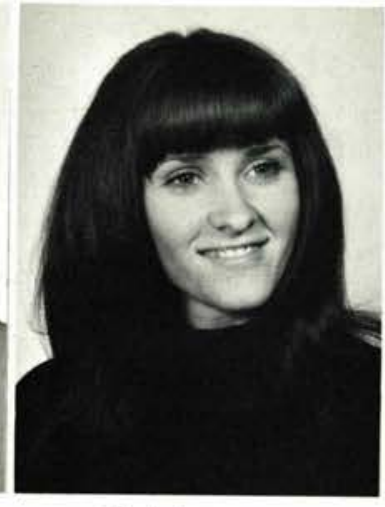

Christine Meyer

Batavia, Ohio

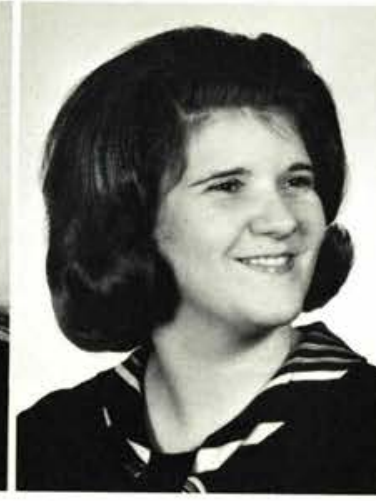

Ruth Michael Renfrew, Pa.

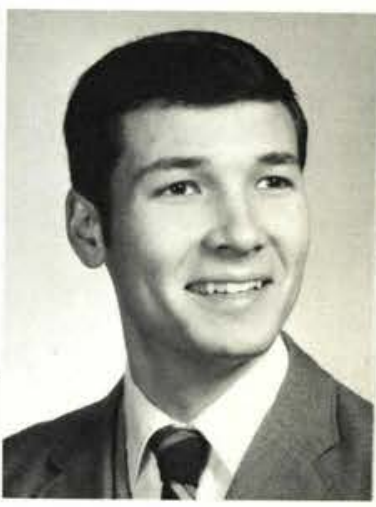

David Miller Springfield, P.

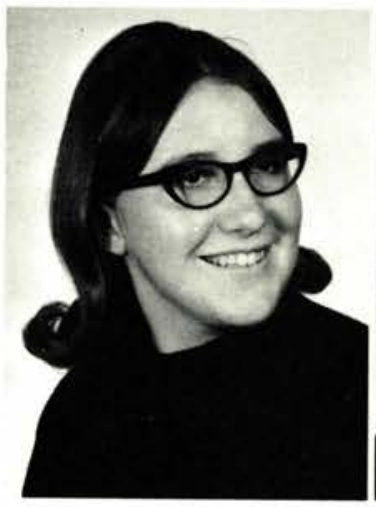

Marilyn Miller Oil City, Pa.

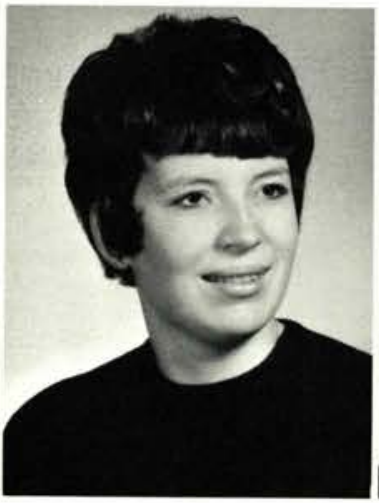

Kathy Modrall Toledo, Ohio

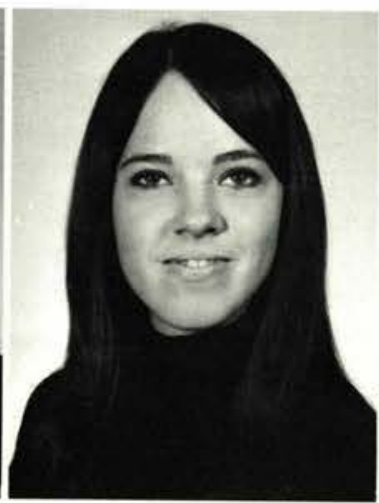

Anita Mogle

Xenia, Ohio

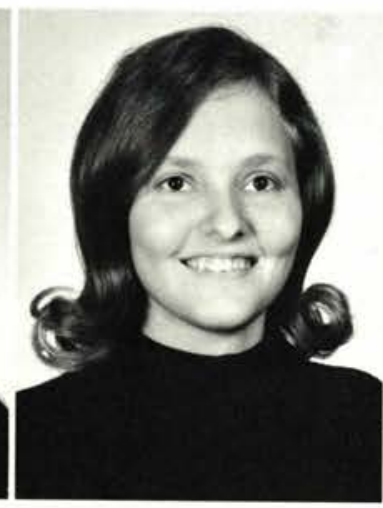

Nola Moistner

Cambridge City, Ind.

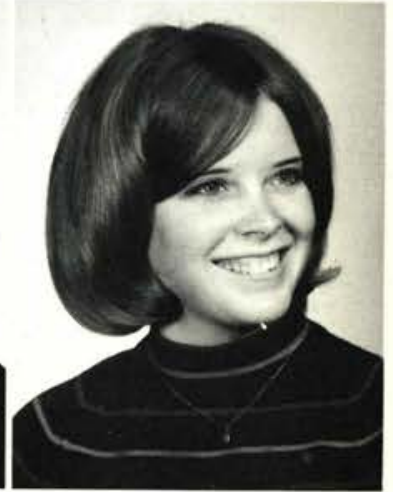

Martha Moore Ypsilanti, Mich.

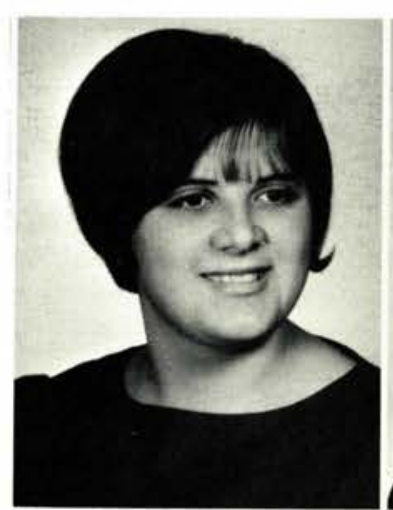

Rebecca Moore Elyria, Ohio

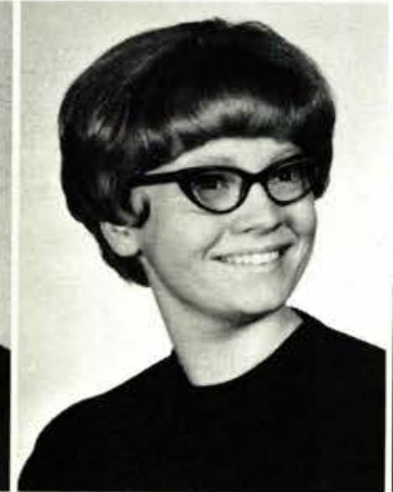

Susan Moore

Jamestown, Ohio

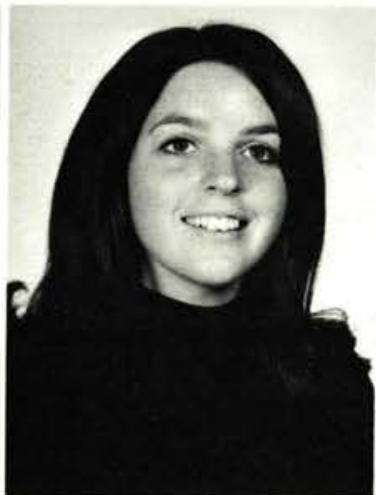

Bonnie Morris

Painesville, Ohio

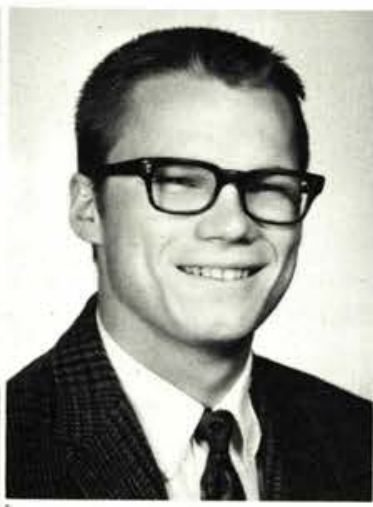

Tom Mudder

Hastings, Minn.

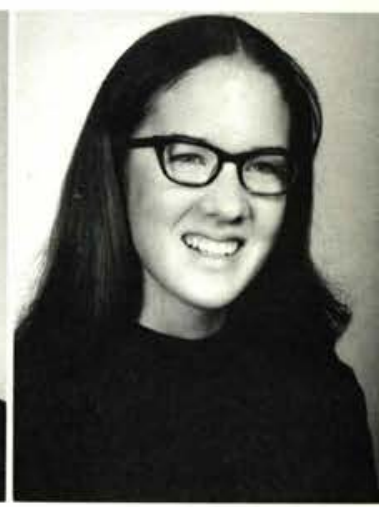

Glennie Murphy

Silver Spring, Md.
Shelly Murry

Columbus, $\mathrm{O}$.

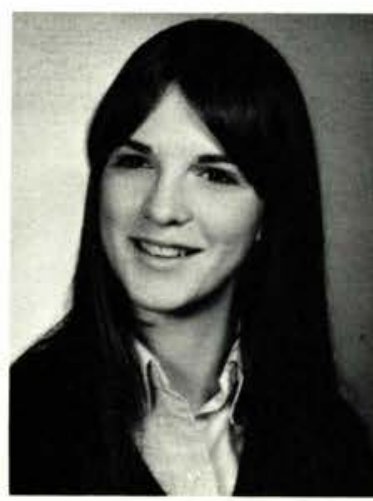

Ranelle Nabring Flint, Mich.

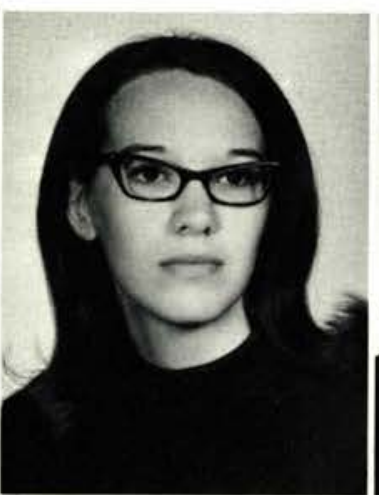

Susan Nauman Otselic, N. Y.

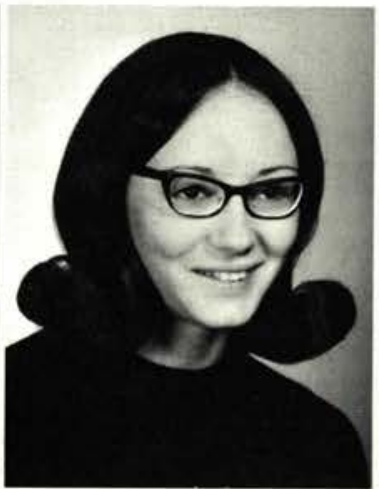

Jeanne Nelson St. Louis, Mo.

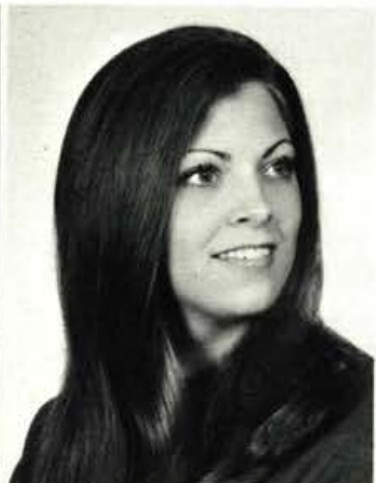

Darlene Nottingham Mansfield, Ohio

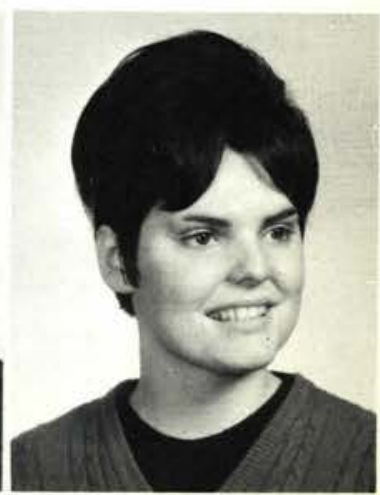




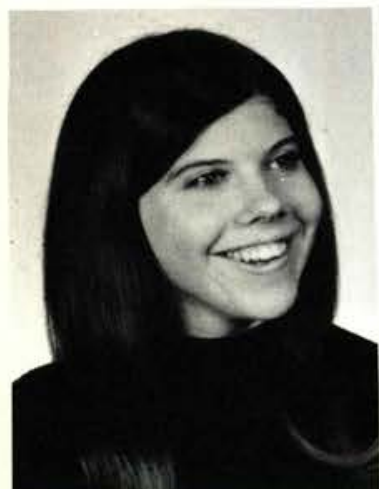

Susan Odegard

Flint, Mich.

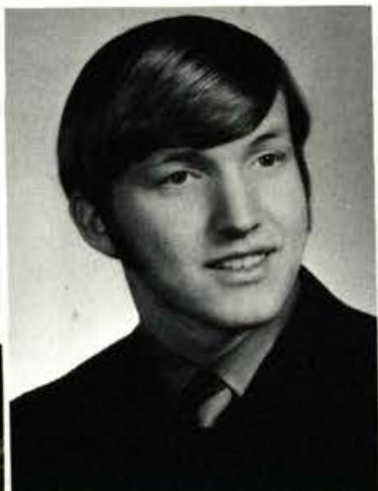

Steve Ogborn

Zionsville, Ind

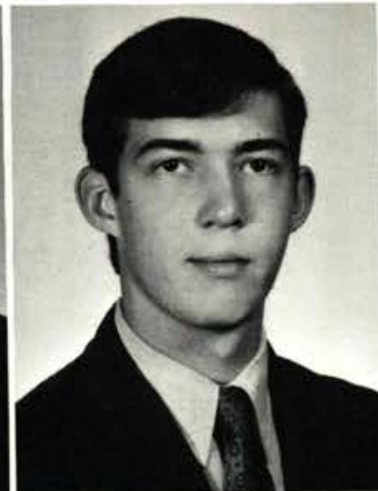

Bruce Olsen Lima, Peru

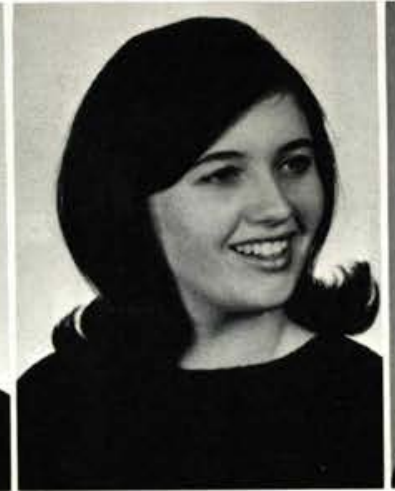

Kaye Partlow Indianapolis, Ind.

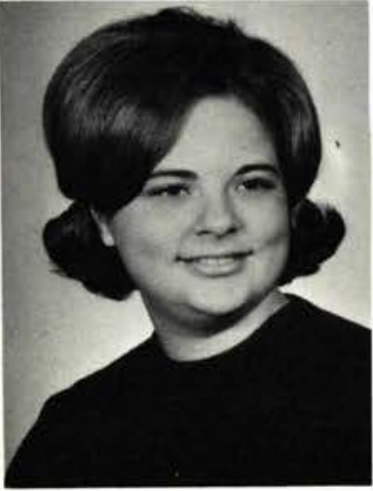

Linda Phelps Lorain, Ohio
Sheldon Philips Portland, Ore.
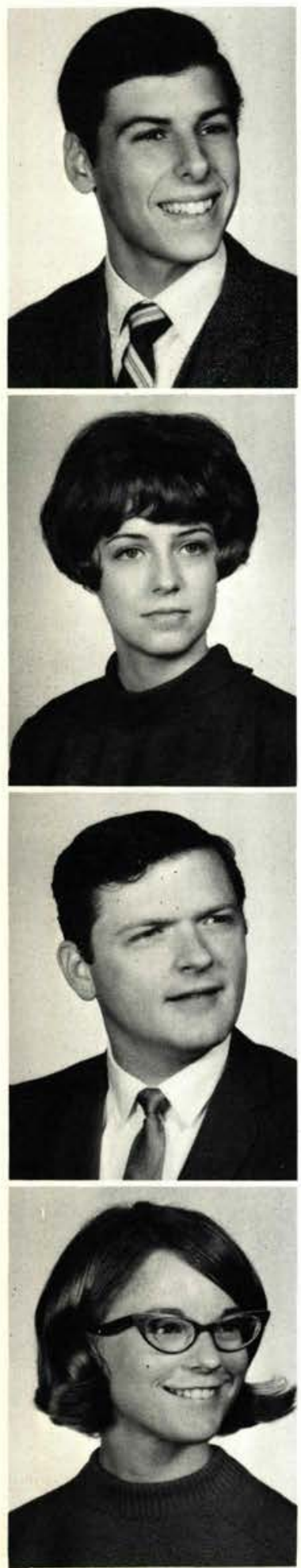

Mary Piach

Yellow Springs, O.

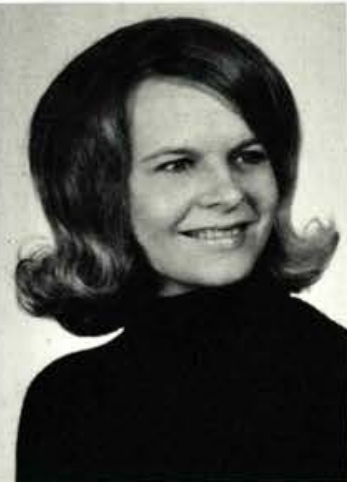

Gale Portz

North Olmsted, O.

Rodney Post

Newark Valley, N. Y.

Jeanette Powley

Lake Orion, Mich.
Gary Pippinger Breman, Indiana
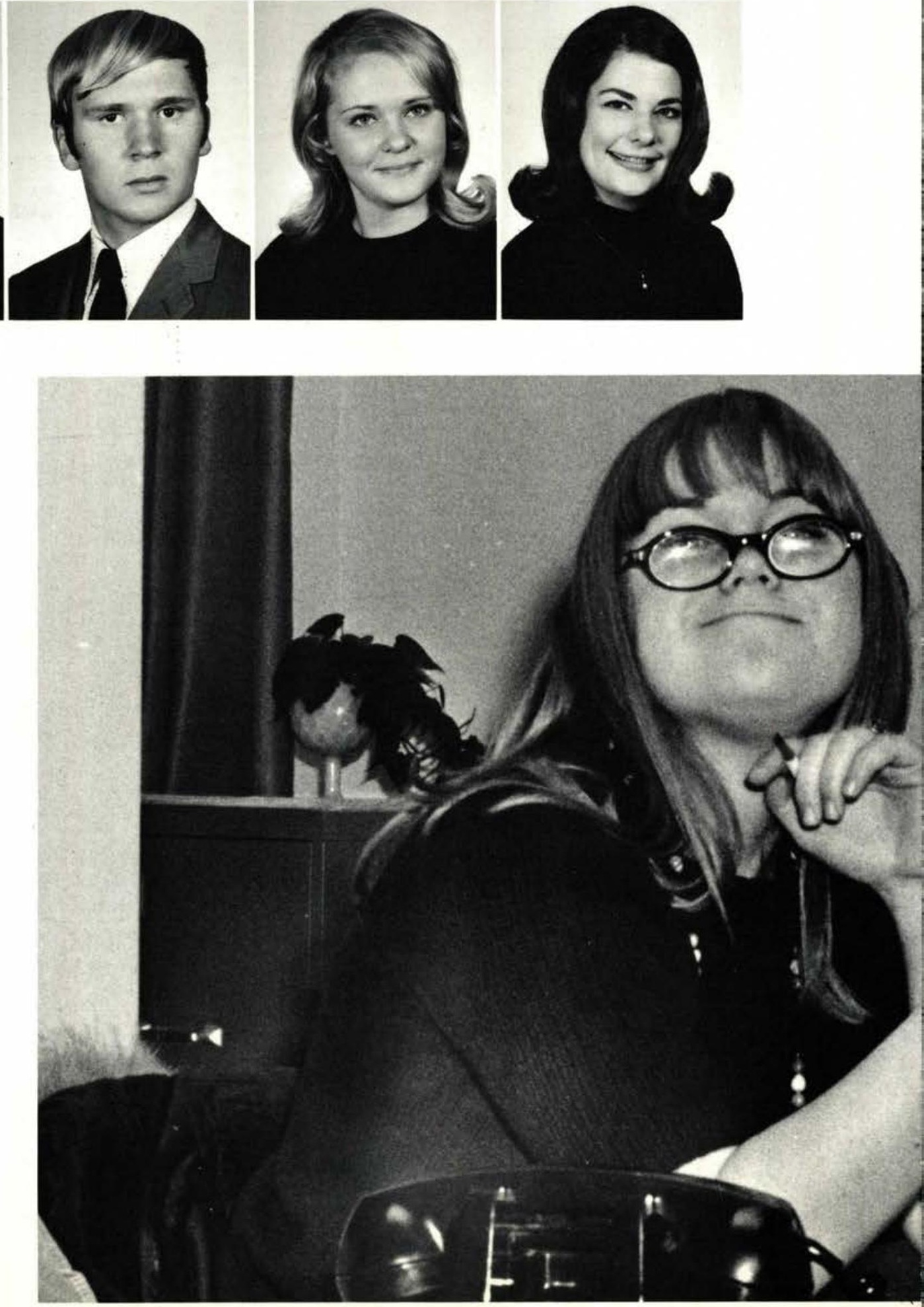


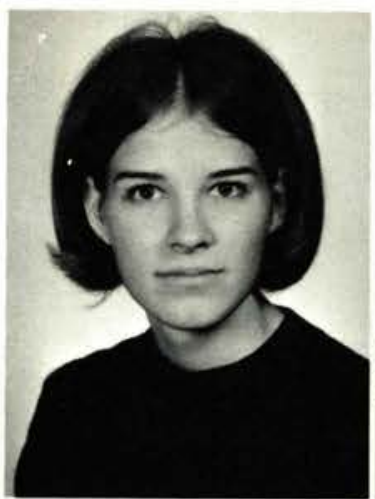

Paula Prater

Washington, D. C.

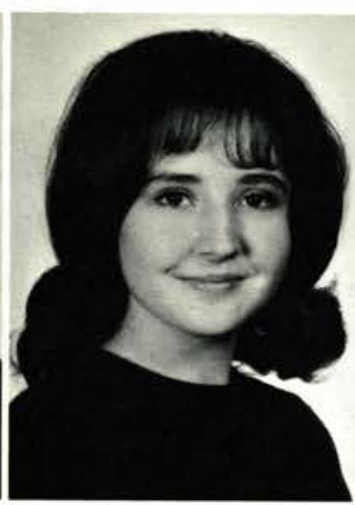

Grace Pruden

Prudenville, Mich.

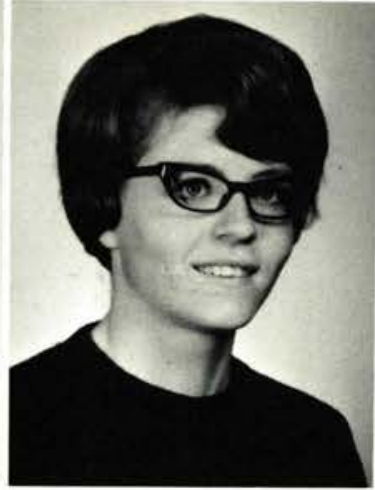

Connie Pumpelly

Newark, Ohio

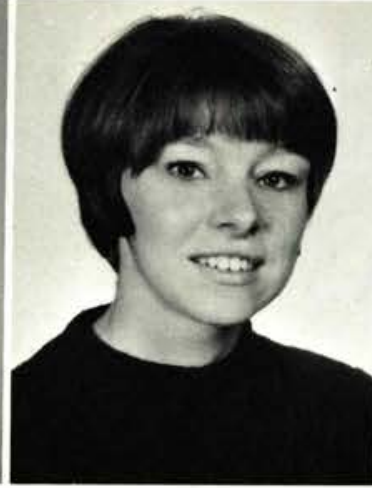

Sally Ray

Mancelona, Mich.

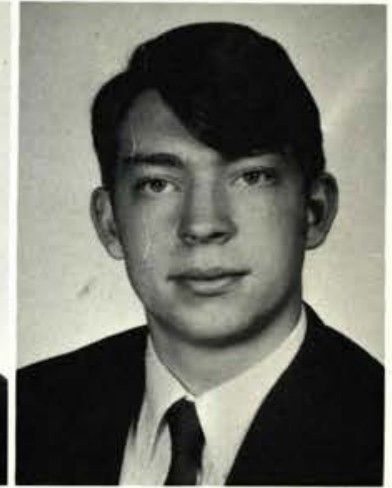

Steven Read Monticello, Ind.
Sharon Reese

Lebanon, Ohio

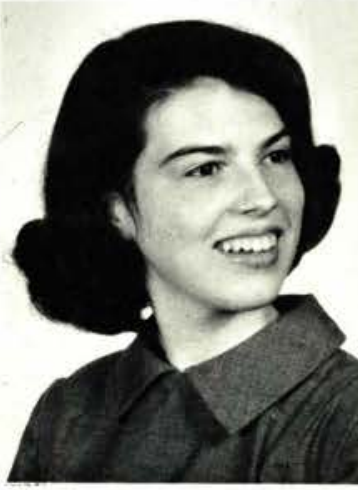

Dean Rendell

Cranford, N. J.

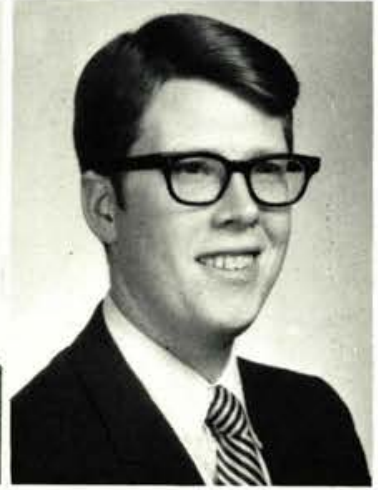

Vicki Reynolds

New Philadelphia, O.
Gary Rhodes

Tawas City, Mich.

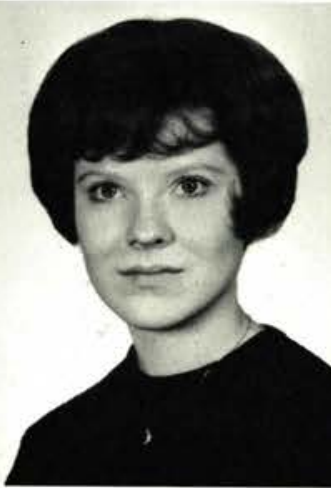

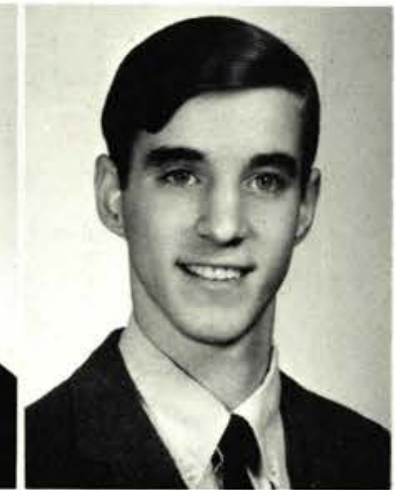

Gale Richardson Euclid, Ohio

Dennis Roberts Bunker Hill, III.

Michael Roden Dorset, Ohio
Gerald Rice

Tamaqua, $\mathrm{Pa}$
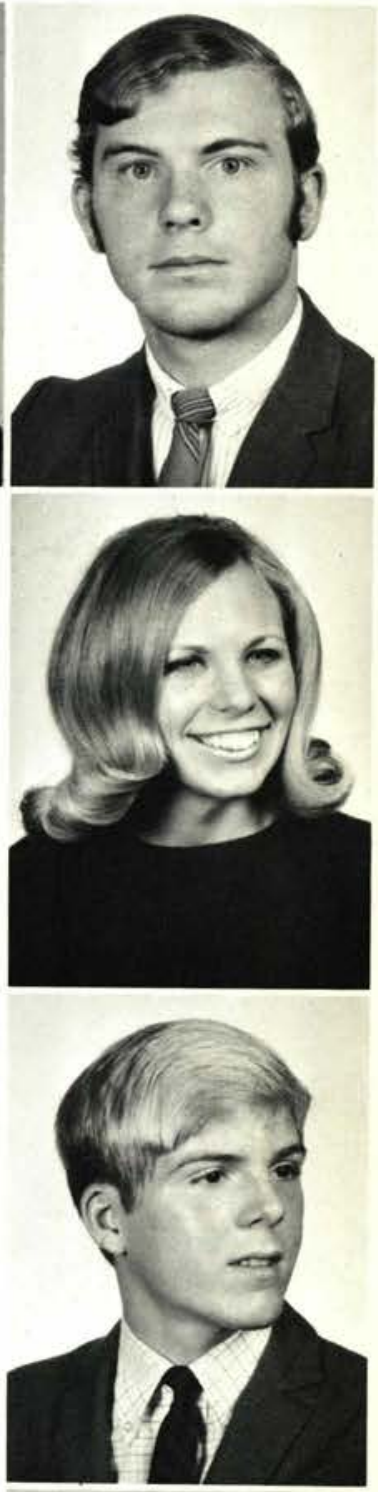

RERERENCE

STenoeralesu

ars

Mann's

CBDARYTL:

COL. $3 \mathrm{GE}$

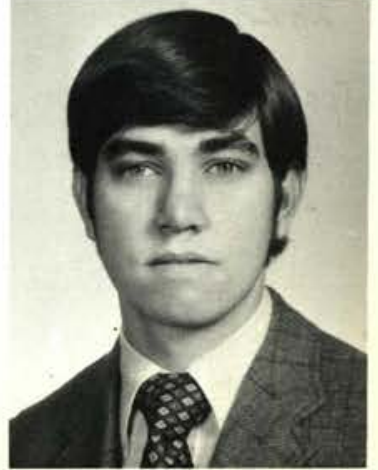




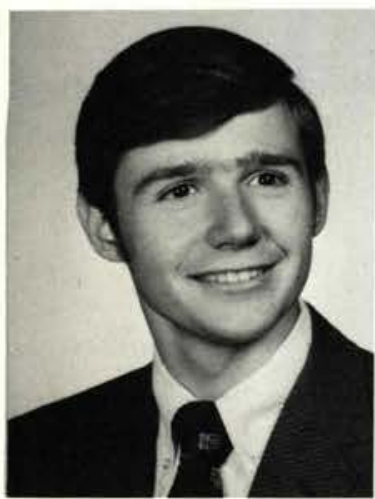

Carl Rodgers

Cuyahoga Falls, O.

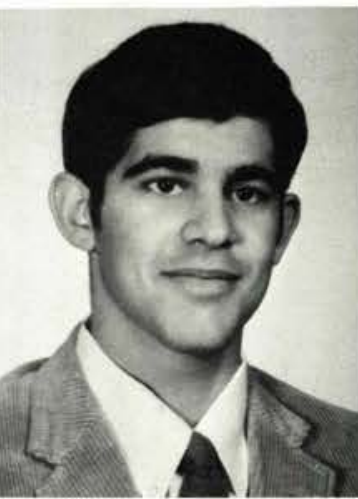

David Rodriguez

Greenlawn, N. Y.

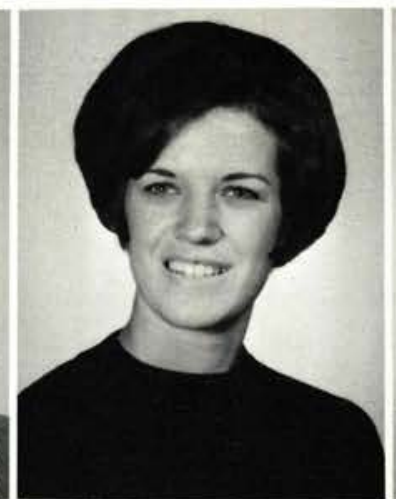

Nancy Ross

Pontiac, Mich.

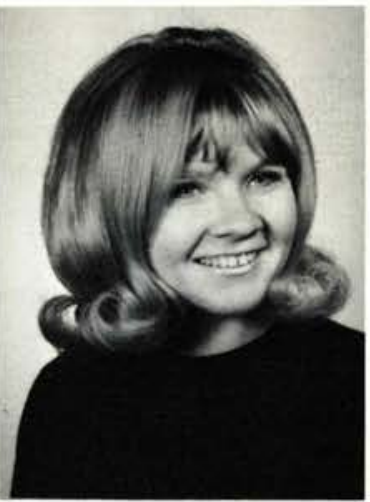

Candy Ruby

Dayton, Ohio

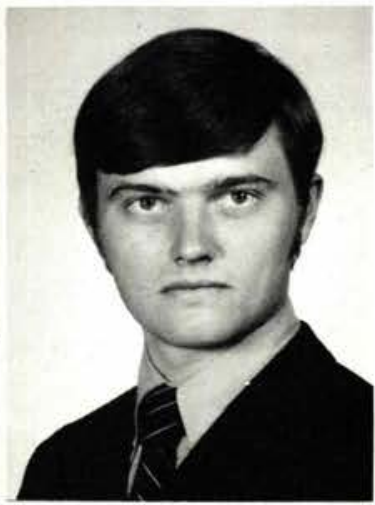

James Samuelson

Allegan, Mich.

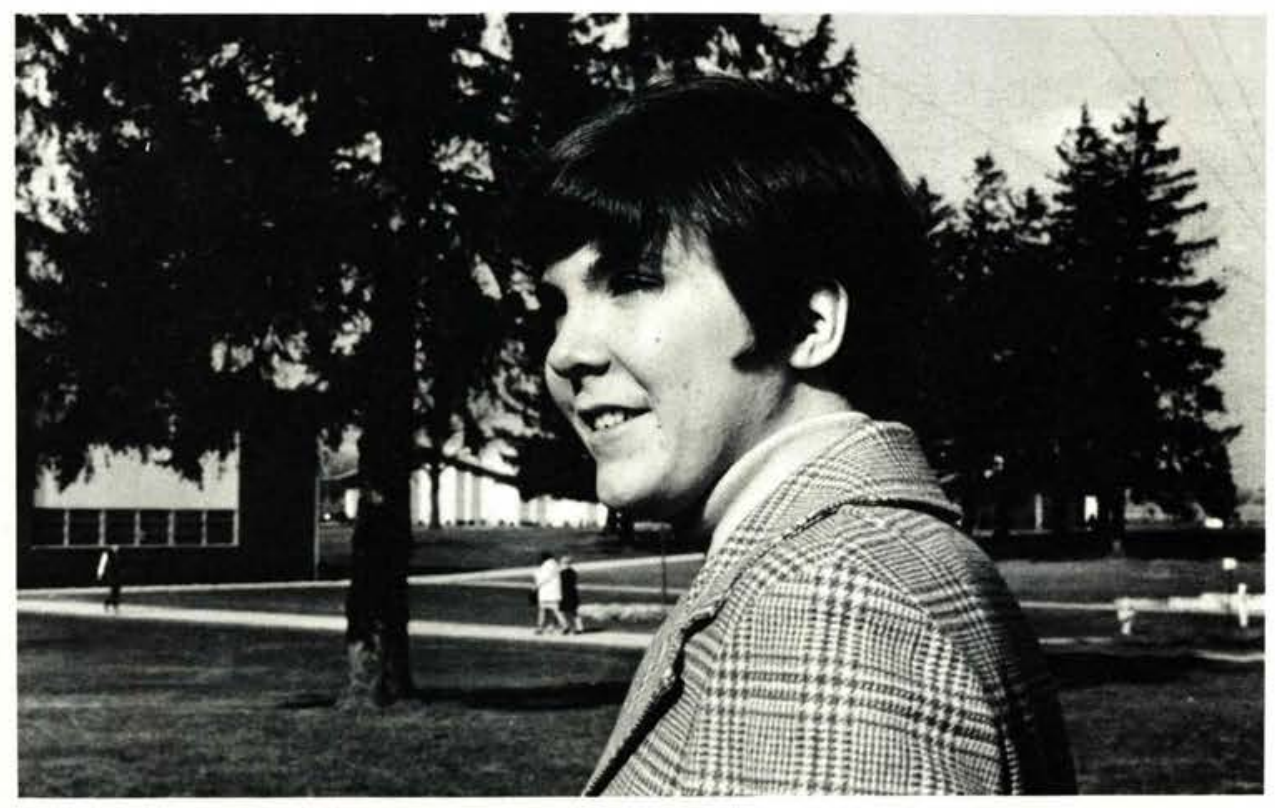

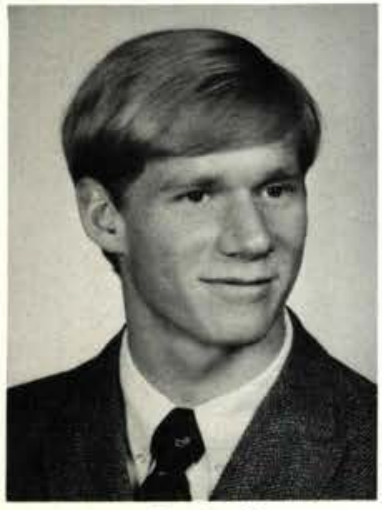

Tim Sanderlin

Portsmouth, $\mathrm{O}$.

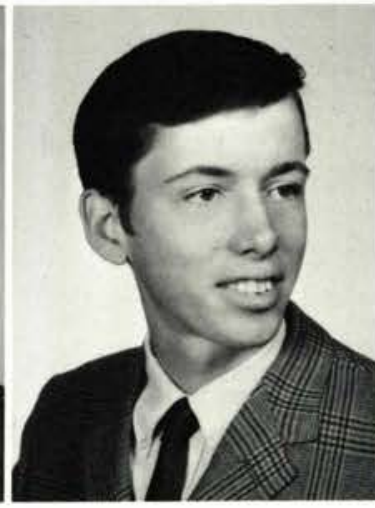

Eldon Sarver

Rose Hill, Iowa

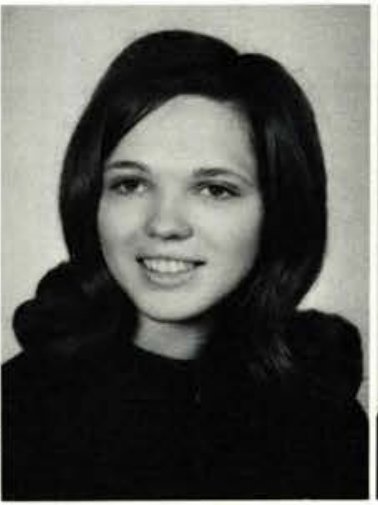

Gretchen Schlechty Lakewood, Ohio

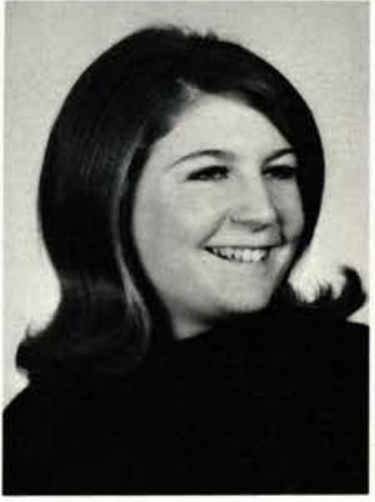

Kathy Schmidt Detroit, Mich

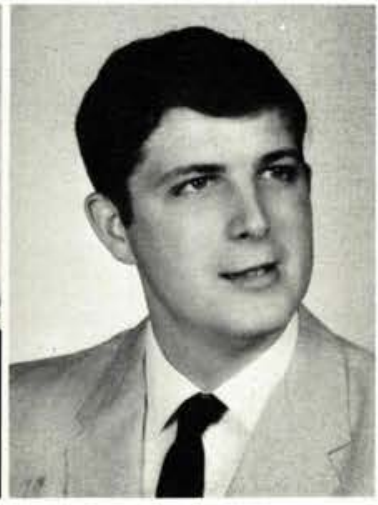

Lloyd Schopmeyer Brazil, Ind.
Don Schultz Midland, Mich.

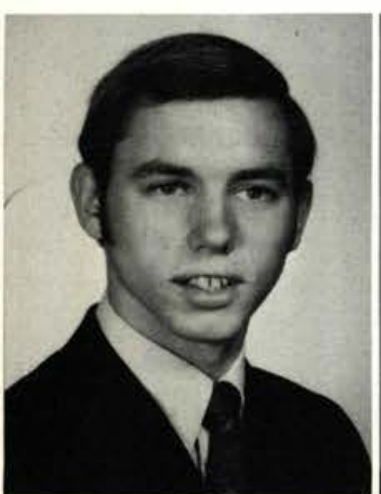

Esther Schultz Milwaukee, Wis.

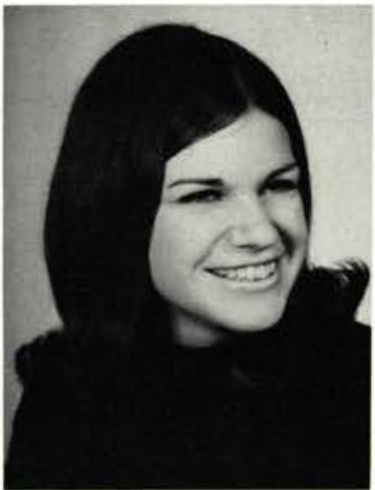

Nancy Sell New Canaan, Conn.

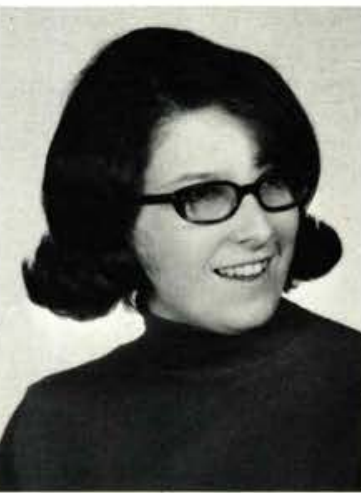

Steve Shank New Madison, $\mathrm{O}$.

Linda Shorey

Akishima Shi, Tokyo, Japan

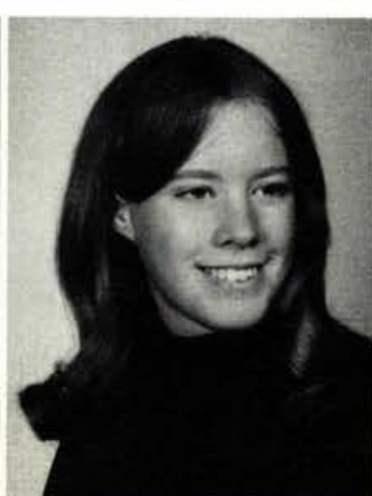




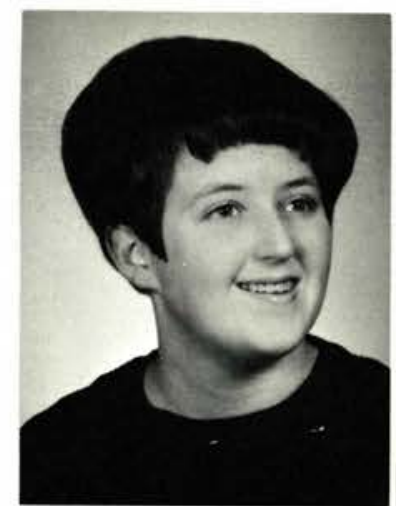

Charlene Sickels

Barberton, Ohio

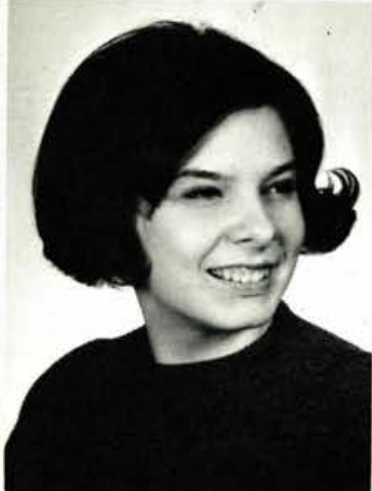

Connie Simpkins

Cambridge City, Ind.

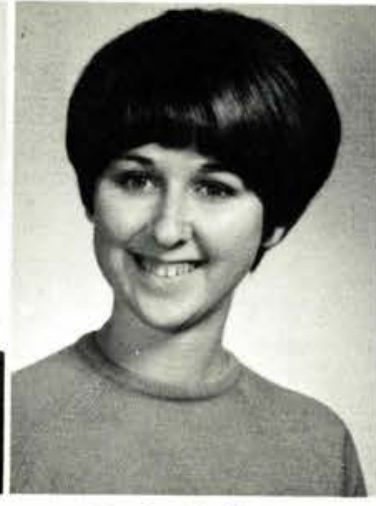

Becky Smith

Dayton, Ohio

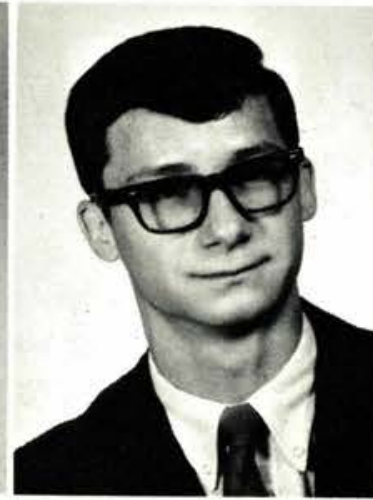

Dale Smith

Celina, Ohio

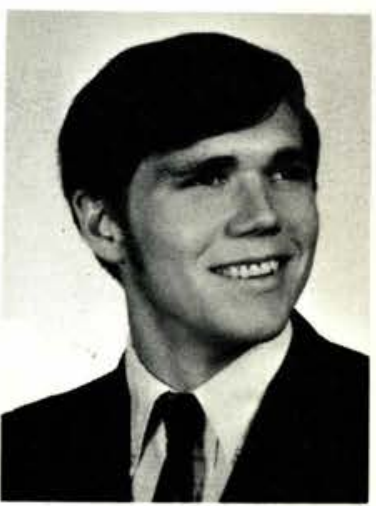

Jonathan Smith Findley, Ohio

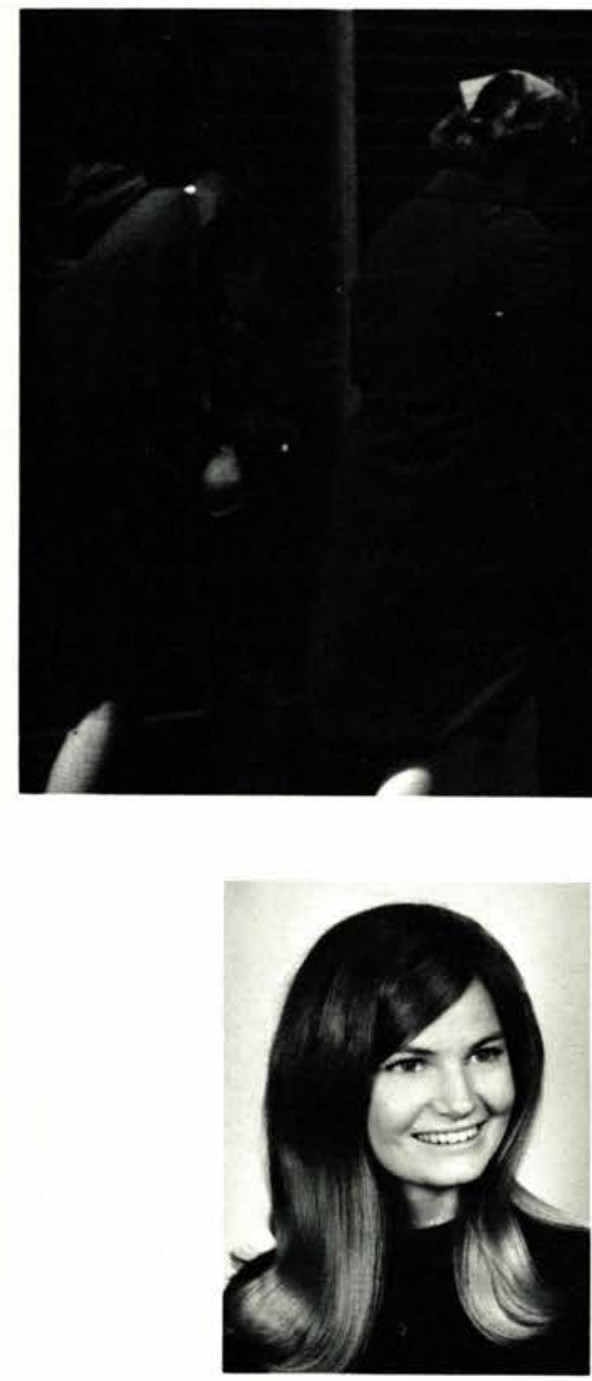

Adria Soeder

Euclid, Ohio
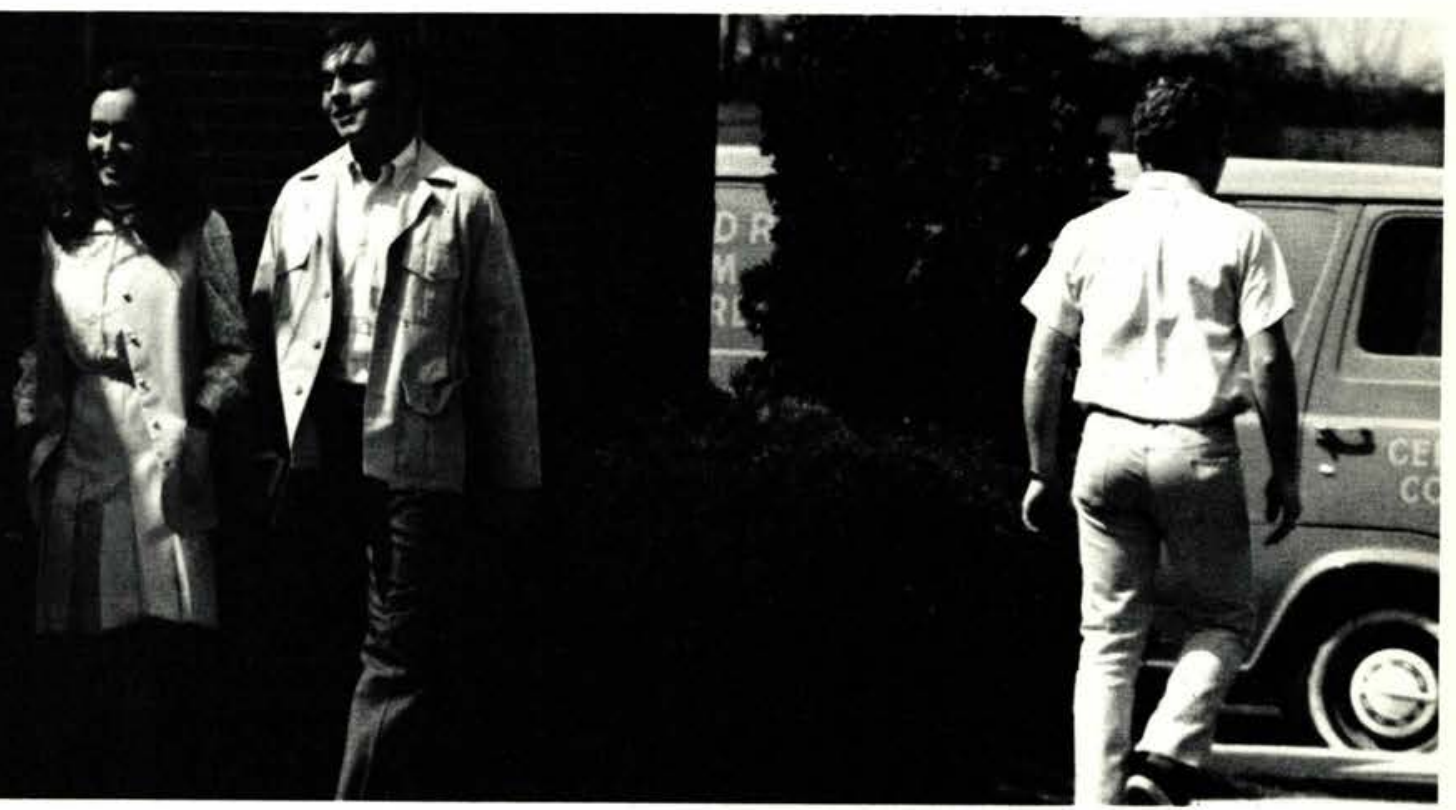

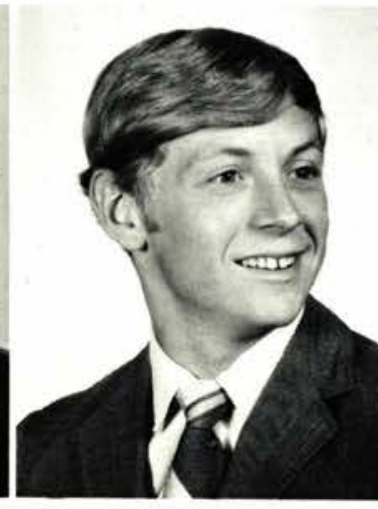

Don Southwell

Mancelona, Mich.

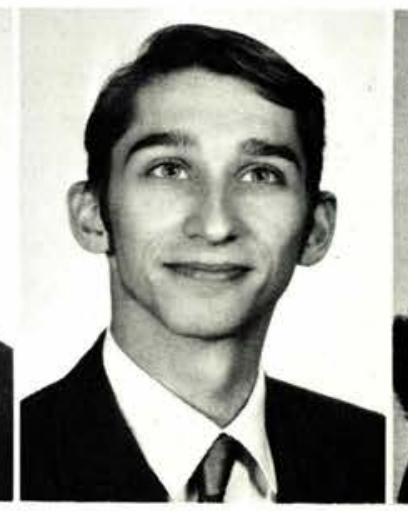

Joseph Spade

Vandelia, Ohio

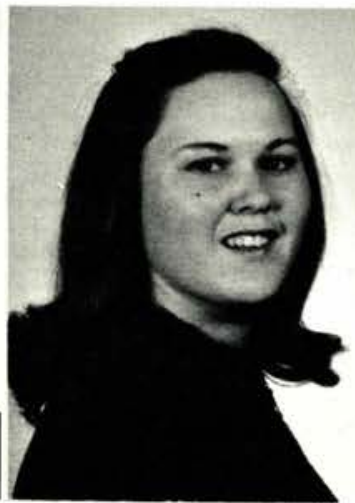

Bonnie Spears

Strongville, $\mathrm{O}$.

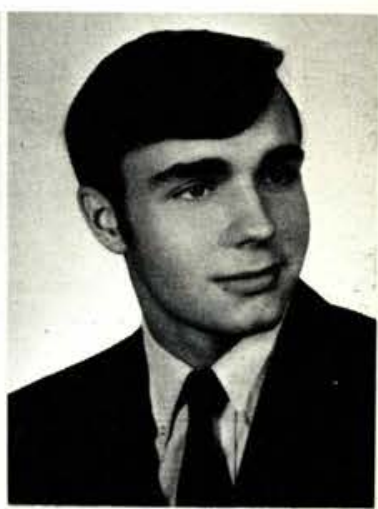

Walter Spencer

Hadden Heights, N. J.
Stephen Spink Belfast, N. Y.

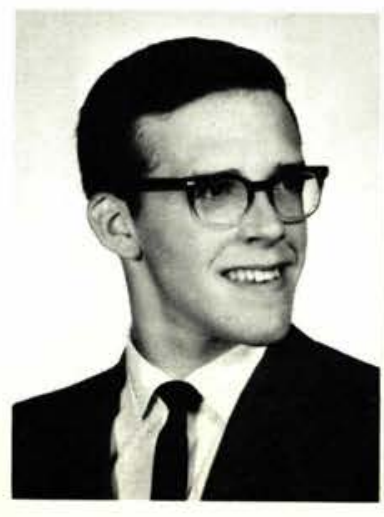

Jan Spradlin

Wheaton, Md.

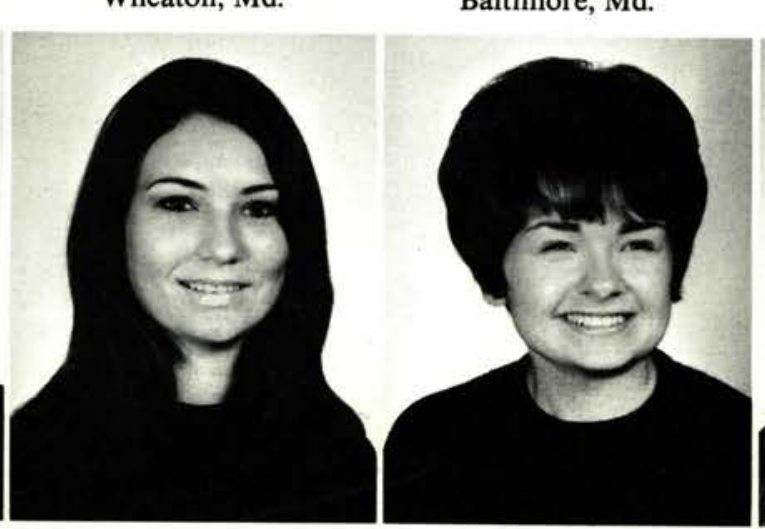

Mark Stephenson Grundy Center, Ia.

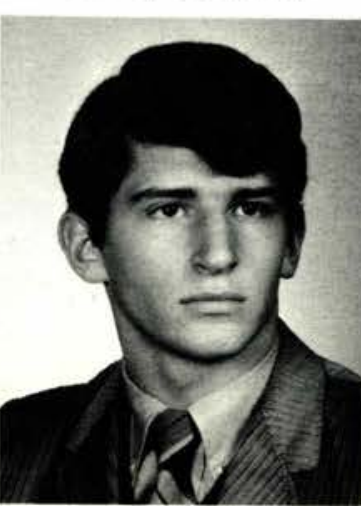

Ruth Steyer

Coshocton, $\mathrm{O}$. 

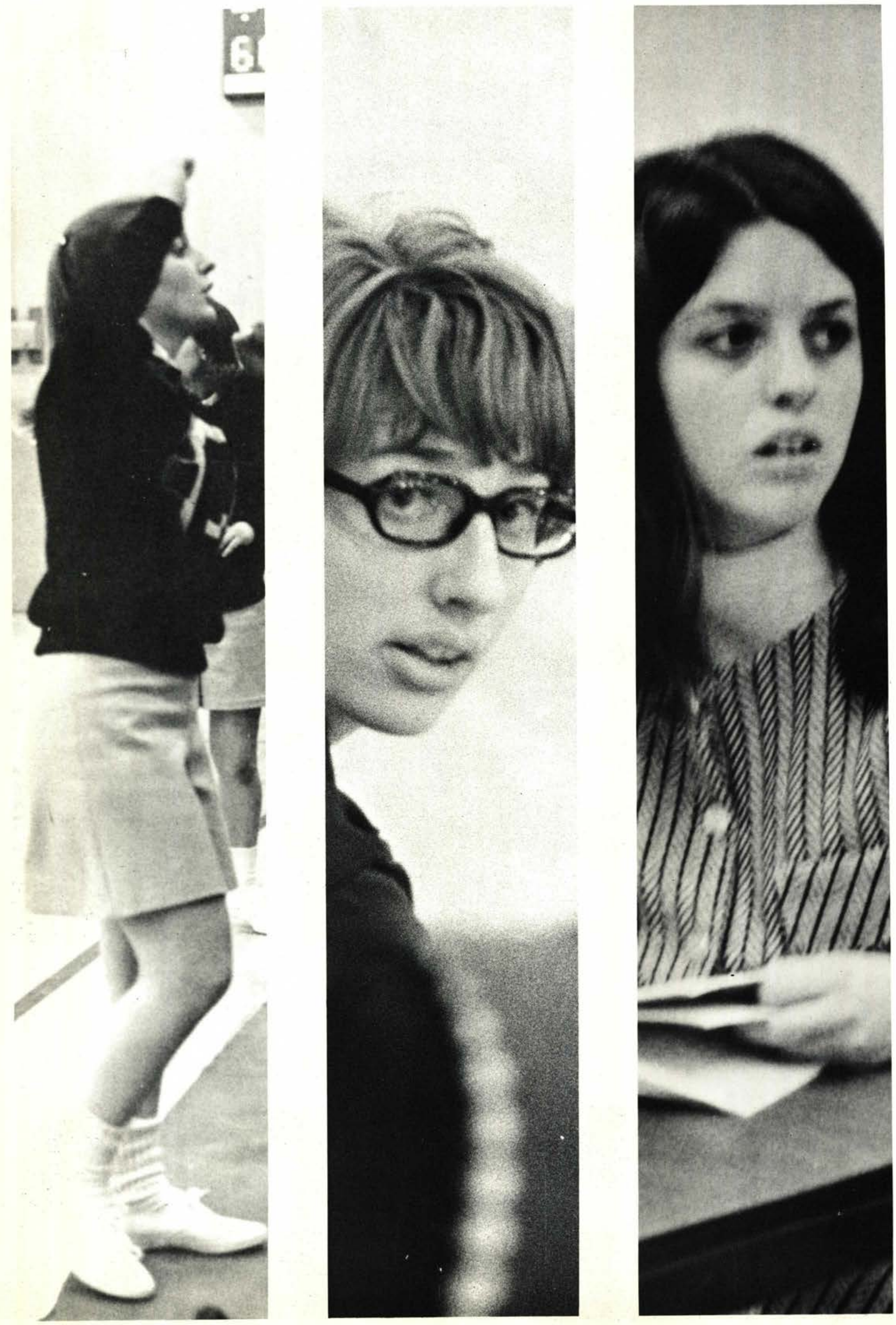


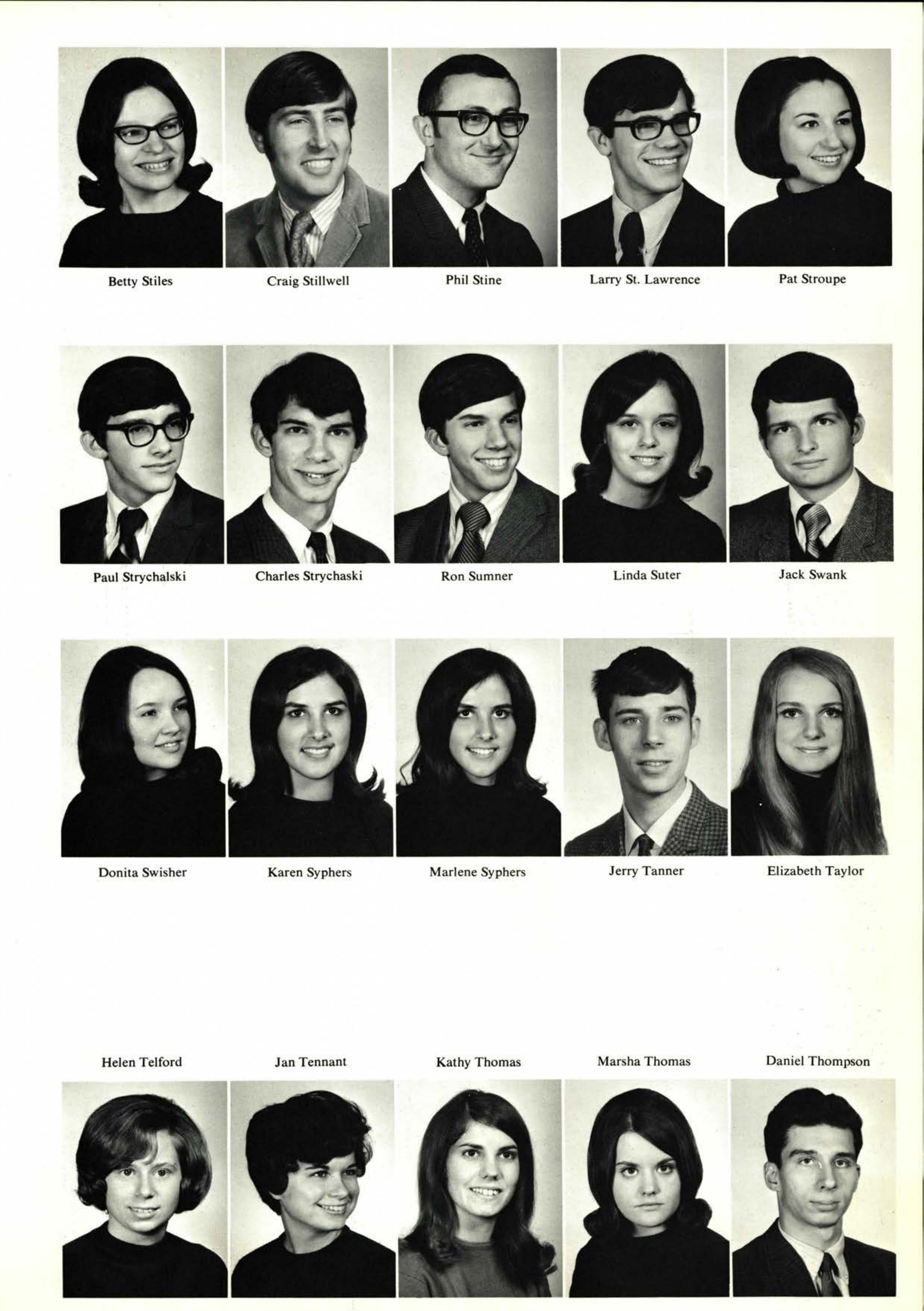




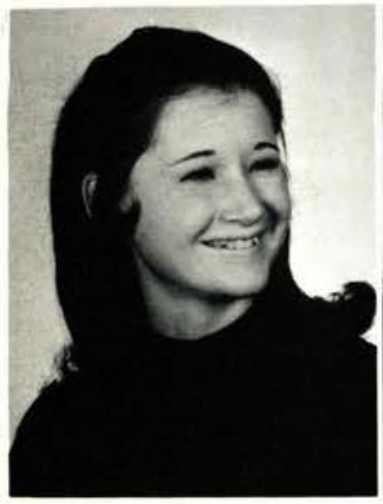

Deborah Thorton

Cedarville, Ohio

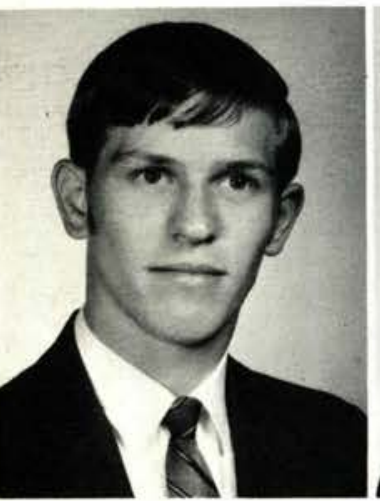

Jacob Tipton

Northfield, Ohio

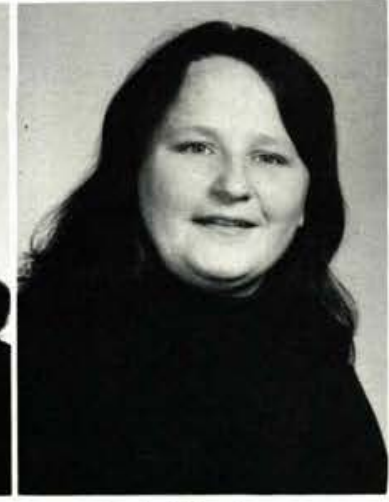

Lois Tingle

Rockton, Pa.

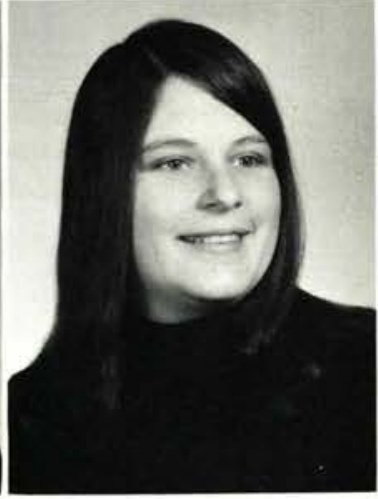

Patricia Tingle Rockton, Pa.

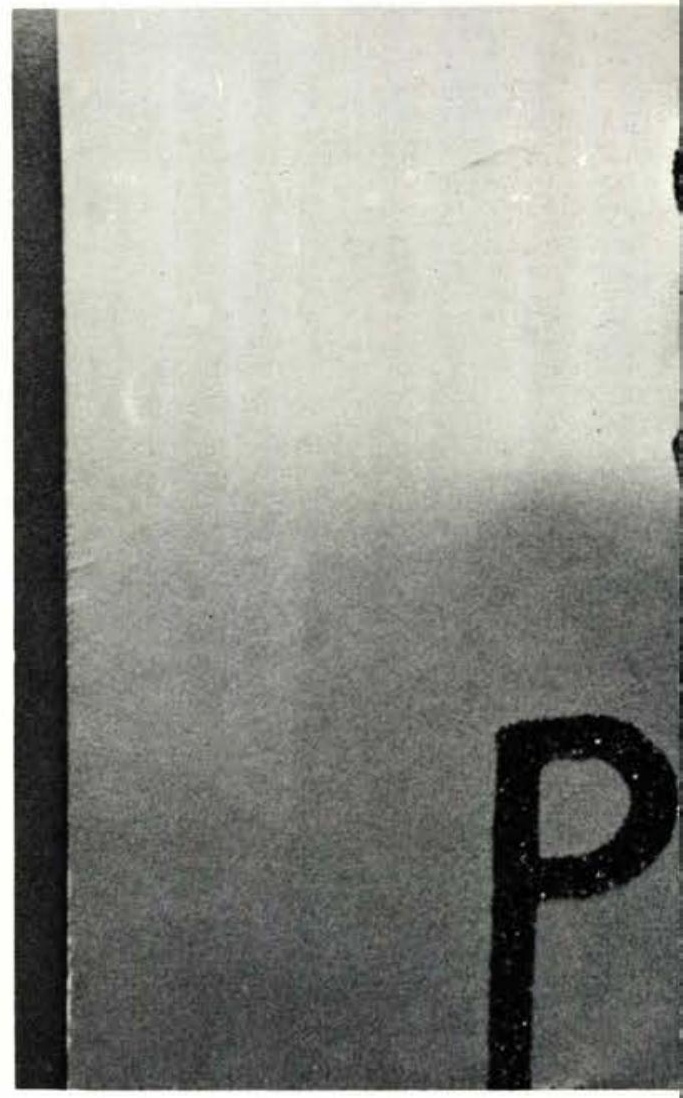

Brenda Tompkins

Sherman, N.Y.

Joy Trefzger

Cedarville, Ohio
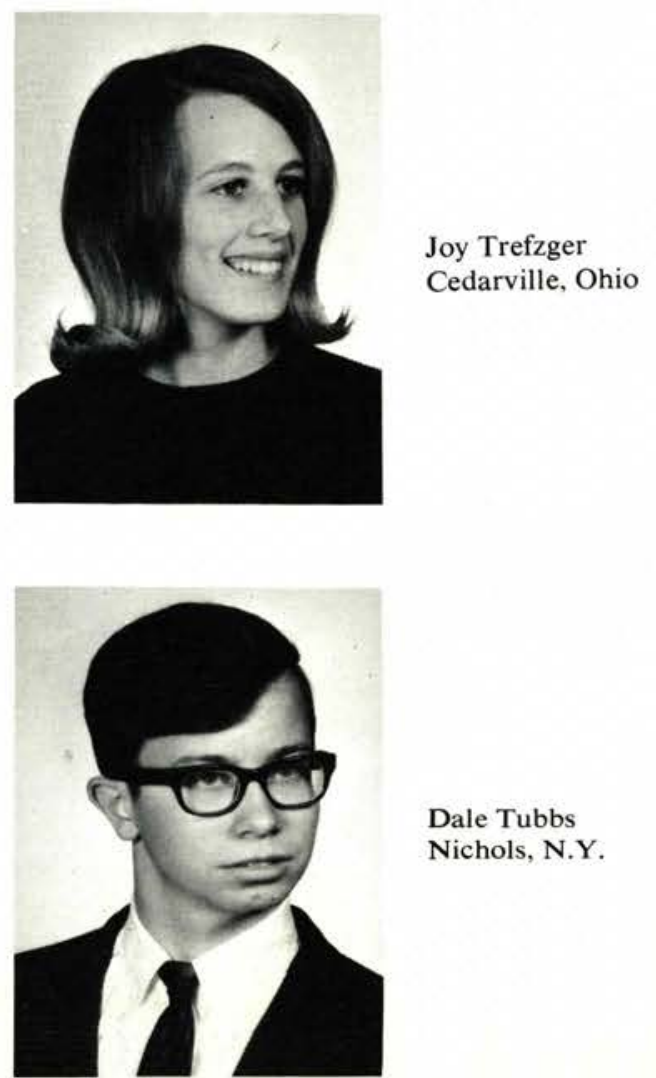

Dale Tubbs

Nichols, N.Y. 


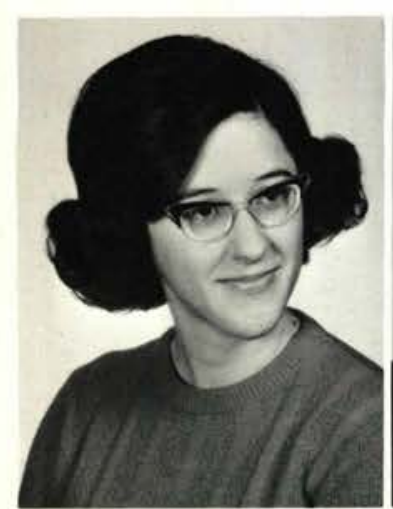

Laura Weddle

Prosperity, $\mathrm{Pa}$.

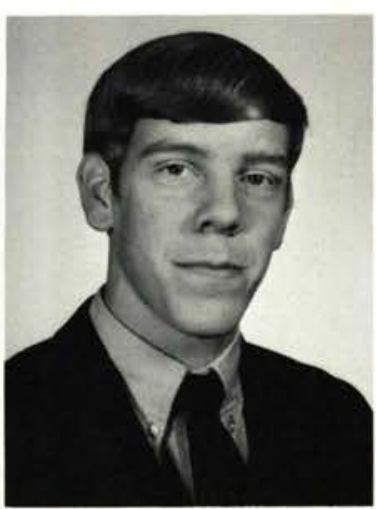

Tim Welmer

Dayton, Ohio

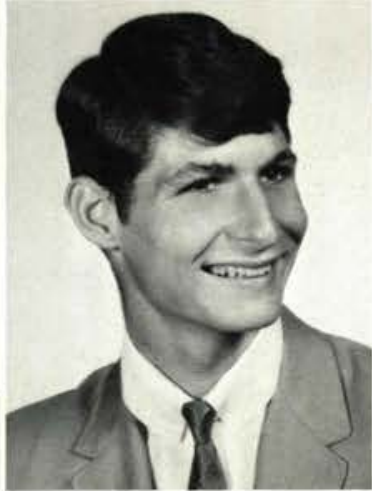

Ernie Welborn Mentone, Ind.

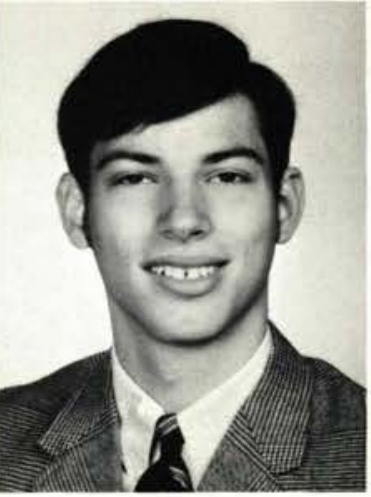

David Weng

Dayton, Ohio

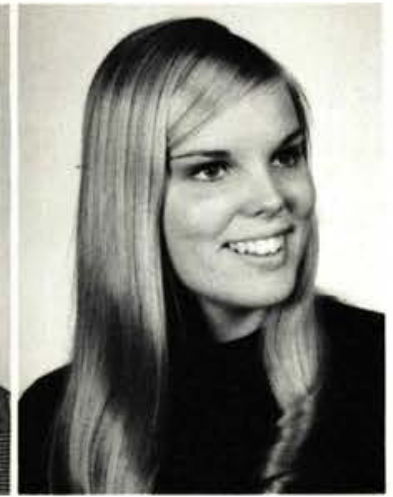

Holly Wenner

New London, O.

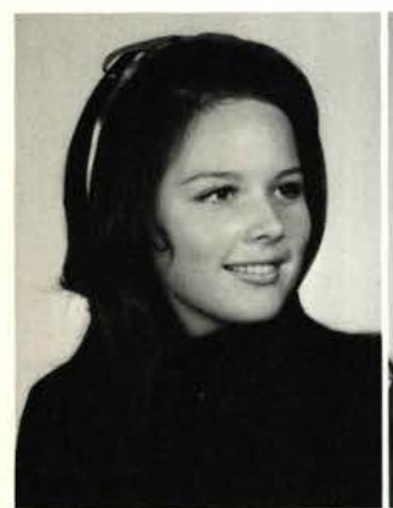

Yvonne West

Monmouth, Ill.

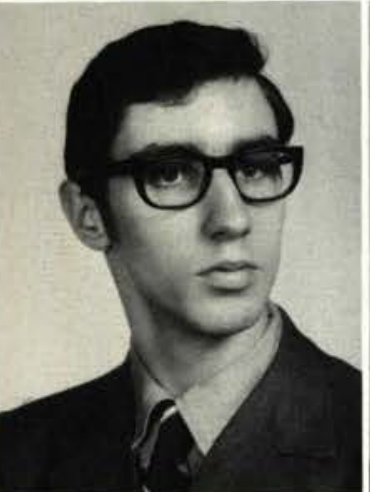

Michael Wilkman

Detroit, Mich.

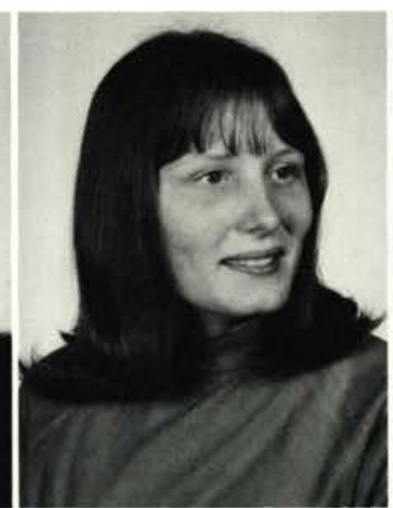

Patricia White

Lewiston, Idaho

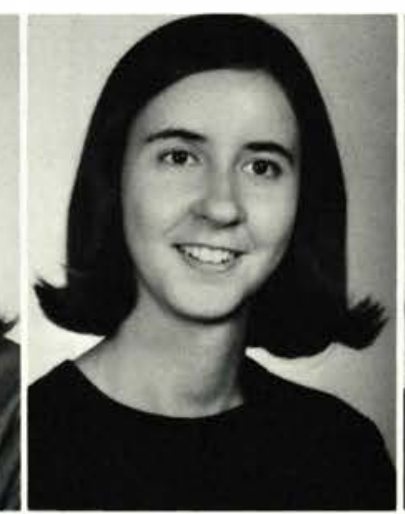

Sandra White

Youngstown, $\mathrm{O}$.

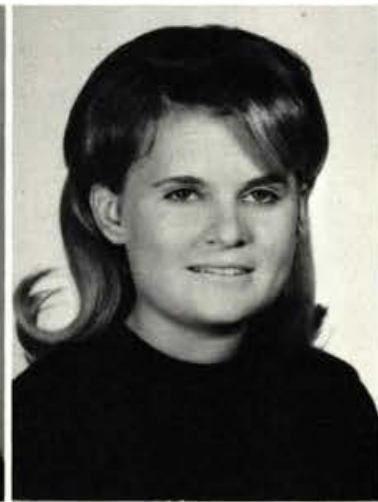

Chery Whitney

Wheaton, Md.

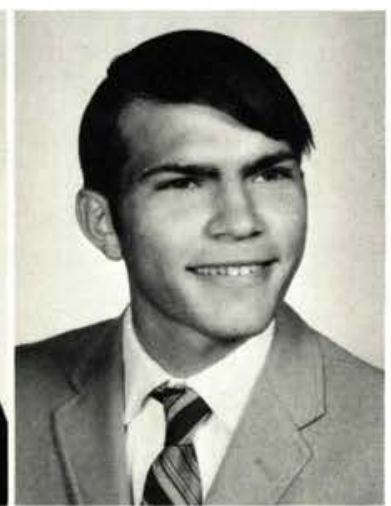

John Wiess

Hamburg, N. Y.

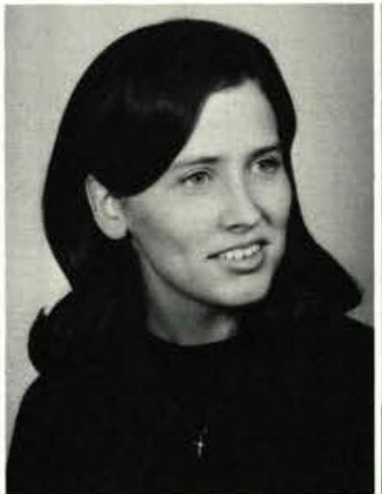

Becky Williams

Wood River, Ill.

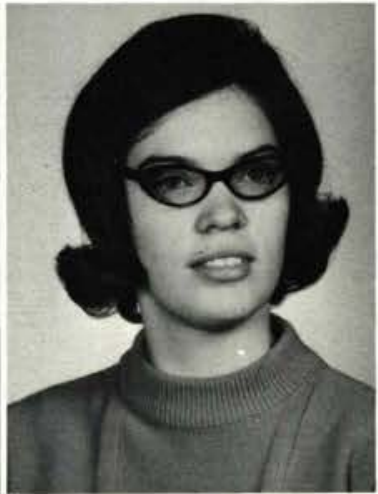

Gay Williams

Lake Park, Florida

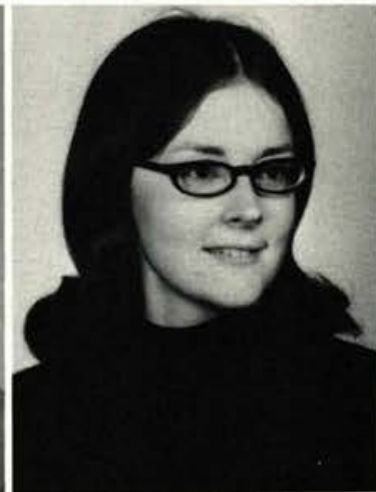

Linda Willms

Warren, Mich.

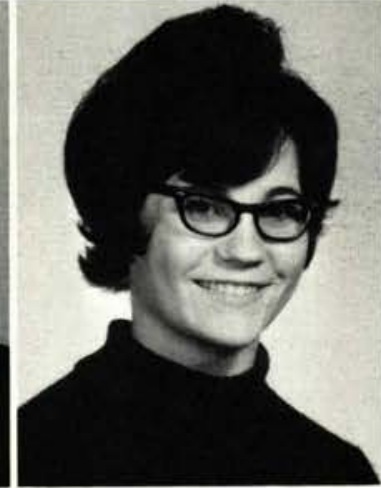

Ardell Witt

Waterloo, Iowa
Freda Witt Libertyville, Iowa

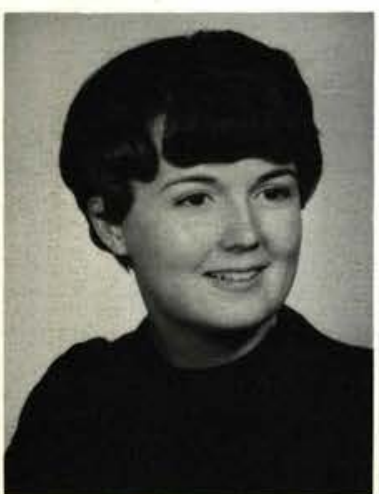

Ronald Wolfe

Farmingdale, N. J.

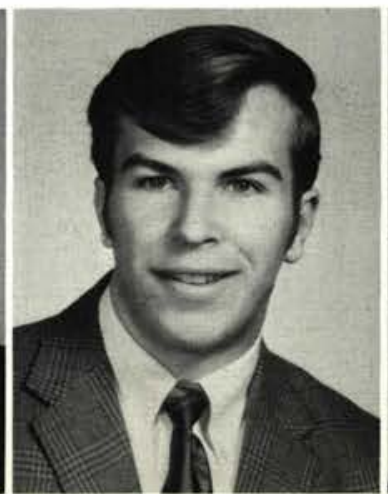

Robert Wood

Clarkston, Wash

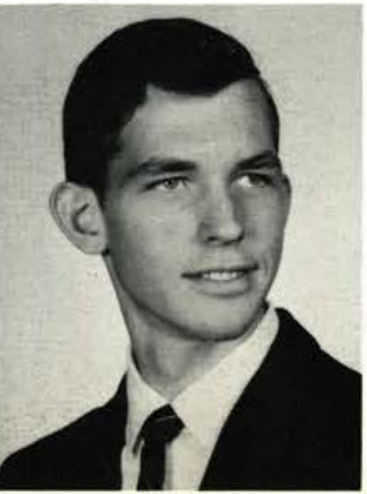

David Work

Coudersport, $\mathrm{Pa}$

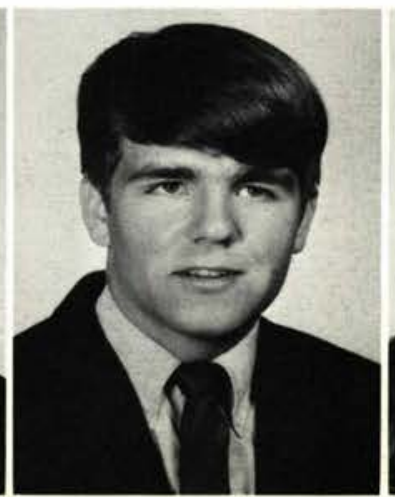

Deborah Wrigh

Fairfax, Va.

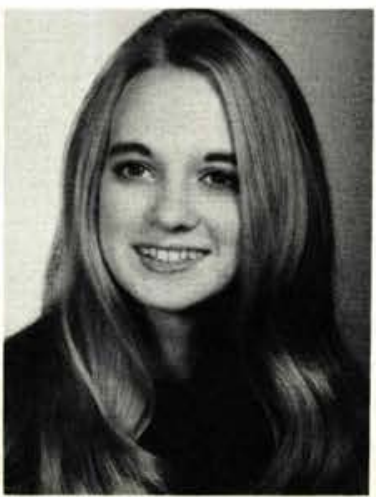




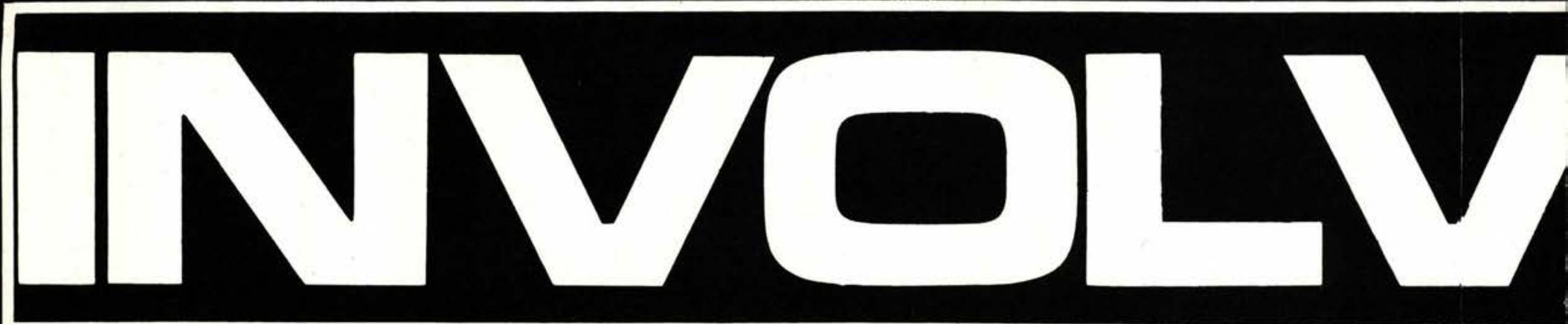

- 8

(1) 11 -

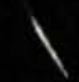

Thuthe

(4tutum

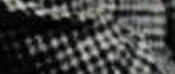

(4)

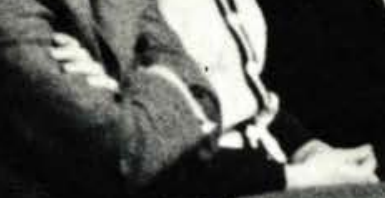

$r_{1}^{-\infty}$
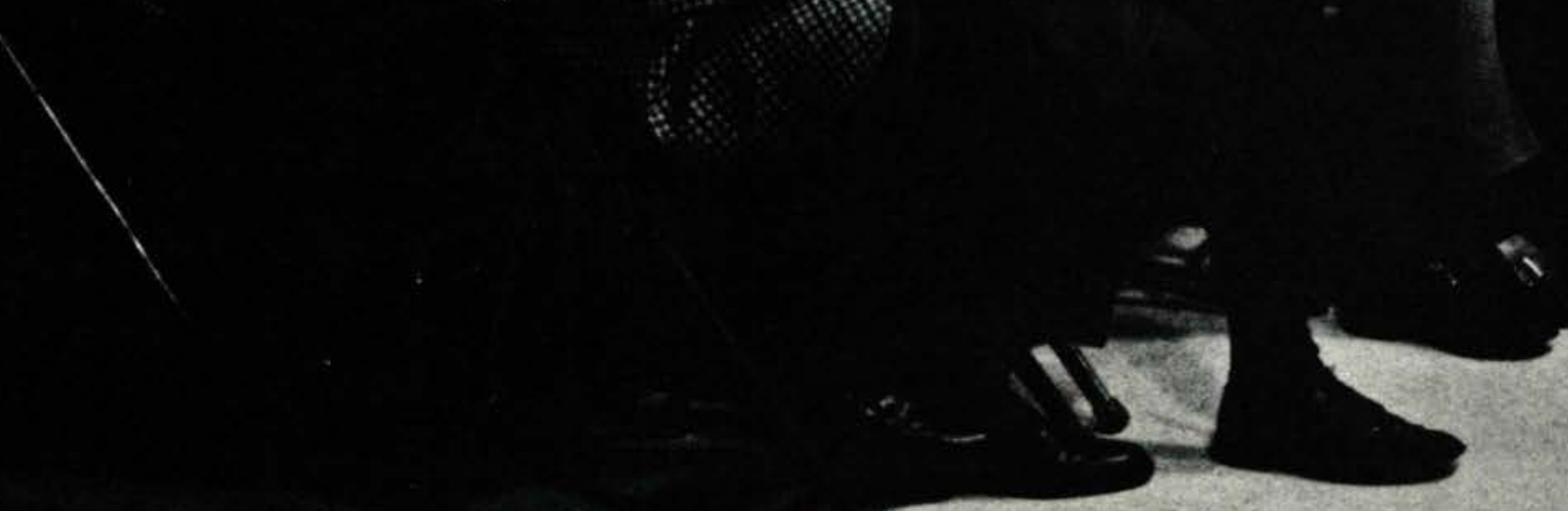

30
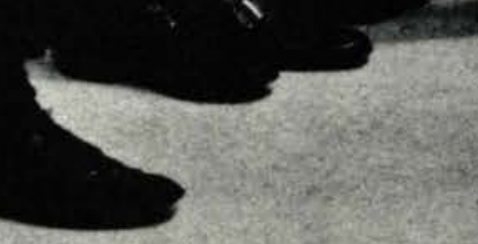

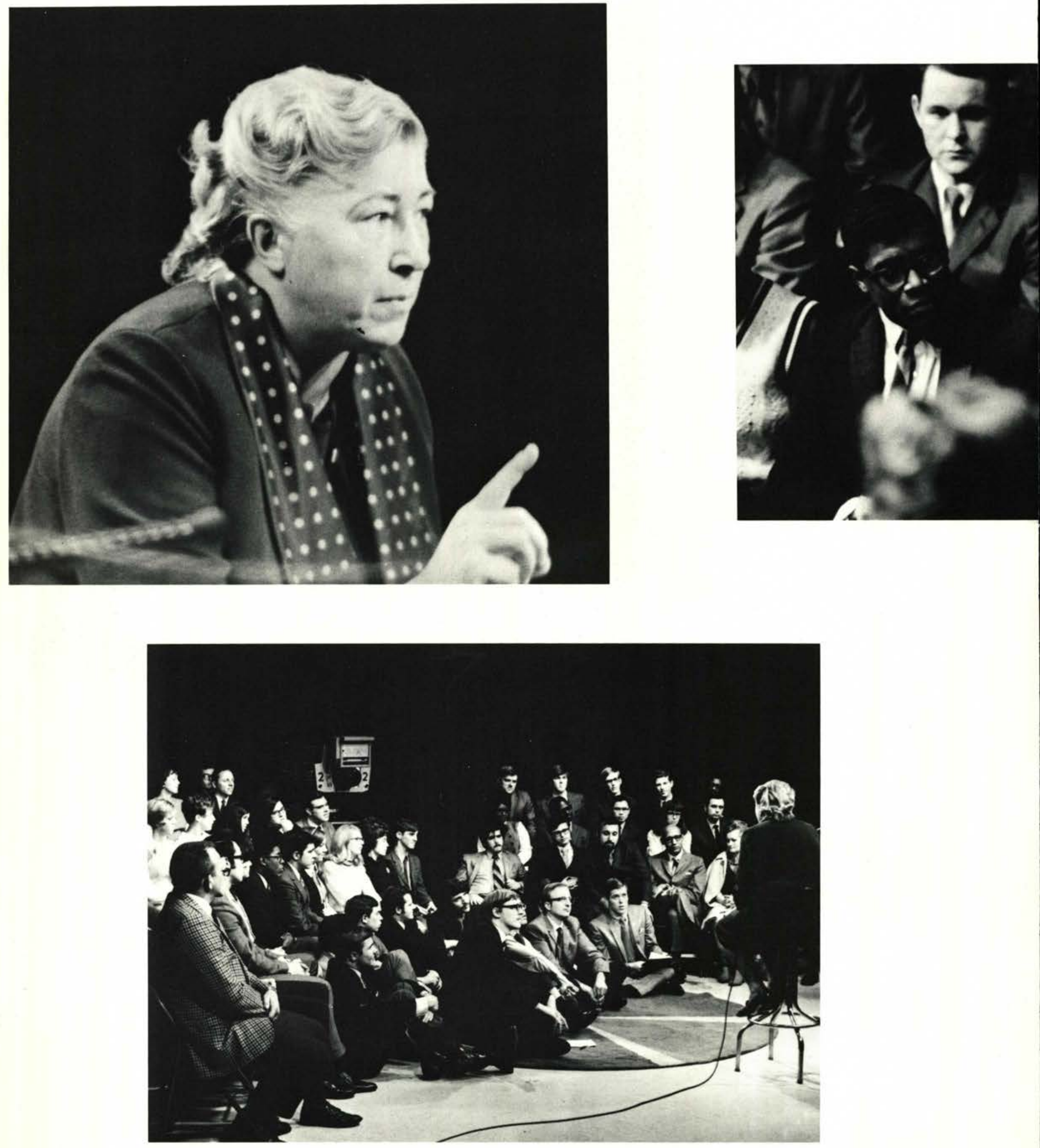


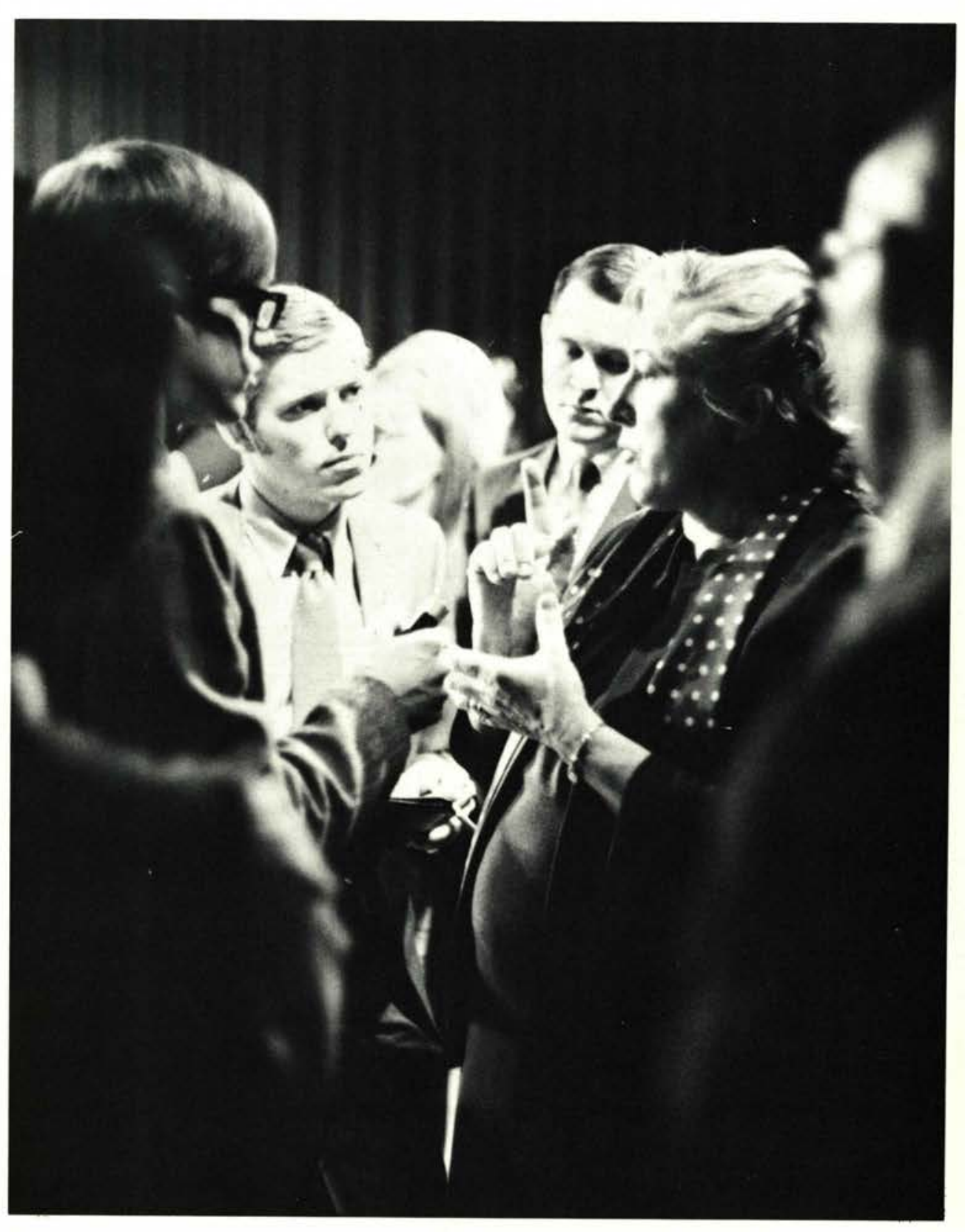

"The fool hath said in his heart, 'There is no God.',"

-Psalm 53:1 


\section{Artigt}
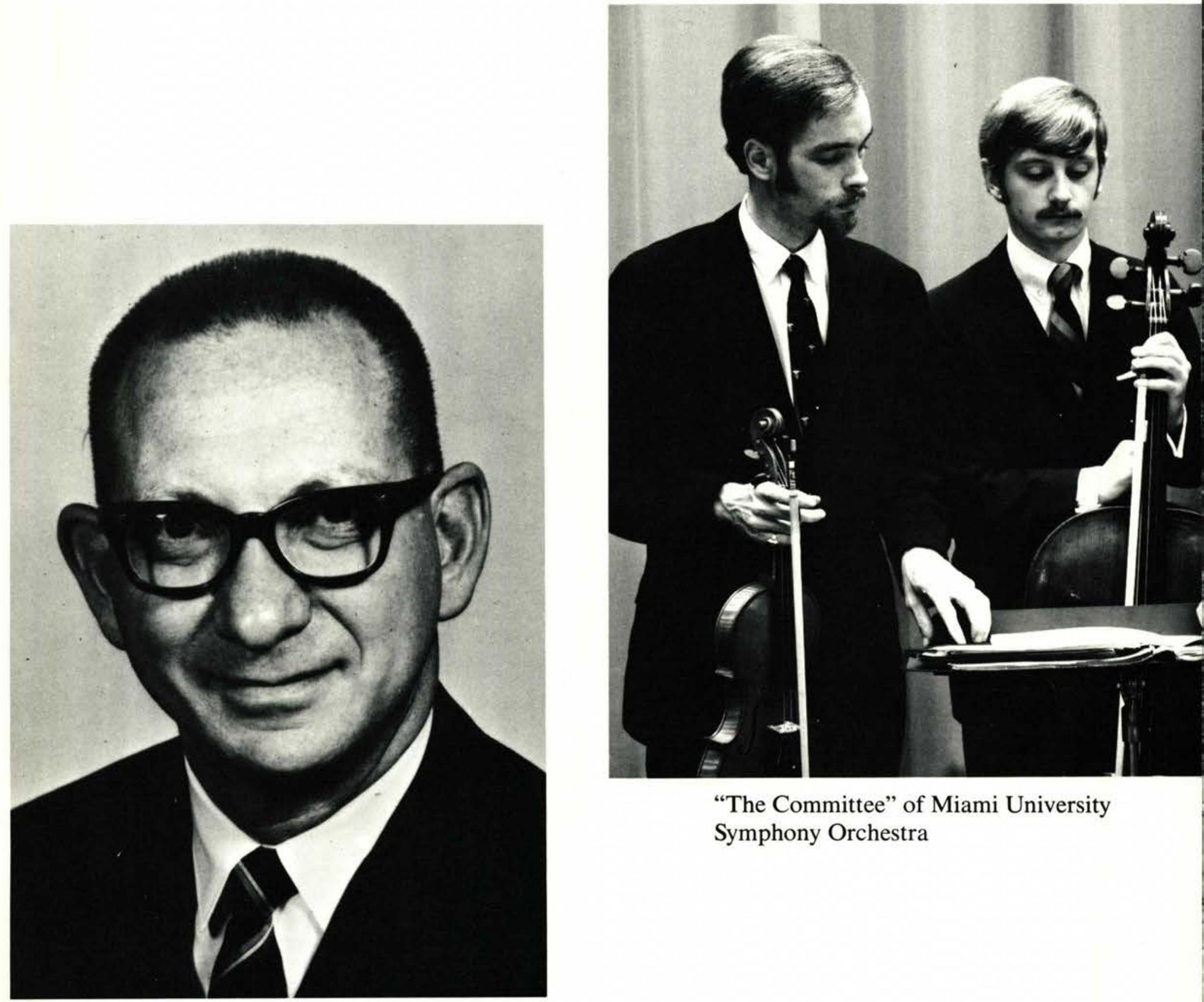

"The Committee" of Miami University Symphony Orchestra

Dr. George A. Seltzer

Director, Miami University Symphony 


\section{$1 \mathfrak{g} \mathfrak{h} \mathfrak{q}-7 \mathfrak{n}$}

Nov. 20 . . . . Miami University Symphony Orchestra Dec. 1 . . . Dr. Karlis Leyasmeyer Feb. 13 . . . . . . . Joyce Jones, Organ Virtuoso Feb. 19 . . . . . . Alan Lightner, "Life in a Moslem Culture" Mar. 24 . . . . University of Akron Concert Band and Chorus Mar. 27 .... . Dr. James Golden, "The Rhetoric of Black Power" April 5....... Wheaton College Concert Band April 17 ......... James Oliver Buswell, IV, Violin Virtuoso 


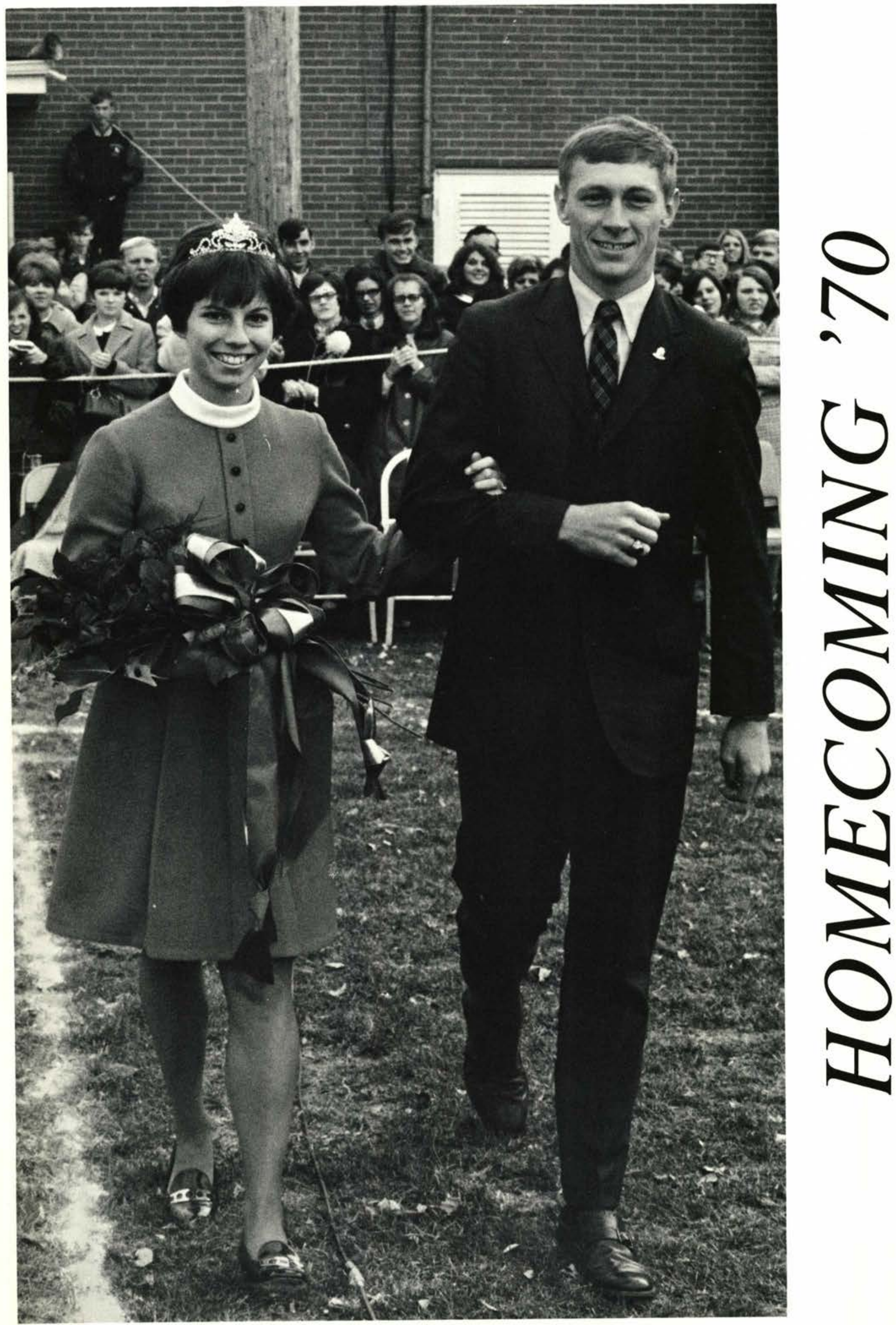


Glowing bonfire (?). . . divergent entertainment . crisp autumn air . . . variegated displays . . . charming queen . . .graceful court . . . sportsmanship . . .
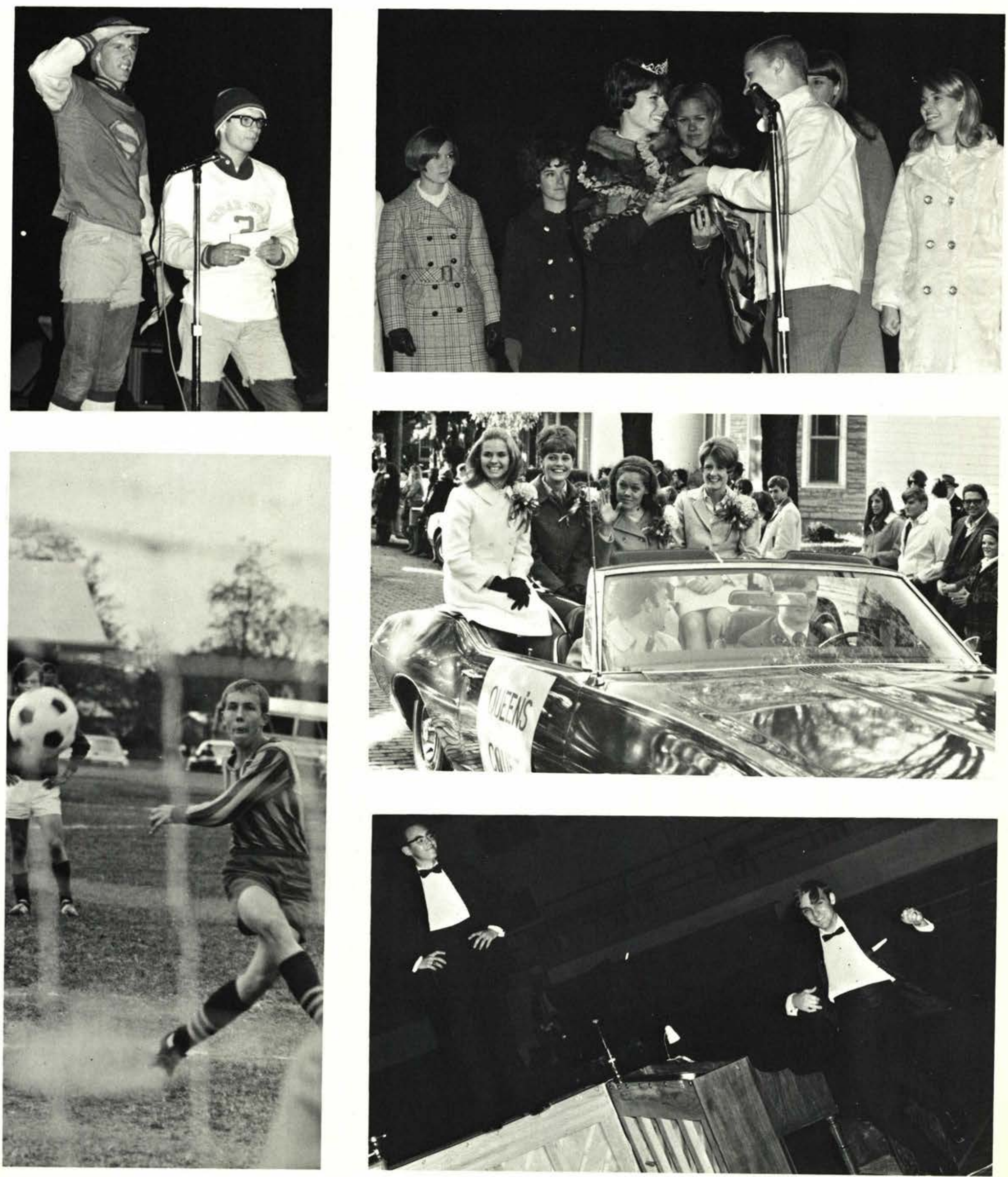

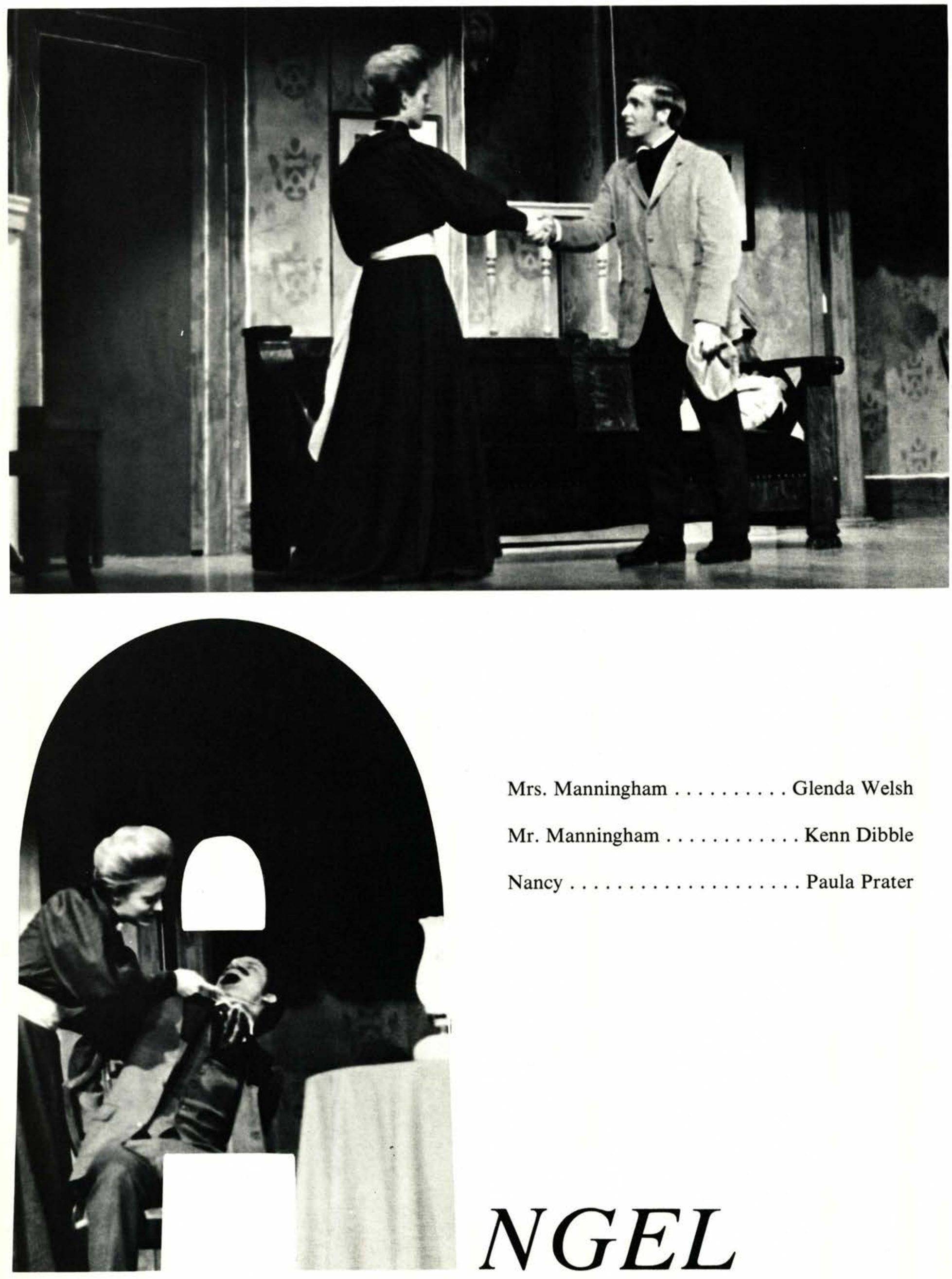

Mrs. Manningham . . . . . . . Glenda Welsh

Mr. Manningham . . . . . . . Kenn Dibble

Nancy . . . . . . . . . . Paula Prater

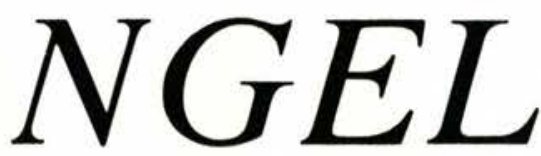



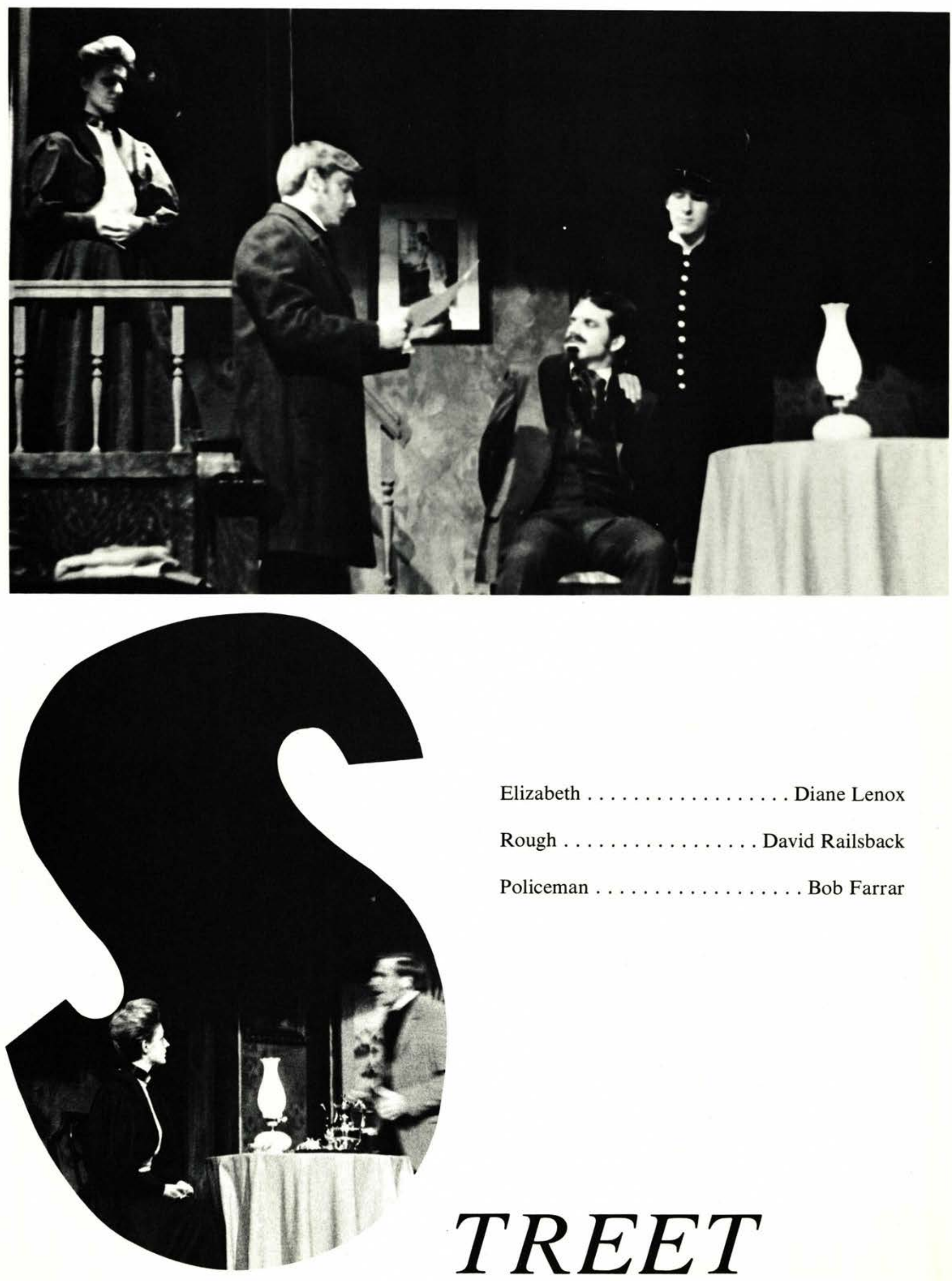

Elizabeth . . . . . . . . . . . Diane Lenox

Rough . . . . . . . . . . David Railsback

Policeman . . . . . . . . . Bob Farrar

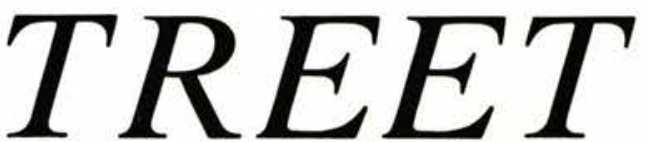


CAST

Dr. Haggett

Susan Haggett

Abby

Mrs. Haggett

Ada Haggett

Warren Creamer

Tallent

Rosen

Maxwell Davenport

Assistant Director
Dan Liechty

Peggy Porterfield Jean King

Susan Gift

Joyce Saemenes

Kenn Dibble

Randall Hamilton

Don Harmon

Dale Shilling

John Kuhns

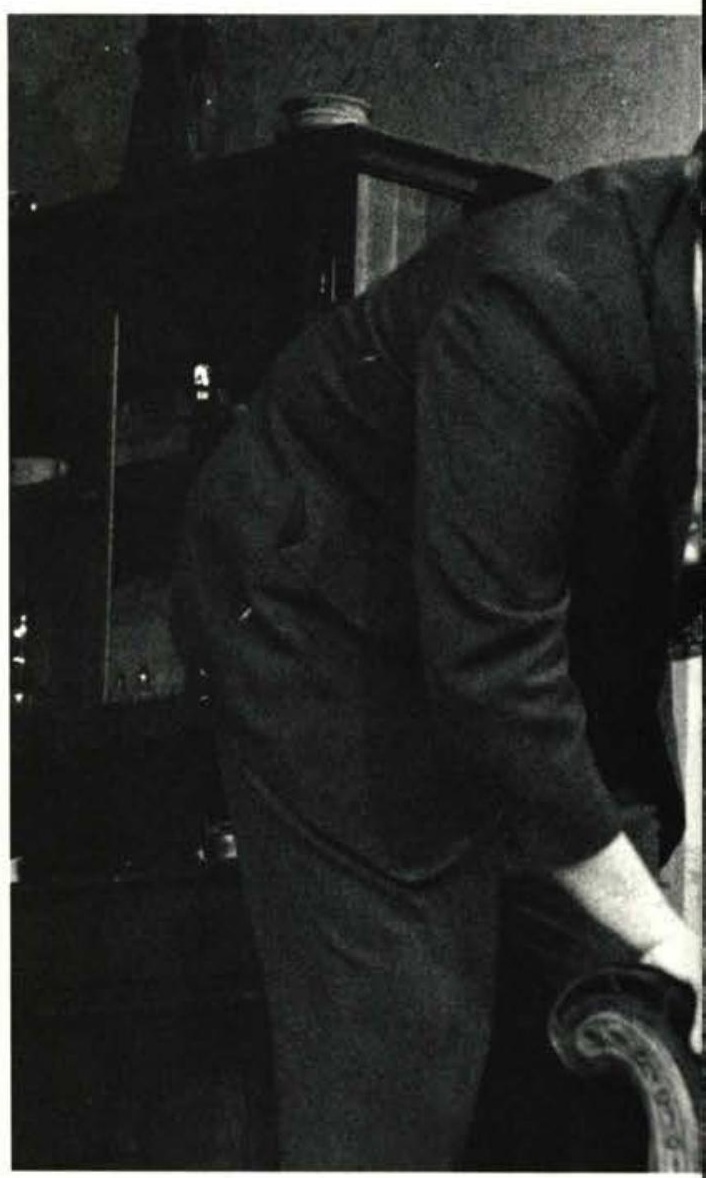

$T H E$

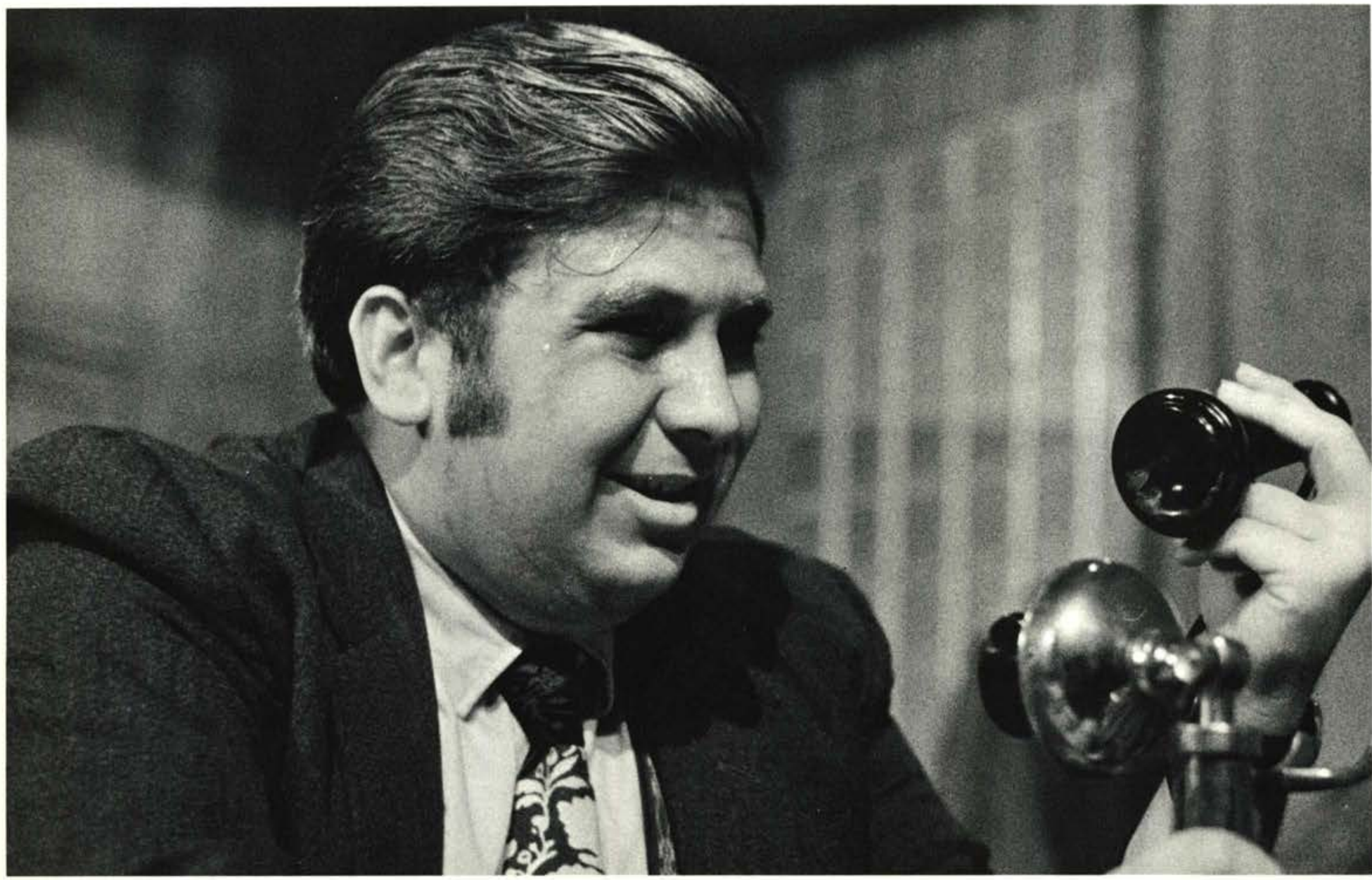




$$
+
$$
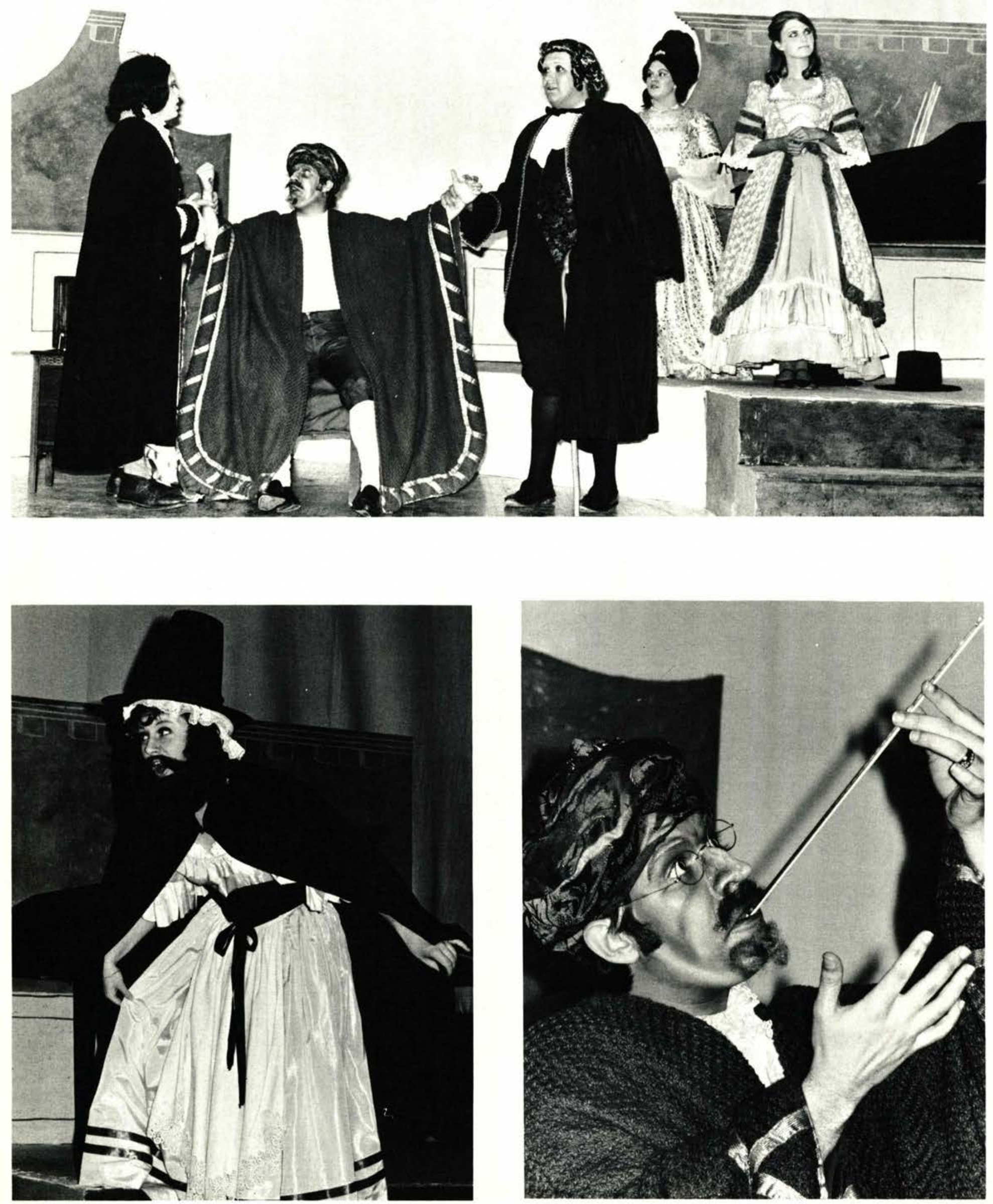


\section{THE IMAGIN ARY INVALID}

CAST

Monsieur Argan . . . . . . . . . Robie Ison Toinette . . . . . . . . . . . . Peggy Porterfield Angelique ............. Ginger Burget Beline . . . . . . . . . . . . . Jean King Monsieur Bonnefoi . . . . . . . . . . Jerry Grayson Cleante . . . . . . . . . . Mark Flint Dr. Diaforus . . . . . . . . Dan Liechty Dr. Thomas Diaforus . . . . . . . . Don Harmon Louise . . . . . . . . . . . Paula Prater Monsieur Beralde . . . . . . . John Bacheller The Apothecary . . . . . . . . . Ron Meyers Dr. Purgon . . . . . . . . . . . . Bill Henry Assistant Director . . . . . . . . Susan Gift
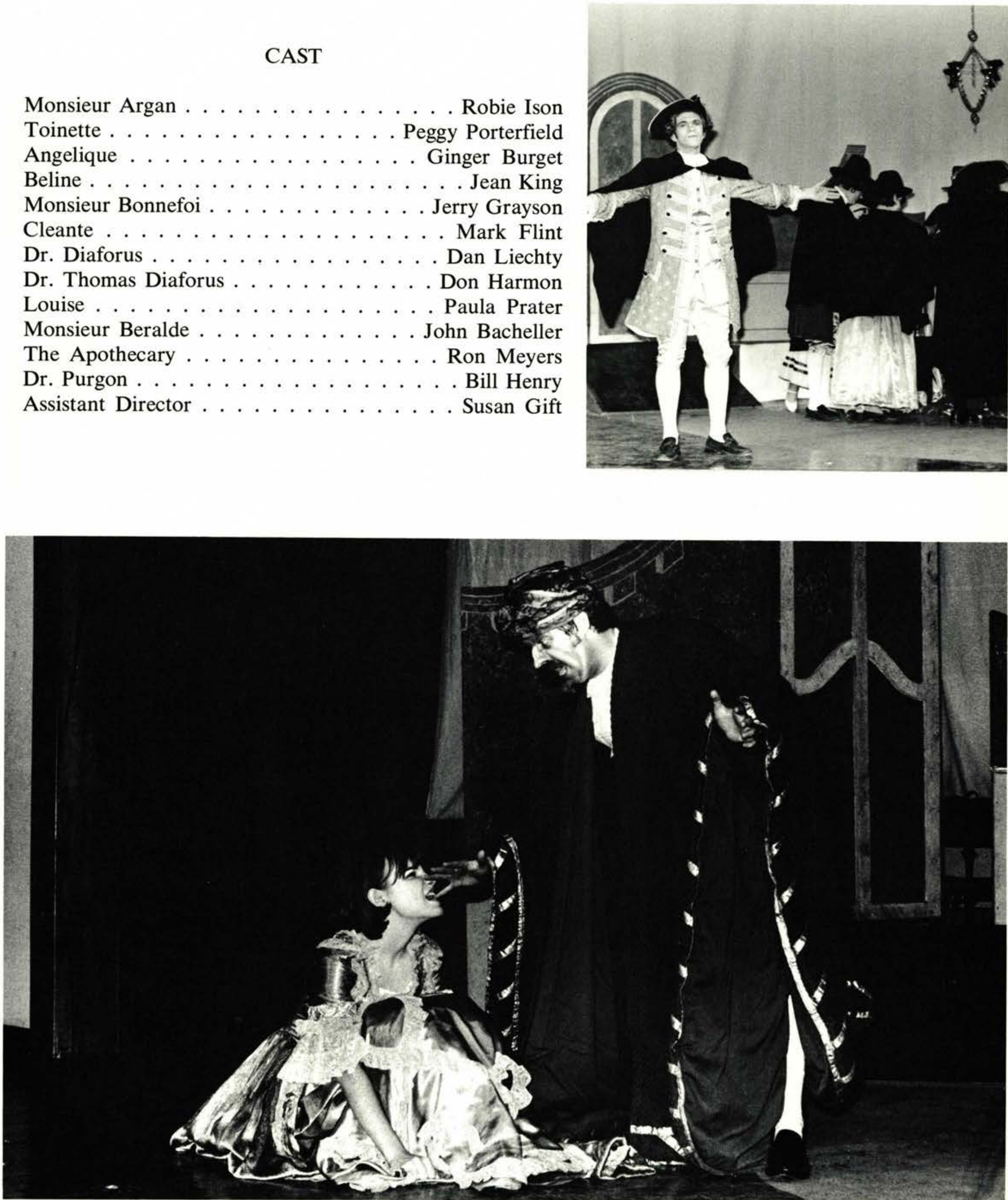

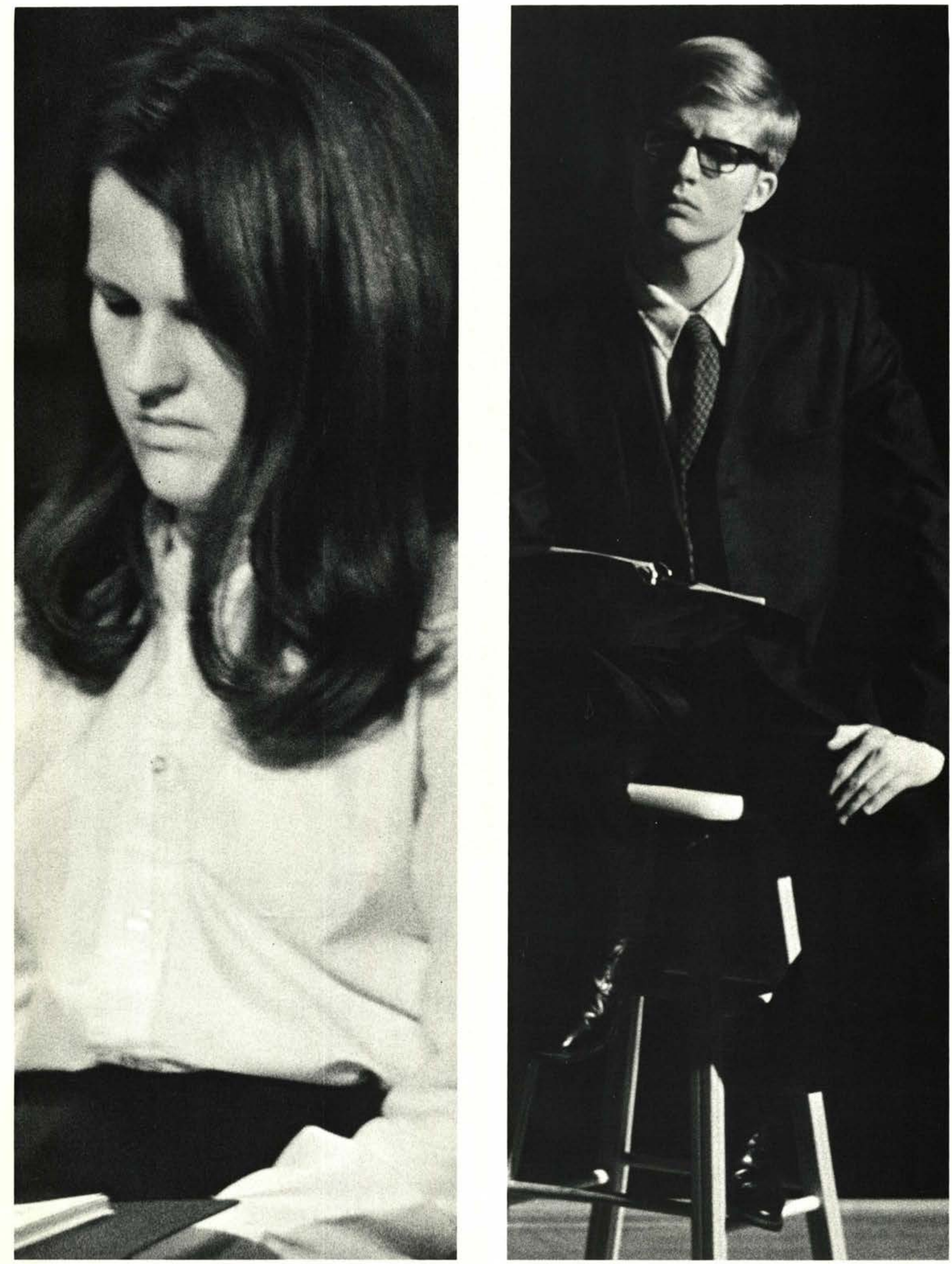


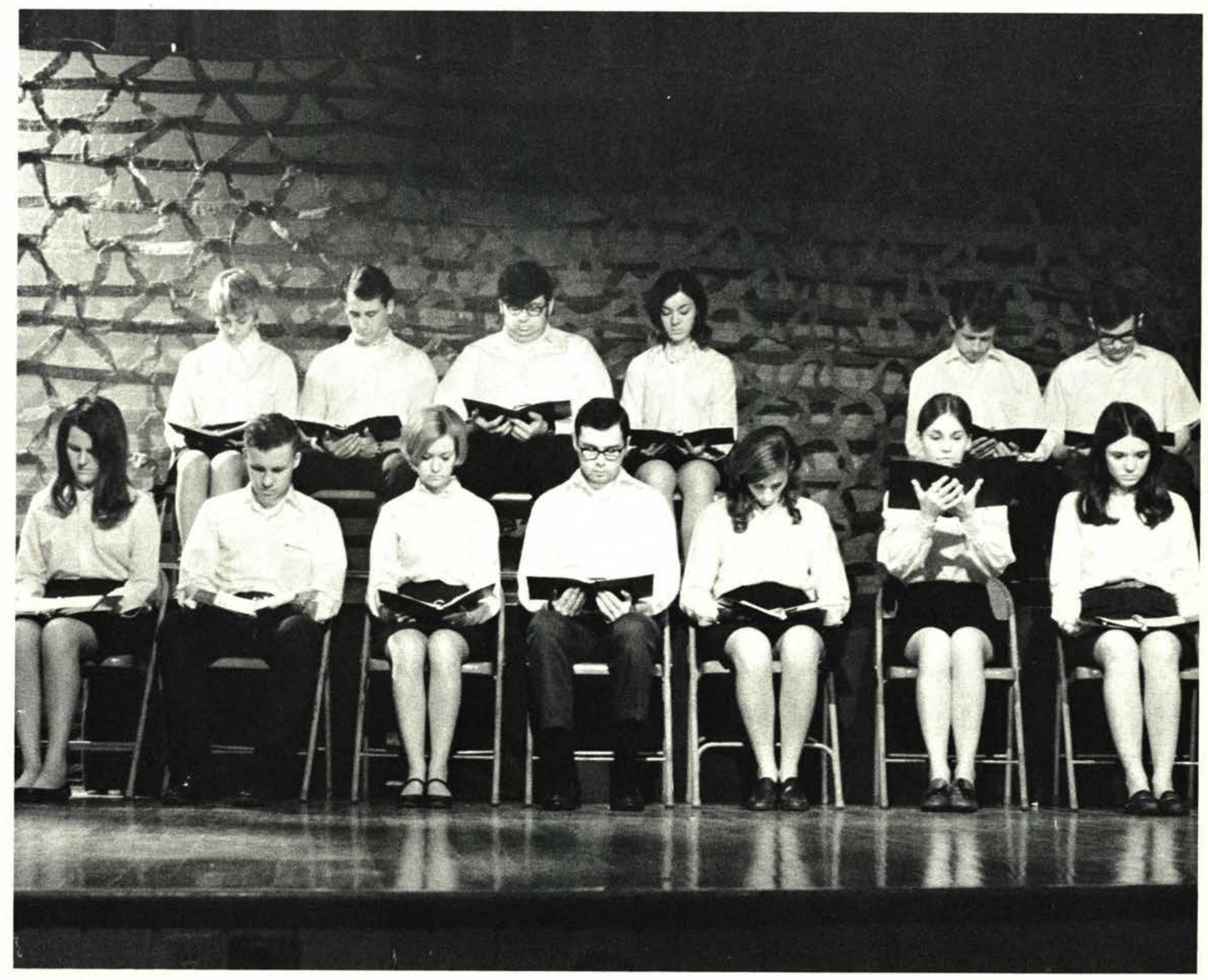

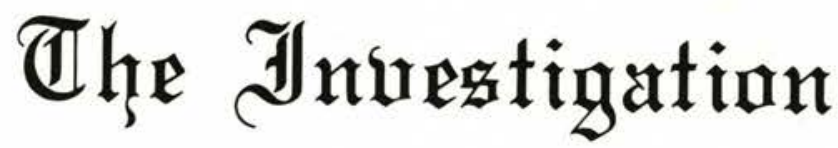




\section{BEYOND}
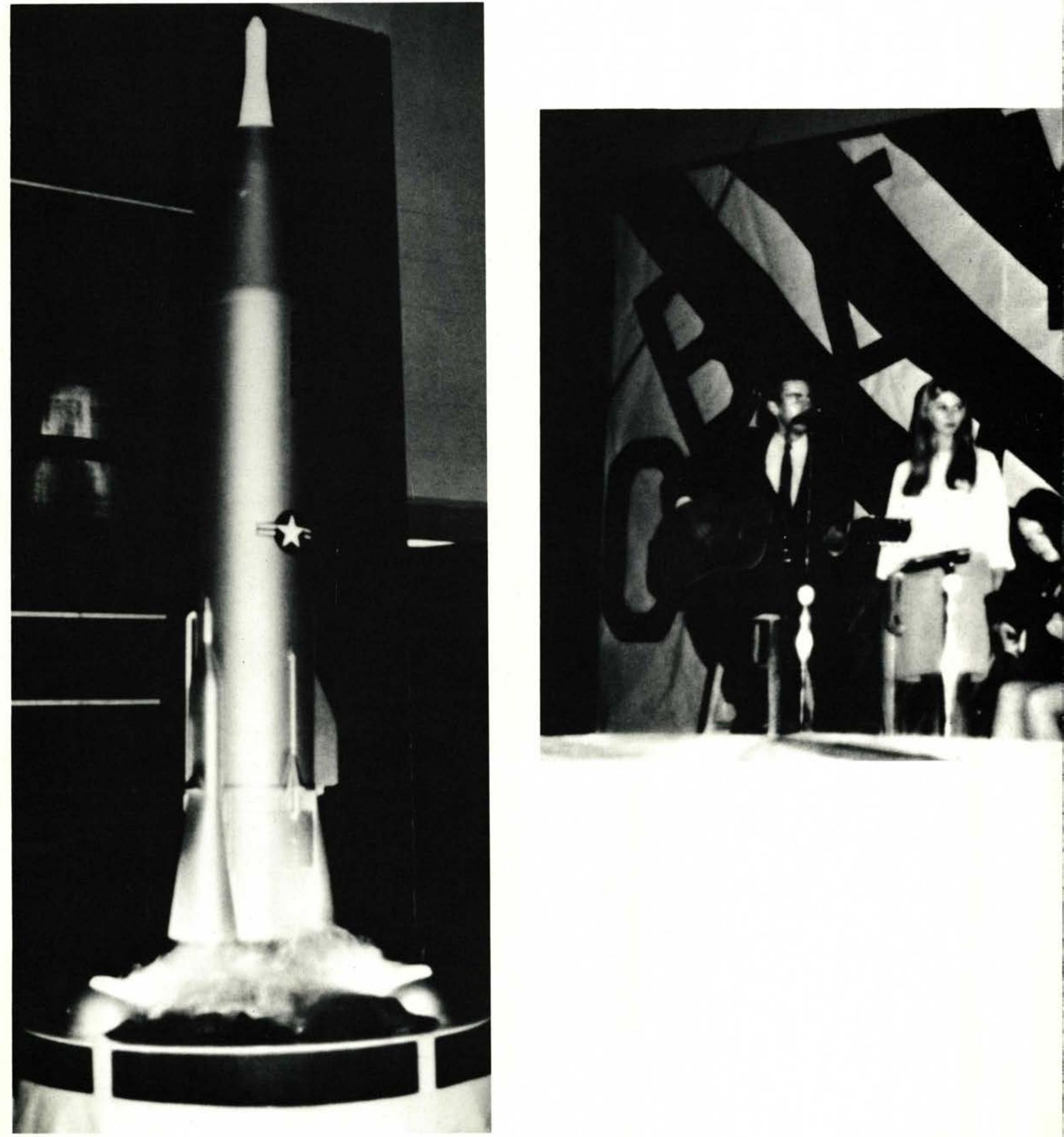


\section{GOD'S EARTH}
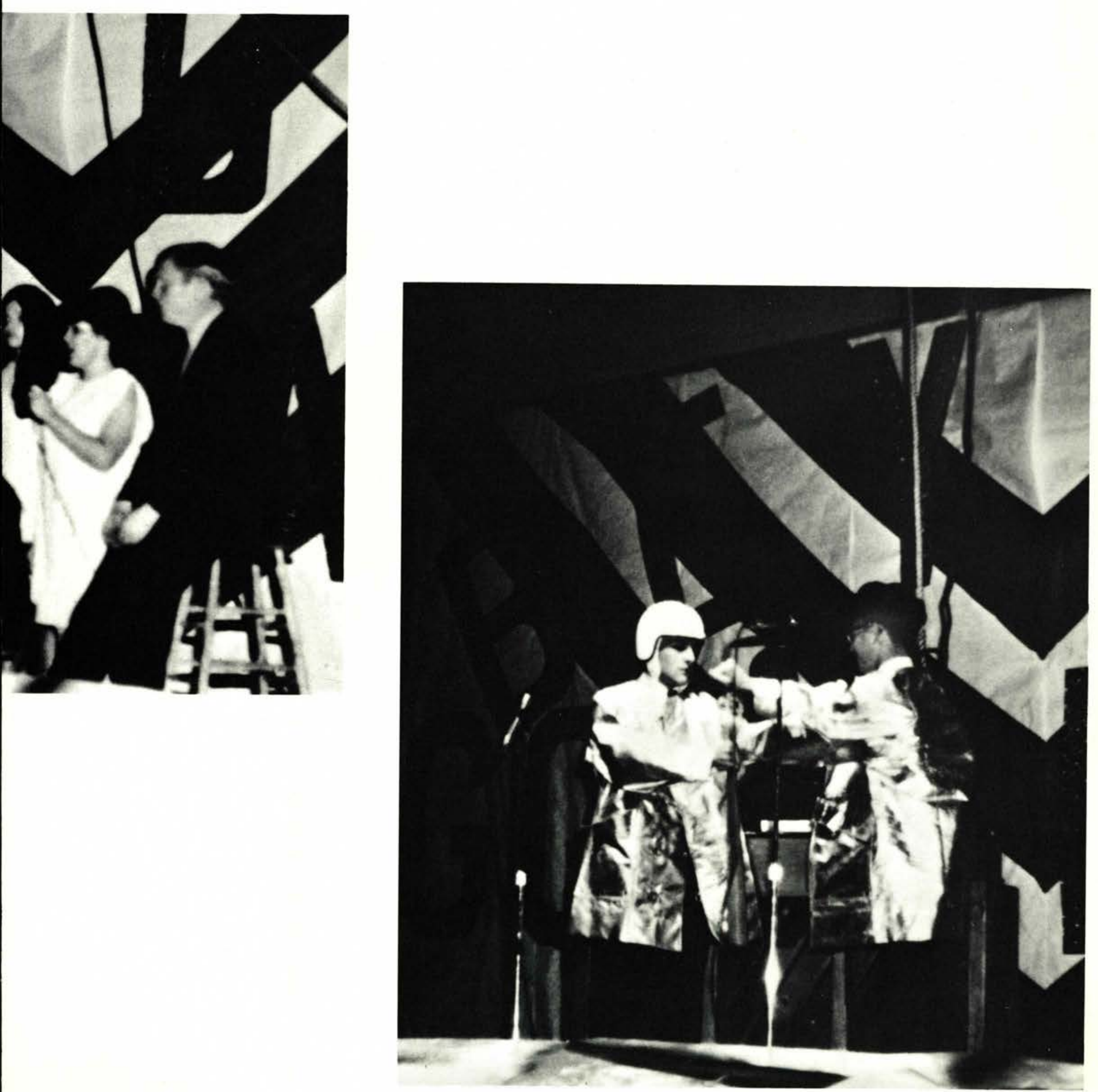


\section{HOLID AY}

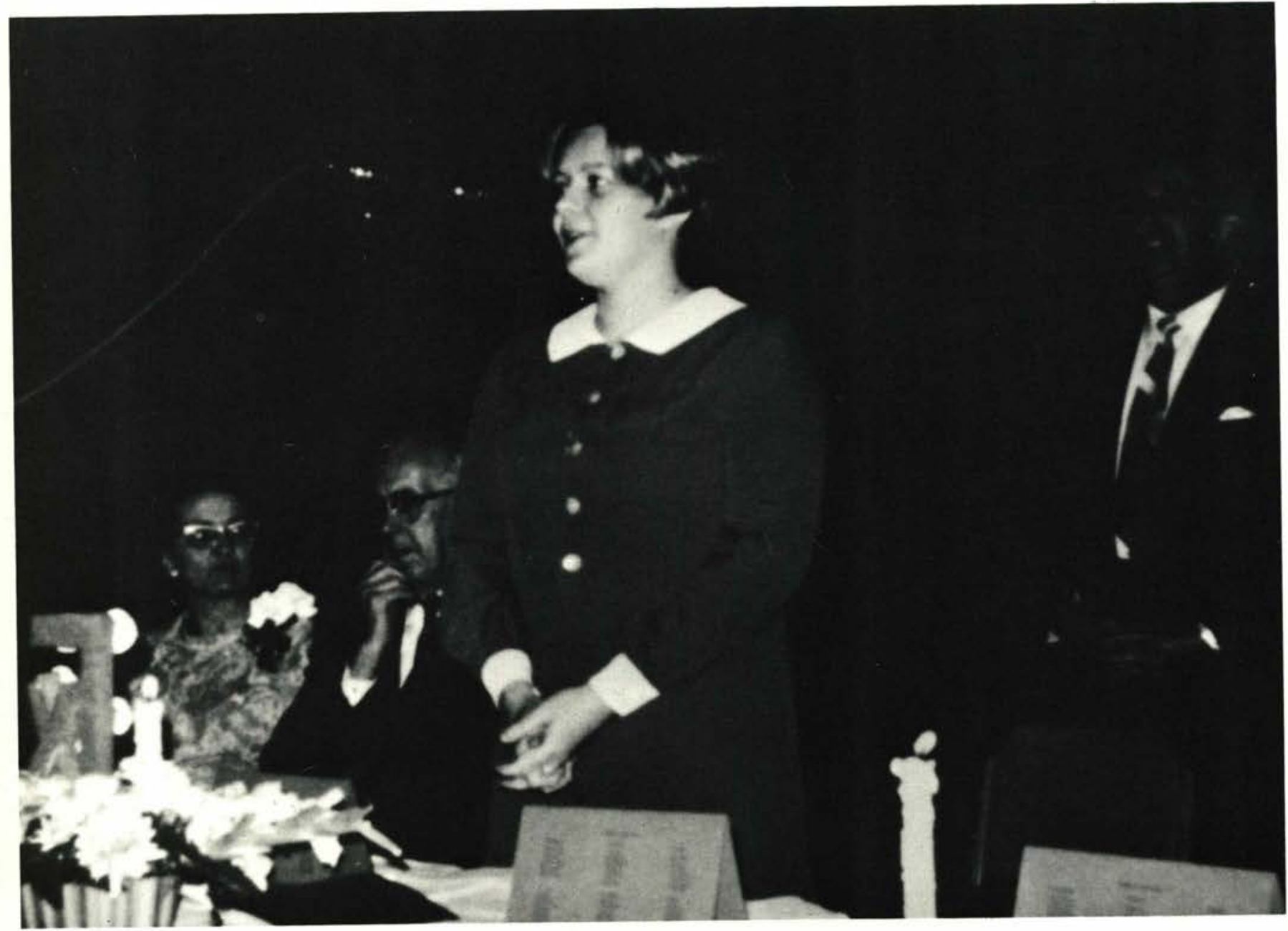




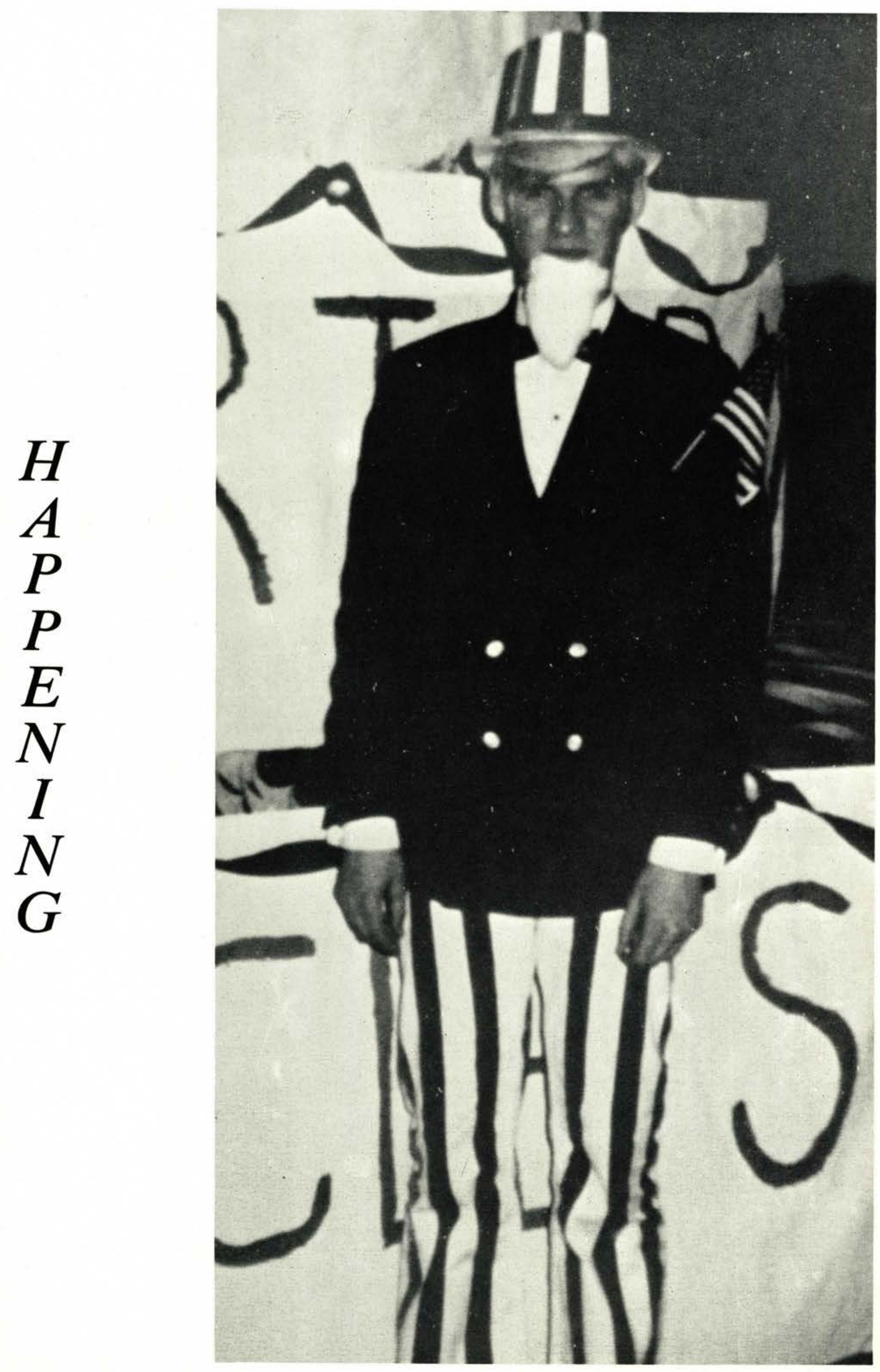




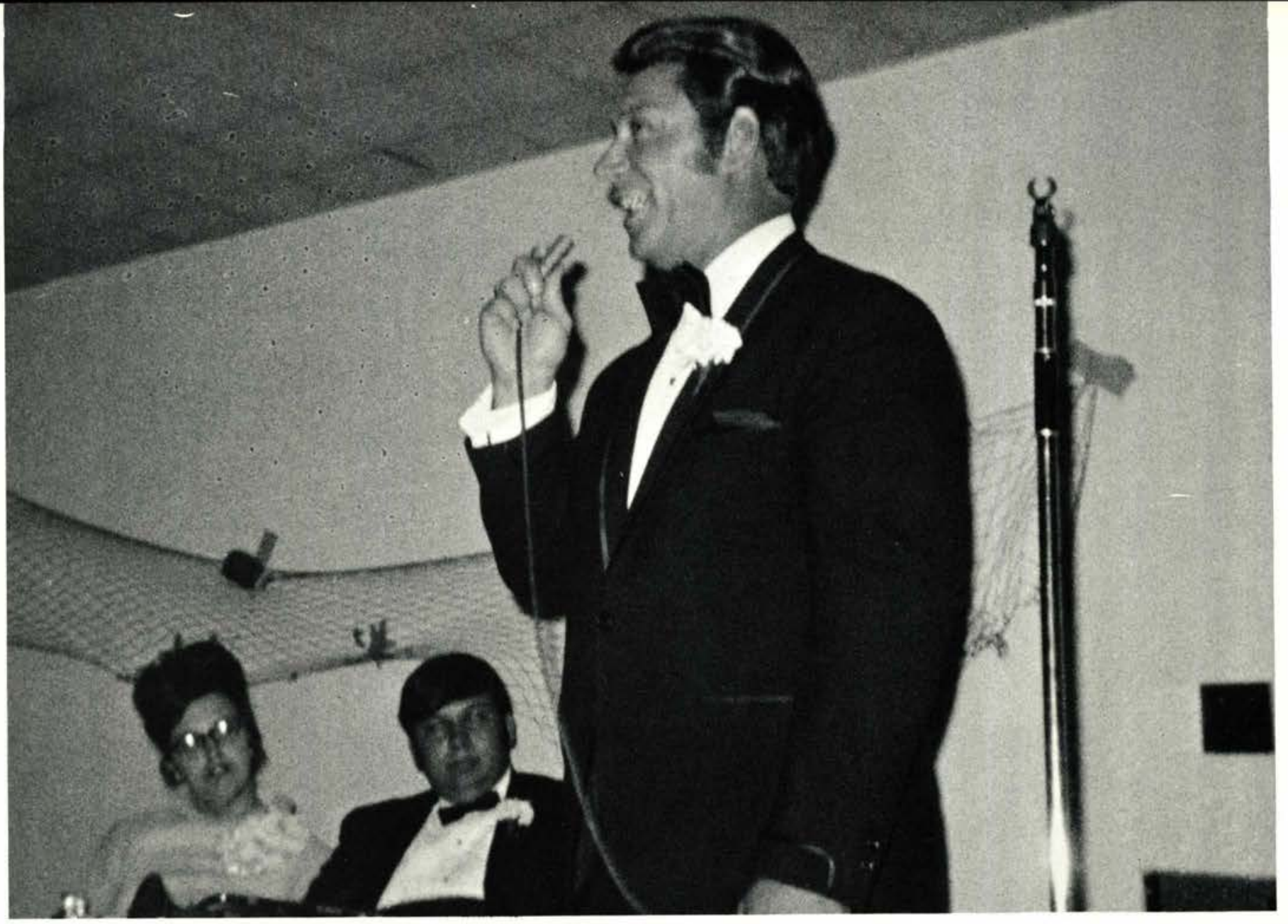

Steve Boalt provided the entertainment.

Dave Matson acted as Master of Ceremonies.

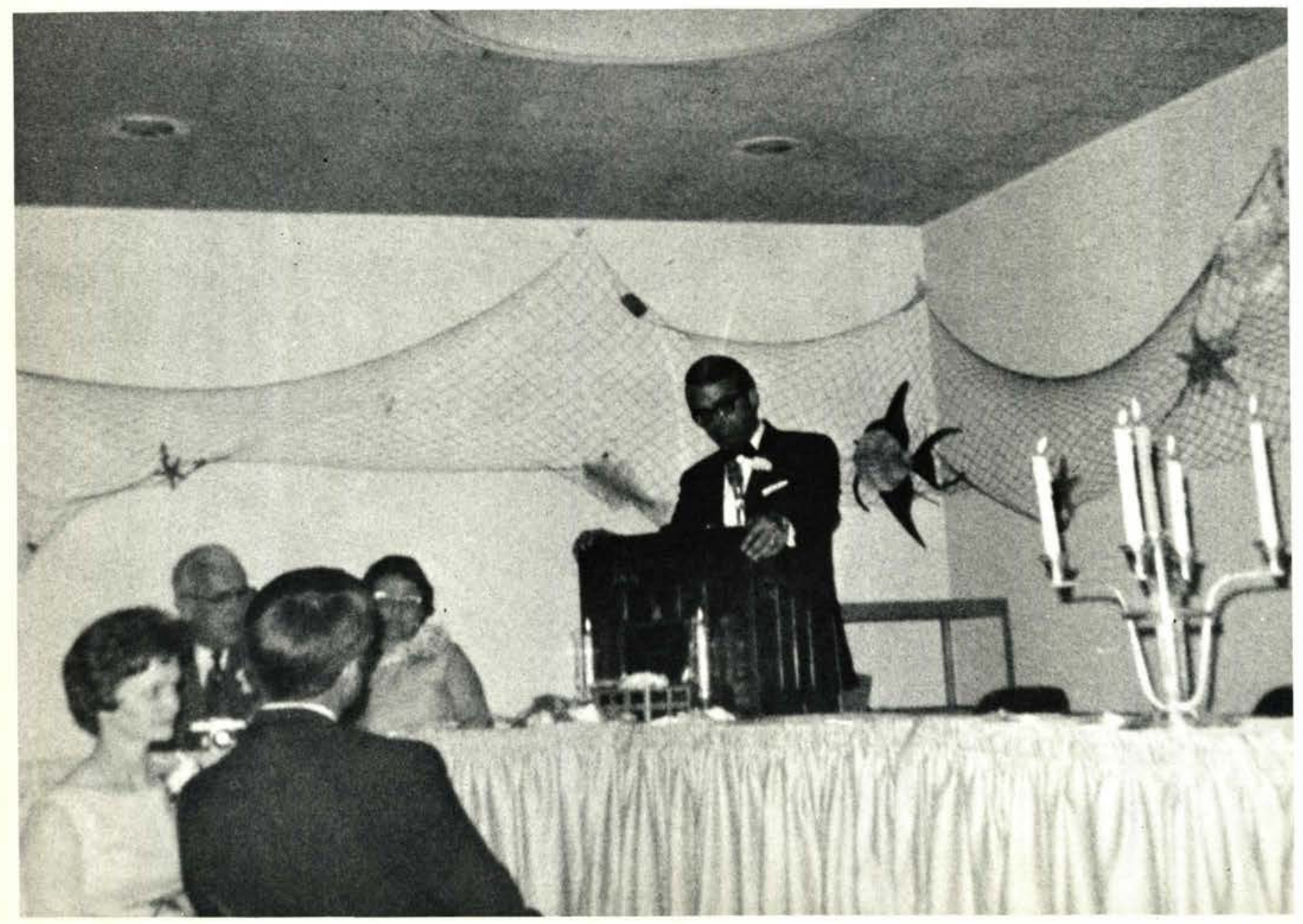




\section{SYMPHONY}

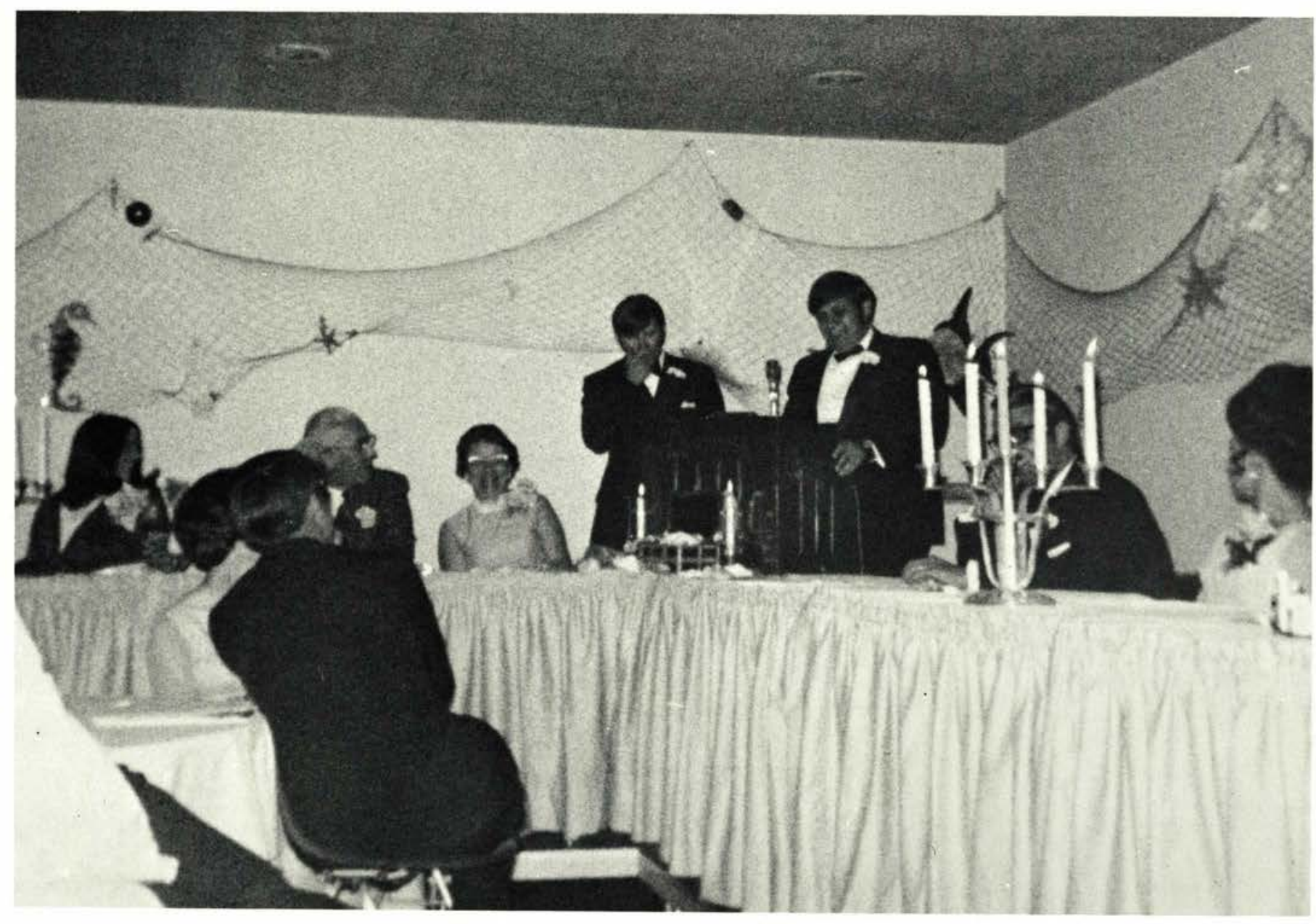

$$
\begin{gathered}
\text { OF THE } \\
\text { SEA }
\end{gathered}
$$




\section{HONORS}

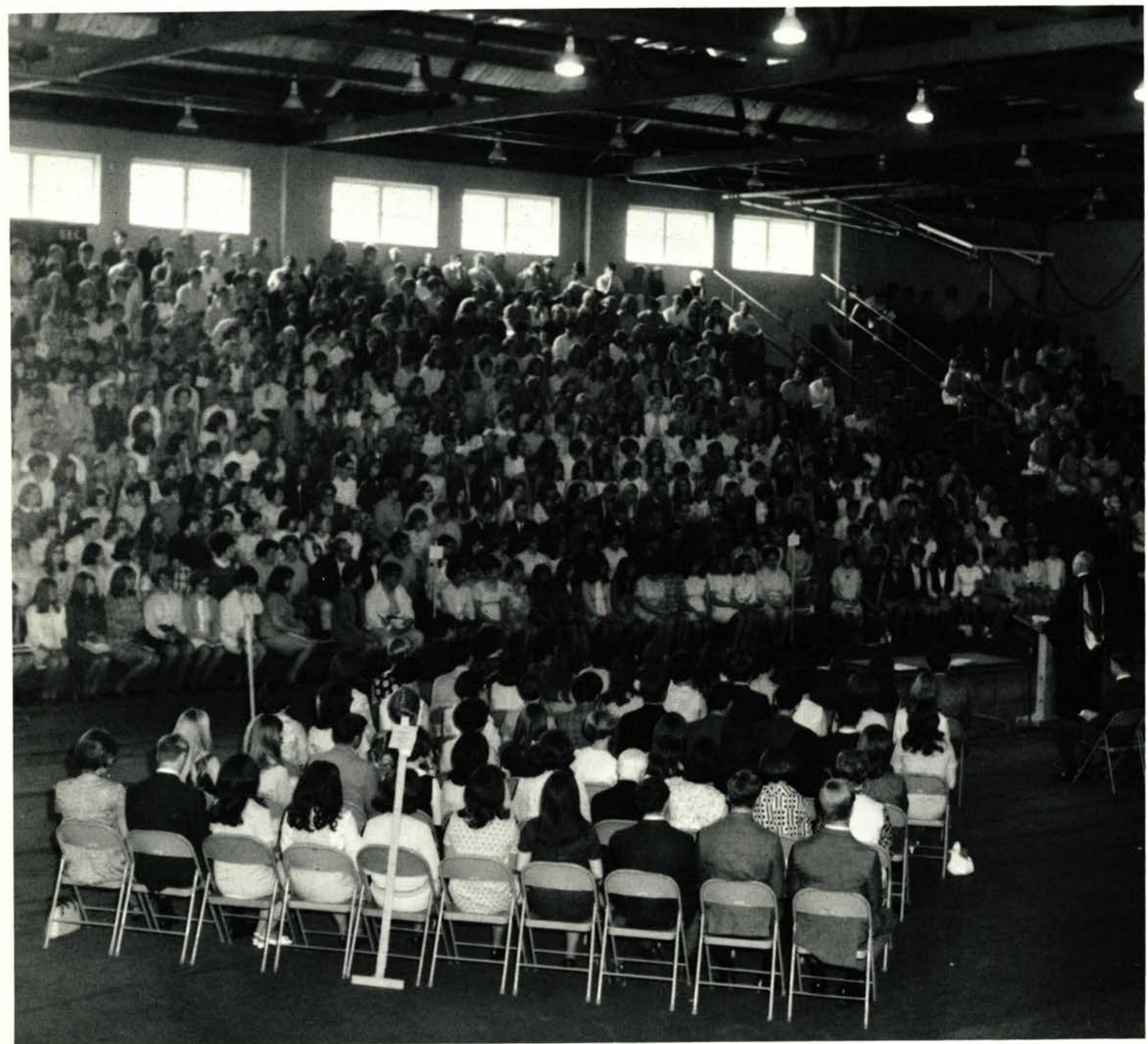




\section{$D A Y 1970$}

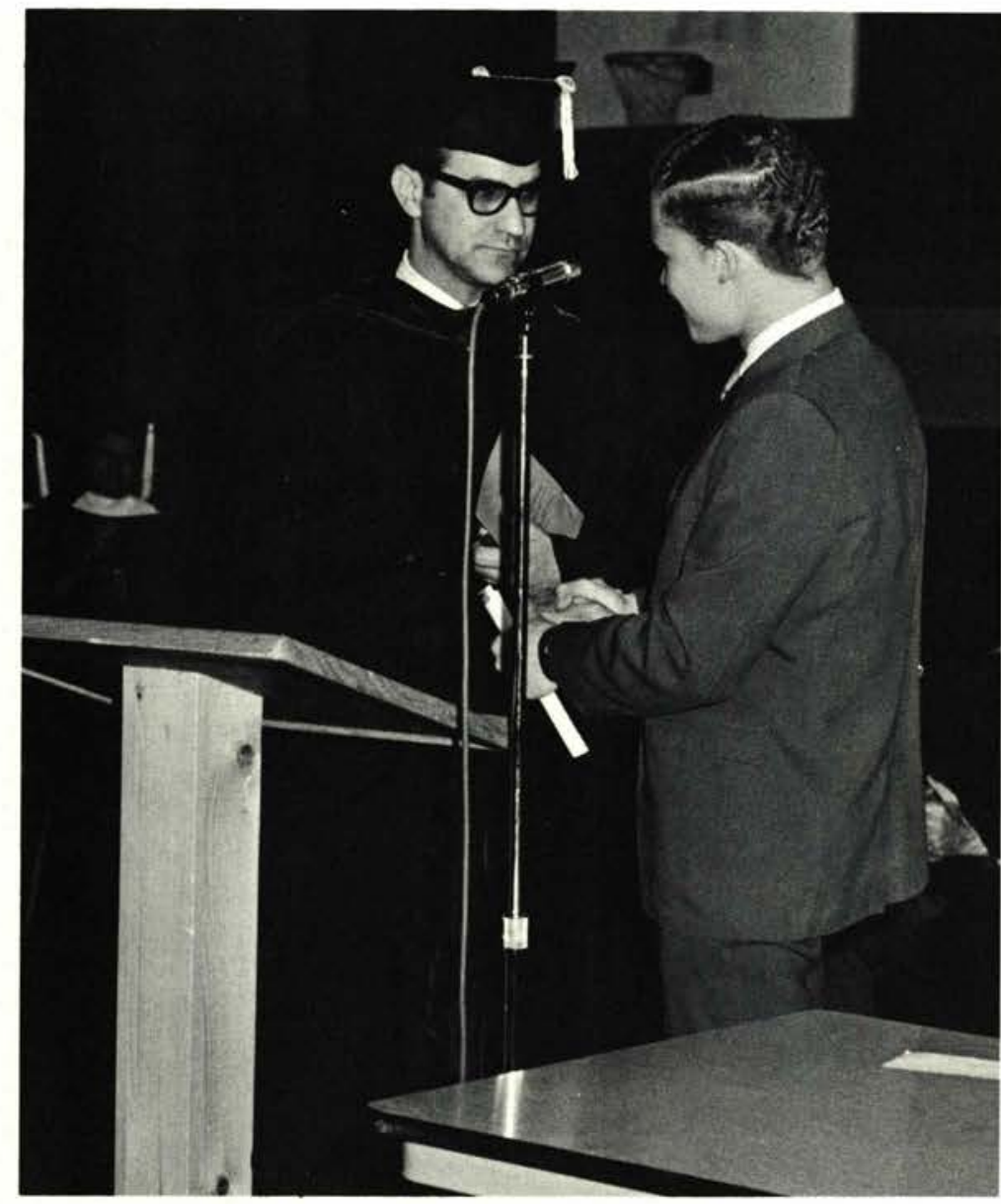

Dr. Reed receives faculty award.

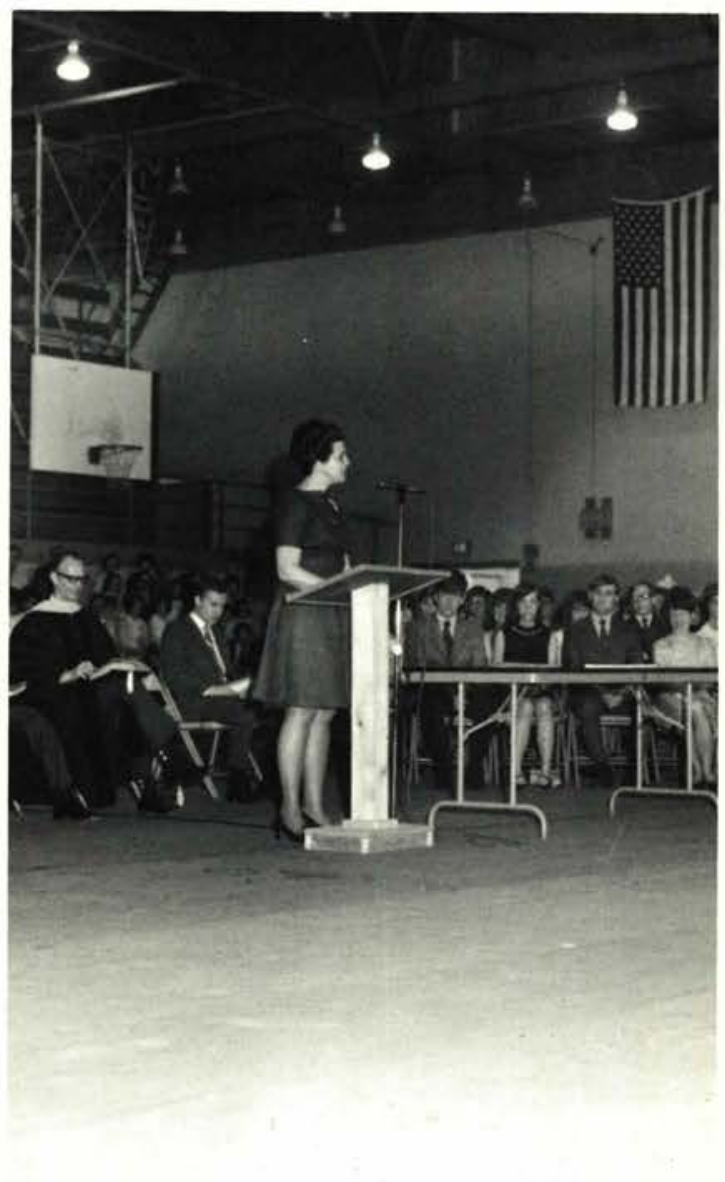

Bernice Bowersox delivers remarks.

Ken Cole receives "Outstanding Debater" award.

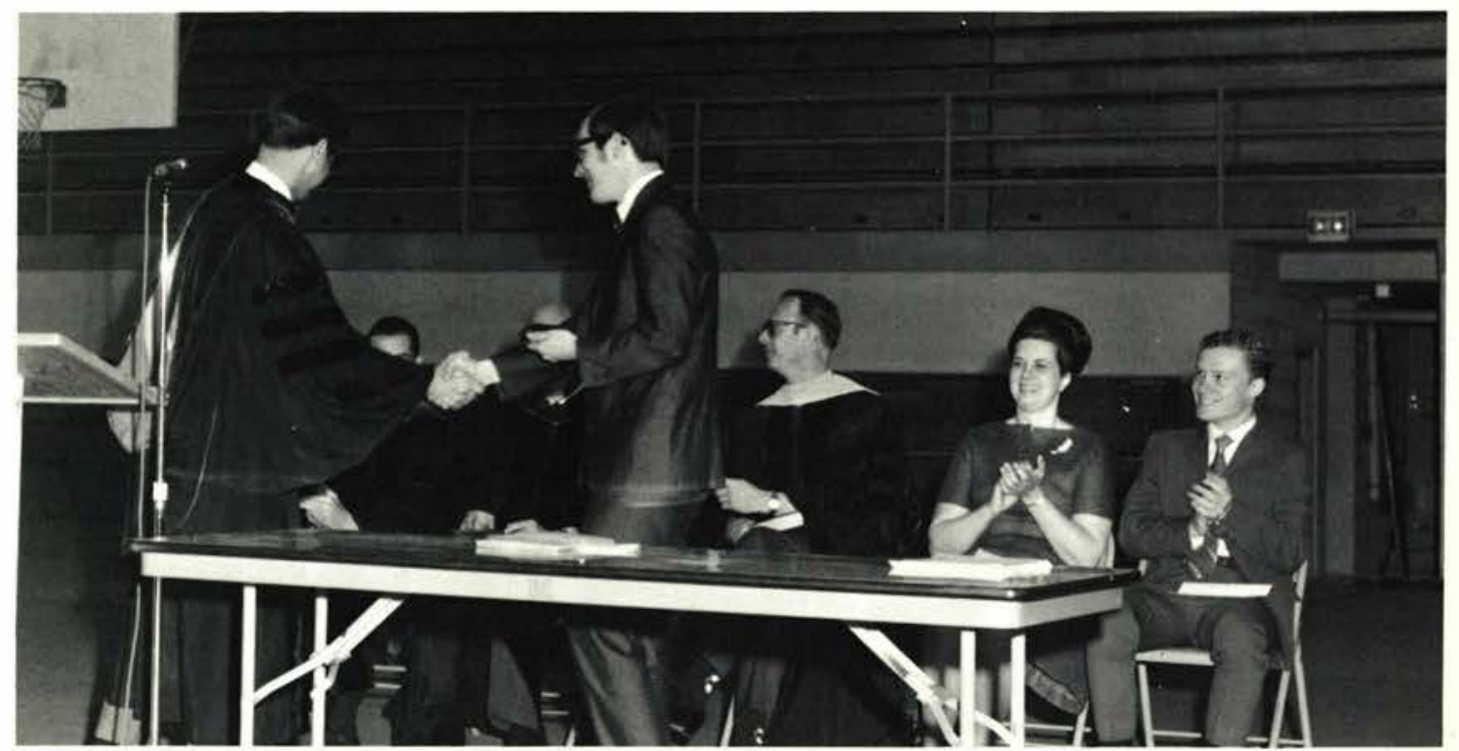




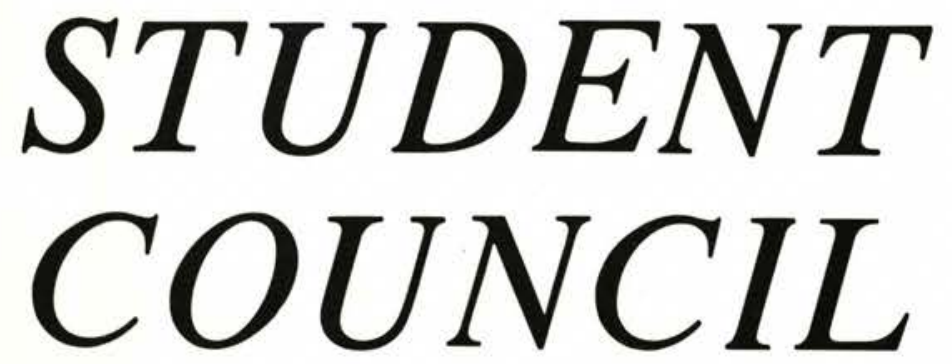

The purpose of Student Council is to assist in providing a wellrounded program of extra-curricular activities, to promote loyalty to the college and to the Word of God, to establish and maintain a harmonious atmosphere on campus, and to take or recommend action deemed necessary for the good of the individual student and the student body as a whole.

The major activities of Student Council this year were of a money-raising nature. The Student Missionary Project raised support for Miss Spradlin, and the Student Body Project raised money to build a barbecue pit and picnic area along the lake.

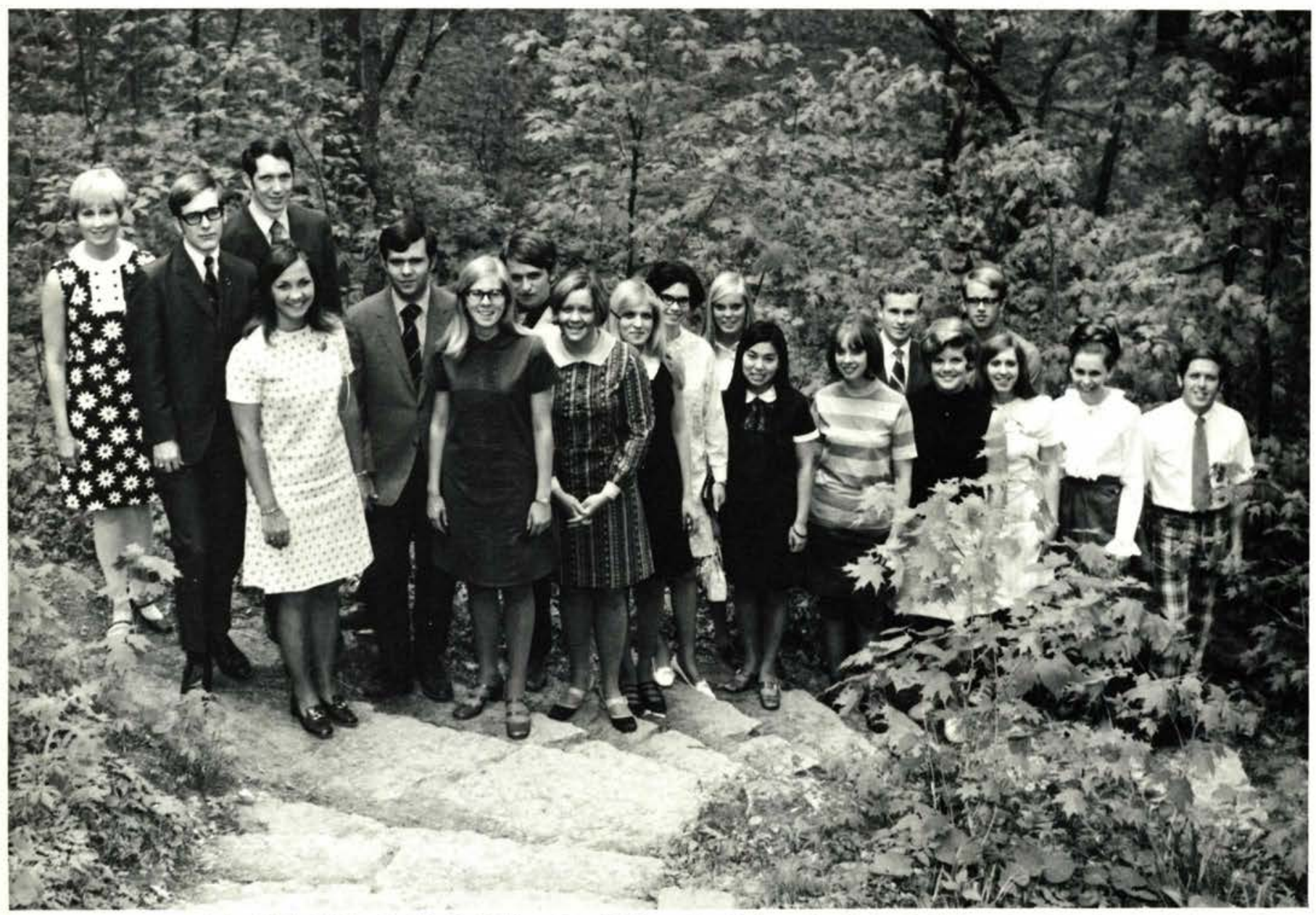

Patti France, Ken Cole, Kirk Hildreth, Vicki Tobias, Stu Walker, Carol Ann Bo, Dale Schilling, Jan Phenix, Gail Sears, Diane Kemp, Holly Wenner, Anne Kusumoto, Connie Busho, Dave Kearney, Carol Bierbaum, Dave Turner, Becky Harmon, Joyce Saemenes, Chip McDaniel 


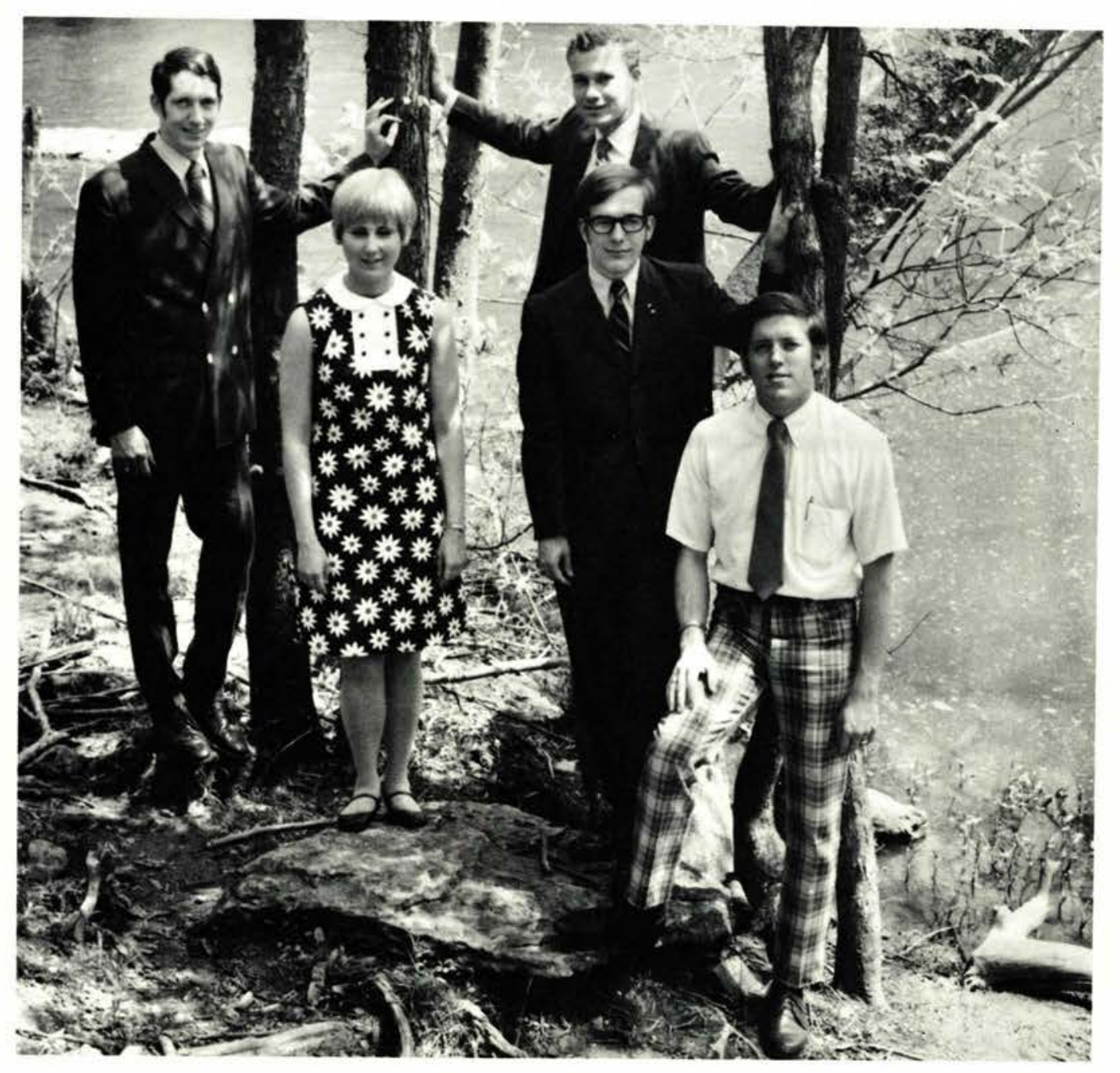

\title{
COUNCIL OFFICERS
}

Kirk Heldreth-Chaplain

Patti France-Secretary

Dave Kearney-President

Ken Cole-Vice President

Chip McDaniel—SBP Chairman

Not Pictured:

Bob Snyder-Treasurer

t

Bob Snyder Treasurer

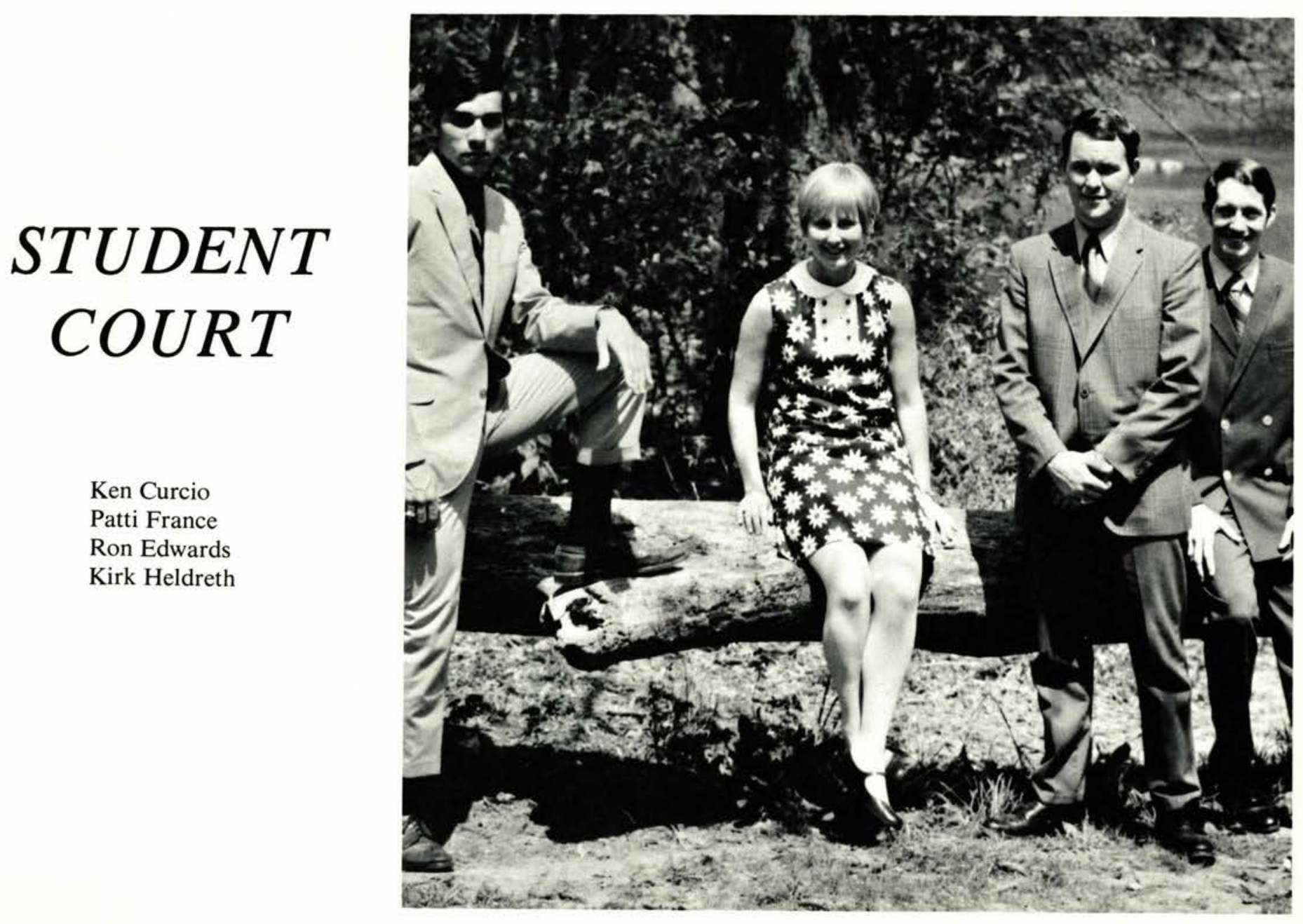




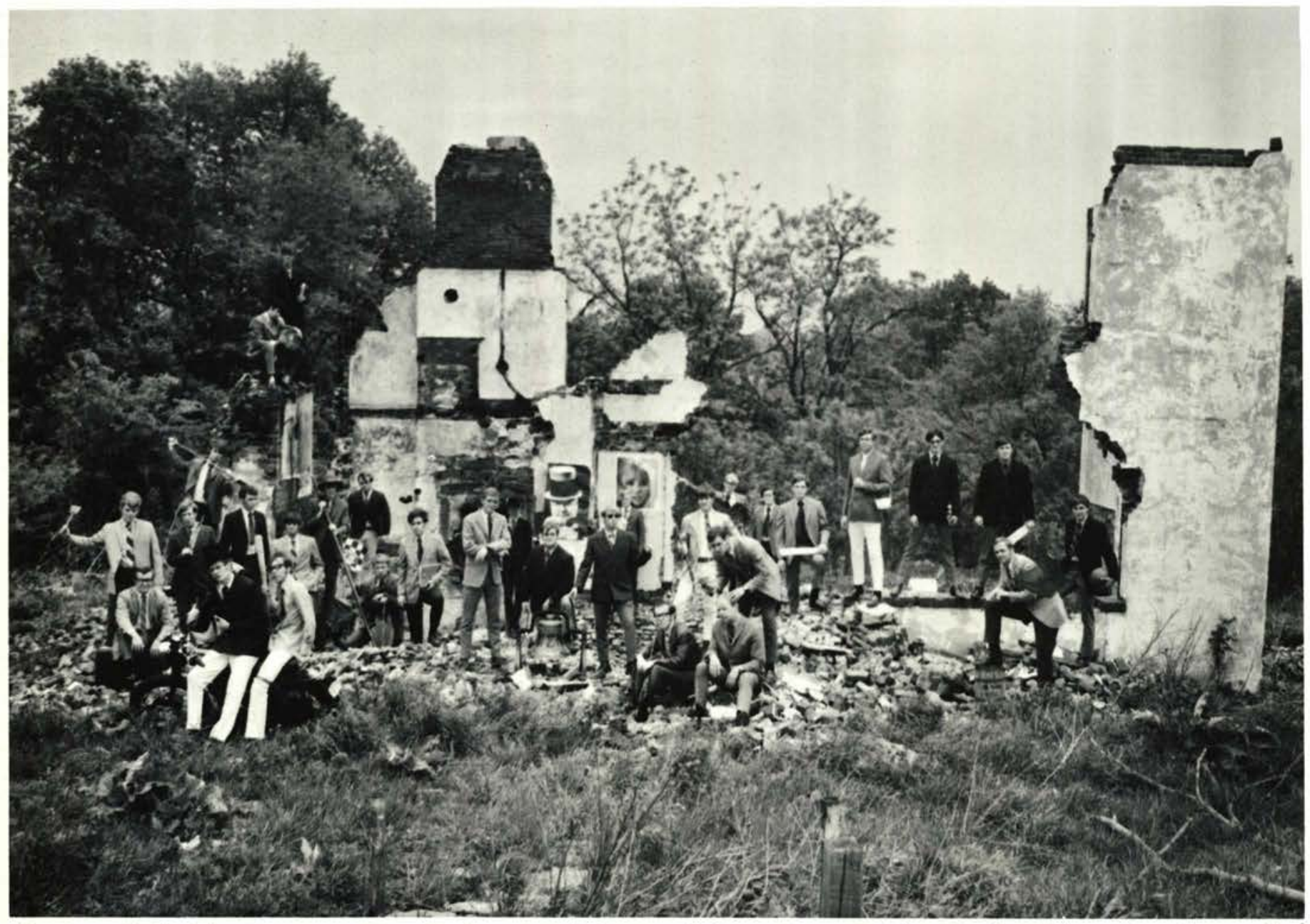

First Row: Philip Stine, Ken Evans, Tony Wall, Jack Holliday, Dennis Stora, Warren Jenkins, Charlie Williams, Dave Brannon Second Row: Bob Ely, Tim Hegg, Tom Kukasky, Rick Ingram, Jim Greening, Dan Ingram, Lynn Howard, Larry Hamilton, Jim Williams Third Row: Randy Bailey, Bob Hoffman, Dan Acton, Ernie Welborn, Les Chambers, Tim Sanderlin, Russ Jenkins Elevated: Warren Pettitt, Bob Austin

Pi Sigma Nu is a men's organization which stands for the spirit, mind, and body of the total man. Pi Sigma Nu endeavors to develop maturity in all aspects of college life and to prepare men to face life after graduation.

Pi Sigma Nu presented their annual school banquet January 31 with the theme of a ski lodge. The club also sponsored a Spring Week-End May 23 which included contests and a variety show in the evening. Special activities for the club members included a swimming party and a barn party.

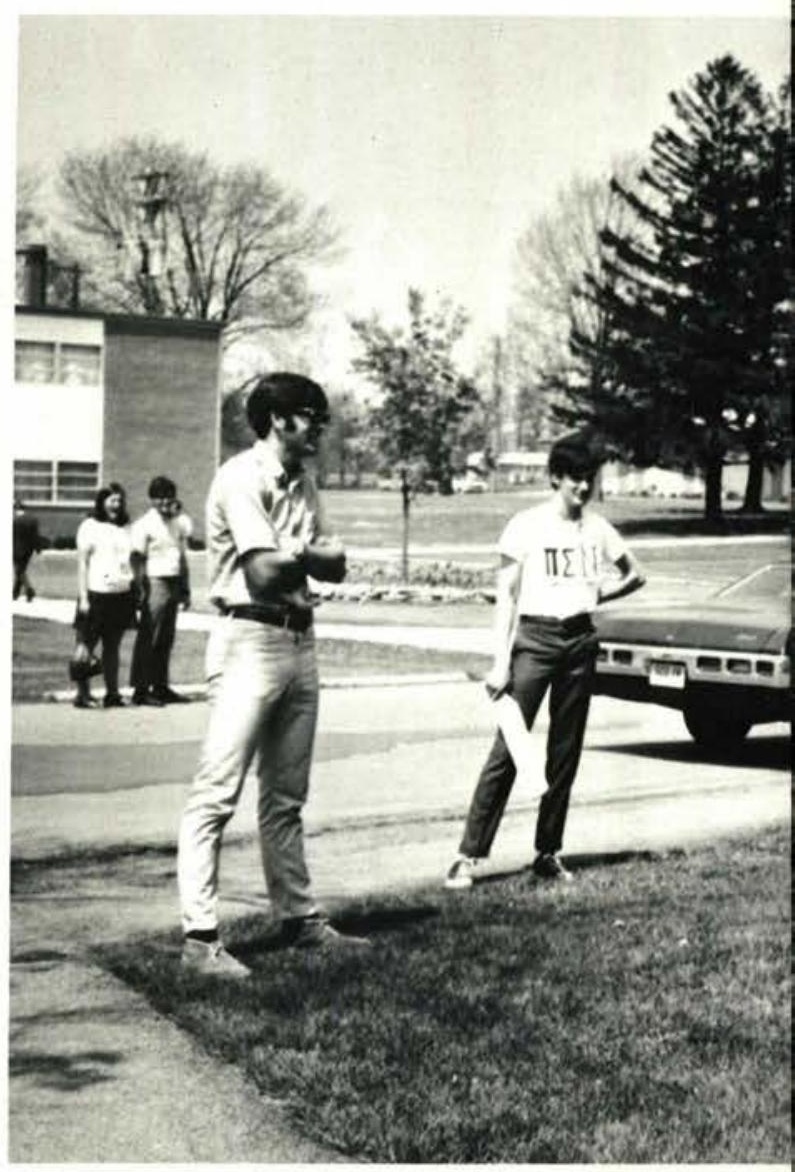




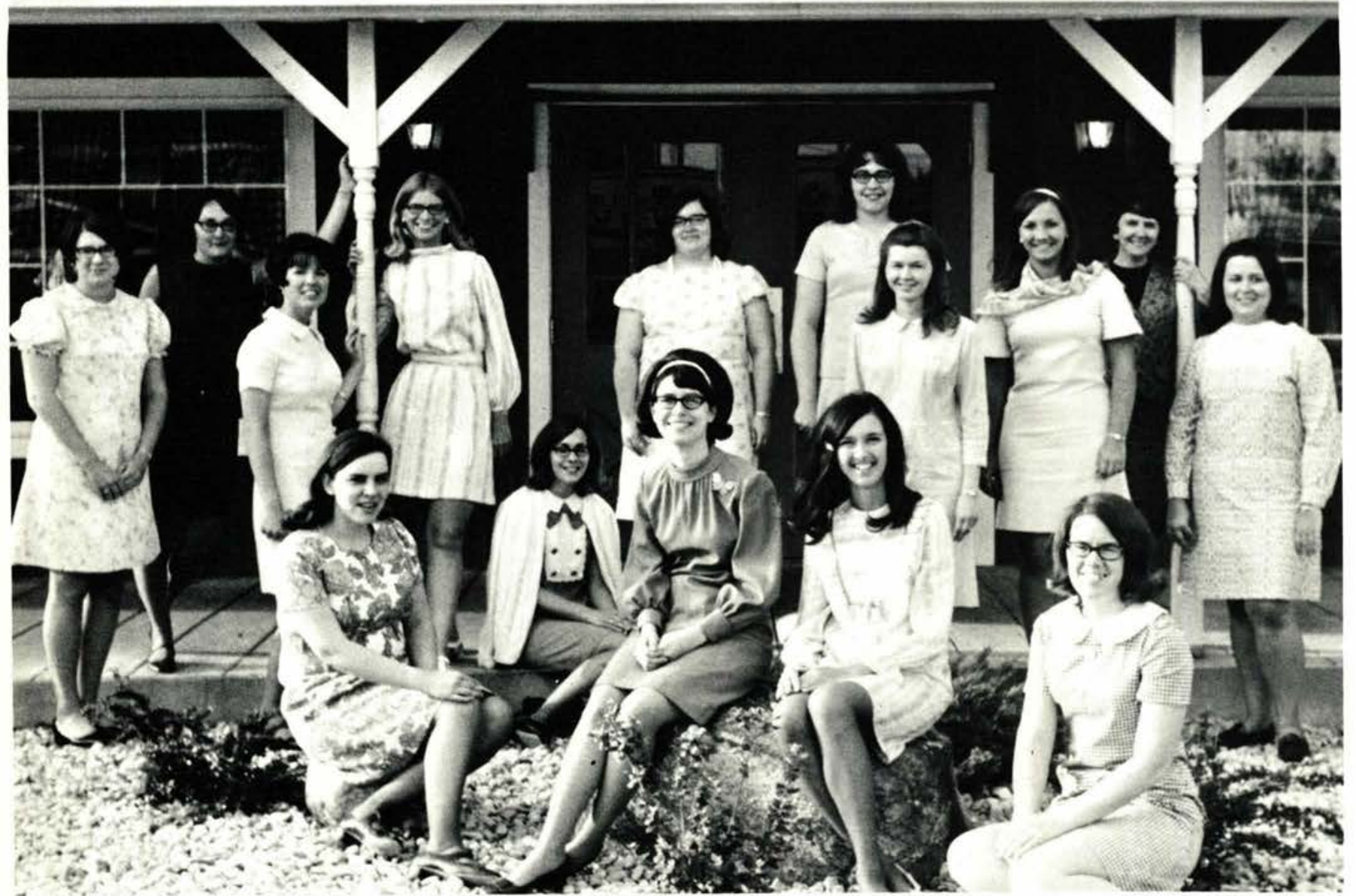

Standing: Joan Boyd, Donita Baker, Tanis McDaniel, Pat Sturdevant, Sandy Lathrop, Vicki Tobias, Mrs. McPheeters Seated: Nola Watson, Sue Lathrop, Edie Phillips, Jacki Barlow, Mary McMurtry

\section{KAPPA DELTA CHI}

\section{OFFICERS}

Mrs. McPheeters, Advisor; Donita Baker, Vice Pres.; Sandy Lathrop, Treasurer; Tanis McDaniel, President; Vicki Tobias, St. Council Rep.

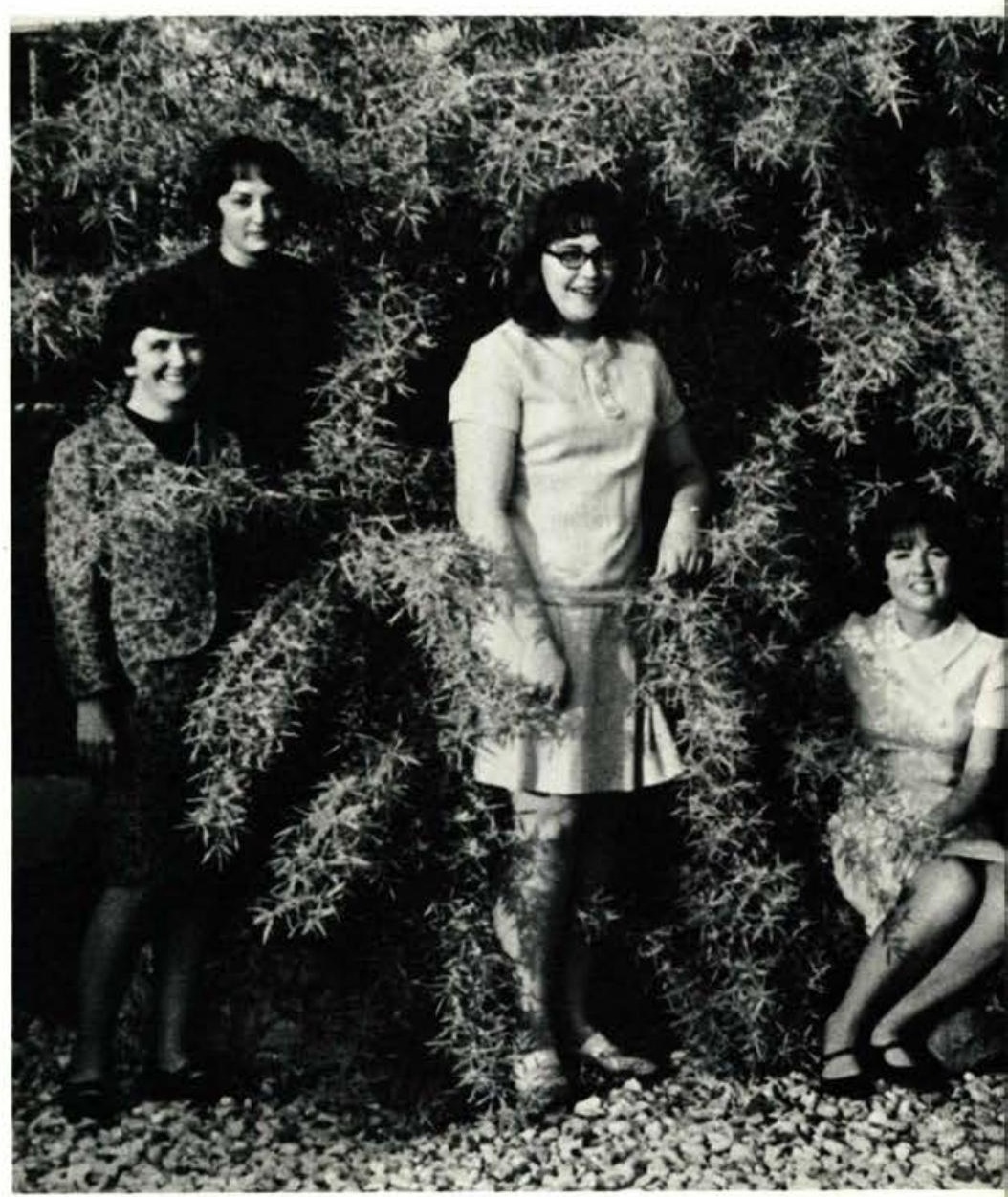


Events of '69-70:

Tea for prospective members Sold mums at Homecoming

Breakfast at Perkins Pancake House Handled coat checks at all home games

Vespers service: "An Evening in Soul" Trip to Tennessee to take clothing to missionary Carola White

End-of-year dinner at Ponderosa

\section{Kappa Delta Chi Rresents:}

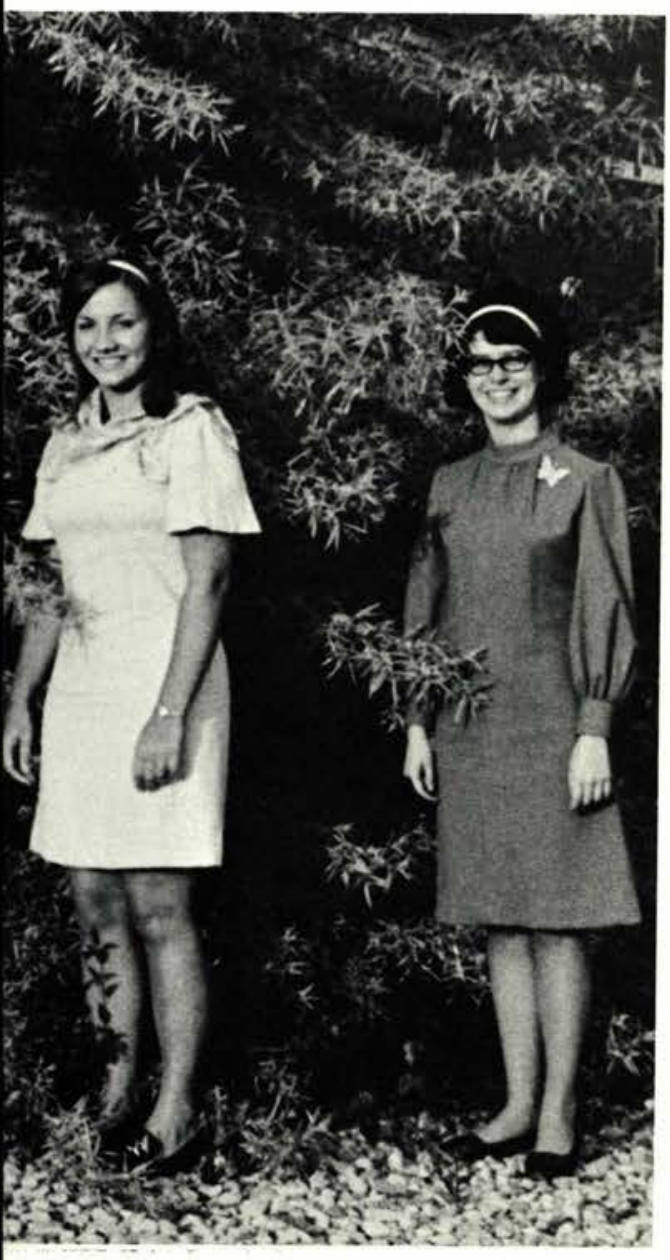

\section{An}

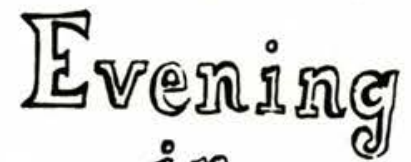
c) Pousl

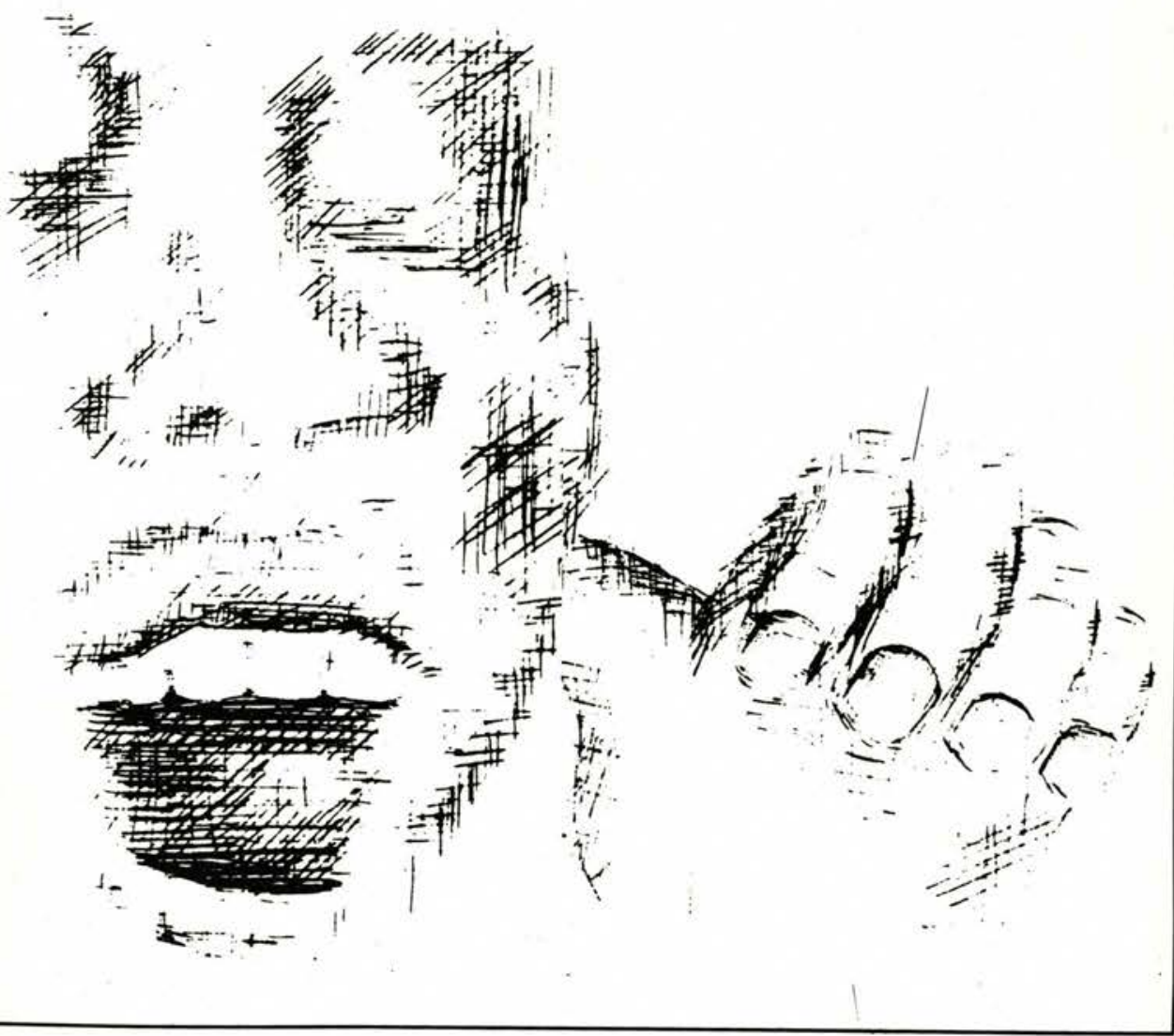

\section{VESPERS}




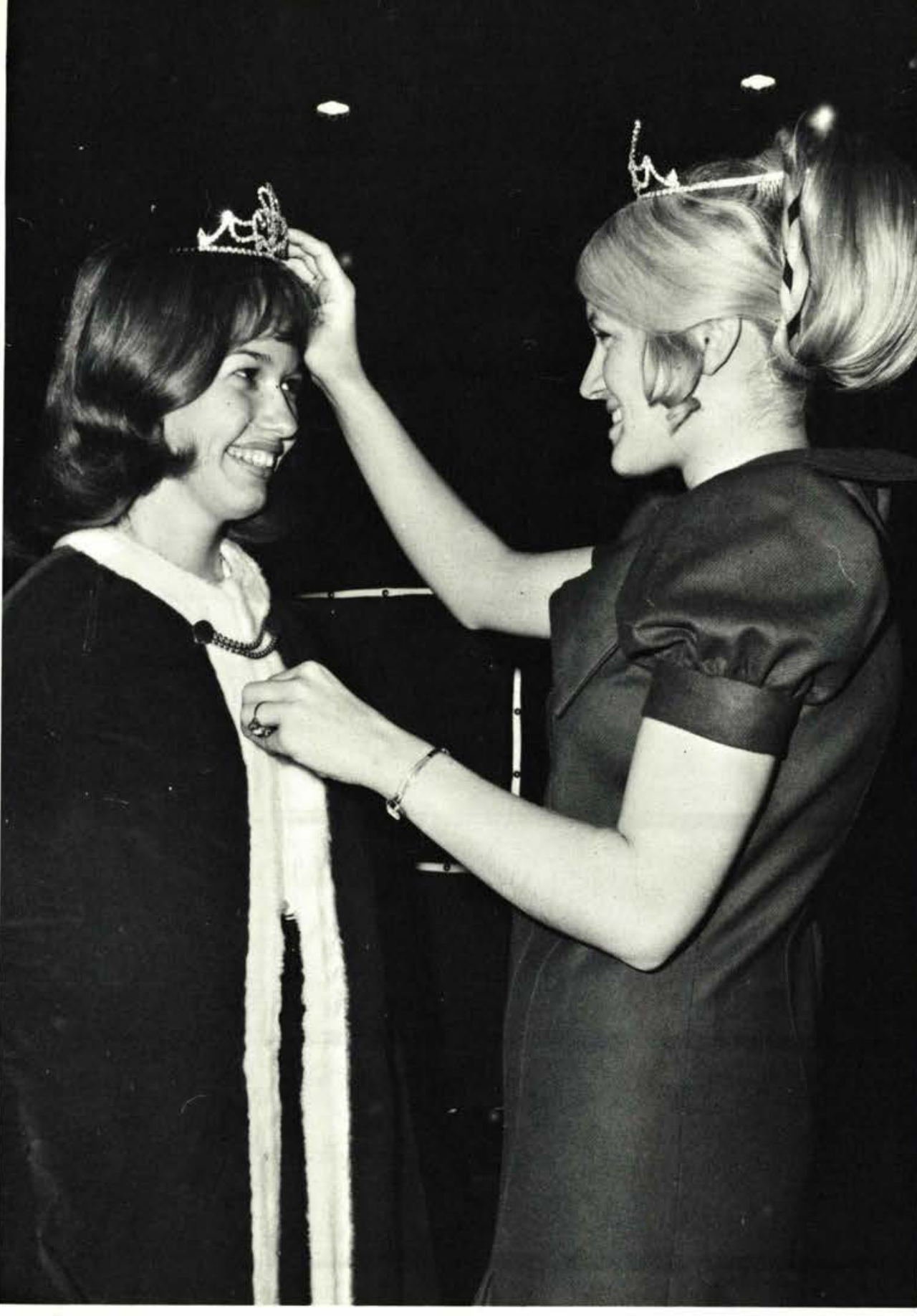

$A L P H A$
CHI

SWEETHEART

\author{
$J A N$ \\ GABRIEL \\ IS \\ CROWNED
}

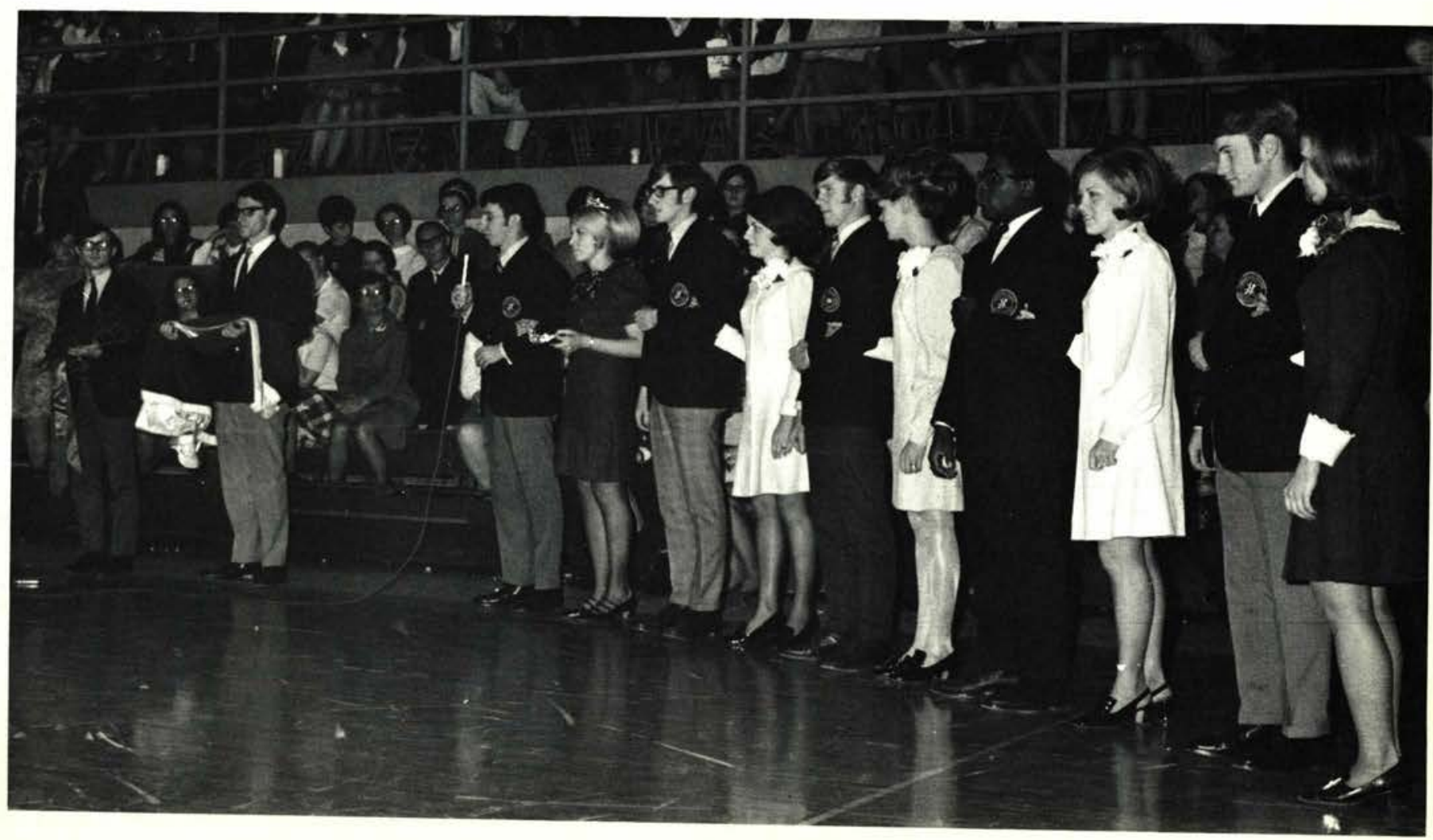




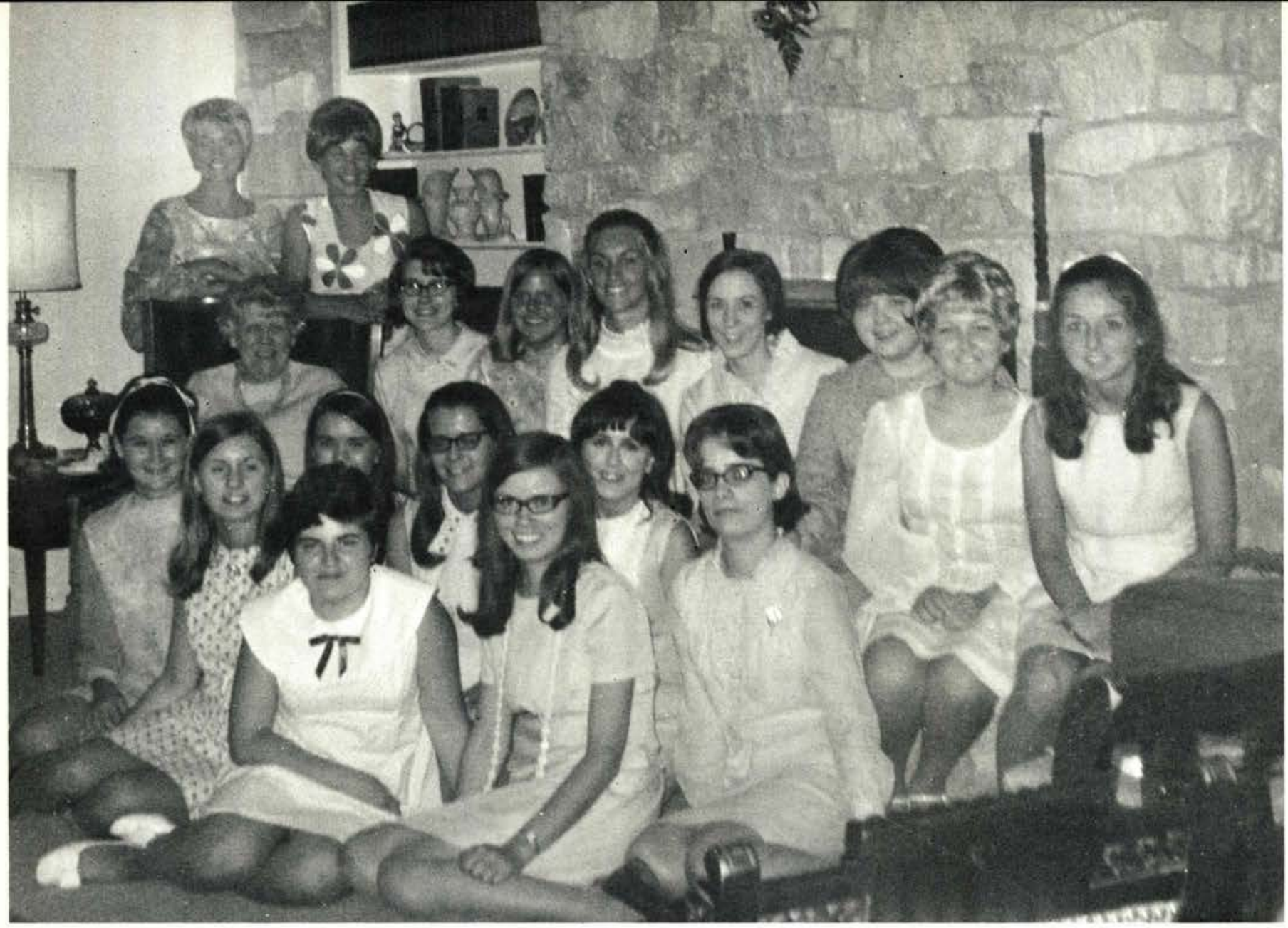

Debbie Good, Rosalie Howard, Debi Thornton, Linda Walter, Linda Billington, Mary Lou Storey, Joyce Motts, Carol Smith, Mrs Maddox, Marsha Osmann, Carol DeBoer, Candy Barr, Bev Maidment, Janet Epps, Linda Browning, Pat Jenkin, Glenda Welch, Judy Leach

\section{GAMMA CHI}

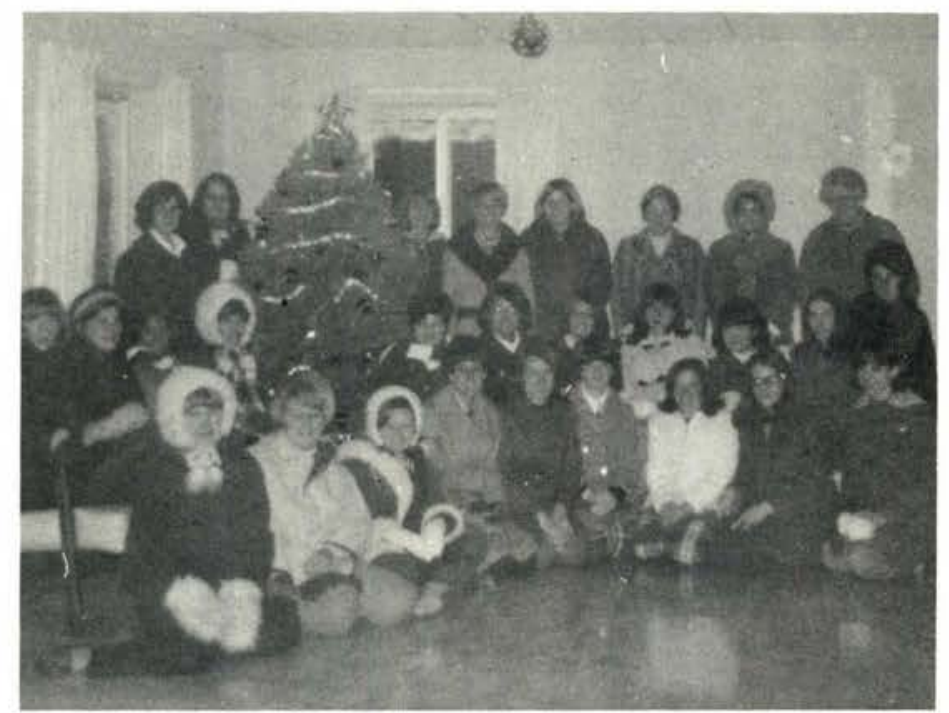




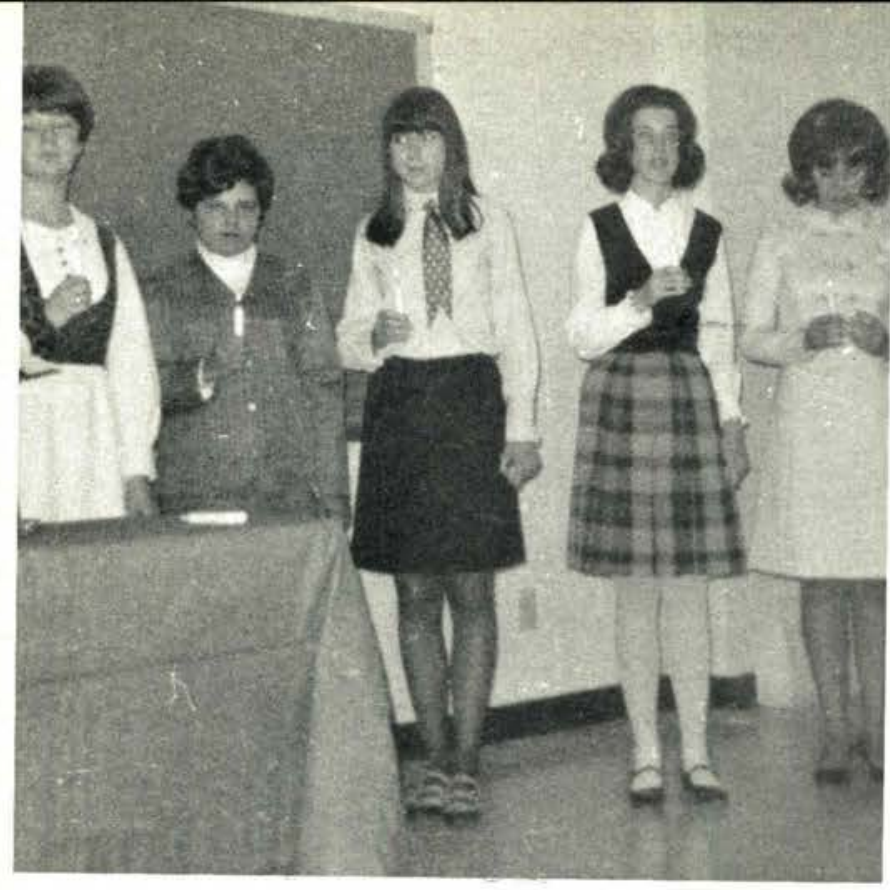

INITIATION

\section{OFFICERS}

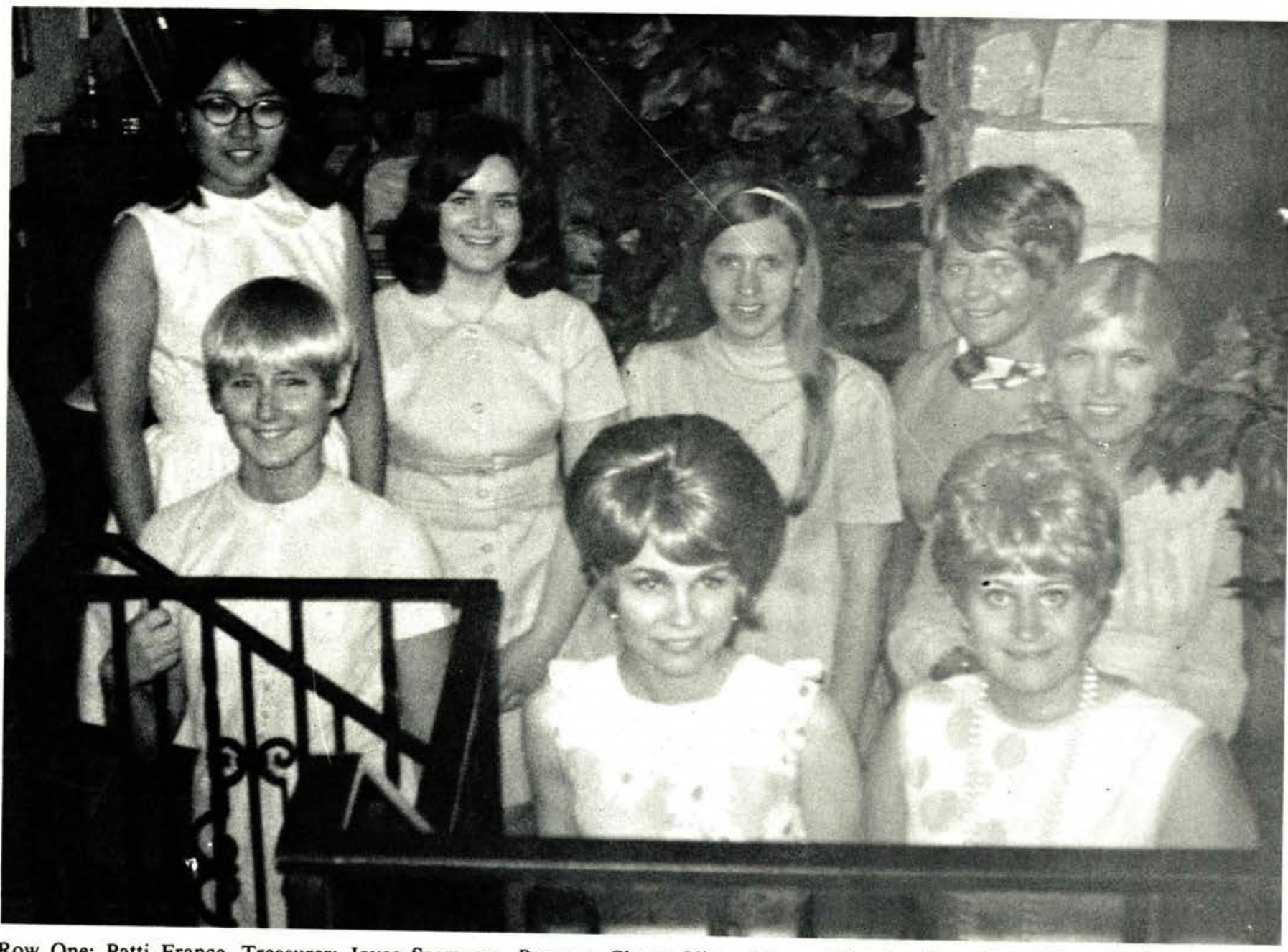

Row One: Patti France, Treasurer; Joyce Saemenes, Program Chrm.; Viann Meyer, Historian Row Two: Mitsu Ishuin, Vice Pres.; Mary Beitz, Parliamentarian; Becky Harman, St. Council Rep.; Jan Phenix, President; Gail Sears, Secretary 


\section{MIRACLE STAFF}

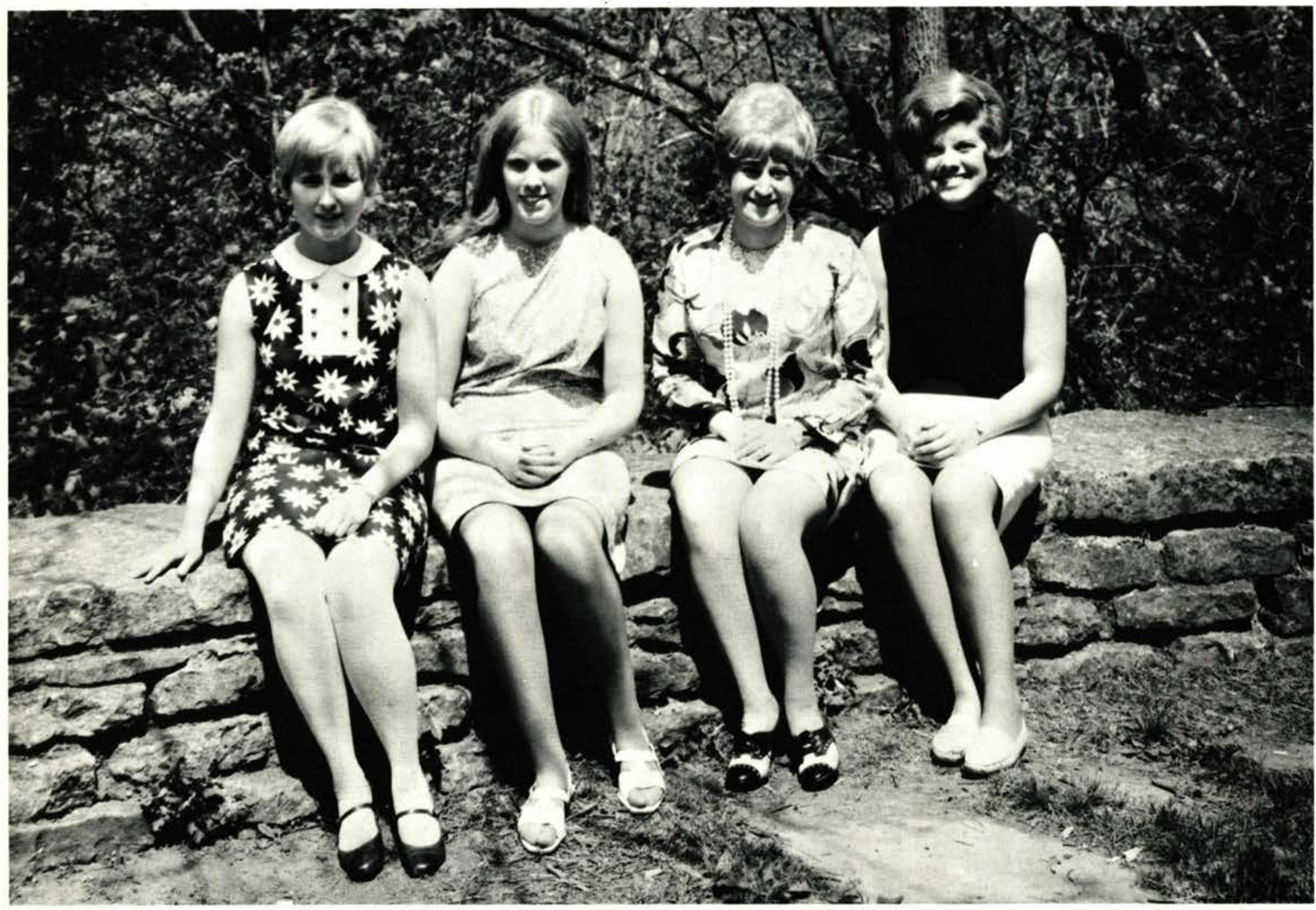

\section{EDITORS}

Patti France ... Editor-in-Chief

Linda Shorey . . . Typing Editor

Viann Meyer . . . Copy Editor

Carol Bierbaum . . . Layout and Asst. Editor 


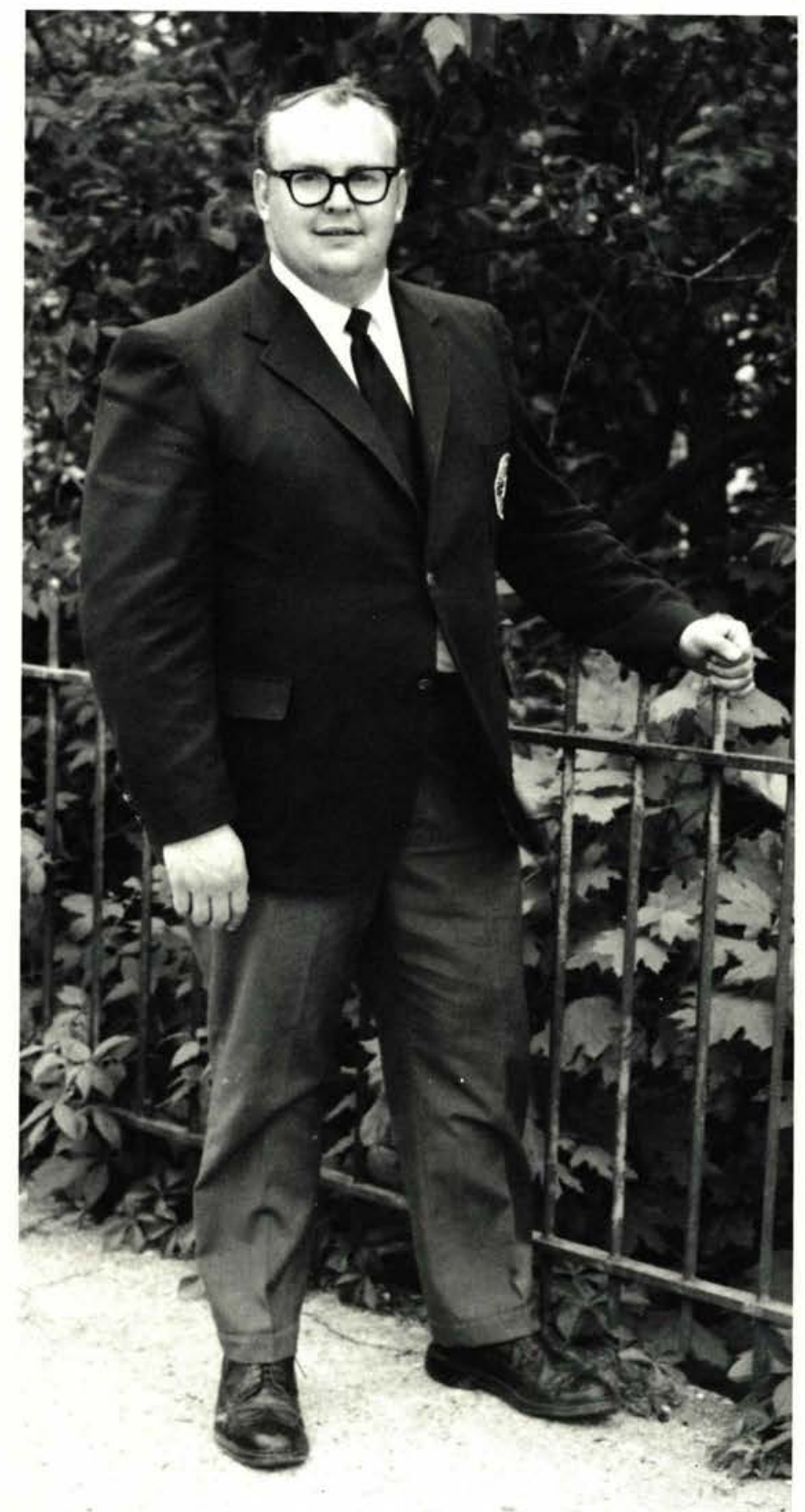

Roy Kern

Photography Editor

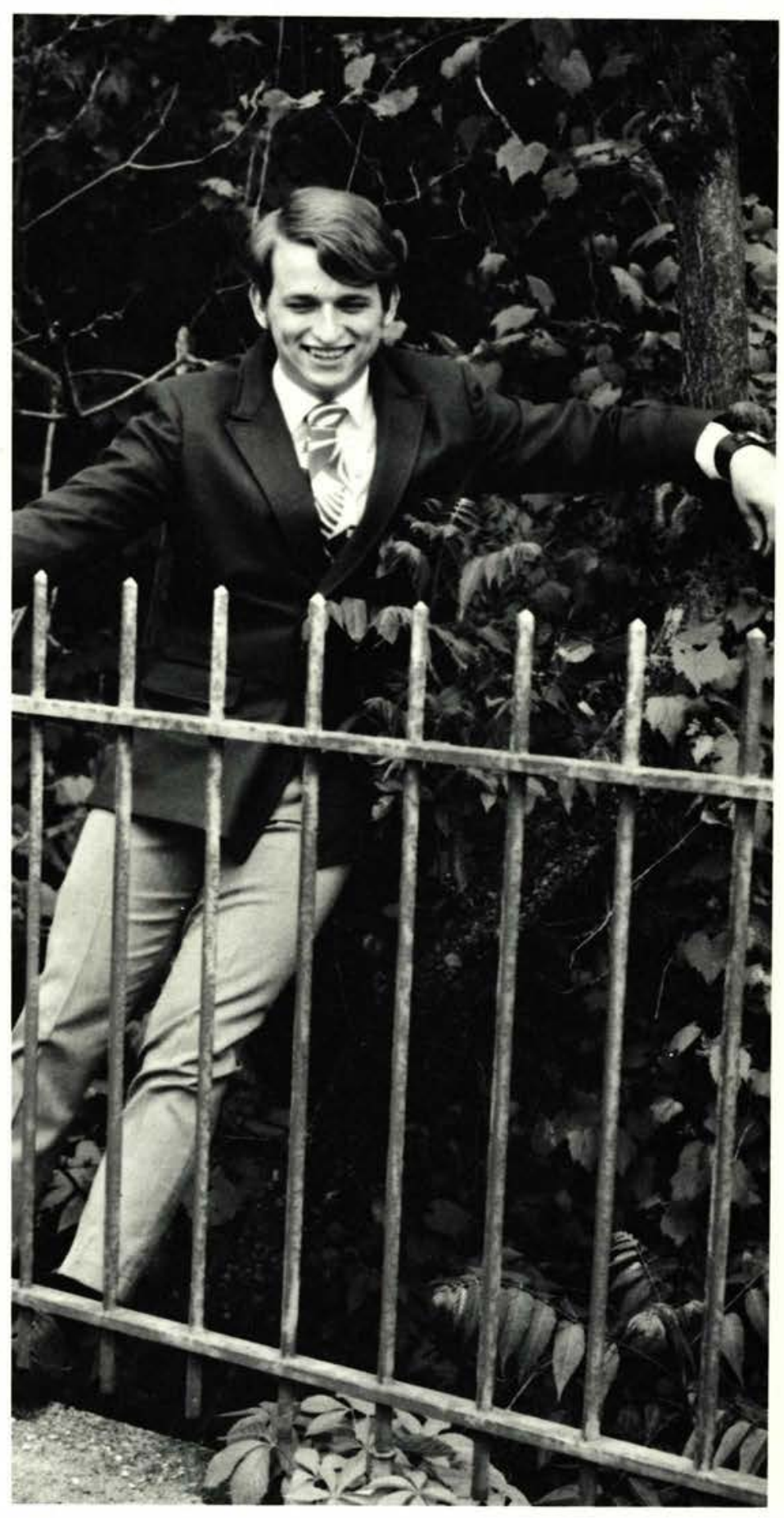

Dale Schilling

Advertising Editor 


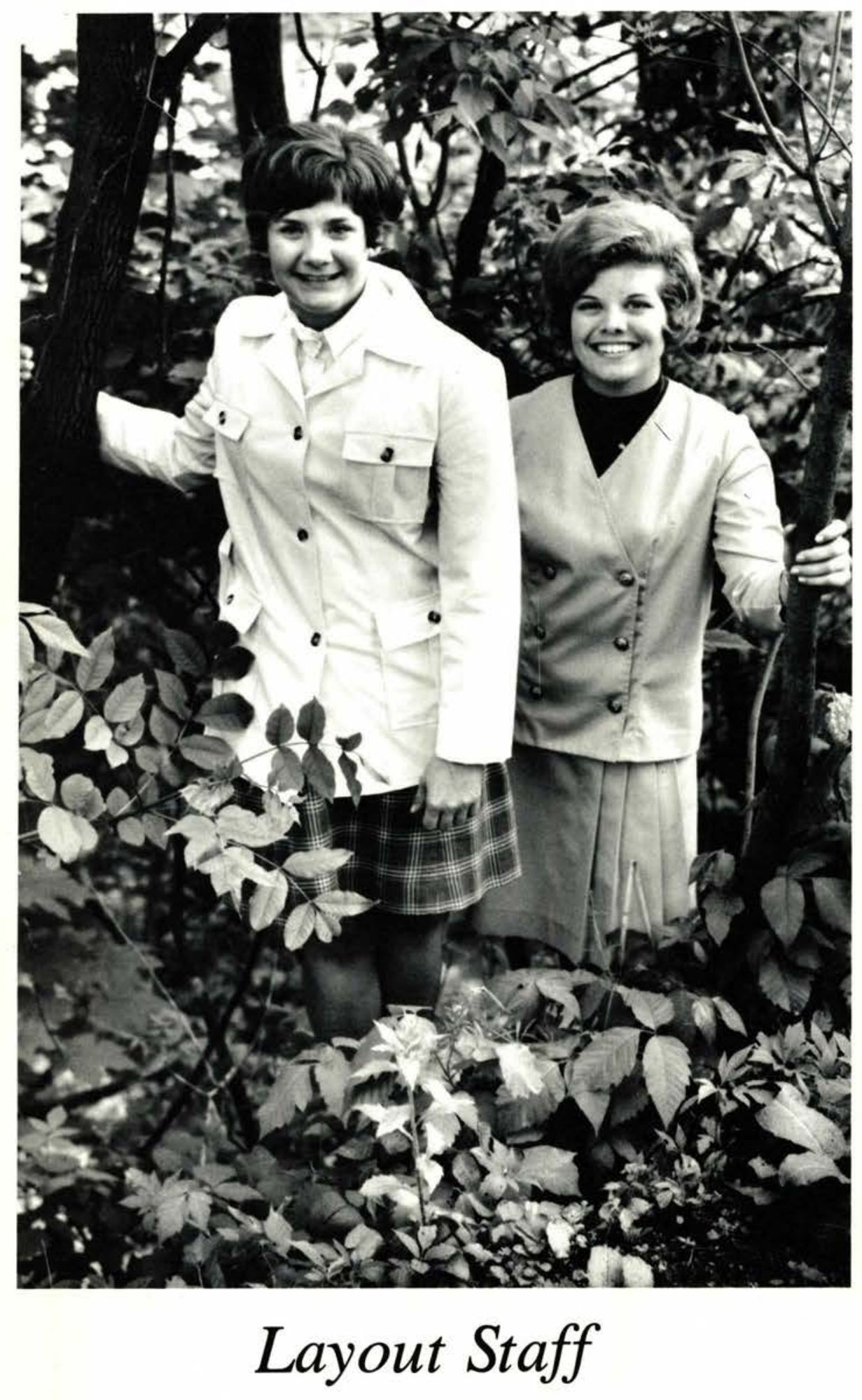

\section{Photography}

Art Shuter

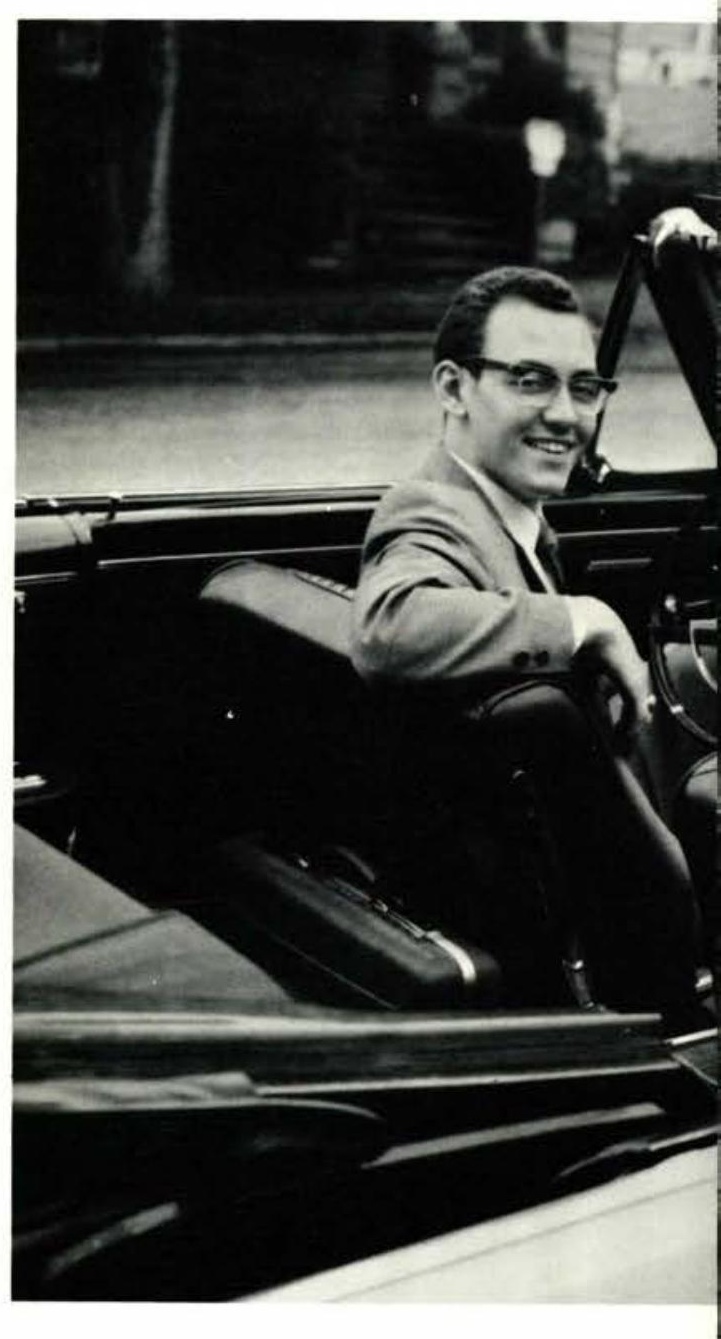




\title{
Copy Staff
}

Staff

\author{
Viann Meyer
}

Debi Thornton

\section{Paula Prater}

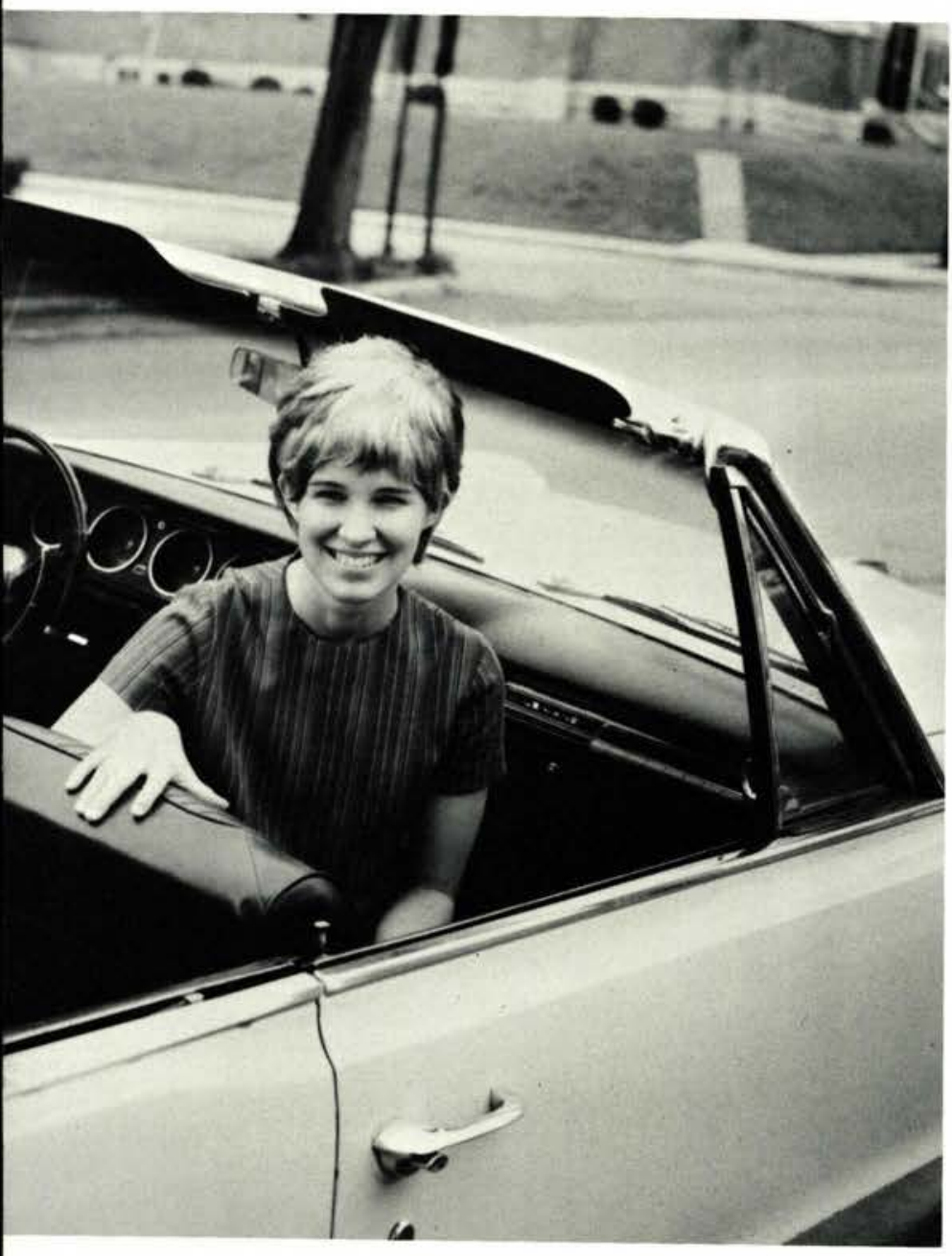

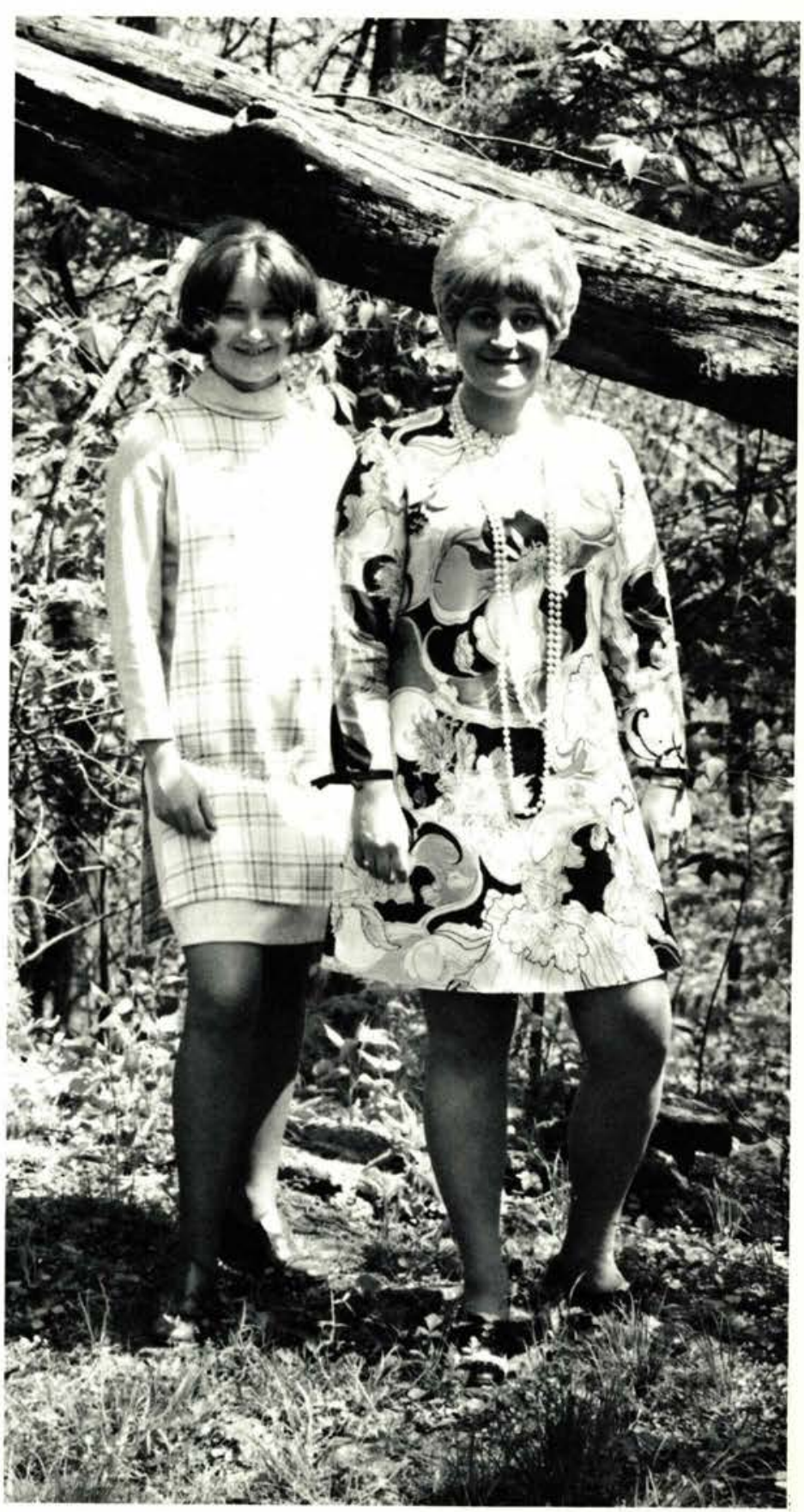




\section{WHISPERING CEDARS STAFF}

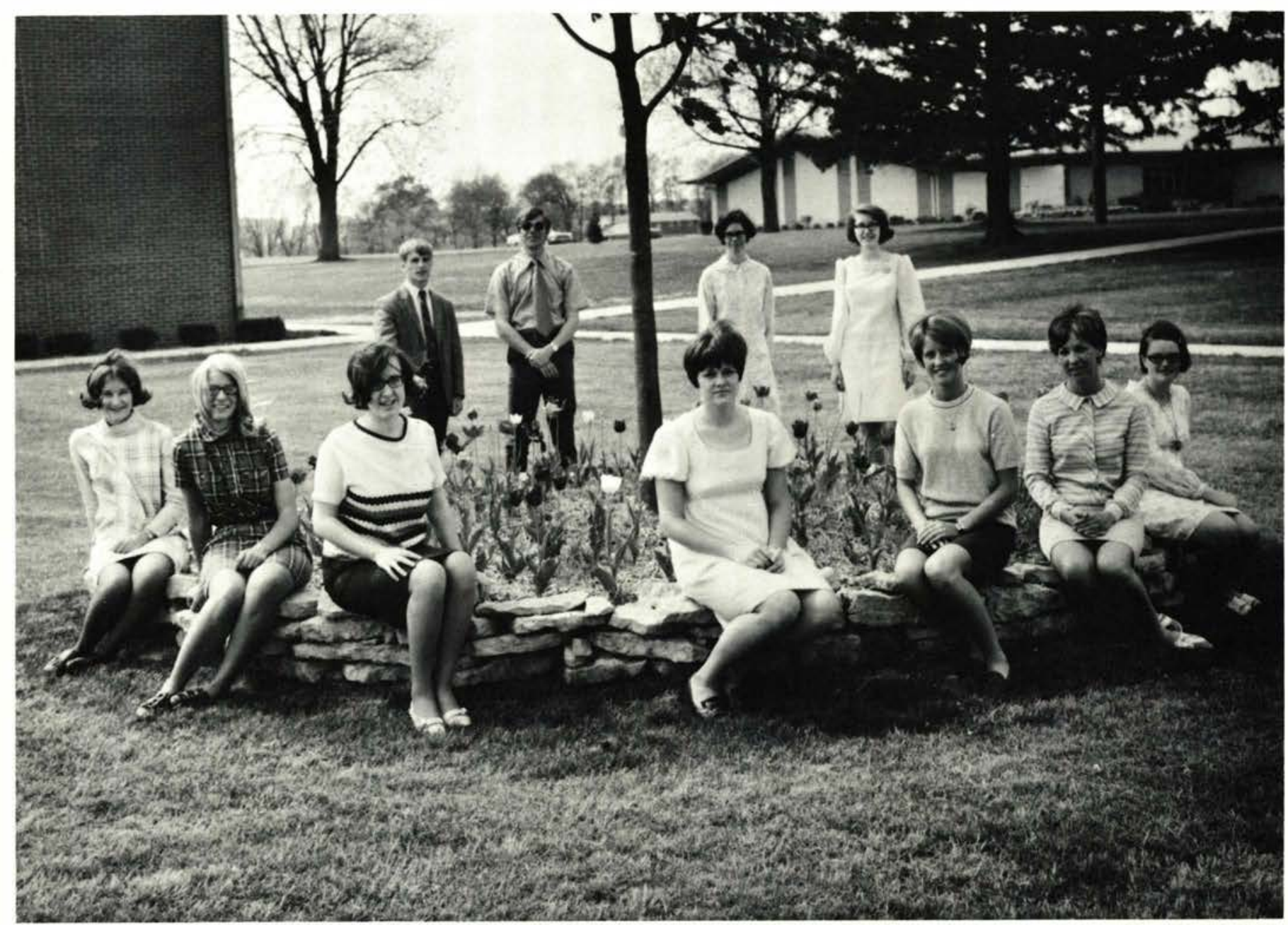

Seated: Debi Thornton, Darlene Rouch, Dawn Myers, Barb Johnson, Pat Wissinger, Judy Leach, Judy Johnson Standing: Lyle Miller, John Smith, Diane Kemp, Marsha Osman

An effective college newspaper is a challenge to not just the editor, but to every contributing member of the staff. The newspaper has a responsibility to present student opinion in the most unbiased manner, regardless of the position held by the author of the opinion. Trying to maintain these standards proves to be a real challenge . . . one which must be met in the college community. 


\section{$W C D R-F M$}

WCDR is a stereo, fully automated, FM, student operated radio station which exists to provide programming for the community and the campus as well as practical experience for students in radio broadcasting. Under the managerial supervision of general manager Paul Gathany, student manager Phil Rohler, and production director Lee Eichelberger, the staff spins records, reports the news, and produces a variety of programs and announcements. This year, for the first time WCDR had a paid secretary which greatly increased the capabilities of other staff members.

Seated: Ginny Heal, Lyle Rodman, Vicki Tobias, Vic Lakes, Bob Carmean, Paul Wheeler, Barb Cooper Standing: Phil Rohler, Cathy Clark, Dane Rohm, Ken Cole, Diane Cockerill, Jan Hillery, Rich DeWalk, Lee Eichelberger, Dan Mead, Judy Johnson, Ellen Young, Tim Andreanopolis, Wayne St. Denis, Carol Bierbaum, Mr. Gathany

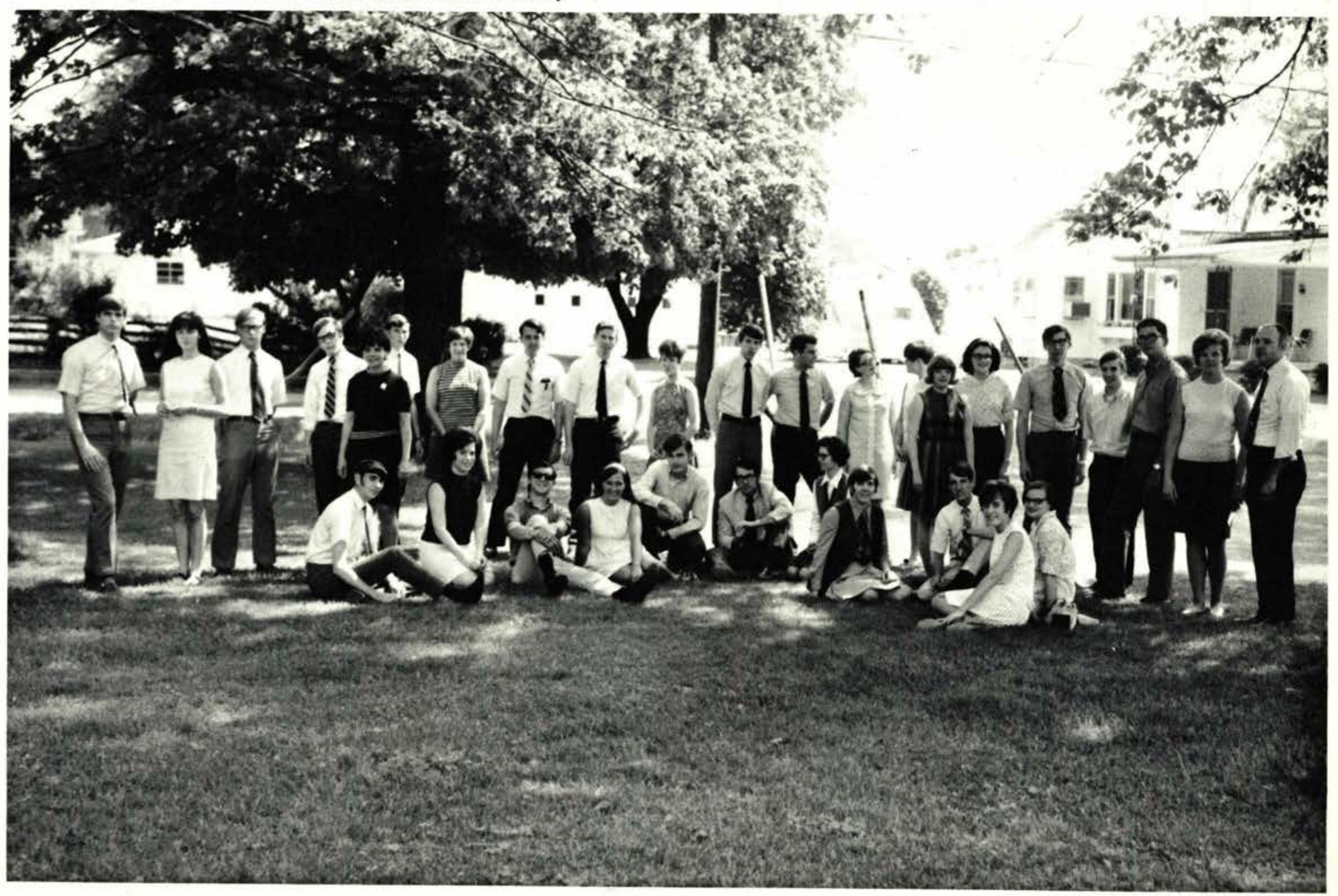




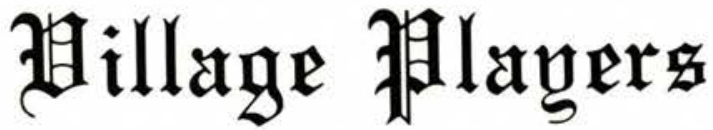

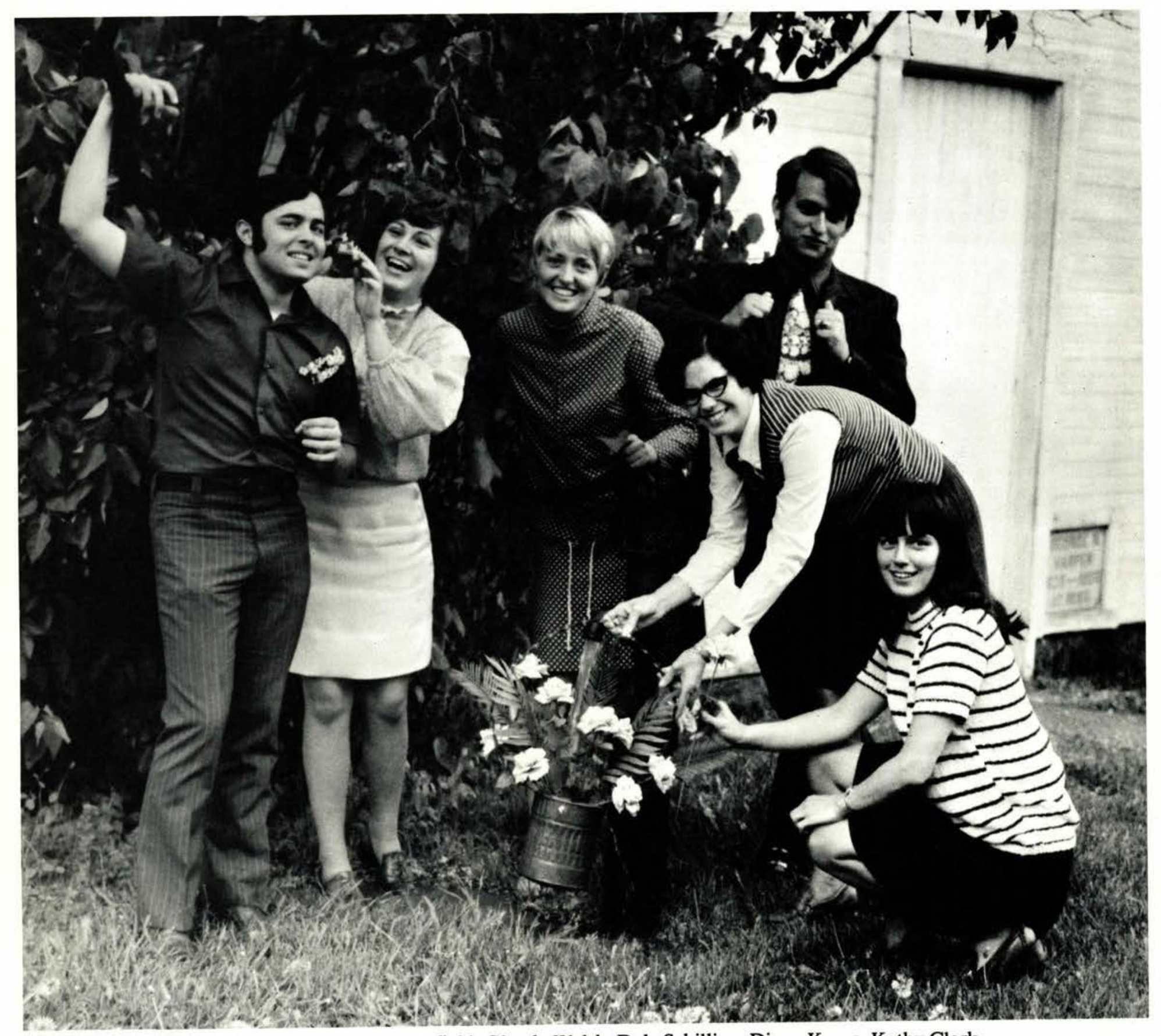

Don Harmon, Peggy Porterfield, Glenda Welsh, Dale Schilling, Diane Kemp, Kathy Clark. 


\section{$M E N C$}

Music Educators National Conference makes available opportunities for professional musical development, acquaints students with privileges and responsibilities of music education, and provides its members an acquaintance with leaders in the music education profession.

This year MENC as an organization attended the State of Ohio Music Educators Association Convention, visited the Selmer Music Factory in Elkhart, Indiana, and visited the Lenord Smith workshop.

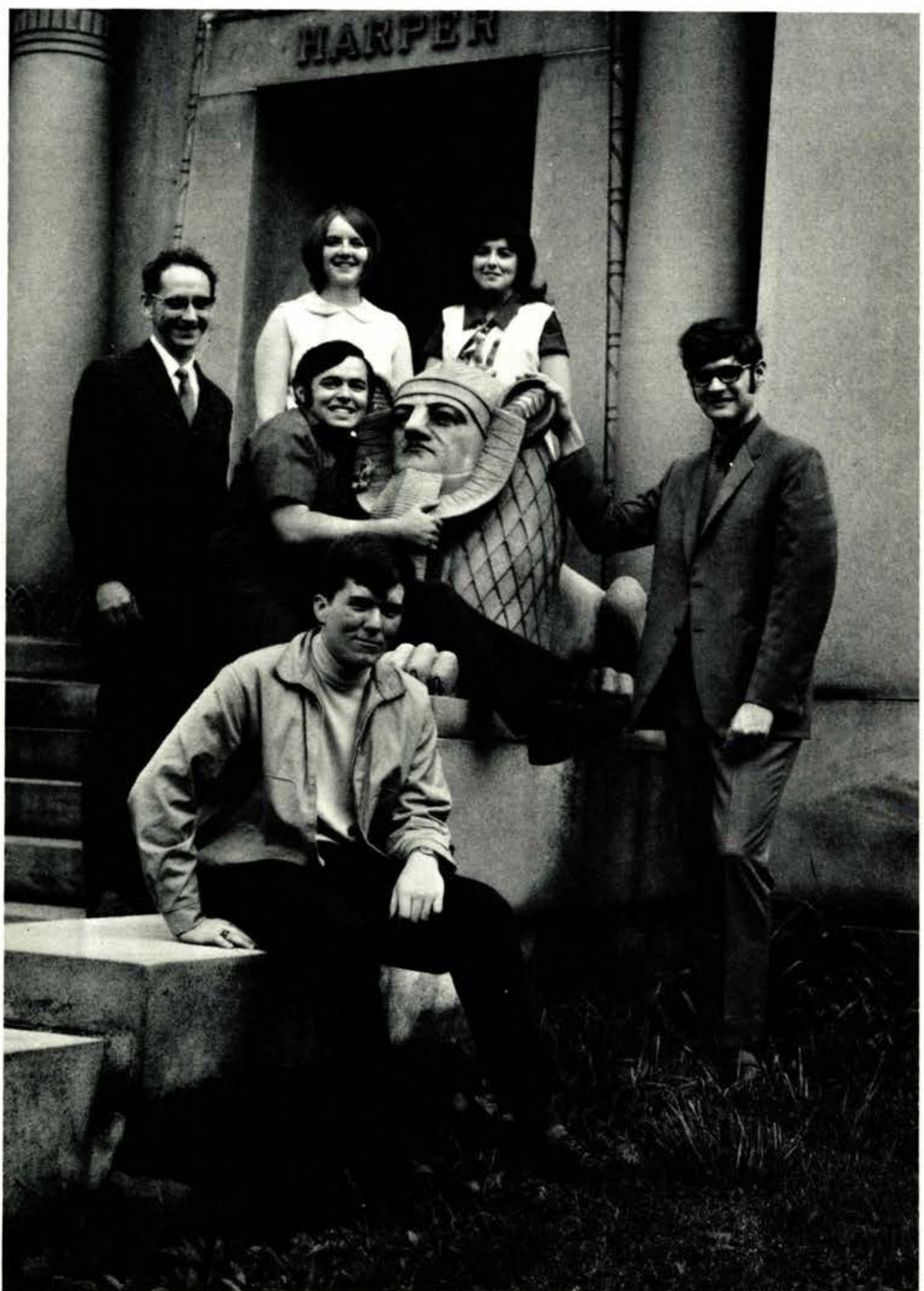

Row One: Larry Alfrey Row Two: Don Harmon Row Three: Mr. Thornton, Judy Grover, Joyce Motts, Ron Spieth 


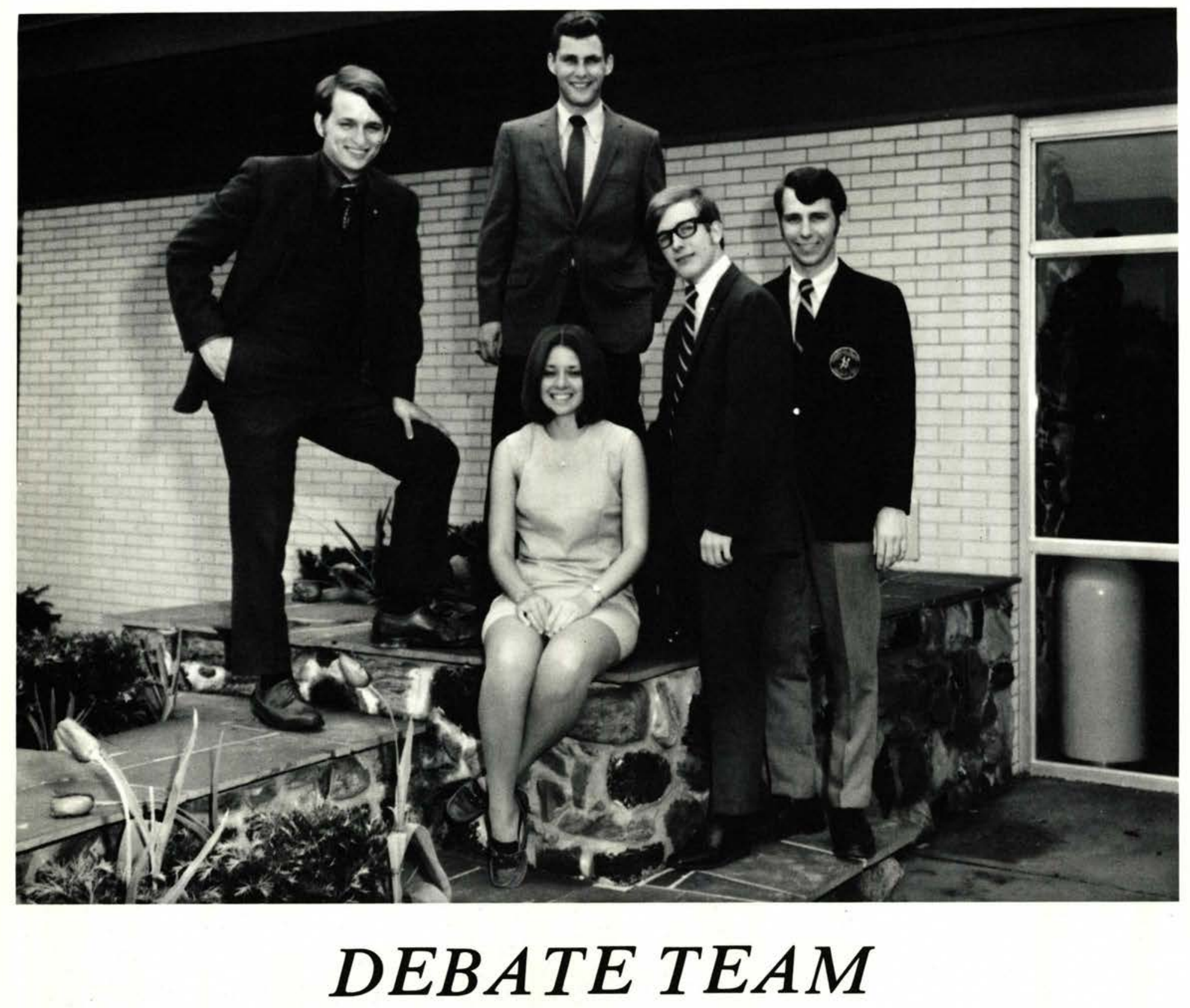

Seated:

Melinda McNiece
Standing:

Dale Schilling

Dan Mead

Ken Cole

Randy Patten 
Delta Sigma Alpha, which stands for Masters of Symphony and Harmony, is the honorary music society on campus. Its purpose is to foster a greater and continued interest in all types of musical performance; to encourage more discriminating habits in listening to and in the performance of musical literature; to increase and advance the spirit of good music and musical knowledge; and to further the reputation of our college as a musical center.

Delta Sigma Alpha sponsored a lecture-demonstration for the school by Mr. Bob Larson, an authority on Rock and Roll.

\section{DELTA SIGMA ALPHA}

Seated:

Lyle Anderson
Standing:

Joyce Motts, Jan Brock

Connie Clark, Becky Hull

Judy Grover, Bob Snyder

Second Row:

Curt Rexroth, Mr. Matson

Ron Spieth

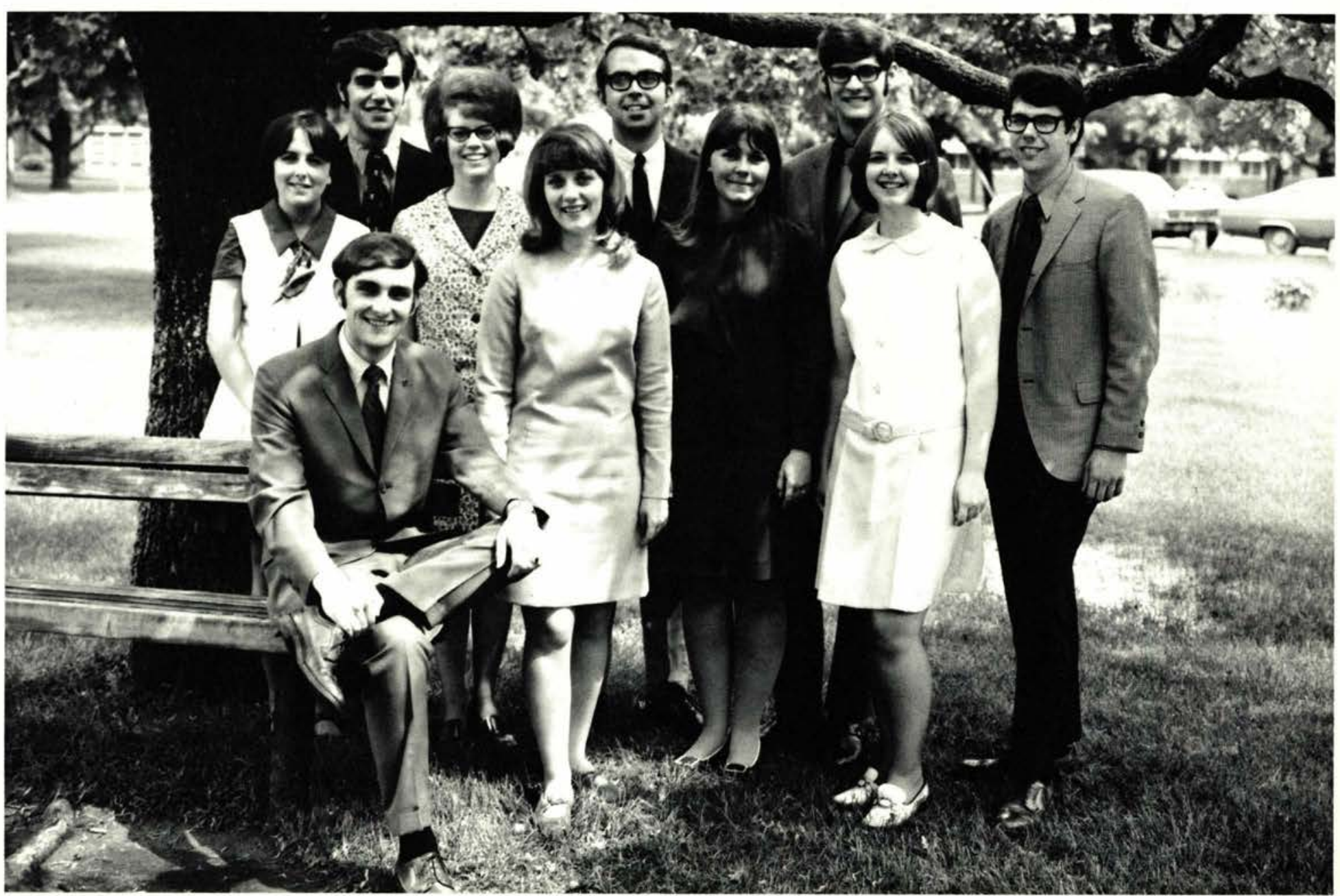




\section{VARSITY “C”}

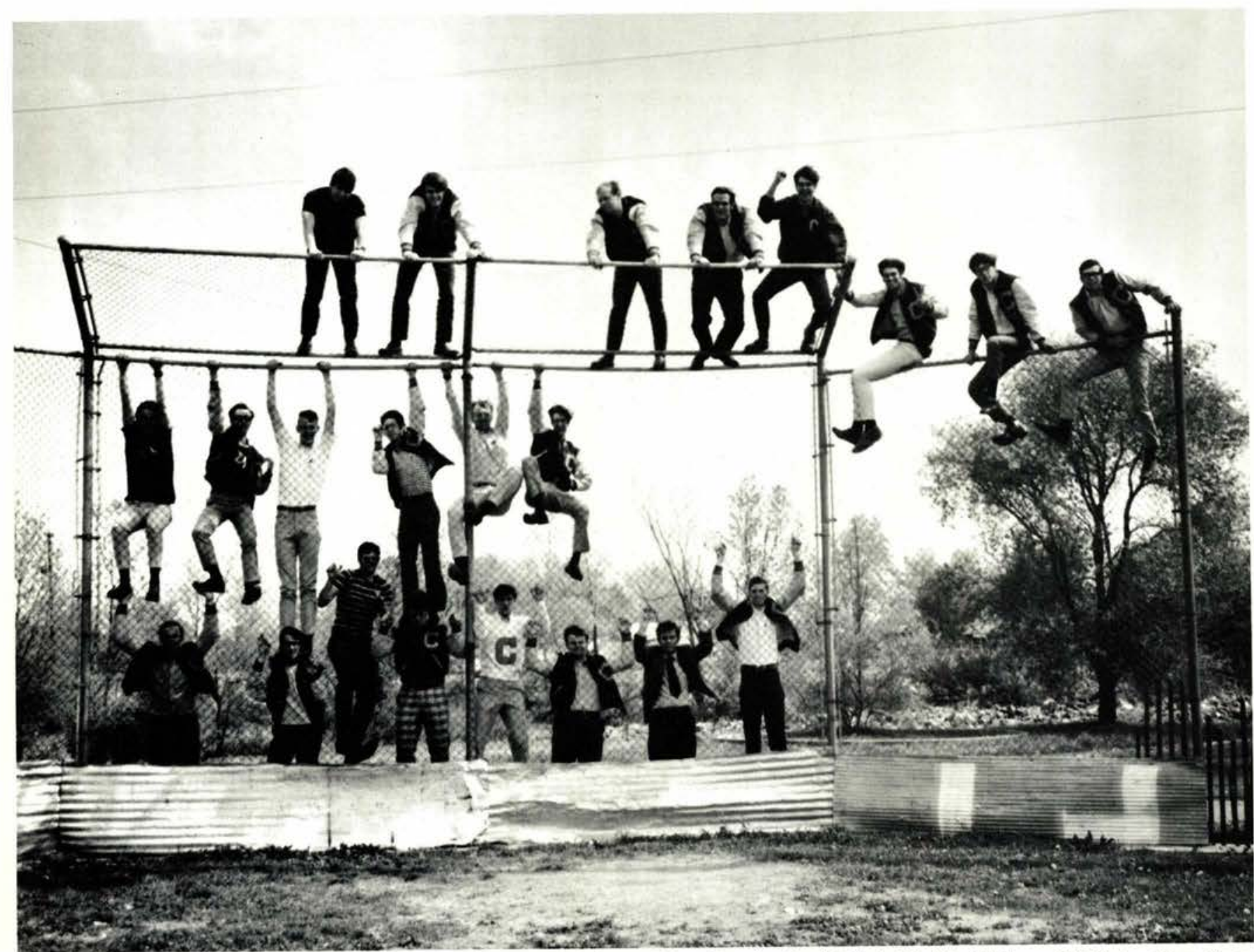

Row One: Charlie Williams, Lee Reno, Bill Trefzger, Phil Johnson, Ken Curcio, Paul Friedrich, Stu Walker, Bill Tripp Row Two: Dave Jewell, Tony Wall, Don Atherton, Warren Pettitt, Bob Lunney, Bob Austin Row Three: Ken Boothe, Darwin Boertje, Denny Stora, Steve Taylor, Dan Inghram, Rick Inghram, Dan Smith, Dee Mays 


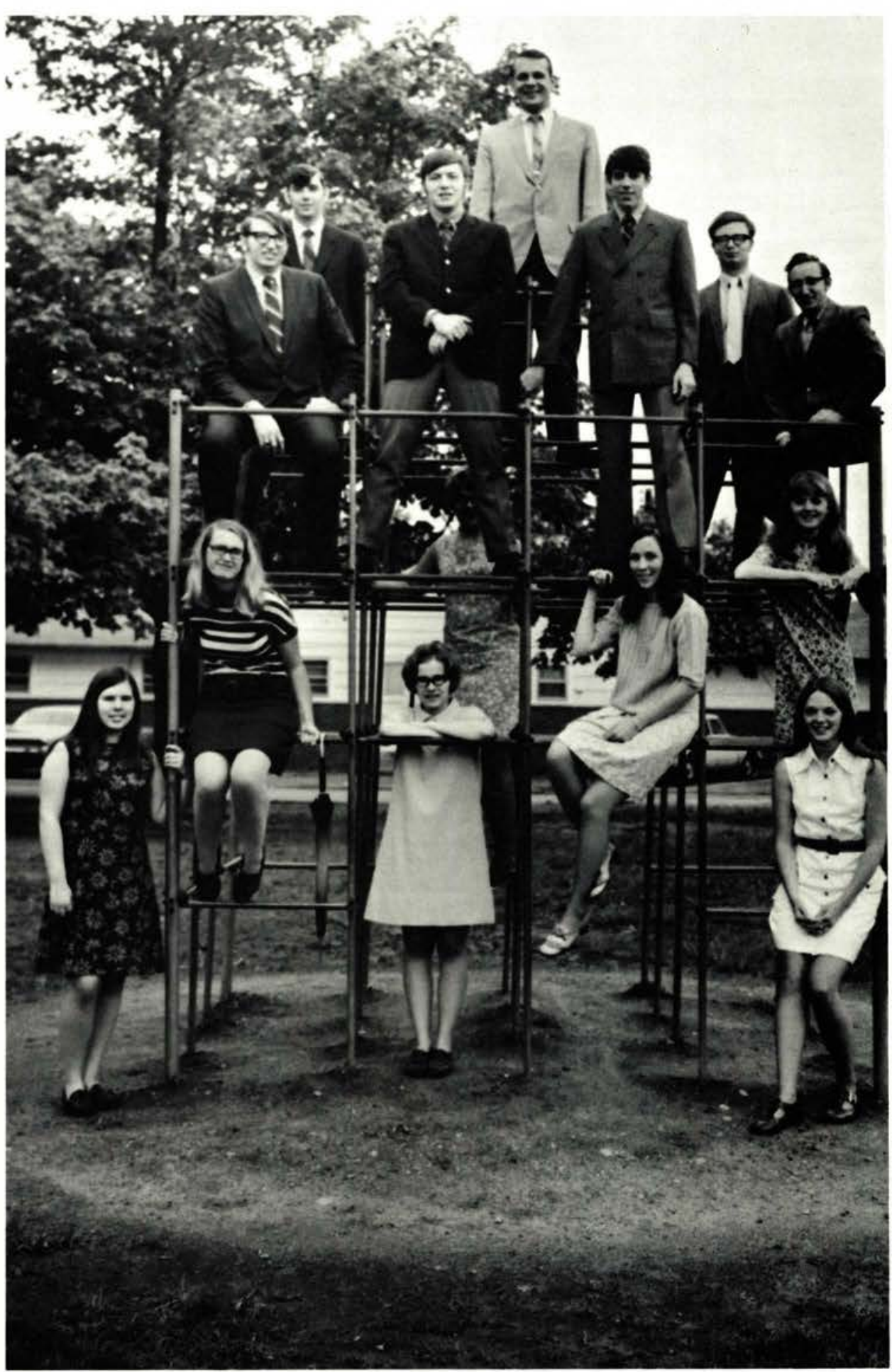

$F B A$
Row One: Sue Harold, Elaine Dixon, Gretchen Schlechty Row Two: Pam Borg, Bev Maidment, Debbie Kitting Row Three: Mr. Wilcoxon, Jim Williams, Don Fleming, Alan Klinger Row Four: Jerry Garland, John Burkholder, Preston Butcher 


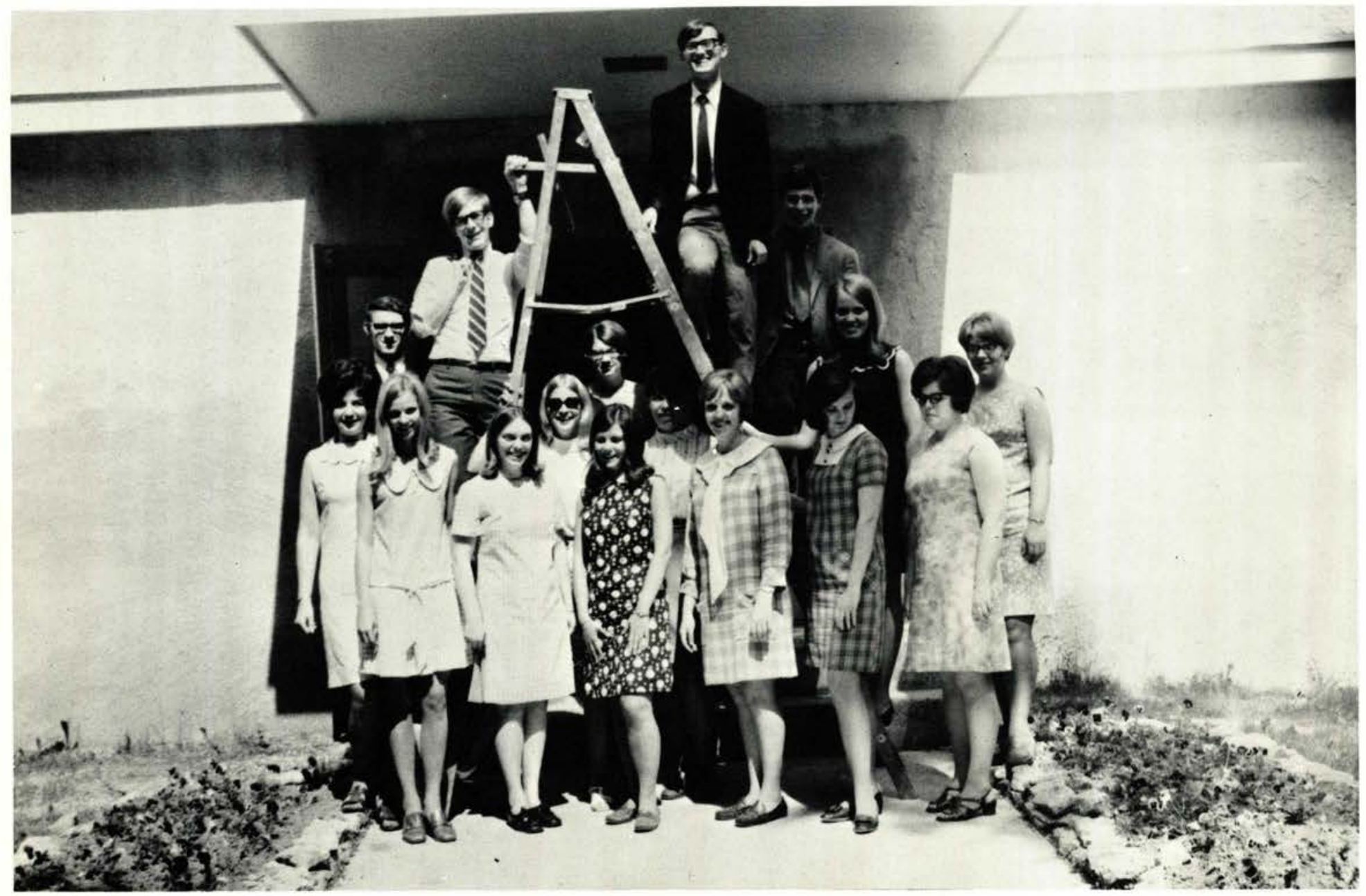

Row One: Karyn Peterson, Cecilia Romine, Sue Webb, Jane Cuttingham, Margaret Muirhead, Nancy Smith Row Two: Sarah Hickam, Darlene Rouch, Joanie Boyd, Cathy Getz, Florence Krikke, Sue Roloff Row Three: Phil Mohler, Ken Cole, Sam McMillen, Ralph Perkins

The responsibilities and opportunities of dorm counselors are many-fold. They are the motivating factor in the residence halls to promote the student to his maximum potential spiritually, academically, and socially. Assisting to communicate the needs and wishes of individuals and groups of students to proper channels is perhaps one of the most difficult tasks these counselors must perform; an effective dorm counselor will, however, have the best interests of all students first in their minds as their biggest responsibility should be to lead and counsel these students spiritually.

\section{DORM \\ COUNSELORS}




\section{OFFICERS}

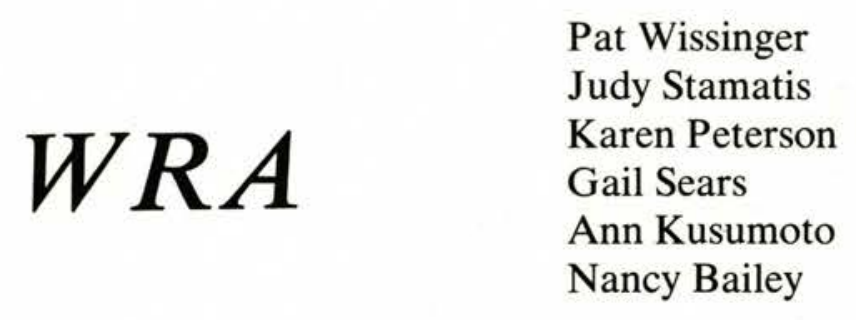

President

Vice President

Secretary

Treasurer

Student Council Rep. Intra-Mural Chairman

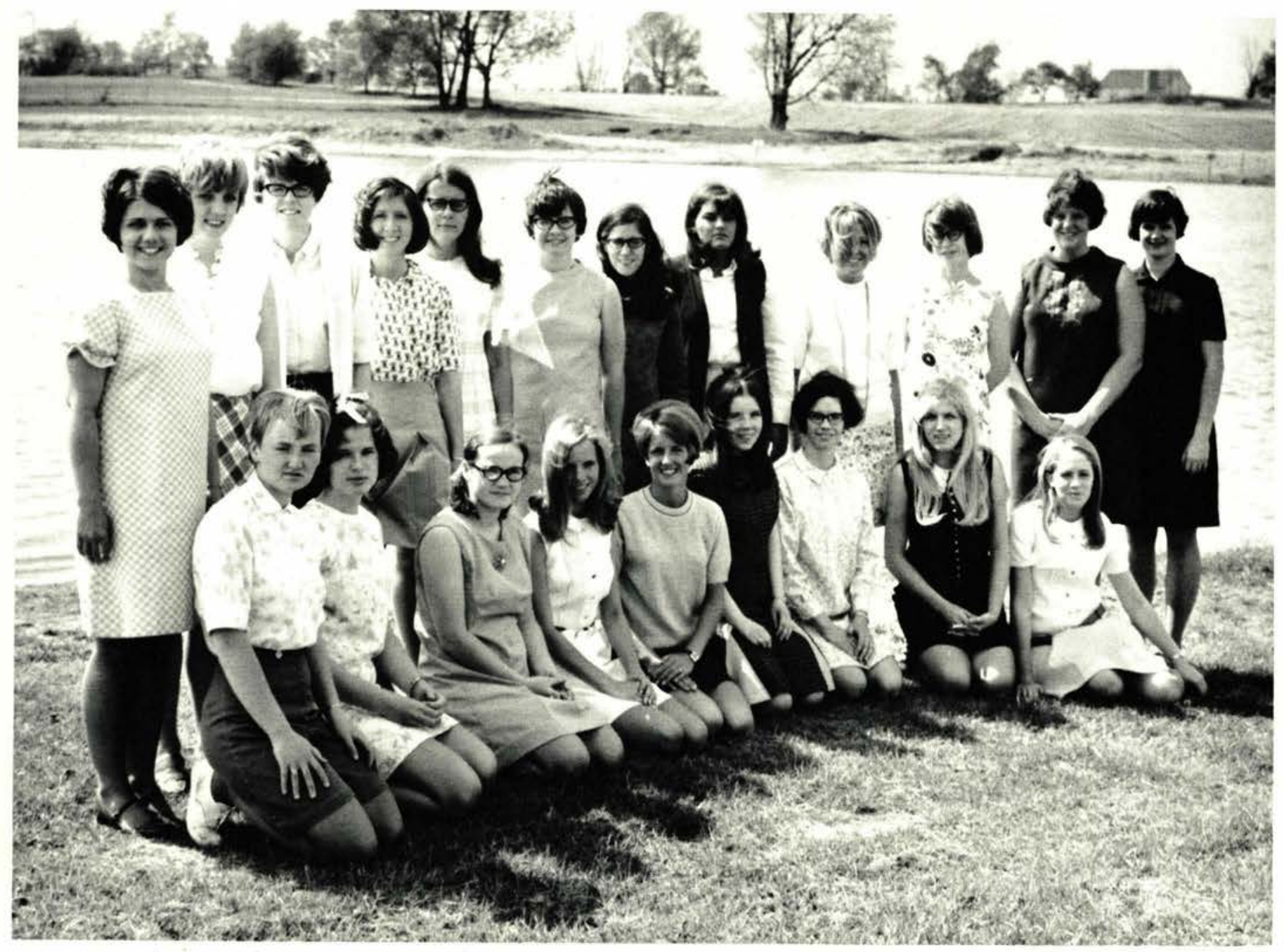

Kneeling: Marsha Zerwicki, Joan Tennant, Elaine Kalnbach, Kayrn Peterson, Pat Wissinger, Verna Tallmon, Dianne Kemp, Gail Sears, Connie Walker. Standing: Billie Thomas, Jane Cunningham, Connie Pumpelly, Landra Lowery, Mary Lou Storey, Pam Branning, Cathy Zwiesler, Brenda Tompkins, Roxanne Davis, Becky Bittner, Karen DeMars, Jan Martin 


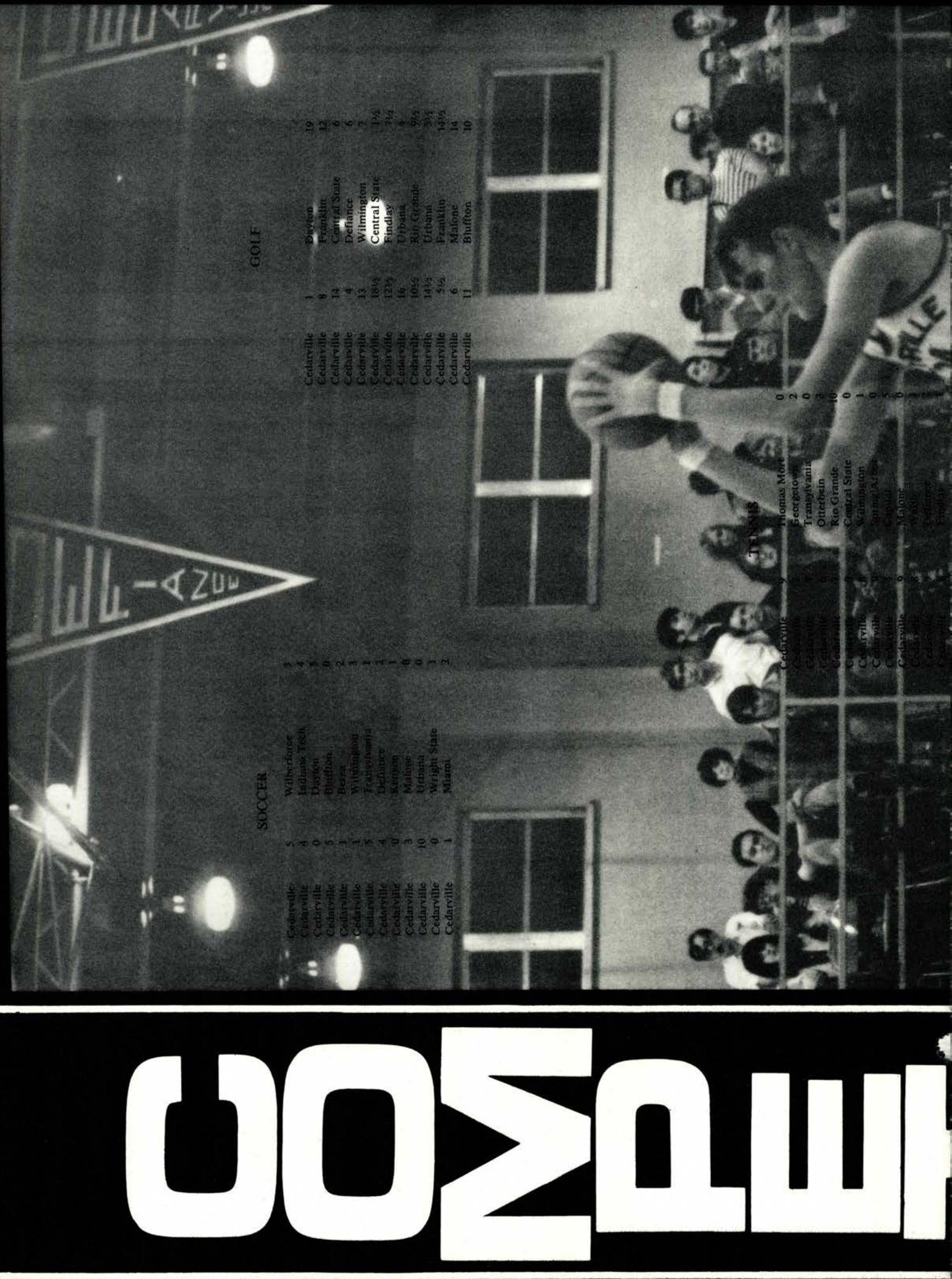




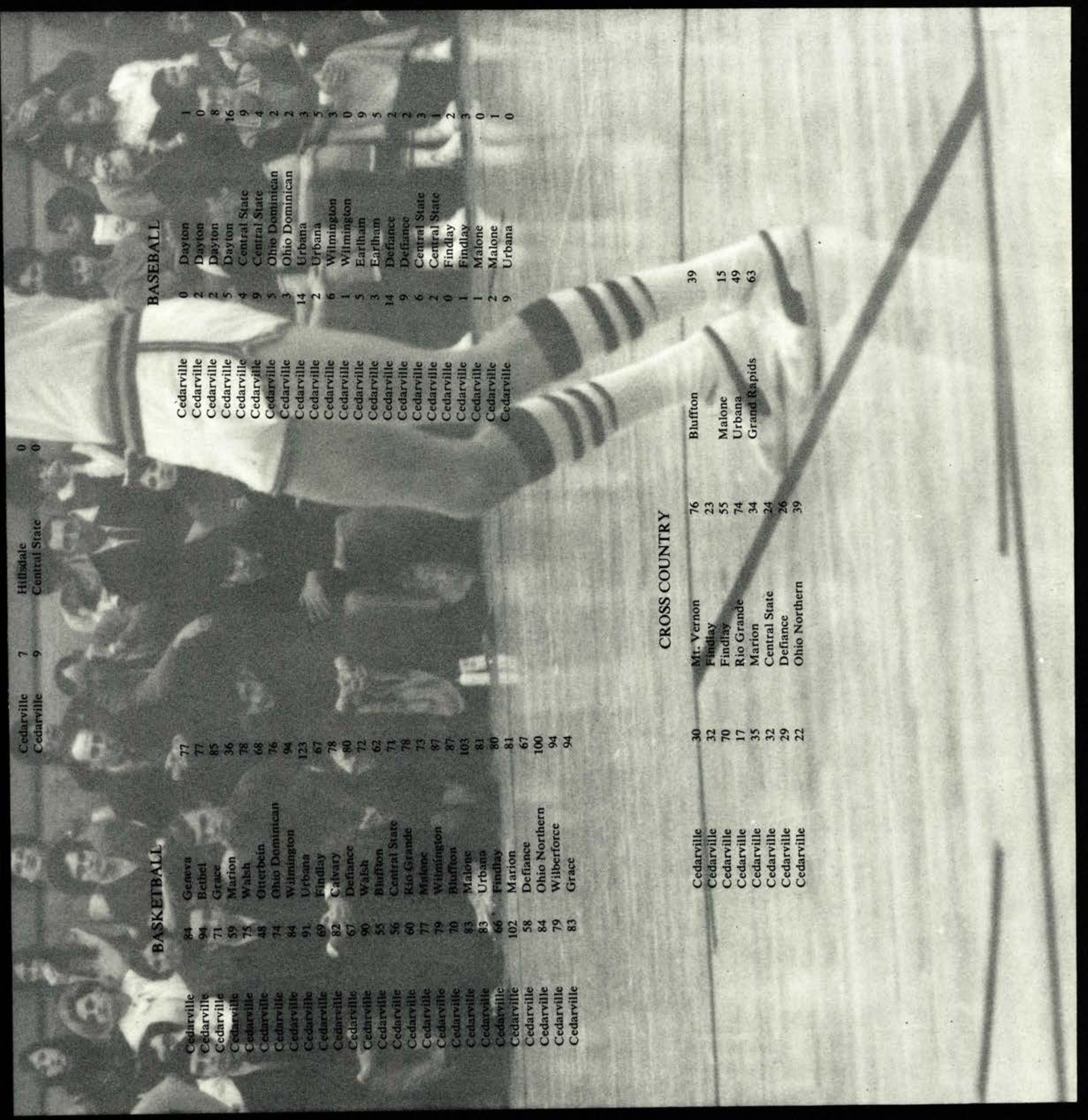

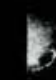
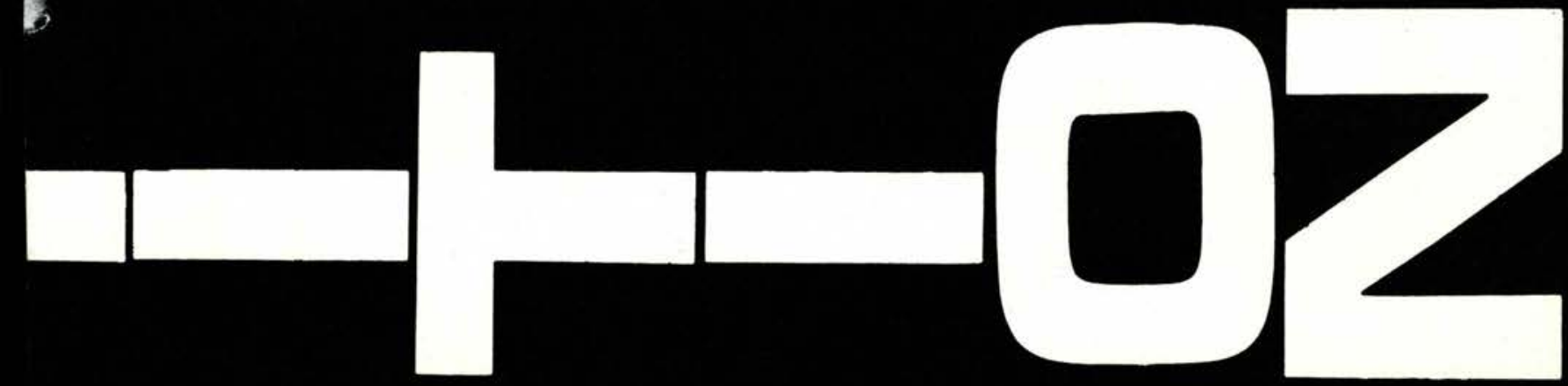


\section{SOCCER}
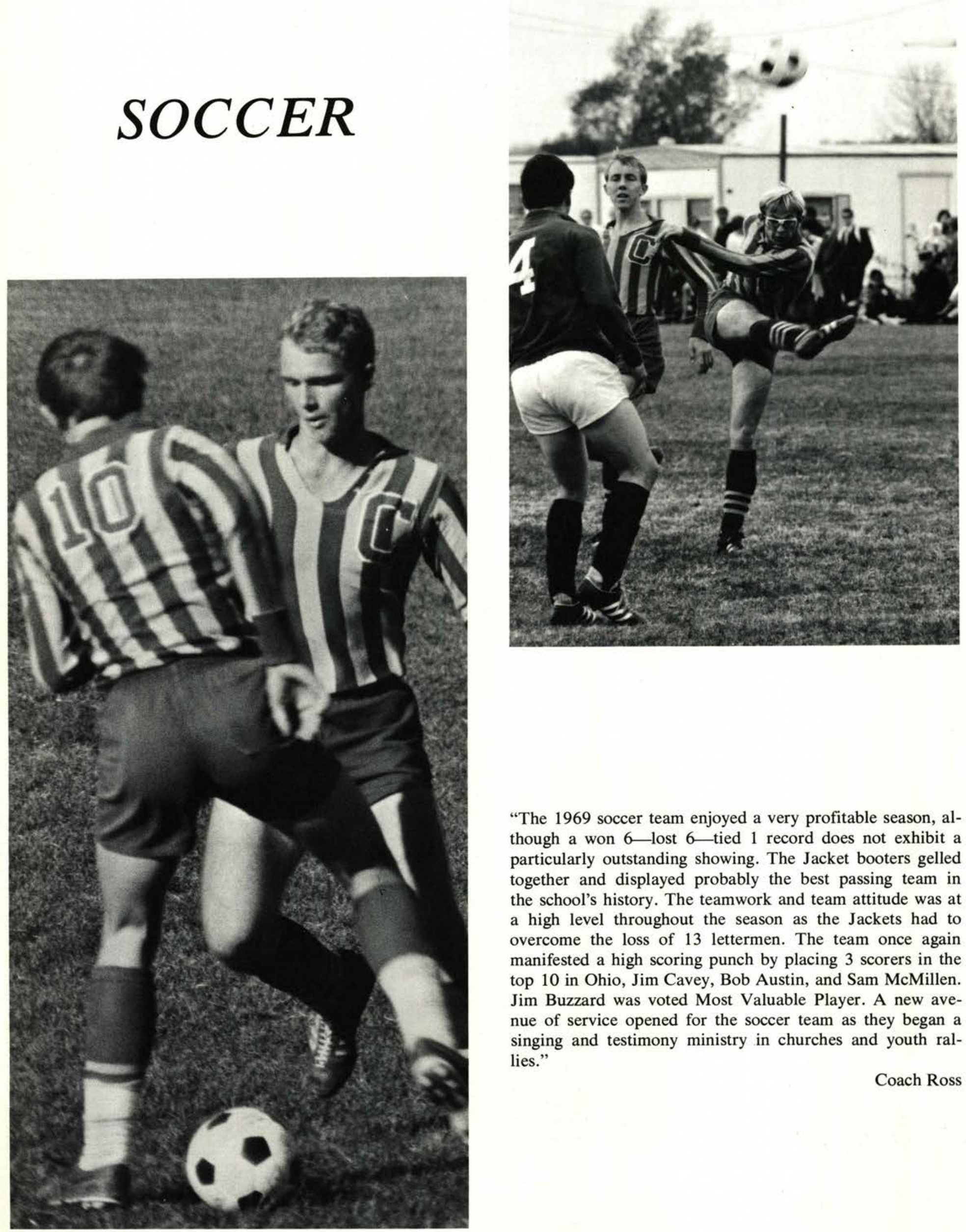

"The 1969 soccer team enjoyed a very profitable season, although a won 6-lost 6 -tied 1 record does not exhibit a particularly outstanding showing. The Jacket booters gelled together and displayed probably the best passing team in the school's history. The teamwork and team attitude was at a high level throughout the season as the Jackets had to overcome the loss of 13 lettermen. The team once again manifested a high scoring punch by placing 3 scorers in the top 10 in Ohio, Jim Cavey, Bob Austin, and Sam McMillen. Jim Buzzard was voted Most Valuable Player. A new avenue of service opened for the soccer team as they began a singing and testimony ministry in churches and youth rallies."

Coach Ross 
$\sum_{n=}^{x}$

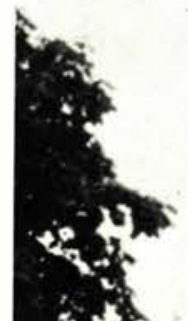

8.

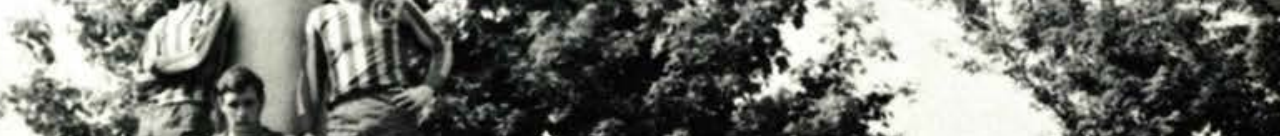
of.

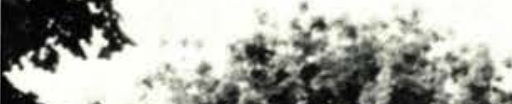
Niver

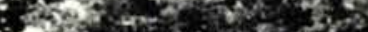

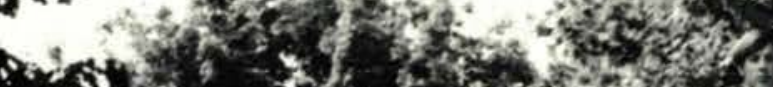

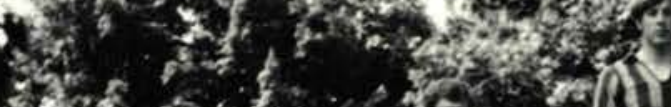

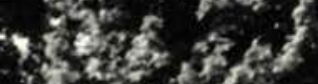

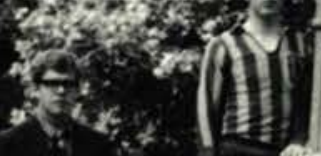

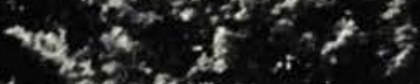

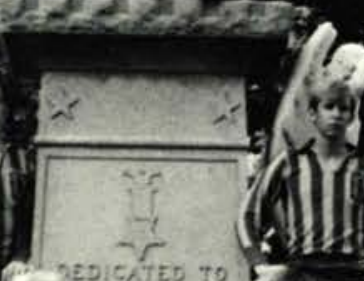

\section{7}

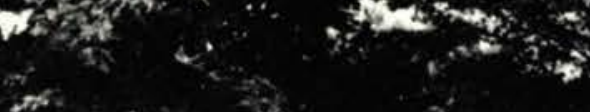

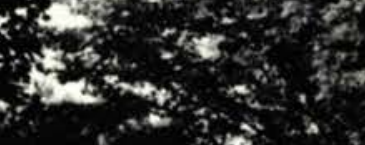

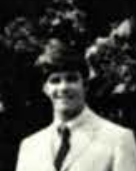

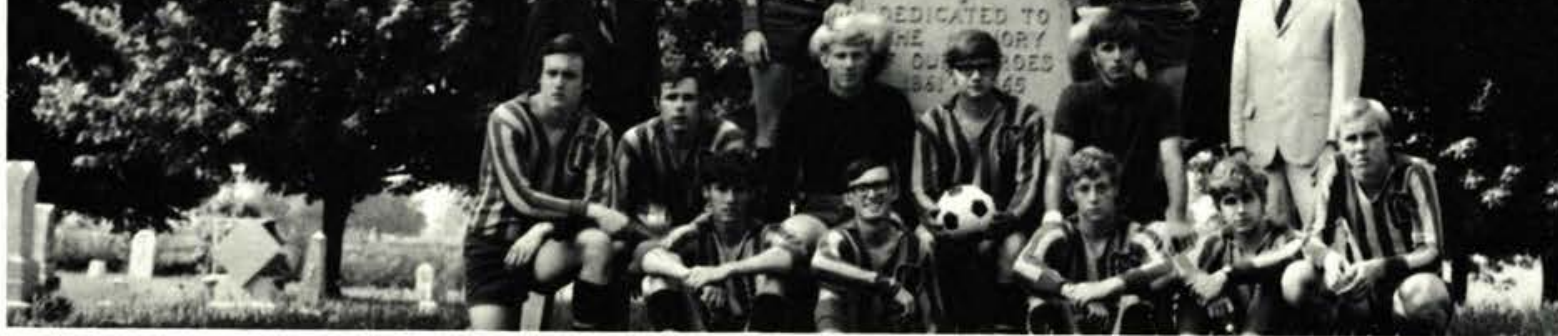

Row One: Bob Hoffman, Sam McMillien, Don Southwell, Bob Senseney, Jim Cavey Row Two: Russ Jenkins, Dave Draxler, Cliff Jensen, Andy Gathany, Jim Buzzard Standing: Buddy McNiece, Tim Hegg, Jim Greening, Coach Ross Elevated: Bob Austin, Warren

Jenkins, Jeff Woodcock
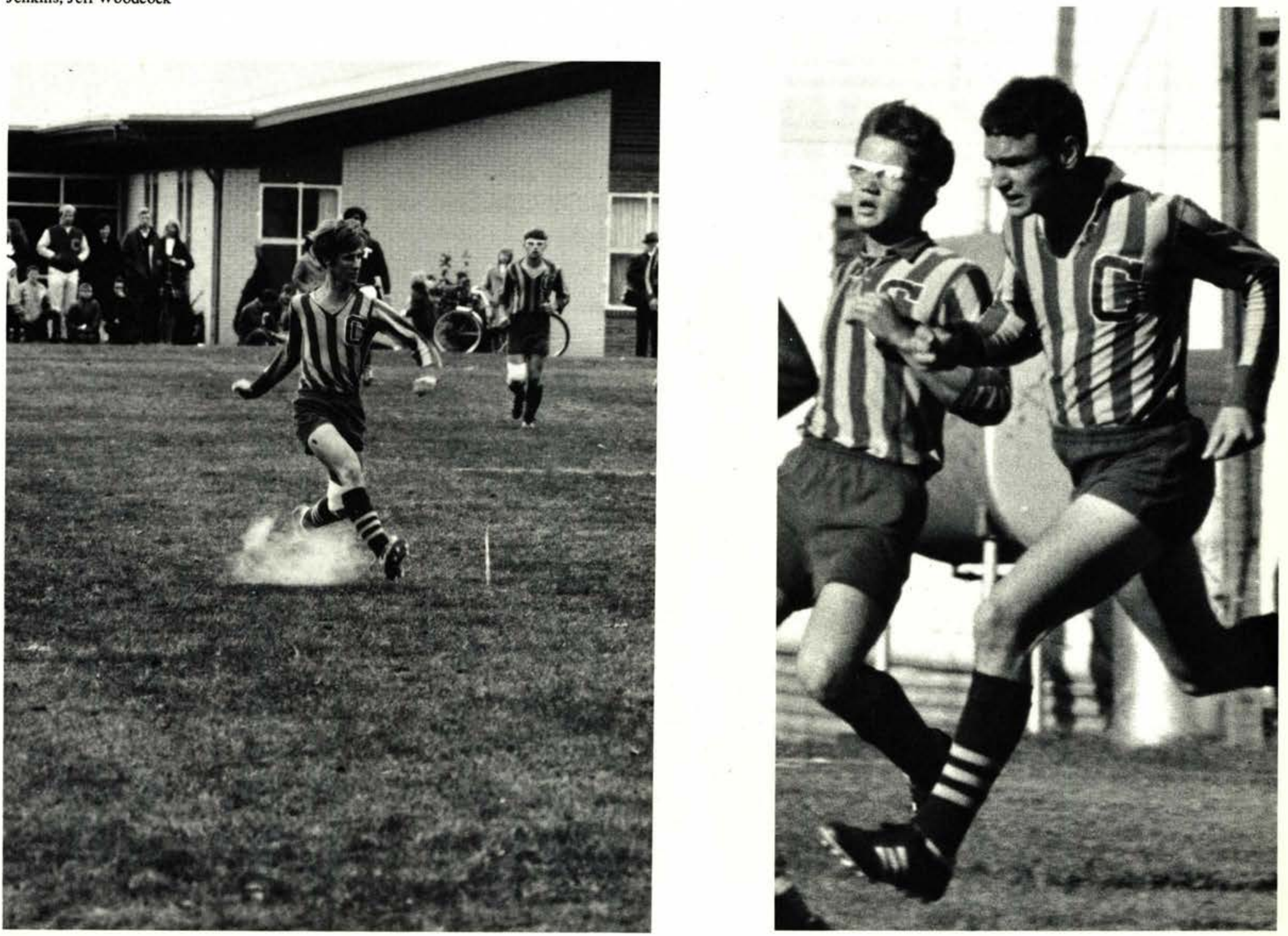


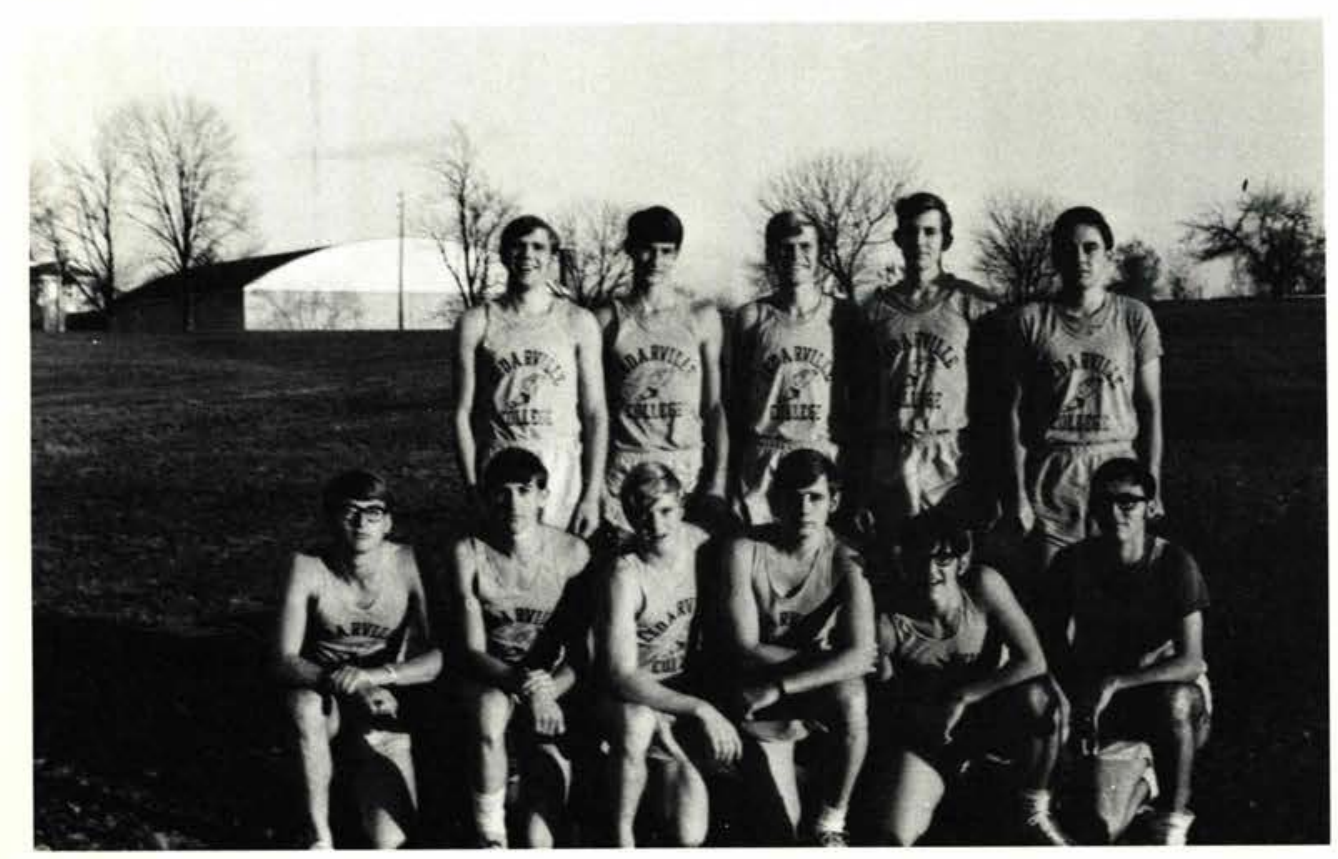

"Our team had good spirit and ran well all year. We were mostly freshmen and therefore inexperienced at the 4 mile distance. With the entire team returning we have a great nucleus for next year's team".

Coach King

\section{TRACK}

Kneeling: Ron Yoder, Tom Farlow, Les Chambers, Carl Rogers, Kim Gerber, Tom Mullins Standing: John Smith, Ernie Welborn, Ken Evans, Bob Wood, Dave Jewell

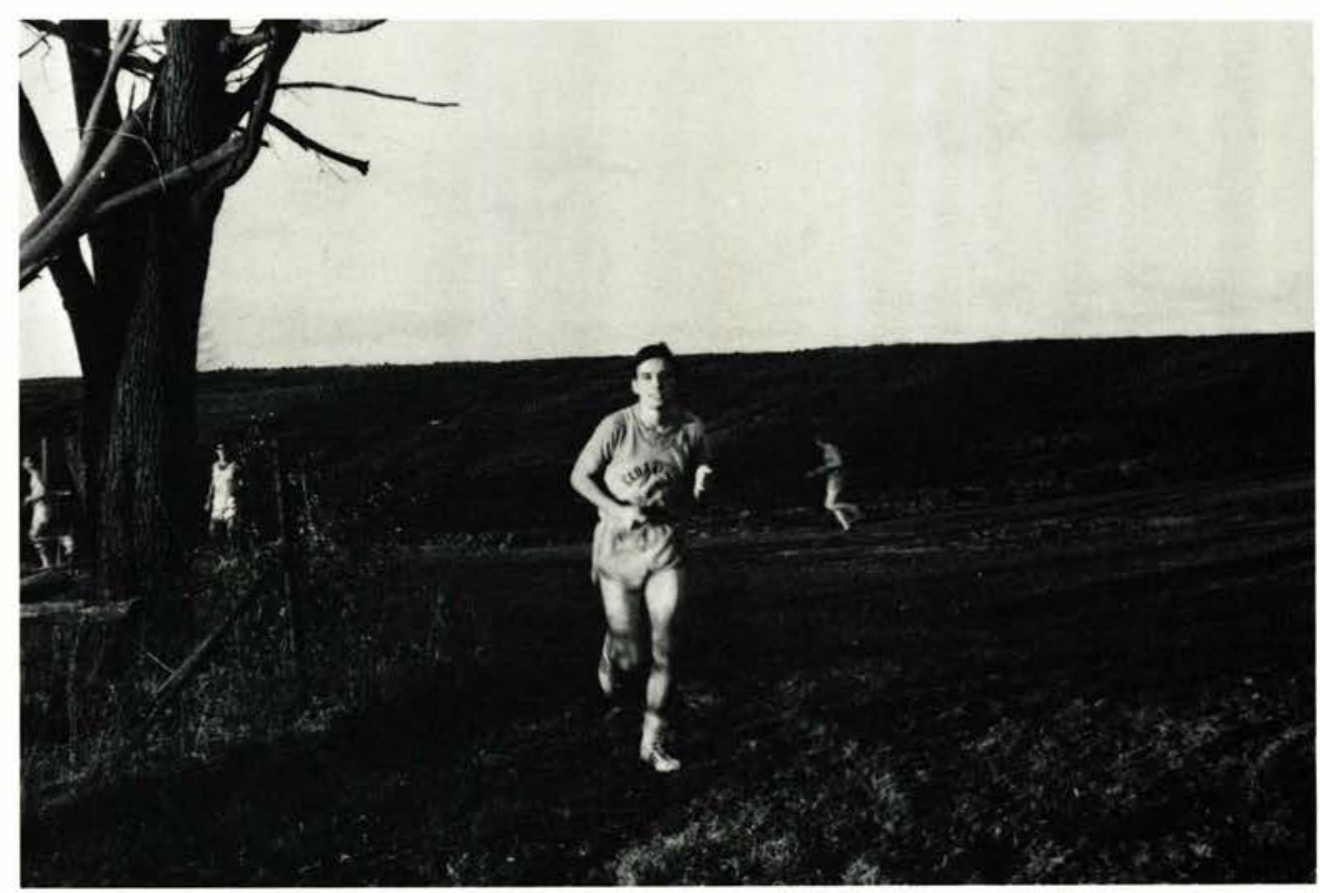




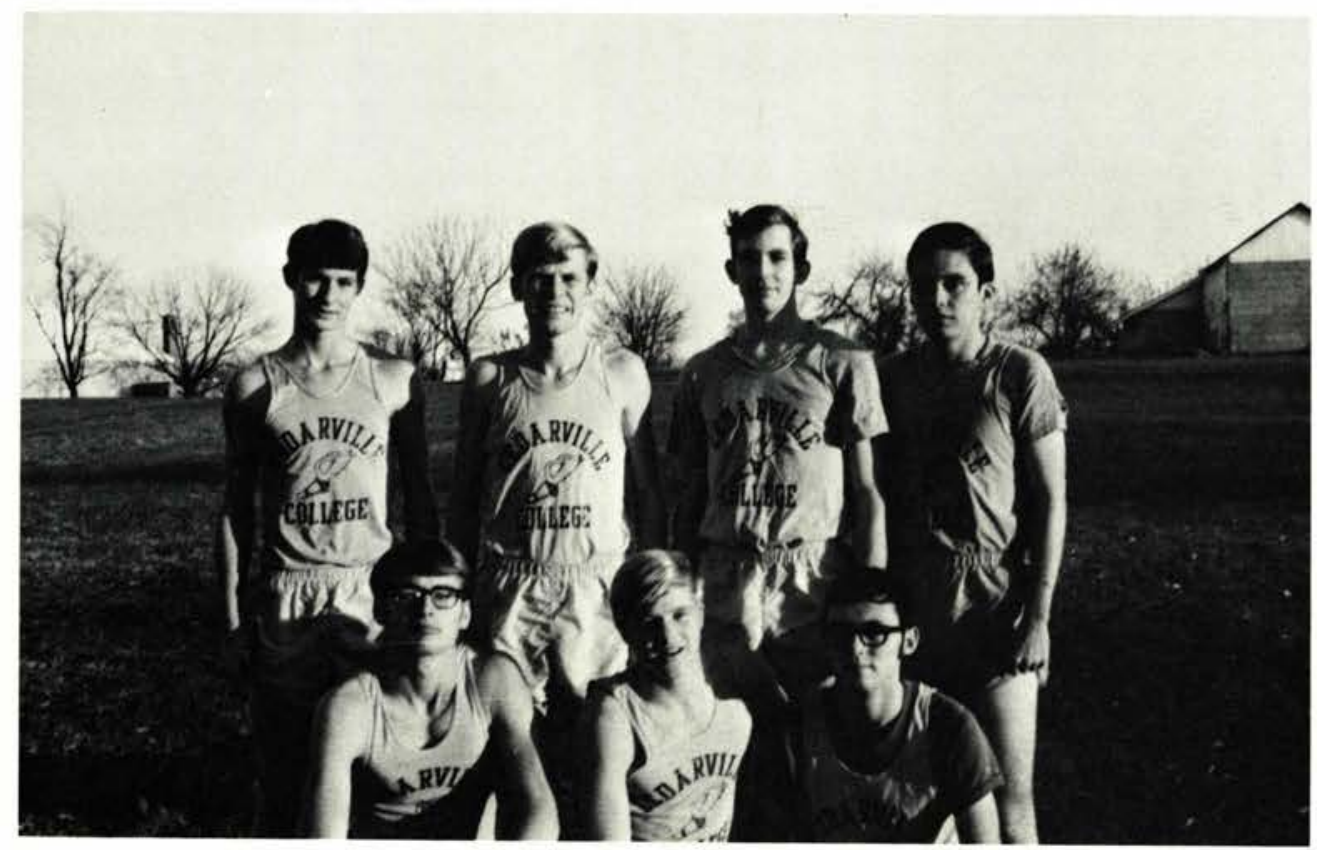

Row One: Ron Yoder, Les Chambers, Tom Mullins Row Two: Ernie Welborn, Ken Evans, Bob Wood, Dave Jewell

\section{CROSS COUNTRY}

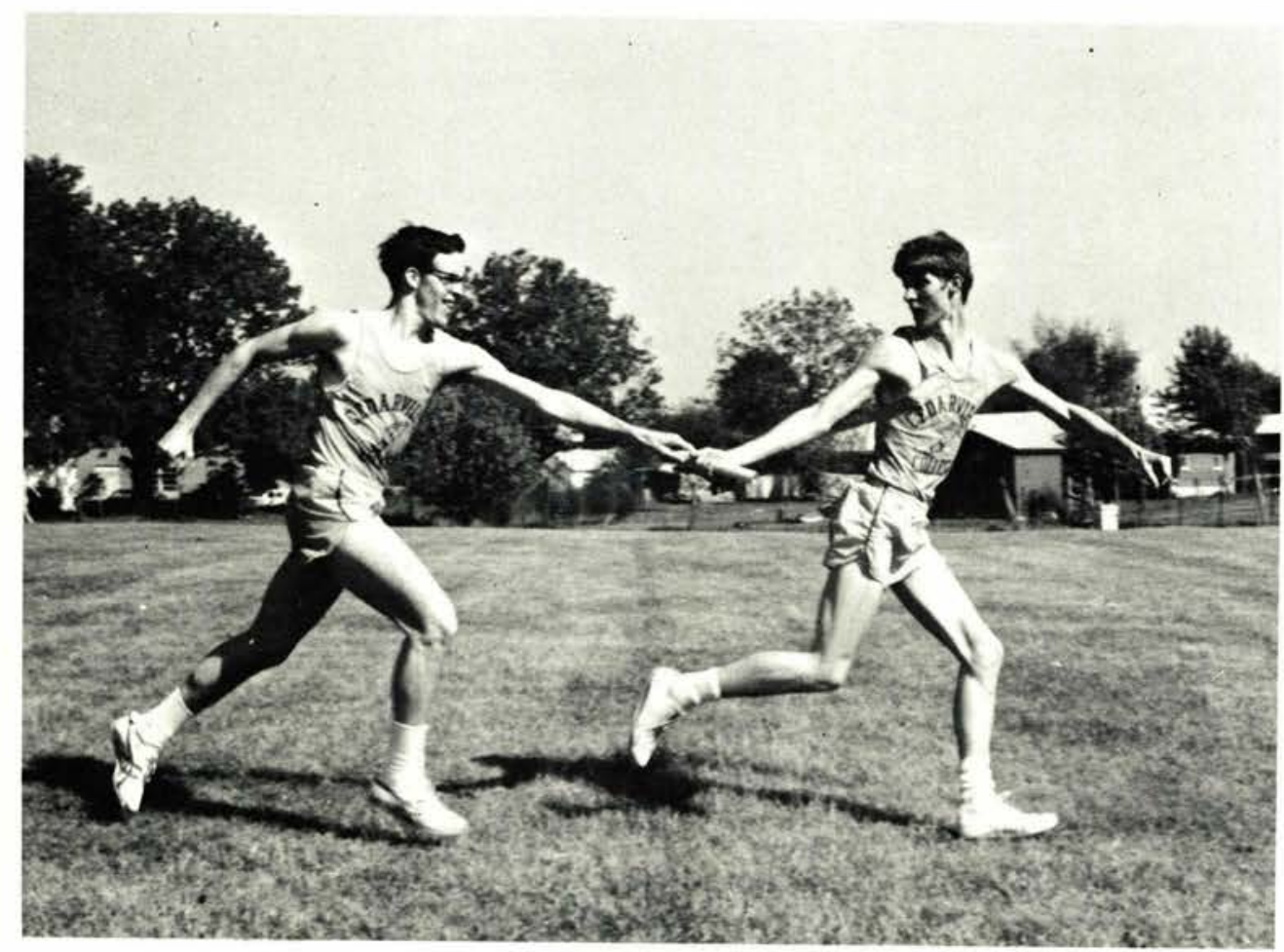



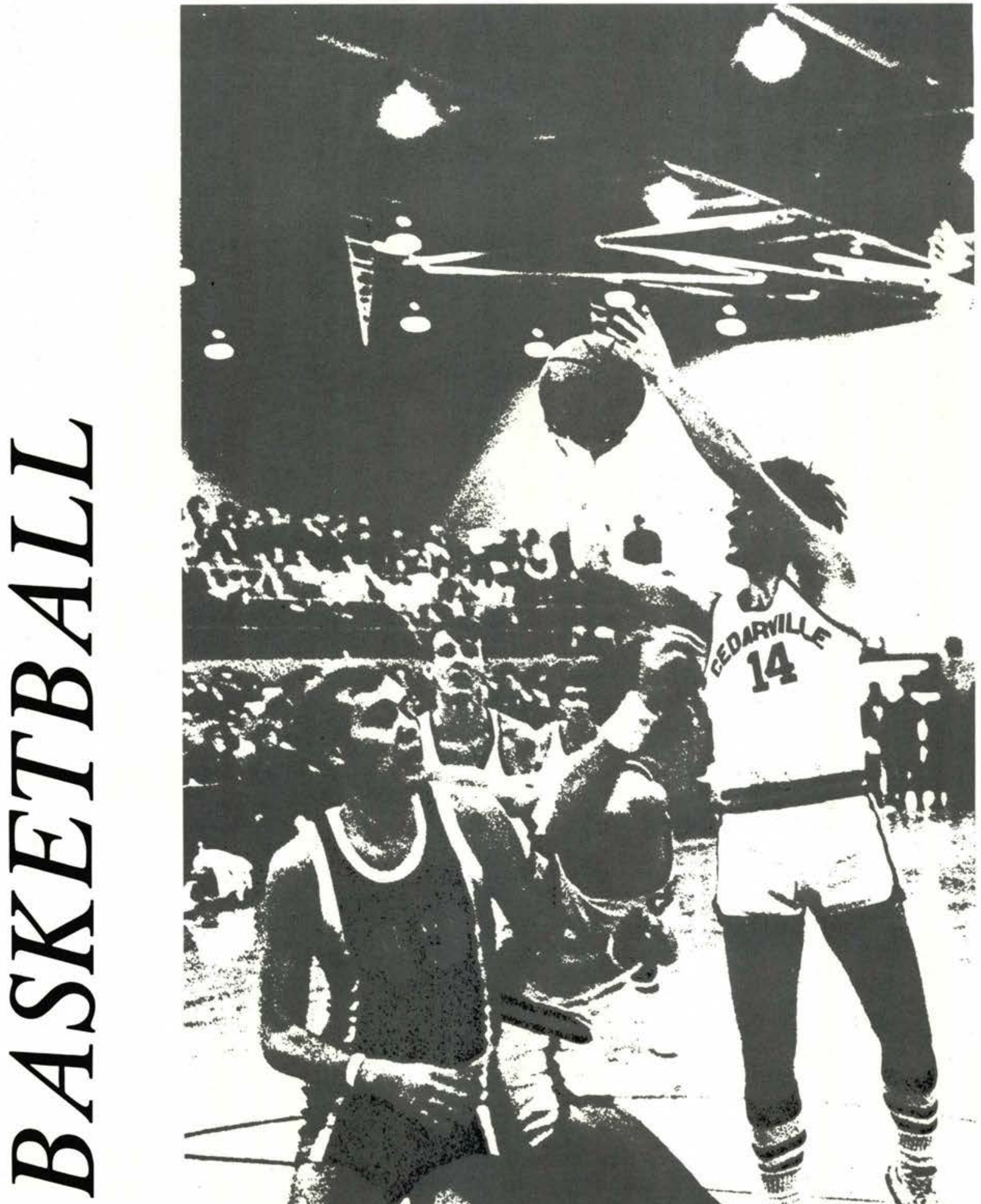

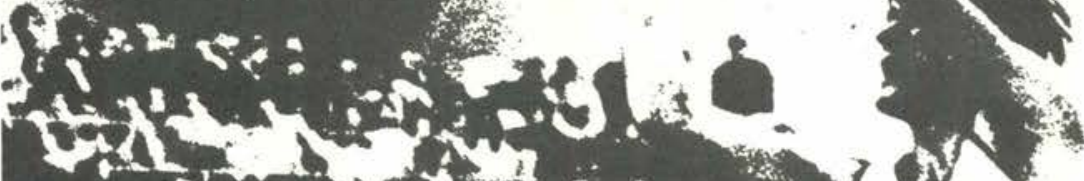

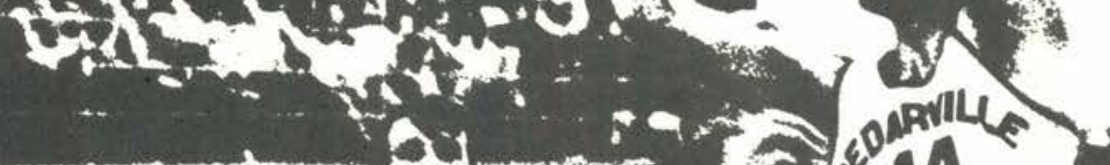
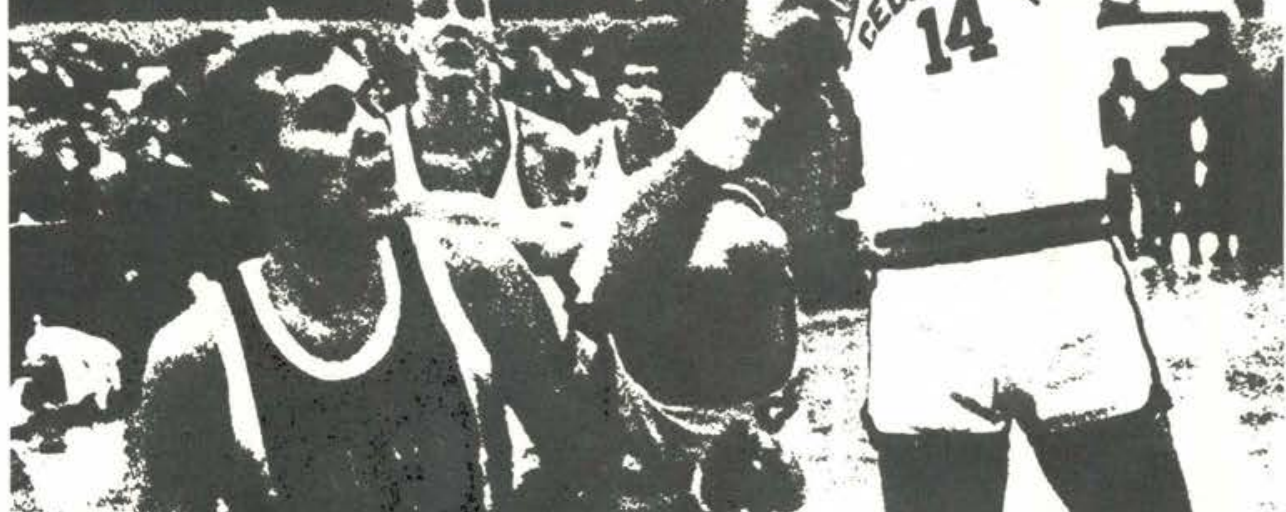

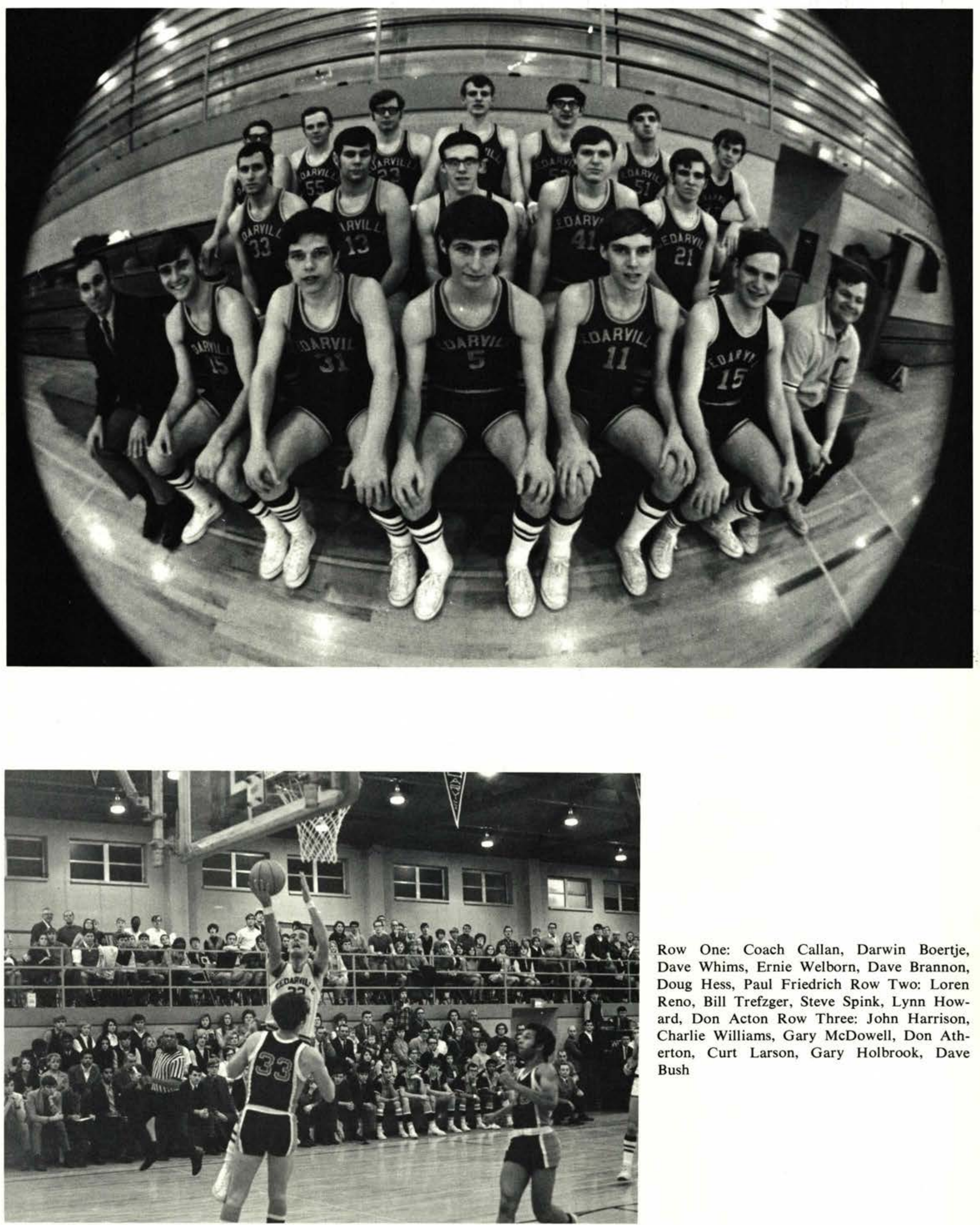

Row One: Coach Callan, Darwin Boertje, Dave Whims, Ernie Welborn, Dave Brannon, Doug Hess, Paul Friedrich Row Two: Loren Reno, Bill Trefzger, Steve Spink, Lynn Howard, Don Acton Row Three: John Harrison, Charlie Williams, Gary McDowell, Don Atherton, Curt Larson, Gary Holbrook, Dave Bush 

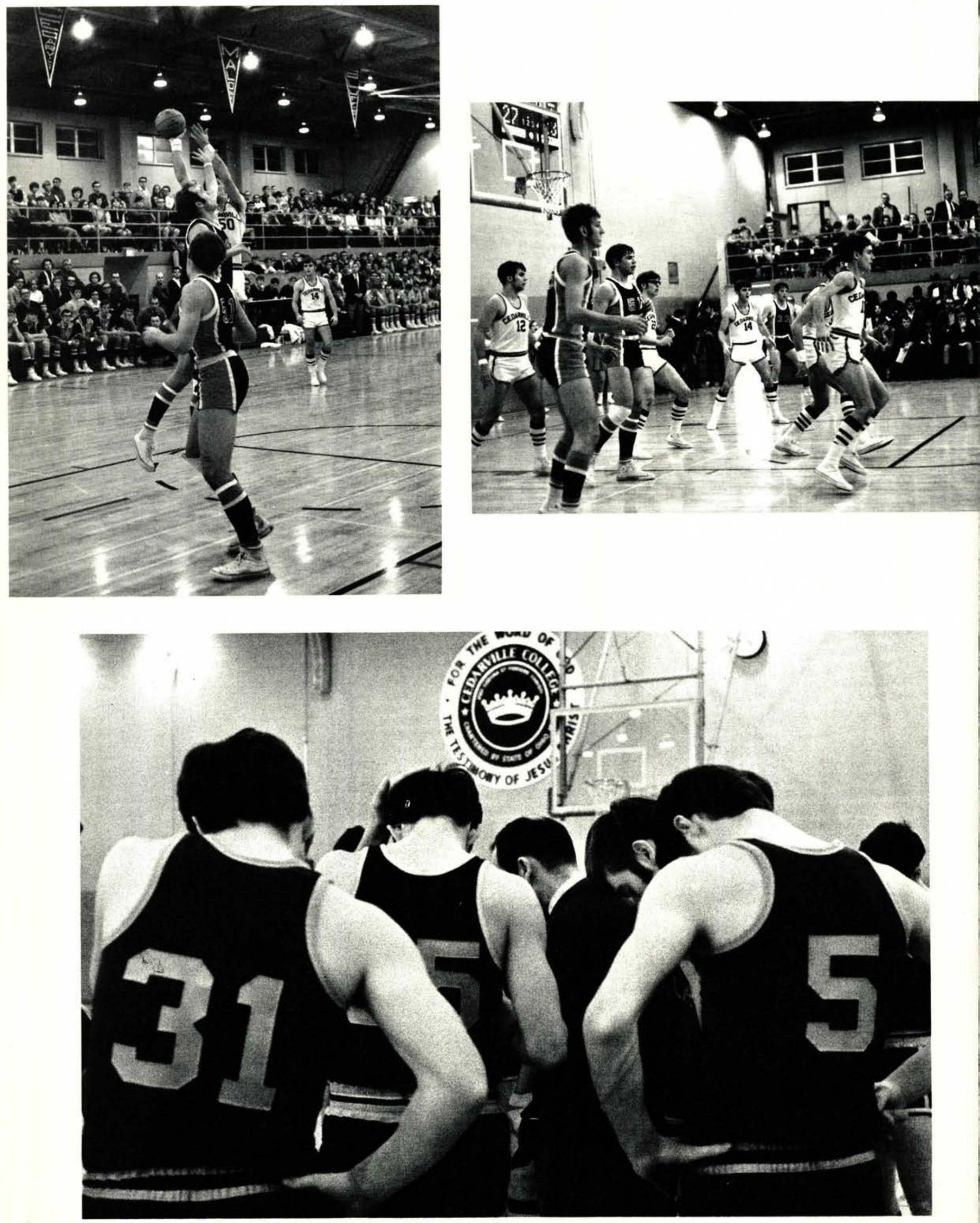


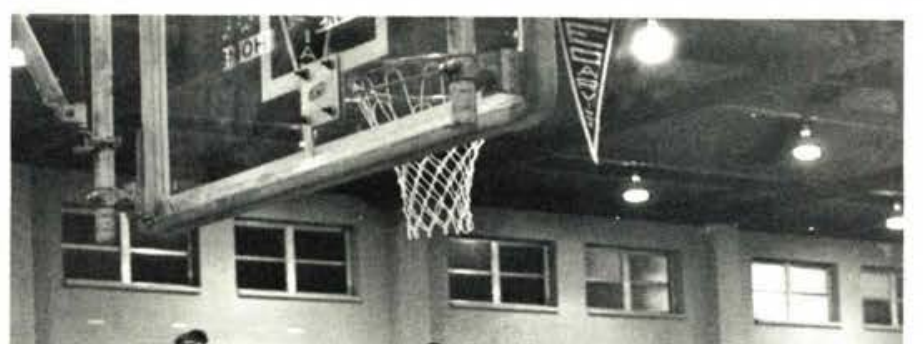

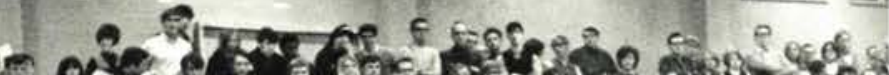

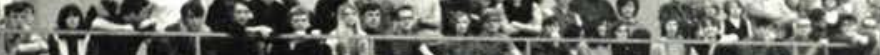

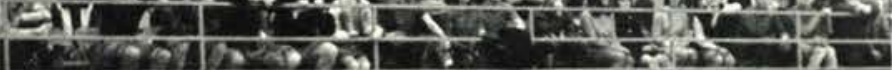

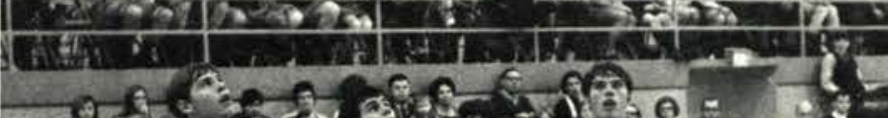
करें

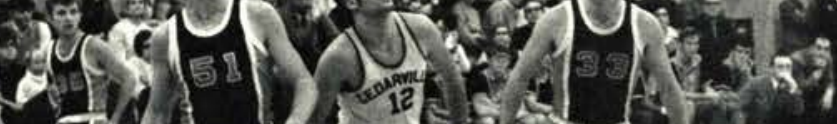

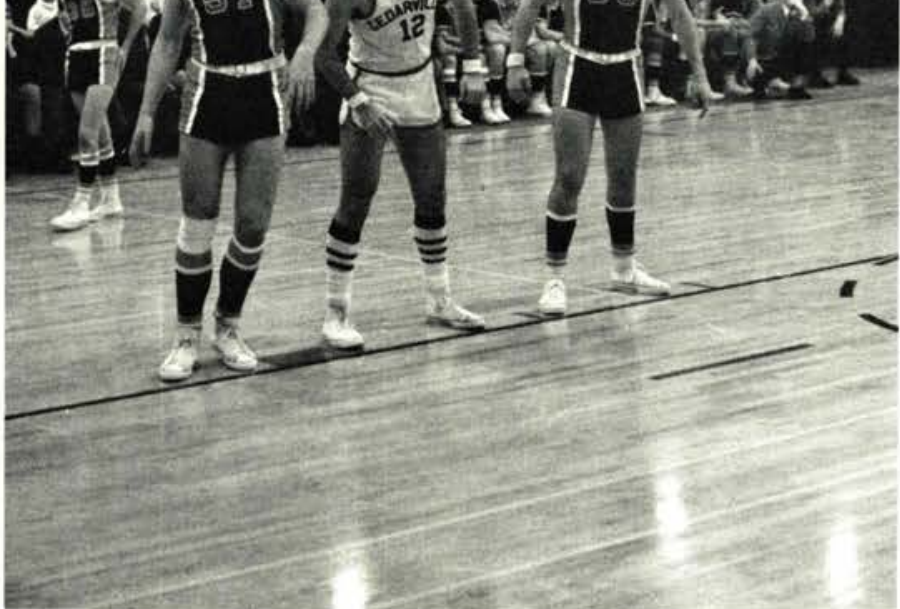

Row One: Ernie Welborn, Doug Hess Row Two: Dave Bush, Steve Spink, Dave Brannon, Lynn Howard Row Three: John Harrison, Charlie Williams, Curt Larson, Don Acton

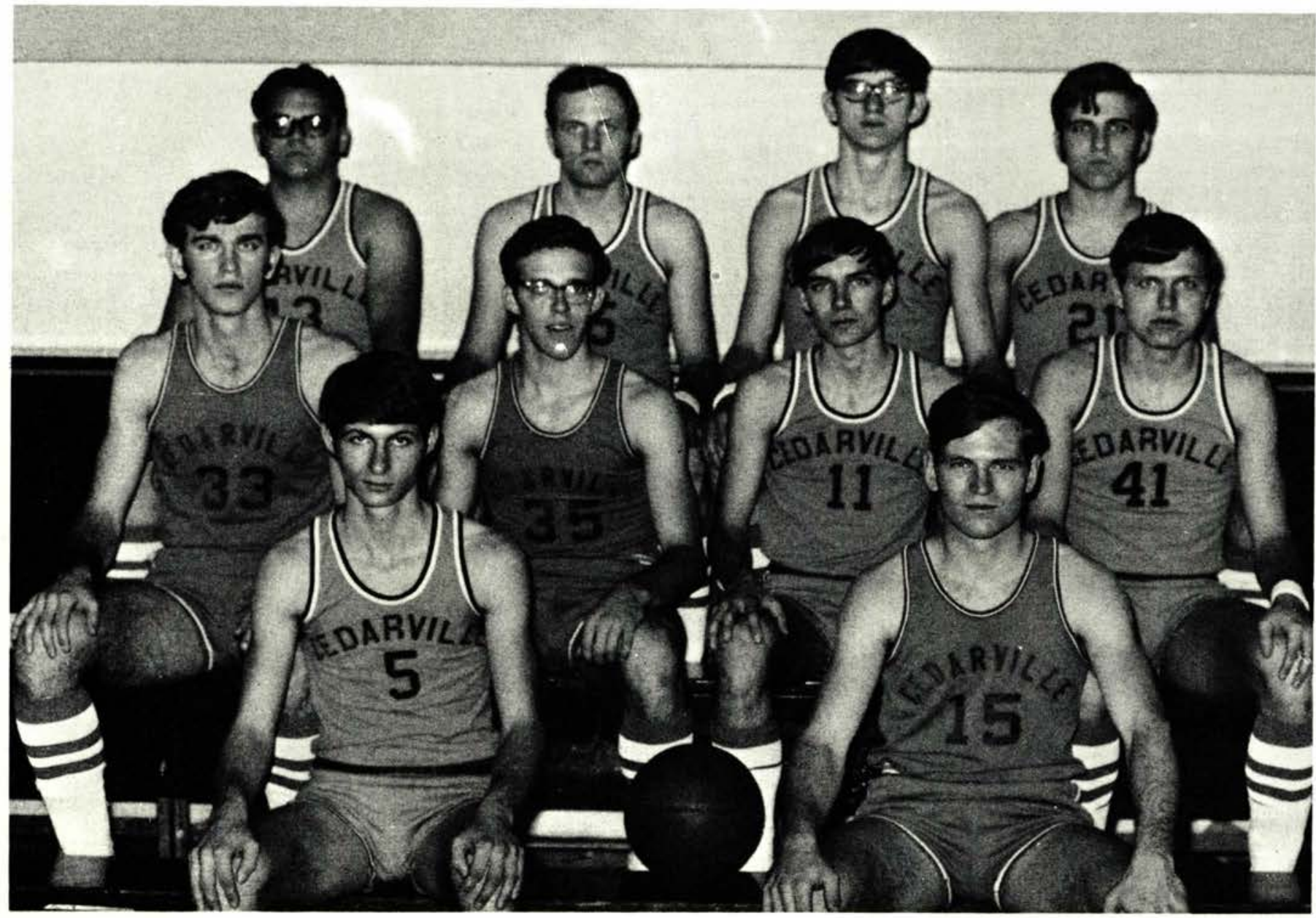




\section{TENNIS}
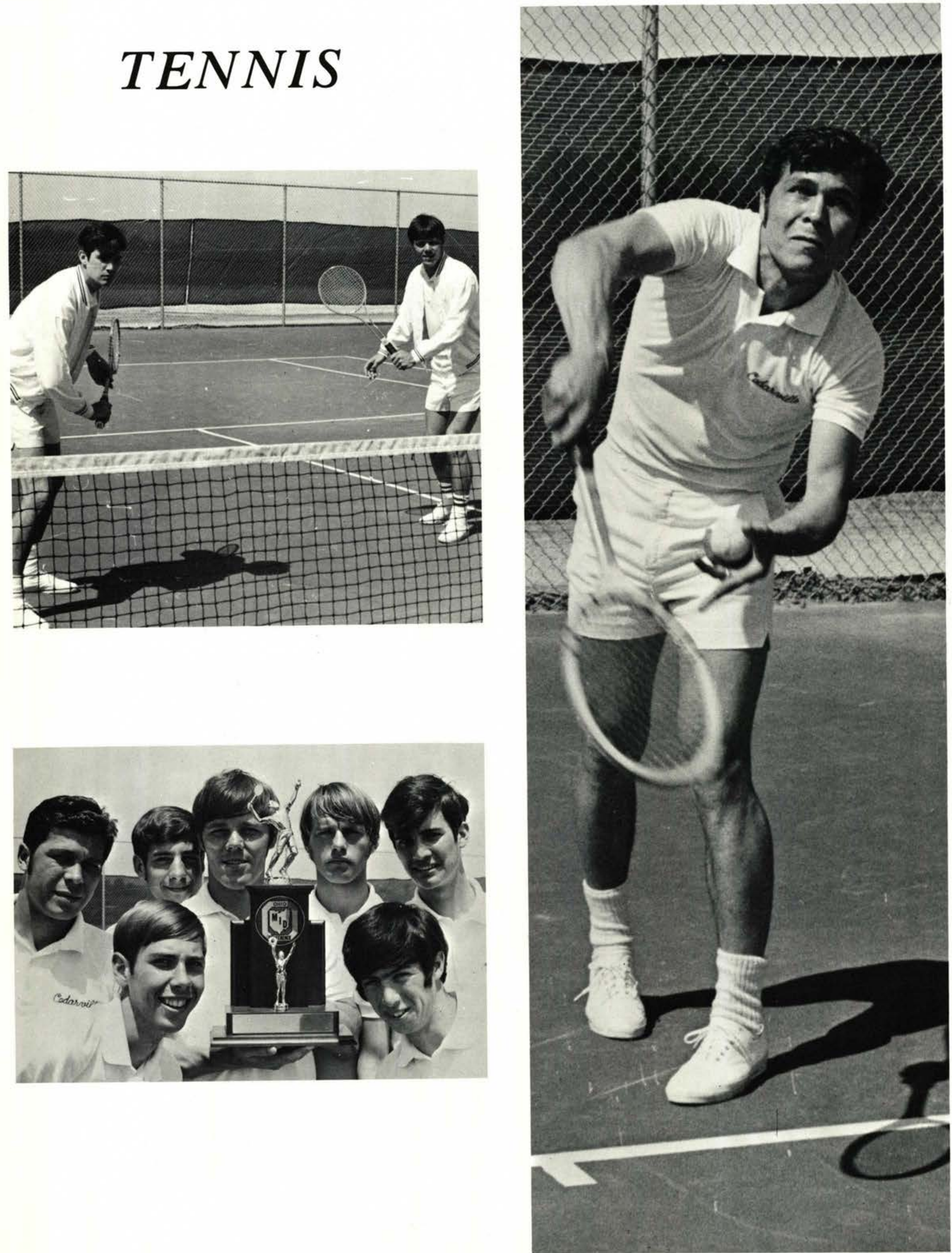


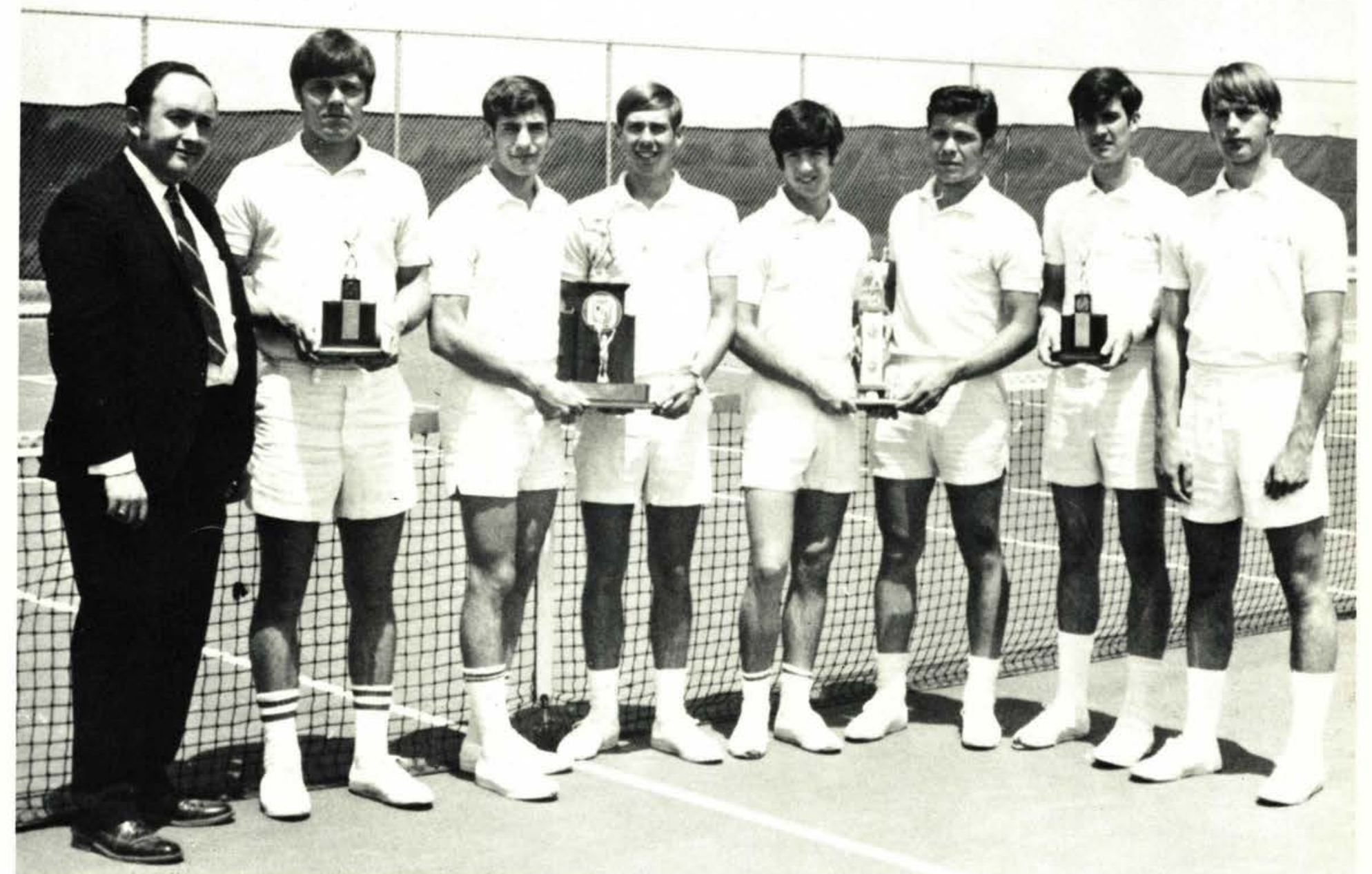

Coach Murdoch, Dan Ruffin, Dave Beck, Dave Nelson, Warren Pettit, Jerry Jones, Dave Durham, Mike Coffman.

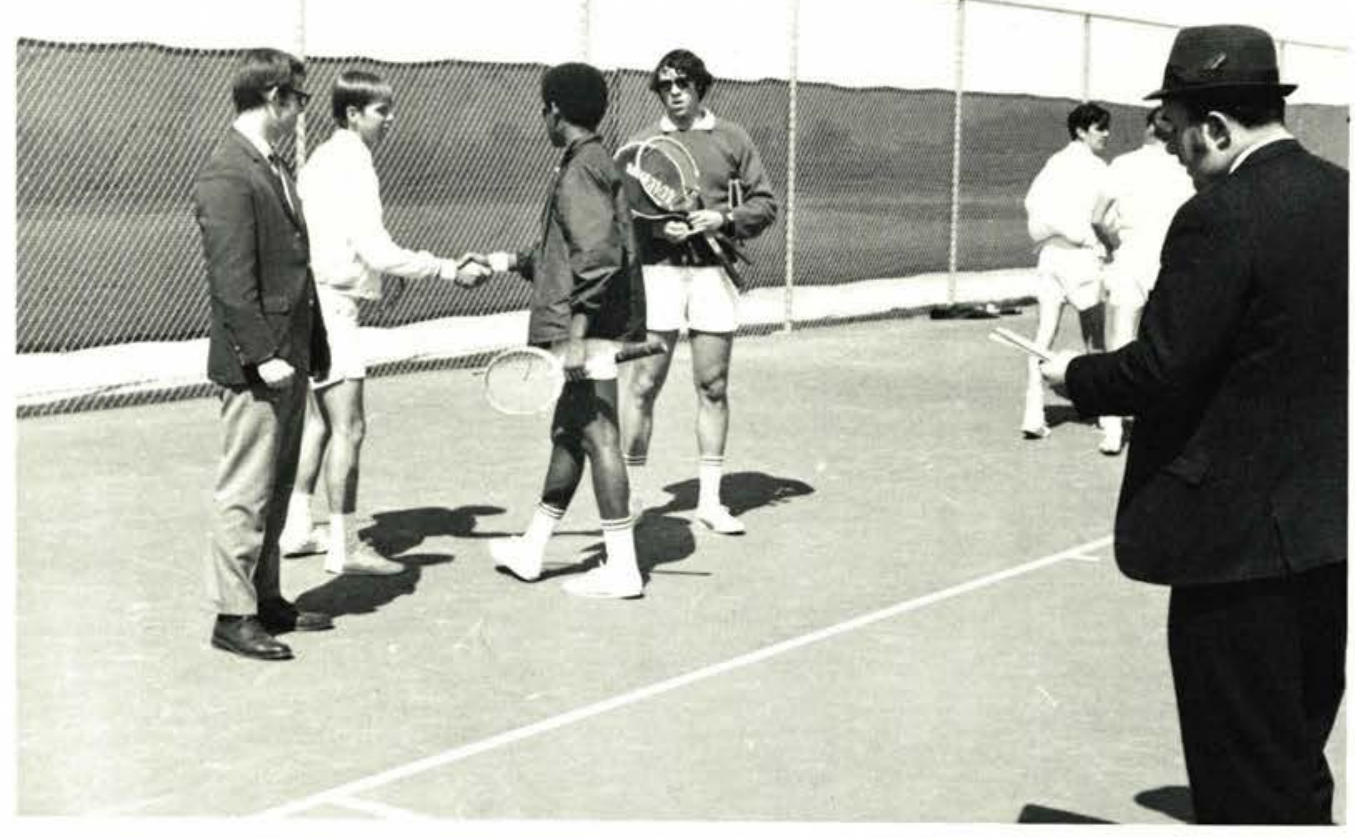




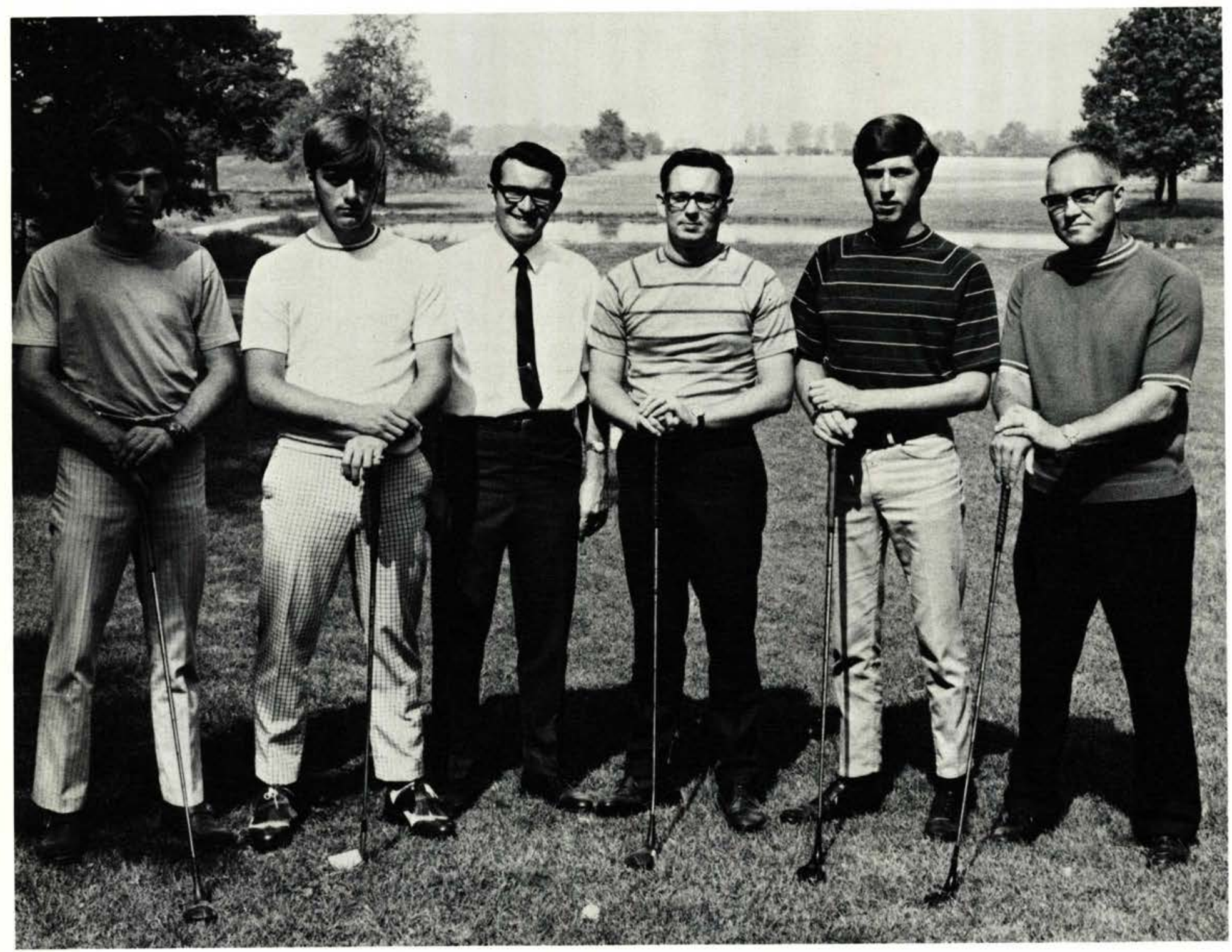

\section{GOLF}

Mark Causey, Jim Buzzard Coach Gromacki, Dan Ricker, Dan Smith, Eric Campfield. 


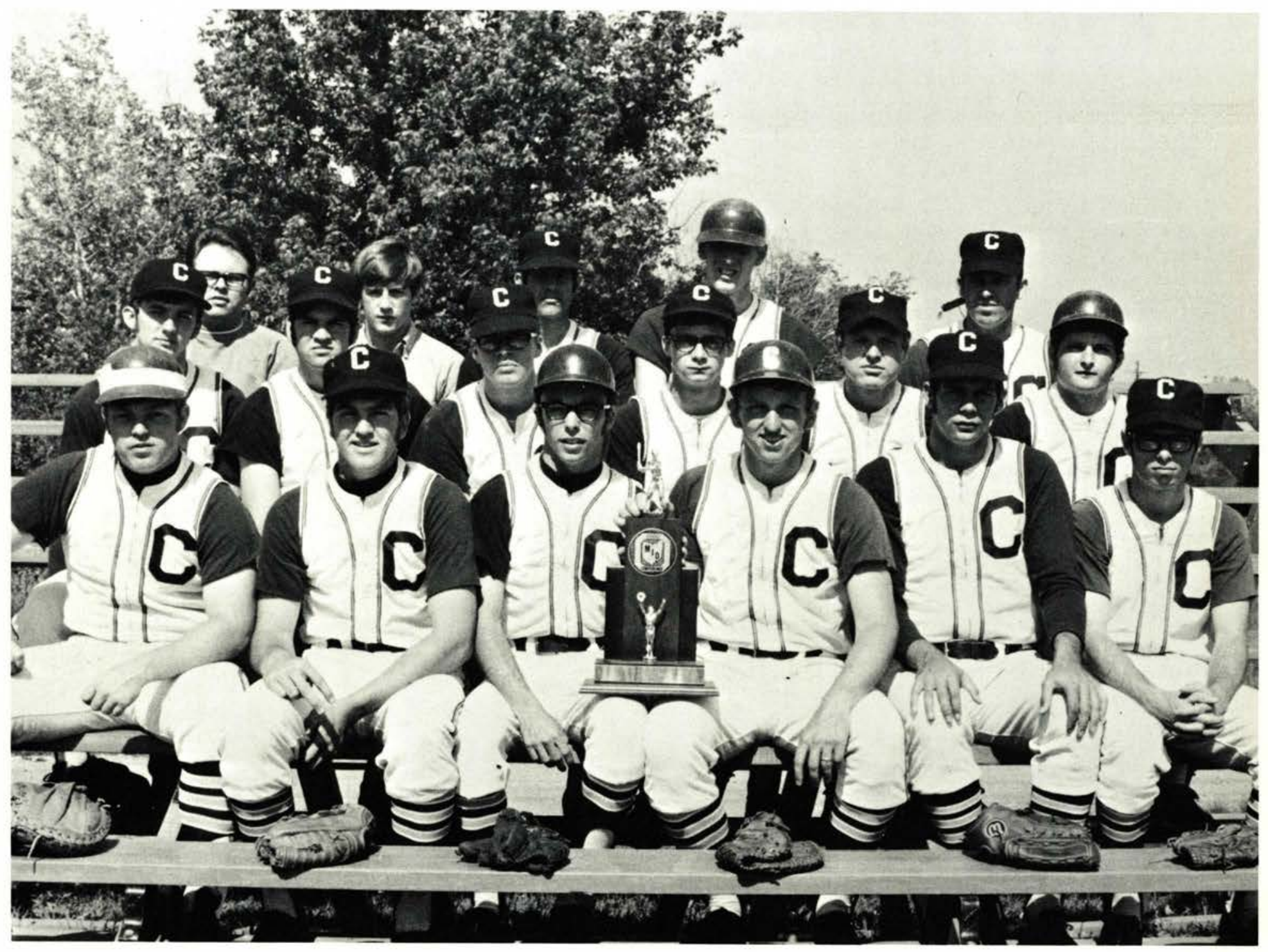

\section{$B A S E B A L L$}

Row One: Phil Johnson, Dan Inghram, Tony Wall, Bob Lunney, Bill Trefzger, Dee Mays Row Two: Dave Grant, Rick Inghram, Buddy McNiece, Lyn Howard, Jack Swank Row Three: Wayne Williams, Jack Campbell, Dave Rodreguez, Don Atherton, Coach Callan 


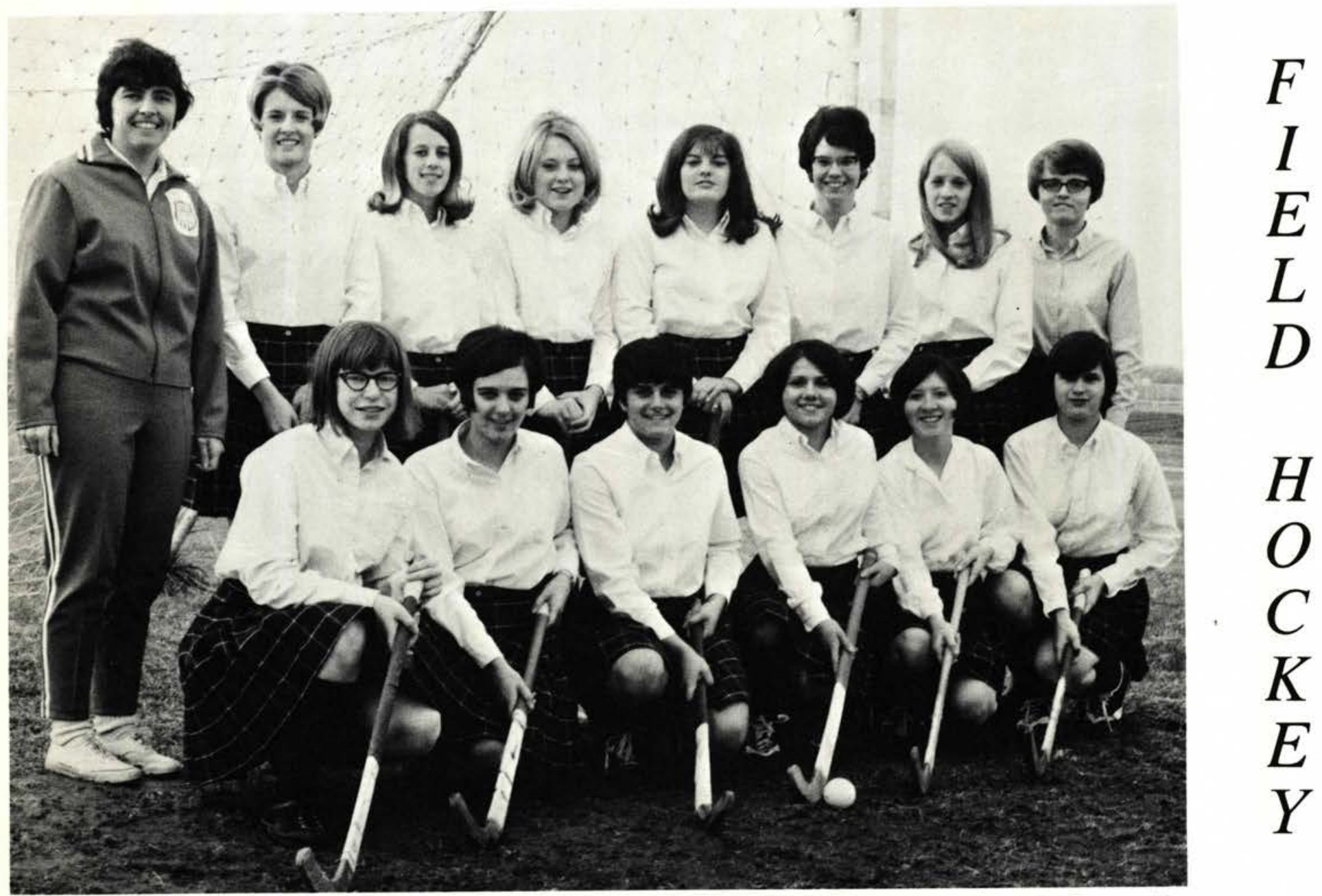

Seated: Becky Bittner, Nancy Bailey, Judy Stamatis, Billie Thomas, Landra Lowery, Janice Martin Standing: Miss Kearney, Pat Wissinger, Joy Trefsger, Roxann Davis, Brenda Tompkins, Dianne Kemp, Connie Walker, Connie Pumpelly

Becky Bittner, Karen DeMars, Wilma Hopkins, Pat Wissinger, Judy Stamatis, Patti Irwin, Joy Trefsger

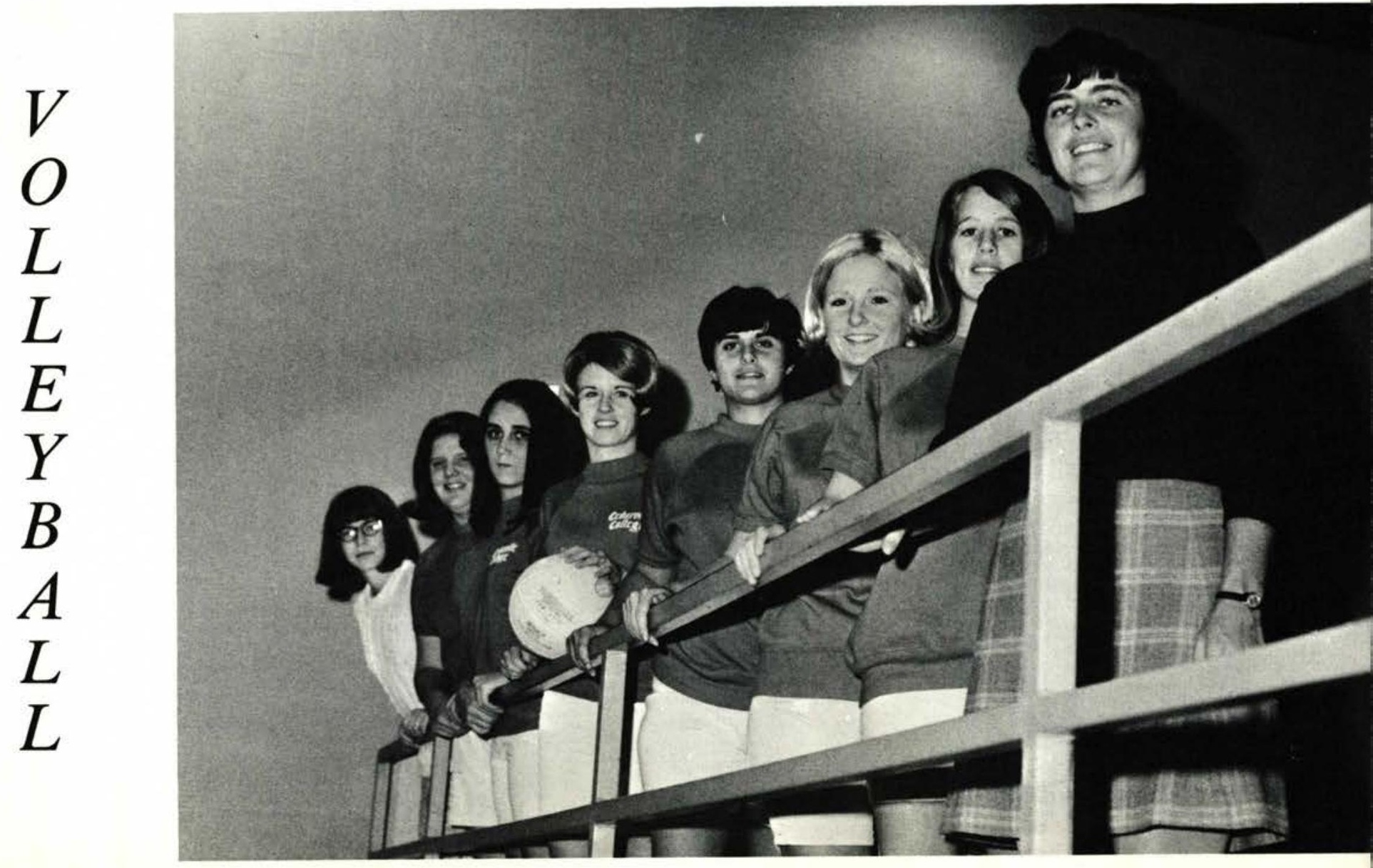


Women's Basketball Statistics

$\begin{array}{llll}\text { Cedarville } & 28 & \text { Manchester } & 38 \\ \text { Cedarville } & 38 & \text { Cincinnati } & 13 \\ \text { Cedarville } & 38 & \text { Dayton } & 40 \\ \text { Cedarville } & 38 & \text { Wilmington } & 15 \\ \text { Cedarville } & 72 & \text { Western } & 13 \\ \text { Cedarville } & 55 & \text { St. Elizabeth's } & 35 \\ \text { Cedarville } & 43 & \text { Ashland } & 31 \\ \text { Cedarville } & 53 & \text { Malone } & 32 \\ \text { Cedarville } & 24 & \text { Mt. St. Joseph } & 39 \\ \text { Cedarville } & 30 & \text { Mt. St. Joseph } & 37 \\ \text { Cedarville } & 61 & \text { Mt. St. Joseph } & 26\end{array}$

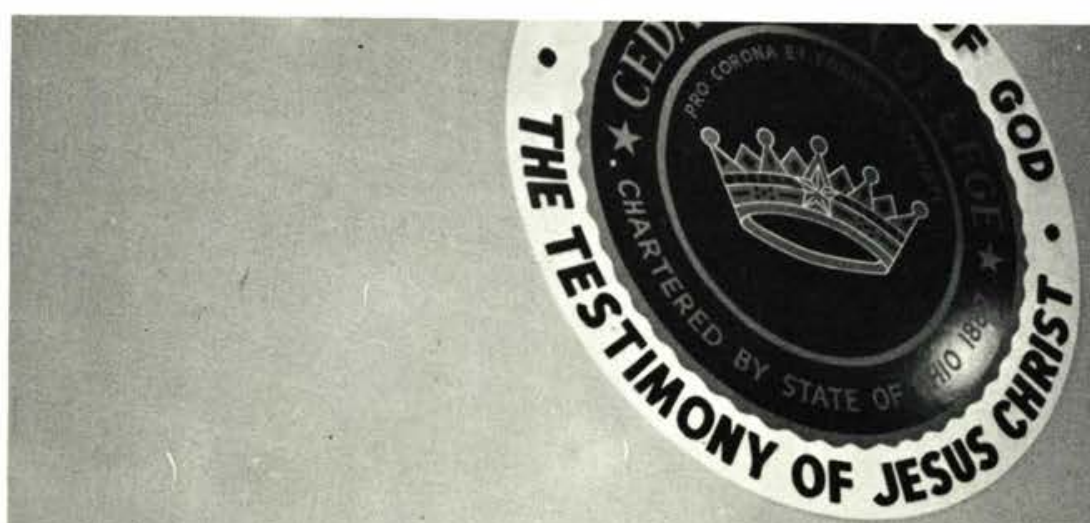

Cedarville $61 \quad$ Mt. St. Joseph

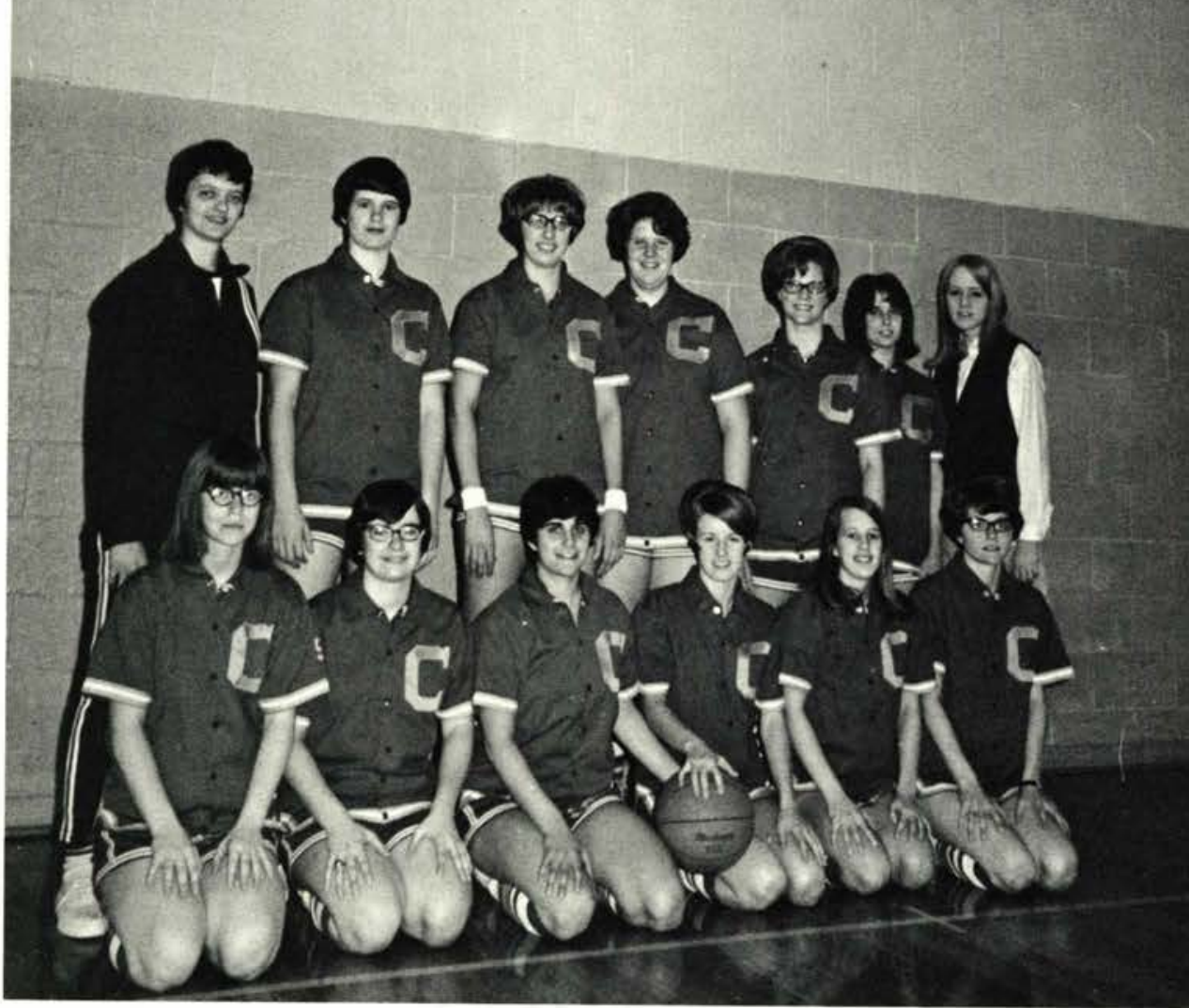

\section{$B A S K E T B A L L$}

Kneeling: Becky Bittner, Pam Branning, Judy Stamatis, Pat Wissinger, Joy Trefzger, Connie Pumpelly Standing: Miss Jeremiah, Janice Martin, Rita Gast, Karen DeMars, Joan Dautel, Darlene Mann, Connie Walker 


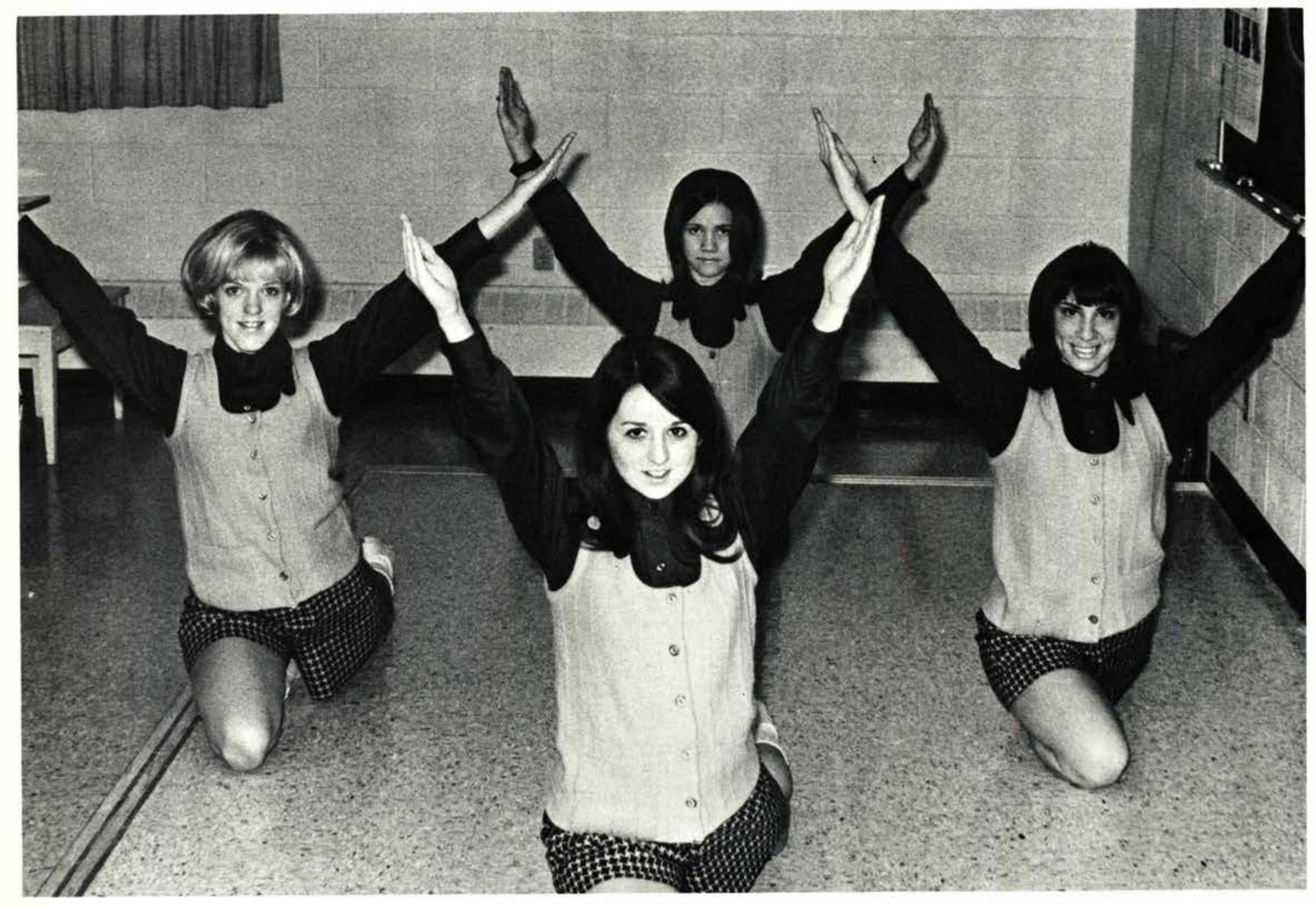

\section{VARSITY CHEERLEADERS}

. . . excitement, noise, crowds, practice victories, disappointments, late trips, Burger Chef, fun, laughter, singing, meeting new friends, finding close friends . . . as my college years come to an end, I realize how important cheerleading has been to me. It has truly been a privilege to represent Cedarville in this way; I will never forget the memories I have from this experience. . .

—Judy McCann

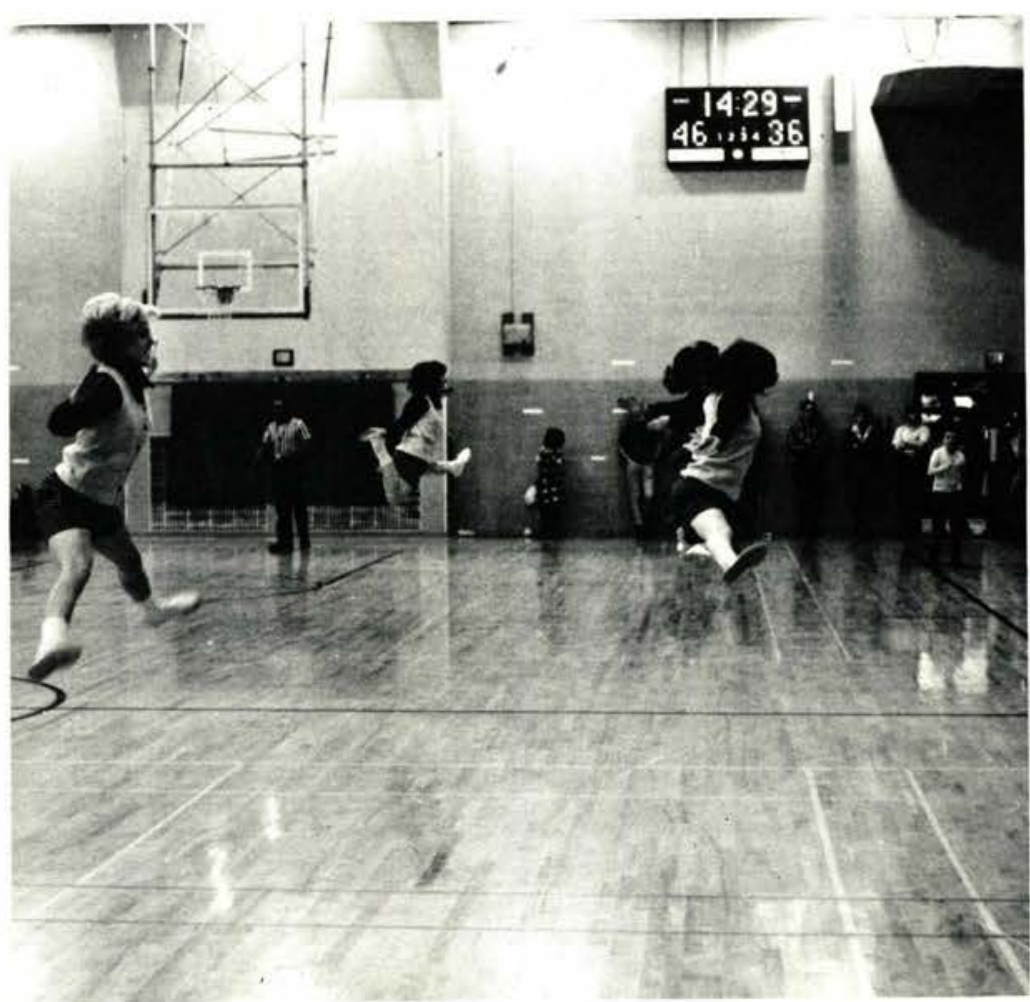




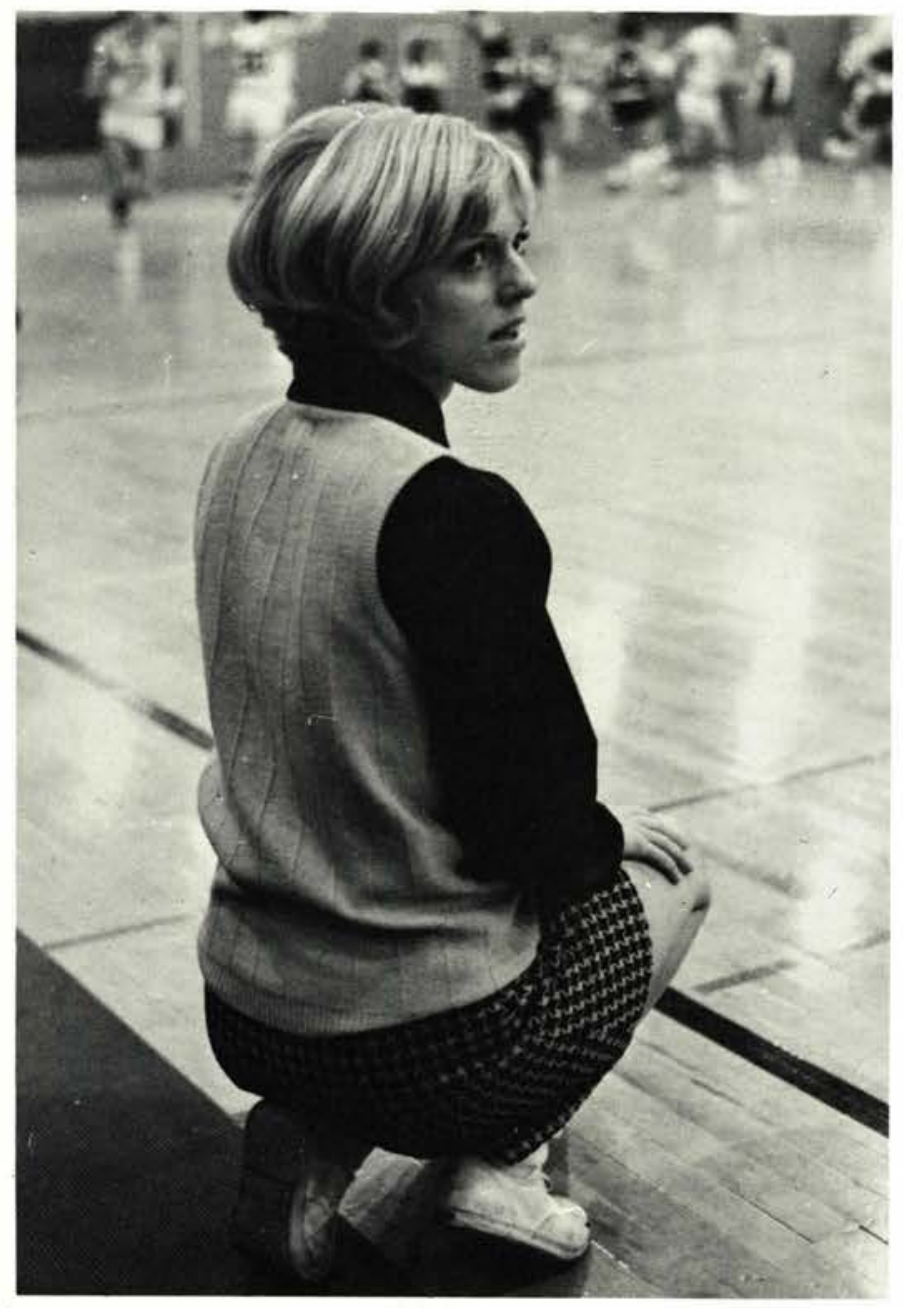

Cheerleading is a fascination of starting a spark of enthusiasm and watching it spread through a grandstand of anxious fans. It is an opportunity to reveal genuine school spirit and to cultivate thorough excitement for Cedarville's great team. Cheerleading means getting involved with the team and knowing that when our boys have met victory, we, along with the spirited fans, are perhaps a small part of that victory.

-Patti Jenkin

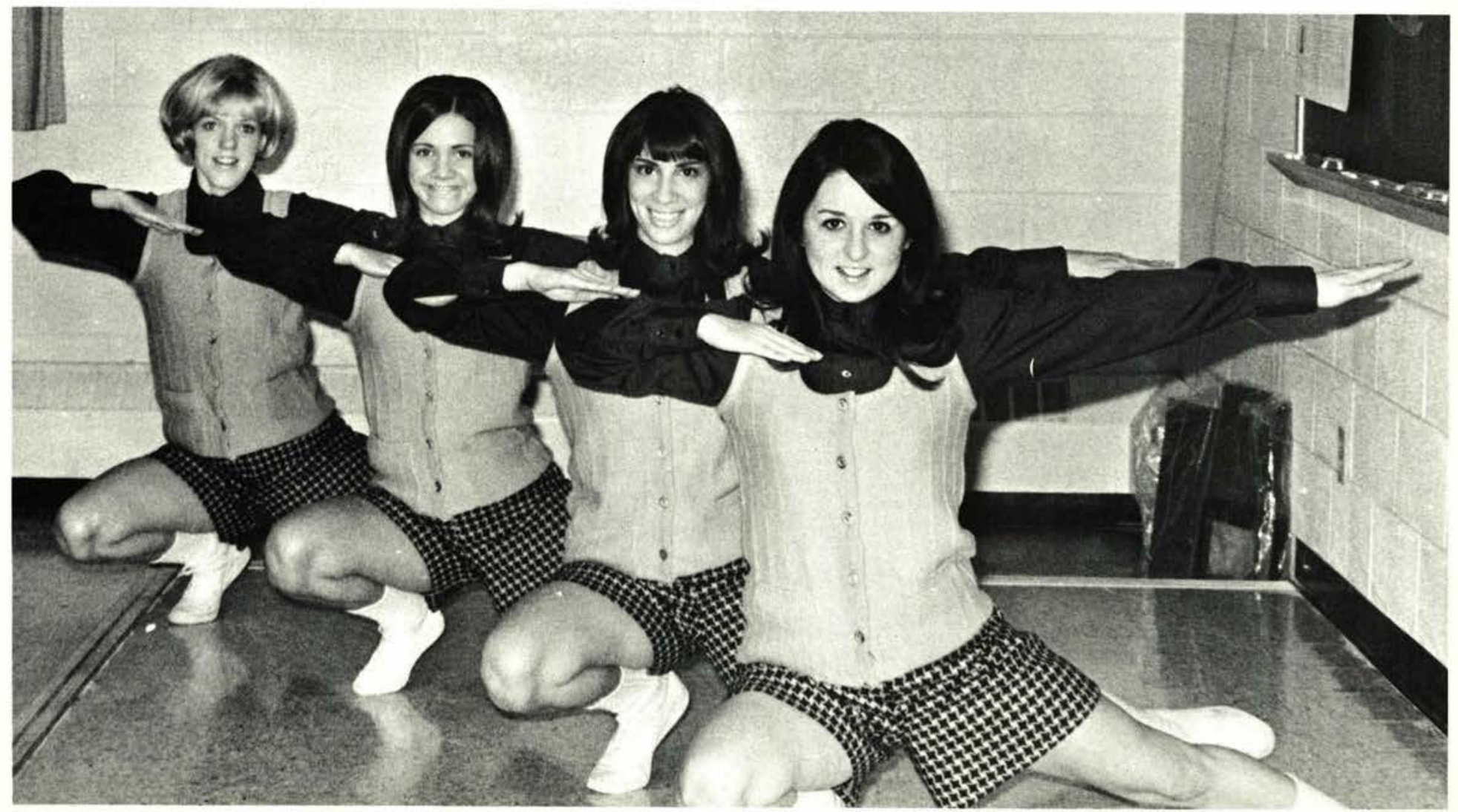



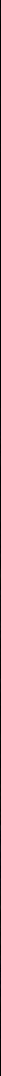

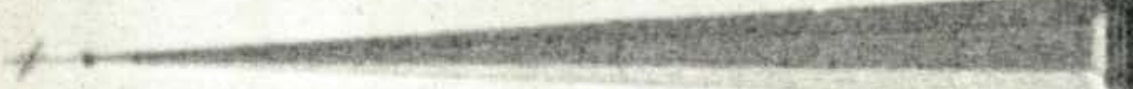
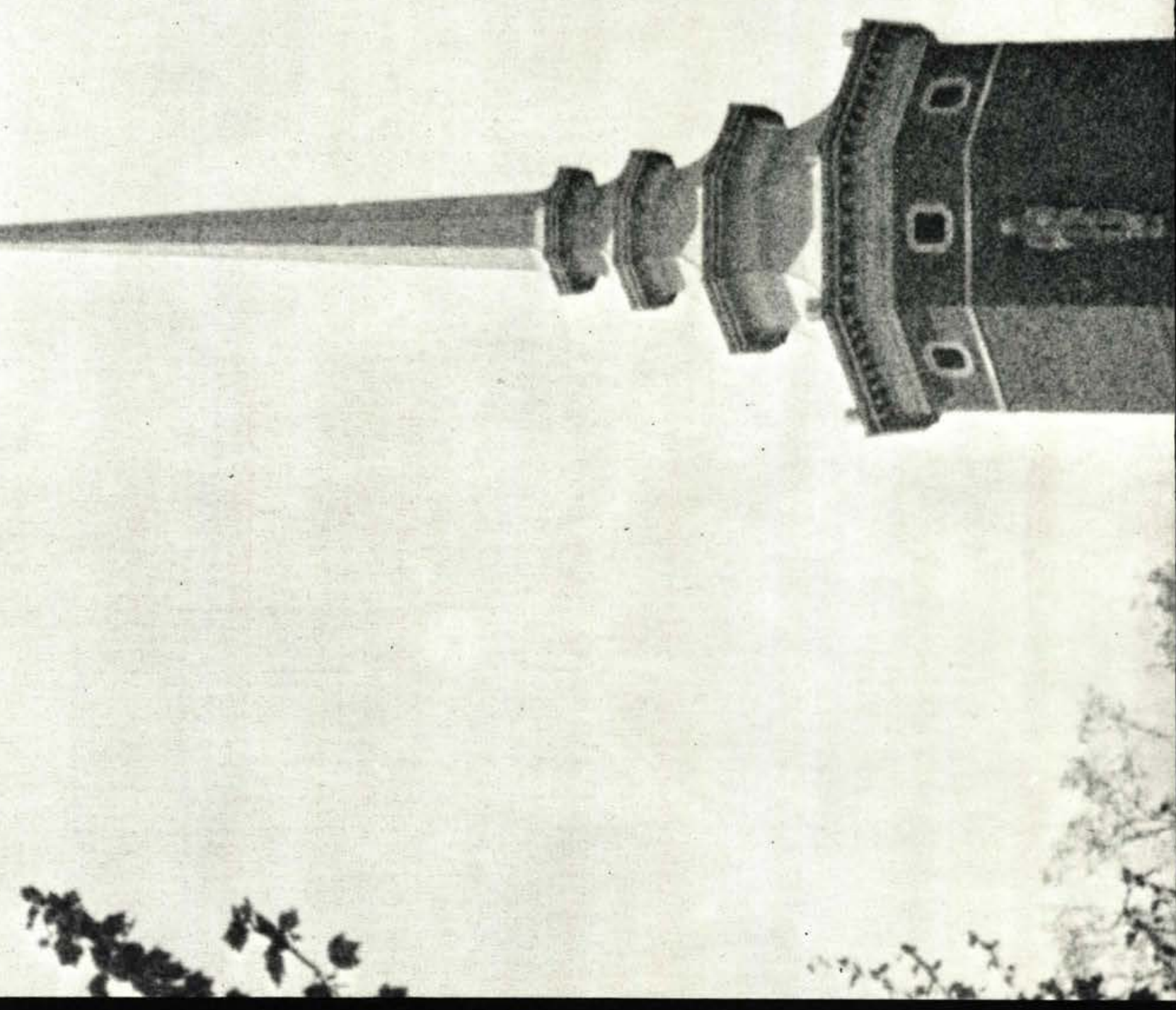

$\rightarrow x x^{2}$

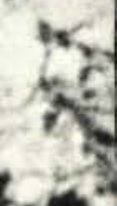




\section{BIBLE CONFERENCE}

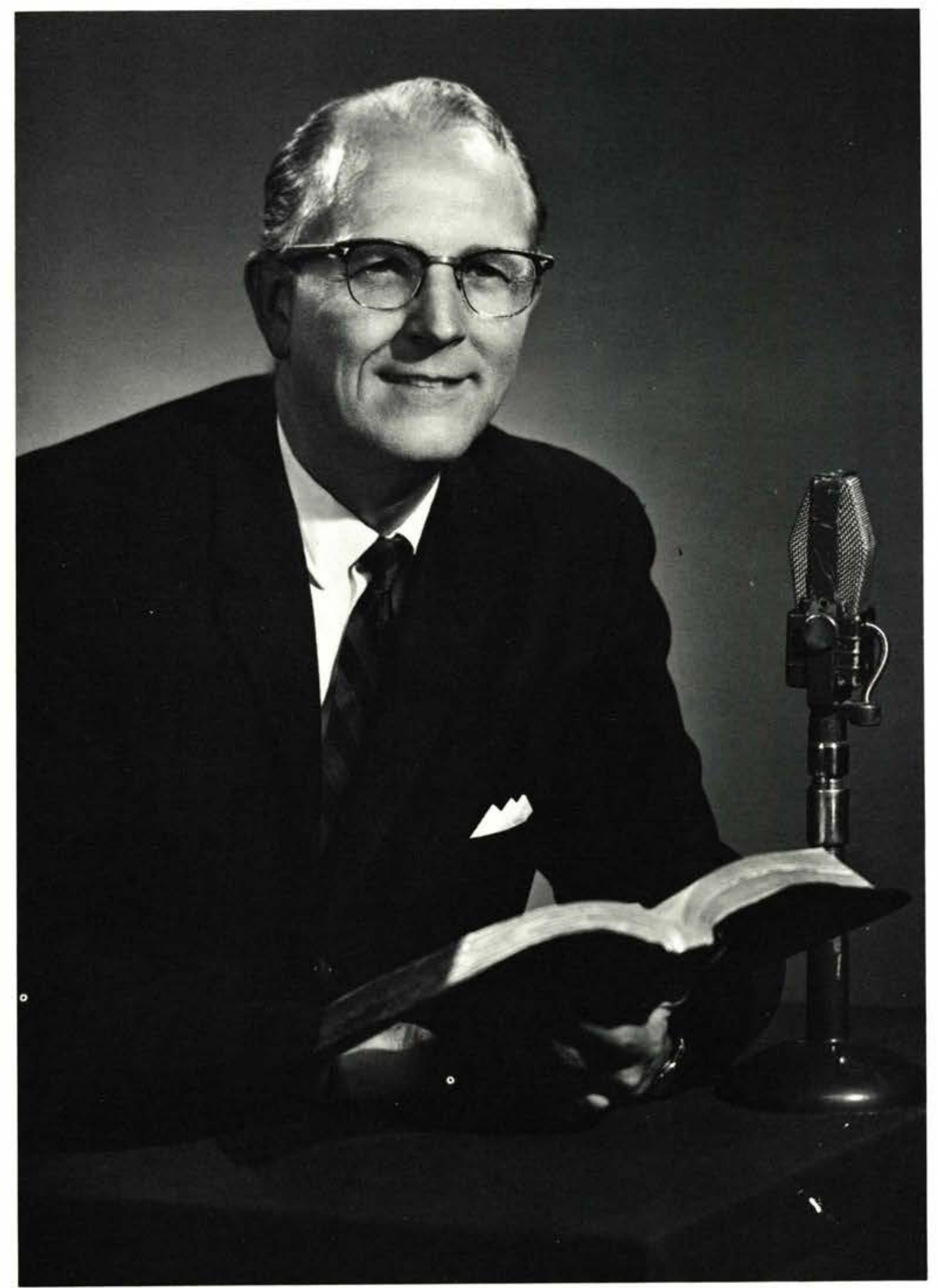

Rev. Paul Levine 


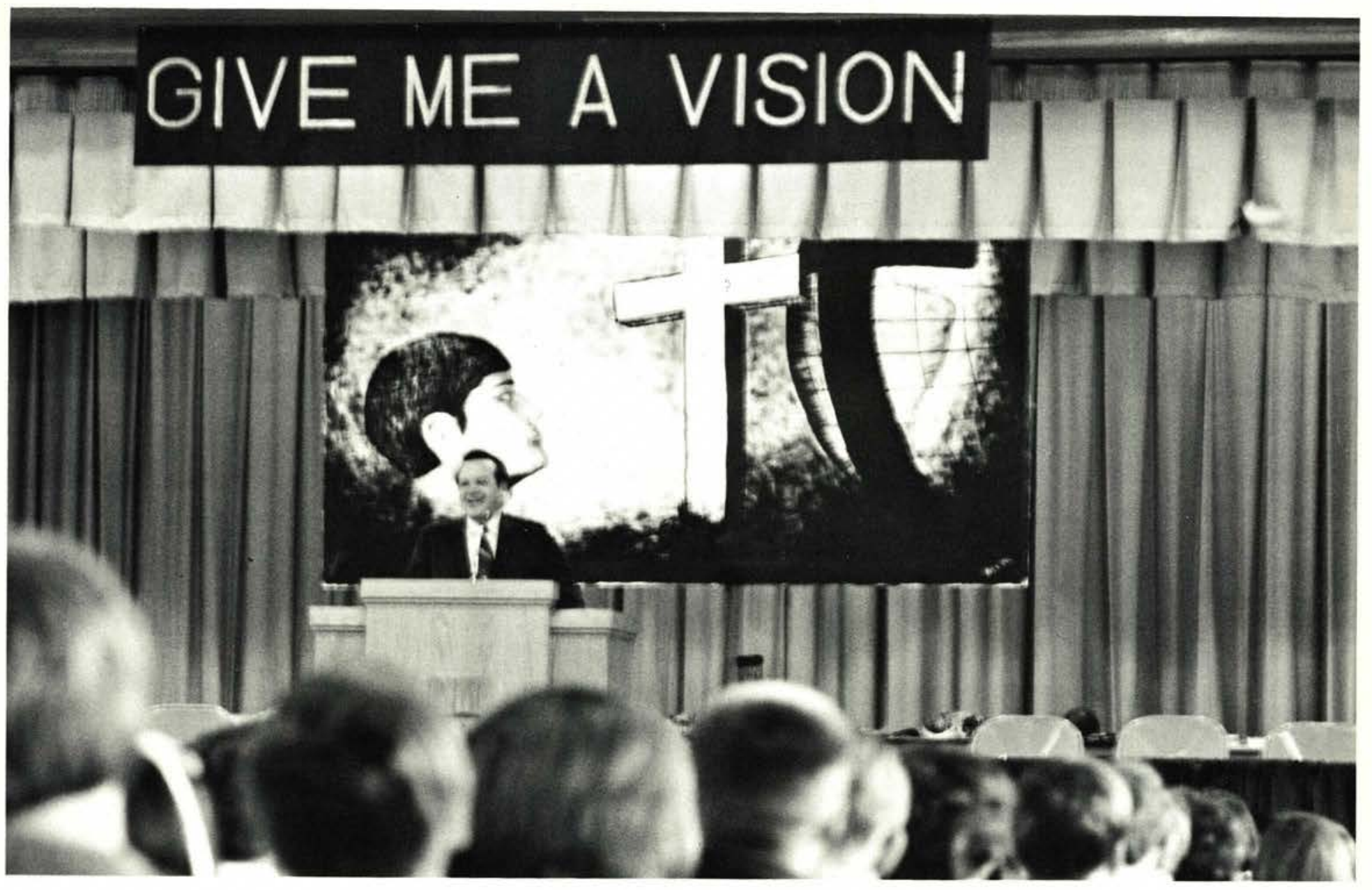

\section{MISSIONARY CONFERENCE}

\section{GIVE ME A VISION}

\subsection{9 is}

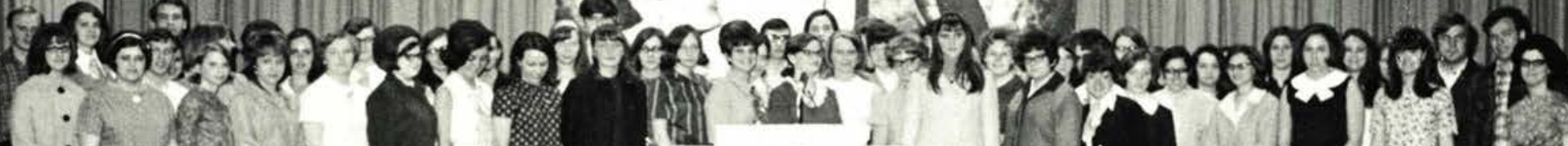

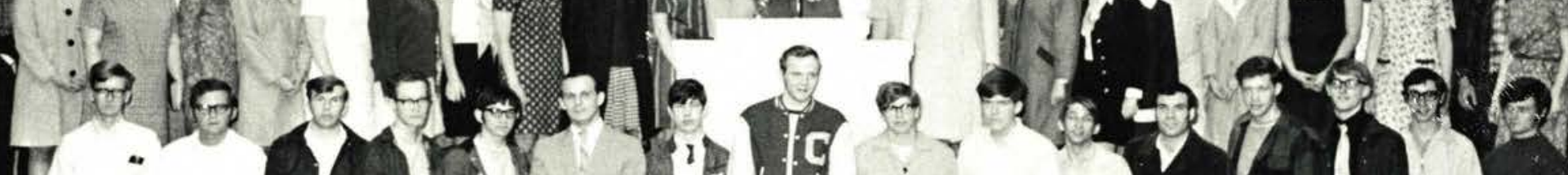
${ }_{7}^{1}$ 


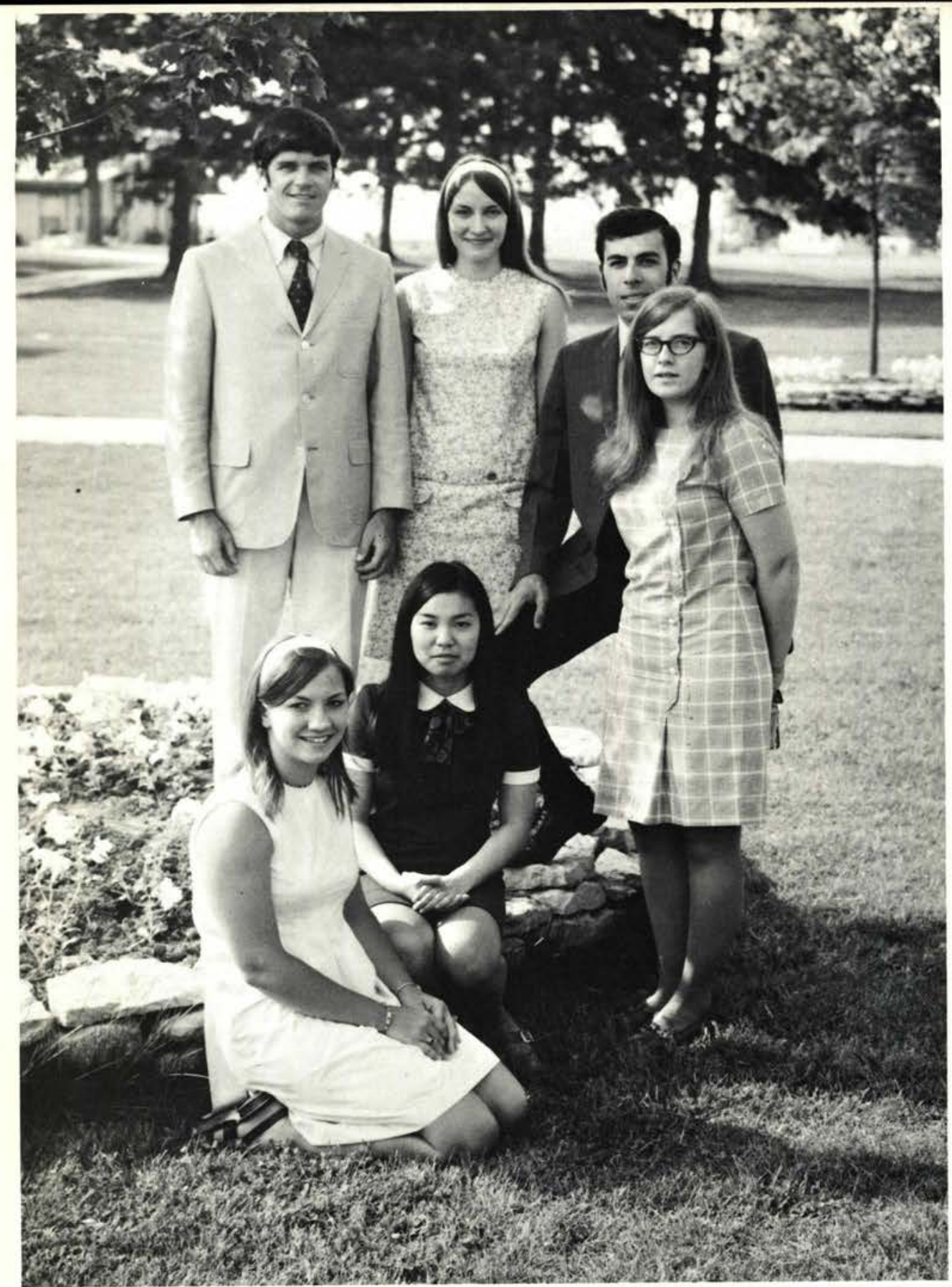

The purpose of MOC is to encourage the memorization of Scripture. Problems in memorizing and the blessings of committing Scripture to memory are discussed. Verses are chosen during the meetings.

Bonnie Smith, Ellen Young, Diane Cockerill, Dr. Ager, Larry Alfrey, Mareanne Beitler, Tim Northey, Nancy Paugh, Arlene Knight, Barb Cooper.

\section{CAMPUS LIFE}

Seated: Vicki Tobias, Ann Kusumoto Standing: Randy Ross, Lin Mason, John Pereira, Mary Bartlett

\section{$M O C$}

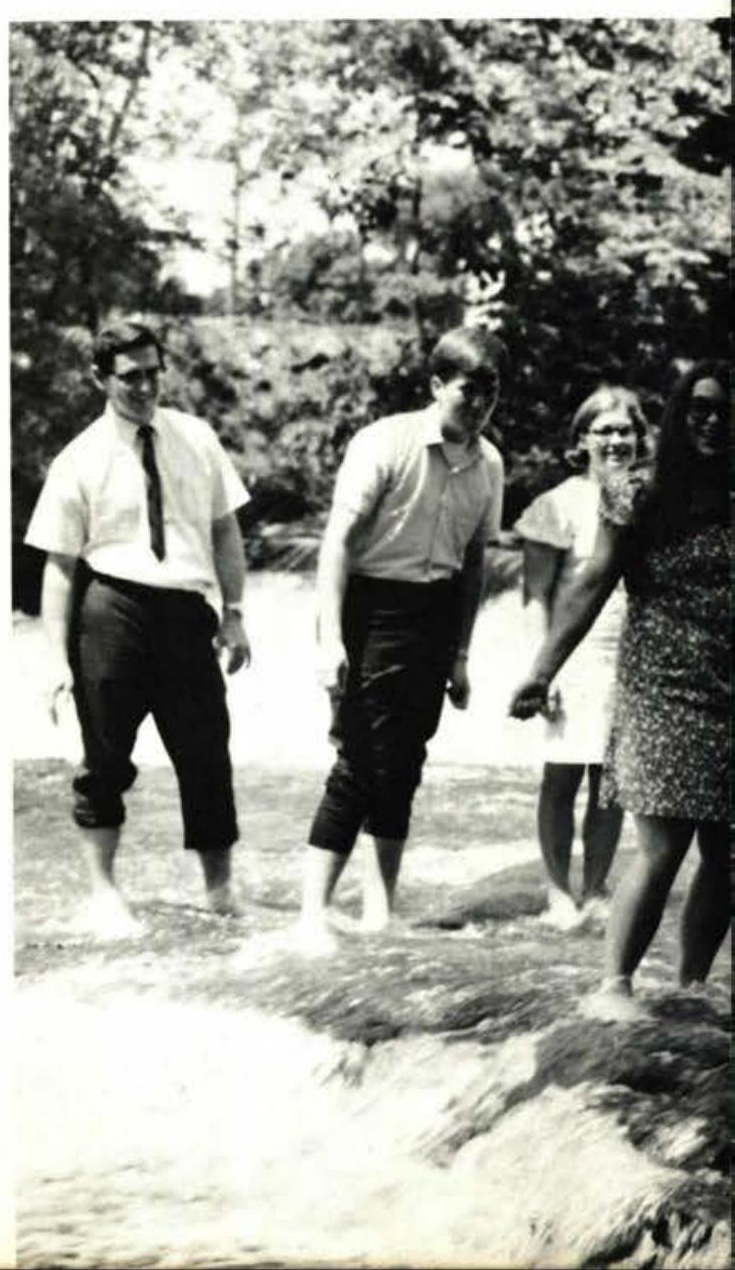




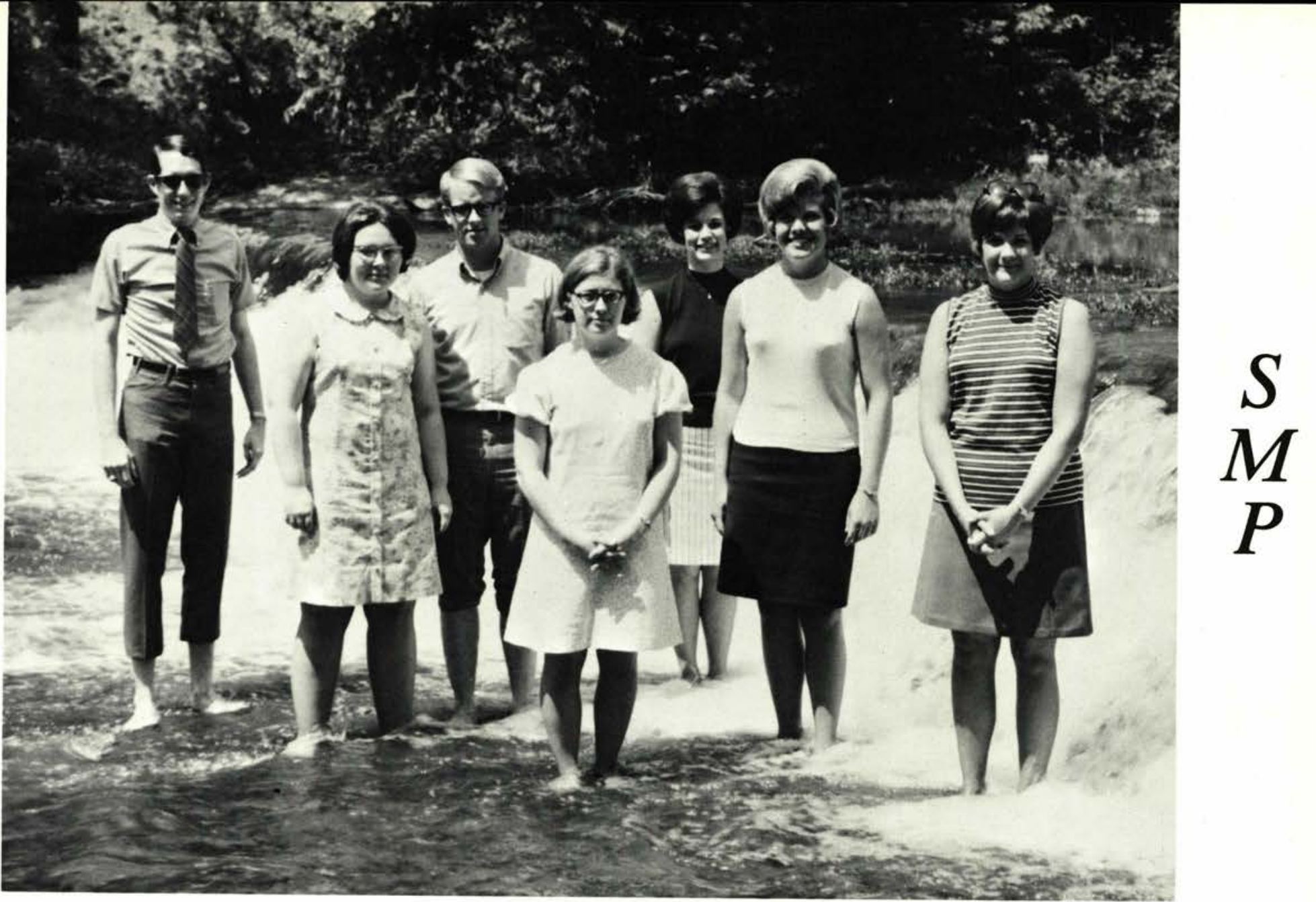

Bev Mix, Mareanne Beitler, Carol Bierbaum, Jan Hillery, Kirk Heldreth, Dave Turner, Ginny Heal
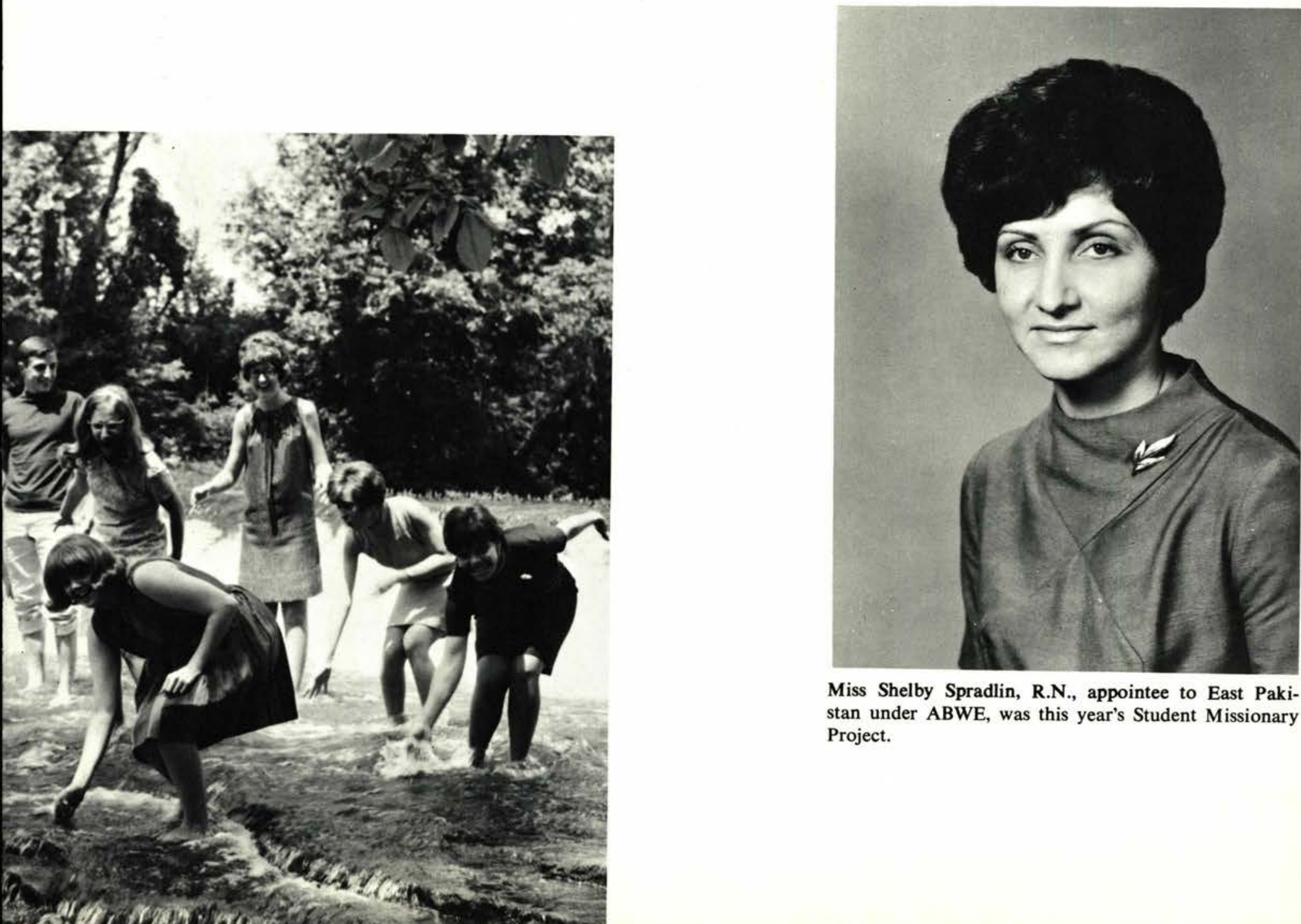

Miss Shelby Spradlin, R.N., appointee to East Pakistan under ABWE, was this year's Student Missionary Project. 


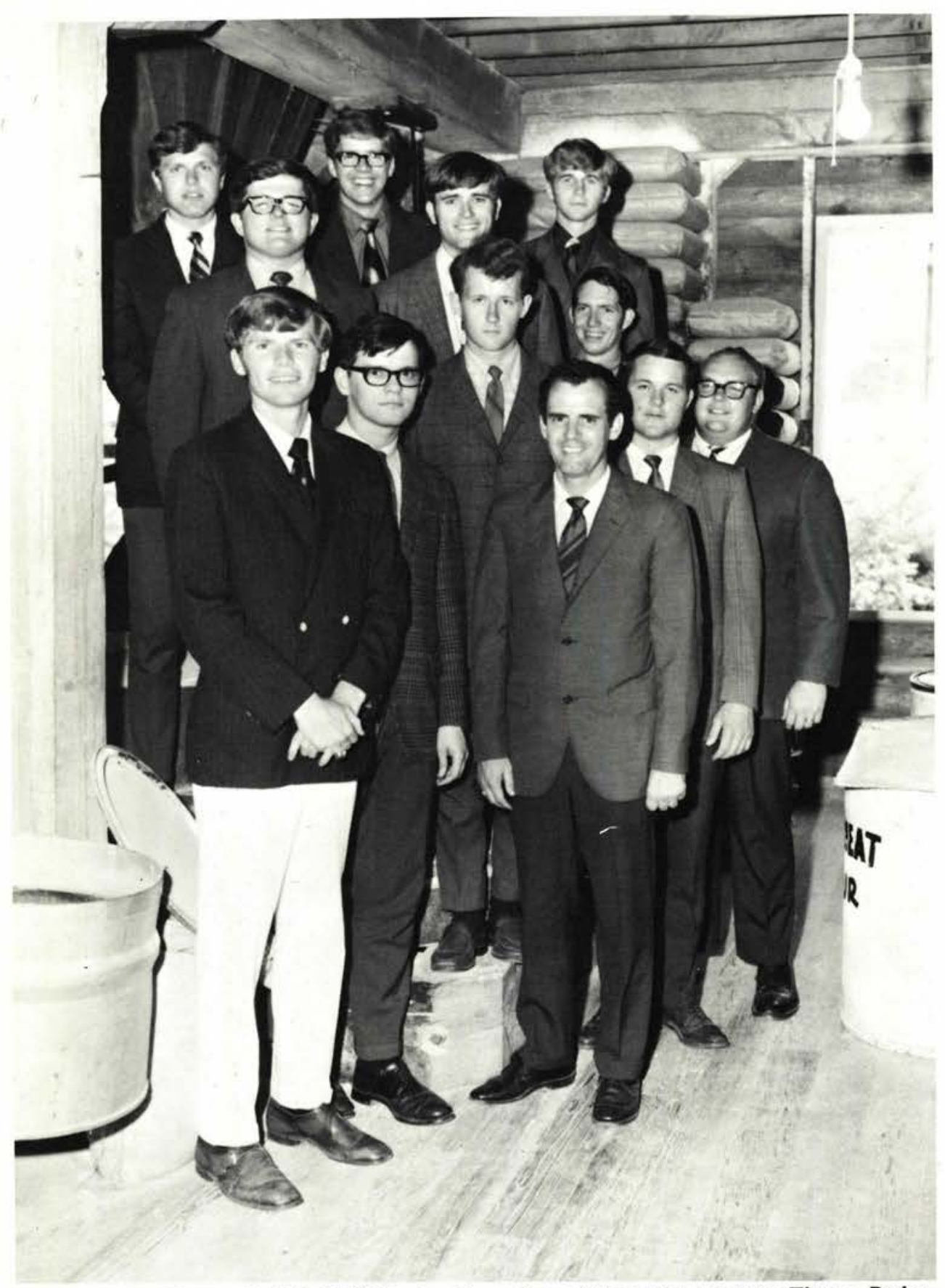

First Row: Ken Evans, Paul Radcliff, Pastor Green Second Row: Bill Amstutz, Thayne Bodenmiller, Ron Edwards Third Row: Darwin Boertje, Kirk Heldreth, Roy Kern Fourth Row: Lynn Howard, William McNiece, Bob Senseney
The purpose of the Cedarville Baptist Fellowship, headed by the Advisory Twelve, is to provide a more natural home-church-like atmosphere for the students during their stay at Cedarville College. The responsibility for the operation of the local fellowship rests upon the students; thus, a first hand experience in the conducting of a church program preparing them for service after graduation. The Advisory Twelve help to prepare and organize a complete church program each week, dividing the responsibilities between the Trustee Board, Sunday School Committee, Music Committee, and Missions Committee.

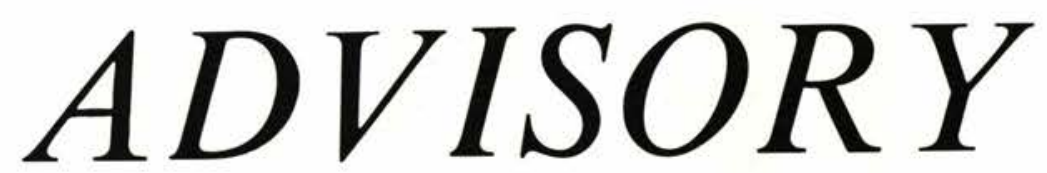




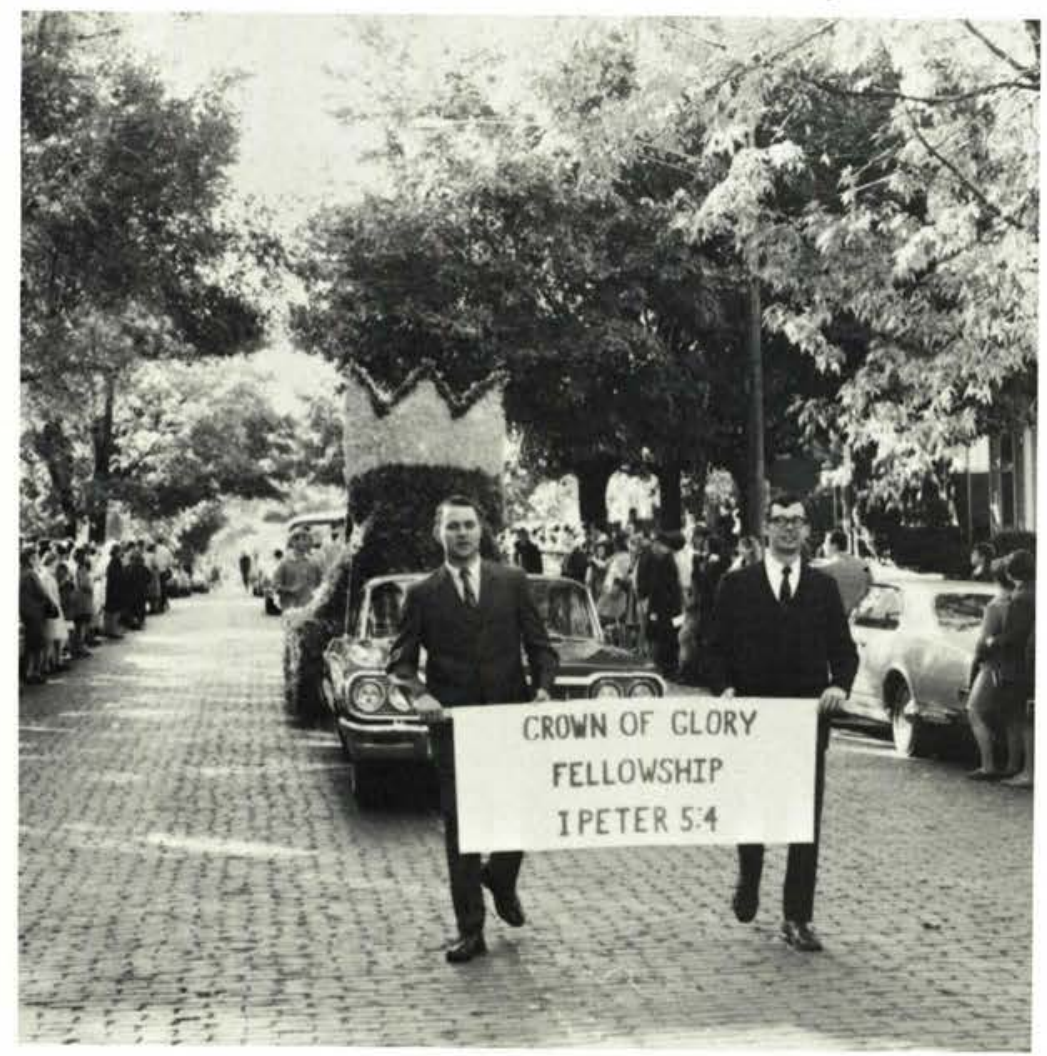

Sigma Delta Kappa is a men's organization providing more practical aspects of Christian service than those offered in the classroom. This organization is designed to help all men going into Christian service with a special emphasis on the future pastor. SDK was the only organization to enter a float in the Homecoming parade this year. Other activities for the organization included a banquet at the Holiday Inn in Springfield sponsored by the Presbyterian Minister's Fund Life Insurance, and programs featuring special speakers including Dr. R. T. Ketchum, Mr. Paul Levine, and Dr. Jack Riggs.

\section{SIGMA DELTA KAPPA}

Row One: Ron Edwards, Denny Dieringer Row Two: Jim Hostetler, Ken Cole, Lyle Miller Row Three: Phil Rohler, Kirk Heldreth, Bob Carmean Elevated: Paul Wheeler

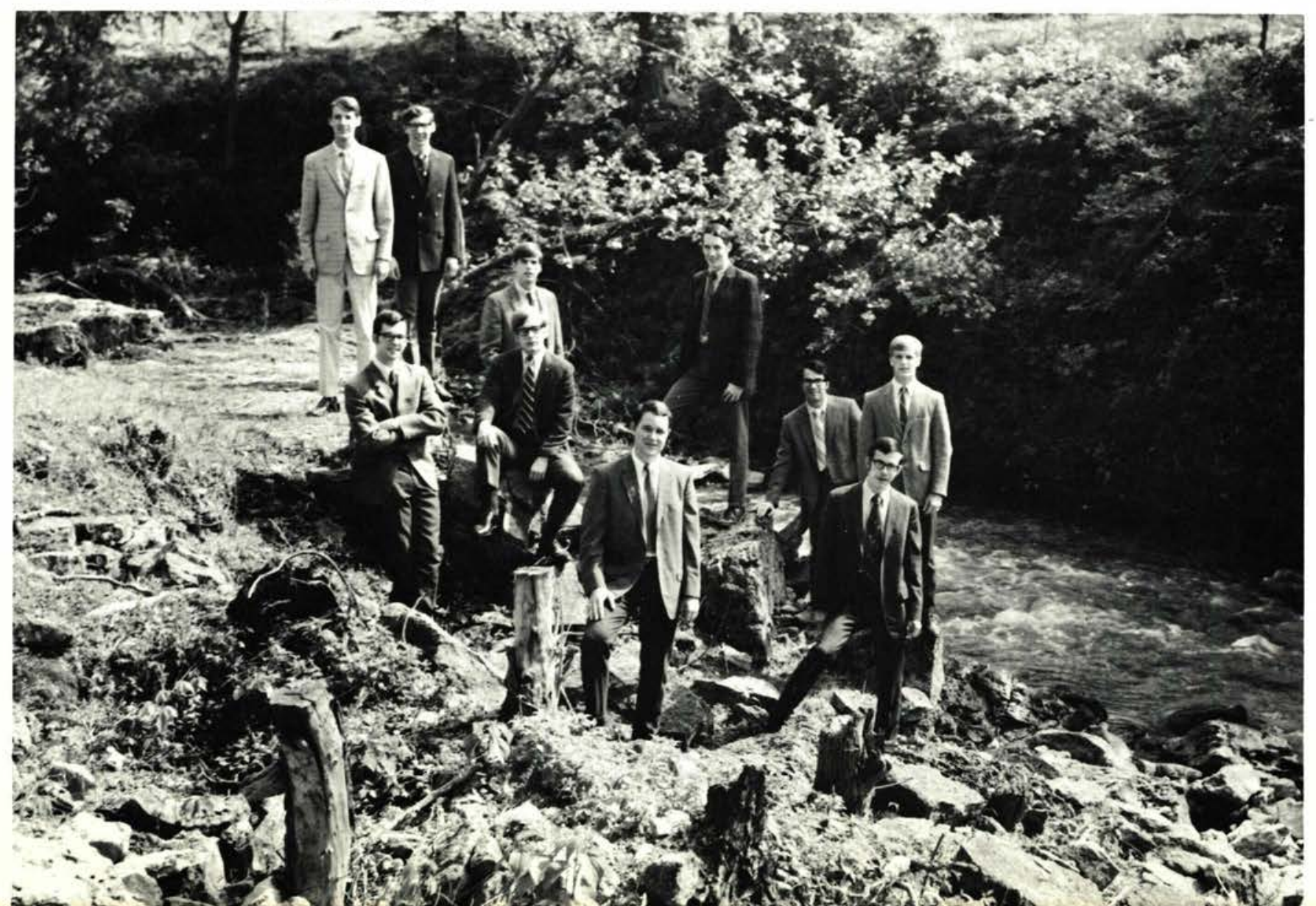




\section{SWORDBEARERS}

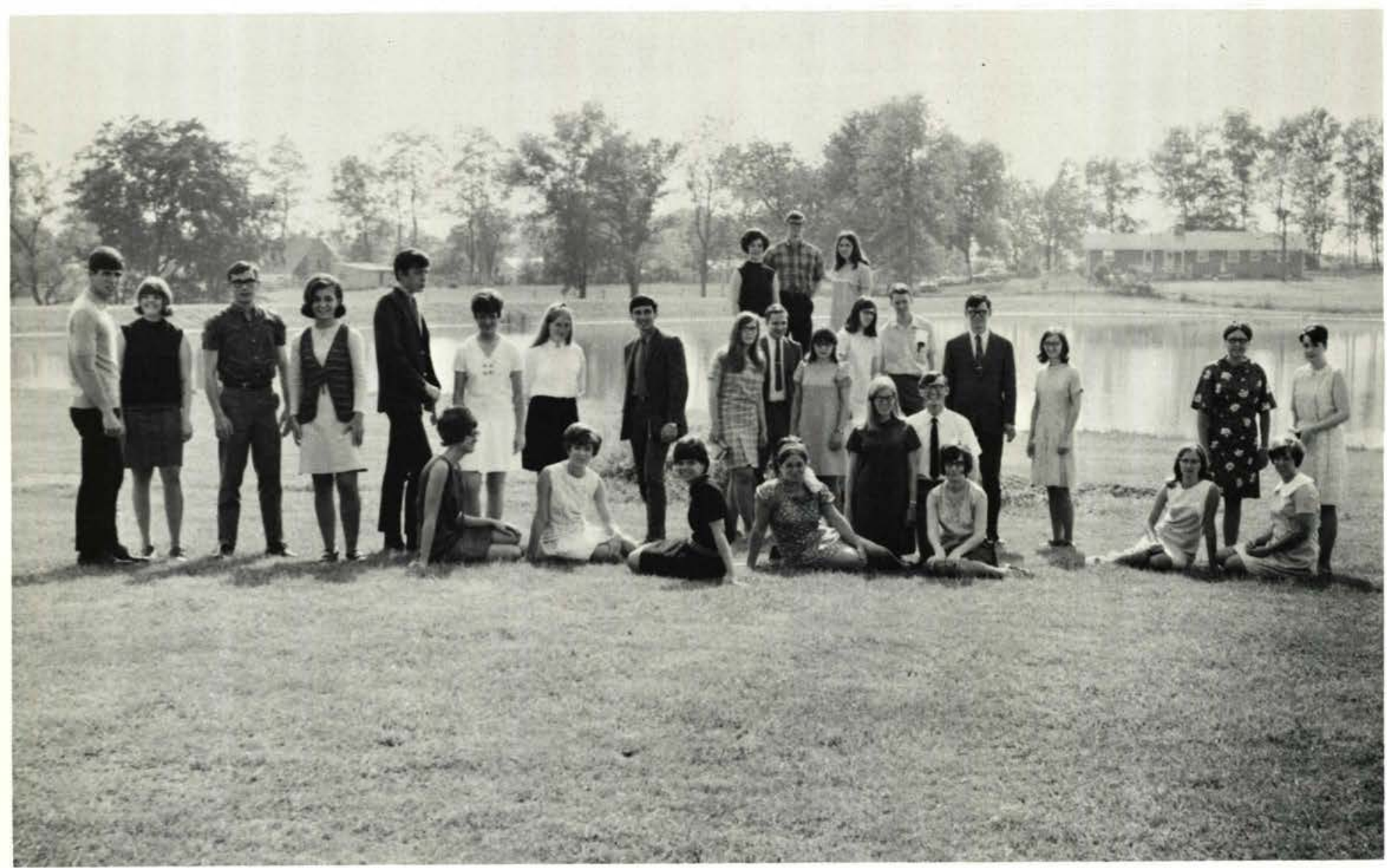

Seated: Arlene Knight, Barb Cooper, Diane Cockerill, Bonnie Smith, Carol Ann Bo, Sam McMillen, Carol Smith, Nancy Paugh Standing: Ken Boothe, Ellen Young, Dave Peters, Kathy Leeser, Eldon Sarver, Vicki Reynolds, Bev Tingle, Chuck Rowe, Mary Bartlett, Bill Smith, Pam Kendrick, Joan Miller, Larry Radcliff, Carolyn Stubrick, Ruth Steyer, Freda Witt Elevated: Ginny Heal, Dave Globig, Becky Williams

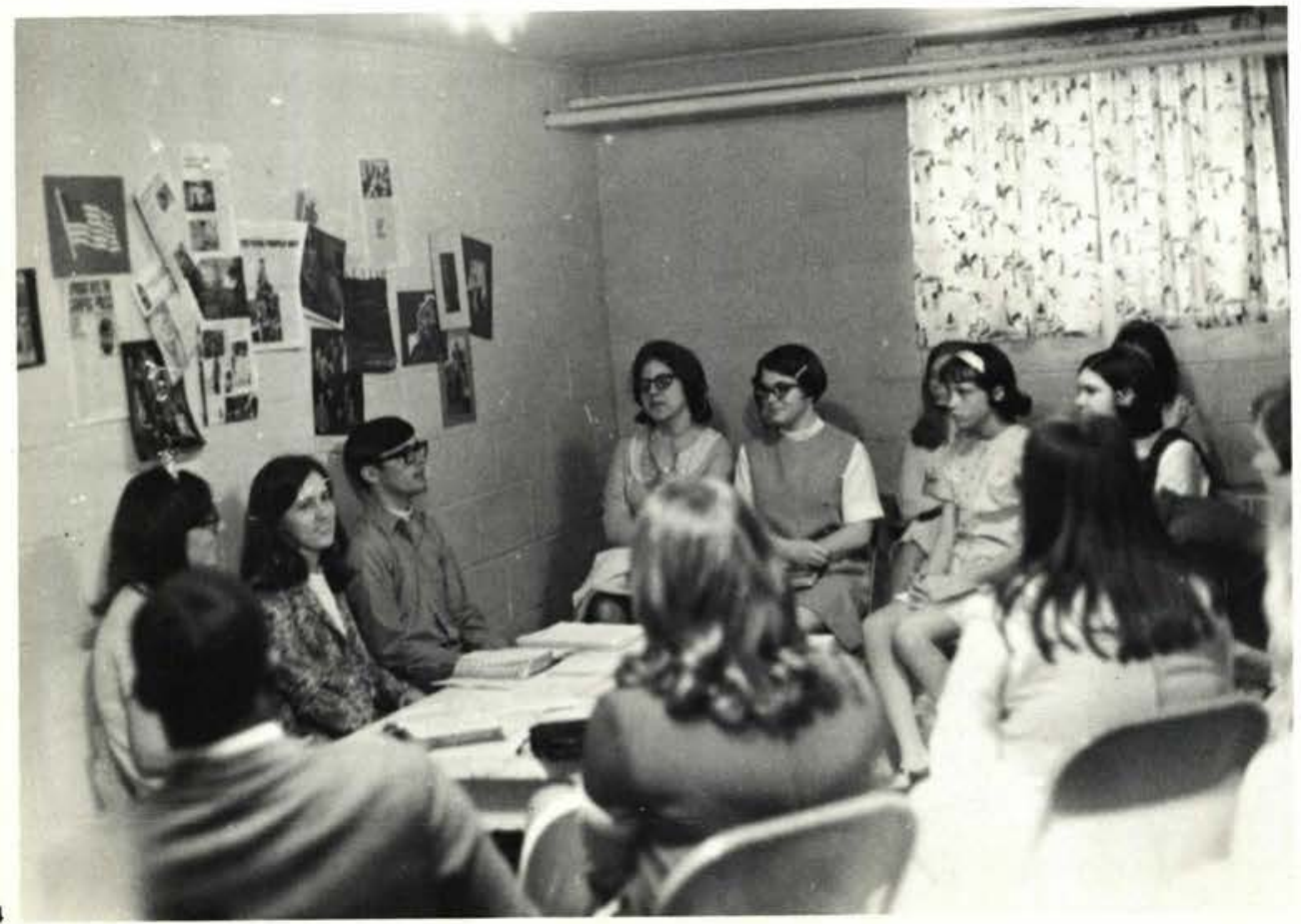

Swordbearer Conference in West Virginia. 


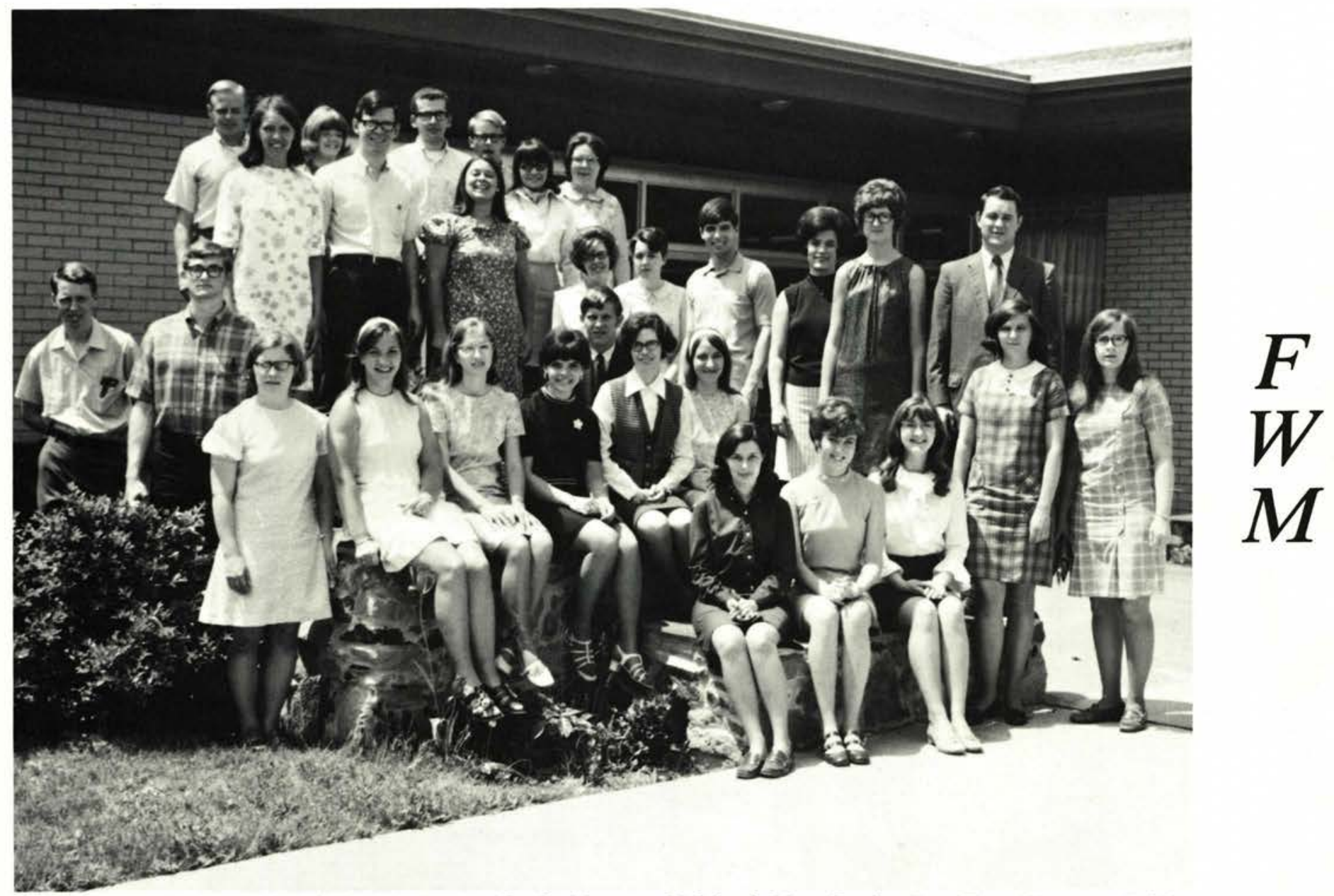

Row One: Marsha Hawley, Barb Cooper, Lynette Davis, Margaret Muirhead, Mary Bartlett Row Two: Mareanne Beitler, Vicki Tobias, Nancy Paugh, Diane Cockerill, Dianne Kemp, Lin Mason Row Three: Larry Radcliff, Dave Globig, Ken Boothe, Ginny Heal, Arlene Knight, Ron Edwards Row Four: Nancy Snider, Paul Radcliff, Bonnie Smith, Linda Weaver, Freda Witt Row Five: Dave Mallinak, Ellen Young, Dave Turner, Pam Kendrick, Bev Mix

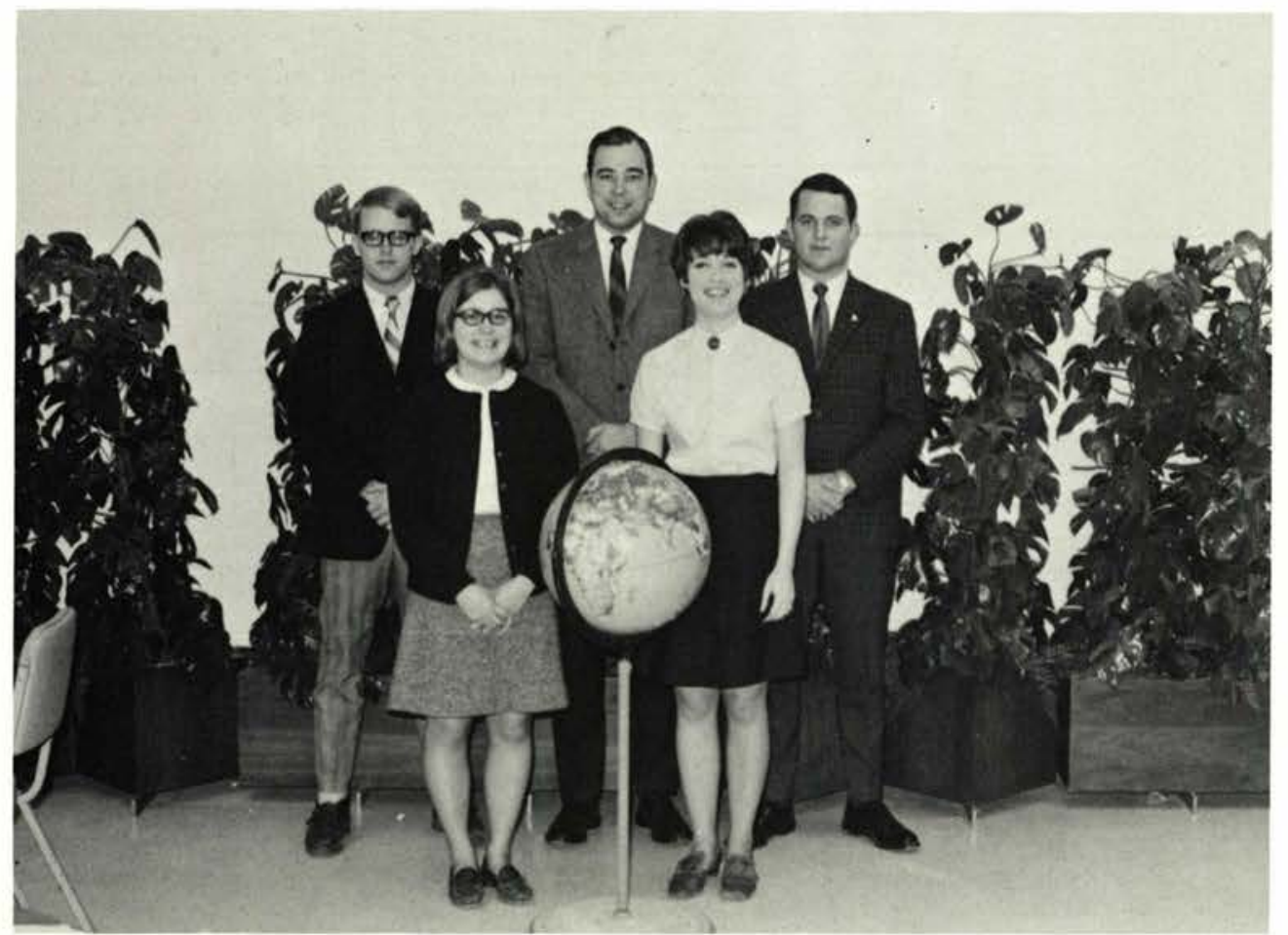

FWM provides a daily time of fellowship for students who are concerned about missions. It informs students of the various ministries and needs of mission fields around the world. It offers a time when students can gather and pray for the needs of missionaries.

Mareanne Beitler, Treasurer; Tanis McDaniel, Secretary; Dave Turner, Vice Pres.; Dr. Riggs, Advisor; Ron Edwards, President. 


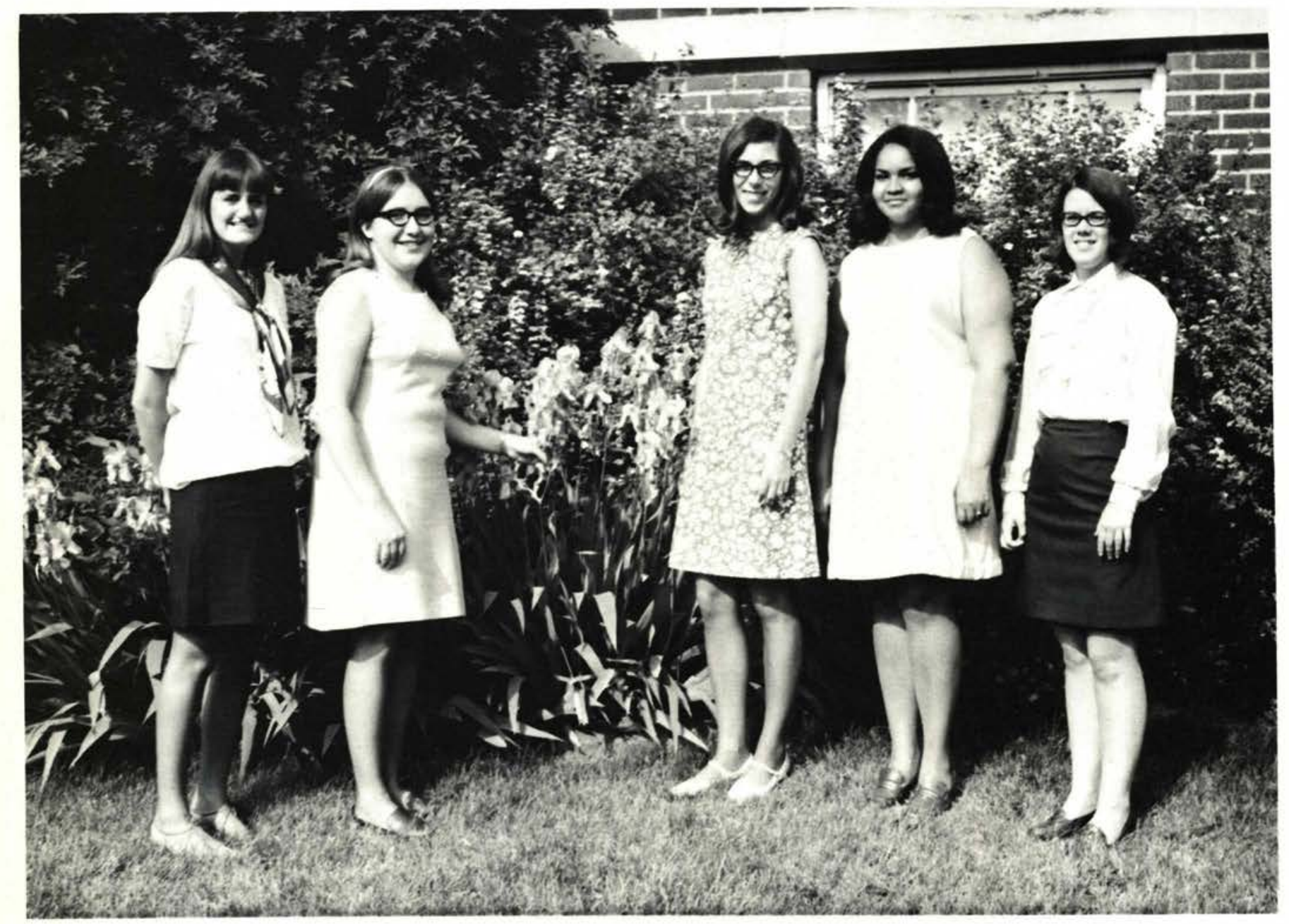

\section{HAPPY TIMERS}

Susan Gustfason, Jan Malmstrom, Sherie Nelthropp, Mary McMurtry

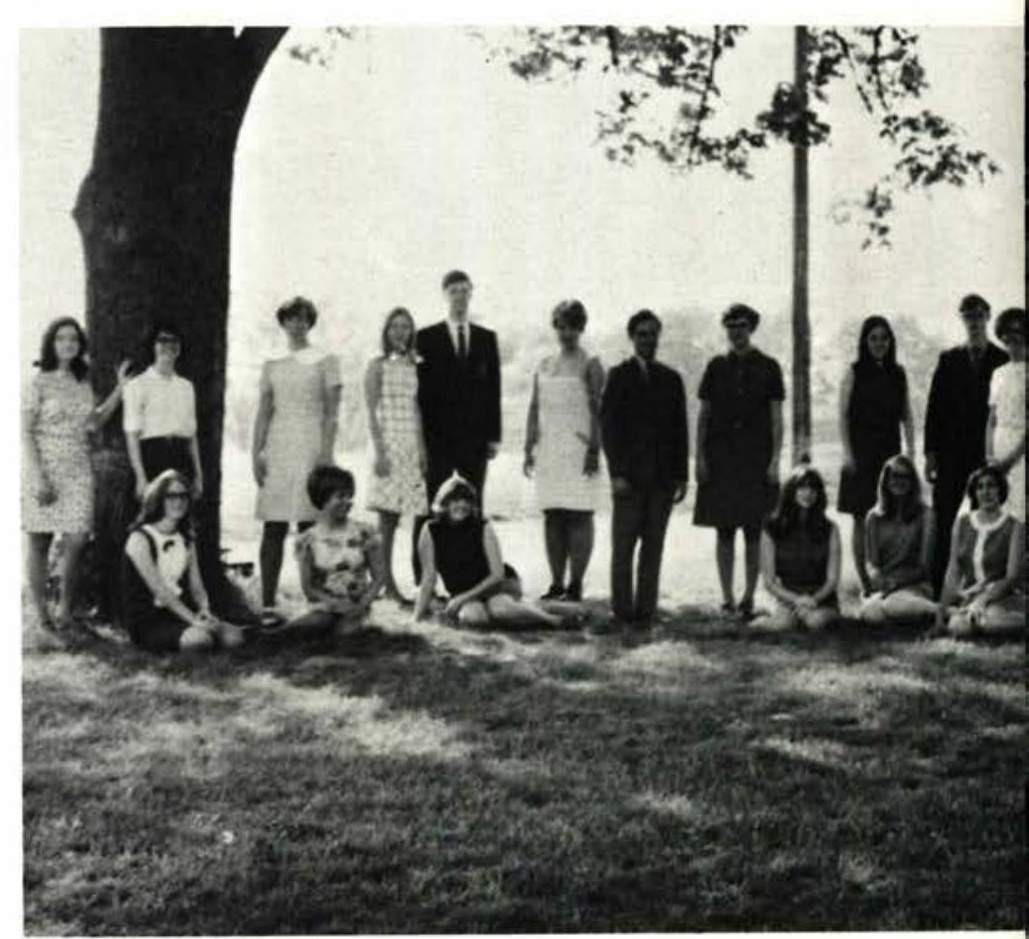

REST

Seated: Ellen Young, Arlene Knight, Vicki Jones, Mitsu Ishuin Standing: Ruth Ransbottom, Edie Phillips, Helen Telford, Judy Price, Sam Olsen 

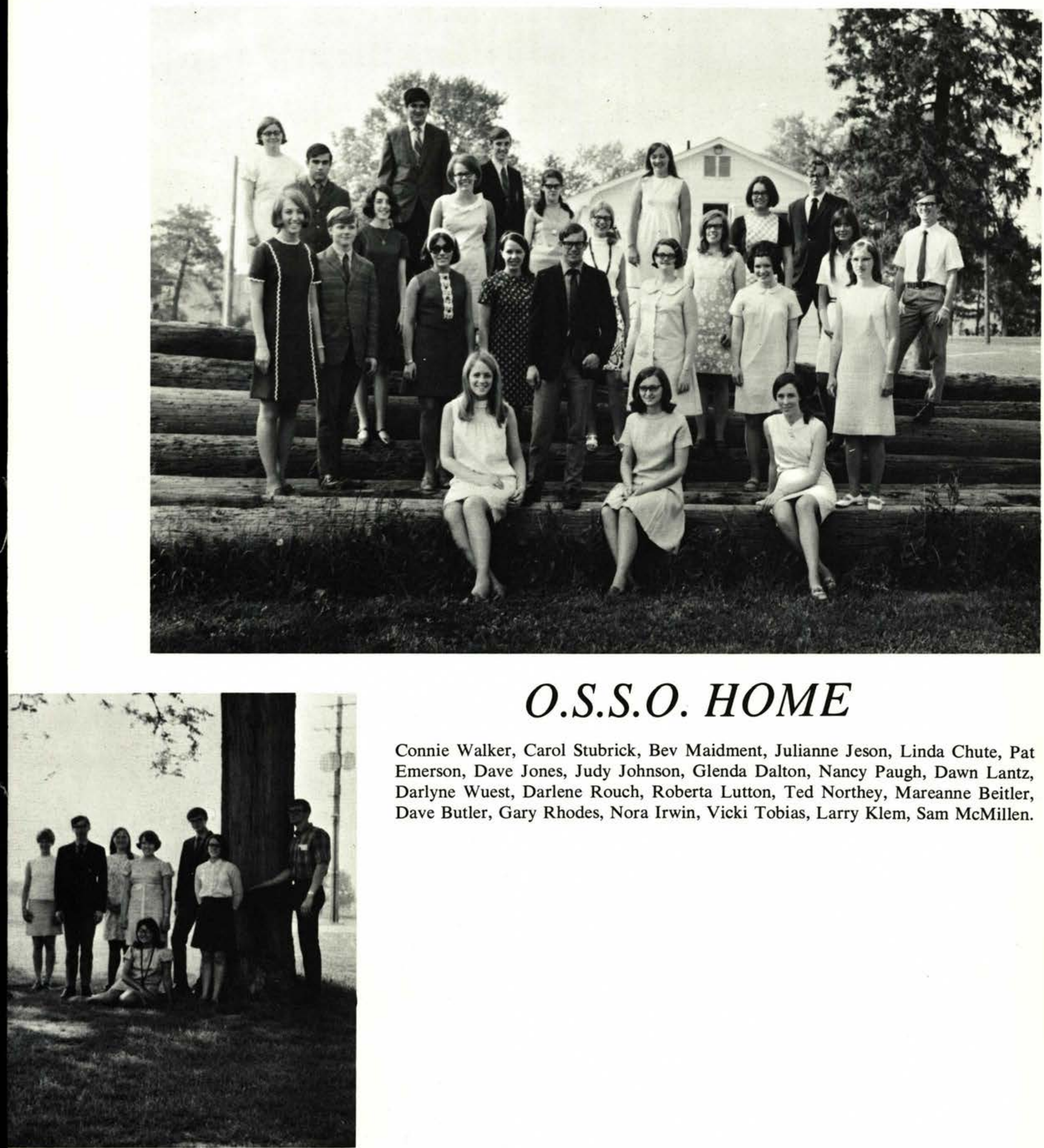

Connie Walker, Carol Stubrick, Bev Maidment, Julianne Jeson, Linda Chute, Pat Emerson, Dave Jones, Judy Johnson, Glenda Dalton, Nancy Paugh, Dawn Lantz, Darlyne Wuest, Darlene Rouch, Roberta Lutton, Ted Northey, Mareanne Beitler, Dave Butler, Gary Rhodes, Nora Irwin, Vicki Tobias, Larry Klem, Sam McMillen.

\section{HOME}

Linda Christensen, Ron Clark, RaeBell Fisher, Verna Tallmon, Bernie Horn, Linda Weaver, Mary Piach, Mike DeBerg, Bonnie Spears, Roger Swanson, Mary McMurtry, Dave Globig 


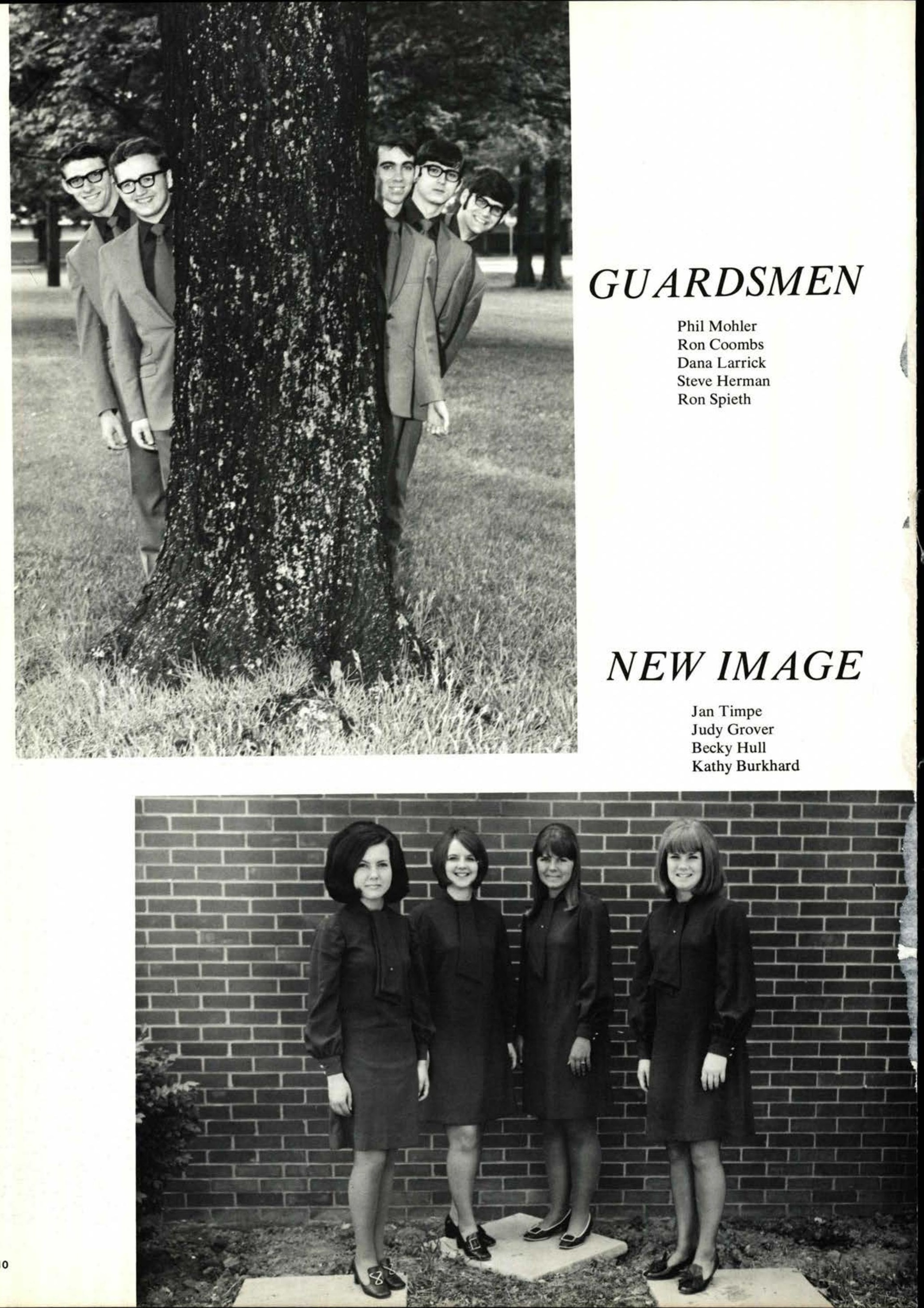




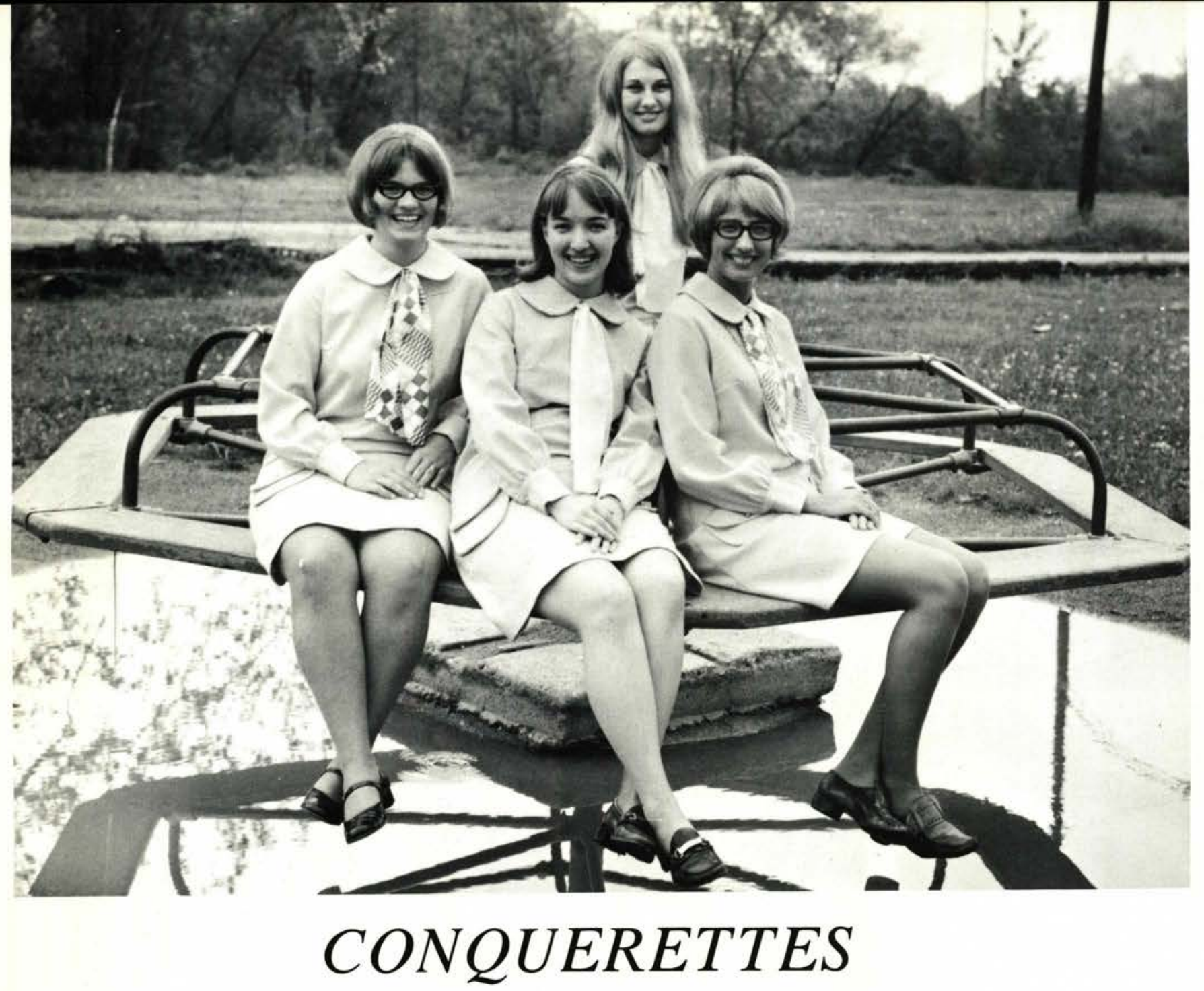

Seated: Linda Betts, Annette Clark, Rita Gast Elevated: Sue Scott

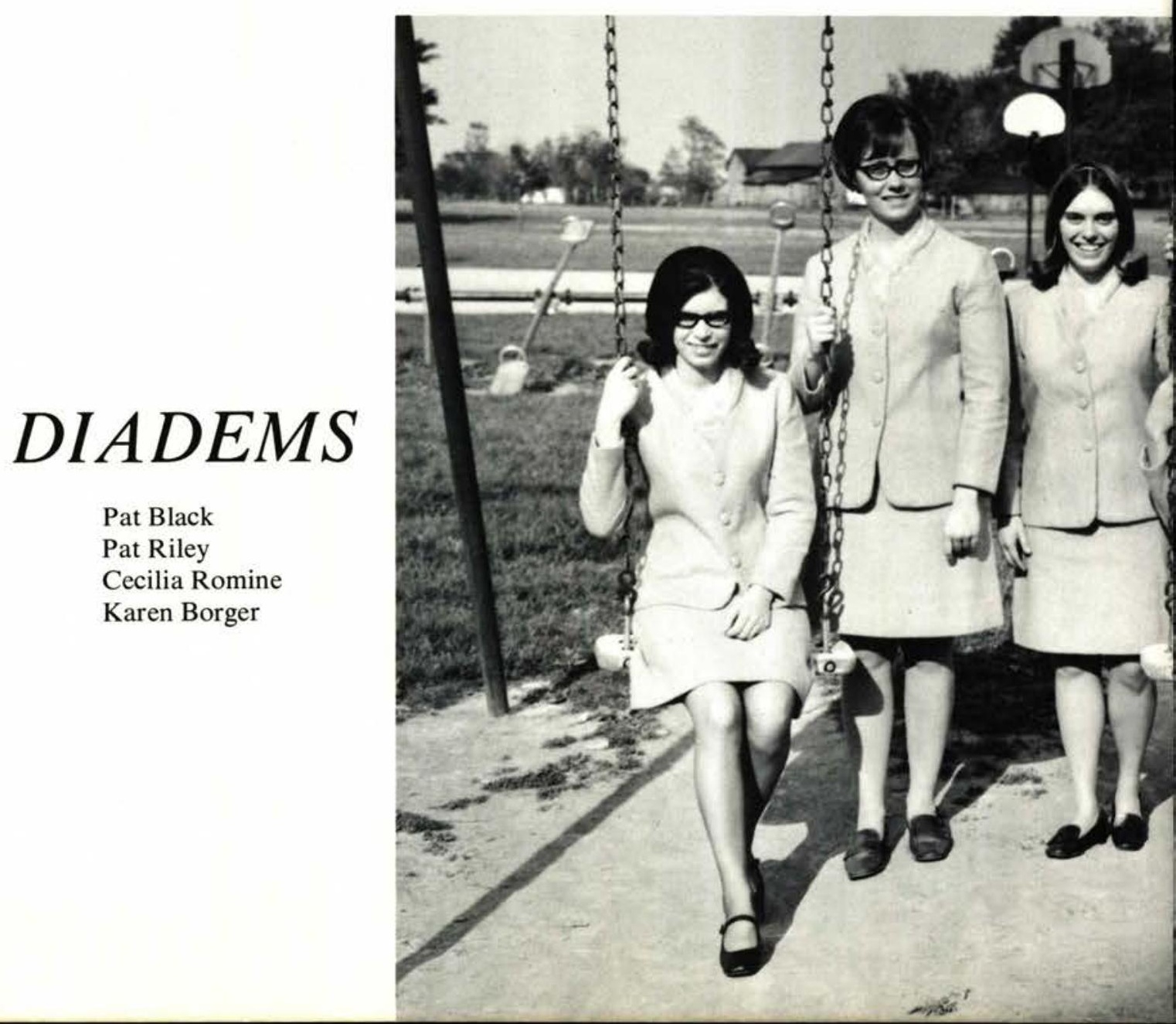




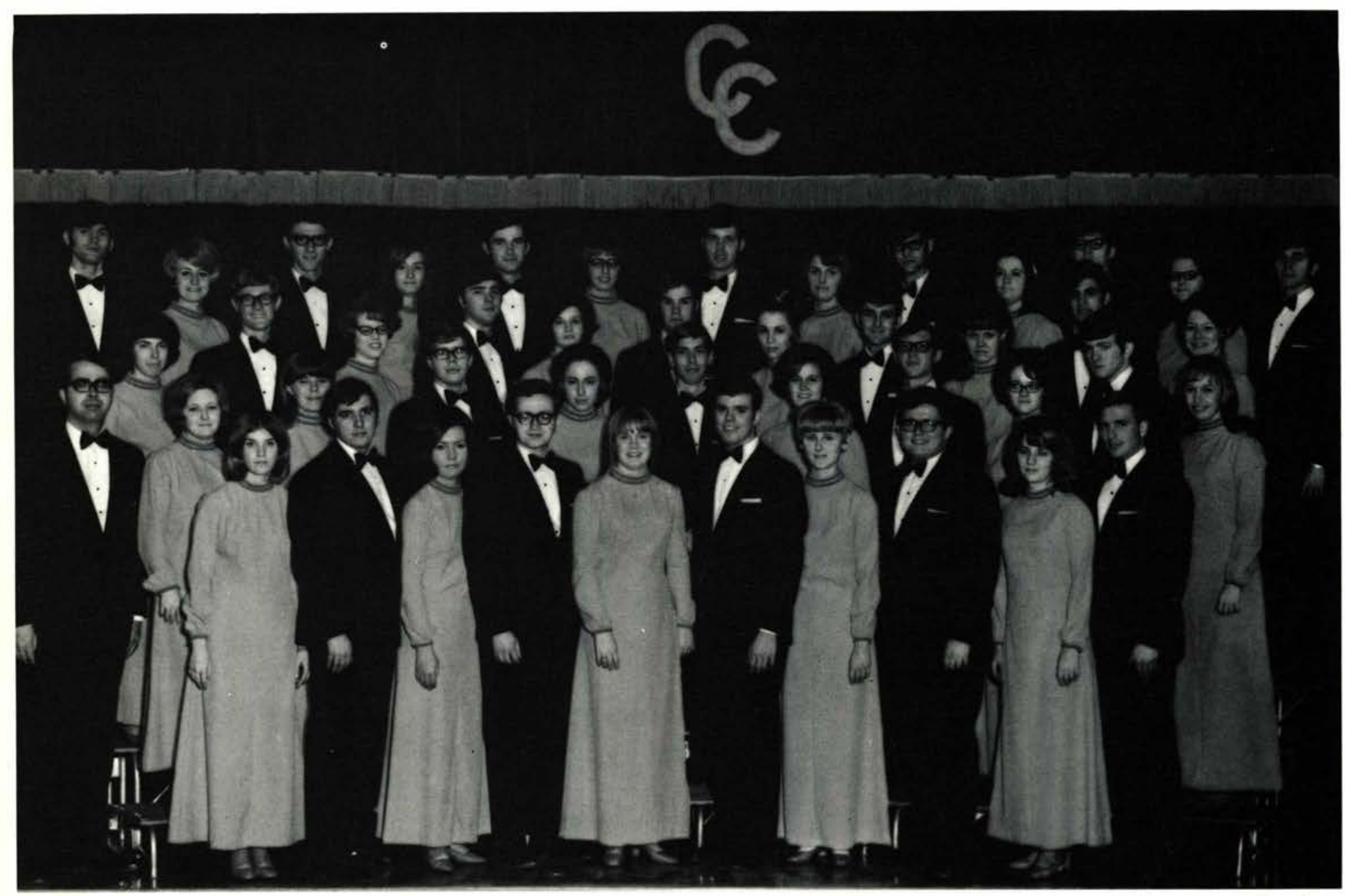

Row One: Paula Prater, Randy Hamilton, Jan Timpe, Ron Coombs, Kathy Burkhard, Bob Brown, Patti France, Bill Amstutz, Connie Clark, Dave Railsback Row Two: Mr. Matson, Linda Browning, Becky Hull, Buddy McNiece, Becky Chandes, Dave Nelson, Val Butler, Lyle Rodman, Marilyn Brown, Jim Jeremiah, Jan Gabriel Row Three: Carol Morse, Jerry Grayson, Jan Brock, Ron Meyers, Karen Borger, Tom Strong, Joyce Saemenes, Lyle Anderson, Jan Beesley, Dave Durham, Cindy Cartner Row Four: John Schneider, Viann Meyer, Phil Mohler, Sue Gift, Victor Lakes, Rita Gast, Sam Olsen, Marikay Schneider, Ron Spieth, Kathy Gelo, Steve Herman, Bev Moore, Bob Senseney

\section{COLLEGE CHOIR}

New music ... hoarseness ... memorization ... prayer ... choir tour ... testimonies ... close Christian fellowship ... "When $\mathrm{He}$ Shall Come" ... and suddenly it is the end of the season for the 1969-70 College Choir. But the glowing memories of sharing Christ through music continue. The choir had the opportunity of performing musical selections of a sacred nature from various periods of music history; all with the goal of getting the message across to those who listened.

Many week-ends were spent singing in Ohio communities and the spring tour led the choir into Illinois, Indiana, Iowa, and Missouri.

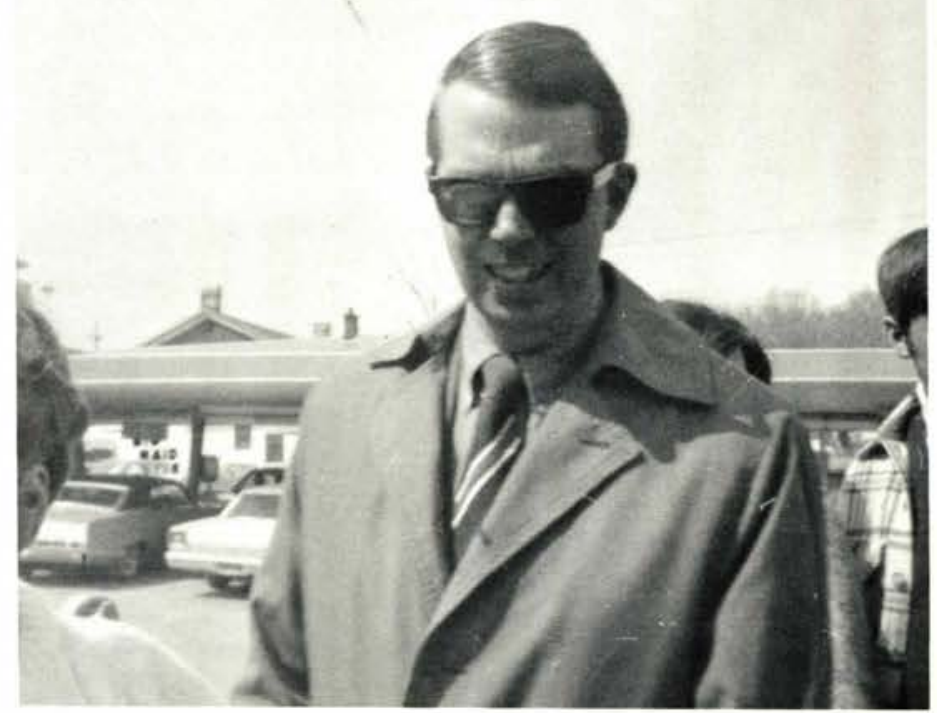




\section{CHOIR MEMBERS}

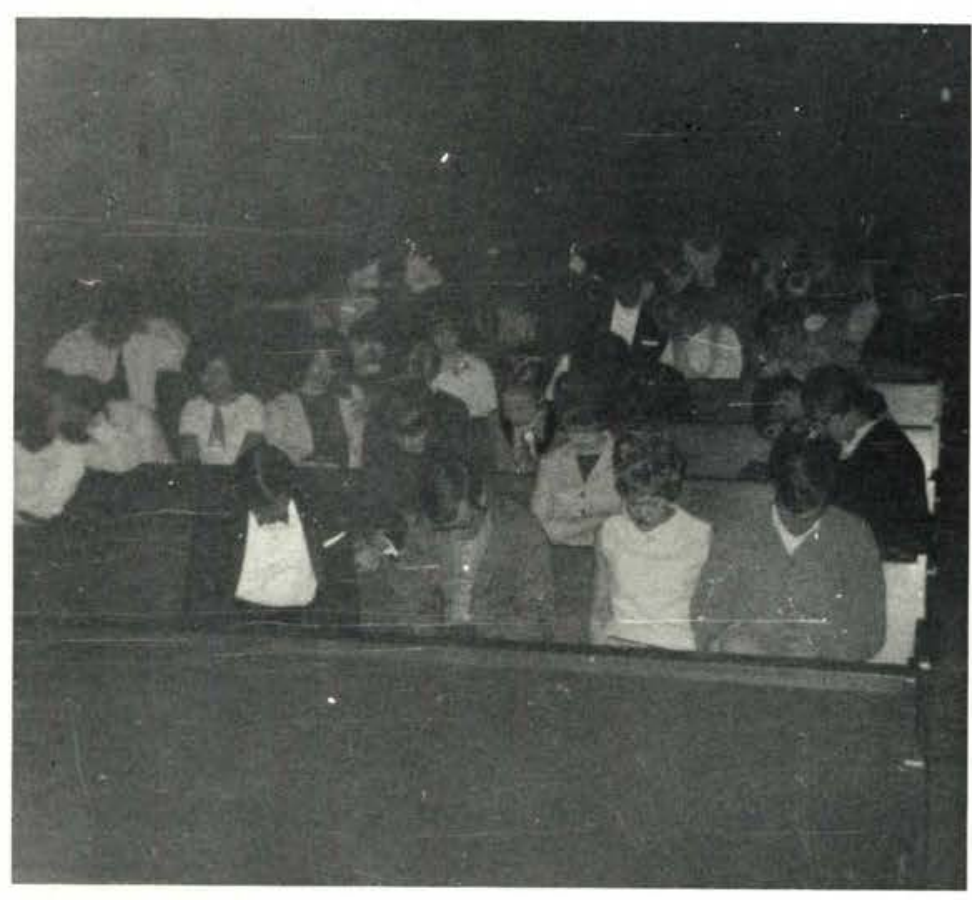

PRAYED

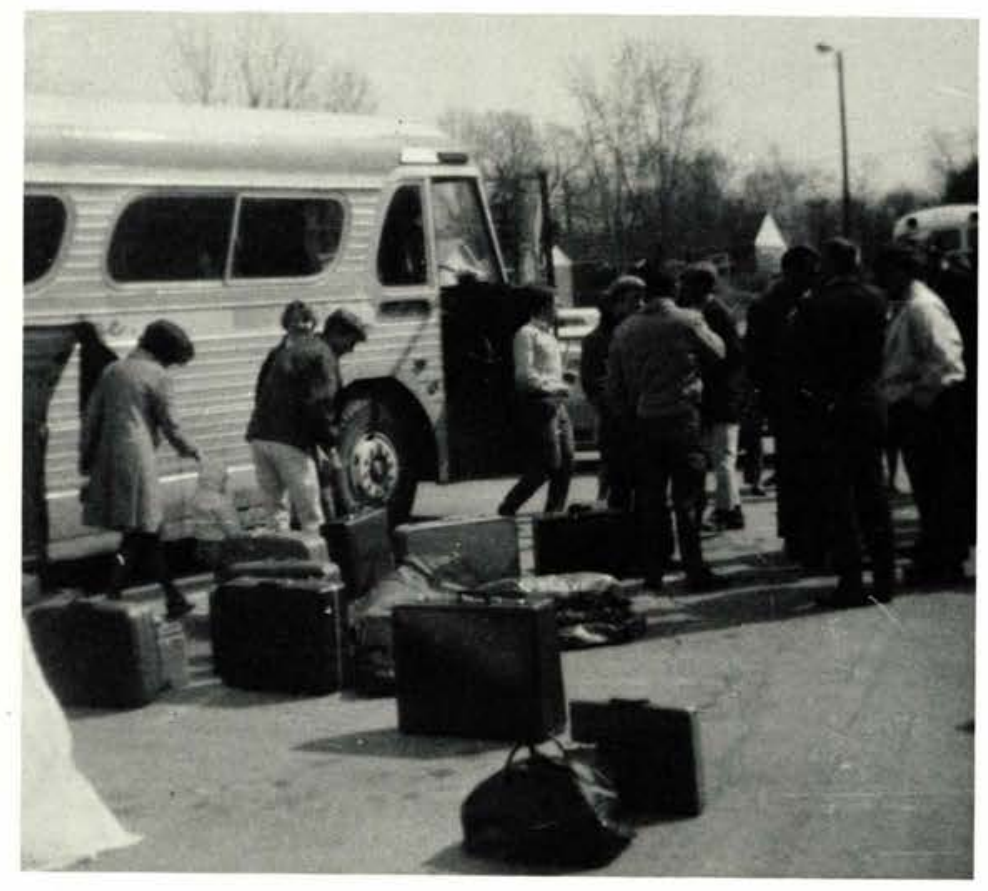

\section{$P A C K E D$}

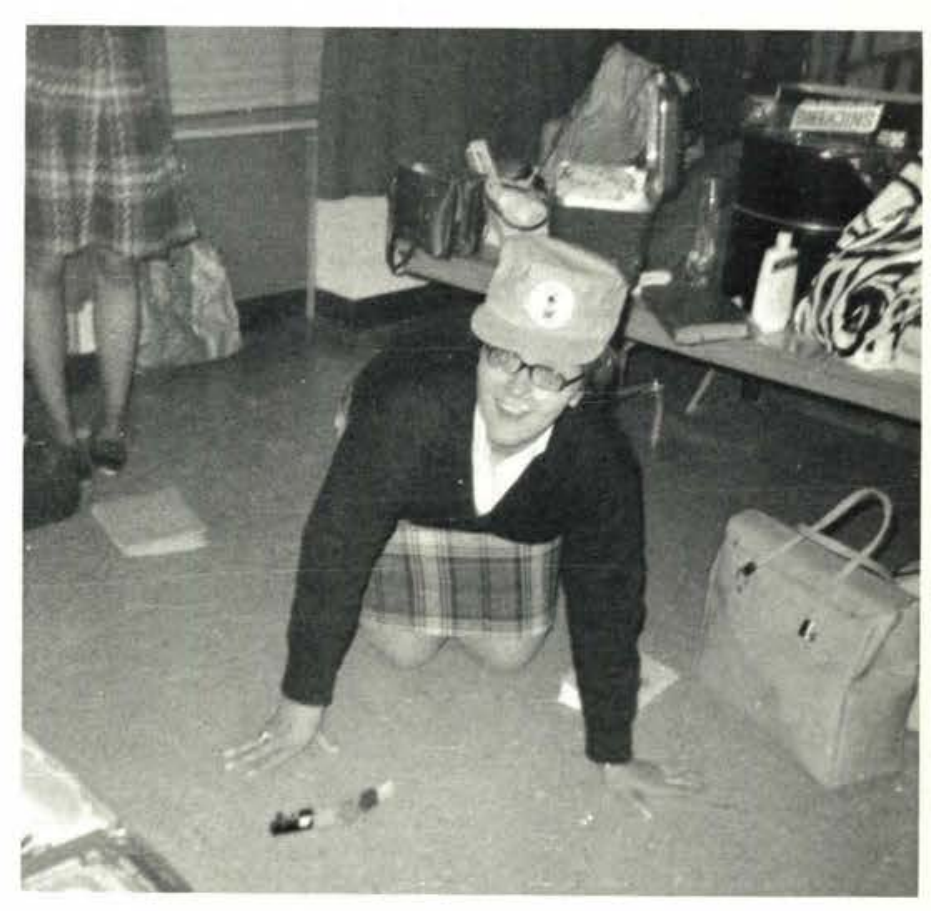

PLAYED

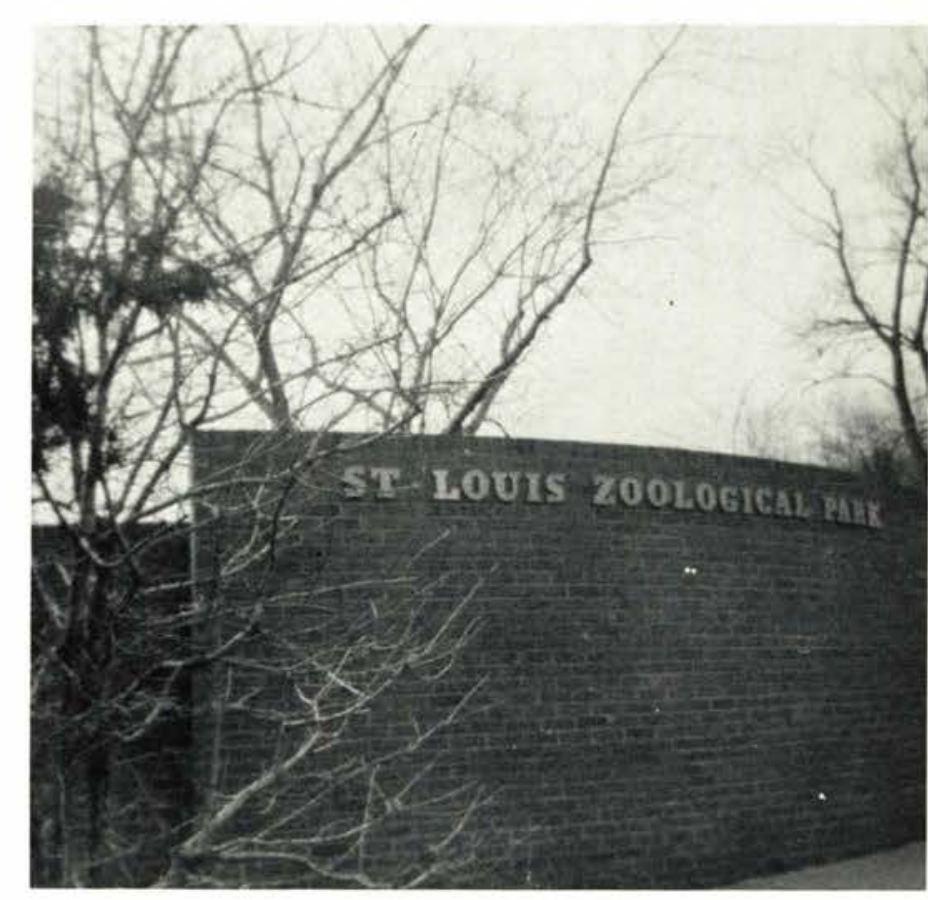

PICNICKED

\section{ON TOUR, 1970}




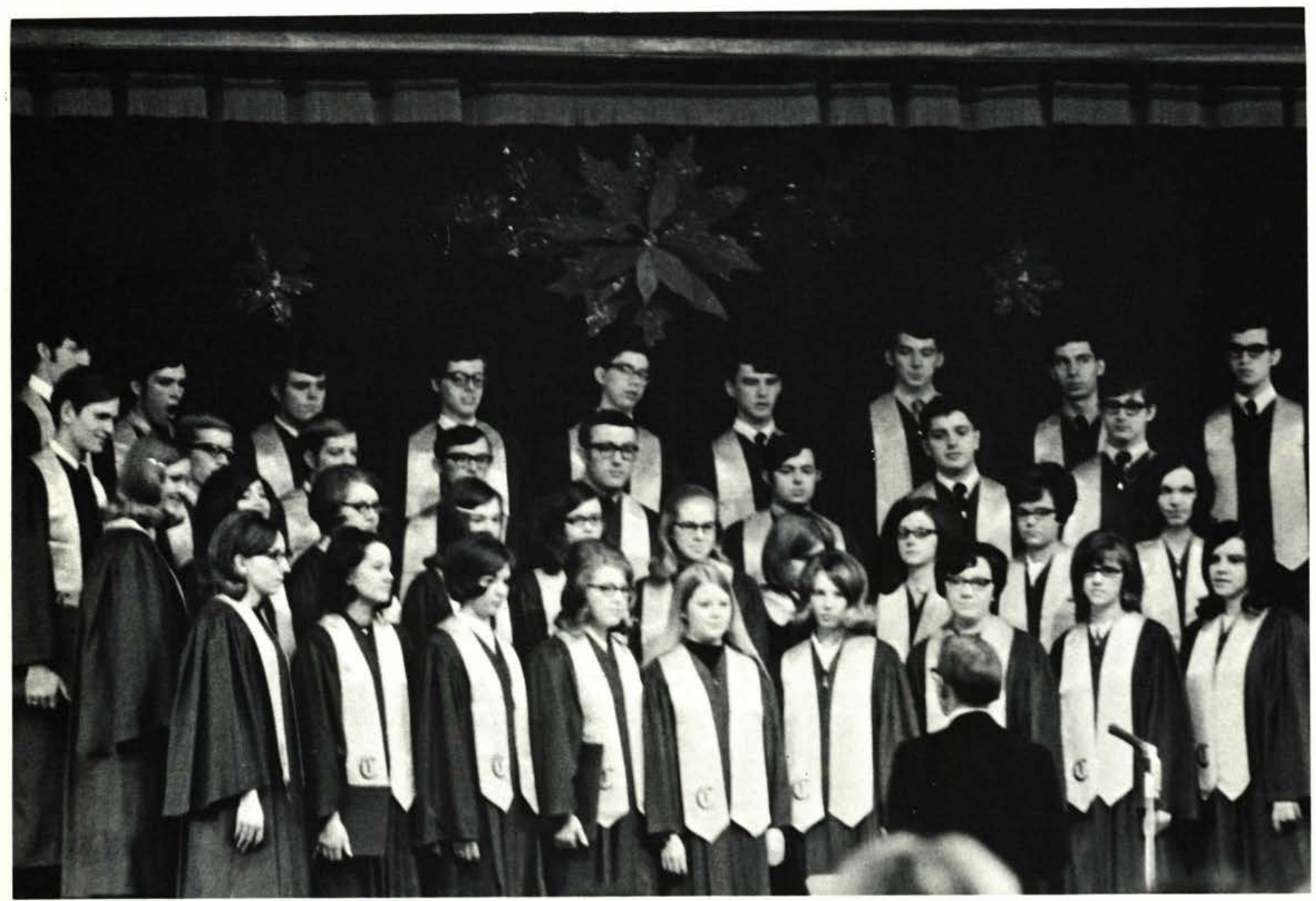

\section{CHRISTMAS CONCERT}

Row One: Mercy Tennant, Corrine Duhon, Ruth Moats, Linda Hershey, Geri Crotty, Laura Jones, Carolyn Lambert, Lois King, Nola Watson Row Two: Joyce Love, Kay Partlow, Ruth Coombs, Becky Fleming, Rosalie Howard, Carol Bo, Martha Moore, Linda Wilms, Brenda Younger, Sharon Gillogly Row Three: Doug Hess, Yonze Miller, Tim Hegg, Sam McMillien, Phil Stine, Don Harmon, Fred Erskine, Steve Herman Row Four: Don Hendrickson, Terry Hignite, Bob Brown, Larry Klemm, Steve Spink, Tim Kline, Tim Andreanopoulos, Dan Thompson, Jim Bucholtz

\section{CHORALAIRES SALUTE GOD AND COUNTRY}


Choralaires desires to raise the level of music appreciation through the performance of good secular music while appropriating this opportunity to represent and glorify Christ through personal testimony.

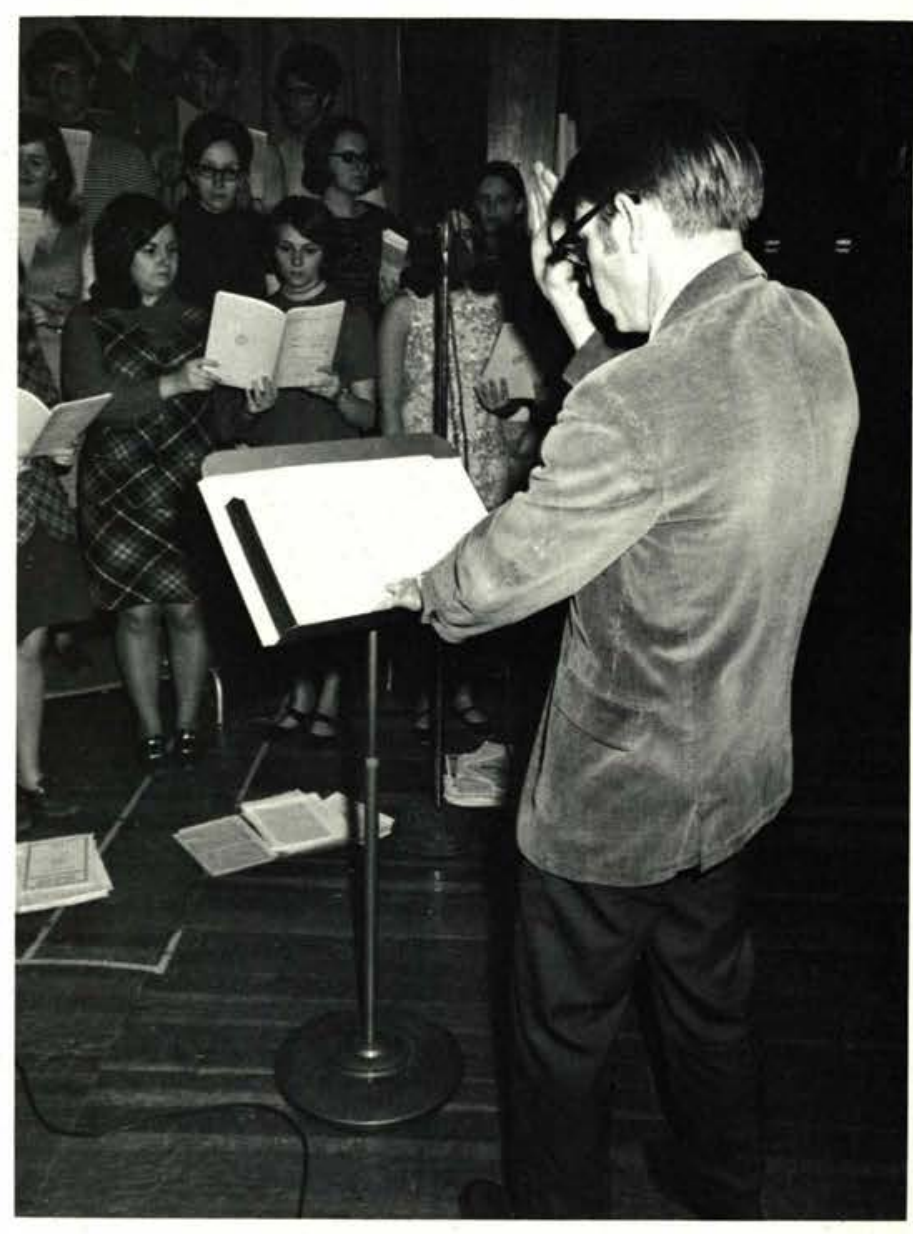

“OUR AMERICAN HERIT AGE”

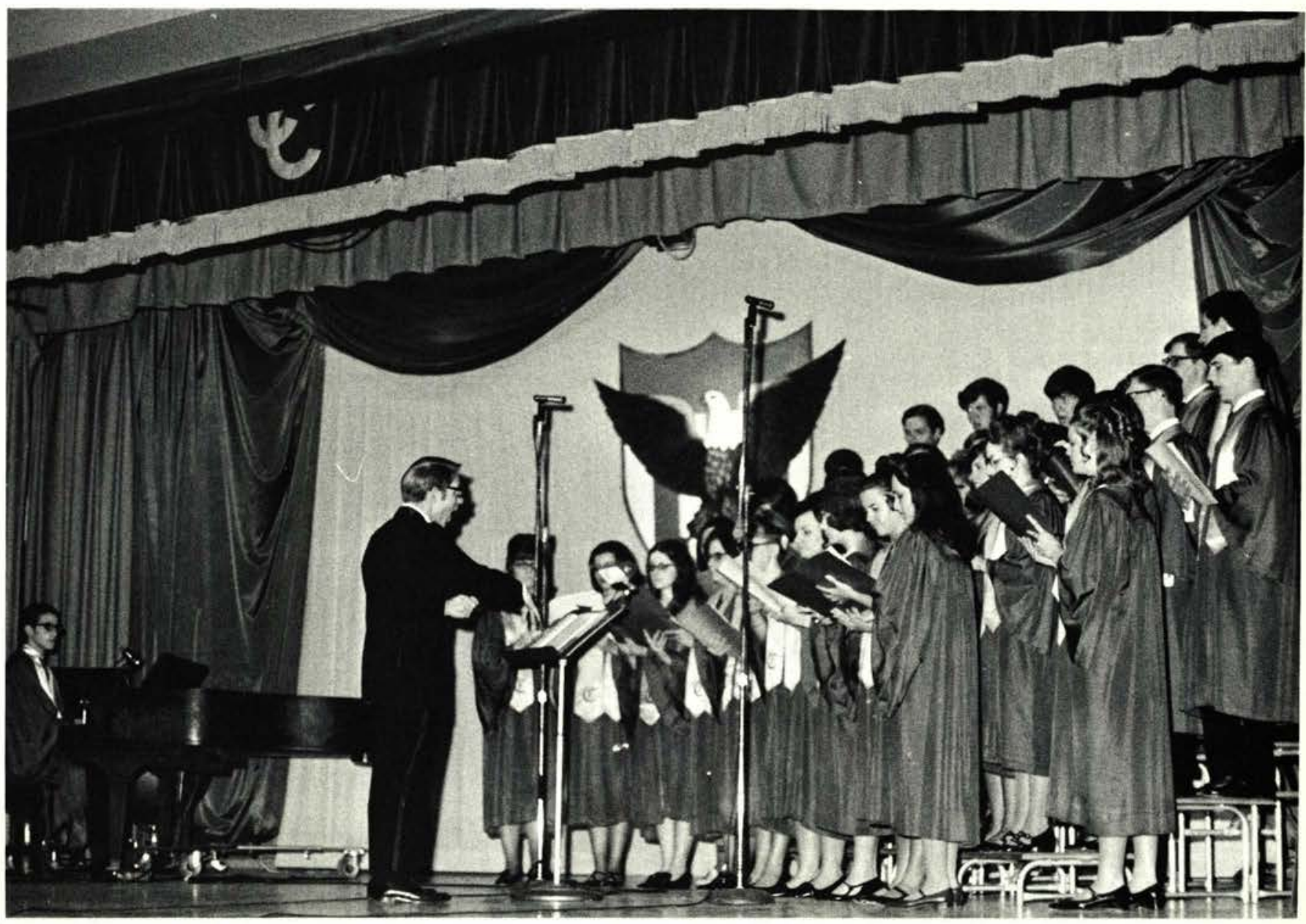




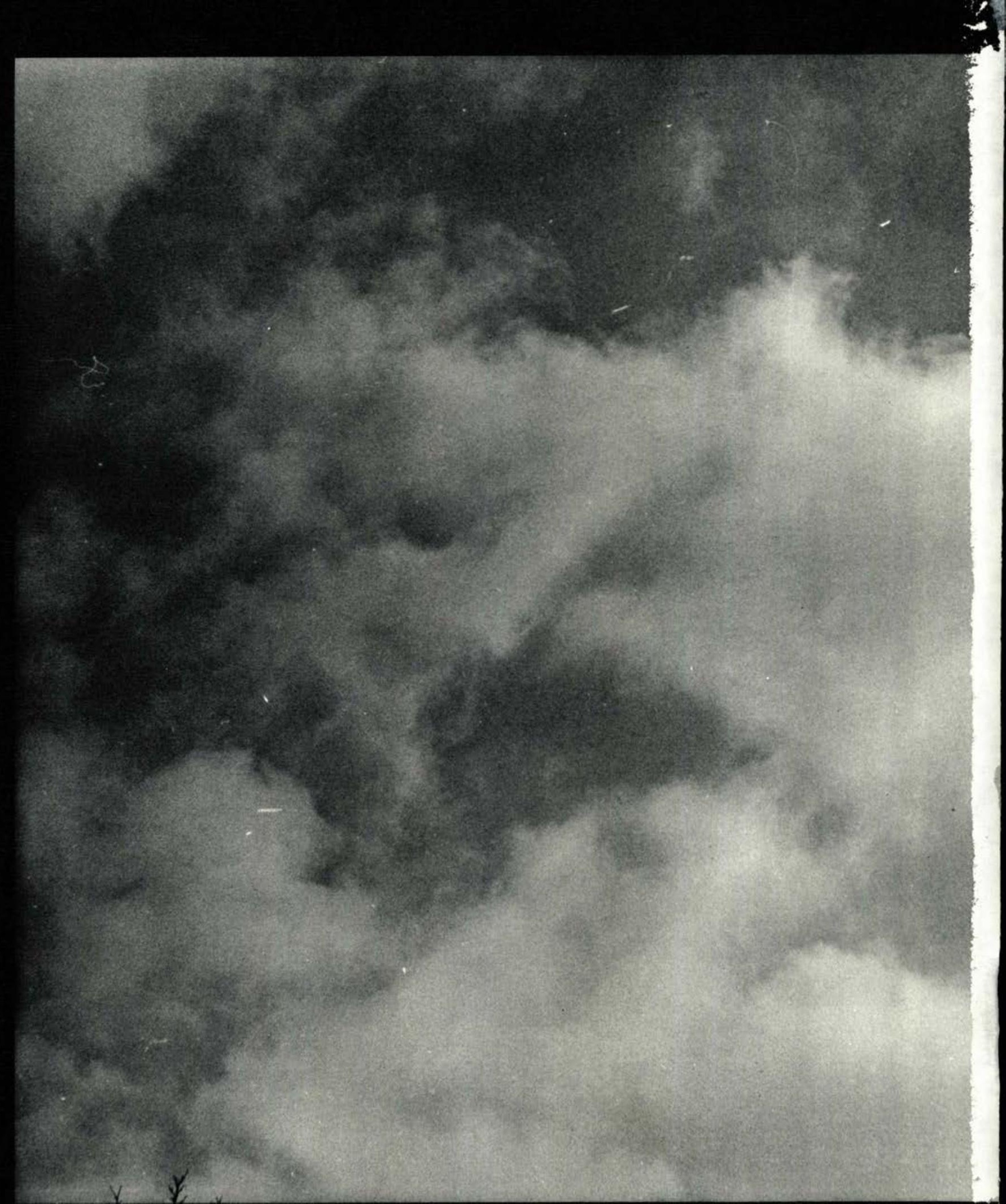




\section{fipilng}

A Miracle. .

That's how it will all end,

T. S. Elliot

Was wrong,

It will not end with a whimper

But with a

Shout

And the trumpet shall sound

And the dead shall be raised inc

And we shall all be changed. .

Changed

From academician garb

From athletic uniform

From choir robe (though gold) a To robes of righteousness. .

Changed

From weariness and disappointn From the flu and sick trays

From deadlines and demerits

From the madman's pace of livir That robs of life,

From 9 months of rain and jello, To the side of the Great I Am . Changed

From a status quo that fosters

Hatred and bigotry

Injustice and disease

Violence and assassinations

And the complacency that allows To the city of gold where the Lar Changed

From a species

That sends Christmas cards

And blows each others' brains ou From a race

That walks the moon

And walks all over darker men,

From a breed

That transplants hearts

And creates Viet Nams,

From a kind

That passes birth control

And defeats gun control,

To the presence of the Prince of 1

What a Miracle

What a Savior!

Even so come,

Lord Jesus .

Patti Fra 
This has always been

one of my favorite places,

My home is just a few minutes away;

When I was a little girl

I used to come here with my dad

And watch the sailboats and the noisy gulls

And the kids fishing and the couples walking

And the old men sleeping in the sun ...

I loved the way the sun shone on the water

And the sound the ripples made

Lapping against the breakwall;

I loved the cool breeze and the scent . . .

But it's not much fun to come here anymore.

The water is murky

The sun clouded

And the scent foul . . .

The gulls choke on industrial waste

And Governor Rhodes declared the fish

Unfit for human consumption . . .

Man has polluted everything:

His environment

His body

His character;

$\mathrm{He}$ has managed to distort the world so cruelly

That today

Our peace is more violent than our war,

Our love is a twisted figment of sensualism,

And our compassion a meaningless code

Of cold ethics and religion . . .

We take instead of give

We scream instead of listen

We curse instead of care

And it seems as if only a miracle could

Change the pollution. .

But it would not take a miracle,

Just people who have had

A miracle worked in their lives,

People who can live together and work together

And tolerate each other's differences

And thereby teach the world . . .

The 1970 Miracle

Is all about people

Their government, their dialogue,

Their competition,

Their involvement with and commitment to

A better way. .

But let us not be satisfied

With an individual miracle,

Let us seek to cause what

Senator Kennedy

Called a "ripple of hope"

That can become a current

Strong enough to dash

The most formidable walls of

Injustice and wrong . . .

Let us work for the miracle of change

In a world that yields most painfully

To change. .
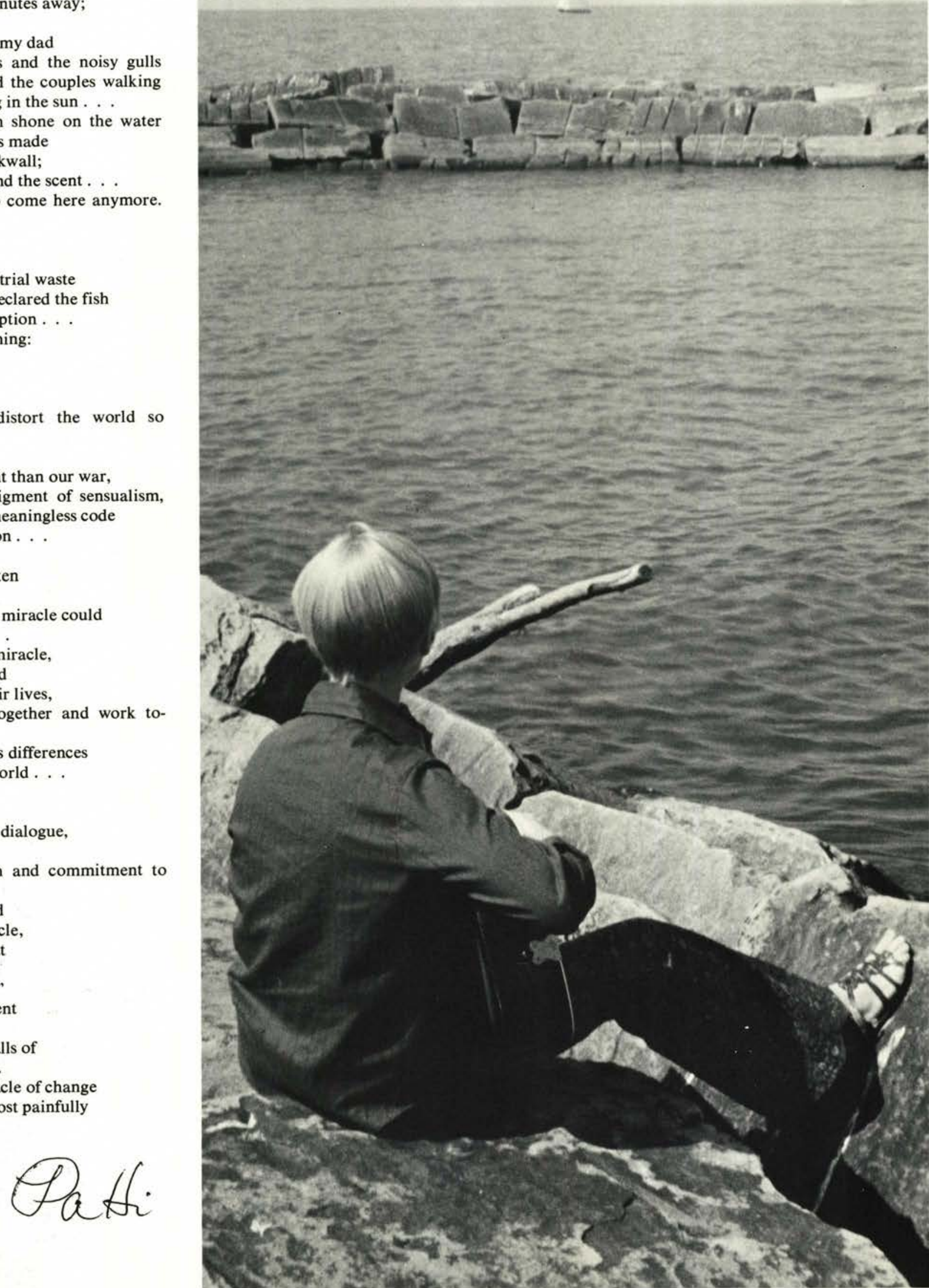


\section{IN APPRECIATION}

The Miracle staff, and especially the editor, wish to acknowledge and thank the following for their much-appreciated contributions to Miracle '70:

Ty Bryant: photography

Charles Miller: ideas and enthusiasm

Dr. Baumann: patience, prodding, perseverance, and omnipresence

Charles Miller: ideas and enthusiasm

Dr. Baumann: patience, prodding, perseverance, and omnipresence 

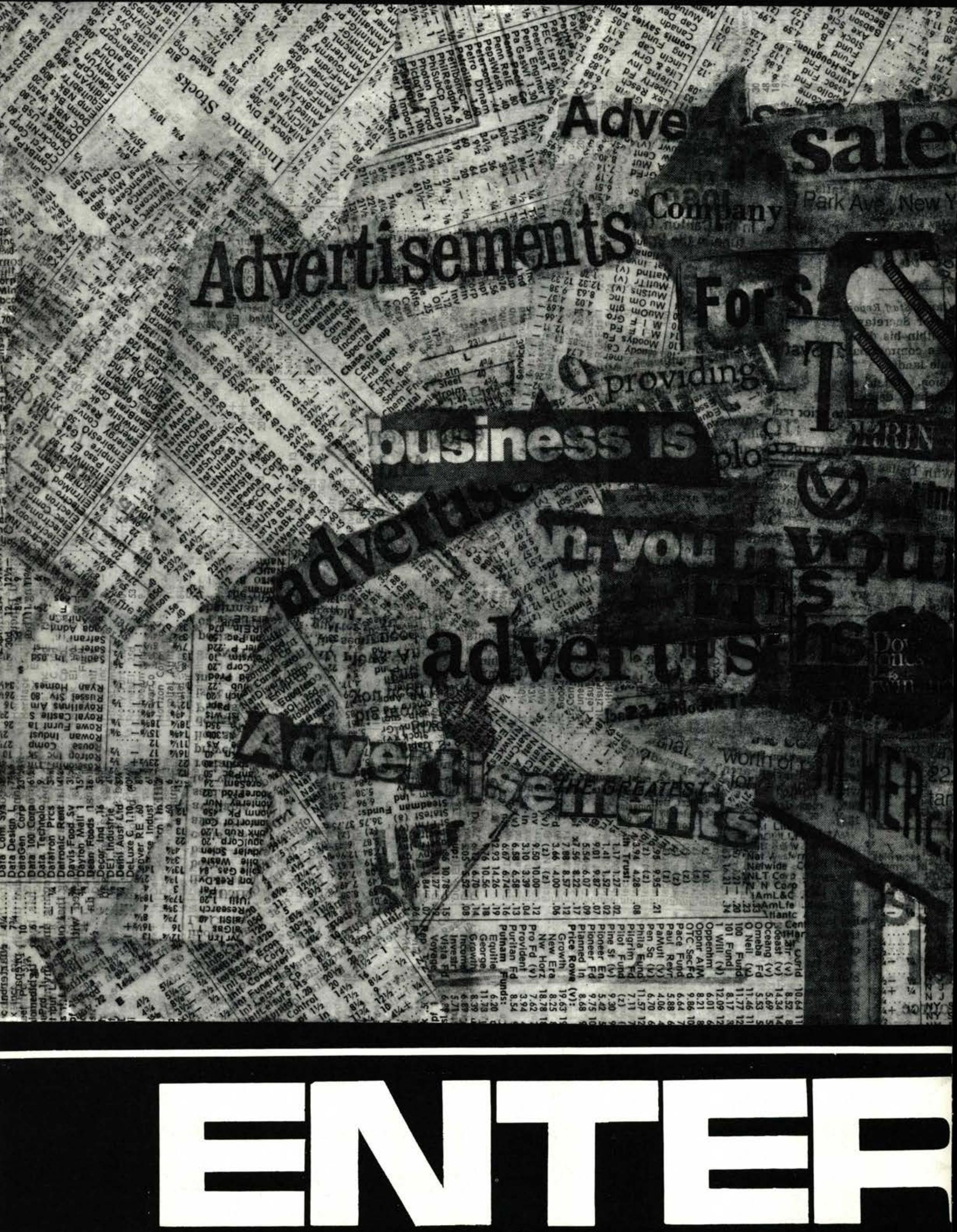
ans

\section{-1 thene}

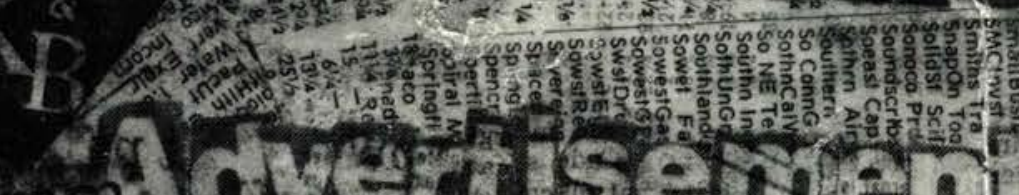

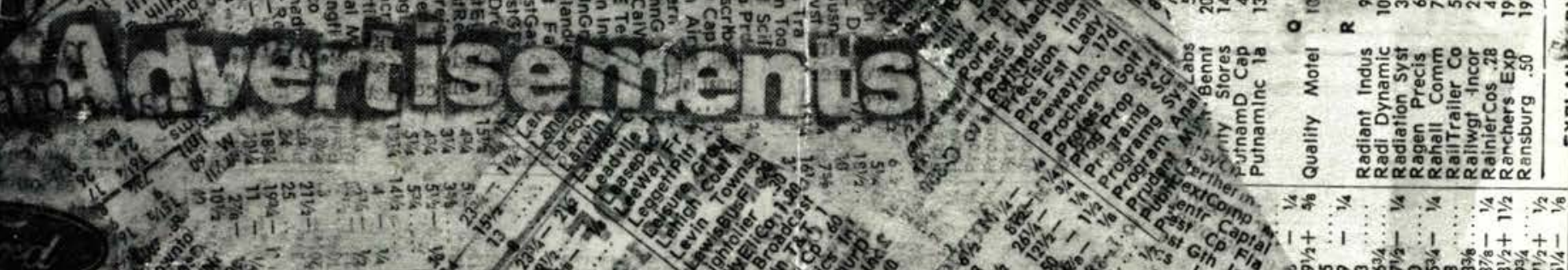

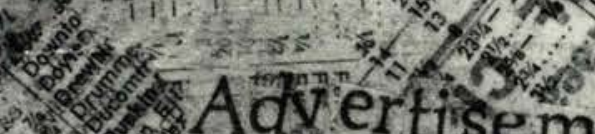
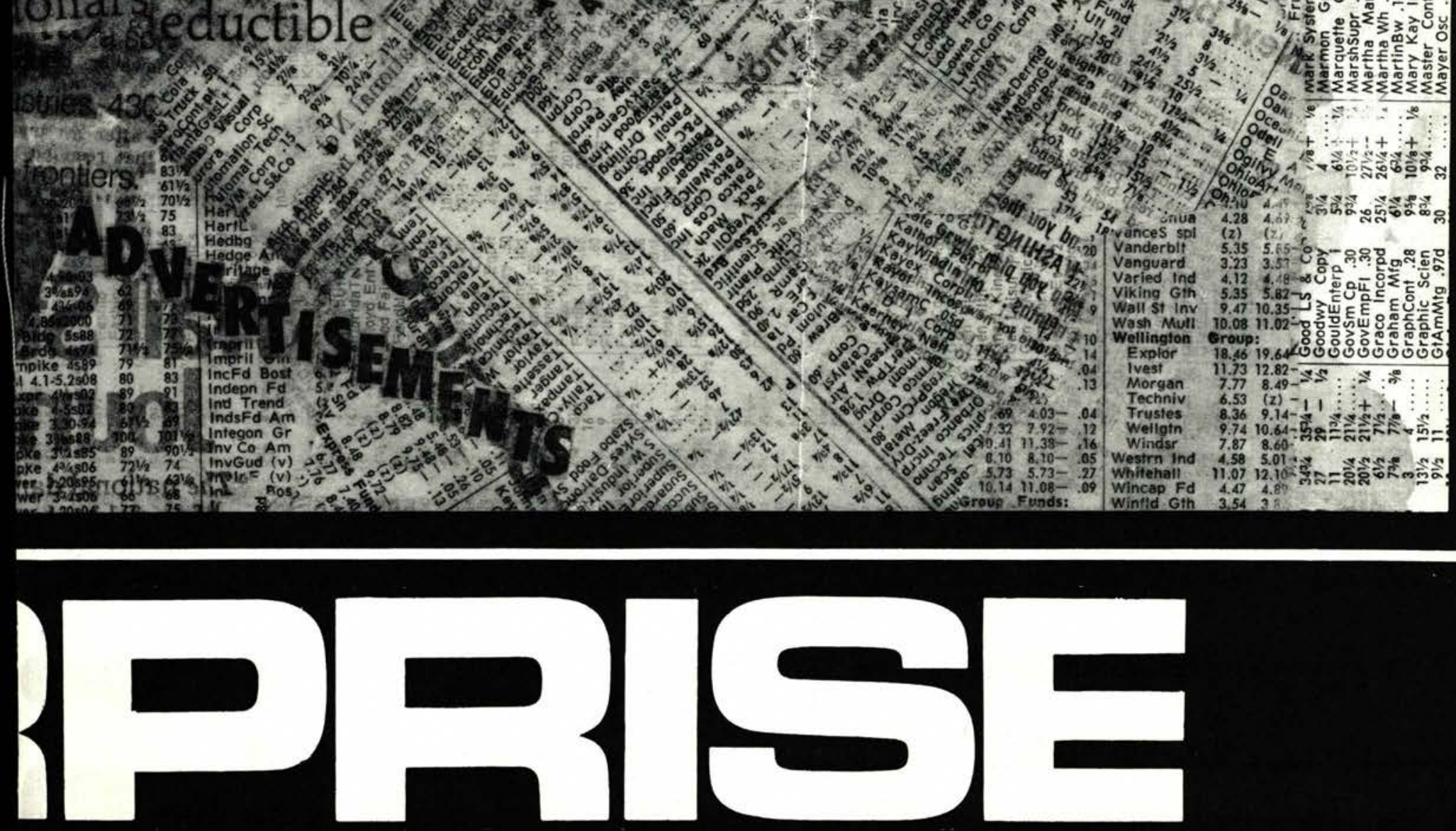


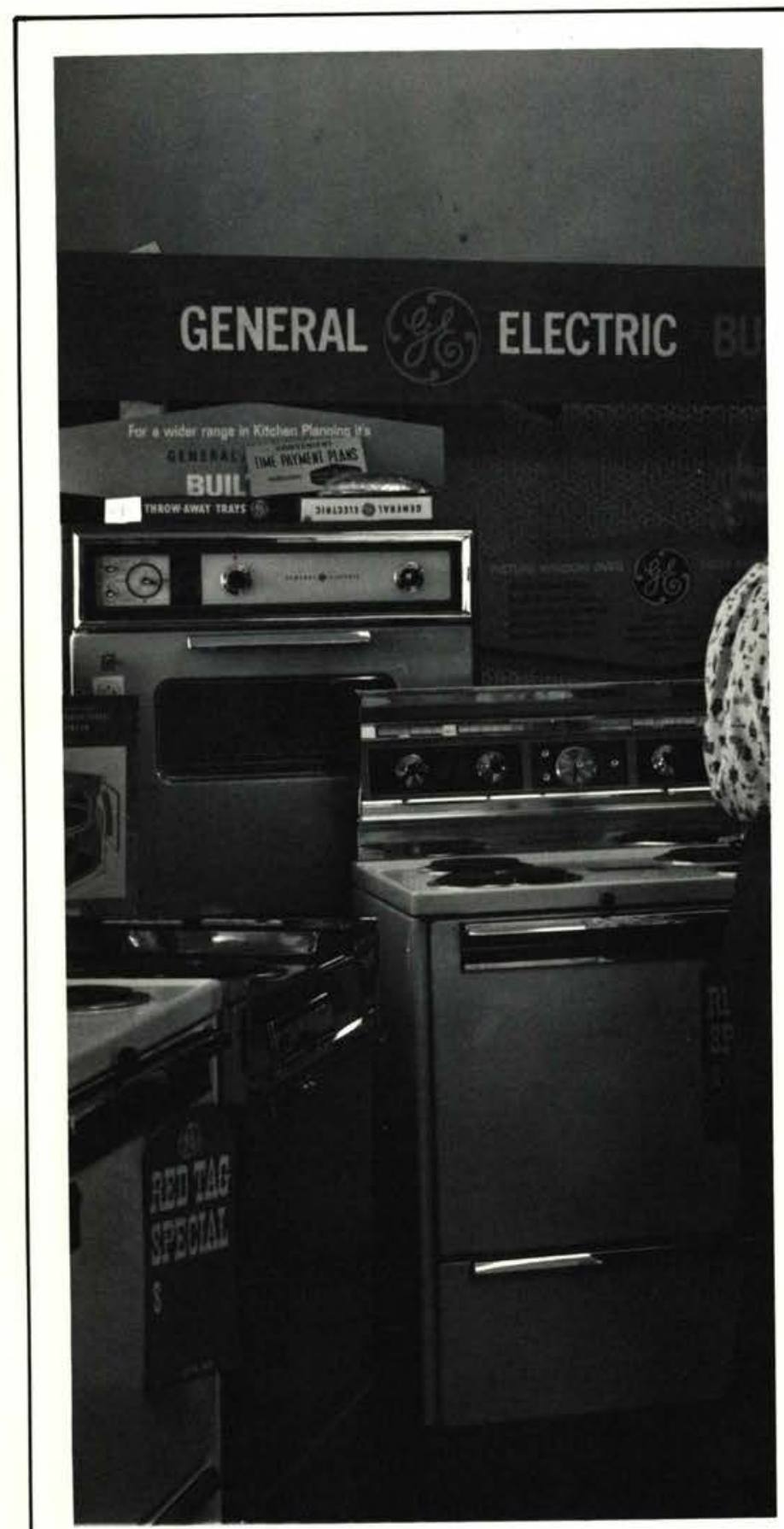

Welcome to:

\section{First Baptist Church}

401 Walnut Blvd.

\section{Rochester, Michigan}

10:00 A.M. Sunday School

11:00 A.M. Morning Worship

5:45 P.M. Youth Groups

7:00 P.M. Evening Service

7:30 P.M. Wednesday Prayer Meeting

Cedarville Hardware

Serving The

Greater Cedarville

Area 


\section{Congratulations}

Frosh

on

Keeping

Your Link

with

Miss Spradlin

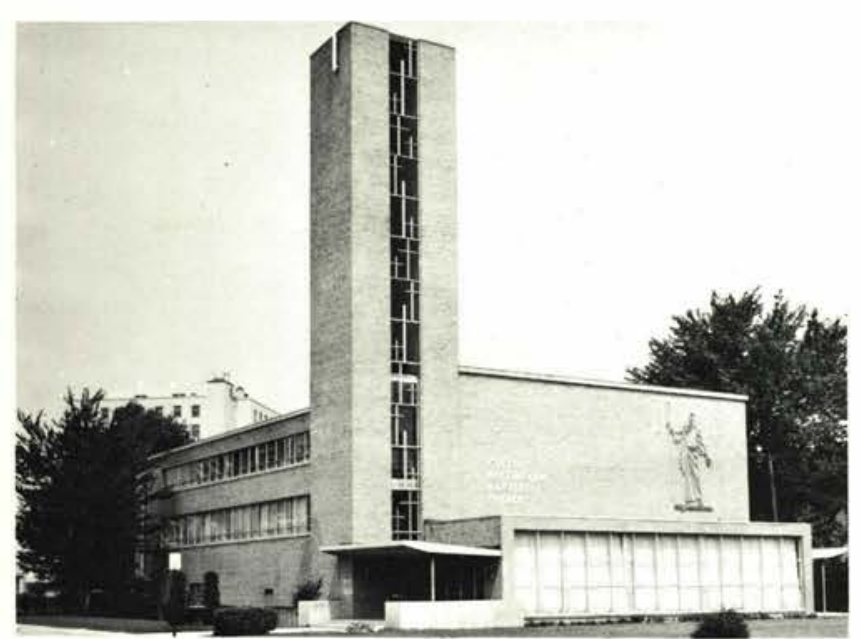

EUCLID-NOTTINGHAM BAPTIST CHURCH 18901 Lake Shore Boulevard Euclid, Ohio 44119

Dr. Wilbur C. Rooke, Pastor

Rev. Gary W. Moosey, Associate

Sunday School-9:45 A.M.

Morning Worship_-11:00 A.M.

Evening Worship_7:00 P.M.

Midweek Family Night-7:00 P.M. Wednesday

\section{Patronage}

Cedarville Farm Implement

Beal's Department Store

Cedar Cliff Beauty Salon

McDormen's Clothing

Band Box

Berean Church Supplies

Cherry's Furnishings

Famous Auto Supply

Hitchcock Jewelers Store

Xenia Home Furniture

Xenia One-Hour Martinizing

Nelson-Bone Florists

Xenia Office Supplies

Rich Jewelers

Sports Cycles of Xenia 


\section{Stephen's Rexall Store}

Cedarville, Ohio

42 N. Main

Phone 766-1771

\section{John Donley Gas \& Oil Discount}

"It's Unique"

Xenia, Ohio

58 E. Main St.

Fairborn, Ohio 500 W. Main

293 S. Main Street

"First Quality Products Only"

"Lowest Gas Prices in Town"

\section{Chaplin Cleaners}

Laundry Service

Cleaning-Shoe Repair

Phone 766-3781

Cedarville, Ohio 


\section{Cedarville Feed and Grain}

Grain, Feed, Seed, Coal, Salt, Wool, Fertilizer, Builders' Supplies and Fencing

Cedarville, Ohio

Phone 766-2021

\section{College Hill IGA}

Serving Cedarville Monday thru Saturday

Route 72, Main Street Cedarville, Ohio

\section{Village Restaurant}

"In the heart of Cedarville"

Complete Food Service 766-5318
Ring enlarged to show detail.

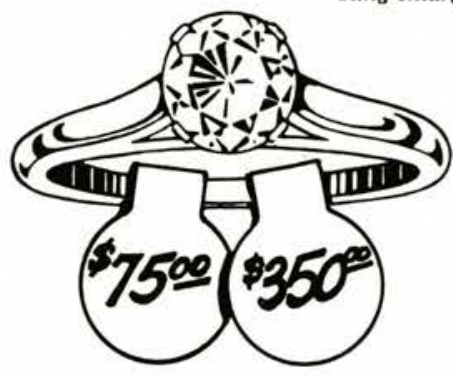

What you should look for in a diamond

Puzzled by the wide variety in diamond pricing? Confused by "discount" promises in mail-order ads and catalogs? Then you need someone you can trust to give you factual information about what to look for in a diamond. As a member firm of the American Gem Society, we have such a diamond specialist on our staff. He will be happy to properly and ethically advise you on the subtle differences in diamond quality that affect the price you pay. Come in and see us.

MEMBER AMERICAN GEM SOCIET

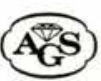

\section{Braun's Jeweler's}

744 E. Main St. Xenia

372-5071 


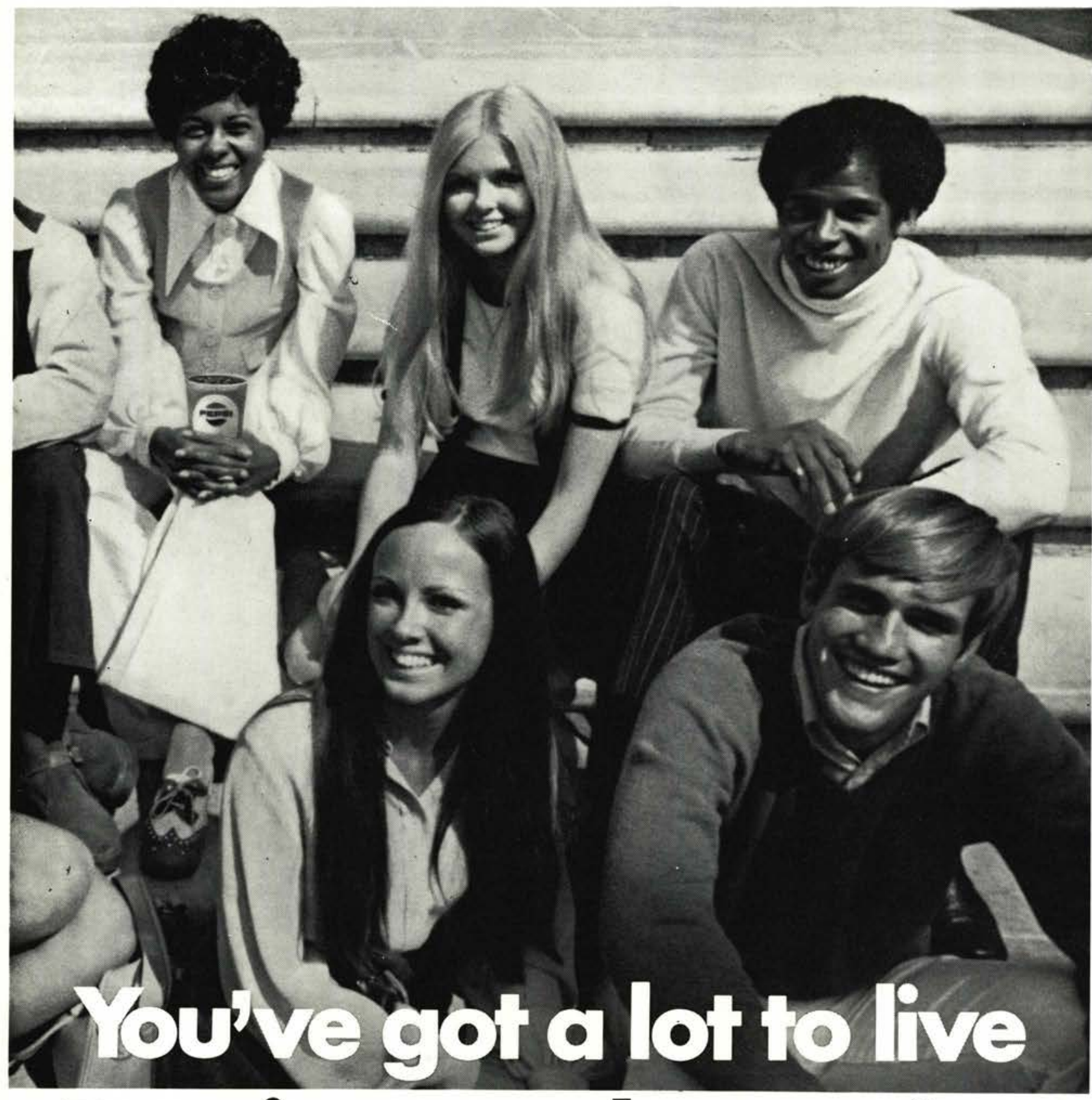

\section{Pepsi's got a lot to give}

Things are getting better.

People are learning to live every

day to the fullest. Pepsi-Cola

belongs in this kind of world.

Enjoy it. You've got a lot to live.

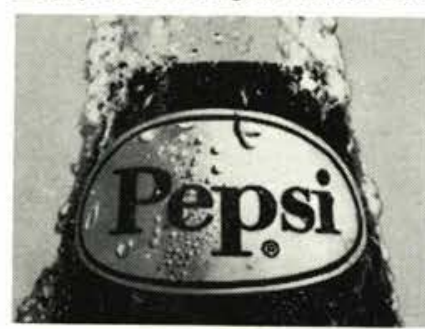




\section{Super Value}

Cedarville

Super Value

Groceries

Produce

Meats

Phone: 766-1201

Cedarville, Ohio

\section{Congratulations \\ and}

\section{Best Wishes}

To the Graduating Class

and

To the Entire School

\section{Immanuel Baptist Church}

\section{Palmetto Avenue \\ Columbus, Ohio 274-2687}

William E. Abernathy, Pastor

Sunday School

Morning Worship

Youth Groups

Evening Service

Prayer Meeting, Wed.

\section{SERVICES}

We are thankful for you!
9:30 A.M. 10:40 A.M. 6:00 P.M. 7:00 P.M. 7:30 P.M!.

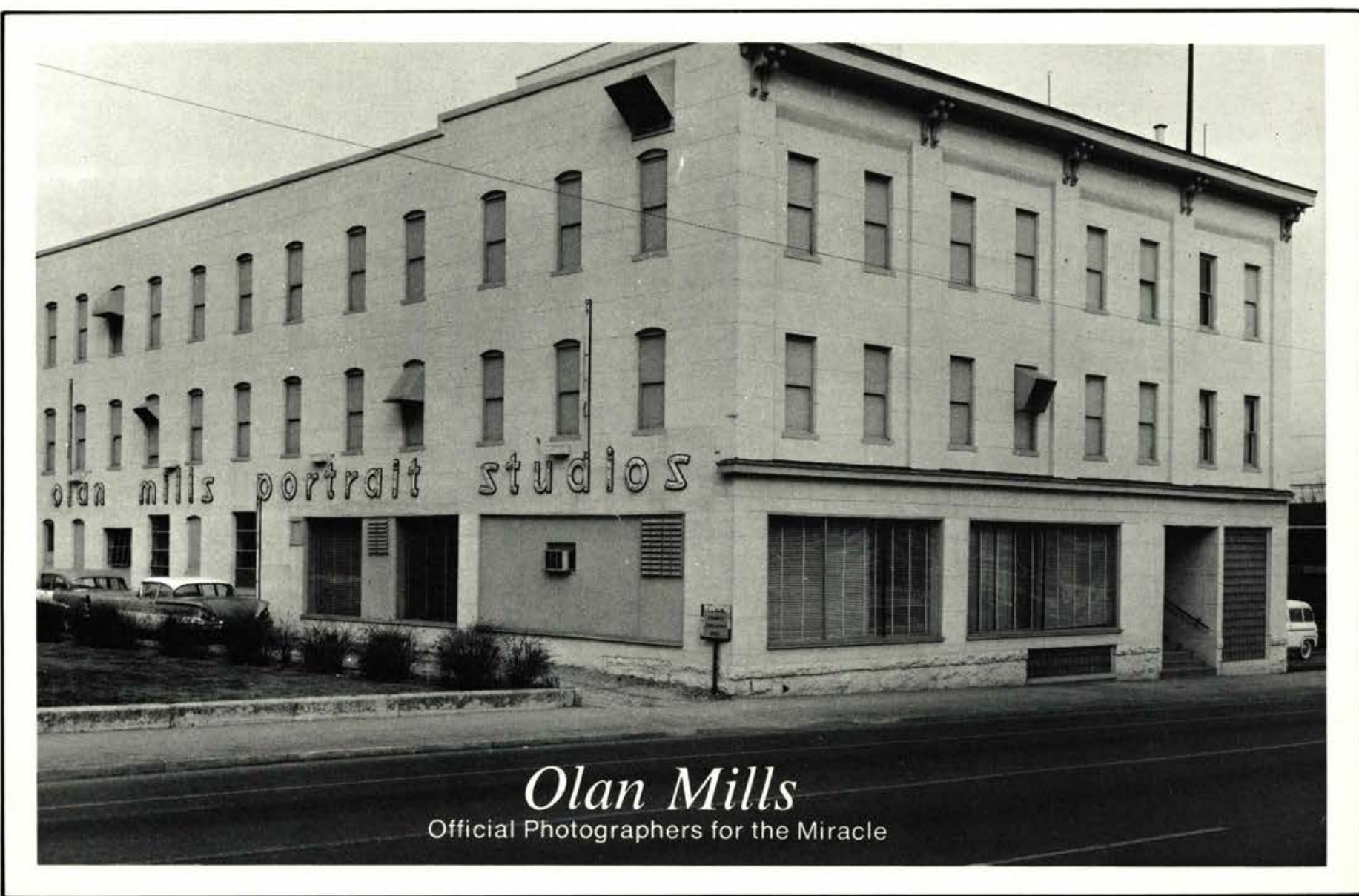




\section{Xenia \\ National Bank}

161 E. Main St.-82 N. Allison Ave.

Xenia, Ohio

Member Federal Deposit

Insurance Corporation

\section{Criterion \\ Xenia, Ohio}

Men's Clothing and Furnishings

Varsity Town-Arrow

MacGregor-Stetson

Palm Beach

Congratulations Seniors

\section{Grace Baptist Church}

23096 Center Ridge Road

Westlake, Ohio 44145

Douglas R. Couch, Pastor

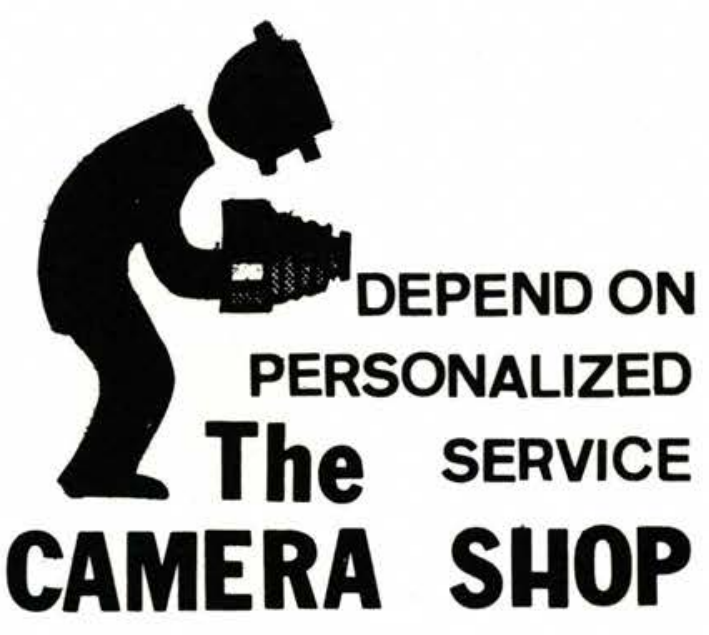

34 W. MAIN XENIA,OHIO

PHONE: 372-1469
First

Baptist

Church

\section{MacDonald, Ohio}

602 Illinois Ave.

Llberty 5-8835

J. Edgar Beckley, Jr.

Pastor 


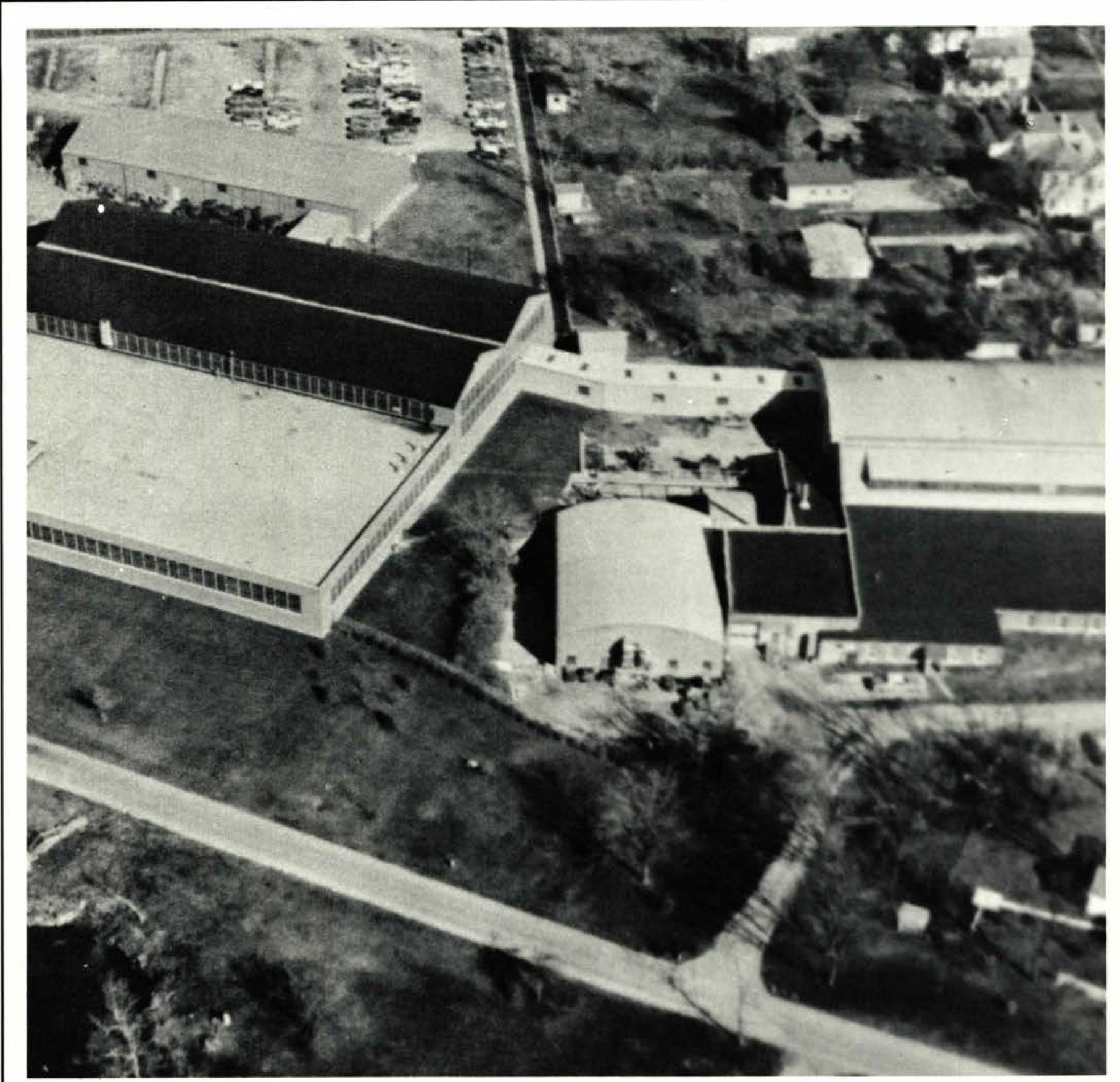

\section{MORRIS BEAN $A N D C O$.}





